\title{
Synthese von Amino- bzw. Hydrazinosilanen und -boranen, Lithiumsalzen, Iminoborenen und Borkationen
}

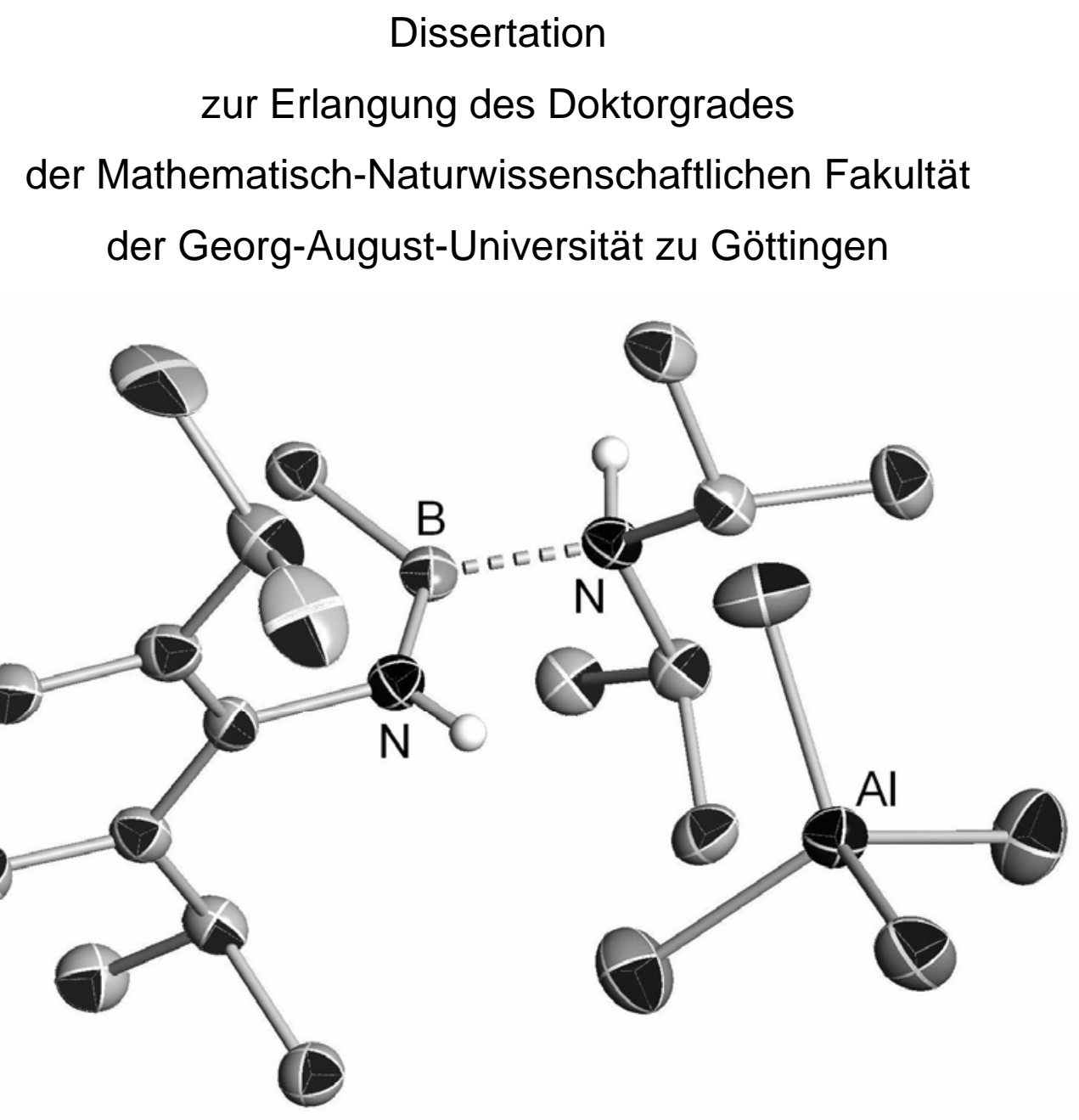

vorgelegt von

Christoph Matthes

aus Eisenach 
D 7

Referent: Prof. Dr. U. Klingebiel

Korreferent: Prof. Dr. J. Magull

Tag der mündlichen Prüfung: 23.01.2008 


\section{Vorwort und Danksagung}

Die vorliegende Arbeit wurde in der Zeit vom Januar 2006 bis Dezember 2007 unter der Leitung von Prof. Dr. U. Klingebiel am Institut für Anorganische Chemie der Georg-August-Universität Göttingen angefertigt.

Herrn Prof. Dr. U. Klingebiel danke ich hierbei für seine Ideen und Unterstützung besonders in schwierigen Zeiten. Vor allem möchte ich inm für die schöne Zeit in seiner Abteilung und die vielen Erinnerungen, die er bei mir geschaffen hat, meinen Dank aussprechen.

Danken möchte ich auch allen Mitarbeitern des Arbeitskreises von Prof. Dr. U. Klingebiel. Vor allem Andreas Fischer, Martin Görth und Christian Klingbeil sei besonderer Dank ausgesprochen. Christian Klingbeil danke ich für die Hilfe bei der Anfertigung der REM-Aufnahmen und EDX-Spektren.

Dem technischen Personal, besonders der kernresonanzspektroskopischen und massenspektroskopischen Abteilung, danke ich für die Unterstützung.

Frau Dr. S. Stückl danke ich für die Anfertigung der ESR-Spektren und der DFTRechnungen.

Herrn Dr. S. Schmatz danke ich für die große Mühe beim Versuch der Aufklärung von Reaktionsmechanismen via DFT- Rechnungen.

Für die Durchführung der Röntgenstrukturanalysen gilt besonders Prof. Dr. J. Magull, A. Hofmeister und A. Ringe, B. Heisen sowie H. Ott und Dr. S. Deuerlein besonderen Dank. Frau A. Hoffmeister sowie Herrn A. Ringe und $\mathrm{H}$. Ott danke ich für die Hilfe bei der Bearbeitung der Kristallstrukturgrafiken.

Herrn Prof. Dr. H. Nöth danke ich für die hilfreichen Diskussionen.

Der DFG danke ich für die finanzielle Unterstützung.

Bedanken möchte ich mich nochmals bei meinem Chemielehrer Herren F. Umlauf und meinem Großvater H. Räder, denen ich mein naturwissenschaftliches Grundwissen, das Interesse und vor allem das Gefühl für die Chemie zu verdanken habe. 
Diese Arbeit ist meinen Großeltern Ute und Hartfried Räder gewidmet, die mich stets unterstützt haben, die mich nie stillstehen haben lassen und die mir gleichzeitig das Gefühl geben, was man Heimat nennt. 


\section{Einleitung}

1.1 Stickstoff-Bor-Chemie 1

1.1.1 Aminoborane 1

1.1.2 Iminoborene 1

1.1.3 Boriniumionen 3

1.2 Stickstoff-Silicium-Chemie 4

1.2.1. Synthese von Silylaminen $\quad 4$

1.2.2 Cyclosilazane $\quad 5$

$\begin{array}{lll}1.2 .3 & \text { Silylhydrazine } & 6\end{array}$

1.2.4 Silatropie $\quad 7$

1.2.4.1 Thermische Umlagerung $\quad 8$

$\begin{array}{ll}\text { 1.2.4.2 Anionische Umlagerung } & 8\end{array}$

1.2.5 Lithiumsalze der Silazane/Silylhydrazine 9

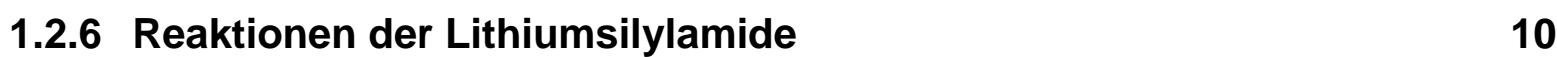

$\begin{array}{lll}1.3 & \text { Keramiken } & 12\end{array}$

2 Problemstellung 13

3 Ergebnisse und Diskussion 15

$\begin{array}{lll}3.1 & \text { Synthese und Reaktionen an Bis(1,1,3-trisila-2- } & 15\end{array}$ aza)ethylendiaminen

3.1.1. Thermische Silylgruppen-Wanderung 15

3.1.2. Bildung des Dilithium-N,N'-bis(1-trimethylsilyl-2-amido-3- 17 dimethylsilyl-trimethylsilyl)ethylendiamins unter anionischer Silylgruppen-Wanderung

3.1.3 Kristallstrukturen der Dilithiumsalze 4- 6

3.1.3.1 Dilithium-bis(1-trimethylsilyl-2-amido-3-dimethylsilyltrimethylsilyl)ethylendiamin, 4

3.1.3.2 Dilithium-bis(1-trimethylsilyl-2-amido-3-dimethylsilyltrimethylsilyl)ethylendiamin-2 THF, 5

3.1.3.3 Dilithium-bis(1-trimethylsilyl-2-amido-3-dimethylsilyltrimethylsilyl)ethylendiamin-2 TMEDA, 6

3.1.4 Bis(1,1,3-trisila-2-aza)- und Bis(1-bora-1,3-disila-2-aza)bis(silyl)ethylendiamine, 7 - 13

3.1.5 1,3-Bis(trimethylsilyl)-2,4-bis(1,1,5,5-tetra-methylpiperidino)- 
3.1.6 Kristallstrukturen der Verbindungen 8 - 12 und 14

3.1.6.1 Kristallstruktur von $8 \quad 24$

3.1.6.2 Kristallstruktur von 9

3.1.6.3 Kristallstruktur von $10 \quad 26$

3.1.6.4 Kristallstruktur von $11 \quad 27$

3.1.6.5 Kristallstruktur von $12 \quad 28$

3.1.7 Kristallstruktur von 14

3.1.8 Synthese der 2,4,7,9-Tetraaza-1-bora-3,8-disila- und 2,4,7,9- 30 Tetraaza-1,3,8-trissila-cyclononane, 15 und 16

3.2 Synthese und Reaktionen am Bis[1,1-bis(trimethylsilyl)-2-aza-3- 32 dimethylsilyl]hydrazin, 17

3.2.1. Bildung des Dilithium-N,N'-bis(1-trimethylsilyl-2-amido-3dimethylsilyl-trimethylsilyl)hydrazins unter anionischer Silylgruppen-Wanderung

3.2.2 Kristallstrukturen der Dilithiumsalze 18 und 19

3.2.2.1 Dilithium- $N, N$ '-bis(1-trimethylsilyl-2-amido-3-dimethylsilyltrimethylsilyl)hydrazin, 18

3.2.2.2 Dilithium-N,N'-bis(1-trimethylsilyl-2-amido-3-dimethylsilyltrimethylsilyl)hydrazin.2 THF, 19

3.2.3 Synthese von 20 sowie der Cycloheptane 21 und 22

3.2.3.1 Kristallstruktur von Verbindung 21

3.2.3.2 ESR-Messungen an einer Diethyletherlösung von 21

3.3 Reaktionen an lithiierten Di(tert-butyl)fluorsilylamiden

3.3.1 Reaktionen des Lithium-tert-butyl-di(tert-butyl)fluorsilylamids mit Trimethylalan

3.3.2 Kristallstruktur des Lithium-tert-butyl-di(tert-butyl)methylsilyl-amids, 25

3.3.3 Reaktionen des Lithium-tert-butyl-di(tert-butyl)fluorsilylamids mit Fluorboranen

3.3.3.1 Kristallstruktur von 27

3.3.4 Reaktionen des Lithium-bis[di(tert-butyl)fluorsilyl]amids mit Alanen und Chlortrimethylsilan 
3.3.4.1 Reaktion des Lithium-bis[di(tert-butyl)fluorsilyl]amids mit Trimethylalan

3.3.4.2 Reaktion des Lithium-bis[di(tert-butyl)fluorsilyl]amids mit Trichloralan

3.3.4.3 Reaktion des Lithium-bis[di(tert-butyl)fluorsilyl]amids mit Chlortrimethylsilan

3.3.4.4 Kristallstruktur des Bis[di(tert-butyl)methylsilyl]amins, 28

3.3.4.5 Kristallstruktur des Bis[di(tert-butyl)chlorsilyl]amins, 29

3.4 Synthese von sekundären Fluorborylaminen 51

3.4.1 Synthese von Fluor-2,6-diisopropylanilino-boranen 51

3.4.2 Kristallstrukturen der Verbindungen 31 - 33 52

3.4.2.1 Kristallstruktur von $\mathbf{3 1}$ 52

3.4.2.2 Kristallstruktur von 32 54

3.4.2.3 Kristallstruktur von 33 55

3.4.3 Umsetzungen von 31 - 33 mit Lithiumorganylen, 34 - 36

3.4.4 Kristallstrukturen der Verbindungen 34 - 36

3.4.4.1 Kristallstruktur von $34 \quad 57$

3.4.4.2 Kristallstruktur von $35 \quad 58$

3.4.4.3 Kristallstruktur von $36 \quad 59$

3.4.5. Synthese der Fluor-triphenylsilyl-borane 37 - $39 \quad 60$

3.4.6 Kristallstrukturen von 37 und 38

3.4.6.1 Kristallstruktur von $37 \quad 61$

3.4.6.2 Kristallstruktur von $38 \quad 62$

3.4.7 Synthese von tert-Butyldimethylsilylamino-fluor-boranen, $\quad 63$ $40-42$

3.4.8 Synthese der tert-Butyldimethylsilylimino-borene, 43 und 44

3.4.9 Additionsreaktionen an $\mathbf{4 4}$ mit Lewis-Basen 65

3.4.10 Kristallstrukturen der Verbindungen 45 und $47 \quad 67$

$\begin{array}{ll}\text { 3.4.10.1 Kristallstruktur von } 45 & 67\end{array}$

$\begin{array}{ll}\text { 3.4.10.2 Kristallstruktur von } 47 & 68\end{array}$ 
3.5.1 Umsetzung des Fluor-bis[bis(trimethylsilyl)amino]borans mit $\mathrm{AlCl}_{3}$

3.5.2 Kernresonanzspektroskopische Untersuchungen an 48

3.5.3 Kristallstruktur des (Trichloralan-trimethylsilyl-amin)bis(trimethylsilyl)amino-borens, 48

3.5.4 Quantenchemische Berechnungen 72

3.5.4.1 Berechnungen zu einem $\mathrm{AlCl}_{3} \mathrm{~F}$-Übergangszustand 73

3.5.4.2 Berechnungen zu einem Übergangszustand mit 73 drei- und vierfach koordinierten Stickstoffatomen

3.5.4.3 Berechnungen zu einem Übergangszustand mit jeweils vierfach koordinierten Stickstoffatomen

3.5.5 Umsetzung des Fluor-bis[bis(trimethylsilyl)amino]borans mit AICIMe $2, \mathrm{AlBr}_{3}$ und $\mathrm{GaCl}_{3}$

3.5.6 Synthese der Verbindungen 52 - 58

3.5.6.1 Kristallstruktur von 58

3.5.7 Umsetzung des tert-Butyltrimethylsilylamino-bis(tri80 methylsilyl)amino-fluor-borans mit $\mathrm{AlCl}_{3}$ und $\mathrm{GaCl}_{3}$

3.5.7.1 Kristallstruktur von 59 81

3.5.7.2 Kristallstruktur von 60

3.5.8 Versuch der Darstellung eines Bis(fluorsilylaminoborans)

3.5.8.1 Kristallstruktur von 61

3.5.9 Synthese arylsubstituierter Fluorsilylaminoborane

3.5.9.1 Kristallstruktur von 63

3.5.10 Umsetzung von 62 und 63 mit $\mathrm{AlCl}_{3}$ 86

3.5.10.1 Kristallstruktur von 64 89

3.5.10.2 Kristallstruktur von 65 89

3.5.10.3 Kristallstruktur von 66 90

3.5.11 Umsetzung von 27 mit $\mathrm{AlCl}_{3}$ in $\mathrm{CH}_{2} \mathrm{Cl}_{2}$

3.5.11.1 Kristallstruktur von 67

3.5.12 Synthese von Fluor-hydrazon- bzw. Fluor-ketazin-

silylamino-boranen ${ }^{[75]}$

3.5.12.1 Kristallstruktur von 68 
3.5.12.3 Kristallstruktur von 70

3.5.12.4 Kristallstruktur von 71

3.5.12.5 Synthese von Hydrazonen mit der Struktureinheit N(Si)-BF-N(Si)

3.5.12.6 Kristallstruktur von 73

3.5.13 Synthese bisher unbekannter Fluorborane als Vor-

stufe für die Bildung von Boreniumionen

3.5.13.1 Kristallstruktur von 74

3.5.13.2 Kristallstruktur von 75

3.6 Keramik 48X

4 Zusammenfassung

5 Ausblick

128

6 Präparativer Teil

6.1 Allgemeine Arbeitsbedingungen

6.2 Arbeitsvorschriften für die dargestellten Verbindungen 1 - 75

6.3 Behandlung und Entsorgung der Abfälle

7 Charakteristische Daten der vorgestellten Verbindungen

7.1 Verwendete Messgeräte und -methoden

7.2 Physikalische Daten der dargestellten Verbindungen 1- 75

7.3 Übersicht über die neuen Verbindungen 225

8 Literaturverzeichnis

Publikationen

Lebenslauf 
Abkürzungen und Einheiten

\begin{tabular}{|c|c|c|c|}
\hline $\mathrm{Bu}$ & Butyl, $\mathrm{C}_{4} \mathrm{H}_{9}$ & {$[\mathrm{M}]^{+}$} & Molekülion \\
\hline bzw. & beziehungsweise & $\mathrm{m}$ & Mulitiplett \\
\hline $\mathrm{C}$ & Celsius & $\mathrm{m} / \mathrm{z}$ & Masse-Ladungsverhältnis \\
\hline $\mathrm{cm}$ & Zentimeter & mbar & Millibar \\
\hline$\delta$ & chemische Verschiebung & $\mathrm{Me}$ & Methyl, $\mathrm{CH}_{3}$ \\
\hline$\Delta$ & Differenz & $\mathrm{mL}$ & Milliliter \\
\hline$d$ & Dublett & $\mu \mathrm{m}$ & Mikrometer \\
\hline d.h. & das heißt & MS & Massenspektroskopie \\
\hline dd & Dublett vom Dublett & $n$ & normal, unverzweigt \\
\hline DFT & Dichtefunktionalmethode & $\mathrm{nm}$ & Nanometer \\
\hline $\mathrm{dt}$ & Dublett vom Triplett & NMR & $\begin{array}{l}\text { Nuclear Magnetic Resonance, } \\
\text { Kernmagnetische Resonanz }\end{array}$ \\
\hline$E$ & Element & ORTEP & Oak Ridge Thermal Ellipsoid Plot \\
\hline$E_{0}$ & Anregungsenergie & $\mathrm{Ph}$ & Phenyl, $\mathrm{C}_{6} \mathrm{H}_{5}$ \\
\hline E.I. & Elektronenstoßionisation & pm & Picometer \\
\hline EDX & $\begin{array}{l}\text { Energy Dispersive X-ray } \\
\text { Analysis, Energiedispersive } \\
\text { Röntgenspektroskop(ie) }\end{array}$ & ppm & parts per million \\
\hline ESR & Elektronenspinresonanz & R, R', R" & Rest, Substituent \\
\hline et al. & und anderen & REM & Rasterelektronenmikroskop(ie) \\
\hline $\mathrm{eV}$ & Elektronenvolt & RT & Raumtemperatur \\
\hline o & Grad & $\Sigma$ & Summe \\
\hline g & Gramm & s & Singulett \\
\hline geb. & geboren(e) & S. & Seite \\
\hline$\Delta \mathrm{G}_{0}$ & freie Reaktionsenthalpie & Sdp. & Siedepunkt \\
\hline $\mathrm{h}$ & Stunde & SEM & Sekundärelektronenmikroskop(ie) \\
\hline Hal & Halogen(id) & sept & Septett \\
\hline $\mathrm{Hz}$ & Hertz & $\mathrm{t}$ & Triplett \\
\hline HMDS & Hexamethyldisilazan & tert & tertiär \\
\hline i.d.R. & in der Regel & THF & Tetrahydrofuran \\
\hline IR & Infrarot & TMEDA & Tetramethylethylendiamin \\
\hline $\mathrm{J}$ & Kopplungskonstante & TMP & 1,1,5,5-Tetramethylpiperidin \\
\hline Jh. & Jahrhundert & u & unit \\
\hline $\mathrm{K}$ & Kelvin & vgl. & Vergleich \\
\hline kcal & Kilokalorie & $\Varangle$ & Winkel \\
\hline $\mathrm{keV}$ & Kiloelektronenvolt & $\mathrm{X}$ & Substituent \\
\hline Lsg. & Lösung & z.B. & zum Beispiel \\
\hline $\mathrm{M}$ & Metallion & $\ddagger$ & nicht isoliertes Zwischenprodukt \\
\hline
\end{tabular}




\section{$1 \quad$ Einleitung}

\subsection{Stickstoff-Bor-Chemie}

\subsubsection{Aminoborane}

Bereits 1881 synthetisierte Mixter mit dem Trifluorboran-Ammoniakaddukt ein stabiles, bei Raumtemperatur unzersetzt isolierbares Boranaddukt ${ }^{[1]}$. Die Darstellung weiterer Borverbindungen, entstehend aus der Reaktion des Trimethylborans mit Hydroxiden, wurde ebenfalls in der zweiten Hälfte des 19. Jh. durch Frankland und Brown beschrieben ${ }^{[2,3]}$.

Erst die Synthese des Borazins 1926 durch Stock und Pohland ${ }^{[4]}$ kann jedoch als Beginn der modernen Bor-Stickstoff-Chemie angesehen werden. Seit dieser Zeit sind zahlreiche Verbindungen dieser Stoffklasse dargestellt worden.

Weitgehend findet man hierbei das Boratom dreifach koordiniert. Auf Grund der vier zur Verfügung stehenden Valenzorbitale ist jedoch auch eine Vierfachkoordination, wie z.B. im oben genannten Trifluorboran-Ammoniakaddukt, möglich. Höhere Koordinationen des Bors wurden ab den 1960er Jahren in Metallaboranen und -boriden sowie in Polyboranen und Carboranen nachgewiesen ${ }^{[5,6]}$ und über Mehrzentrenbindungen erklärt ${ }^{[7,8]}$.

\subsubsection{Iminoborene*}

Verbindungen mit sp-hybridisiertem, zweifach koordiniertem Bor sind thermodynamisch instabil. Zwar sind z.B. Iminoborene isoelektronisch gegenüber den Alkinen und stimmen auch in der Summe ihrer Atomradien mit ihnen überein, jedoch führt die Polarität der B-N Bindung i.d.R. zu einer Oligomerisierung.

\footnotetext{
* Vergleichbar zur Nomenklatur der organischen Chemie werden Doppelbindungssysteme in der anorganischen Chemie zunehmend analog benannt. So werden Verbindungen mit zweifach koordiniertem Stickstoff ( $I \mathrm{~min}$ ) und dreifach gebundenem Silicium (Silen) bzw. zweifach koordiniertem Bor (Boren) im Folgenden als Iminosilene (statt Iminosilane) bzw. Iminoborene (statt Iminoborane) bezeichnet.
} 
Die ersten Boroxane ${ }^{[9]}$, Borthiane ${ }^{[10]}$, Borselenane ${ }^{[11]}$, Alkylidenborane ${ }^{[12]}$ oder Iminoborene ${ }^{[13]}$ konnten in den 1970er Jahren zunächst in der Gasphase nachgewiesen werden.

Folgende Studien, besonders in den Arbeitskreisen Paetzold und Nöth, zeigen, dass weniger elektronische Effekte, sondern vielmehr eine sterische Abschirmung des Stickstoff- und vor allem des Boratoms zu einer kinetischen Stabilisierung der Iminoborene führt, was seit Mitte der 1980er Jahren die Isolierung zahlreicher Vertreter dieser Stoffklasse ermöglichte ${ }^{[13,14]}$.

Hierbei wurden unterschiedlichste Synthesewege beschritten. Neben der Spaltung von Ringen und Umlagerungsreaktionen stellte sich die thermisch oder chemisch induzierte Eliminierung von Molekülen (z.B. Stickstoff, Halogensilanen oder Halogenwasserstoff) aus den entsprechenden Vorstufen als besonders effektive Darstellungsmethode heraus ${ }^{[15]}$.

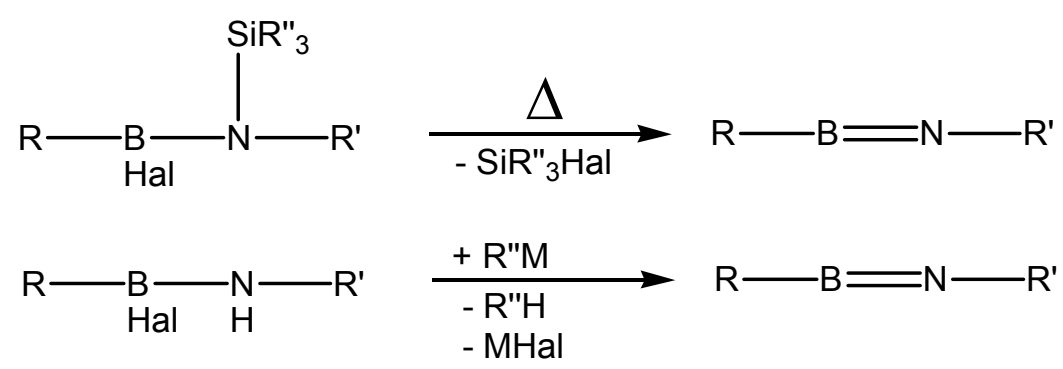

Gleichung 1: Darstellung von Iminoborenen über thermische und chemische Eliminierungsreaktionen

Zahlreiche Folgereaktionen sind seitdem bekannt ${ }^{[13]}$. So verhalten sich die Iminoborene bei Cycloadditions-, Insertions-, En- oder Adduktreaktionen ähnlich den Alkinen oder auch Iminosilenen. Bei Reaktionen an heteronuclearen Verbindungen findet man auf Grund der unterschiedlichen Polaritäten stets eine selektive Reaktivität vor, indem das Boratom als Lewis-Säure und der Stickstoff als entsprechende Base fungieren. Elektrophile, wie Trihalogenalane oder -gallane lagern sich als Addukte an den Stickstoff an ${ }^{[16]}$. Ein röntgenstruktureller Nachweis dieser Verbindungsklasse konnte bisher nicht erbracht werden.

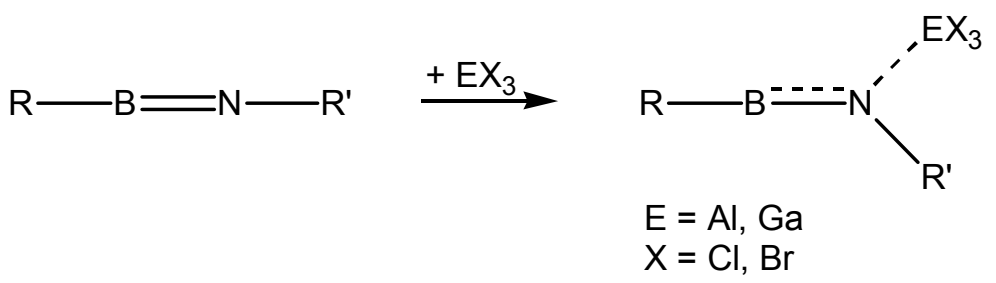

Gleichung 2: Adduktbildungen von Trihalogenalanen bzw. -gallanen an Iminoborenen 
Bei Anlagerungen der Halogenborane an Aminoiminoborenen findet hingegen eine Cyclisierung mit Halogenid-Wanderung statt. Im Gegensatz zur oben beschriebenen Anlagerung der Trihalogenalane oder -gallane bleibt hierbei das Boratom nicht in der Zweifachkoordination bestehen. Kernresonanz- und röntgenspektroskopische Untersuchungen zeigen eine Drei- bzw. Vierfachkoordination der Boratome ${ }^{[15,17]}$.
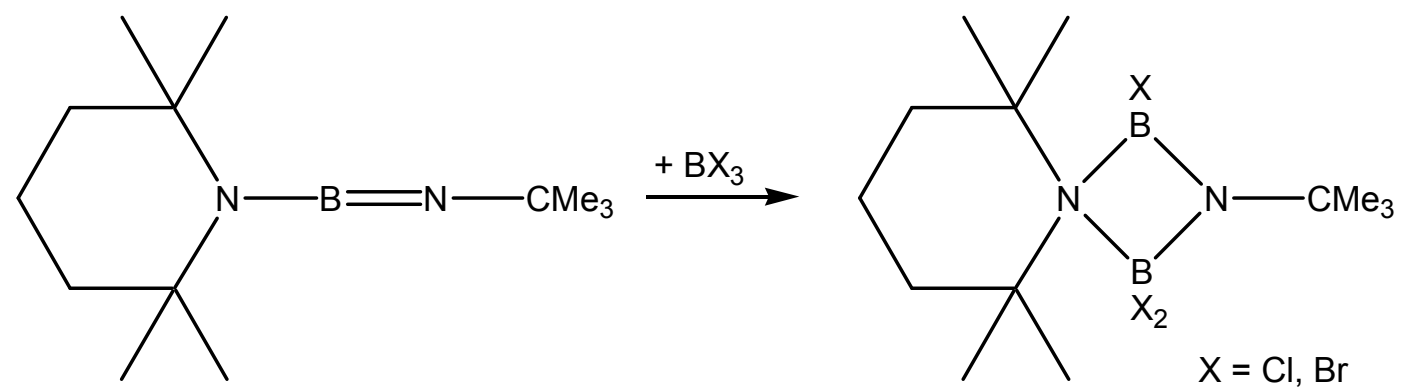

Gleichung 3: Cycloadditionsreaktion am (2.2.6.6-Tetramethylpiperidino)-tert-butyliminoboren ${ }^{\text {[17] }}$

\subsubsection{Boriniumionen}

Neben den Iminoborenen wurde Bor in der Zweifachkoordination bereits $1906{ }^{[18]}$ als Kation postuliert und Ende der 1950er Jahre erstmals von Parry ${ }^{[19]}$ als Spaltprodukt des Diborans nachgewiesen. Besonders im Arbeitskreis Nöth konnten seit den 1970er Jahren zahlreiche Boriniumionen dargestellt und strukturell gesichert werden ${ }^{[20]}$. Bei der Synthese wird von Dihalogenboranen mittels einer Lewis-Säure das Halogenanion abgespalten und somit eine Trennung der Spezies in An- und Kationen erreicht ${ }^{[20]}$.

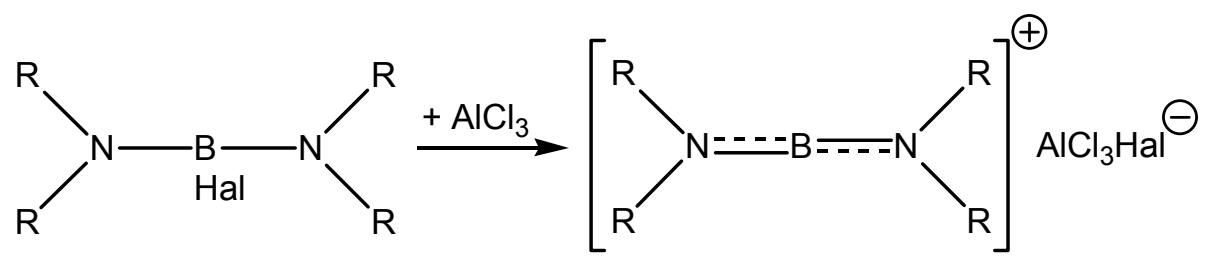

Gleichung 4: Mögliche Darstellung von Boriniumionen

Wie bei den Iminoborenen zeigen Röntgenstrukturanalysen auch hier ein linear umgebenes Borkation. Ist dieses an Stickstoffatome gebunden, so findet man eine Bindungsverkürzung der $\mathrm{B}-\mathrm{N}$-Bindung auf Grund des $p_{\Pi}-p_{\Pi}-$ Rückbindungsanteils vom freien Elektronenpaar des Stickstoffs zum Bor. Auch hier sind sterisch 
anspruchsvolle Substituenten für die Stabilität solcher Kationen weitaus entscheidender als ein Absenken der Bindungspolarität durch push (am Bor)- bzw. pull (am Stickstoff)- Effekte ${ }^{[20]}$.

\subsection{Stickstoff-Silicium-Chemie}

70 Jahre vor der Darstellung des ersten Bor-Stickstoff-Addukts synthetisierte Davis 1812 das $\mathrm{SiF}_{4} \cdot 2 \mathrm{NH}_{3}$-Addukt ${ }^{[21]}$, welches Ladenburg als die erste Stickstoff-Siliciumverbindung beschreibt ${ }^{[22]}$. Seitdem wurde in der Chemie damit begonnen, siliciumorganische Verbindungen gezielt herzustellen und zu untersuchen. Um 1955 waren jedoch erst 150 Stickstoff-Siliciumverbindungen bekannt ${ }^{[23]}$. In der zweiten Hälfte des 20. Jh. wurde die Forschung bezüglich der Silazane intensiviert, sodass 1995 über 10.000 Verbindungen bekannt waren.

\subsubsection{Synthese von Silylaminen}

Die Synthese von Silylaminen kann auf unterschiedliche Weise erfolgen. Am einfachsten ist die Aminolyse von Chlor-, Brom- oder Jodsilanen ${ }^{\text {[23]. }}$

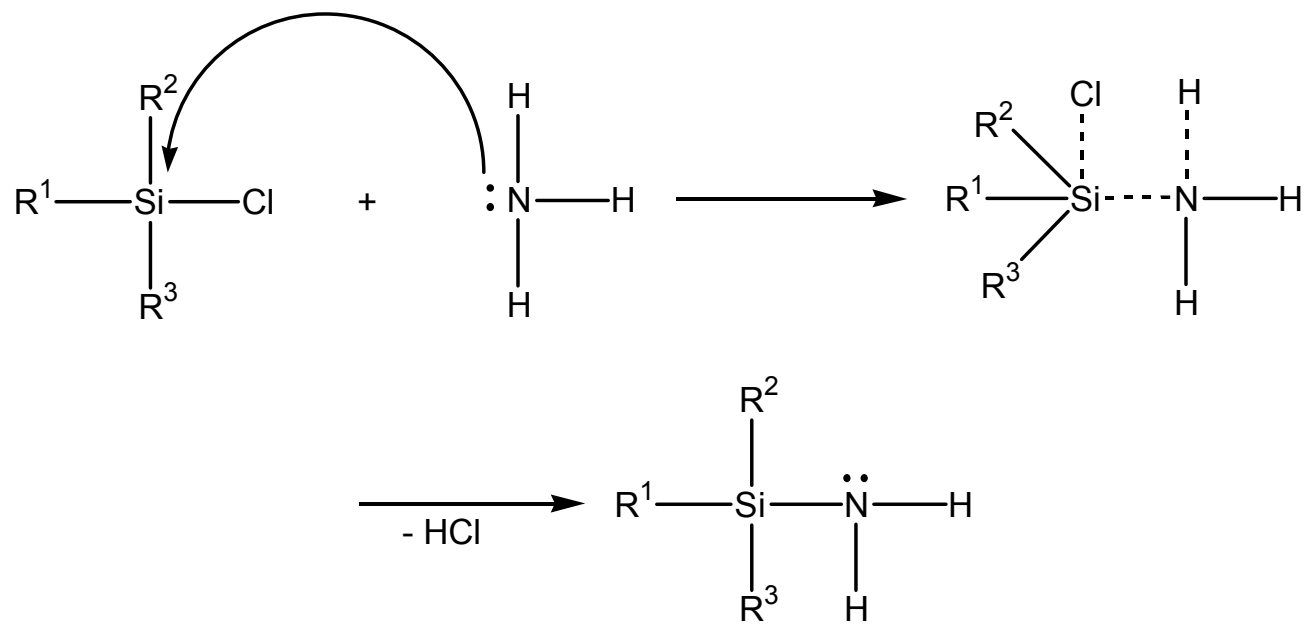

Gleichung 5: Synthese von Silylaminen über Chlorsilane

Neben Ammoniak können auch primäre oder sekundäre Amine zur Reaktion gebracht werden. Sterisch anspruchsvollere Substituenten am Stickstoff behindern eine Reaktion. Durch Metallierung des Amins mit Alkalimetallen oder Magnesiumderivaten wird die Substitutionsreaktion erleichtert ${ }^{[24]}$. 
Reaktionen von Brom- oder lodderivaten können höhere Ausbeuten liefern. Fluorsilane tendieren auf Grund der stabilen Si-F-Bindung nicht zur Abspaltung von Fluorwasserstoff.

Durch eine Metallierung des Amins z.B. mit Butyllithium wird jedoch, auf Grund der erhöhten Basizität des Stickstoffs, die Reaktivität mit Fluorsilanen erhöht:<smiles>[R]N([R])[Si]([R])([R])N([R])[R]</smiles>

Gleichung 6: Synthese von Silylaminen über Fluorsilane

\subsubsection{Cyclosilazane}

Neben den kettenförmigen Silazanen, welche über Kondensations- oder Substitutionsreaktionen an Silylaminen hergestellt werden können, sind inzwischen zahlreiche Cyclosilazane bekannt. Die ersten wurden 1948 von Brewer und Haber durch Umsetzung von Dialkyldichlorsilanen mit Ammoniak bei $-78^{\circ} \mathrm{C}$ hergestellt ${ }^{[25]}$.<smiles>[R][Si]([R])(Cl)Cl</smiles><smiles>[R][Si]([R])(NC(C)C)C(C)(C)C</smiles>

Gleichung 7: Synthese von Cyclotri- bzw. Cycloteterasilazanen ${ }^{[25]}$

Die ersten viergliedrigen Cyclodisilazane wurden 1961 von Fink et al. durch Thermolyse acyclischer Silazane dargestellt ${ }^{[26,27]}$. Synthesewege von vier- bzw. sechsgliedrigen Ringen laufen über Kondensationsreaktionen von Aminosilanen ab ${ }^{[28]}$. Heteroatome wie Bor, Phosphor oder Germanium können ebenfalls an oder in die Ringe gebunden werden ${ }^{[28]}$. 


\subsubsection{Silylhydrazine}

Aylett und Wannagat isolierten Mitte der 1950er Jahre die ersten stabilen Silylhydrazine, wobei ähnlich zur Synthese der Silylamine zum einen die Umsetzung von wasserfreiem Hydrazin mit Chlor-, Brom- oder lodsilanen unter Zugabe eines

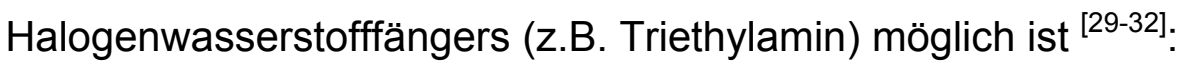

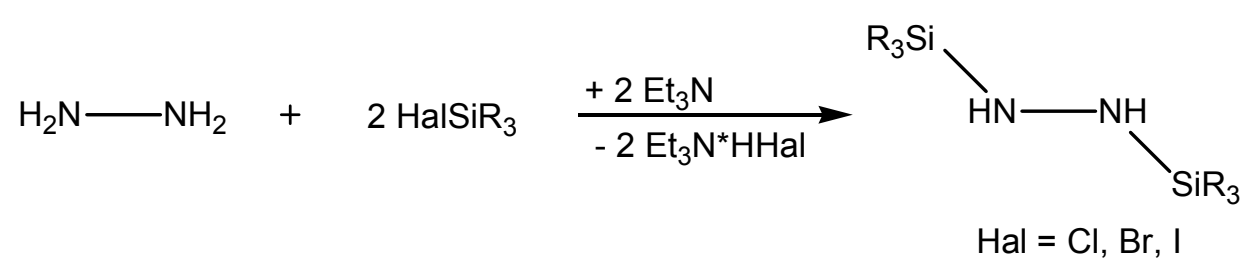

Gleichung 8: Synthese von Silylhydrazinen

Zum anderen besteht auch hier die Möglichkeit, über Reaktionen von metallierten Hydrazinen mit Halogensilanen das gewünschte Silylhydrazin zu erhalten:

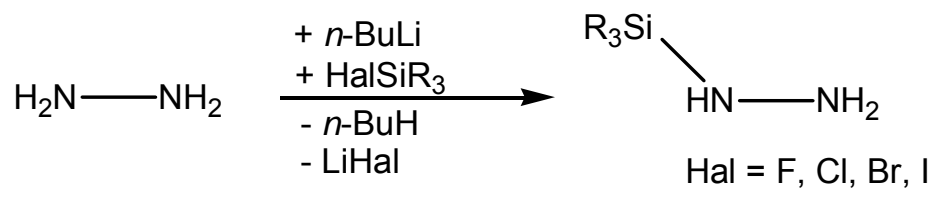

Gleichung 9: Synthese von Silylhydrazinen

Vorteile der letztgenannten Methode liegen, neben der oftmals vereinfachten Synthese, auch in der Möglichkeit, asymmetrisch substituierte Bis-, Tris- bzw. Tetrakis(silyl)hydrazine mit sterisch anspruchsvollen Substituenten über die Reaktion

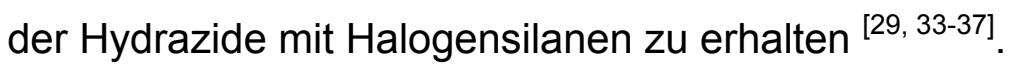




\subsubsection{Silatropie}

Als Silatropie bezeichnet man das "Wandern" von Silylgruppen innerhalb eines Moleküls. Silylgruppen lösen sich dabei nach einem $\mathrm{S}_{\mathrm{N}} 2$-Mechanismus vom schwächer basischen Atom ab und lagern sich am stärker basischen Zentrum an.

Beim Kohlenstoff sind ebenfalls intramolekulare Umlagerungen von Phenyl- und Methylgruppen bekannt. Im Vergleich hierzu weisen Silylgruppen jedoch eine höhere Mobilität auf. Dies ist hauptsächlich darauf zurückzuführen, dass Silicium im Vergleich zu Kohlenstoff pentakoordinierte Übergangszustände bilden kann, was die 1,3-sigmatrope oder dyotrope Umlagerung eines Silylsubstituenten erleichtert ${ }^{[38,39]}$.

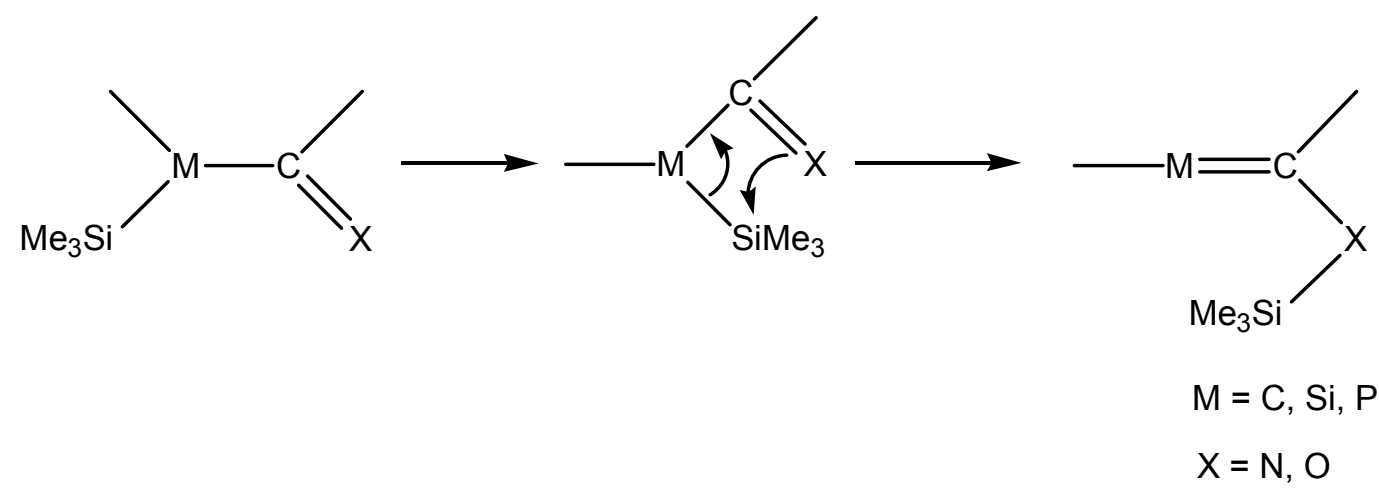

Gleichung 10: Elektrophile 1,3-sigmatrope Umlagerung am Beispiel einer Keto-Enol-Tautomerie

Man unterscheidet mehrere Arten der Silatropie:

- Thermische Umlagerungen

- Anionische Umlagerungen

- Saure Umlagerungen

- Silatropien zwischen Heteroatomen

- Radikalische Umlagerungen

- Dyotrope Umlagerungen 


\subsubsection{Thermische Umlagerung}

U. Wannagat beobachtete 1961, dass beim Aminodimethylsilyl-bis(trimethylsilyl)amin bei $200-240^{\circ} \mathrm{C}$ eine Isomerisierung zum 1,1,1,3,3,5,5,5-Octamethyl-1,3,5trisila-2,4-diazan durch Wanderung einer Trimethylsilylgruppe stattfindet ${ }^{[23]}$.

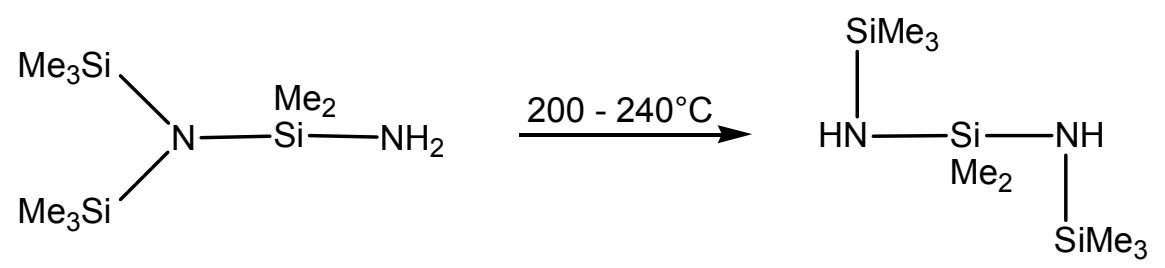

Gleichung 11: Thermische Umlagerung beim Aminodimethylsilyl-bis(trimethylsilyl)-amin

\subsubsection{Anionische Umlagerung}

Durch Metallierung eines Amins, z.B. mit Alkalimetallen oder mit organometallischen Verbindungen wie Butyllithium, spaltet sich das Proton am Stickstoff ab. Es bildet sich molekularer Wasserstoff bzw. das Proton verbindet sich mit dem Organylrest hier zu Butan.

Durch die Metallierung entsteht ein Amid, welches im Vergleich zum Amin stärker basisch ist. Auf Grund der unterschiedlichen Basizitäten des Stickstoffs wird der Energieniveauunterschied der beiden Isomere vergrößert. Die geringere Aktivierungsenergie, die für eine Silylgruppenwanderung nötig ist, zeigt sich im Vergleich zu den Umlagerungstemperaturen der gleichen oder ähnlichen unmetallierten Silylamine ${ }^{[27,39,40]}$.

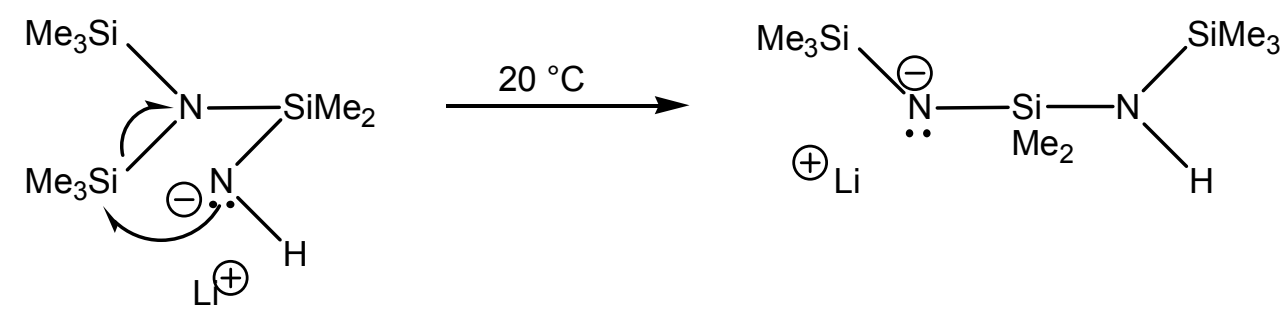

Gleichung 12: Anionische Umlagerung beim 4-Lithium-

1,1,1,3,3,4-Hexamethyl-2-trimethylsilyl-1,3,-disila-2-aza-4-amid ${ }^{[27,39,40]}$ 


\subsubsection{Lithiumsalze der Silazane/Silylhydrazine}

Wie bereits beschrieben, lässt sich die $\mathrm{NH}$-Gruppe der Silazan- oder Silylhydrazinverbindungen mit elementaren (Erd)Alkalimetallen, deren Hydriden oder mit Metallorganylverbindungen metallieren (siehe 1.2.4.2). Interessant sind dabei oftmals die Strukturen der erhaltenen Salze. Je nach der Art des Metallions, der Substituenten am Stickstoff oder des Lösungsmittels zeigen die Kristallstrukturen unterschiedlichste Koordinationen der Metall- bzw. Amidionen ${ }^{[41-44]}$.
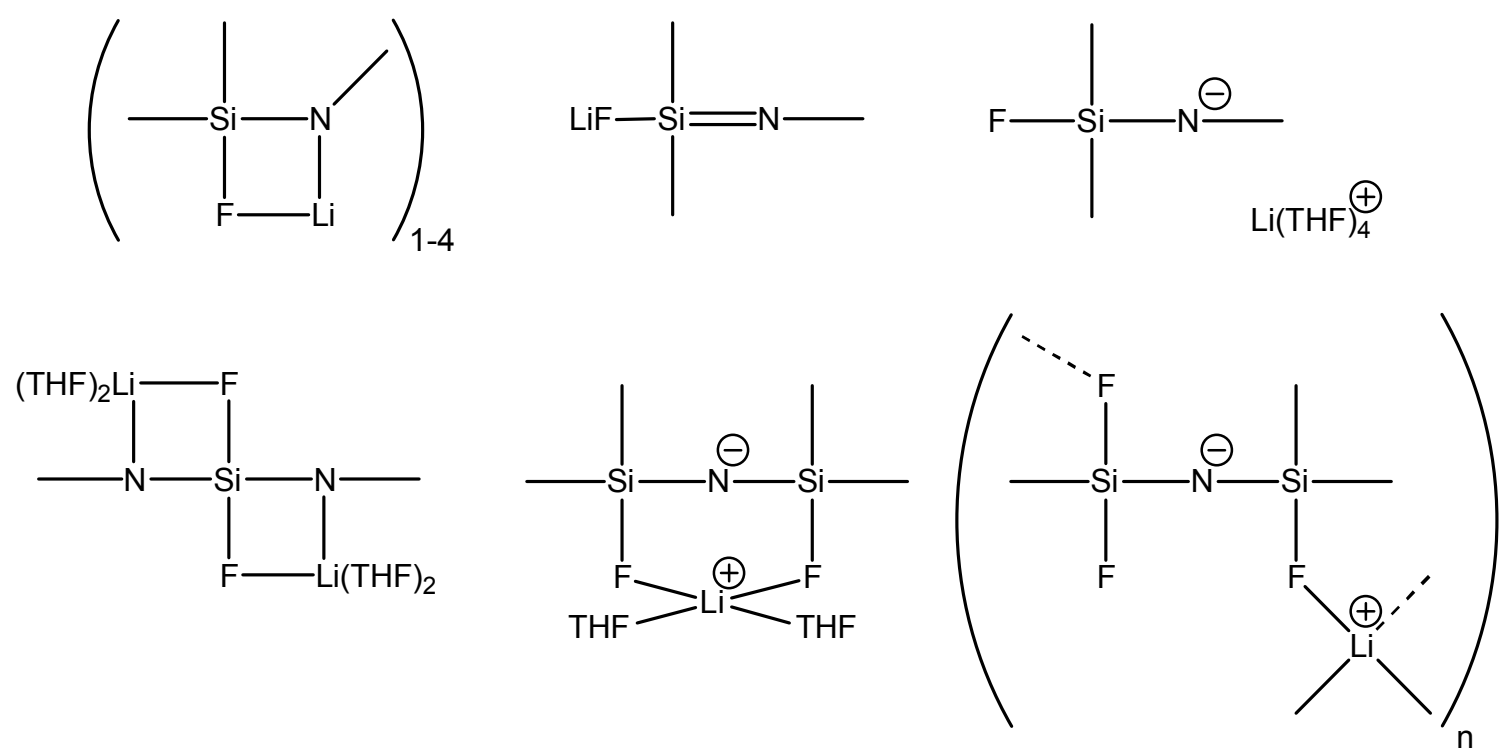

Abbildung 1: Beispiele möglicher Koordinationen des Lithiums in Lithiumsilylamiden

Lithiumsalze der Silylhydrazine zeigen, u.a. abhängig von der Größe der Silylsubstituenten, monomere, dimere, tetramere oder hexamere Einheiten. Die Lithiumatome können weiterhin "end-on" oder "side-on" gebunden sein ${ }^{[29,45,46] \text {. }}$<smiles>[R]N([R])N([R])[Al]</smiles>

"end-on"<smiles>[R]N1[Al]N1[R]</smiles>

"side-on" 


\subsubsection{Reaktionen der Lithiumsilylamide}

Besonders interessante Lithiumsalze sind halogenhaltige Silylamide. In einer intramolekularen Salzeliminierungsreaktion können hier oftmals Iminosilene oder deren Oligomerisationsprodukte (Cyclosilazane) erhalten werden ${ }^{[47]}$.<smiles>[R]N([Si])[SiH]([R])[R]</smiles><smiles></smiles><smiles>[R]N=[Si]([R])[R]</smiles><smiles>[Y10]CC</smiles><smiles>[R]N1[Si]([R])([R])N([R])[Si]1([R])[R]</smiles>

Gleichung 13: Bildung von Iminosilenen bzw. Cyclodisilazanen über Lithiumhalogenidabspaltung

An Iminoborene wie auch an Iminosilene können Aluminiumverbindungen addieren (siehe Gleichung 2). Werden Halogensilylamide mit Aluminiumchloriden oder Methylalanen umgesetzt, zeigen diese ein vergleichbares Reaktionsverhalten, wobei ebenfalls eine intramolekulare Lithiumhalogenidabspaltung zu beobachten ist ${ }^{[48,49]}$.<smiles>[R][Si]([R])(F)N(C)[AlH]</smiles><smiles>[R][C@H]1[AlH]N(C(C)(C)C)[Si]1([R])[R]</smiles> 
Teilweise kann hierbei auch eine intermolekulare Lithiumchloridabspaltung als Konkurrenzreaktion beobachtet werden ${ }^{[50]}$.<smiles></smiles><smiles>[R][Si]1([R])N(C)[Si](Cl)(Cl)N1COCCO</smiles>

Gleichung 15: Reaktion von Fluorsilylamiden und Aluminiumchlorid unter LiCl-Abspaltung ${ }^{\text {[50] }}$

Substitutionsreaktionen der Lithiumsalze können ebenfalls mit Halogensilanen oder -boranen durchgeführt werden, wobei Silazane ${ }^{[51]}$ oder Borazane ${ }^{[52,53]}$ erhalten werden.<smiles>[R]N=[Si]([R])([R])[R]</smiles>

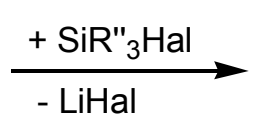<smiles>[R]N([AsH2])[Si]([R])([R])[R]</smiles>

Gleichung 16: Substitution von Silylamiden mit Halogensilanen

Werden Dilithiumsalze mit mehrfach halogenierten Boranen oder Silanen umgesetzt, können neben offenkettigen Produkten oftmals auch Ringe isoliert werden ${ }^{[51]}$.

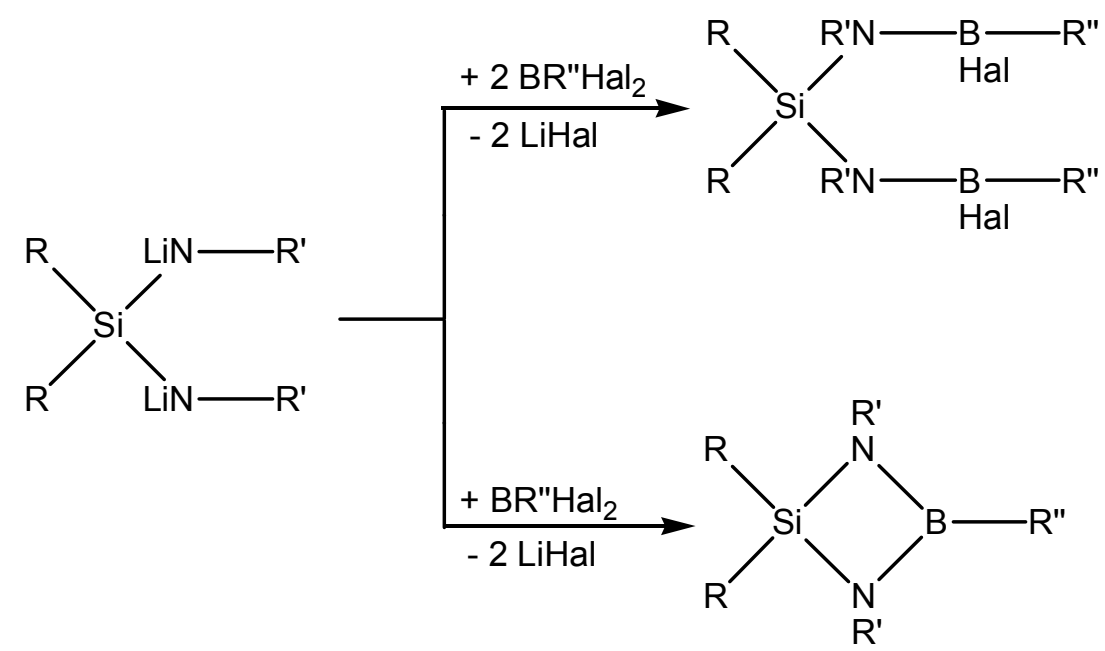

Gleichung 17: Disubstitution bzw. Ringschlussreaktion von Dilithiumsalzen mit Boranen 


\subsection{Keramiken}

Keramiken sind nach Definition der Deutschen Keramischen Gesellschaft anorganische Werkstoffe, die wasserunlöslich, nichtmetallisch und zu mindestens 30\% kristallin sind ${ }^{[54]}$. Häufig eingesetzte Keramiken bestehen aus Nichtmetallen (C, N, O) und/oder Halbmetallen (B, Al, Si).

Besonderes Interesse gilt dabei Materialien mit hoher thermischer Beständigkeit (bis $2200{ }^{\circ} \mathrm{C}$ ), geringer Dichte (bis zu 2,0 g/ $\mathrm{cm}^{3}$ ) hoher Härte (z.B. kubisches Bornitrid) oder auch elektrischer Leitfähigkeit (z.B. Aluminiumnitrid) ${ }^{[55-57]}$.

Die industrielle Herstellung von Keramiken basiert entweder auf der Synthese aus den Elementen (wobei nur binäre Keramiken erhalten werden können) oder aus der Reaktion von Molekülen bei hohen Temperaturen, wobei hier neben dem Sol-GelProzess besonders die chemische Gasphasen- (Chemical-Vapor-Deposition), Festphasen- oder Polymerpyrolyse angewendet werden ${ }^{[58-60]}$. Bei letzterem Verfahren können auch ternäre Keramiken erhalten werden ${ }^{[61]}$.

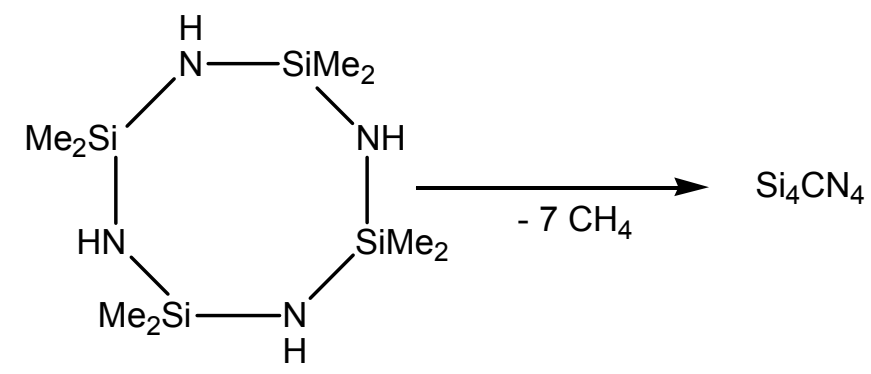

Gleichung 18: Darstellung von $\mathrm{Si}_{4} \mathrm{CN}_{4}$ aus der Polymerisation von Cyclosilazanen [61] 


\section{$2 \quad$ Problemstellung}

Im Rahmen dieser Arbeit soll versucht werden, neuartige Silylamine und Aminoborane durch folgenden Reaktionsweg herzustellen.

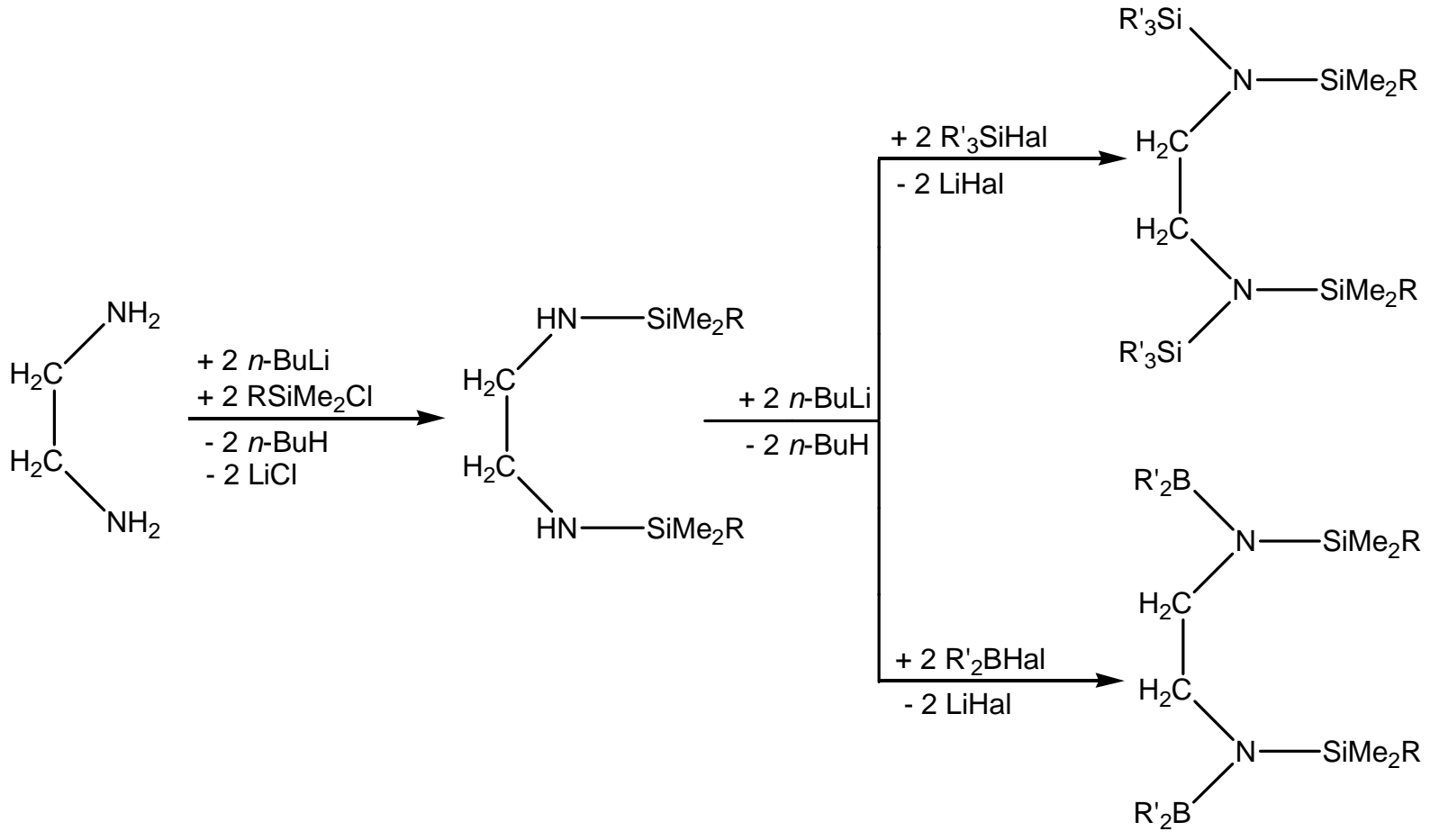

Gleichung 19: Syntheseweg von Bis(silyl)ethylendiaminen

Neben offenkettigen Substitutionsprodukten sollen Ringschlussreaktionen mit Boranen und Silanen durchgeführt werden.

Alternativ zum Ethylendiamin soll Hydrazin als Edukt verwendet werden.

Die Silylamine und Silylhydrazine sollen auf thermische und anionische Silatropie untersucht werden. 
Weiterhin sollen lithiierte Bis(di-tert-butylfluorsilyl)amine mit Halogenboranen und mit Alanen umgesetzt werden.

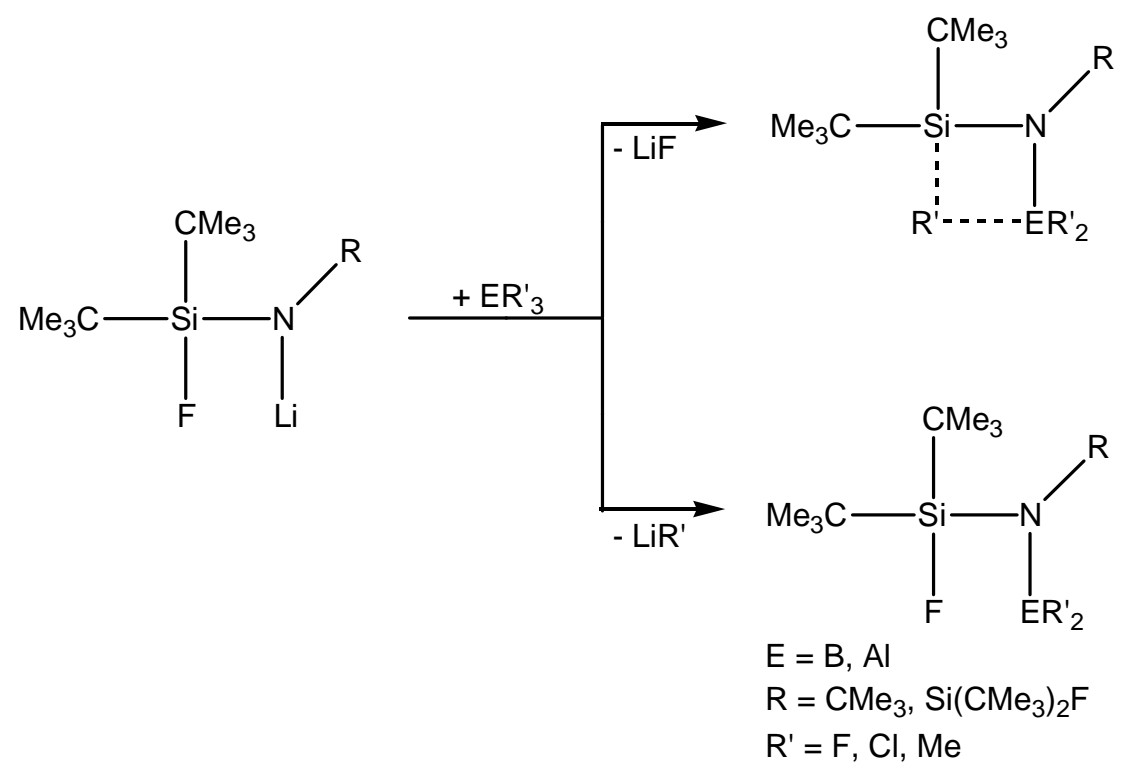

Gleichung 20: Substitutionsreaktionen an Lithium-di(tert-butyl)fluorsilylamiden

Dazu vergleichend soll überprüft werden, ob $\mathrm{NH}$-funktionelle Fluorborane ebenso lithiiert und mit Alanen umgesetzt werden können.

Wenn möglich, sollen Moleküle synthetisiert werden, welche sich als Vorstufen für Al/B/N/Si-haltige Keramiken eignen. Dabei sollen Verbindungen gefunden werden, bei denen nach thermischer Abspaltung von Halogensilanen ideale Verhältnisse der gewünschten Elemente $\mathrm{B}, \mathrm{N}$ und Al vorzufinden sind, z. B. B : Al : N = 1: $1: 2$.

Letztlich ist es Ziel dieser Arbeit leicht zugängliche Silylhalogenborazane direkt mit Alanen umzusetzen, wobei Boriniumionen erhalten werden sollen.
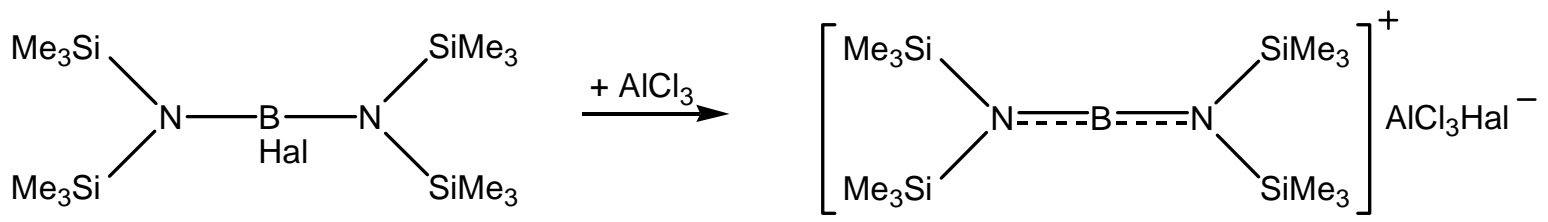

Gleichung 21: Bildung von Boriniumionen

Wenn möglich, werden die erhaltenen Verbindungen röntgenstrukturanalytisch untersucht. 


\section{$3 \quad$ Ergebnisse und Diskussion}

Die in dieser Arbeit vorgestellten Verbindungen wurden bis auf 64, 66 und 67 vorwiegend kernresonanz- und massenspektroskopisch charakterisiert.

In diesem Kapitel werden Reaktionsmechanismen sowie charakteristische Verschiebungen und Kopplungen der einzelnen Verbindungen vorgestellt und diskutiert. Des Weiteren sollen ausgewählte Bindungslängen und -winkel der geröntgten Verbindungen sowie REM(SEM)-Bilder und EDX-Spektren diskutiert werden.

\subsection{Synthese und Reaktionen an $N, N^{\prime}$-Bis(1,1,3-trisila-2-aza)ethylendi- aminen}

Das Dilithiumsalz des Ethylendiamins reagiert nicht mit den chlorfunktionellen Tris(silyl)aminen $\mathrm{ClSiMe}_{2} \mathrm{~N}\left(\mathrm{SiMe}_{3}\right) \mathrm{SiMe}_{2} \mathrm{R}$, [R = Me (1), $\mathrm{CMe}_{3}$ (2)] zu N,N'-Bis(1,1,3trisila-2-aza)ethylendiaminen. In Reaktionen mit den chlorfunktionellen Tris(silyl)aminen wurde daher Ethylendiamin im äquimolaren Verhältnis eingesetzt, so dass ein Teil als HCl-Fänger fungierte.
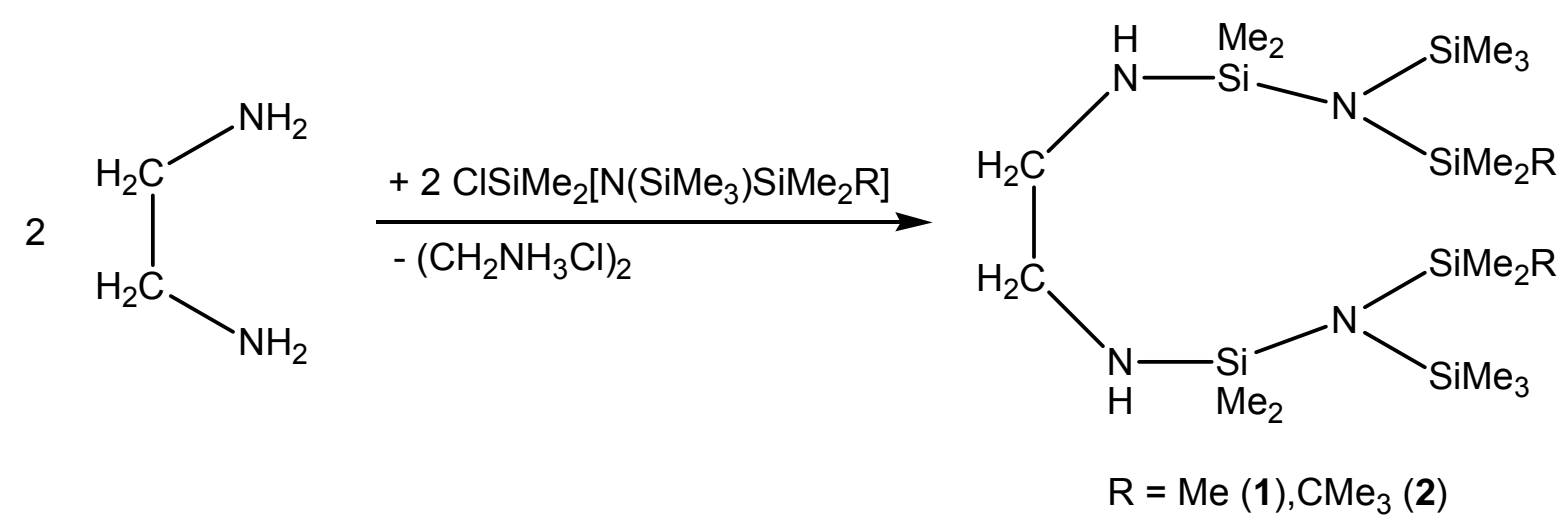

Gleichung 22: Synthese der N,N'-Bis(1,1,3-trisila-2-aza)ethylendiamine 1 und 2

\subsubsection{Thermische Silylgruppen-Wanderung}

Verbindung 1 wird durch Destillation des Rohproduktes bei vermindertem Druck rein erhalten. Beim Versuch $\mathbf{2}$ zu destillieren, wurde quantitativ das Strukturisomer $\mathbf{3}$ isoliert, d. h. die $\mathrm{SiMe}_{2} \mathrm{CMe}_{3}$ - und nicht die $\mathrm{SiMe}_{3}$-Gruppe ist vom Tris(silyl)amin- zum Ethylenaminstickstoff gewandert. 


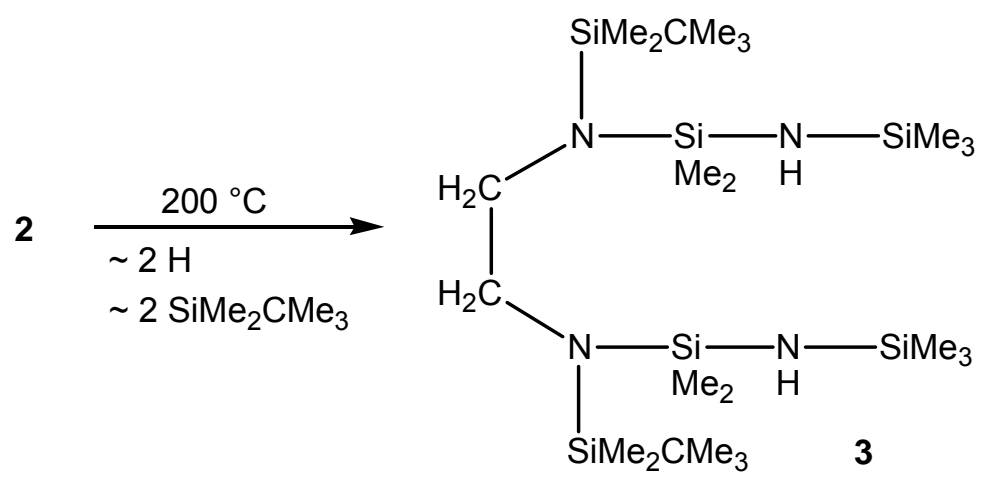

Gleichung 23: Thermisch induzierte Silatropie bei 2

Dieser Befund wurde erwartet, da das Siliciumatom der $\mathrm{SiMe}_{2} \mathrm{CMe}_{3}$-Gruppe auf Grund seiner Substitution elektronenärmer und somit Lewis-saurer ist als das der $\mathrm{SiMe}_{3}$-Gruppe. Experimentelle und theoretische Studien des Isomerisierungsverhaltens der Aminodimethylsilyl-bis(silyl)-amine ${ }^{[40]}$ sind daher auf die $N, N^{\prime}$ Bis(1,1,3-trisila-2-aza)ethylendiamine übertragbar. Auf Grund der unterschiedlichen Größe des Wasserstoffatoms und der Silylgruppe lässt sich auch hier auf einen zweistufigen Umlagerungsprozess schließen. Der erste Übergangszustand ist durch eine verbrückende Silylgruppe und der zweite durch ein verbrückendes Wasserstoffatom gekennzeichnet. Das Intermediat zwischen den Übergängen ist ein Zwitterion mit zweifachkoordiniertem, negativem Amido- und vierfachkoordiniertem, positiven Amino-Stickstoffatom.

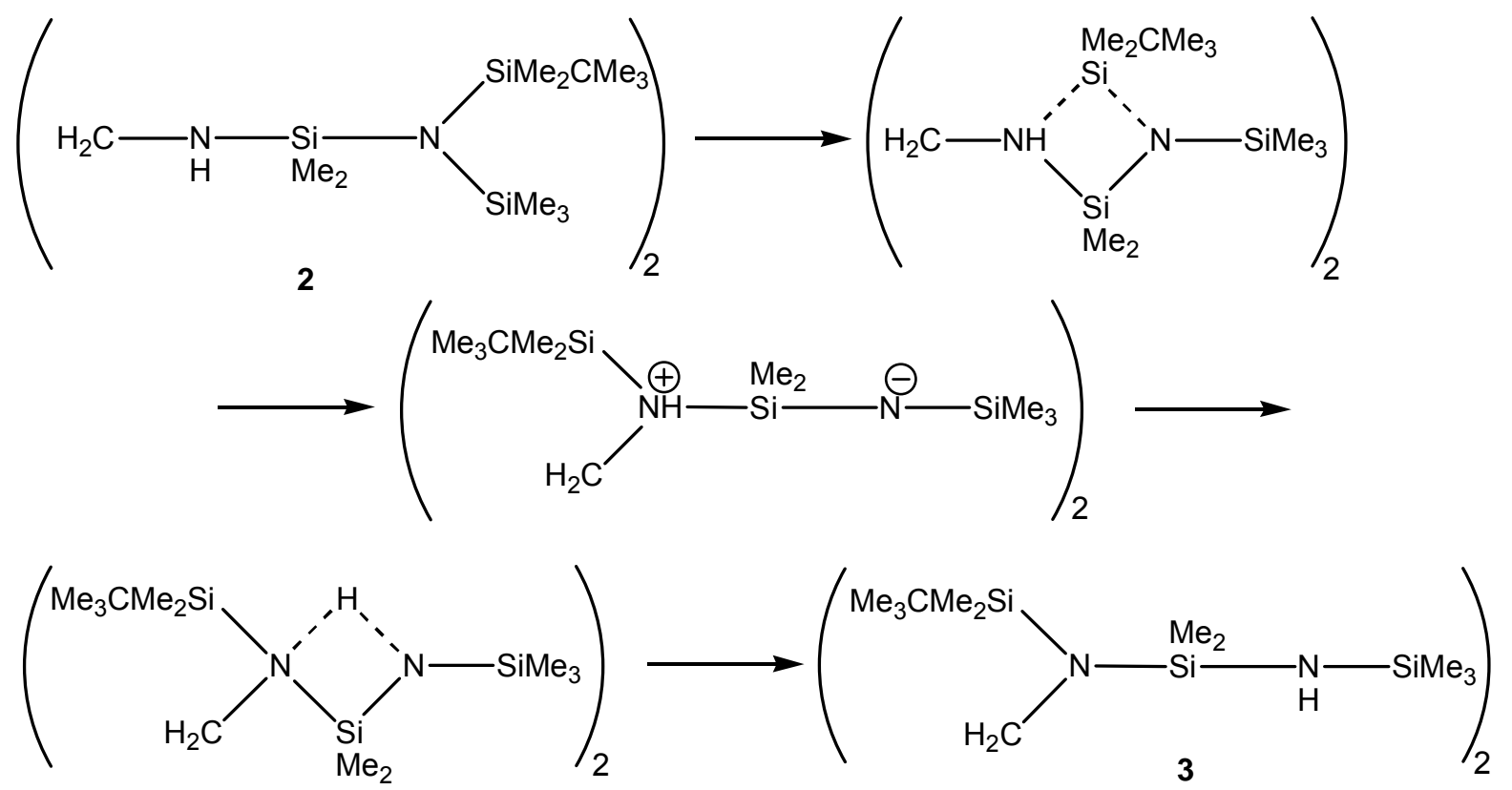




\subsubsection{Bildung des Dilithium-N,N'-bis(1-trimethylsilyl-2-amido-3-dimethylsilyl- trimethylsilyl)ethylendiamins unter anionischer Silylgruppen- Wanderung}

Verbindung 1 reagiert mit $n$-Butyllithium im molaren Verhältnis 1:2 unter Butanbildung zu einem Dilithiumsalz, das sich bereits bei Raumtemperatur zu 4 umlagert. 4 wird destillativ und durch Kristallisation aus $n$-Hexan rein erhalten.

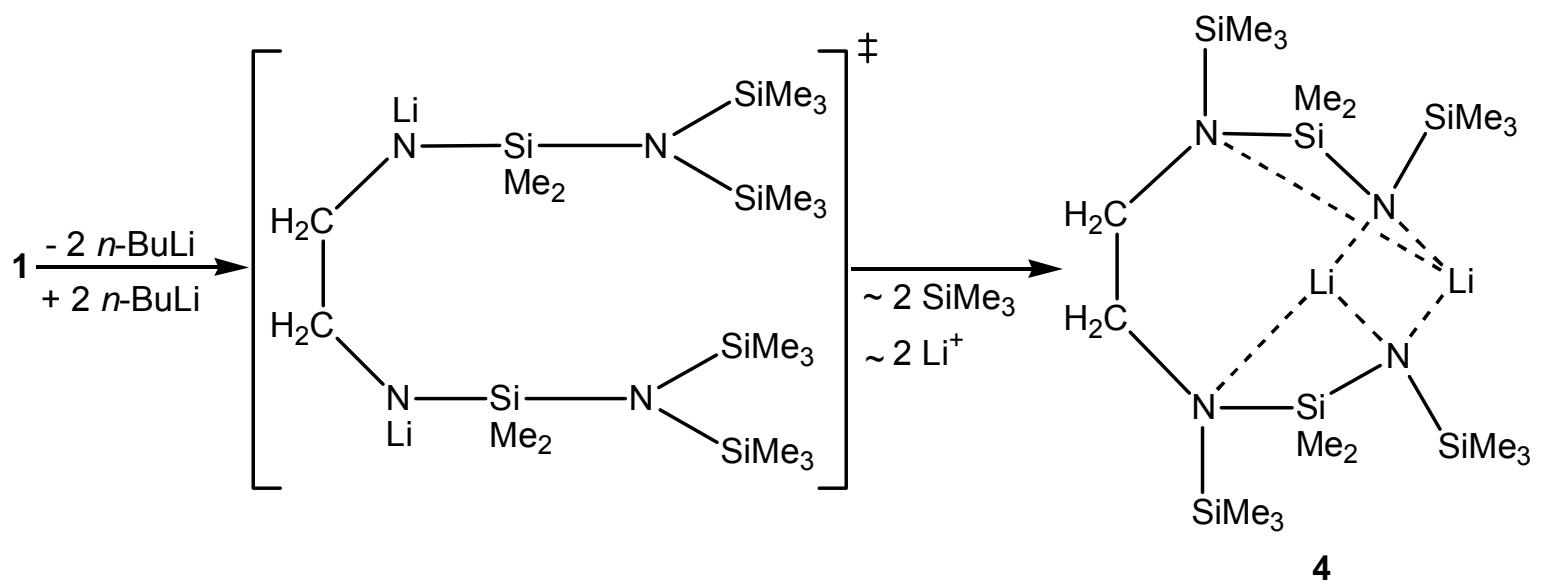

Gleichung 24: Synthese des Dilithiumsalzes 4

Wird $\mathbf{4}$ in siedendem THF gelöst und bei Raumtemperatur auskristallisiert, addiert $\mathbf{4}$ je Lithiumion ein THF-Molekül. Während Lithiumsalze mit der Struktureinheit $\mathrm{SiMe}_{3}-$ $\mathrm{NLi}^{-S i M e}{ }_{2}$ oftmals als Tri- oder Dimere kristallisieren ${ }^{[62]}$, sind 4, 5 und 6 Monomere.

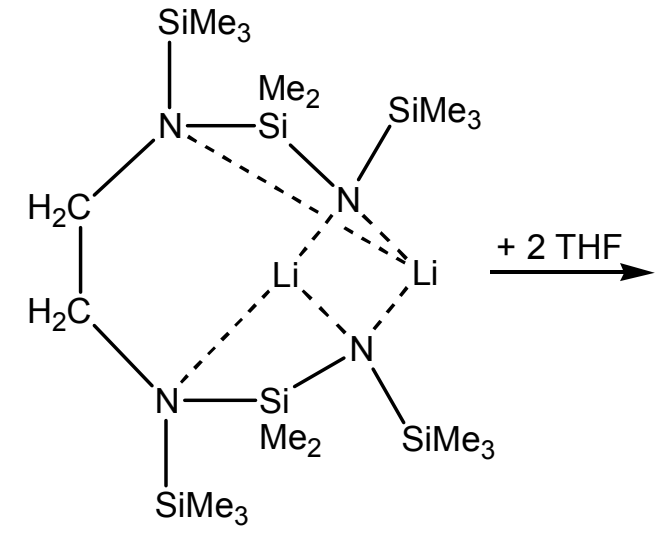

4

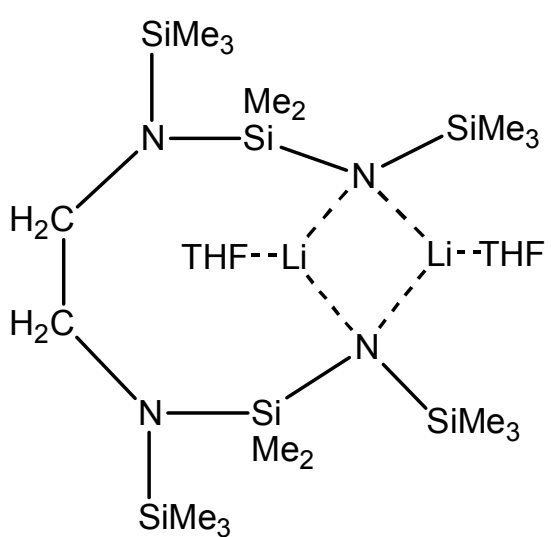

5

Gleichung 25: Addition von 2 THF-Molekülen an 4

In $\mathbf{4}$ sind die Lithiumionen des viergliedrigen ( $\mathrm{LiN})_{2}$-Rings auf Grund der Koordination zu den Ethylenamin-Stickstoffatomen weitgehend trigonal pyramidal umgeben. Der 
elektrostatische Kontakt zu diesen Stickstoffatomen fehlt in 5, so dass hier die Lithiumionen eine planare Koordination haben.

Die Verklammerung der beiden Amidionen in $\mathbf{4}$ und $\mathbf{5}$ wird bei Verwendung des zweizähnigen Liganden TMEDA gelöst. Es entsteht das offenkettige Dilithiumsalz 6. Die Lithiumionen sind durch die Stickstoffatome der TMEDA-Moleküle und die Amidogruppen dreifach planar koordiniert.

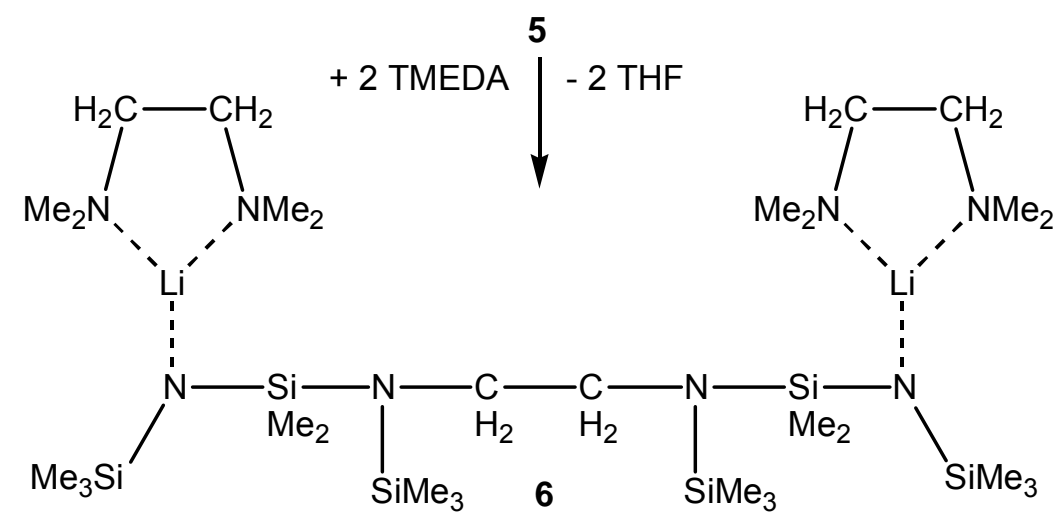

Gleichung 26: Synthese des 2-TMEDA-Addukts 6

\subsubsection{Kristallstrukturen der Dilithiumsalze 4 - 6}

\subsubsection{Dilithium- $N, N$ '-bis(1-trimethylsilyl-2-amido-3-dimethylsilyl-trimethyl- silyl)ethylendiamin, 4}

Das Dilithiumsalz 4 kristallisiert aus $n$-Hexan monoklin in der Raumgruppe $P 2_{1} / n$.

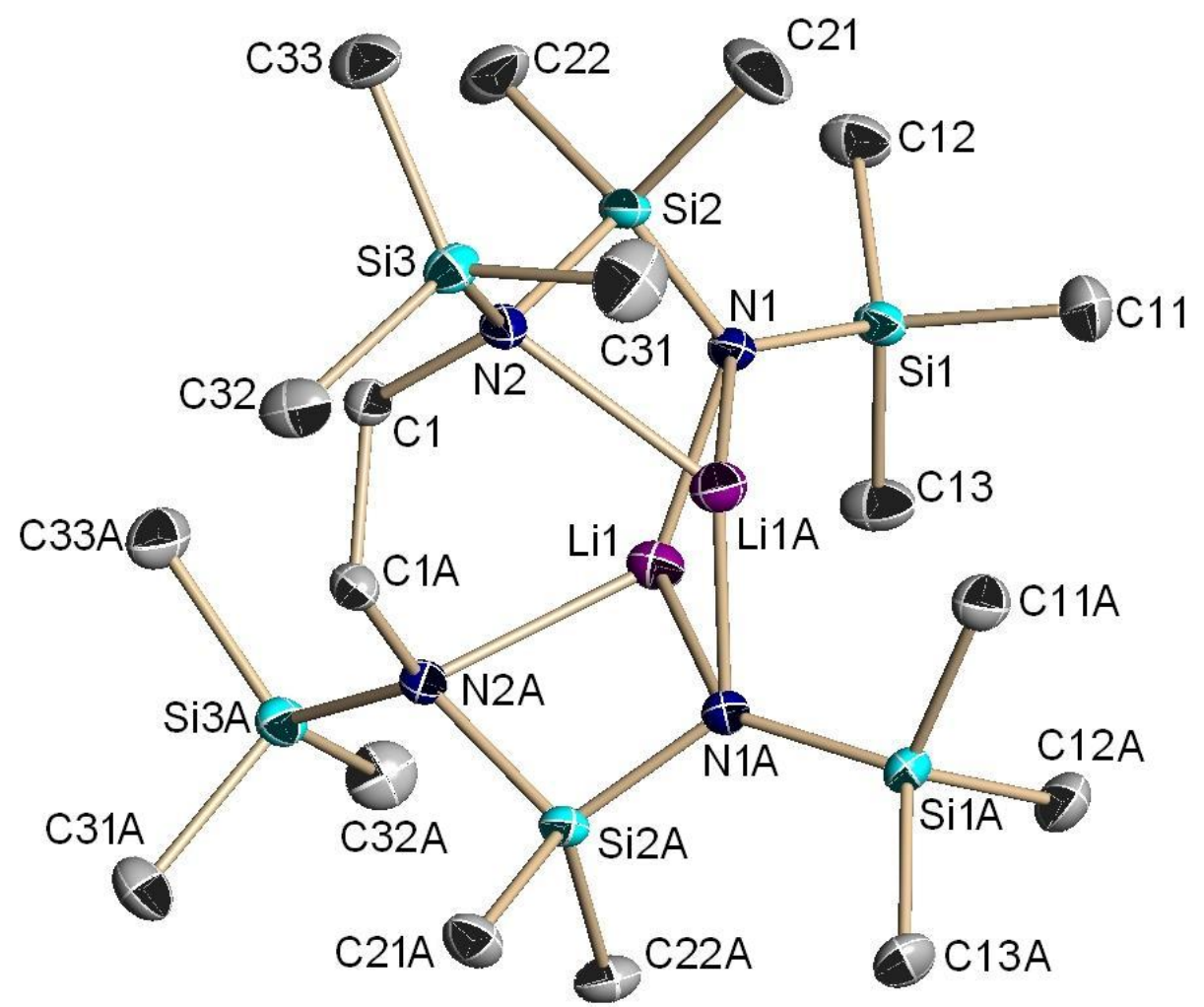

Abbildung 3: Kristallstruktur von Verbindung 4 ohne Wasserstoffatome 
Bindungslängen/pm mit Standardabweichung

\begin{tabular}{|ll|ll|ll|}
\hline Si1-N1 & $169,54(11)$ & Si3-N2 & $175,42(11)$ & N2-Li1A & $224,2(2)$ \\
\hline Si1-C1 & $187,58(14)$ & N1-Li1 & $195,8(2)$ & N1A-Li1A & $197,4(2)$ \\
\hline Si2-N1 & $167,95(11)$ & N1-Li1A & $207,6(2)$ & C1-C1A & $154,26(17)$ \\
\hline Si2-N2 & $178,75(11)$ & N2-C1 & $150,38(15)$ & & \\
\hline
\end{tabular}

Tabelle 1: Ausgewählte Bindungslängen der Verbindung 4

Bindungswinkel ${ }^{\circ}$ mit Standardabweichung

\begin{tabular}{|ll|ll|ll|}
\hline Si2-N1-Si1 & $128,56(6)$ & Si1-N1-Li1A & $131,98(9)$ & Si3-N2-Si2 & $124,14(6)$ \\
\hline Si2-N1-Li1 & $125,56(9)$ & Li1-N1-Li1A & $70,53(9)$ & N2-C1-C1A & $115,33(10)$ \\
\hline Si1-N1-Li1 & $101,25(8)$ & C1-N2-Si3 & $114,24(8)$ & Si2-N1-Li1A & $87,37(8)$ \\
\hline C1-N2-Si2 & $109,66(8)$ & & & & \\
\hline
\end{tabular}

Tabelle 2: Ausgewählte Bindungswinkel der Verbindung 4

Im Dilithiumsalz 4 werden die endständigen Amidionen (N1, N1A) durch die Lithiumionen ( $\mathrm{Li1}$, Li1A) zu einem viergliedrigen ( $\mathrm{LiN})_{2}$-Ring verklammert. Die Lithiumionen haben zusätzlich einen schwächeren Kontakt zu jeweils einem Stickstoffatom des Ethylenamins (Li1A-N2 $=224,2 \mathrm{pm}$ ), so dass zwei viergliedrige $\left(\mathrm{LiN}_{2} \mathrm{Si}\right)$-Ringe und, ausgehend von der $\mathrm{CH}_{2}-\mathrm{CH}_{2}$-Einheit, zwei siebengliedrige ( $\left.\mathrm{C}_{2} \mathrm{NSiNLiN}\right)$-Ringe gebildet werden. Die Lithiumionen haben eine pyramidale Umgebung mit Winkelsummen von $311^{\circ}$ und $312^{\circ}$. Der Lithium-Amino-Kontakt führt ebenfalls bei den AminStickstoffatomen zum Verlust der Planarität. Das an zwei Lithiumionen koordinierte Amidion hat eine tetraedrische Umgebung. Die Amido-Silicium-Abstände (168 pm) sind im Vergleich zu den Amino-Silicium-Abständen erwartungsgemäß um ca. 10 pm verkürzt. Die $\mathrm{NSi}_{2}$-Winkel der Amidogruppe sind mit $128^{\circ} \mathrm{im}$ Vergleich zu dem $\mathrm{NSi}_{2}$-Winkel der Aminogruppe $\left(124^{\circ}\right)$ leicht aufgeweitet. 


\subsubsection{Dilithium-bis(1-trimethylsilyl-2-amido-3-dimethylsilyl-trimethylsilyl)- ethylendiamin 2 THF, 5}

Das THF-Addukt des Dilithiumsalzes (5) wird durch Kristallisation von $\mathbf{4}$ aus THF isoliert. 5 kristallisiert als Monomer triklin in der Raumgruppe $P \overline{1}$.

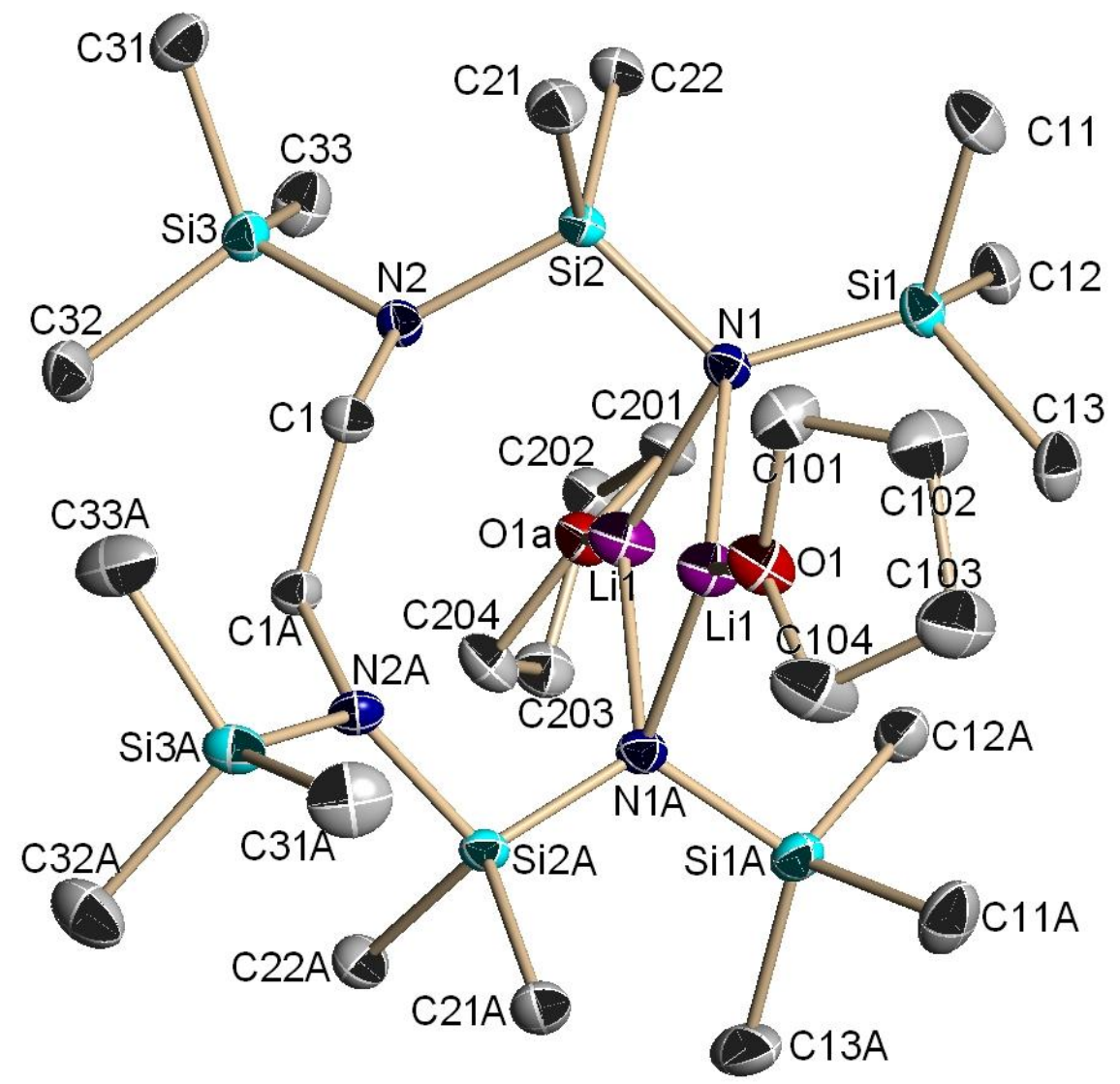

Abbildung 4: Kristallstruktur des Dilithiumsalzes 5 ohne Wasserstoffatome

Bindungslängen/pm mit Standardabweichung

\begin{tabular}{|ll|ll|ll|}
\hline Si1-N1 & $170,73(11)$ & Si2A-N2A & $176,77(10)$ & N1-Li1A & $202.8(2)$ \\
\hline Si2-N1 & $170,07(1)$ & Si3A-N2A & $175,07(10)$ & N1-Li1 & $208,6(2)$ \\
\hline Si2-N2 & $175,84(11)$ & O1-C44 & $144,43(16)$ & N2-C1 & $148,52(15)$ \\
\hline Si3-N2 & $174,30(11)$ & O1-Li1 & $192,2(2)$ & N1A-Li1A & $204,0(2)$ \\
\hline Si1A-N1A & $170,79(10)$ & O1A-C41A & $143,97(17)$ & N1A-Li1 & $206,3(2)$ \\
\hline Si2A-N1A & $169,74(10)$ & O1A-Li1A & $191,0(2)$ & N2A-C1A & $148,96(15)$ \\
\hline
\end{tabular}

Tabelle 3: Ausgewählte Bindungslängen der Verbindung 5

Bindungswinkel $/^{\circ}$ mit Standardabweichung

\begin{tabular}{|ll|ll|ll|}
\hline N1-Si2-N2 & $109,95(5)$ & C1A-N2A-Si3A & $115,20(8)$ & N1A-Li1-N1 & $105,12(10)$ \\
\hline Li1A-N1-Li1 & $72,45(9)$ & C1A-N2A-Si2A & $110,93(8)$ & O1A-Li1A-N1A & $120,40(12)$ \\
\hline C1-N2-Si3 & $111,03(8)$ & Si3A-N2A-Si2A & $123,27(6)$ & N2-C1-C1A & $113,32(10)$ \\
\hline Si2A-N1A-Si1A & $119,87(6)$ & O1-Li1-N1A & $129,35(12)$ & & \\
\hline Li1A-N1A-Li1 & $72,71(9)$ & O1-Li1-N1 & $122,04(12)$ & & \\
\hline
\end{tabular}


Verbindung 5 unterscheidet sich von Verbindung 4 durch die Koordination eines THF-Moleküls pro Lithiumion. Dadurch wird der Lithiumkontakt zum Amin-Stickstoffatom (N2) gelöst. Die Lithiumionen sind mit Winkelsummen von $356,5^{\circ}$ (Li1) und $359,9^{\circ}$ (Li1A) fast trigonal planar umgeben. Die Amido-Stickstoffionen haben eine tetraedrische- und die Amino-Stickstoffatome mit Winkelsummen von $353,5^{\circ}$ (N2) bzw. $349,4^{\circ}$ (N2A) eine pyramidale Umgebung.

\subsubsection{Dilithium-bis(1-trimethylsilyl-2-amido-3-dimethylsilyl-trimethylsilyl)- ethylendiamin-2 TMEDA, 6}

Wird das Dilithiumsalz $\mathbf{5}$ in THF in der Siedehitze gelöst und der zweizähnige Ligand TMEDA zugetropft, werden die koordinierenden THF-Moleküle durch je ein TMEDAMolekül substituiert. Die Lithiumionen bleiben dreifach koordiniert, verlieren jedoch den Kontakt zum zweiten Amidion. $\mathbf{6}$ kristallisiert monoklin in der acyclischen Raumgruppe $P 2_{1} / \mathrm{n}$.

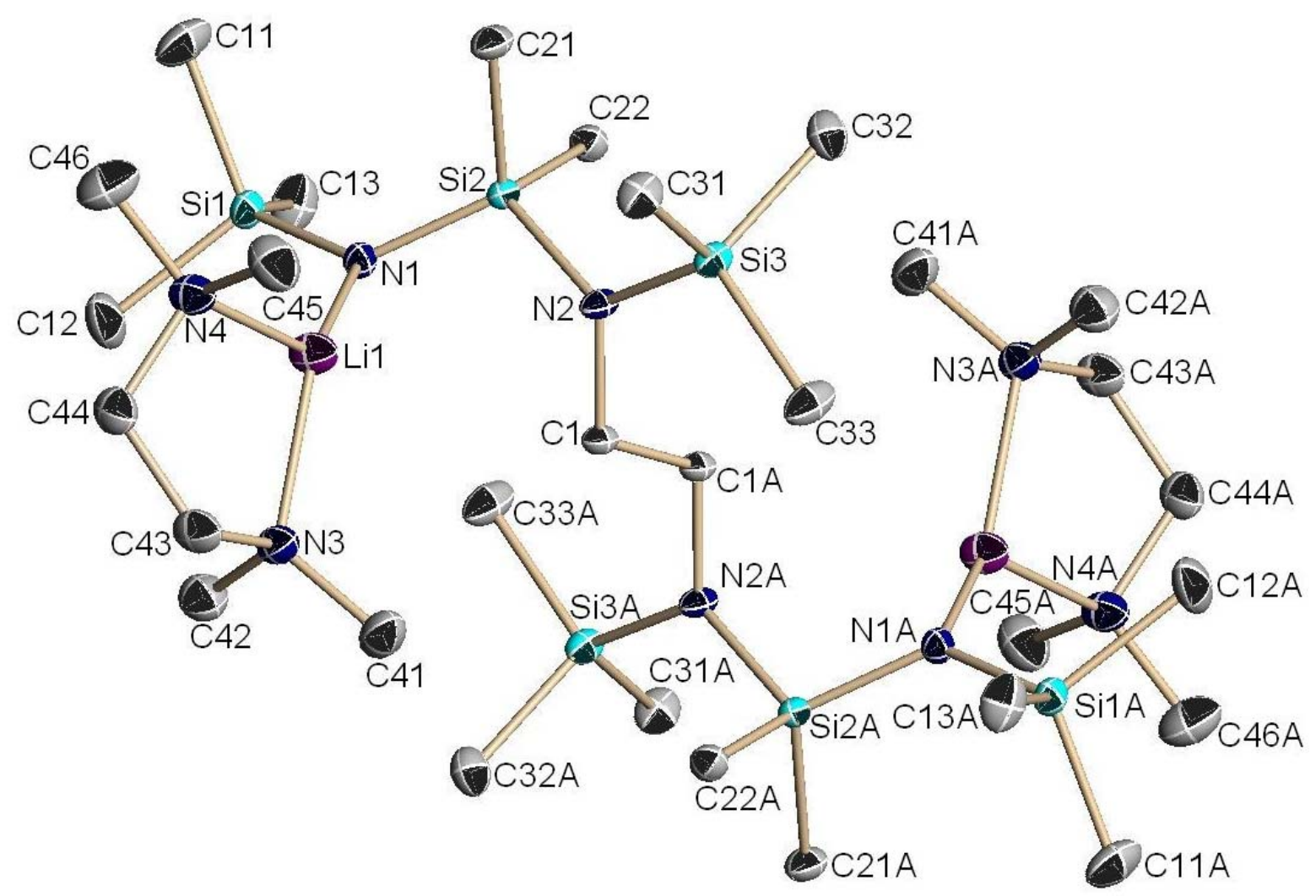

Abbildung 5: Kristallstruktur des Dilithiumsalzes 6 ohne Wasserstoffatome

Bindungslängen/pm mit Standardabweichung

\begin{tabular}{|ll|ll|ll|}
\hline Si1-N1 & $168,20(8)$ & Si2-N2 & $177,23(8)$ & N1-Li1 & $192,2(2)$ \\
\hline Si2-N1 & $167,65(8)$ & Si3-N2 & $174,06(9)$ & N2-C1 & $148,76(12)$ \\
\hline
\end{tabular}

Tabelle 5: Ausgewählte Bindungslängen der Verbindung 6 
Bindungswinkel $/^{\circ}$ mit Standardabweichung

\begin{tabular}{|ll|ll|ll|}
\hline N1-Si2-N2 & $109,13(4)$ & Si1-N1-Li1 & $120,20(8)$ & N1-Li1-N3 & $140,26(11)$ \\
\hline Si2-N1-Si1 & $127,32(5)$ & Si3-N2-Si2 & $124,53(5)$ & N4-Li1-N3 & $86,36(8)$ \\
\hline Si2-N1-Li1 & $107,66(7)$ & N1-Li1-N4 & $128,49(11)$ & & \\
\hline
\end{tabular}

Tabelle 6: Ausgewählte Bindungswinkel der Verbindung 6

Die Winkelsumme am Amidstickstoff N1 beträgt $355,2^{\circ}$, die des Aminstickstoffes N2 $358,9^{\circ}$. Beide sind somit nahezu planar umgeben. Der Abstand Li1-N1 von $192 \mathrm{pm}$ ist ca. 20 pm kürzer als zu den TMEDA-Stickstoffatomen (Li1-N3, Li1-N4). Etwa um $10 \mathrm{pm}$ verkürzen sich die Amido-Silicium-Abstände (Si1-N1 $=168,20$ pm, Si2-N1 $=167,65 \mathrm{pm}) \quad$ im Vergleich $\mathrm{zu}$ den Amino-Silicium-Bindungslängen $(\mathrm{Si} 2-\mathrm{N} 1=167,6 \mathrm{pm}, \mathrm{Si} 2-\mathrm{N} 2=177,2 \mathrm{pm})$.

\subsubsection{N,N'-Bis(1,1,3-trisila-2-aza)- und $N, N^{\prime}$-Bis(1-bora-1,3-disila-2-aza)- bis(silyl)-ethylendiamine, 7 - 13}

Die Reaktivität der Dilithiumsalze steigt von Verbindung 4 zu Verbindung 6. Wird 5 im molaren Verhältnis 1:2 hydrolytisch aufgearbeitet (7), mit Halogensilanen (8 - 10) oder -boranen (11 - 13) versetzt, erfolgt in quantitativer Reaktion die Substitution der Lithiumionen durch Silyl- oder Borylgruppen bzw. bei Verbindung $\mathbf{7}$ - dem Isomer von 1 - durch Protonen.

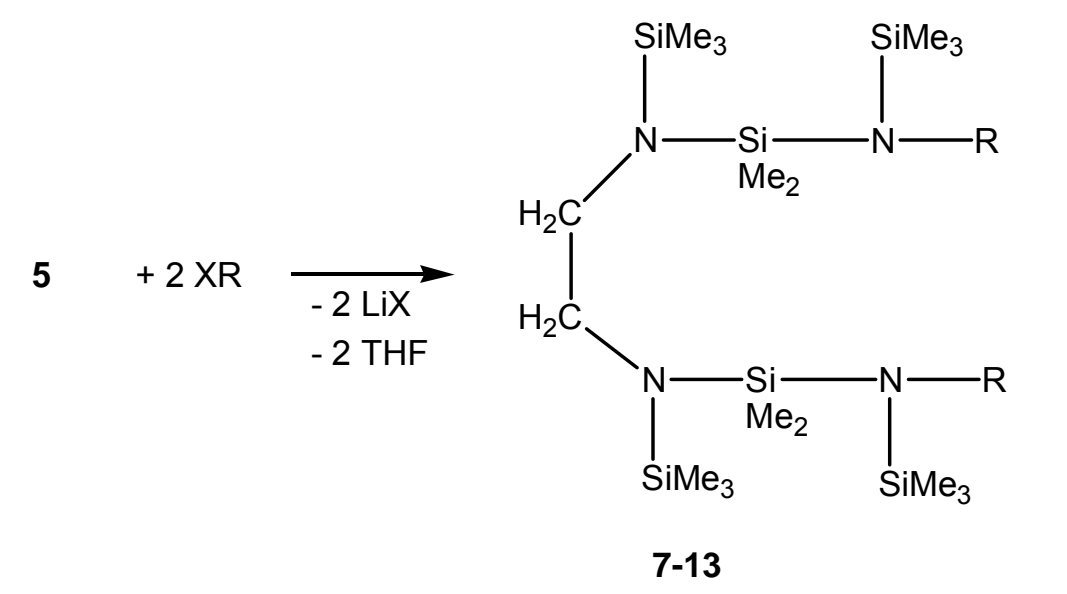

\begin{tabular}{|c|c|c|c|c|c|c|c|}
\hline & $\mathbf{7}$ & $\mathbf{8}$ & $\mathbf{9}$ & $\mathbf{1 0}$ & $\mathbf{1 1}$ & $\mathbf{1 2}$ & $\mathbf{1 3}$ \\
\hline $\mathrm{R}$ & $\mathrm{H}$ & $\mathrm{F}_{2} \mathrm{SiCMe}_{3}$ & $\mathrm{FSiMe}_{2}$ & $\mathrm{SiMe}_{3}$ & $\mathrm{FBN}\left(\mathrm{CMe}_{2} \mathrm{CH}_{2}\right)_{2} \mathrm{CH}_{2}$ & $\mathrm{FBN}\left(\mathrm{SiMe}_{3}\right)_{2}$ & $\mathrm{FBN}\left(\mathrm{CHMe}_{2}\right)_{2}$ \\
\hline $\mathrm{X}$ & $\mathrm{OH}$ & $\mathrm{F}$ & $\mathrm{F}$ & $\mathrm{Cl}$ & $\mathrm{F}$ & $\mathrm{F}$ & $\mathrm{F}$ \\
\hline
\end{tabular}

Gleichung 27: Synthese der Verbindungen 7-13

Die Verbindungen sind mit Ausnahme von $\mathbf{1 1}$ thermisch stabil, können im Hochvakuum unzersetzt destilliert werden und kristallisieren nach der Destillation. 


\subsubsection{1,3-Bis(trimethylsilyl)-2,4-bis(1,1,5,5-tetramethylpiperidino)-1,3-diaza-}

\section{2,4-dibora-cyclobutan, 14}

Beim Versuch der destillativen Aufarbeitung der Tetramethylpiperidin-Verbindung $\mathbf{1 1}$ erfolgte die Fluorierung einer und zum Teil beider $\mathrm{SiMe}_{3}$-Gruppen durch die Fluorborylgruppe. Isoliert wurde das 1,3-Diaza-2,4-dibora-cyclobutan 14.

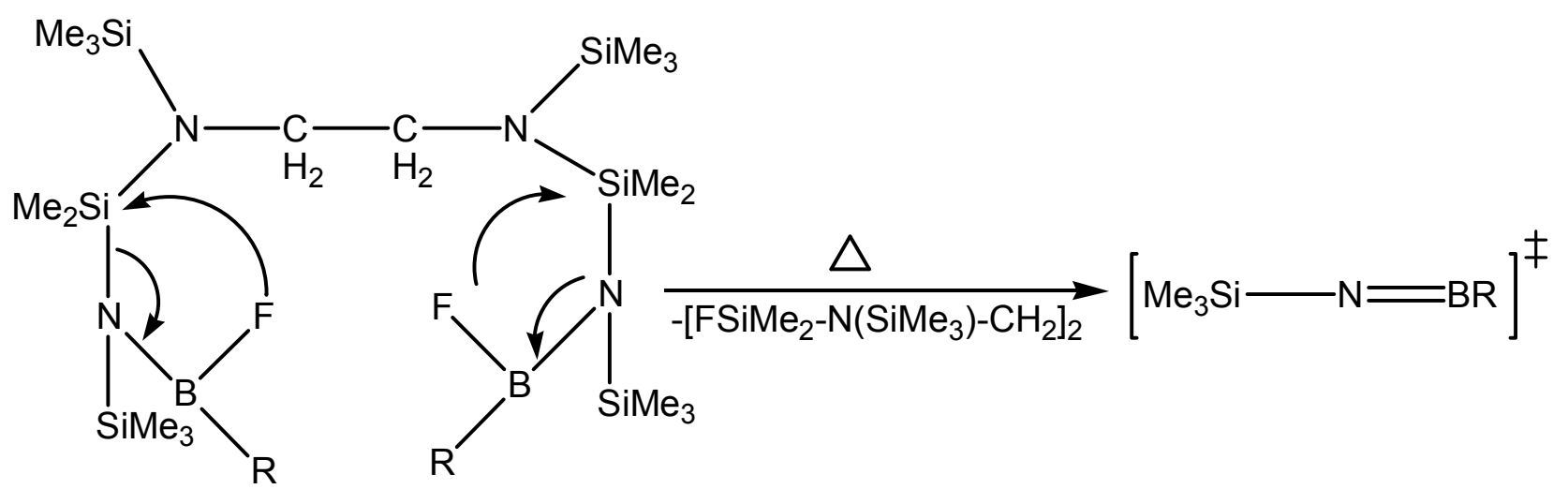

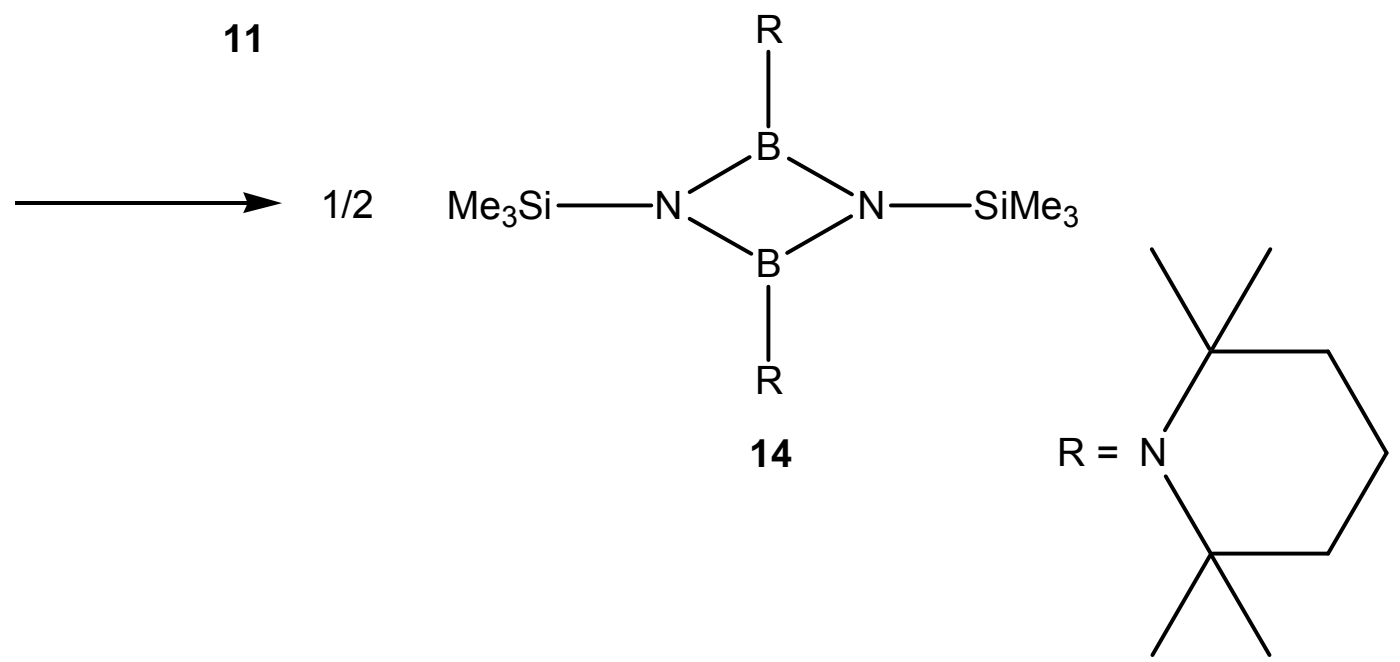




\subsubsection{Kristallstrukturen der Verbindungen 8 - 12 und 14}

\subsubsection{Kristallstruktur von 8}

Verbindung 8 kristallisiert aus $n$-Hexan triklin in der Raumgruppe $P \overline{1}$. Die Stickstoffatome haben eine planare Umgebung $\left(\Sigma \Varangle \mathrm{N} 1=360,0^{\circ}, \Sigma \nless \mathrm{N} 2=359,2^{\circ}\right)$. Der elektronenziehende Effekt der Fluoratome führt zu einer Bindungsverkürzung der N1-Si4-Bindung auf 170,6 pm.

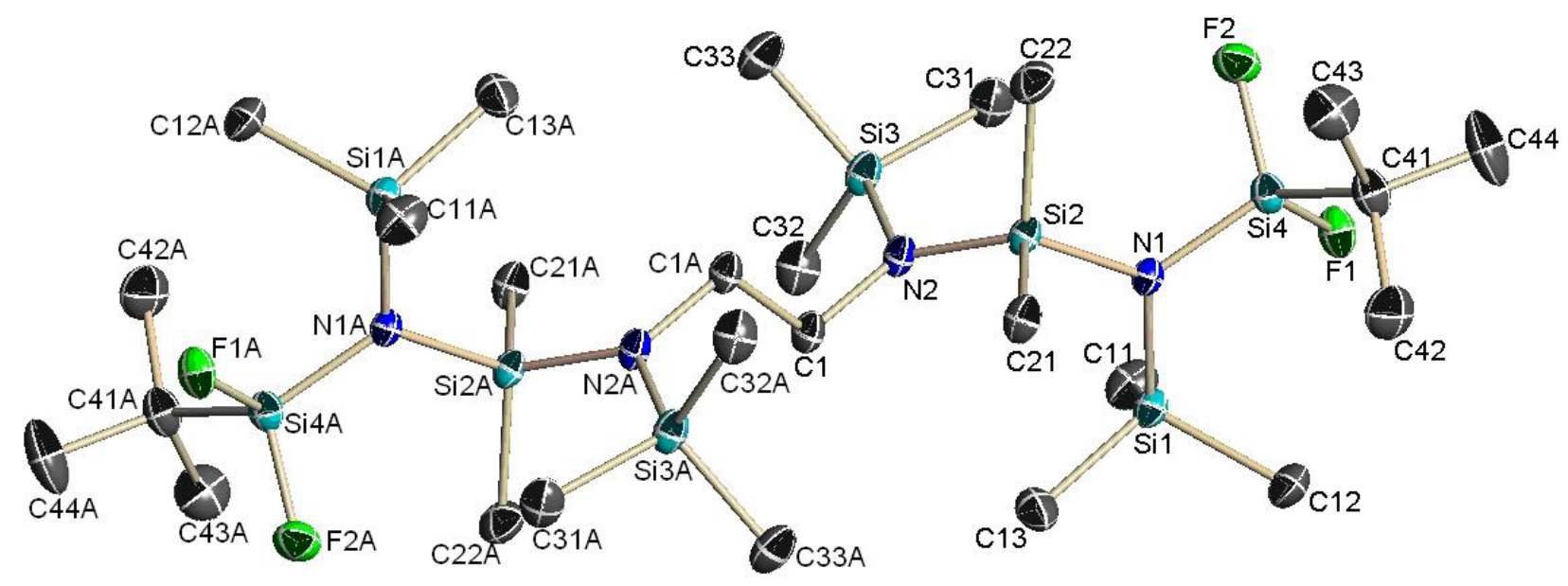

Abbildung 6: Kristallstruktur von 8 ohne Wasserstoffatome

Bindungslängen/pm mit Standardabweichung

\begin{tabular}{|ll|ll|ll|}
\hline F1-Si4 & $159,44(10)$ & N1-Si2 & $176,57(13)$ & N2-Si3 & $175,58(13)$ \\
\hline F2-Si4 & $158,78(10)$ & N1-Si1 & $177,61(13)$ & C1-N2 & $148,70(2)$ \\
\hline N1-Si4 & $170,58(13)$ & N2-Si2 & $173,53(14)$ & C1-C1A & $153,90(3)$ \\
\hline
\end{tabular}

Tabelle 7: Ausgewählte Bindungslängen der Verbindung 8

Bindungswinkel $/^{\circ}$ mit Standardabweichung

\begin{tabular}{|ll|ll|ll|}
\hline Si4-N1-Si2 & $121,75(8)$ & C1-N2-Si3 & $116,59(10)$ & F1-Si4-N1 & $106,10(6)$ \\
\hline Si4-N1-Si1 & $117,53(7)$ & Si2-N2-Si3 & $123,09(8)$ & N2-Si2-N1 & $112,15(6)$ \\
\hline Si2-N1-Si1 & $120,72(7)$ & F2-Si4-F1 & $103,45(6)$ & & \\
\hline C1-N2-Si2 & $119,59(10)$ & F2-Si4-N1 & $111,31(6)$ & & \\
\hline
\end{tabular}

Tabelle 8: Ausgewählte Bindungswinkel der Verbindung 8 


\subsubsection{Kristallstruktur von 9}

Verbindung 9 kristallisiert aus $n$-Hexan monoklin in der Raumgruppe $P 2_{1} / n$.

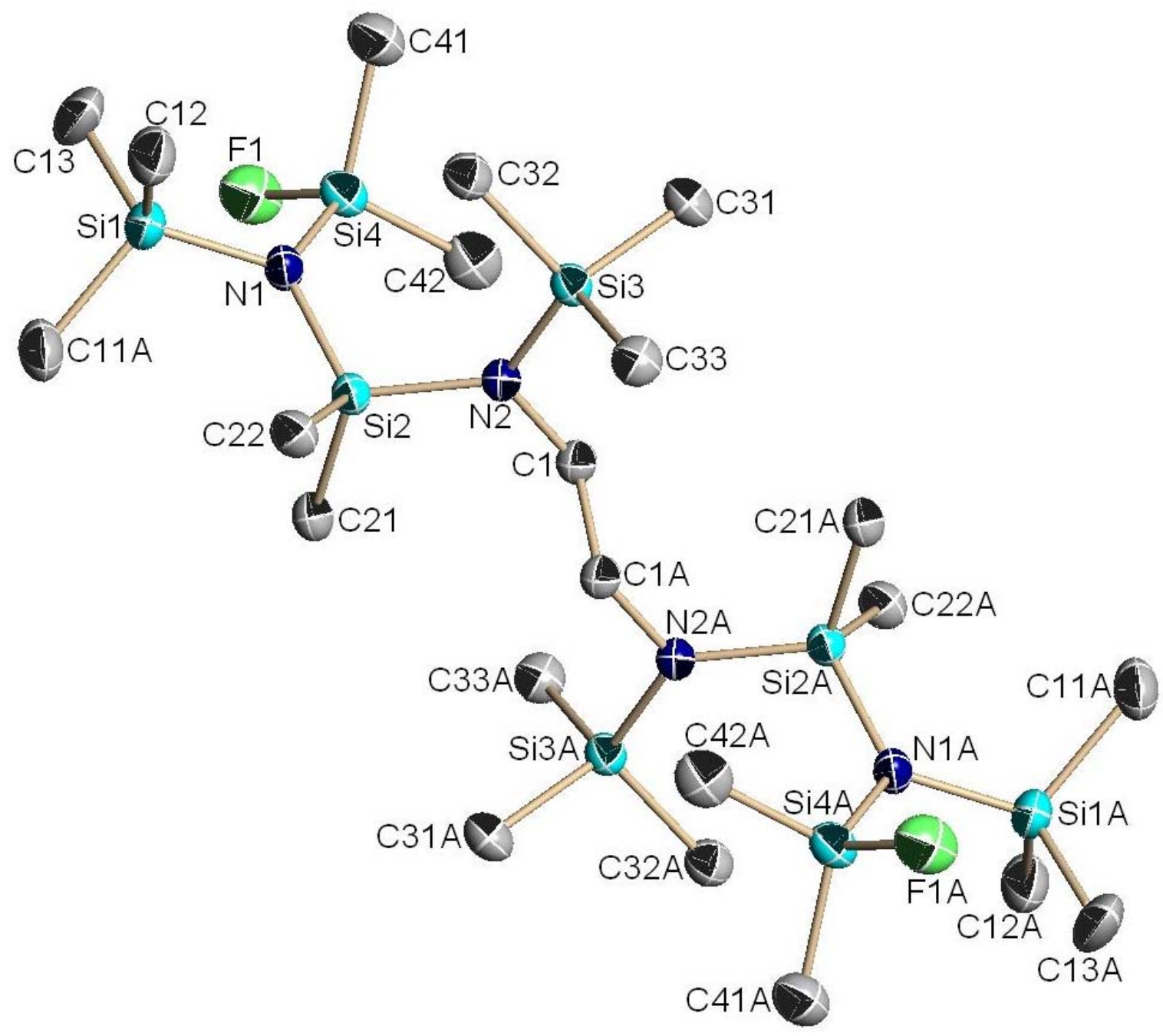

Abbildung 7: Kristallstruktur von 9 ohne Wasserstoffatome

Bindungslängen/pm mit Standardabweichung

\begin{tabular}{|ll|ll|ll|}
\hline F1-Si4 & $162,2(2)$ & Si2-N1 & $175,9(2)$ & C1-C1A & $153,2(5)$ \\
\hline Si1-N1 & $176,5(2)$ & Si3-N2 & $174,5(2)$ & & \\
\hline Si2-N2 & $174,2,(2)$ & N2-C1 & $148,7(3)$ & \\
\hline
\end{tabular}

Tabelle 9: Ausgewählte Bindungslängen der Verbindung 9

Bindungswinkel $/{ }^{\circ}$ mit Standardabweichung

\begin{tabular}{|ll|ll|ll|}
\hline Si4-N1-Si2 & $120,88(13)$ & Si2-N1-Si1 & $120,45(13)$ & C1-N2-Si3 & $116,75(16)$ \\
\hline Si4-N1-Si1 & $118,17(14)$ & C1-N2-Si2 & $118,44(16)$ & Si2-N2-Si3 & $124,53(13)$ \\
\hline
\end{tabular}

Tabelle 10: Ausgewählte Bindungswinkel der Verbindung 9 


\subsubsection{Kristallstruktur von 10}

Verbindung 10 kristallisiert aus $n$-Hexan triklin in der Raumgruppe $P \overline{1}$.

Aus sterischen Gründen weicht die Winkelsumme von $\mathrm{N} 1\left(\Sigma \nless \mathrm{N} 1=357,8^{\circ}\right)$ um $2,2^{\circ}$ von der Planarität ab.

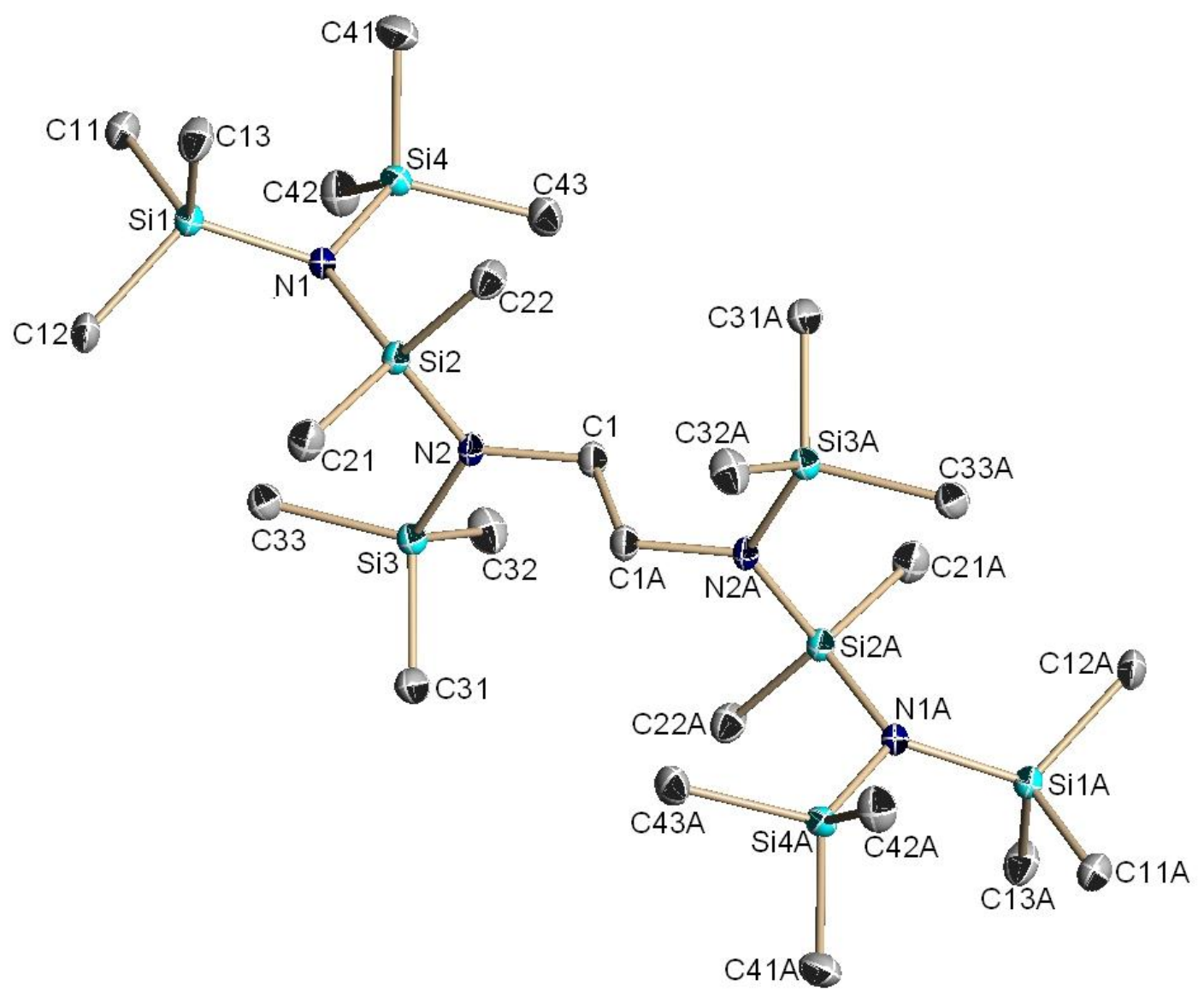

Abbildung 8: Kristallstruktur von 10 ohne Wasserstoffatome

Bindungslängen/pm mit Standardabweichung

\begin{tabular}{|ll|ll|ll|}
\hline Si1-N1 & $175,71(15)$ & Si3-N2 & $174,82(14)$ & C1-C1A & $152,24(3)$ \\
\hline Si2-N2 & $174,00(14)$ & Si4-N1 & $176,14(14)$ & \\
\hline Si2-N1 & $175,32(14)$ & N2-C1 & $148,9(2)$ & & \\
\hline
\end{tabular}

Tabelle 11: Ausgewählte Bindungslängen der Verbindung 10

Bindungswinkel $/{ }^{\circ}$ mit Standardabweichung

\begin{tabular}{|ll|ll|ll|}
\hline N2-Si2-N1 & $111,71(7)$ & Si1-N1-Si4 & $117,70(8)$ & Si2-N2-Si3 & $124,13(8)$ \\
\hline Si2-N1-Si1 & $121,45(8)$ & C1-N2-Si2 & $119,53(11)$ & N2-C1-C1A & $113,53(17)$ \\
\hline Si2-N1-Si4 & $118,26(8)$ & C1-N2-Si3 & $116,20(11)$ & & \\
\hline
\end{tabular}

Tabelle 12: Ausgewählte Bindungswinkel der Verbindung 10 


\subsubsection{Kristallstruktur von 11}

Verbindung $\mathbf{1 1}$ kristallisiert aus $n$-Hexan triklin in der Raumgruppe $P \overline{1}$. Röntgenstrukturtaugliche Kristalle von $\mathbf{1 1}$ werden durch Kristallisation aus $n$-Hexan gewonnen. Die Stickstoff- und Boratome haben eine planare Umgebung $\left(\Sigma \Varangle B 1=360,0^{\circ}\right.$, $\left.\Sigma \Varangle \mathrm{N} 1=358,7^{\circ}, \Sigma \Varangle \mathrm{N} 2=358,1^{\circ}, \Sigma \Varangle \mathrm{N} 3=359,2^{\circ}\right)$.

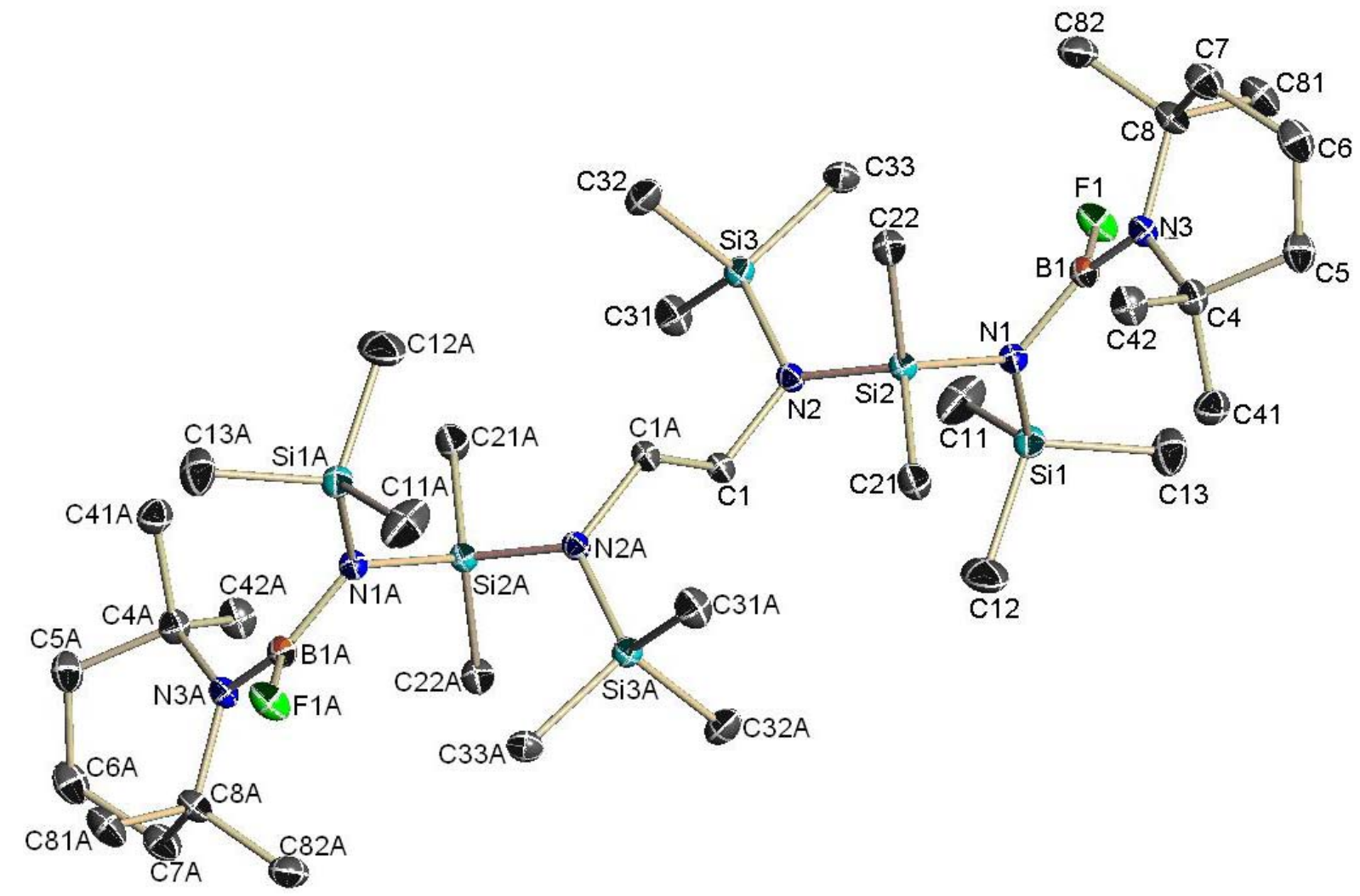

Abbildung 9: Kristallstruktur von 11 ohne Wasserstoffatome

Bindungslängen/pm mit Standardabweichung

\begin{tabular}{|ll|ll|ll|}
\hline B1-F1 & $137,16(17)$ & Si2-N2 & $175,26(12)$ & C4-C42 & $153,90(2)$ \\
\hline B1-N3 & $142,43(19)$ & Si3-N2 & $176,69(12)$ & C1-C1A & $154,30(3)$ \\
\hline B1-N1 & $145,02(19)$ & Si1-N1 & $175,53(12)$ & & \\
\hline Si2-N1 & $174,72(12)$ & N2-C1 & $148,75(17)$ & & \\
\hline
\end{tabular}

Tabelle 13: Ausgewählte Bindungslängen der Verbindung 11

Bindungswinkel $/{ }^{\circ}$ mit Standardabweichung

\begin{tabular}{|ll|ll|ll|}
\hline F1-B1-N3 & $115,71(12)$ & N3-B1-N1 & $130,62(13)$ & B1-N3-C8 & $117,80(12)$ \\
\hline F1-B1-N1 & $113,67(12)$ & B1-N3-C4 & $123,93(12)$ & C4-N3-C8 & $117,42(11)$ \\
\hline
\end{tabular}

Tabelle 14: Ausgewählte Bindungswinkel der Verbindung 11 


\subsubsection{Kristallstruktur von 12}

Verbindung 12 kristallisiert aus $n$-Hexan monoklin in der Raumgruppe $P 2_{1} /$ c. Die

Stickstoffatome N2 und N3 haben Winkelsummen von $\Sigma \Varangle N 2=358,4^{\circ}$ bzW.

$\Sigma \nless \mathrm{N} 3=358,6^{\circ}$ und weichen somit geringfügig von einer planaren Umgebung ab.

Sterische Effekte bewirken relativ große Bindungslängen, z. B. Si4-N3 = 177,5 pm.

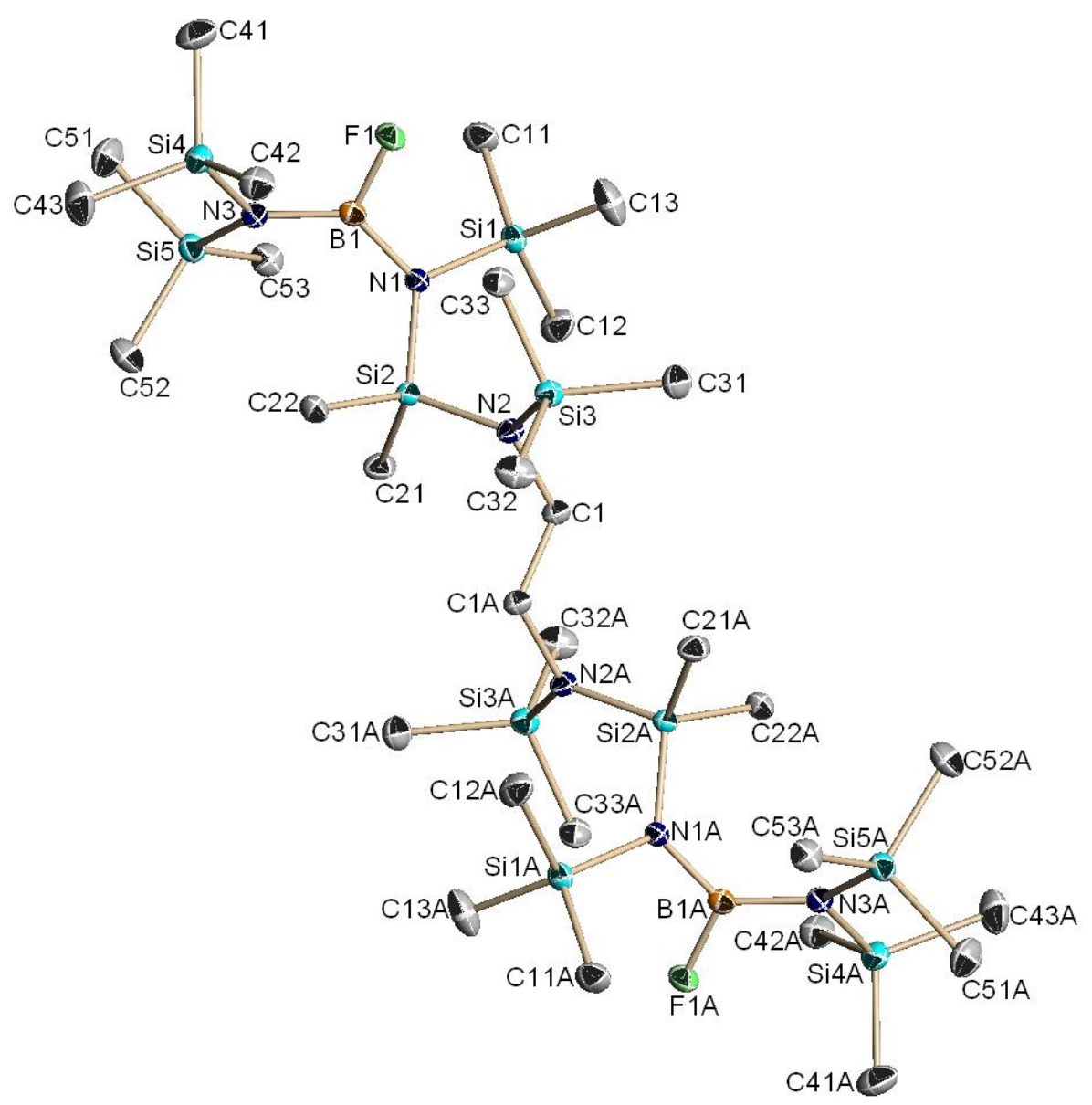

Abbildung 10: Kristallstruktur von 12 ohne Wasserstoffatome

Bindungslängen/pm mit Standardabweichung

\begin{tabular}{|ll|ll|ll|}
\hline Si3-N2 & $175,43(9)$ & Si4-N3 & $177,45(9)$ & B1-F1 & $137,13(13)$ \\
\hline Si2-N2 & $174,78(9)$ & Si5-N3 & $176,14(9)$ & C1-C1A & $154,8(2)$ \\
\hline Si2-N1 & $175,68(9)$ & N1-B1 & $144,21(14)$ & & \\
\hline Si1-N1 & $177,29(9)$ & N3-B1 & $143,36(14)$ & & \\
\hline
\end{tabular}

Tabelle 15: Ausgewählte Bindungslängen der Verbindung 12

Bindungswinkel $/^{\circ}$ mit Standardabweichung

\begin{tabular}{|ll|ll|ll|}
\hline C1-N2-Si2 & $119,14(7)$ & B1-N1-Si2 & $115,54(7)$ & Si5-N3-Si4 & $120,20(5)$ \\
\hline C1-N2-Si3 & $115,91(7)$ & Si2-N1-Si1 & $122,25(5)$ & F1-B1-N3 & $115,42(9)$ \\
\hline Si2-N2-Si3 & $123,39(5)$ & B1-N3-Si5 & $122,60(7)$ & F1-B1-N1 & $114,94(9)$ \\
\hline B1-N1-Si2 & $121,65(7)$ & B1-N3-Si4 & $115,78(7)$ & N3-B1-N1 & $129,64(9)$ \\
\hline
\end{tabular}

Tabelle 16: Ausgewählte Bindungswinkel der Verbindung 12 


\subsubsection{Kristallstruktur des 1,3-Bis(trimethylsilyl)-2,4-bis(1,1,5,5-tetramethyl- piperidino)-1,3-diaza-2,4-dibora-cyclobutan, 14}

Verbindung 14 kristallisiert aus $n$-Hexan orthorhombisch in der Raumgruppe Pbca.

Das Ringsystem ist planar. Die exocyclischen B-N-Bindungen sind mit 144,8 pm für B1-N2 ca. 3,4 pm kürzer als die endocyclischen. Die Boratome und das Stickstoffatom N2 sind mit Winkelsummen von $359,93^{\circ}$ bzw. $359,88^{\circ}$ planar umgeben. Die Silylgruppen am endocyclischen Stickstoffatom N1 zum planaren $(B N)_{2}$-Ring mit $12,8^{\circ}$ trans-ständig angeordnet.

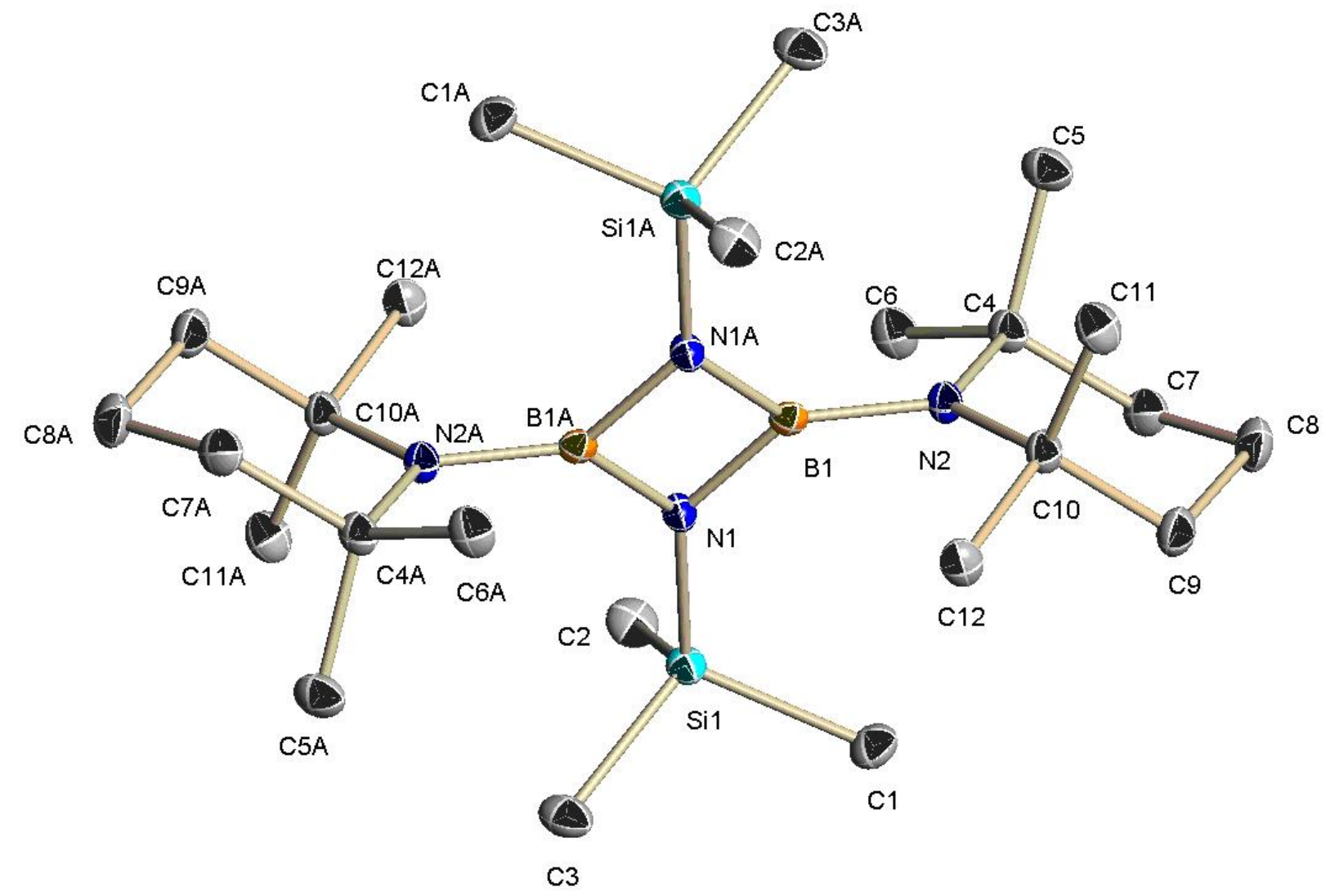

Abbildung 11: Kristallstruktur von 14 ohne Wasserstoffatome

Bindungslängen/pm mit Standardabweichung

\begin{tabular}{|ll|ll|ll|}
\hline Si1-N1 & $173,12(11)$ & N2-B1 & $144,77(17)$ & N1-B1 & $148,09(16)$ \\
\hline C4-N2 & $149,27(15)$ & N1-B1A & $148,05(16)$ & B1-N1A & $148,05(16)$ \\
\hline
\end{tabular}

Tabelle 17: Ausgewählte Bindungslängen der Verbindung 14

Bindungswinkel $/{ }^{\circ}$ mit Standardabweichung

\begin{tabular}{|ll|ll|ll|}
\hline B1-N2-C4 & $122,26(10)$ & B1A-N1-B1 & $83,35(10)$ & N2-B1-N1A & $131,73(11)$ \\
\hline B1-N2-C10 & $117,80(10)$ & B1A-N1-Si1 & $137,99(9)$ & N2-B1-N1 & $131,55(11)$ \\
\hline C4-N2-C10 & $119,82(9)$ & B1-N1-Si1 & $135,49(9)$ & N1A1-B1-N1 & $96,65(10)$ \\
\hline
\end{tabular}

Tabelle 18: Ausgewählte Bindungswinkel der Verbindung 14 


\subsubsection{Synthese der 2,4,7,9-Tetraaza-1-bora-3,8-disila- und 2,4,7,9-Tetraaza-}

\section{1,3,8-trissila-cyclononane, 15 und 16}

Die Reaktion des Dilithiumsalzes 4 mit $\mathrm{SiF}_{4}$ oder $\mathrm{BF}_{3}\left(\mathrm{Et}_{2} \mathrm{O}\right)$ im molaren Verhältnis 1:1 bzw. 1:3 führt zur Bildung der neungliedrigen Ringverbindungen $\mathbf{1 5}$ und 16.

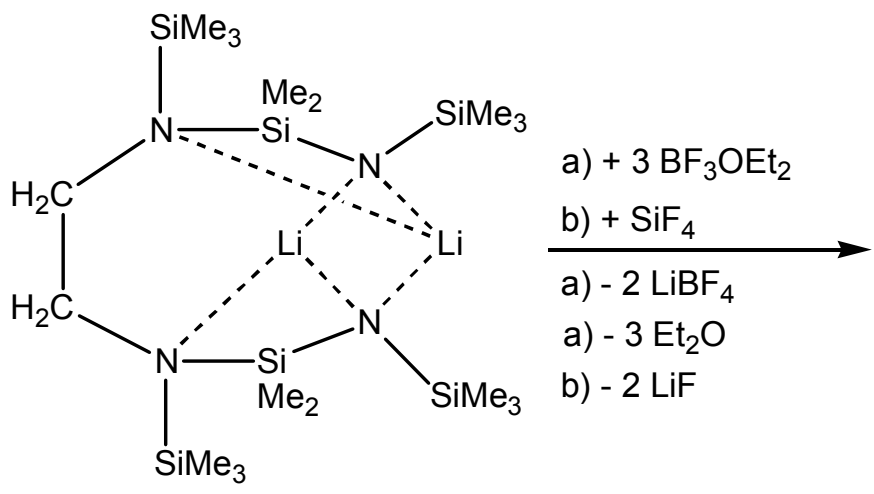

4

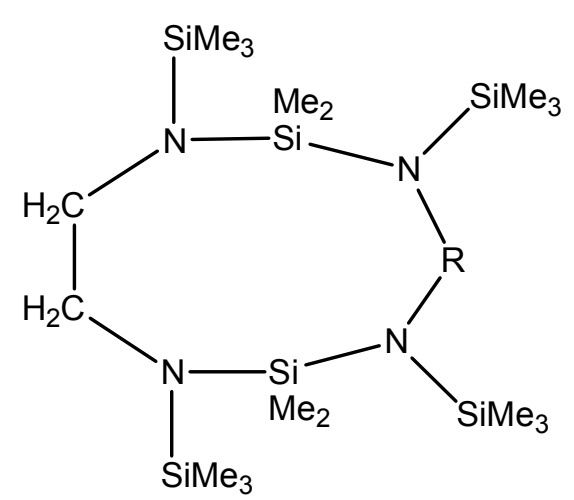

a) $R=B F(15)$,

b) $\mathrm{R}=\mathrm{SiF}_{2}(\mathbf{1 6})$

Gleichung 29: Synthese der Cyclononane 15 und 16

15 und 16 sind bei Raumtemperatur farblose Feststoffe, die im Vakuum unzersetzt destilliert werden können.

Durch Umkristallisation aus $n$-Hexan können Einkristalle erhalten werden. Die gefundene Struktur bestätigt die des kernresonanz- und massenspektroskopisch charakterisierten neungliedrigen Ringes. Fehlordnungsprobleme verhinderten jedoch das vollständige Lösen der Struktur. Auch das Wiederholen der Messung von 9, aus Diethylether und Toluol umkristallisierter Kristalle an unterschiedlichen Diffraktometern (Cu-Ka- statt Mo-Ka-Strahlung), konnte keine leichter zu lösenden Daten liefern. Im Rahmen dieser Arbeit wird deshalb die ermittelte Struktur als ball-and-stick Modell abgebildet. Auf Angaben über Bindungslängen und -winkel wird verzichtet, da keine verlässlichen Aussagen getroffen werden können. 


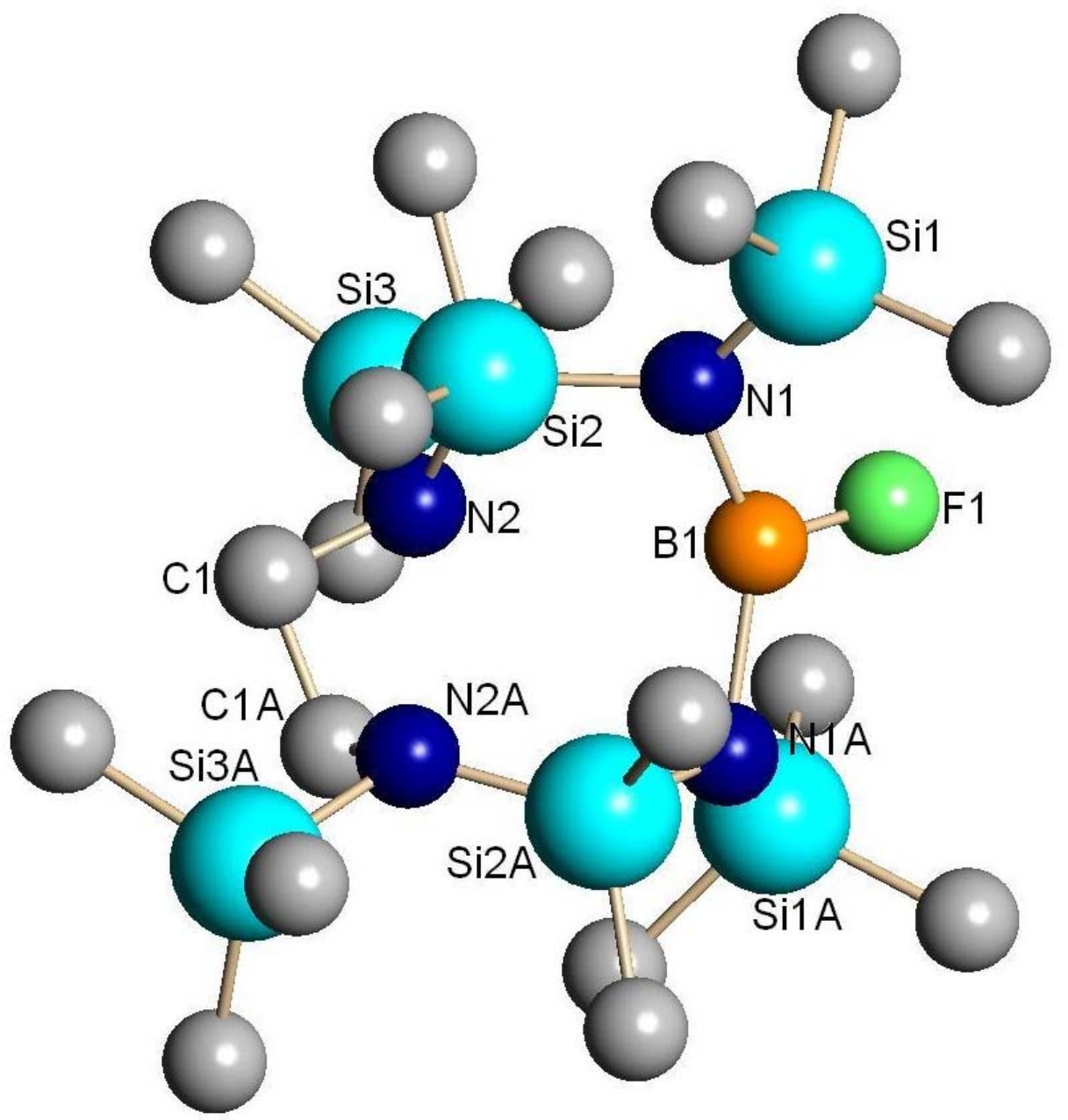

Abbildung 12: Ball-and-stick Modell der Kristallstruktur von 15 ohne Wasserstoffatome

Im ${ }^{1} \mathrm{H}-\mathrm{NMR}$-Spektrum konnte ausschließlich eine ${ }^{5} \mathrm{~J}_{\mathrm{HF}}-$ Kopplung der Protonen der Trimethylsilylgruppe (hier Si1 bzw. Si1A) mit dem Fluoratom festgestellt werden. Wie in Abbildung 12 zu erkennen ist, kann diese Raumkopplung durch den geringen Abstand der Trimethylsilylgruppe zum Fluoratom begründet werden. Auch die mit $3,5 \mathrm{~Hz}$ gefundene ${ }^{4} \mathrm{~J}_{\mathrm{CF}}-$ Kopplung und das Dublett des Siliciumsignals $\left({ }^{3} \mathrm{~J}_{\mathrm{SiF}}=9,1 \mathrm{~Hz}\right)$ sind um 1,9 Hz bzw. $2,5 \mathrm{~Hz}$ größer als die der Dimethylsilylgruppe, was ebenfalls auf eine größere Distanz zur BF-Einheit auch bei Raumtemperatur hinweist. 


\subsection{Synthese und Reaktionen am N,N'-Bis[1,1-bis(trimethylsilyl)-2-aza-3- dimethylsilyl]hydrazin, 17}

Wie unter 3.1 beschrieben, konnte Ethylendiamin in quantitativen Ausbeuten mit den beschriebenen chlorfunktionellen Silylaminen umgesetzt werden. Ein vergleichbares Reaktionsverhalten zeigt auch wasserfreies Hydrazin, wobei das Hydrazin ebenfalls zum Teil als $\mathrm{HCl}-F a ̈ n g e r$ fungiert.
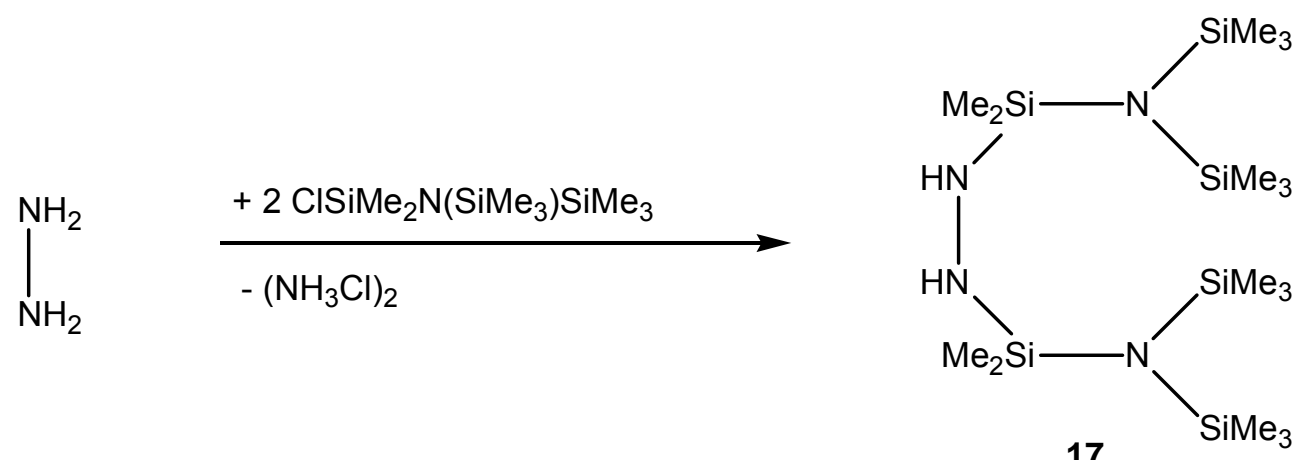

Gleichung 30: Synthese des N,N'-Bis(1,1-bistrimethylsilyl-3-dimethylsilyl-1,3-disila-2-aza)-hydrazins 17

Während die Trimethylsilylgruppen der Ethylendiaminverbindung 1 und der Hydrazinverbindung 17 ähnliche Verschiebungen im ${ }^{29} \mathrm{Si}-\mathrm{NMR}$-Spetrum zeigen, ist die Dimethylsilylgruppe von 17 um ca. 1,8 pm Tieffeld verschoben. Dies ist auf den verstärkten Elektronenzug des zweiten Stickstoffatoms anstelle einer $\mathrm{CH}_{2}$-Gruppe zurückzuführen.

Das erhaltene Hydrazin kann unzersetzt destilliert werden und zeigt keine thermisch induzierte Silylgruppenwanderung (ab $270^{\circ} \mathrm{C}$ Zersetzung). Zwar zeigen die ${ }^{15} \mathrm{~N}-\mathrm{NMR}$-Verschiebungen des Hydrazins im Vergleich zu 1 ein um 25 ppm Hochfeld verschobenes Signal, jedoch ist der Hydrazinstickstoff formal zweifach negativ geladen und dementsprechend weniger Lewis-basisch als der Aminstickstoff (-III). 


\subsubsection{Bildung des Dilithium-N,N'-bis(1-trimethylsilyl-2-amido-3-dimethylsilyl- trimethylsilyl)hydrazins unter anionischer Silylgruppen-Wanderung}

Versuche, die Monolithiumsalze von 1 und $\mathbf{1 7}$ darzustellen, schlugen fehl. Stattdessen wird bei einer äquimolaren Umsetzung mit $n$-Butyllithium zur Hälfte das $N, N^{\prime}$-Dilithium-bis[1,1-bis(trimethylsilyl)-2-amino-3-dimethylsilyl)hydrazid erhalten, welches bereits bei Raumtemperatur durch anionische Silatropie zu 18 umlagert. Das Supramolekül (ionischer Kern, große kovalente Hülle ${ }^{[40]}$ ) kann destillativ gereinigt werden.

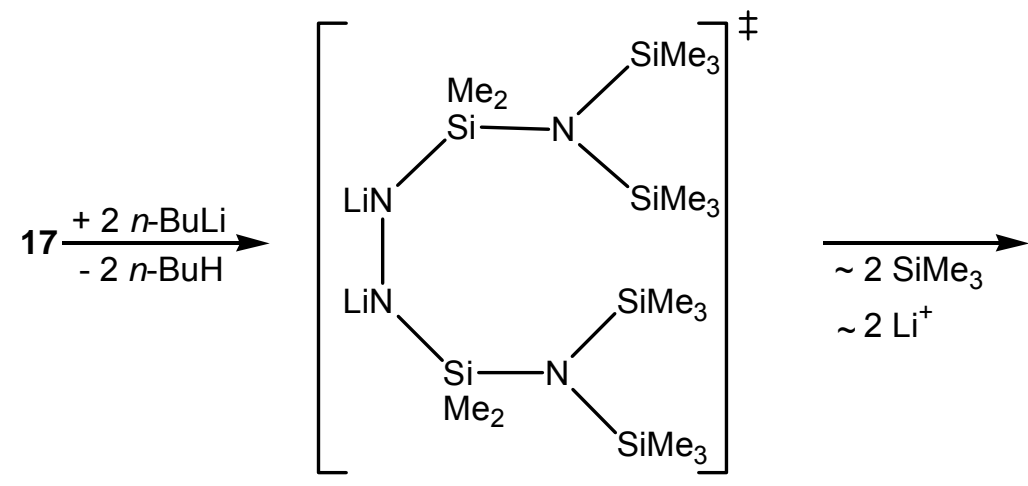

Gleichung 31: Synthese von 18

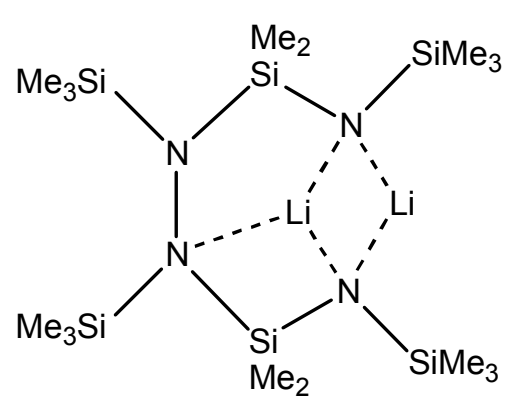

18

Wird 18 in siedendem THF gelöst und bei Raumtemperatur auskristallisiert, addiert 18 je Lithiumion ein THF-Molekül. Es entsteht das ebenfalls monomere Dilithiumsalz 19.

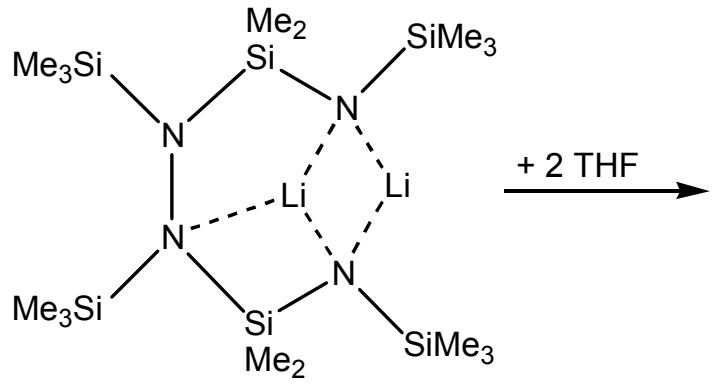

18

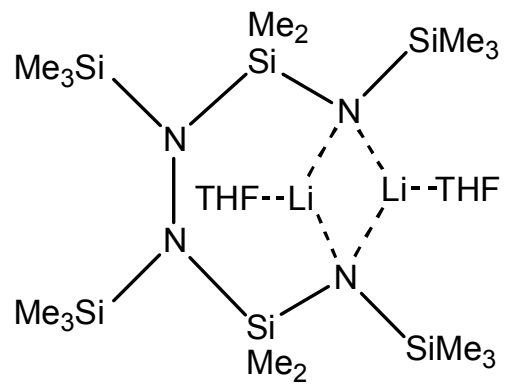

19

Gleichung 32: Synthese von 19 


\subsubsection{Kristallstrukturen der Dilithiumsalze 18 und 19}

\subsubsection{Dilithium- $N, N$ '-bis(1-trimethylsilyl-2-amido-3-dimethylsilyl-trimethyl- silyl)hydrazin, 18}

18 kristallisiert aus $n$-Hexan orthorhombisch in der Raumgruppe $P 2_{1}$.

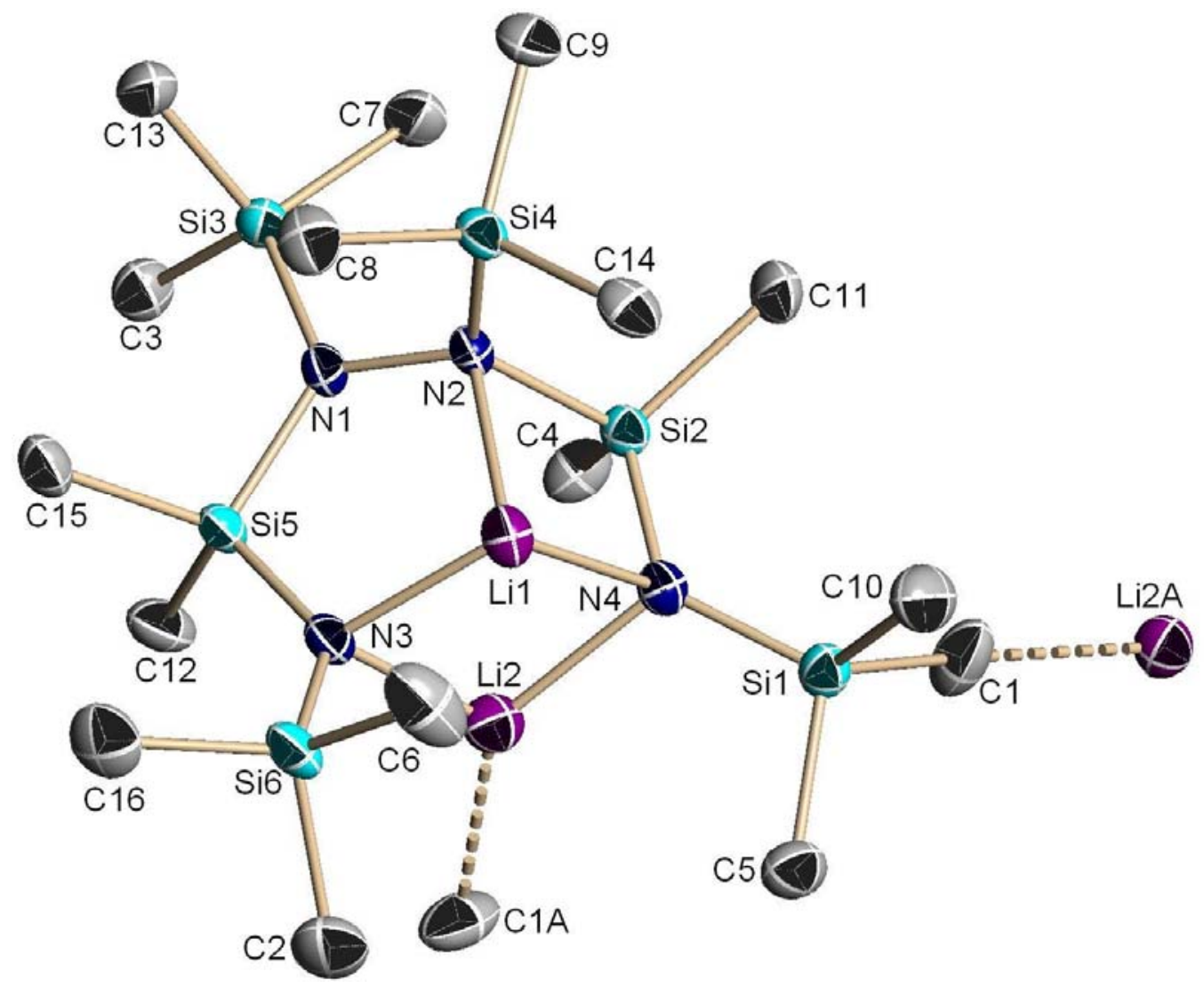

Abbildung 13: Kristallstruktur von Verbindung 18 ohne Wasserstoffatome

Bindungslängen (-) bzw. intermolekulare Atomabstände (...)/pm mit Standardabweichung

\begin{tabular}{|ll|ll|ll|}
\hline Li1-N3 & $195,7(4)$ & Li2...C1A & $252,4(5)$ & Si3-N1 & $174,9(2)$ \\
\hline Li1-N4 & $199,3(4)$ & C1 ..Li2A & $252,4(5)$ & Si4-N2 & $175,4(2)$ \\
\hline Li1-N2 & $216,7(4)$ & Si1-N4 & $168,6(2)$ & Si2-N2 & $178,6(2)$ \\
\hline Li2-N4 & $200,9(4)$ & Si1-C1 & $189,5(3)$ & Si5-N1 & $178,1(2)$ \\
\hline Li2-N3 & $201,9(5)$ & Si1-C5 & $187,0(2)$ & Si5-N3 & $168,5(2)$ \\
\hline Si6-N3 & $169,6(2)$ & Si1-C10 & $187,2(3)$ & N1-N2 & $150,0(2)$ \\
\hline
\end{tabular}

Tabelle 19: Ausgewählte Bindungslängen bzw. intermolekulare Atomabstände der Verbindung 18

Bindungswinkel $/^{\circ}$ mit Standardabweichung

\begin{tabular}{|ll|ll|ll|}
\hline N3-Li1-N4 & $105,82(18)$ & N3-Li1-N2 & $94,23(16)$ & N4-Li1-N2 & $83,85(14)$ \\
\hline N4-Li2-N3 & $102,92(19)$ & N4-Li2-C1A & $131,6(2)$ & N3-Li2-C1A & $124,55(19)$ \\
\hline N2-N1-Si3 & $116,21(12)$ & N2-N1-Si5 & $116,77(12)$ & Si3-N1-Si5 & $126,54(9)$ \\
\hline N1-N2-Si4 & $117,43(12)$ & N1-N2-Si2 & $116,23(12)$ & Si4-N2-Si2 & $125,76(10)$ \\
\hline N1-N2-Li1 & $107,76(14)$ & Si4-N2-Li1 & $89,93(12)$ & Si2-N2-Li1 & $81,20(12)$ \\
\hline Si1-C1-Li2A & $174,3(2)$ & & & & \\
\hline
\end{tabular}

Tabelle 20: Ausgewählte Bindungswinkel der Verbindung 18 
Im Dilithiumsalz 18 werden die endständigen Amido-Stickstoffionen (N3, N4) durch die Lithiumionen (Li1, Li2) zu einem viergliedrigen ( $\mathrm{LiN})_{2}$-Ring verklammert. Das Lithiumion Li1 hat zusätzlich einen schwächeren Kontakt zum Stickstoffatom des Hydrazins (Li1-N2 $=216,7 \mathrm{pm}$ ), so dass ein viergliedriger $\left(\mathrm{LiN}_{2} \mathrm{Si}\right)$ - und ein fünfgliedriger ( $\mathrm{LiN}_{3} \mathrm{Si}$ )-Ring gebildet werden. Das Lithiumion Li1 hat somit eine pyramidale Umgebung mit einer Winkelsumme von $283,9^{\circ}$. Der Lithium-Hydrazin-Kontakt führt beim Hydrazinstickstoff N2 zum Verlust der Planarität. Der an zwei Lithiumionen koordinierte Amidostickstoff hat eine tetraedrische Umgebung. Die Amido-SiliciumAbstände (168 - 170 pm) sind im Vergleich zu den Hydrazin-Silicium-Abständen erwartungsgemäß um 5 - 10 pm verkürzt. Während beim Dilithiumsalz 4 beide Lithiumionen einen Kontakt zum Aminostickstoff haben, zeigt in $\mathbf{1 8}$ das Lithiumion Li2 eine Wechselwirkung mit der Methylgruppe(C1A) des benachbarten Moleküls. Dieser Abstand von $252 \mathrm{pm}$ ist relativ lang, bedingt aber eine Bindungsverlängerung der Si1-C1-Bindung um $2 \mathrm{pm}$. Weiterhin führt dies zu einer Zick-Zack-Anordnung der Moleküle im Kristall, wobei jedes mit zwei weiteren verbunden ist (in Abbildung 14 über C1 und Li2).

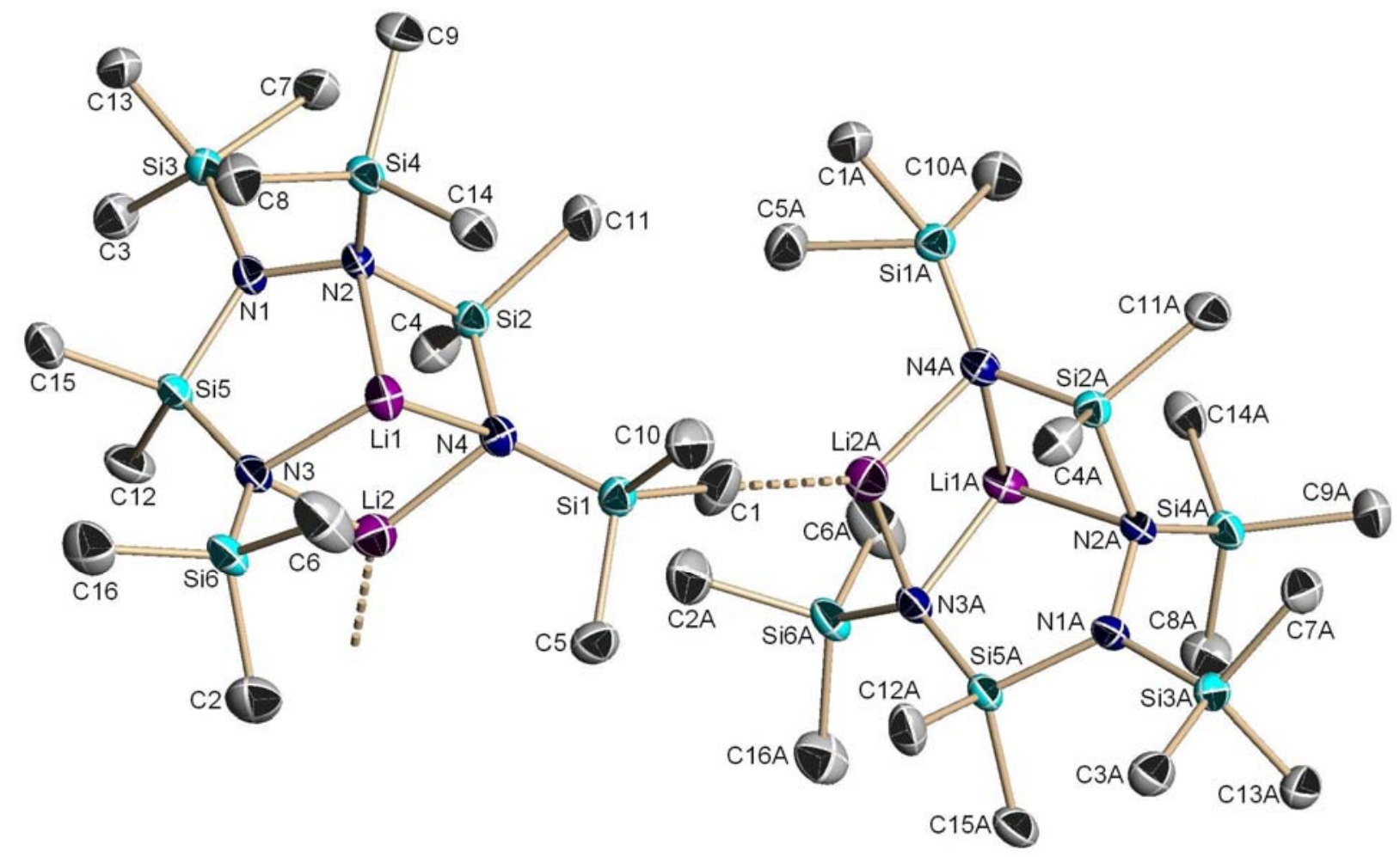

Abbildung 14: Anordnung zweier Moleküle von 18 ohne Wasserstoffatome 


\subsubsection{Dilithium- $N, N$ '-bis(1-trimethylsilyl-2-amido-3-dimethylsilyl-trimethyl- silyl)hydrazin·2 THF, 19}

Das THF-Addukt des Dilithiumsalzes (19) wird durch Kristallisation von 18 aus THF isoliert. 19 kristallisiert monoklin in der Raumgruppe $P \overline{1}$. Auf Grund einer nicht vollständig gelösten Fehlordnung eines THF-Moleküls wird O2 nicht als Thermalellipsoid dargestellt.

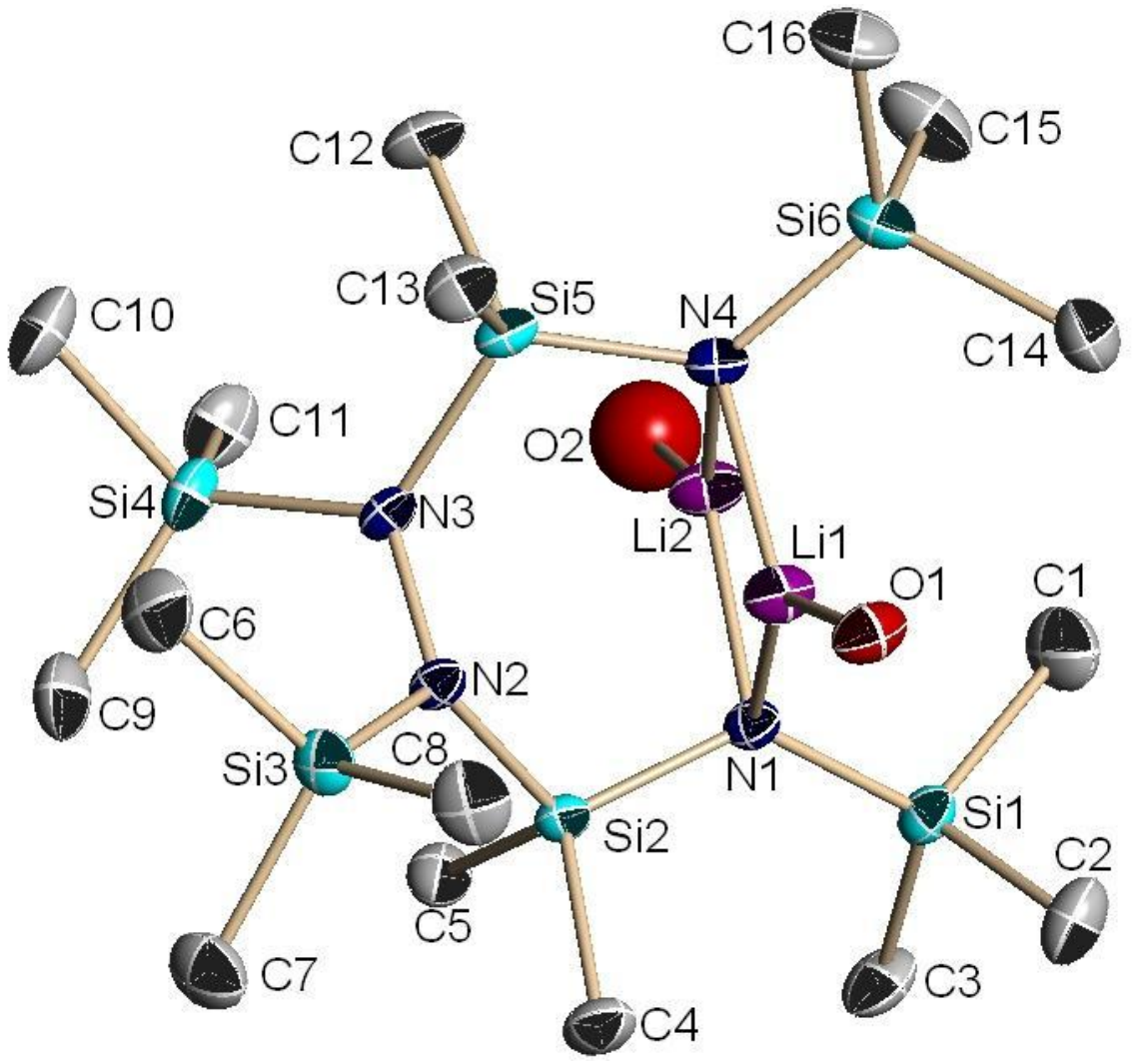

Abbildung 15: Kristallstruktur des Dilithiumsalzes 19 ohne Wasserstoffatome und THF-Methylgruppen

Bindungslängen/pm mit Standardabweichung

\begin{tabular}{|ll|ll|ll|}
\hline Si1-N1 & $169,4(1)$ & Si5-N4 & $168,1(2)$ & N1-Li2 & $198,3(3)$ \\
\hline Si2-N1 & $168,7(2)$ & Si5-N3 & $177,9(2)$ & N1-Li1 & $202,5(3)$ \\
\hline Si2-N2 & $178,0(1)$ & Si6-N4 & $169,2(2)$ & N2-N3 & $151,3(2)$ \\
\hline Si3-N2 & $176,3(1)$ & O1-Li1 & $195,0(3)$ & N4-Li1 & $197,4(3)$ \\
\hline Si4-N3 & $176,6(1)$ & N4-Li2 & $202,0(3)$ & & \\
\hline
\end{tabular}

Tabelle 21: Ausgewählte Bindungslängen der Verbindung 19

Bindungswinkel $/{ }^{\circ}$ mit Standardabweichung

\begin{tabular}{|ll|ll|ll|}
\hline Si2-N1-Si1 & $127,80(9)$ & Li2-N1-Li1 & $80,23(13)$ & Si4-N3-Si5 & $122,81(8)$ \\
\hline Si2-N1-Li2 & $108,39(12)$ & N3-N2-Si3 & $119,29(10)$ & N2-N3-Si5 & $115,78(10)$ \\
\hline Si1-N1-Li2 & $113,28(12)$ & N3-N2-Si2 & $114,95(10)$ & O1-Li1-N1 & $138,60(17)$ \\
\hline Si2-N1-Li1 & $97,34(11)$ & Si3-N2-Si2 & $123,07(8)$ & O1-Li1-N4 & $121,28(16)$ \\
\hline Si1-N1-Li1 & $119,15(12)$ & N2-N3-Si4 & $119,21(11)$ & N4-Li1-N1 & $99,51(14)$ \\
\hline
\end{tabular}

Tabelle 22: Ausgewählte Bindungswinkel der Verbindung 19 
Durch die Koordination eines THF-Moleküls pro Lithiumion wird im Vergleich zu 18 der Lithiumkontakt zum Hydrazinstickstoff und zur Methylgruppe des benachbarten Moleküls gelöst. Die Lithiumionen sind mit Winkelsummen von $359.4^{\circ}$ (Li1) und $359.8^{\circ}$ (Li2) trigonal planar umgeben, die Amido-Stickstoffionen haben eine tetraedrische und die Hydrazin-Stickstoffatome eine beinahe planare Umgebung $\left(\Sigma \nless \mathrm{N} 2=357,3^{\circ}, \Sigma \nless \mathrm{N} 3=357,8^{\circ}\right)$.

\subsubsection{Synthese von 20 sowie der Cycloheptane 21 und 22}

Wird das Dilithiumsalz 18 hydrolysiert, erhält man das thermodynamisch stabilere Isomer zu Verbindung 17. Im Vergleich der ${ }^{29}$ Si-NMR-Verschiebungen von 20 mit der Ethylendiaminverbindung 7 sind die an der Hydrazineinheit gebundenen Silylgruppen weiter Hochfeld verschoben, was auf den verstärkten -I-Effekt des zweiten Stickstoffatoms anstelle der Ethylgruppe zurückzuführen ist.

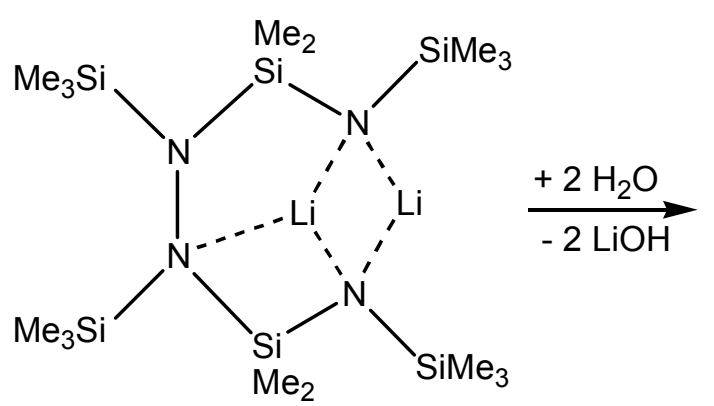

18

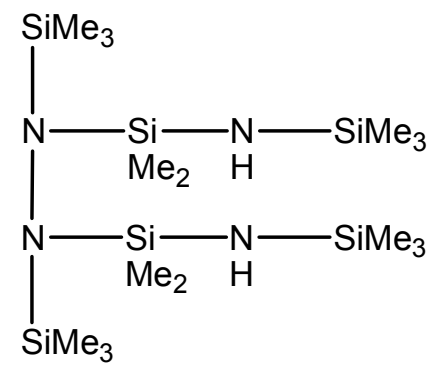

20

Gleichung 33: Synthese von Verbindung 20

Die Reaktion des Dilithiumsalzes 18 mit $\mathrm{SiF}_{4}$ oder $\mathrm{BF}_{3} \mathrm{OEt}_{2}$ im molaren Verhältnis 1:1 bzw. 1:3 führt zur Bildung der siebengliedrigen Ringverbindungen 21 und 22.

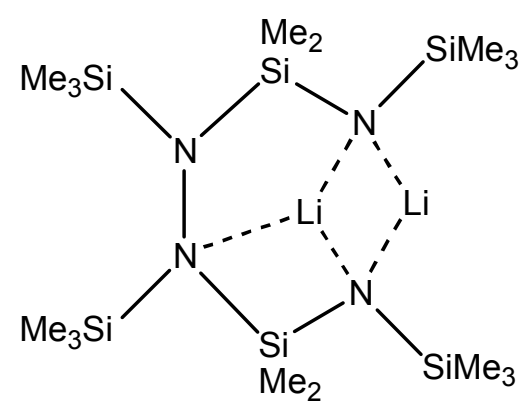

18 a) $+3 \mathrm{BF}_{3} \mathrm{OEt}_{2}$

b) $+\mathrm{SiF}_{4}$

a) - $2 \mathrm{LiBF}_{4}$ $-3 \mathrm{Et}_{2} \mathrm{O}$

b) $-2 \mathrm{LiF}$

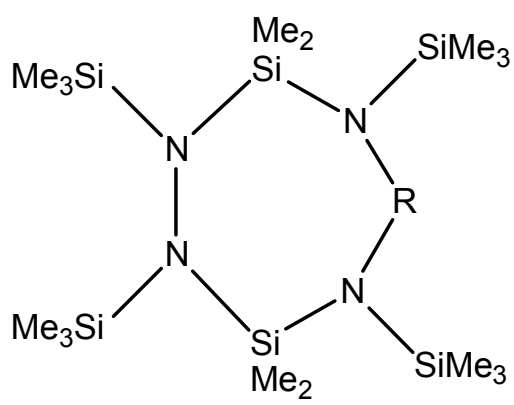

$\mathrm{R}=\mathrm{BF}(\mathbf{2 1}), \mathrm{SiF}_{2}(\mathbf{2 2})$ 
21 und 22 sind bei Raumtemperatur farblose Feststoffe, die im Vakuum unzersetzt destilliert werden können.

Durch Umkristallisation aus $n$-Hexan werden Einkristalle von 21 erhalten, die röntgenstrukturanalytisch untersucht wurden.

\subsubsection{Kristallstruktur von Verbindung 21}

Verbindung 21 kristallisiert aus $n$-Hexan monoklin in der Raumgruppe $P 2_{1} / \mathrm{n}$. Die Borund Stickstoffatome sind bis auf N2 $\left(\Sigma \nless N 2=356,5^{\circ}\right)$ planar umgeben $\left(\Sigma \nless \mathrm{B} 1=359,9^{\circ}, \Sigma \nless \mathrm{N} 1=359,3^{\circ}, \Sigma \nless \mathrm{N} 3=359,9^{\circ}, \Sigma \nless \mathrm{N} 4=358,8^{\circ}\right)$. Die Hydrazinstickstoff-Siliciumbindungen sind kürzer als die Aminstickstoff-Siliciumabstände.

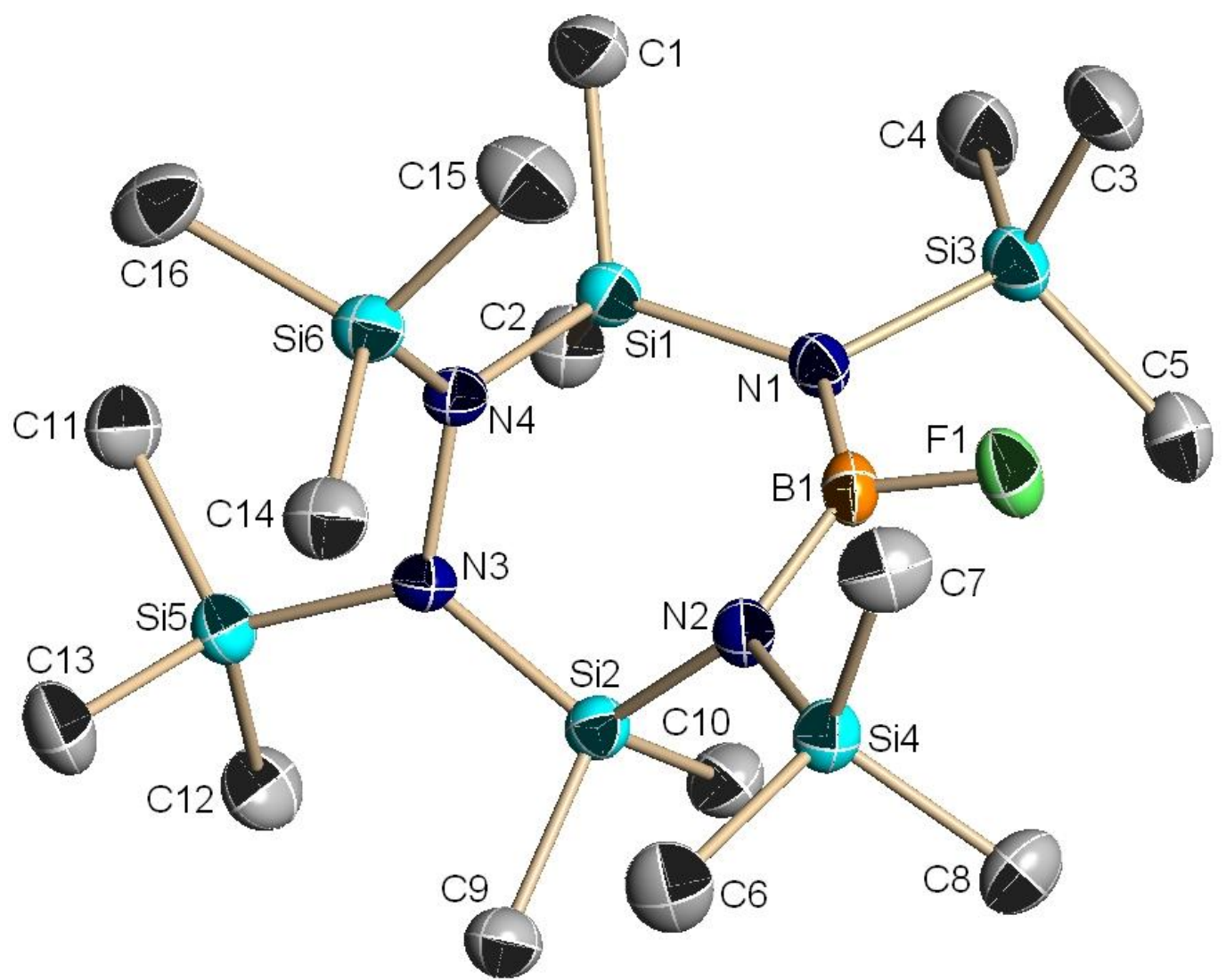

Abbildung 16: Kristallstruktur von 21 ohne Wasserstoffatome

Bindungslängen/pm mit Standardabweichung

\begin{tabular}{|ll|ll|ll|}
\hline B1-F1 & $136,4(3)$ & Si1-N4 & $172,7(2)$ & Si3-N1 & $178,1(2)$ \\
\hline B1-N1 & $143,3(3)$ & Si1-N1 & $175,6(2)$ & Si4-N2 & $177,5(2)$ \\
\hline B1-N2 & $144,8(3)$ & Si2-N3 & $173,8(2)$ & Si5-N3 & $175,6(2)$ \\
\hline N3-N4 & $149,0(2)$ & Si2-N2 & $175,1(2)$ & Si6-N4 & $174,8(2)$ \\
\hline
\end{tabular}

Tabelle 23: Ausgewählte Bindungslängen der Verbindung 21 
Bindungswinkel $/{ }^{\circ}$ mit Standardabweichung

\begin{tabular}{|ll|ll|ll|}
\hline F1-B1-N1 & $114,82(18)$ & F1-B1-N2 & $114,2(2)$ & N1-B1-N2 & $130,9(2)$ \\
\hline B1-N1-Si1 & $123,38(14)$ & B1-N1-Si3 & $113,34(14)$ & Si1-N1-Si3 & $122,56(10)$ \\
\hline B1-N2-Si2 & $116,37(15)$ & B1-N2-Si4 & $114,46(15)$ & Si2-N2-Si4 & $125,66(10)$ \\
\hline N4-N3-Si2 & $116,24(12)$ & N4-N3-Si5 & $116,92(13)$ & Si2-N3-Si5 & $126,69(10)$ \\
\hline N3-N4-Si1 & $111,77(12)$ & N3-N4-Si6 & $117,40(12)$ & Si1-N4-Si6 & $129,66(10)$ \\
\hline N1-Si1-N4 & $108,44(8)$ & N2-Si2-N3 & $108,60(9)$ & & \\
\hline
\end{tabular}

Tabelle 24: Ausgewählte Bindungswinkel der Verbindung 21

In seitlicher Ansicht zeigt der siebengliedrige Ring eine twistartige Konformation, da Stickstoff und Bor beinahe planar umgeben sind und auch die endocyclischen Siliciumatome Tetraederwinkel zeigen $\left(\mathrm{N} 4-\mathrm{Si} 1-\mathrm{N} 1=108.44^{\circ} ; \mathrm{N} 3-\mathrm{Si} 2-\mathrm{N} 2=108.60^{\circ}\right)$.

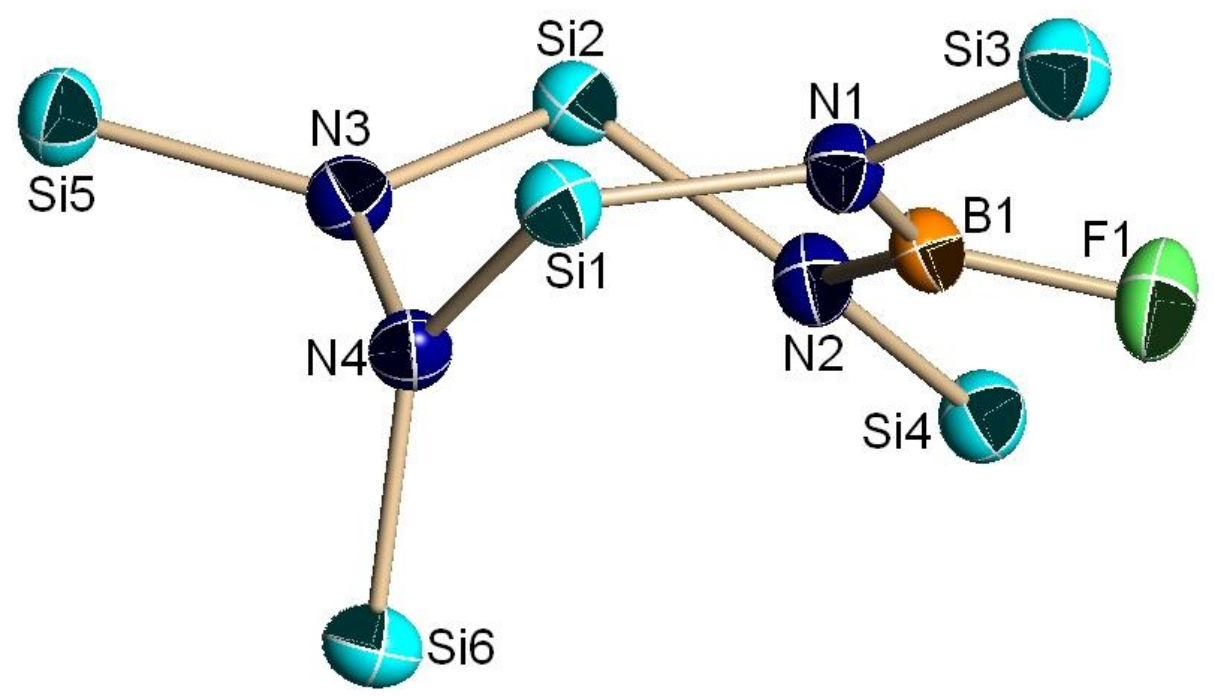

Abbildung 17: Seitenansicht von 22 ohne Methylgruppen

Im Vergleich der ${ }^{1} \mathrm{H}$-, ${ }^{13} \mathrm{C}$ - und ${ }^{29} \mathrm{Si}-\mathrm{NMR}$-Verschiebungen von 21 findet man bei der BF-nahen Trimethylsilylgruppe größere Fluorkopplungen als bei der Dimethylsilylgruppe, was auf eine geringere Distanz zum Fluoratom hinweist und durch die Röntgenstrukturdaten (zumindest bei $133 \mathrm{~K}$ ) bestätigt werden konnte. 


\subsubsection{ESR-Messungen an einer Diethyletherlösung von 21}

Beim Versuch, Einkristalle von $\mathbf{2 1}$ aus einer gesättigten Lösung in Diethylether zu erhalten, kann eine temperaturabhängige, reversible Farbänderung festgestellt werden. Während die Lösung bei Raumtemperatur zunächst farblos erscheint, färbt sich das Gemisch bei Temperaturen unter $0^{\circ} \mathrm{C}$ nach 2 Tagen blaugrün. Ein Erhitzen der Probe auf $35^{\circ} \mathrm{C}$ führt zur erneuten Entfärbung.

Um dieses Phänomen näher zu untersuchen, wurden ESR-Spektren der farblosen $\left(\mathbf{2 1} 1_{\mathrm{E} 1}\right)$ und blaugrünen Probe (21 $\left.1_{\mathrm{E} 2}\right)$ aufgenommen.

Während sich bei $\mathbf{2} \mathbf{1}_{\mathrm{E} 1}$ kein ESR-Signal findet, wird bei $\mathbf{2} \mathbf{1}_{\mathrm{E} 2}$ das Auftreten eines relativ komplizierten Signals im ESR-Spektrum beobachtet. Eine Ursache für dieses reversible Phänomen ist wahrscheinlich in den unterschiedlichen Oxidationsstufen der Amino- und Hydrazinostickstoffatome begründet, jedoch kann auch eine Beteiligung der Lewis-Säure Bor in diesem Prozess nicht ausgeschlossen werden. Ohne Verwendung eines basischen Lösungsmittels - hier Diethylether - ist dieser Effekt nicht zu beobachten. Wird beispielsweise die Anregung zum Triplett als elektronische Konfiguration angenommen, belegen derzeit durchgeführte DFT-Rechnungen eine Aufweitung der N-N-Bindung um ca. 100 pm im Vergleich zur Kristallstruktur (siehe Abbildung 16). Eine Aufklärung dieser Befunde ist weiteren Studien vorbehalten. 


\subsection{Reaktionen lithiierter Di(tert-butyl)fluorsilylamide}

\subsubsection{Reaktionen des Lithium-tert-butyl-di(tert-butyl)fluorsilyl-amids mit Trimethylalan}

Wird Lithium-tert-butyl-di(tert-butyl)fluorsilyl-amid ${ }^{[42,44]}$ mit Chloralanen umgesetzt, finden eine Lithiumchlorid- und eine Lithiumfluoridabspaltung statt (siehe 1.2.6, Gleichungen 14 und 15). Die Röntgenstrukturen zeigen beim tert-Butyl-di(tert-butyl)silyltrichloralan-amin ein verbrückendes Chloratom zum Aluminium und zum Silicium ${ }^{[44]}$. Im Rahmen dieser Arbeit sollte untersucht werden, ob bei einem Austausch der Trichloralangruppe durch Trimethylalan die Darstellung von dreifach koordiniertem Silicium oder Aluminium erreicht werden kann.

Bei der Reaktion entsteht in sehr guten Ausbeuten ausschließlich die Dimethylalanverbindung 23, was in den ${ }^{1} \mathrm{H}$ - sowie ${ }^{13} \mathrm{C}$-Spektren durch das Auftreten von zwei Methylgruppen am Aluminum und einer am Silicium deutlich wird. Das intermediär wahrscheinlich entstehende Silylkation ist somit die stärkere Lewis-Säure. Dies bedingt die Wanderung einer Methanidgruppe vom Aluminium zum Silicium.

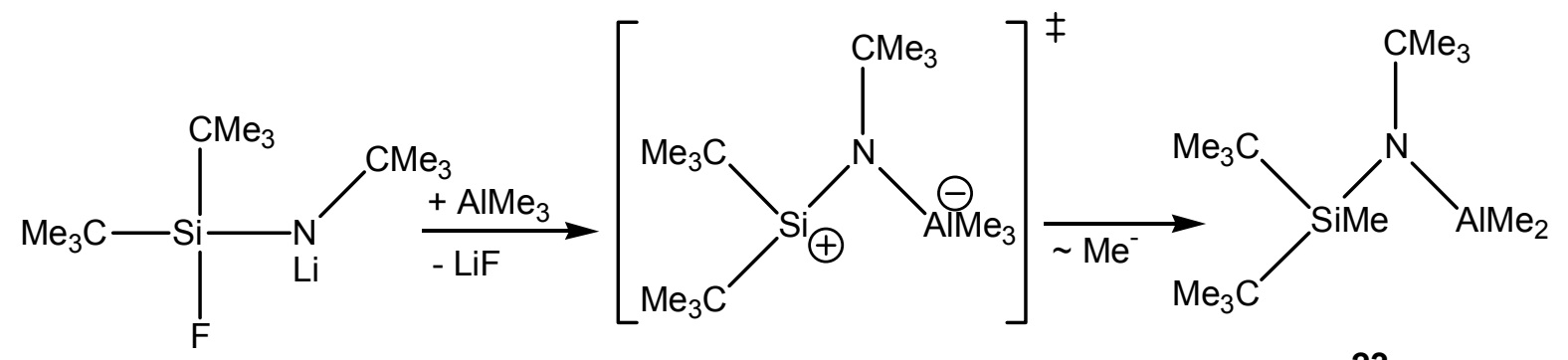

23

Gleichung 35: Reaktion des Lithium-tert-Butyl-di(tert-butyl)fluorsilyl-amids mit Trimethylalan

Die wachsartige, extrem hygroskopische Verbindung 23 kann bei schonender Hydrolyse zu 24 umgesetzt werden. Das hierbei kein Silanol gebildet wird, ist ein weiterer Hinweis auf eine vollständige Anlagerung einer Methylgruppe an das Siliciumatom (siehe Gleichung 36). 


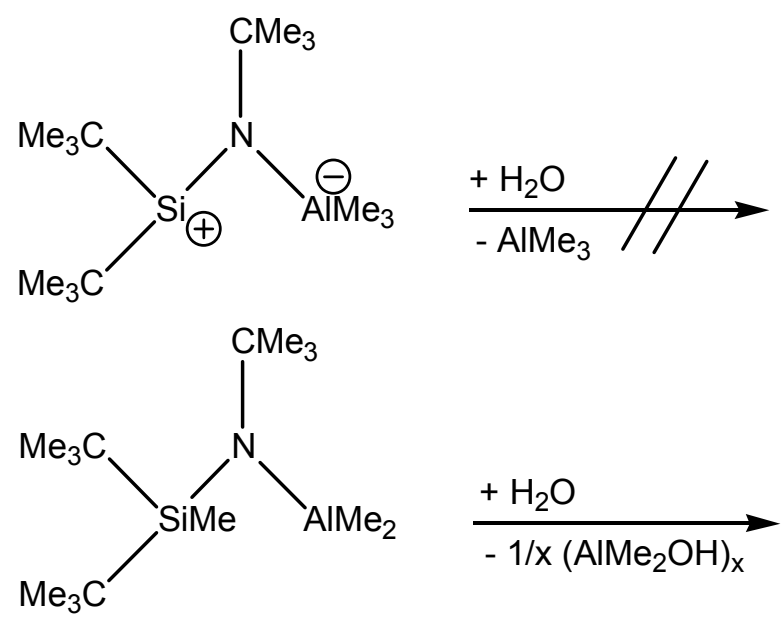

23<smiles>CC(C)(C)NC(C)(C)O</smiles><smiles>CC(C)(C)N[As](C)(C)C</smiles>

24

Gleichung 36: Hydrolyse von 23

Verbindung 24 kann mit $n$-Butyllithium lithiiert werden. Durch Kristallisation aus $n$-Hexan werden farblose Kristalle erhalten (25), welche röntgenstrukturanalytisch untersucht wurden.<smiles>CN[Si](C)(C)C</smiles>

24<smiles></smiles>

25

Gleichung 37: Synthese des Lithiumsalzes 25 


\subsubsection{Kristallstruktur des Lithium-tert-butyl-di(tert-butyl)methylsilyl-amids,} 25

Verbindung 25 kristallisiert aus $n$-Hexan monoklin in der Raumgruppe $P 2_{1} / \mathrm{n}$. Die Lithium- und Stickstoffatome bilden einen viergliedrigen Ring, welcher geringfügig von der Planarität abweicht ( $\Sigma$ Ringinnenwinkel $\left.=358,6^{\circ}\right)$.

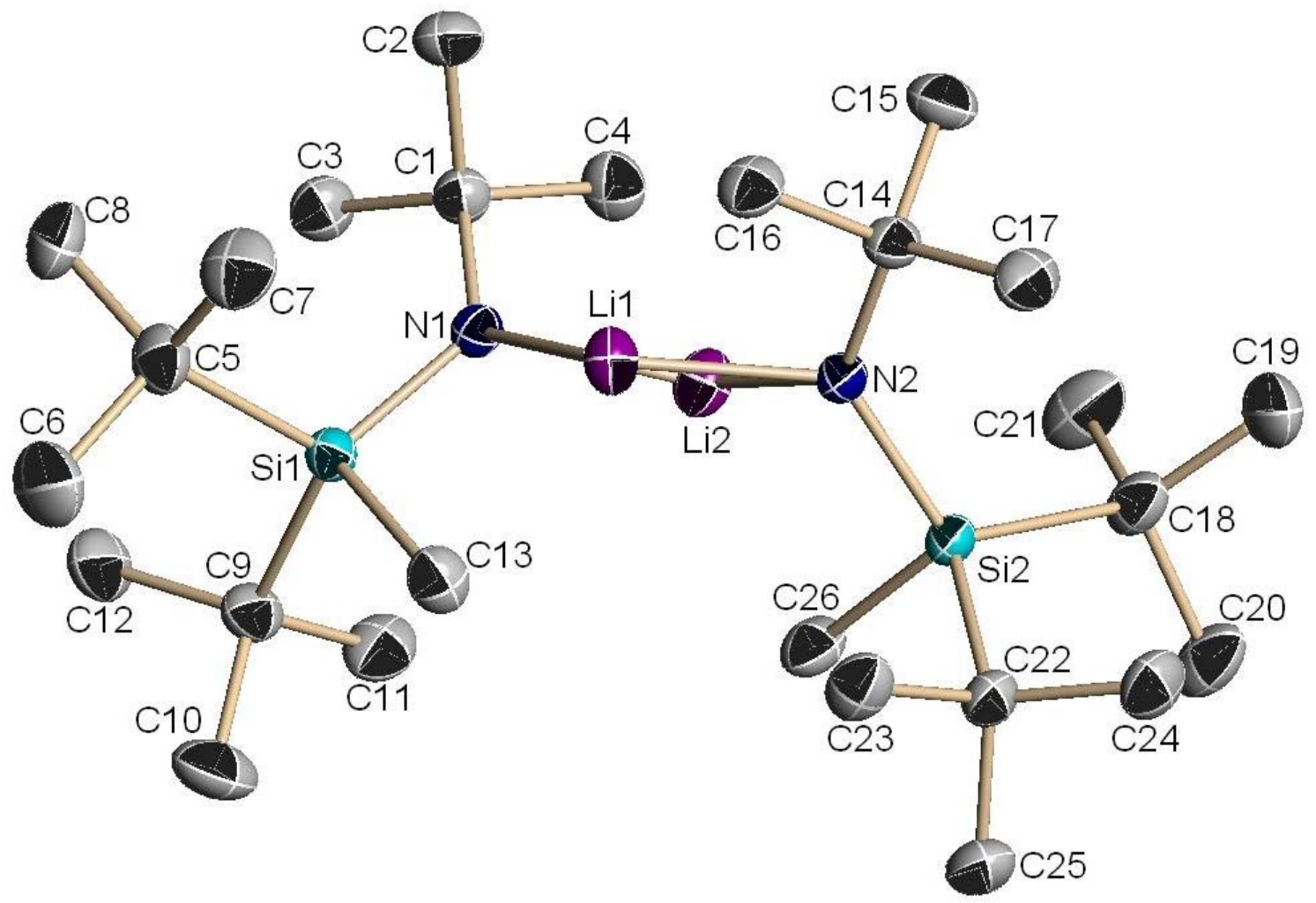

Abbildung 18: Kristallstruktur von 25 ohne Wasserstoffatome

Bindungslängen (-) bzw. intramolekulare Atomabstände (...)/pm mit Standardabweichung

\begin{tabular}{|ll|ll|ll|}
\hline N1-Li2 & $199,9(2)$ & N2-Li2 & $200,2(3)$ & N2-Li1 & $199,5(2)$ \\
\hline N1-Li1 & $200,4(2)$ & Si1-N1 & $170,8(1)$ & N1-C1 & $148,4(2)$ \\
\hline Li1 ...Li2 & $220,3(3)$ & Li1 ..C13 & 236,7 & Li1..C16 & 227,5 \\
\hline Li2 ...C4 & 229,7 & Li2..C26 & 241,5 & & \\
\hline
\end{tabular}

Tabelle 25: Ausgewählte Bindungslängen bzw. intramolekulare Atomabstände der Verbindung 25

Bindungswinkel $/{ }^{\circ}$ mit Standardabweichung

\begin{tabular}{|ll|ll|ll|}
\hline C1-N1-Si1 & $128,40(8)$ & C1-N1-Li2 & $96,72(10)$ & Si1-N1-Li2 & $128,18(9)$ \\
\hline C1-N1-Li1 & $131,33(10)$ & Si1-N1-Li1 & $93,26(8)$ & Li2-N1-Li1 & $66,80(9)$ \\
\hline C14-N2-Si2 & $128,01(8)$ & C14-N2-Li1 & $95,64(9)$ & Si2-N2-Li1 & $129,43(8)$ \\
\hline C14-N2-Li2 & $130,19(10)$ & Si2-N2-Li2 & $94,82(8)$ & Li1-N2-Li2 & $66,91(10)$ \\
\hline N2-Li1-N1 & $112,49(11)$ & N1-Li2-N2 & $112,40(11)$ & & \\
\hline
\end{tabular}

Tabelle 26: Ausgewählte Bindungswinkel der Verbindung 25 
Sowohl die tert-Butyl- als auch die Di(tert-butyl)methylsilylgruppen stehen unerwartet cis-ständig zueinander. Ebenfalls überraschend ist die Zweifachkoordination der Lithiumatome. Während ${ }^{1} \mathrm{H}$ - und ${ }^{13} \mathrm{C}$-NMR-Messungen von 25 in $\mathrm{C}_{6} \mathrm{D}_{6}$-Lösung bei Raumtemperatur äquivalente Methylgruppen zeigen, orientieren sich die silylgebundenen Methylgruppen (C13 und C26) bzw. die Kohlenstoffatome C4 und C16 im Kristall auf Grund elektrostatischer Wechselwirkungen zu den Lithiumatomen. Die Abstände liegen zwischen 227 - 242 pm. Intermolekulare Wechselwirkungen (wie bei Verbindung 18) werden nicht gefunden.

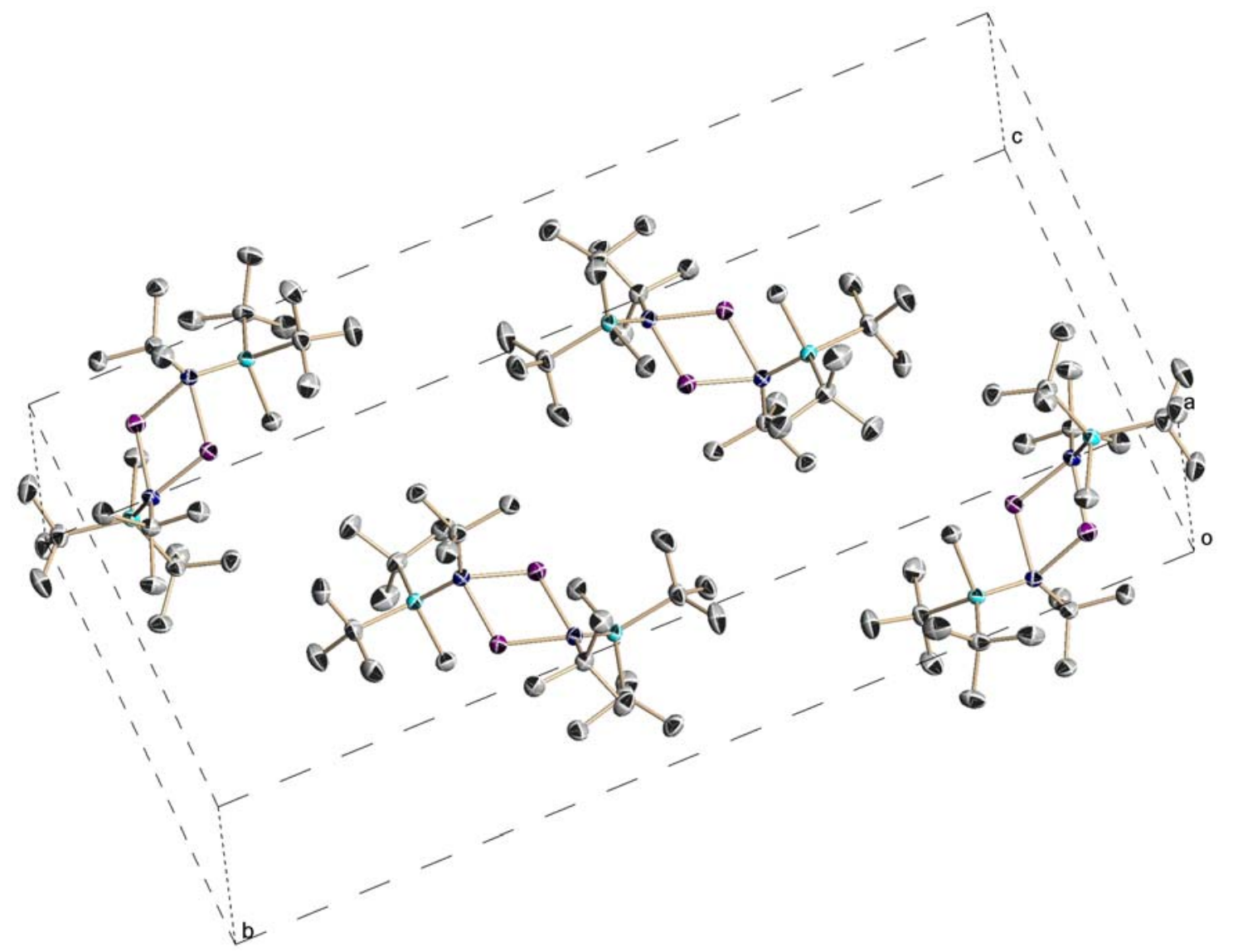

Abbildung 19: Anordnung der Moleküle von 25 in der Elementarzelle ohne Wasserstoffatome 


\subsubsection{Reaktionen des Lithium-tert-butyl-di(tert-butyl)fluorsilyl-amids mit Fluorboranen}

Lithium-tert-butyl-di(tert-butyl)fluorsilyl-amid wurde mit Trifluorborandiethyletherat bzw. mit Difluor-bis(trimethylsilyl)amino-boran in THF zur Reaktion gebracht.

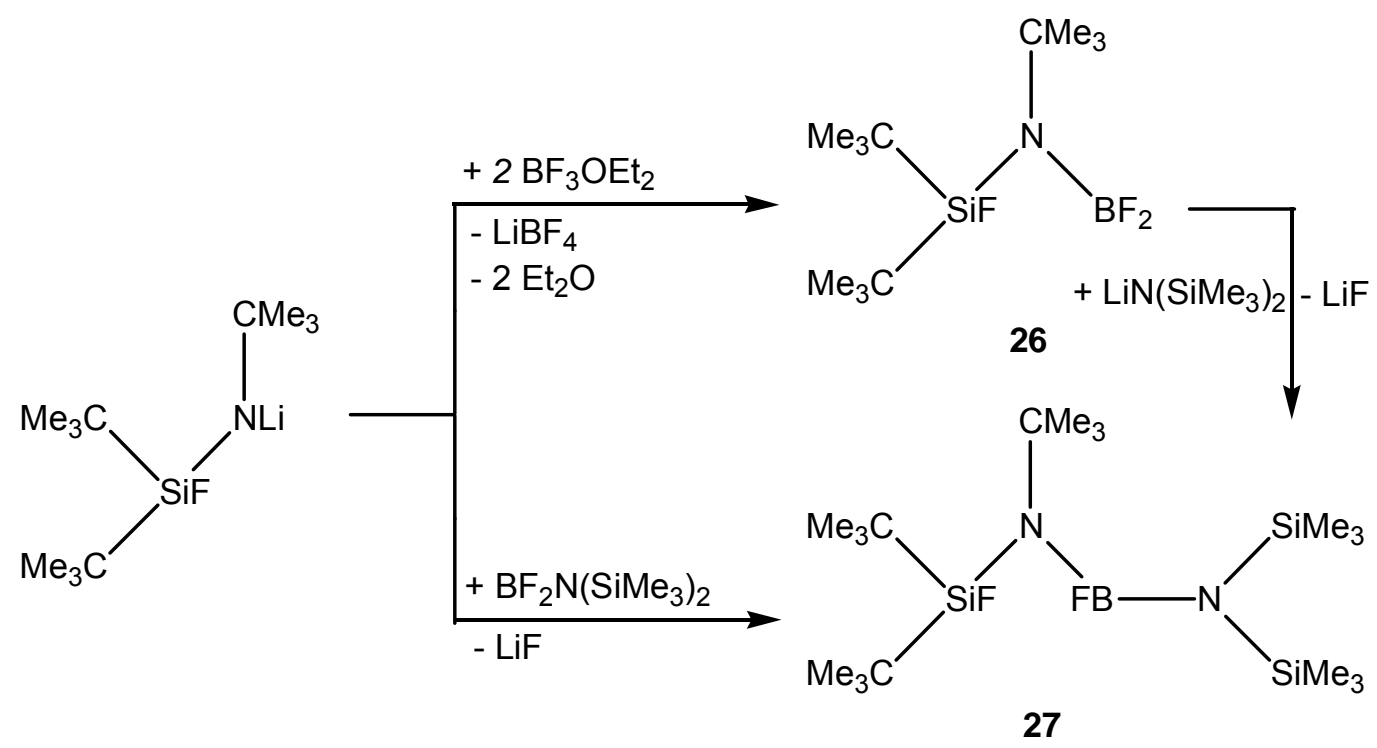

Gleichung 38: Synthese der Verbindungen 26 und 27

Wird 26 mit Lithium-bis(trimethylsilyl)amid umgesetzt, kann ebenfalls 27 erhalten werden. Es findet aus sterischen Gründen keine Substitution des Silylfluors statt.

Die Verbindung 27 kann unzersetzt destilliert werden und zeigt eine große thermische Stabilität. Erst bei $300^{\circ} \mathrm{C}$ wird eine Fluorsilanabspaltung beobachtet, wobei nicht Trimethylfluorsilan, sondern Di(tert-butyl)difluorsilan abgespalten wird. Dies kann durch die größere Lewis-Acidität des fluorsubstituierten Siliciums im Vergleich zum methylsubstituierten Silicium $\left({ }^{29} \mathrm{Si}: \underline{\operatorname{SiF}}\left(\mathrm{CMe}_{3}\right)_{2}=5,58 ; \underline{\mathrm{SiMe}_{3}}=2,05\right)$, durch die geringere Distanz zur BF-Einheit und durch die längere (schwächere) Bindung zum Stickstoff (Si1-N1) erklärt werden (siehe 3.3.4, Abbildung 20). 


\subsubsection{Kristallstruktur von 27}

27 kristallisiert aus $n$-Hexan triklin in der Raumgruppe $P \overline{1}$. N1 und B1 sind exakt planar umgeben. N2 weicht aus elektronischen und sterischen Gründen um $5,2^{\circ}$ von der Planarität ab.

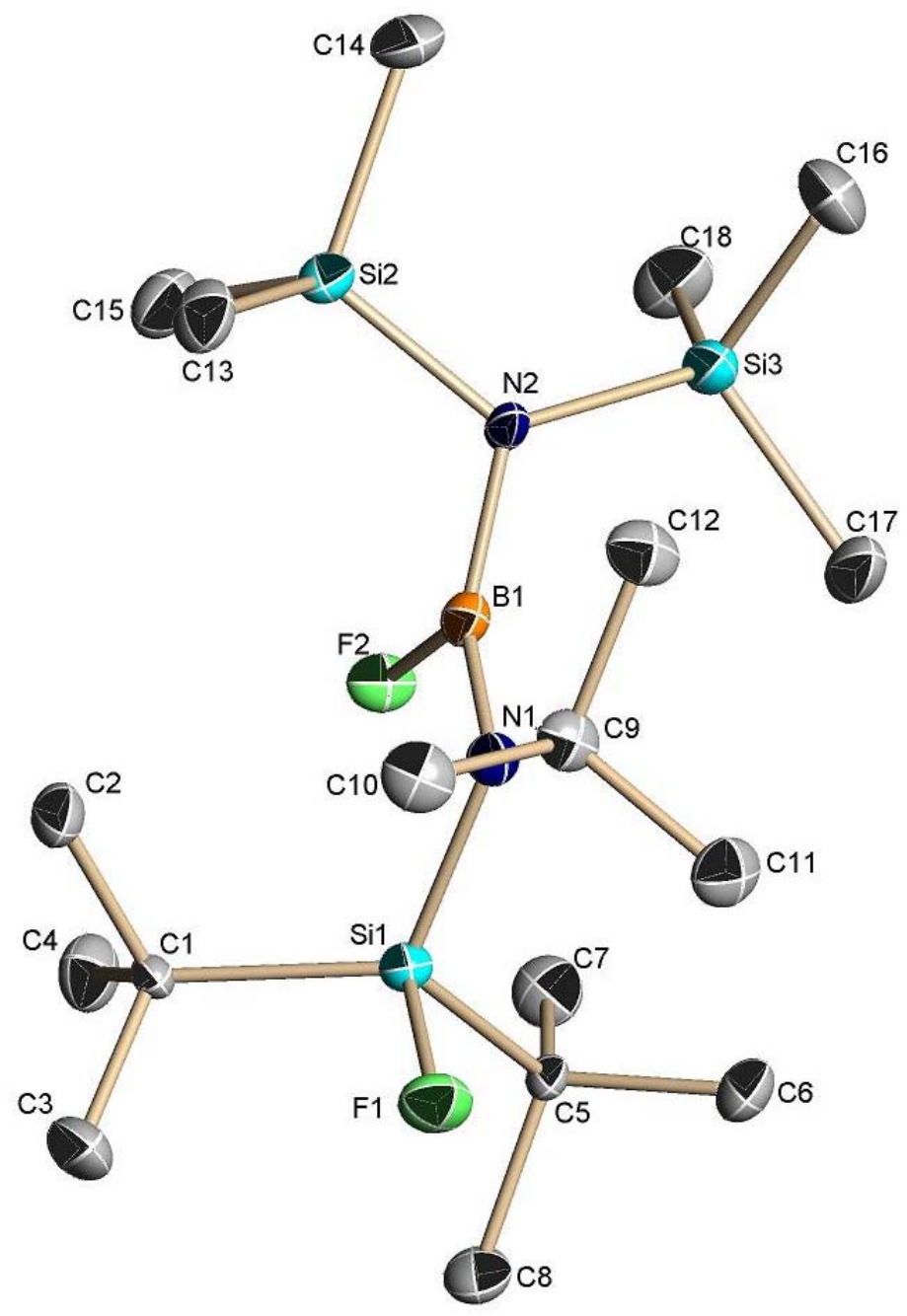

Abbildung 20: Kristallstruktur von 27 ohne Wasserstoffatome

Bindungslängen (-) bzw. intramolekulare Atomabstände (...)/pm mit Standardabweichung

\begin{tabular}{|ll|ll|ll|}
\hline Si1-F1 & $161,54(10)$ & B1-F2 & $137,85(19)$ & F2 ..Si1 & 256,9 \\
\hline Si1-N1 & $178,72(13)$ & B1-N2 & $146,5(2)$ & F2 ..Si2 & 341,3 \\
\hline Si2-N2 & $174,69(13)$ & B1-N1 & $141,5(2)$ & F2 ..Si3 & 329,6 \\
\hline Si3-N2 & $174,92(13)$ & & & & \\
\hline
\end{tabular}

Tabelle 27: Ausgewählte Bindungslängen bzw. intramolekulare Atomabstände der Verbindung 27

Bindungswinkel $/{ }^{\circ}$ mit Standardabweichung

\begin{tabular}{|ll|ll|ll|}
\hline F2-B1-N1 & $110,56(13)$ & B1-N1-C9 & $126,46(13)$ & B1-N2-Si2 & $117,26(10)$ \\
\hline F2-B1-N2 & $116,03(14)$ & B1-N1-Si1 & $111,06(10)$ & B1-N2-Si3 & $115,30(10)$ \\
\hline N1-B1-N2 & $133,41(15)$ & C9-N1-Si1 & $122,24(10)$ & Si2-N2-Si3 & $122,28(7)$ \\
\hline
\end{tabular}

Tabelle 28: Ausgewählte Bindungswinkel der Verbindung 27 


\subsubsection{Reaktionen des Lithium-bis[di(tert-butyl)fluorsilyl]amids mit Alanen und Chlortrimethylsilan}

Wird beim tert-Butyl-di(tert-butyl)fluorsilyl-amin die tert-Butylgruppe am Stickstoffatom durch eine weitere Di(tert-butyl)fluorsilylgruppe ersetzt, erhält man ein leicht lithiierbares Bis(difluorsilyl)amin ${ }^{[63]}$ dessen THF- und TMEDA-Struktur aufgeklärt werden konnte ${ }^{[63,64]}$.

Im Rahmen dieser Arbeit sollte untersucht werden, ob in der Reaktion dieses Lithiumamids mit Alanen ebenfalls, unter Lithiumfluoridabspaltung, die jeweiligen Addukte der Fluorsilyliminosilene entstehen (vgl. 3.3.3). Bei Zugabe von Chlortrimethylsilan wurde eine Umchlorierung und Fluortrimethylsilanabspaltung erwartet, was zur Entstehung eines freien Iminosilens führen sollte.

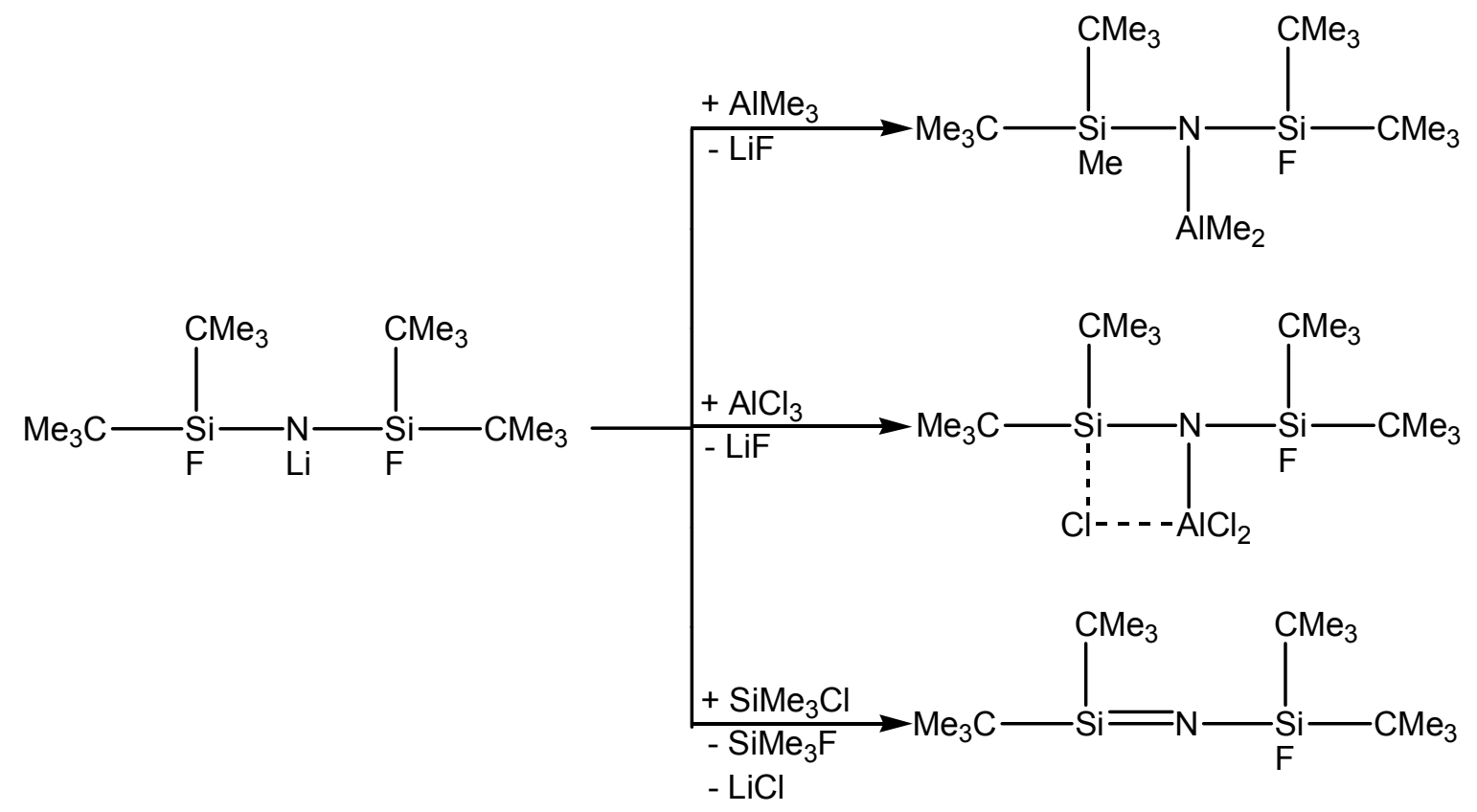

Gleichung 39: Erwarteter Reaktionsverlauf bei der Umsetzung von Lithium-bis[di(tert-butyl)fluorsilyl]amid mit $\mathrm{AlMe}_{3}, \mathrm{AlCl}_{3}$ und $\mathrm{SiMe}_{3} \mathrm{Cl}$

\subsubsection{Reaktion des Lithium-bis[di(tert-butyl)fluorsilyl]amids mit $\mathrm{AlMe}_{3}$}

Auf Grund der sterisch anspruchsvollen Di(tert-butyl)fluorsilylgruppen ist ein nucleophiler Angriff des Amidstickstoffs am Aluminium kinetisch gehemmt. Dies erklärt unter anderem die langen Reaktionszeiten, vor allem bei der Umsetzung mit Trimethylalan. Erst nach einer Woche kann nach dem destillativem Abtrennen des Lösungsmittels und des Edukts Bis[di(tert-butyl)fluorsilyl]amin bei der Aufarbeitung Verbindung 28 isoliert werden (siehe Schema 2). 
Unerwartet bildet sich nicht das fluorhaltige $\mathrm{AlMe}_{2}$-Addukt (siehe Gleichung 39). Da die Ausgangssubstanz zurückerhalten wurde, kann beim Reaktionsmechanismus von einer Umlithiierung ausgegangen werden.

Da weiterhin eine Zersetzung des THF beobachtet wurde, ist eine Protonierung des alan- oder lithiumsubstituierten Amins durch eine THF-Spaltung plausibel, welche oftmals bei Reaktionen von Chlorsilanen mit Lithiumamiden auftritt ${ }^{[65]}$. Der vollständige Mechanismus ist, wie auch bei den Synthesen von 29 und 30, noch nicht vollständig geklärt. Deshalb werden keine Reaktionsgleichungen aufgeführt.

Ohne Zugabe von THF bzw. beim Versuch der Reaktion in Diethylether konnte keine Reaktion des Amids mit Trimethylalan beobachtet werden.

\subsubsection{Reaktion des Lithium-bis[di(tert-butyl)fluorsilyl]amids mit Trichloralan}

Bei der Reaktion mit $\mathrm{AlCl}_{3}$ konnte nicht das THF-stabilisierte Zwischenprodukt charakterisiert werden. Wird das Reaktionsgemisch destillativ aufgearbeitet, kann neben dem, mit 28 vergleichbaren, Bis[di(tert-butyl)chlorsilyl]amin $\left(29^{[66]}\right)$ auch das Di(tertbutyl)chlorsilyl-di(tert-butyl)fluorsilyl-amin $\left(30^{[67]}\right)$ isoliert werden.

Auch hier kann der Reaktionsmechanismus durch eine, im ersten Schritt auftretende, Umlithiierung erklärt werden. Ungewöhnlich ist die LiF-Abspaltung anstelle der Lithiumchlorideliminierung nach der erfolgten Ummetallierung.

Verbindung $29^{[66]}$ und $30^{[67]}$ wurden zur Vervollständigung der Charakterisierung ${ }^{15} \mathrm{~N}$ - und ${ }^{19} \mathrm{~F}-\mathrm{NMR}$-analytisch untersucht.

\subsubsection{Reaktion des Lithium-bis[di(tert-butyl)fluorsilyl]amids mit Trimethyl- chlorsilan}

Wird das Bis(silyl)amid mit Chlortrimethylsilan umgesetzt, so kann weder eine Substitution der Trimethylsilylgruppe am Stickstoff, noch eine Fluorsilan- bzw. Lithiumchloridabspaltung festgestellt werden. Stattdessen wird ebenfalls Verbindung $\mathbf{3 0}$ isoliert. 
<smiles>CC(C)(C)[Si](C)(C)[Si](N[Si](C)(C)C)(C(C)(C)C)C(C)(C)C</smiles>

29<smiles>C[Si](C)(C)N[Si](C)(C)C</smiles>

28<smiles>C[Si](C)(C)N[Si](C)(C)C</smiles>

30

Schema 2: Synthese der Verbindungen 28 - 30

Ein vergleichbares Phänomen beobachteten Popowski et al. bei der Reaktion von Lithium-bis[di(tert-butyl)silyl]amid mit Chlortrimethylsilan in THF, wobei das dichlorsubstituierte Bis(silyl)amin (29) entstand. Der Reaktionsmechanismus ist hierbei noch nicht aufgeklärt ${ }^{[66]}$.

\subsubsection{Kristallstruktur des Bis[di(tert-butyl)methylsilyl]amins, 28}

Verbindung 28 kristallisiert aus $n$-Hexan orthorhombisch in der Raumgruppe Pbca.

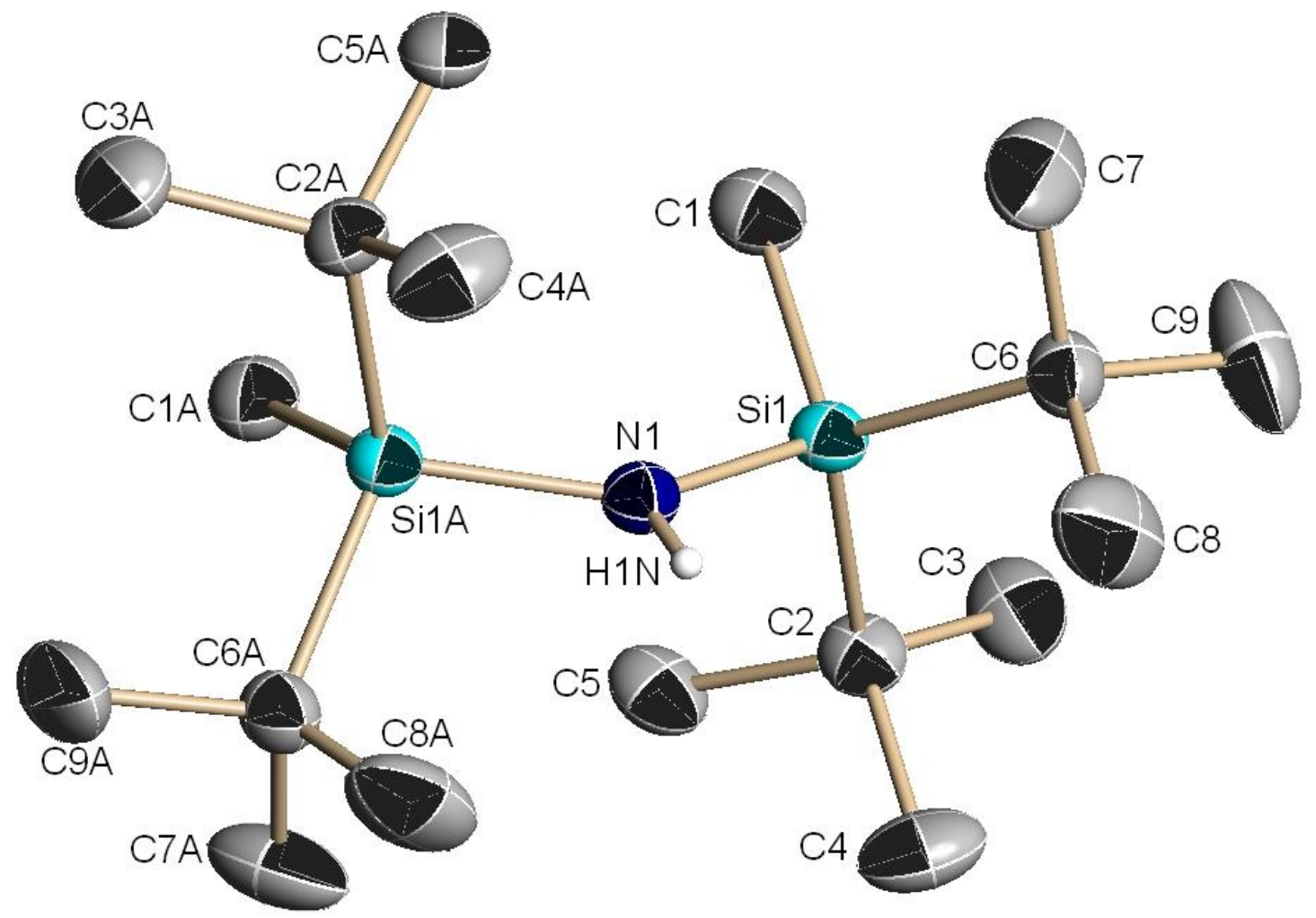

Abbildung 21: Kristallstruktur von $\mathbf{2 8}$ ohne Methylwasserstoffatome 
Bindungslängen/pm mit Standardabweichung

\begin{tabular}{|ll|ll|ll|}
\hline Si1-N1 & $173,5(1)$ & N1-H1N & 78,6 & Si1-C1 & $184,5(3)$ \\
\hline Si1-C2 & $190,9(3)$ & Si1-C6 & $191,7(3)$ & & \\
\hline
\end{tabular}

Tabelle 29: Ausgewählte Bindungslängen der Verbindung 28

Bindungswinkel $/{ }^{\circ}$ mit Standardabweichung

\begin{tabular}{|ll|ll|ll|}
\hline Si1-N1-Si1A & $140,5(2)$ & Si1A-N1-H1N & 109,74 & Si1-N1-H1N & 109,74 \\
\hline N1-Si1-C1 & $110,76(14)$ & N1-Si1-C2 & $109,48(9)$ & C1-Si1-C2 & $107,02(14)$ \\
\hline N1-Si1-C6 & $106,61(12)$ & C1-Si1-C6 & $108,56(13)$ & C2-Si1-C6 & $114,44(12)$ \\
\hline
\end{tabular}

Tabelle 30: Ausgewählte Bindungswinkel der Verbindung 28

Die äquivalenten Silylgruppen sind punktsymmetrisch um den Stickstoff angeordnet.

Dieser ist exakt planar umgeben $\left(\Sigma \Varangle \mathrm{N} 1=360,0^{\circ}\right)$. Die langen Bindungen der Siliciumatome zu den tertiären Kohlenstoffatomen sind auf sterische und elektronische Effekte zurückzuführen. Der Si-N-Si-Winkel $\left(140,5^{\circ}\right)$ ist durch die voluminösen Silylsubstituenten aufgeweitet.

\subsubsection{Kristallstruktur des Bis[di(tert-butyl)chlorsilyl]amins, 29}

Auf Grund der unzureichenden Kristallqualität können keine verlässlichen Aussagen über Bindungslängen und -winkel getroffen werden, weshalb lediglich die Kristallstruktur abgebildet wird.

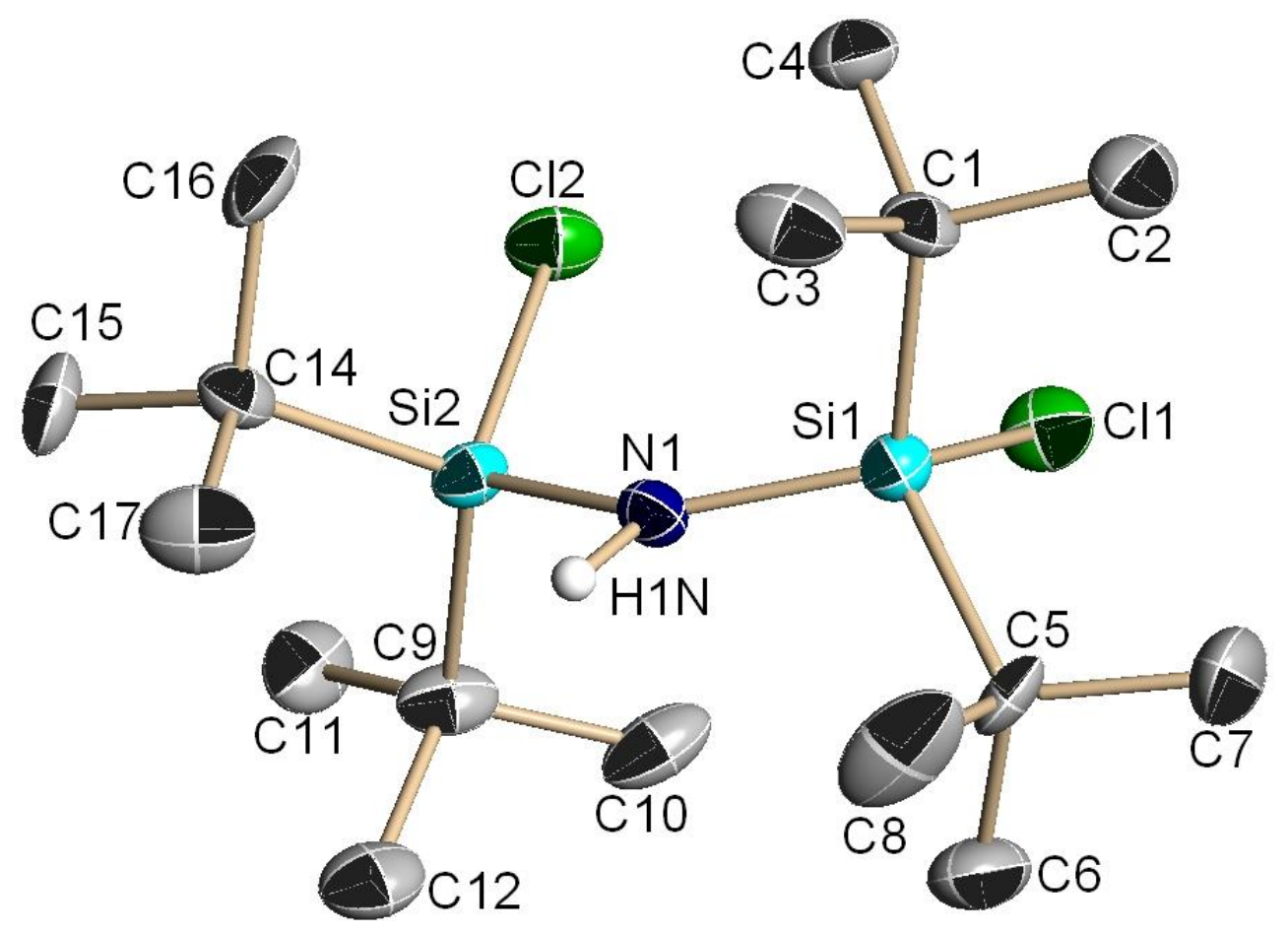

Abbildung 22: Kristallstruktur von 29 ohne Methylwasserstoffatome 


\subsection{Synthese von Fluorborylaminen}

Im Rahmen dieser Arbeit wurden 2,6-Diisopropylanilin, Triphenylsilylamin sowie tertButyldimethylsilylamin lithiiert und mit verschiedenen Difluorboranen zur Reaktion gebracht. Ziel war es, bisher unbekannte Fluorborylamine zu erhalten, welche in einer Folgereaktion mit Lithiumorganylen am Stickstoff metalliert werden sollten.

\subsubsection{Synthese von Fluor-2,6-diisopropylanilino-boranen}

Wird lithiiertes 2,6-Diisopropylanilin in THF zu den äquimolaren Mengen an Difluor(methyl-trimethylsilyl)amino-boran, Difluor-di(iso-butyl)amino-boran und Difluor-diisopropylamino-boran gegeben, werden in quantitativen Ausbeuten die Verbindungen $31{ }^{[68]}, 32$ und $\mathbf{3 3}$ erhalten.

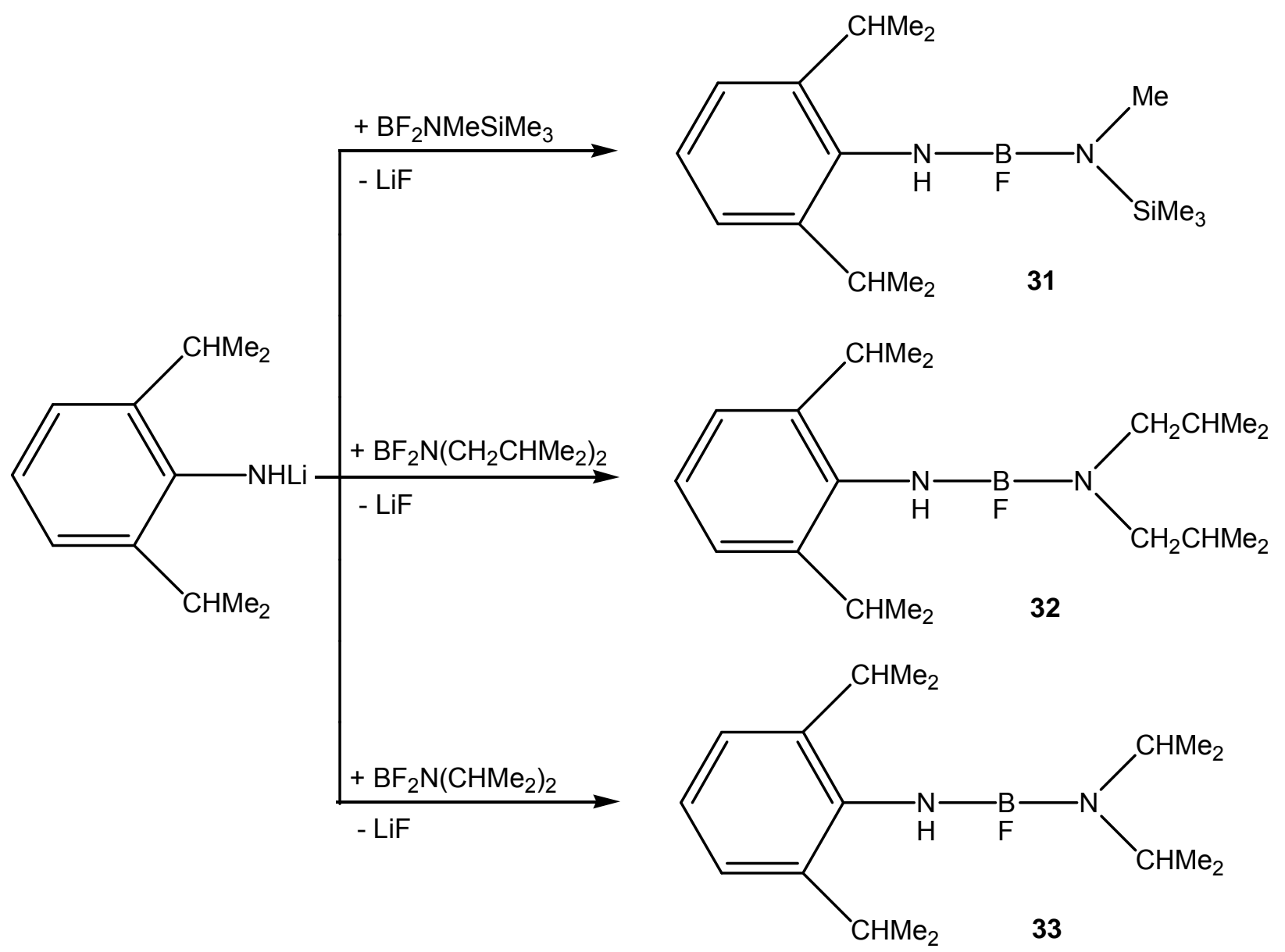

Gleichung 40: Synthese von $31^{[68]}, 32$ und 33 


\subsubsection{Kristallstrukturen der Verbindungen 31 - 33}

Bei allen drei geröntgten Verbindungen liegen die Bor-, Stickstoff- und C1-Atome in einer Ebene (siehe Abbildung 24). Der Phenylring steht fast senkrecht zu dieser Ebene. Die Methylgruppen der Aryldiisopropylsubstituenten sind von der N-BF-NHEinheit abgewandt. Die ORTEP's (Abbildung 23 - 27) lassen den kinetischen Einfluss dieser Isopropylgruppen erkennen, welche die NH-Einheit abschirmen.

\subsubsection{Kristallstruktur von $\mathbf{3 1}$}

Auf Grund fehlender Röntgenstrukturdaten, der im AK Meller bereits synthetisierten Verbindung $31^{[68]}$, wurden Einkristalle röntgenographisch vermessen. 31 kristallisiert aus $n$-Hexan monoklin in der Raumgruppe $P 2_{1} / \mathrm{c}$. Bor, Stickstoff sowie $\mathrm{C} 1$ sind planar umgeben $\left(\Sigma \Varangle \mathrm{B} 1=360,0^{\circ}, \Sigma \nless \mathrm{N} 1=359,7^{\circ}, \Sigma \Varangle \mathrm{N} 2=357,4^{\circ}, \Sigma \Varangle \mathrm{C} 1=360,0^{\circ}\right)$.

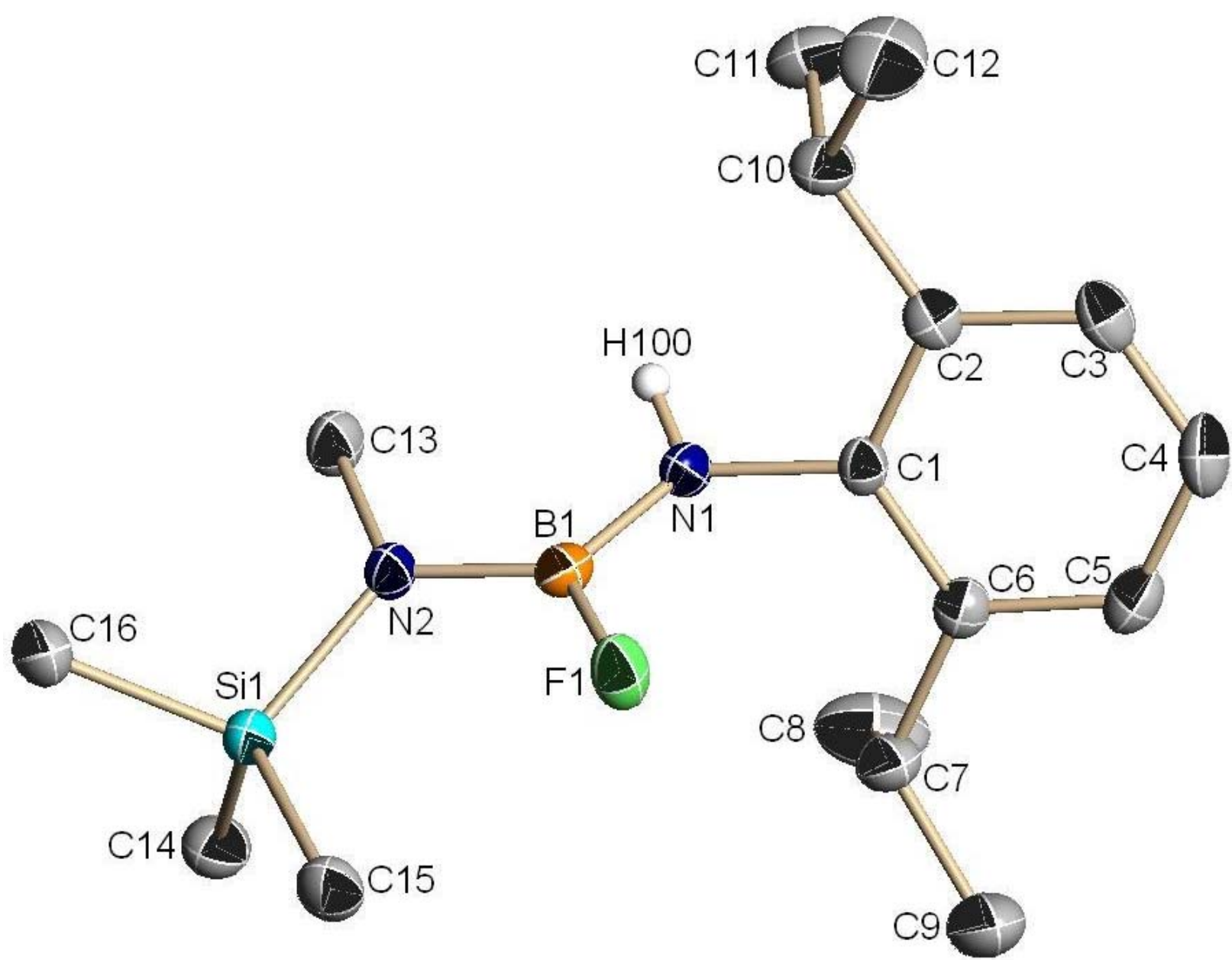

Abbildung 23: Kristallstruktur von 31 ohne $\mathrm{CH}$-Wasserstoffatome 
Bindungslängen/pm mit Standardabweichung

\begin{tabular}{|ll|ll|ll|}
\hline Si1-N2 & $174,53(17)$ & N1-B1 & $140,3(3)$ & N2-B1 & $141,4(3)$ \\
\hline Si1-N2 & $174,53(17)$ & N1-C1 & $143,4(2)$ & N1-H100 & 89,0 \\
\hline
\end{tabular}

Tabelle 31: Ausgewählte Bindungslängen der Verbindung 31

Bindungswinkel $/{ }^{\circ}$ mit Standardabweichung

\begin{tabular}{|ll|ll|ll|}
\hline F1-B1-N1 & $116,34(19)$ & B1-N1-C1 & $126,75(17)$ & B1-N2-C13 & $115,76(17)$ \\
\hline F1-B1-N2 & $118,9(2)$ & B1-N1-H100 & 120,93 & B1-N2-Si1 & $126,53(14)$ \\
\hline N1-B1-N2 & $124,8(2)$ & C1-N1-H100 & 112,02 & C13-N2-Si1 & $115,12(13)$ \\
\hline
\end{tabular}

Tabelle 32: Ausgewählte Bindungswinkel der Verbindung 31

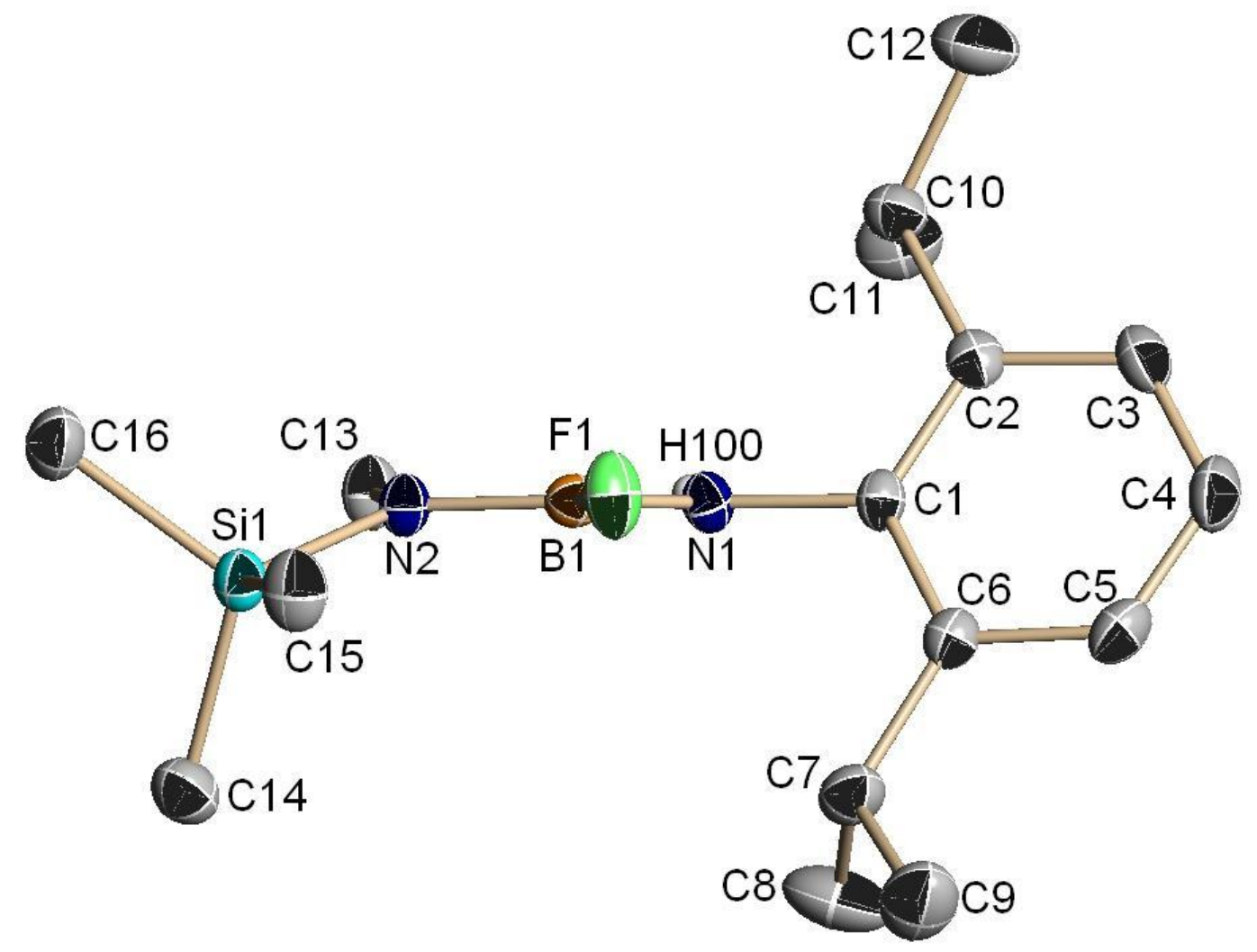

Abbildung 24: Seitenansicht von 31 ohne $\mathrm{CH}$-Wasserstoffatome 


\subsubsection{Kristallstruktur von 32}

Verbindung 32 kristallisiert aus $n$-Hexan monoklin in der Raumgruppe $P 2_{1} / \mathrm{c}$. Die Winkelsummen der Bor- und Stickstoffatome sind jeweils $360,0^{\circ}$.

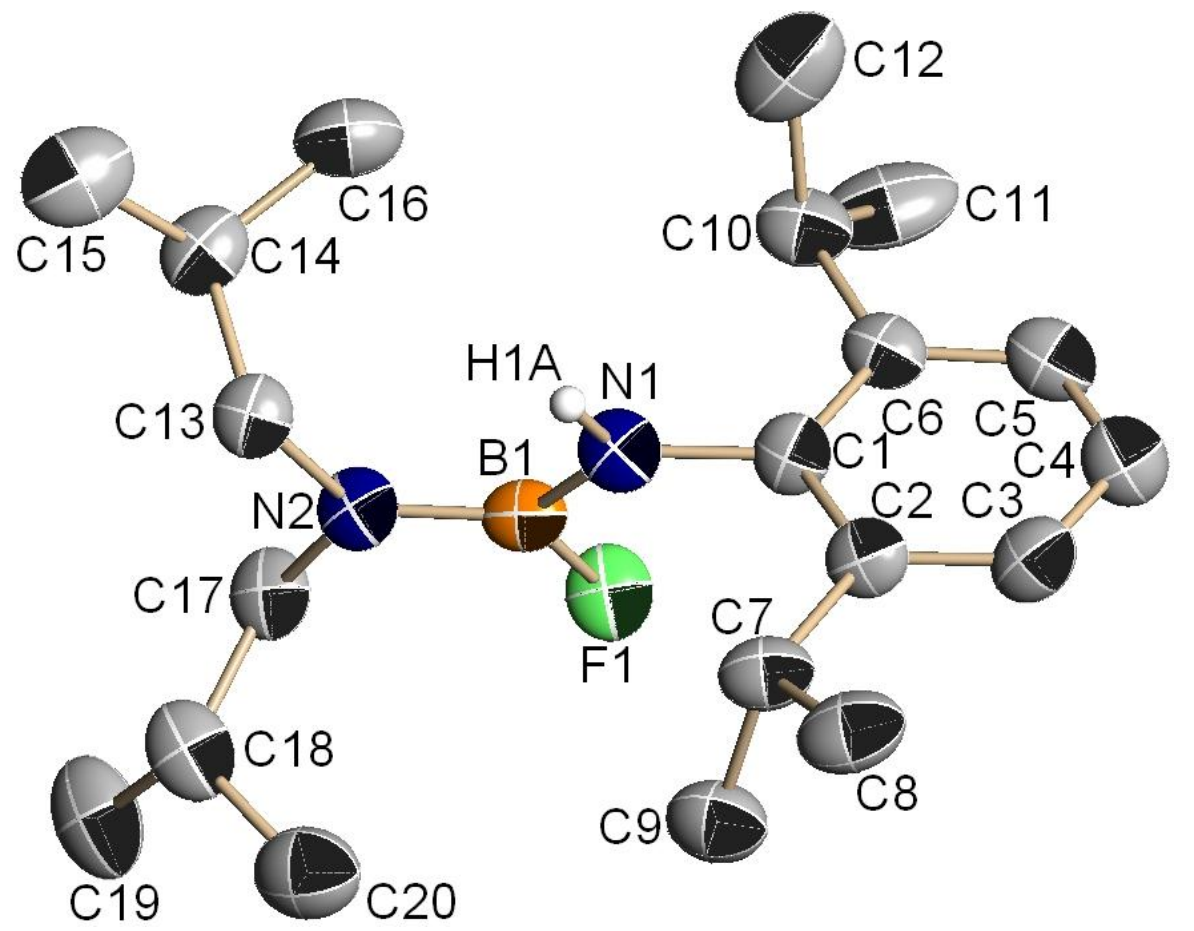

Abbildung 25: Kristallstruktur von 32 ohne $\mathrm{CH}$-Wasserstoffatome

Bindungslängen/pm mit Standardabweichung

\begin{tabular}{|ll|ll|ll|}
\hline F1-B1 & $137,0(4)$ & B1-N1 & $142,1(5)$ & B1-N2 & $138,9(5)$ \\
\hline N1-C1 & $143,6(4)$ & N2-C13 & $147,5(4)$ & N1-H1A & 88,0 \\
\hline
\end{tabular}

Tabelle 33: Ausgewählte Bindungslängen der Verbindung 32

Bindungswinkel $/^{\circ}$ mit Standardabweichung

\begin{tabular}{|ll|ll|ll|}
\hline F1-B1-N2 & $118,2(3)$ & F1-B1-N1 & $115,2(3)$ & N2-B1-N1 & $126,6(3)$ \\
\hline B1-N1-C1 & $125,7(3)$ & B1-N1-H1A & 117,2 & H1A-N1-C1 & 117,1 \\
\hline B1-N2-C13 & $124,0(3)$ & B1-N2-C17 & $122,0(3)$ & C13-N2-C17 & $114,0(3)$ \\
\hline
\end{tabular}

Tabelle 34: Ausgewählte Bindungswinkel der Verbindung 32 


\subsubsection{Kristallstruktur von 33}

Verbindung 33 kristallisiert aus $n$-Hexan monoklin in der Raumgruppe C2/c. Die Borund Stickstoffatome sind exakt planar umgeben: $\Sigma \Varangle \mathrm{B} 1=\Sigma \Varangle \mathrm{N} 1=\Sigma \Varangle \mathrm{N} 2=360,0^{\circ}$.

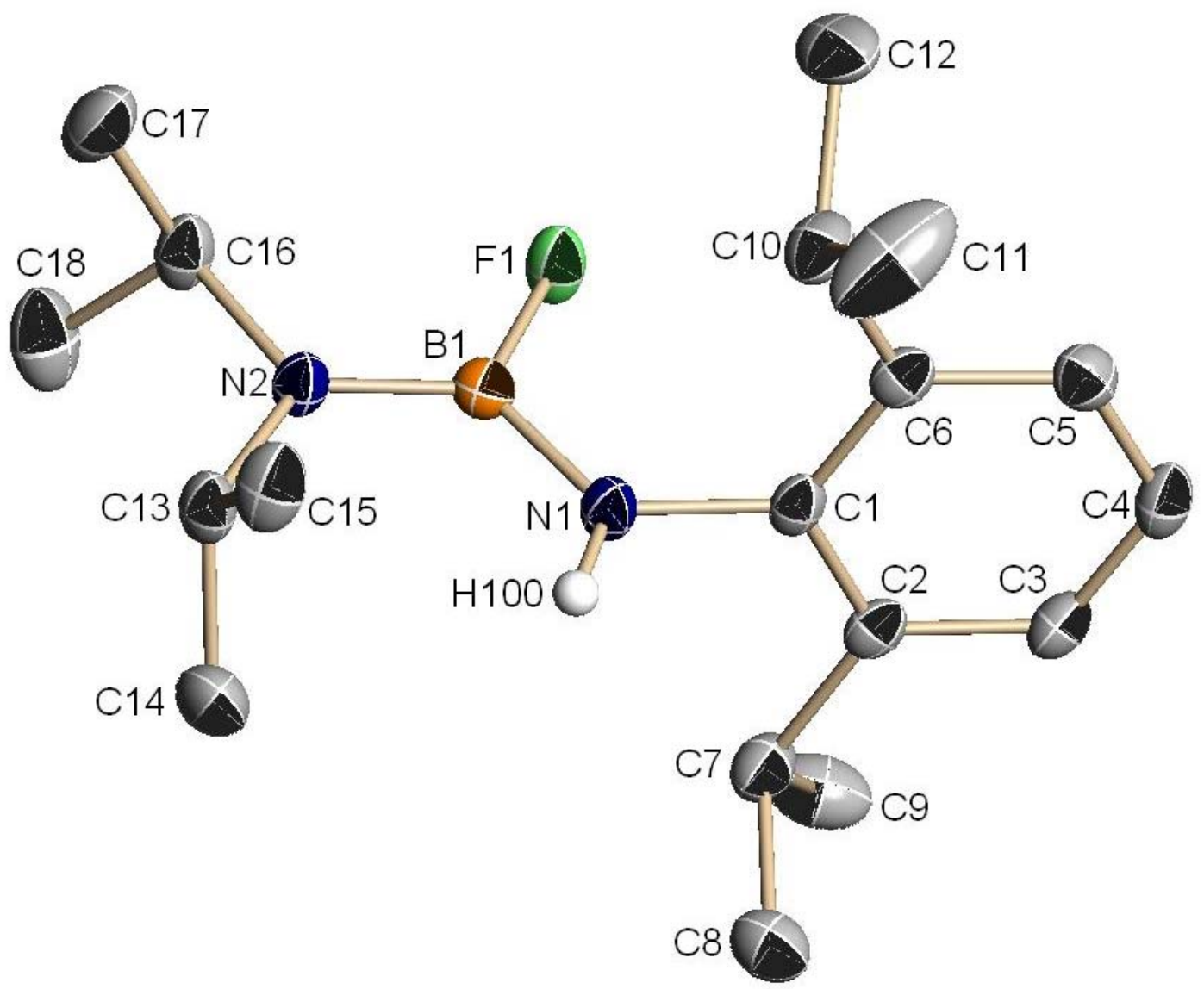

Abbildung 26: Kristallstruktur von 33 ohne $\mathrm{CH}$-Wasserstoffatome

Bindungslängen/pm mit Standardabweichung

\begin{tabular}{|ll|ll|ll|}
\hline N1-B1 & $141,4(2)$ & N1-H100 & 83,2 & N2-C13 & $148,00(18)$ \\
\hline N1-C1 & $143,36(19)$ & N2-B1 & $139,6(2)$ & B1-F1 & $136,81(18)$ \\
\hline
\end{tabular}

Tabelle 35: Ausgewählte Bindungslängen der Verbindung 33

Bindungswinkel $/^{\circ}$ mit Standardabweichung

\begin{tabular}{|ll|ll|ll|}
\hline F1-B1-N2 & $118,43(13)$ & B1-N1-C1 & $125,44(13)$ & B1-N2-C16 & $121,18(12)$ \\
\hline F1-B1-N1 & $114,67(14)$ & B1-N1-H100 & 121,19 & B1-N2-C13 & $123,68(12)$ \\
\hline N2-B1-N1 & $126,89(14)$ & C1-N1-H100 & 113,33 & C16-N2-C13 & $115,13(11)$ \\
\hline
\end{tabular}

Tabelle 36: Ausgewählte Bindungswinkel der Verbindung 33 


\subsubsection{Umsetzungen von $\mathbf{3 1}$ - 33 mit Lithiumorganylen, 34 - 36}

Um eine Metallierung des Aminstickstoffatoms zu erreichen, werden die Aminofluorborane (31 - 33) bei möglichst tiefen Temperaturen mit Lithiumorganylen umgesetzt (beide Edukte auf $-78^{\circ} \mathrm{C}$ gekühlt). Neben $n$-Butyllithium und tert-Butyllithium wurden sterisch anspruchsvolle Lithiumamide als Metallierungsreagenzien gewählt, die eine Substitution am Bor verhindern sollten ${ }^{[15]}$.

Trotz (bzw. wegen) des großen Raumbedarfs der verwendeten Amide können lediglich die, am Bor substituierten, Verbindungen 34 - 36 isoliert werden, was durch die sterische Abschirmung der $\mathrm{NH}$-Einheit begründet werden kann (siehe Abbildungen 23 - 27).

Auch $n$-Butyllithium und tert-Butyllithium zeigen hauptsächlich eine Butylsubstitution anstelle einer Butanbildung. Die Lithiumsalze der Aminofluorborane konnten in keinem Fall isoliert werden. Das Lithiumfluorid spaltete sich bereits bei tiefen Temperaturen $\mathrm{ab}\left(-50^{\circ} \mathrm{C}\right)$. Bei Raumtemperatur konnten weiterhin die Dimere der intermediär entstandenen Iminoborene ${ }^{[68]}$ massenspektroskopisch nachgewiesen werden. Demnach ist die sterische Abschirmung der Substituenten am Bor zu gering, um die Niederkoordination kinetisch zu stabilisieren.

\subsubsection{Kristallstrukturen der Verbindungen 34 - 36}

Die 2,6-Diisopropylanilin-, Bis(trimethylsilyl)amin- und tert-Butyl-trimethylsilyl-aminsubstituierten Verbindungen von $\mathbf{3 2}$ bzw. $\mathbf{3 3}$ wurden röntgenstrukturanalytisch untersucht. Die Bor-, Stickstoff- und C1-Atome sind annäherend planar umgeben. Sie bilden (wie auch bei 31 - 33) eine Ebene.

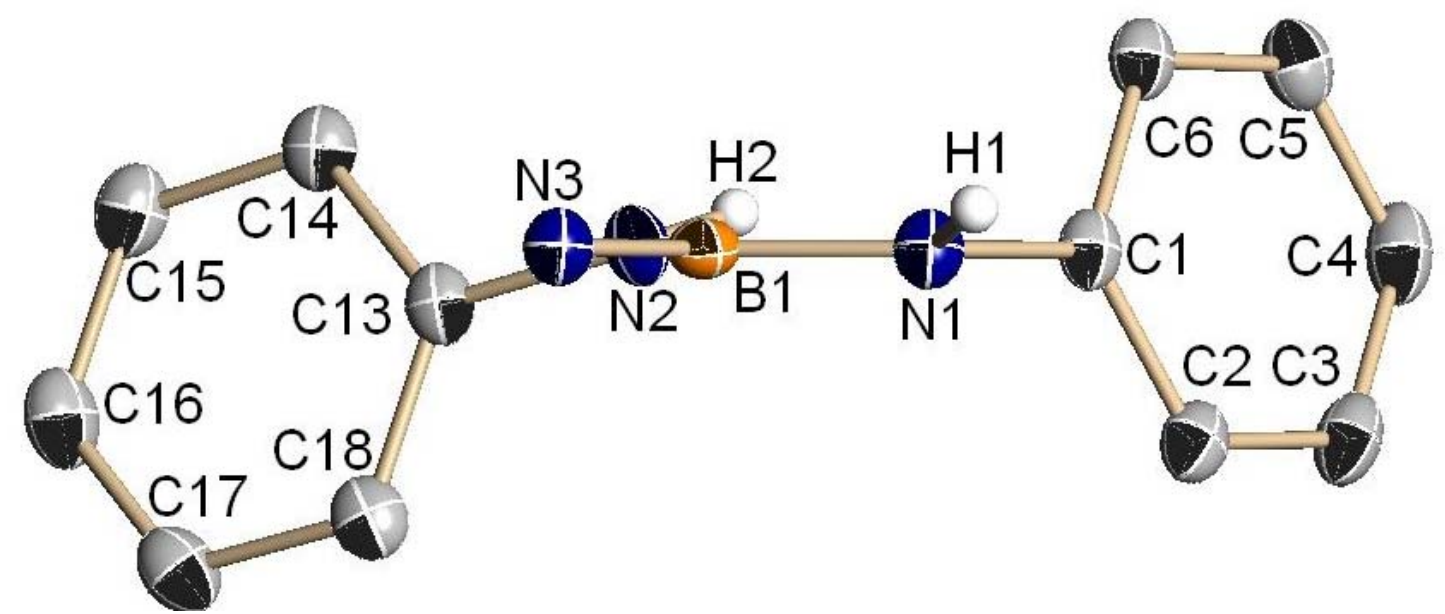

Abbildung 27: Seitenansicht auf $\mathbf{3 4}$ ohne Isopropyl- und Iso-butylgruppen sowie $\mathrm{CH}$-Wasserstoffatome 


\subsubsection{Kristallstruktur von 34}

Verbindung 34 kristallisiert aus $n$-Hexan orthorhombisch in der Raumgruppe Pbca $\left(\Sigma \nless \mathrm{B} 1=360,0^{\circ}, \Sigma \nless \mathrm{N} 1=357,3^{\circ}, \Sigma \nless \mathrm{N} 2=359,8^{\circ}, \Sigma \nless \mathrm{N} 3=359,9^{\circ}, \Sigma \nless \mathrm{C} 1=360,0^{\circ}\right)$.

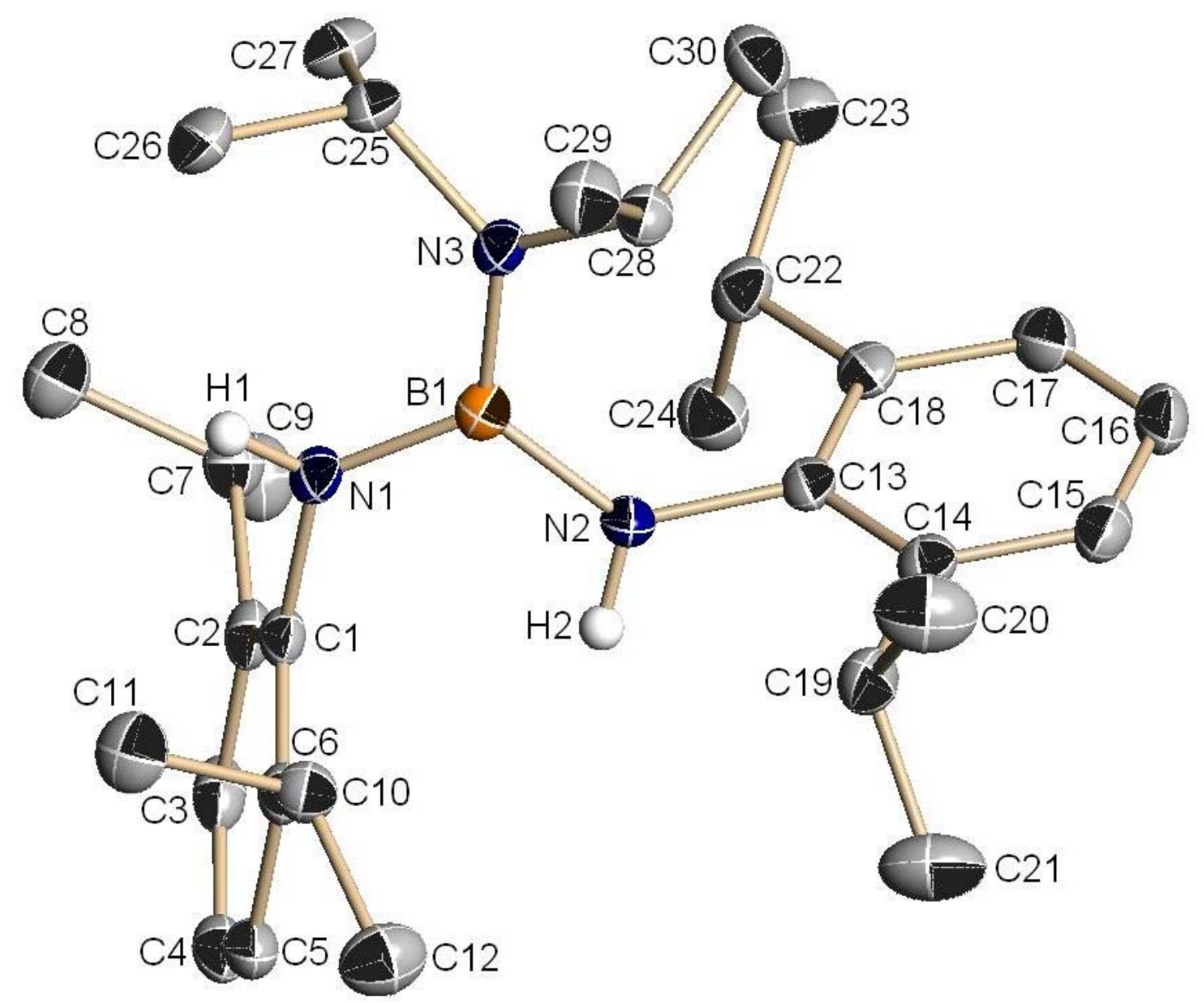

Abbildung 28: Kristallstruktur von 34 ohne $\mathrm{CH}$-Wasserstoffatome

Bindungslängen/pm mit Standardabweichung

\begin{tabular}{|ll|ll|ll|}
\hline B1-N3 & $142,93(17)$ & N1-C1 & $143,25(16)$ & N2-C13 & $143,06(16)$ \\
\hline B1-N1 & $143,42(17)$ & N1-H1 & 84,2 & N3-C28 & $147,40(16)$ \\
\hline B1-N2 & $143,73(17)$ & N2-H2 & 86,4 & N3-C25 & $147,72(16)$ \\
\hline
\end{tabular}

Tabelle 37: Ausgewählte Bindungslängen der Verbindung 34

Bindungswinkel $/^{\circ}$ mit Standardabweichung

\begin{tabular}{|ll|ll|ll|}
\hline N3-B1-N1 & $120,71(11)$ & N3-B1-N2 & $123,15(11)$ & N1-B1-N2 & $116,13(11)$ \\
\hline C1-N1-B1 & $125,91(11)$ & C1-N1-H1 & 113,10 & B1-N1-H1 & 118,29 \\
\hline C13-N2-B1 & $131,31(11)$ & C13-N2-H2 & 113,58 & B1-N2-H2 & 114,92 \\
\hline B1-N3-C28 & $120,86(10)$ & B1-N3-C25 & $124,06(10)$ & C28-N3-C25 & $114,93(10)$ \\
\hline
\end{tabular}

Tabelle 38: Ausgewählte Bindungswinkel der Verbindung 34 


\subsubsection{Kristallstruktur von 35}

36 kristallisiert als Monomer triklin in der Raumgruppe $P \overline{1}\left(\Sigma \nless \mathrm{B} 1=360,0^{\circ}\right.$, $\left.\Sigma \nless \mathrm{N} 1=358,0^{\circ}, \Sigma \Varangle \mathrm{N} 2=357,0^{\circ}, \Sigma \Varangle \mathrm{N} 1=360,0^{\circ}\right)$.

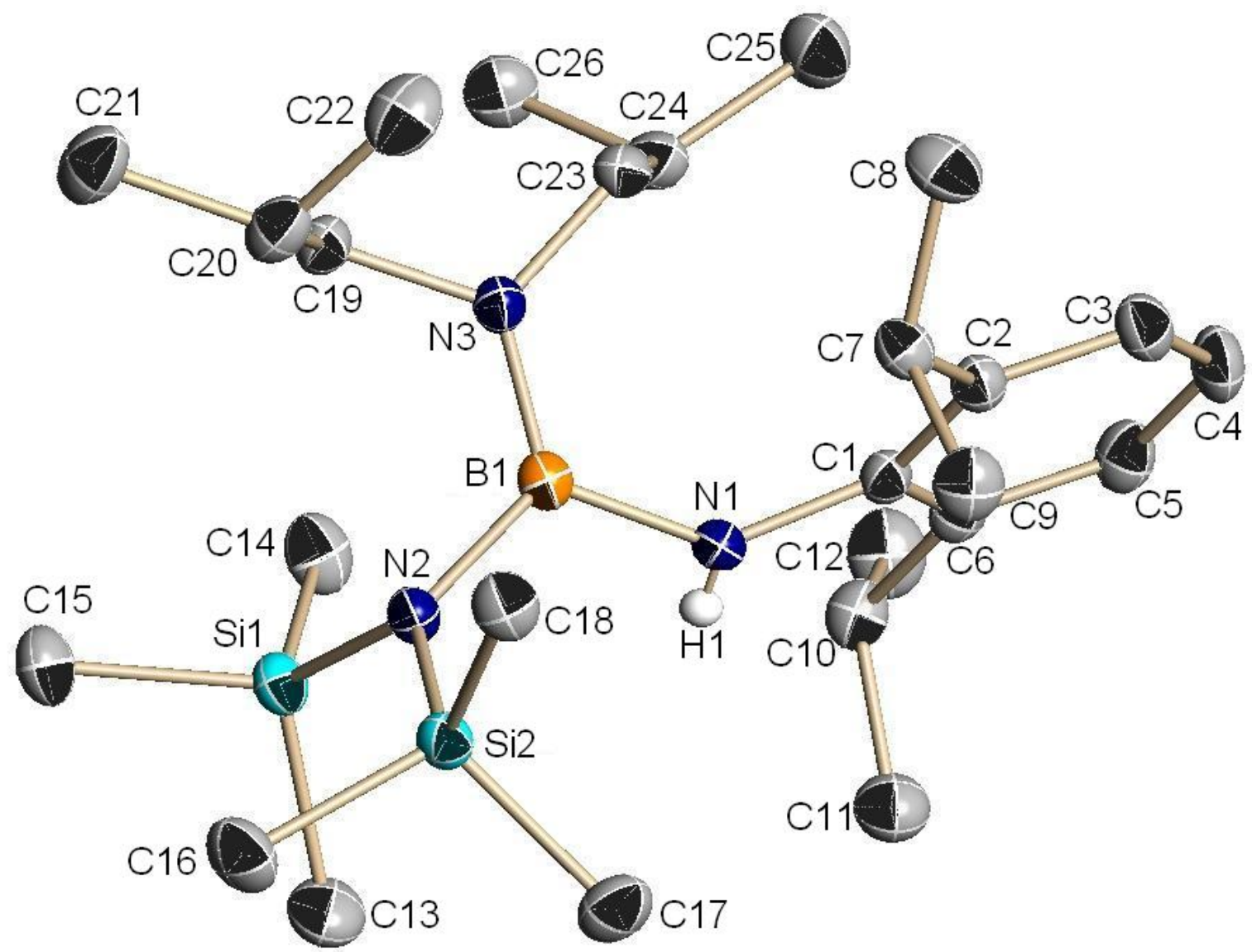

Abbildung 29: Kristallstruktur von 35 ohne $\mathrm{CH}$-Wasserstoffatome

Bindungslängen/pm mit Standardabweichung

\begin{tabular}{|ll|ll|ll|}
\hline Si1-N2 & $174,54(11)$ & N1-H1 & 83,8 & N3-C19 & $146,99(16)$ \\
\hline Si2-N2 & $174,69(11)$ & N1-B1 & $144,43(18)$ & N3-C23 & $147,18(16)$ \\
\hline N1-C1 & $143,26(16)$ & N2-B1 & $148,97(17)$ & N3-B1 & $142,01(18)$ \\
\hline
\end{tabular}

Tabelle 39: Ausgewählte Bindungslängen der Verbindung 35

Bindungswinkel $/{ }^{\circ}$ mit Standardabweichung

\begin{tabular}{|ll|ll|ll|}
\hline C1-N1-B1 & $137,14(11)$ & C1-N1-H1 & 109,31 & H1-N1-B1 & 111,50 \\
\hline B1-N2-Si1 & $119,08(8)$ & B1-N2-Si2 & $116,75(8)$ & Si1-N2-Si2 & $121,20(6)$ \\
\hline B1-N3-C19 & $121,73(10)$ & B1-N3-C23 & $122,81(11)$ & C19-N3-C23 & $115,45(10)$ \\
\hline N3-B1-N1 & $124,34(11)$ & N3-B1-N2 & $121,36(11)$ & N1-B1-N2 & $114,30(11)$ \\
\hline
\end{tabular}

Tabelle 40: Ausgewählte Bindungswinkel der Verbindung 35 


\subsubsection{Kristallstruktur von $\mathbf{3 6}$}

Verbindung 37 kristallisiert triklin in der Raumgruppe $P \overline{1}$. Das Boratom wie auch die

Stickstoffatome sind planar umgeben $\left(\Sigma \Varangle \mathrm{B} 1=\Sigma \nless \mathrm{N} 1=360,0^{\circ}, \quad \Sigma \nless \mathrm{N} 2=359,3^{\circ}\right.$, $\left.\Sigma \nless N 3=358,1^{\circ}\right)$. Auf Grund einer nicht vollständig gelösten Fehlordnung einer isoButylgruppe (C13 - C16) wird diese nicht als Thermalellipsoide dargestellt.

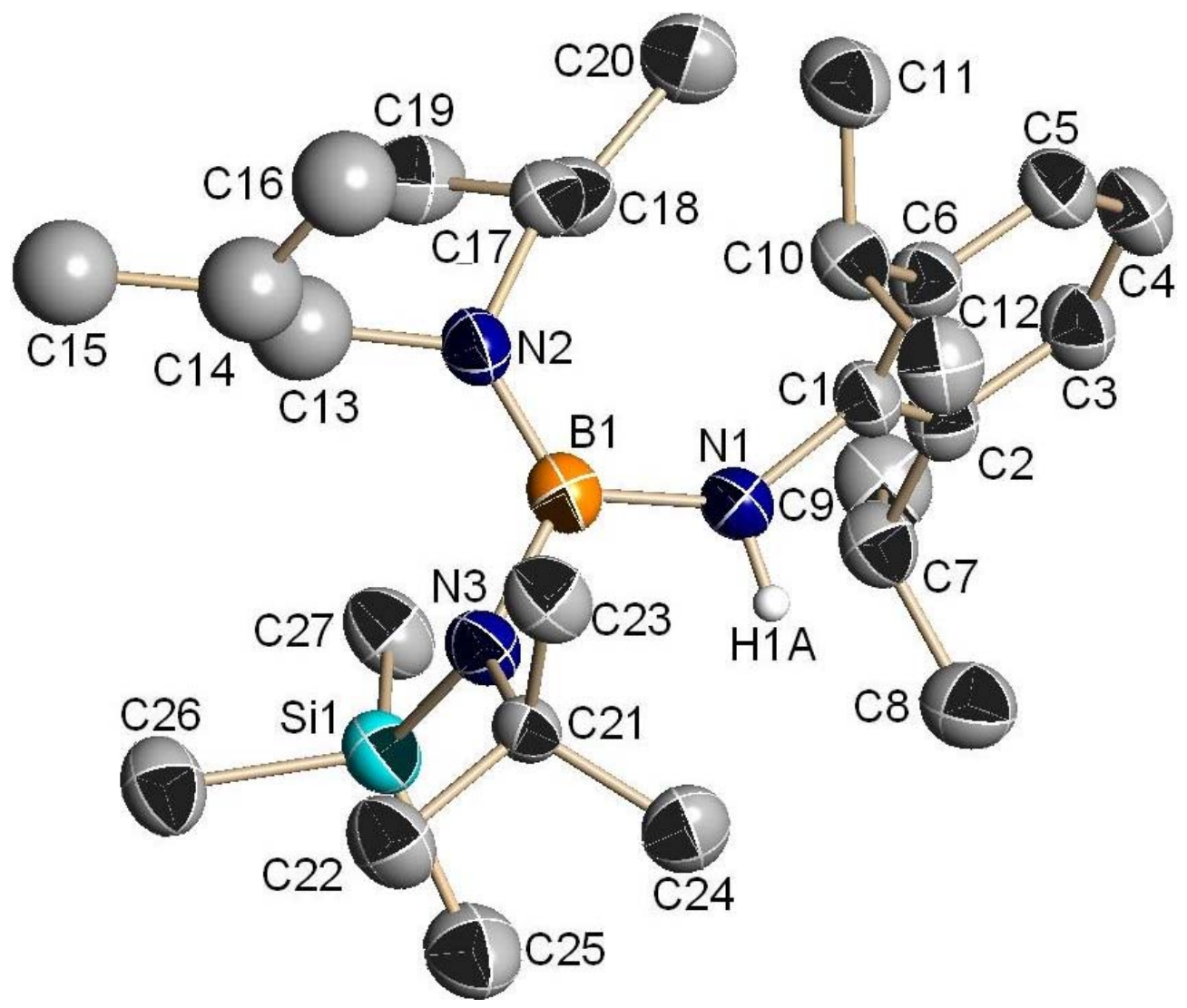

Abbildung 30: Kristallstruktur von 36 ohne $\mathrm{CH}$-Wasserstoffatome

Bindungslängen/pm mit Standardabweichung

\begin{tabular}{|ll|ll|ll|}
\hline B1-N2 & $142,1(4)$ & B1-N1 & $144,9(3)$ & B1-N3 & $148,7(3)$ \\
\hline Si1-N3 & $172,7(2)$ & N1-C1 & $143,0(3)$ & N1-H1A & 88,0 \\
\hline N2-C13 & $147,6(8)$ & N2-C17 & $147,2(3)$ & N3-C21 & $150,5(3)$ \\
\hline
\end{tabular}

Tabelle 41: Ausgewählte Bindungslängen der Verbindung 36

Bindungswinkel $/^{\circ}$ mit Standardabweichung

\begin{tabular}{|ll|ll|ll|}
\hline N1-B1-N3 & $115,4(2)$ & N2-B1-N1 & $123,0(2)$ & N2-B1-N3 & $121,6(2)$ \\
\hline B1-N1-C1 & $138,3(2)$ & B1-N1-H1A & 110,87 & C1-N1-H1A & 110,87 \\
\hline B1-N2-C17 & $122,9(2)$ & B1-N2-C13 & $126,0(4)$ & C17-N2-C13 & $110,4(4)$ \\
\hline B1-N3-C21 & $118,54(19)$ & B1-N3-Si1 & $118,79(16)$ & C21-N3-Si1 & $120,74(15)$ \\
\hline
\end{tabular}

Tabelle 42: Ausgewählte Bindungswinkel der Verbindung 36 
Um eine bevorzugte Lithiierung am Stickstoff zu erreichen, wurden kleinere Stickstoffsubstituenten gewählt. Die gewählten Silyl- statt Arylgruppen sollten weiterhin, auf Grund des +M-Effekts des Stickstoffs (pull), die Polarität der B-N-Bindung reduzieren, so dass die Oligomerisierung der erwarteten Iminoborene unterbunden werden sollte.

\subsubsection{Synthese der Fluor-triphenylsilyl-borane 37 - 39}

Es wurde das Triphenylsilylamin als Substituent am Bor gewählt, welches mit $n$-Butyllithium leicht zu metallieren ist und mit Difluor-bis(trimethylsilyl)amino-boran, Difluor-diisopropylamino-boran und Difluor-1,1,5,5-tetra-methylpiperidino-boran in quantitativen Ausbeuten zu den jeweiligen Fluor-triphenylsilylamino-boranen (37 - 39) reagiert. Wahrscheinlich auf Grund einer kinetischen Hemmung kann keine Reaktion des Triphenylsilylamids mit 2,4,6-Tri(tert-butyl)phenyl-difluor-boran beobachtet werden.

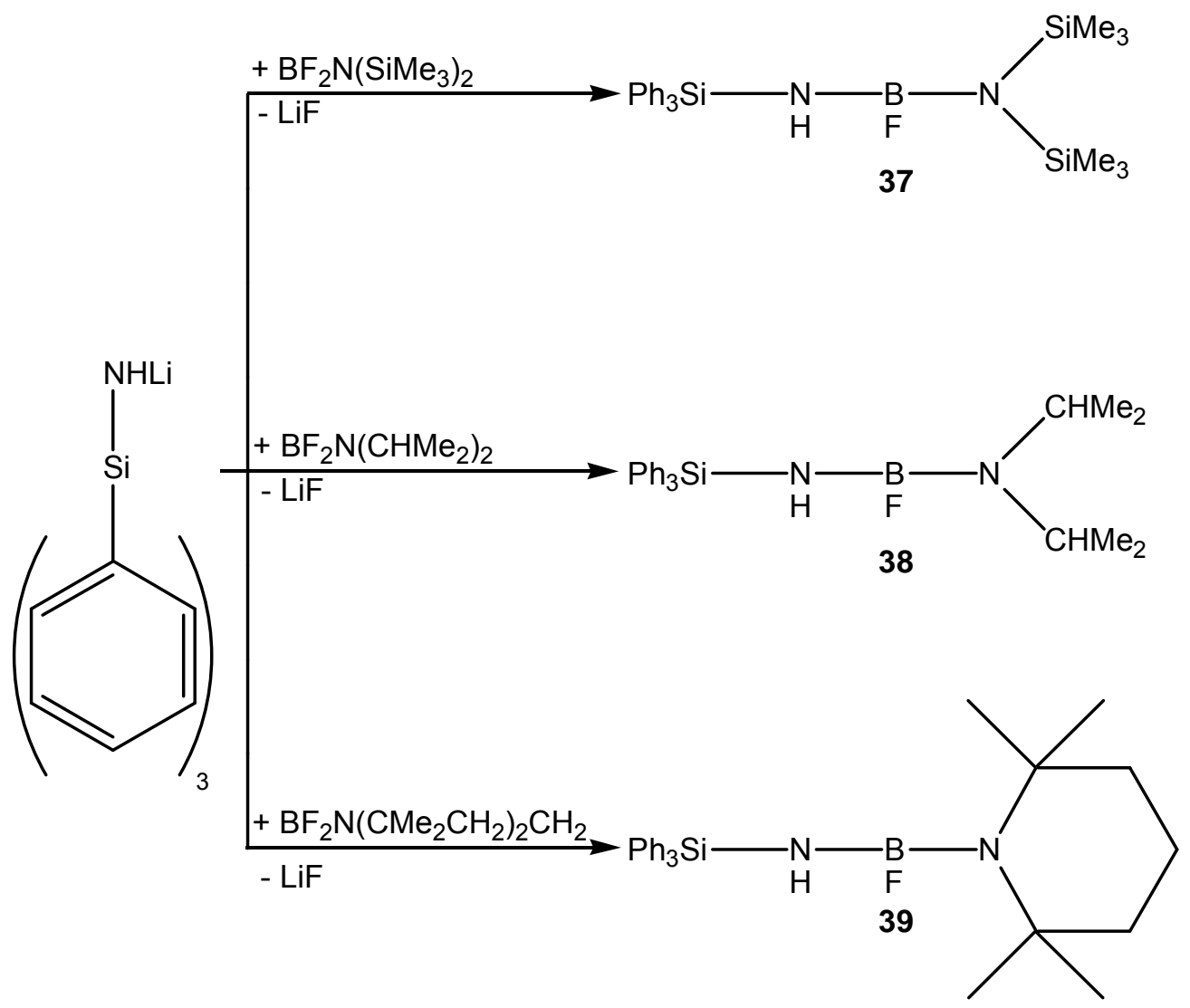

Gleichung 41: Synthese von 37 - 39 


\subsubsection{Kristallstrukturen von $\mathbf{3 7}$ und $\mathbf{3 8}$}

Die Stickstoff- und Boratome der röntgenstrukturanalytisch untersuchten Verbindungen bilden eine Ebene. Die drei planaren Phenylringe sind jeweils fast orthogonal zueinander angeordnet.

\subsubsection{Kristallstruktur von 37}

Verbindung 37 kristallisiert aus $n$-Hexan monoklin in der Raumgruppe $P 2_{1} / \mathrm{n}$. Die Borund Stickstoffatome sind planar umgeben $\left(\Sigma \nless \mathrm{B} 1=360,0^{\circ}, \quad \Sigma \nless \mathrm{N} 1=359,7^{\circ}\right.$, $\left.\Sigma \nless \mathrm{N} 2=360,0^{\circ}\right)$.

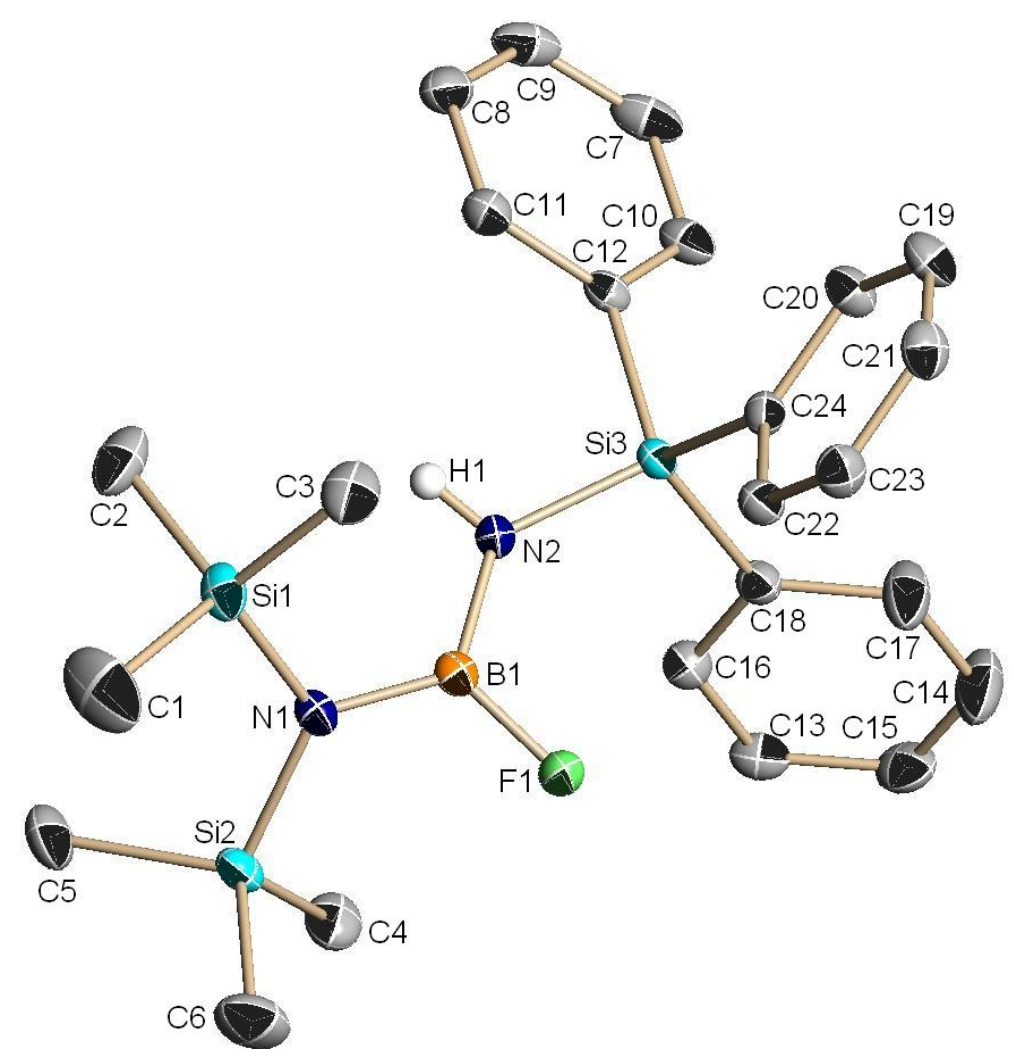

Abbildung 31: Kristallstruktur von 37 ohne $\mathrm{CH}$-Wasserstoffatome

Bindungslängen/pm mit Standardabweichung

\begin{tabular}{|c|c|c|c|c|c|}
\hline B1-F1 & $135,44(15)$ & N1-Si1 & $175,53(10)$ & $\mathrm{N} 2-\mathrm{H} 1$ & 81,9 \\
\hline B1-N2 & $141,43(16)$ & N1-Si2 & $175,54(11)$ & & \\
\hline B1-N1 & $143,74(16)$ & N2-Si3 & $173,67(10)$ & & \\
\hline
\end{tabular}

Tabelle 43: Ausgewählte Bindungslängen der Verbindung 37

Bindungswinkel ${ }^{\circ}$ mit Standardabweichung

\begin{tabular}{|ll|ll|ll|}
\hline B1-N1-Si1 & $118,33(8)$ & B1-N1-Si2 & $117,94(8)$ & Si1-N1-Si2 & $123,43(6)$ \\
\hline B1-N2-Si3 & $133,42(9)$ & Si1-N2-H1 & 112,66 & H1-N2-B1 & 113,92 \\
\hline F1-B1-N2 & $116,16(11)$ & F1-B1-N1 & $119,21(10)$ & N2-B1-N1 & $124,63(11)$ \\
\hline
\end{tabular}

Tabelle 44: Ausgewählte Bindungswinkel der Verbindung 37 


\subsubsection{Kristallstruktur von $\mathbf{3 8}$}

Verbindung 38 kristallisiert aus $n$-Hexan monoklin in der Raumgruppe $P 2_{1} / n$. Die Borund Stickstoffatome sind planar umgeben $\left(\Sigma \Varangle \mathrm{B} 1=360,0^{\circ}, \quad \Sigma \Varangle \mathrm{N} 1=359,2^{\circ}\right.$, $\left.\Sigma \Varangle \mathrm{N} 2=359,6^{\circ}\right)$

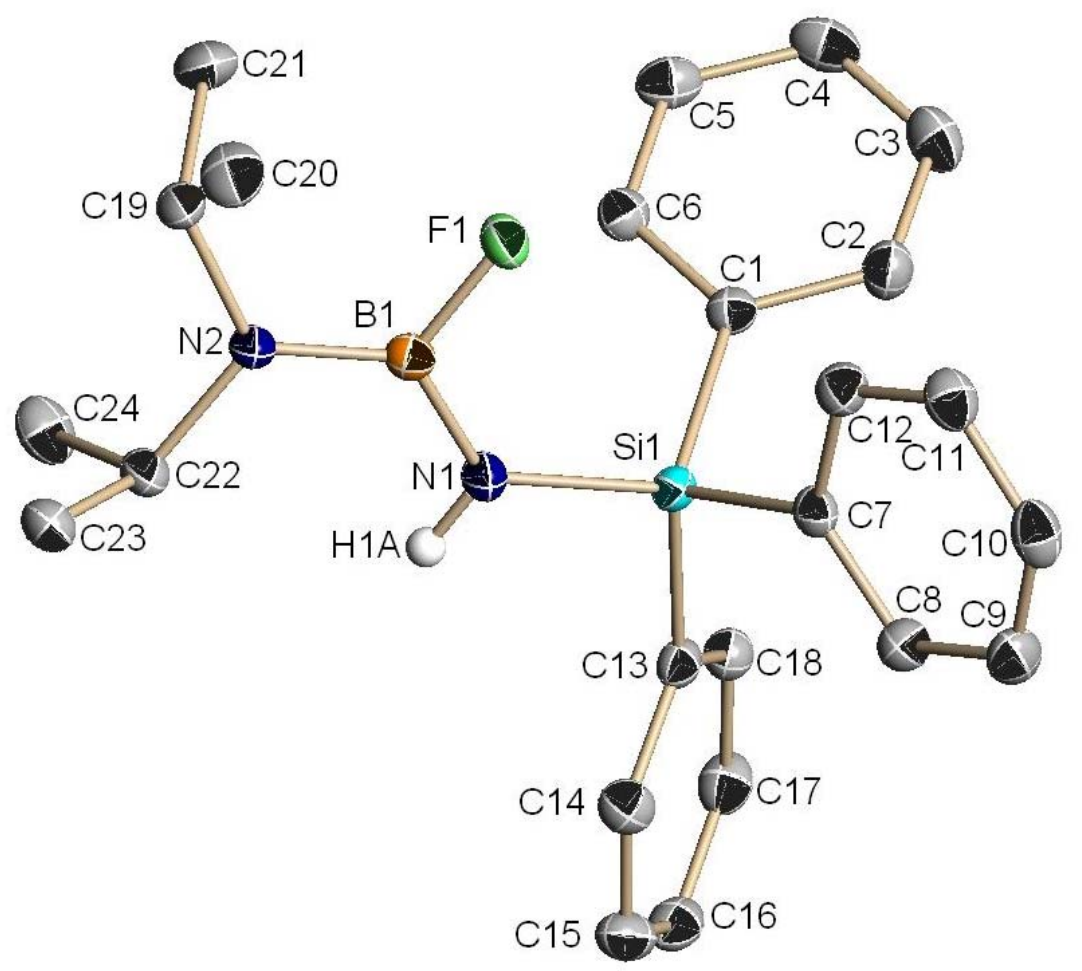

Abbildung 32: Kristallstruktur von 38 ohne $\mathrm{CH}$-Wasserstoffatome

Bindungslängen/pm mit Standardabweichung

\begin{tabular}{|ll|ll|ll|}
\hline B1-F1 & $136,25(15)$ & N1-Si1 & $172,60(10)$ & C22-N2 & $147,64(14)$ \\
\hline B1-N2 & $140,07(16)$ & N1-H1A & 86,7 & C1-Si1 & $187,18(12)$ \\
\hline B1-N1 & $141,98(16)$ & C19-N2 & $147,92(14)$ & & \\
\hline
\end{tabular}

Tabelle 45: Ausgewählte Bindungslängen der Verbindung $\mathbf{3 8}$

Bindungswinkel ${ }^{\circ}$ mit Standardabweichung

\begin{tabular}{|ll|ll|ll|}
\hline B1-N1-Si1 & $129,05(9)$ & Si1-N1-H1A & 112,77 & H1A-N1-B1 & 117,39 \\
\hline F1-B1-N2 & $118,21(10)$ & F1-B1-N1 & $114,97(10)$ & N2-B1-N1 & $126,82(11)$ \\
\hline B1-N2-C22 & $120,86(10)$ & B1-N2-C19 & $122,82(10)$ & C22-N2-C19 & $115,96(9)$ \\
\hline
\end{tabular}

Tabelle 46: Ausgewählte Bindungswinkel der Verbindung 38 


\subsubsection{Synthese der tert-Butyldimethylsilylamino-fluor-borane, 40 - 42}

Werden Difluor-bis(trimethylsilyl)amino-boran, Difluor-diisopropylamino-boran und Difluor-1,1,5,5-tetramethylpiperidino-boran jeweils mit Lithium-tert-butyldimethylsilylamid umgesetzt, können die erwarteten Aminofluorborane $\mathbf{4 0}$ - $\mathbf{4 2}$ isoliert werden.

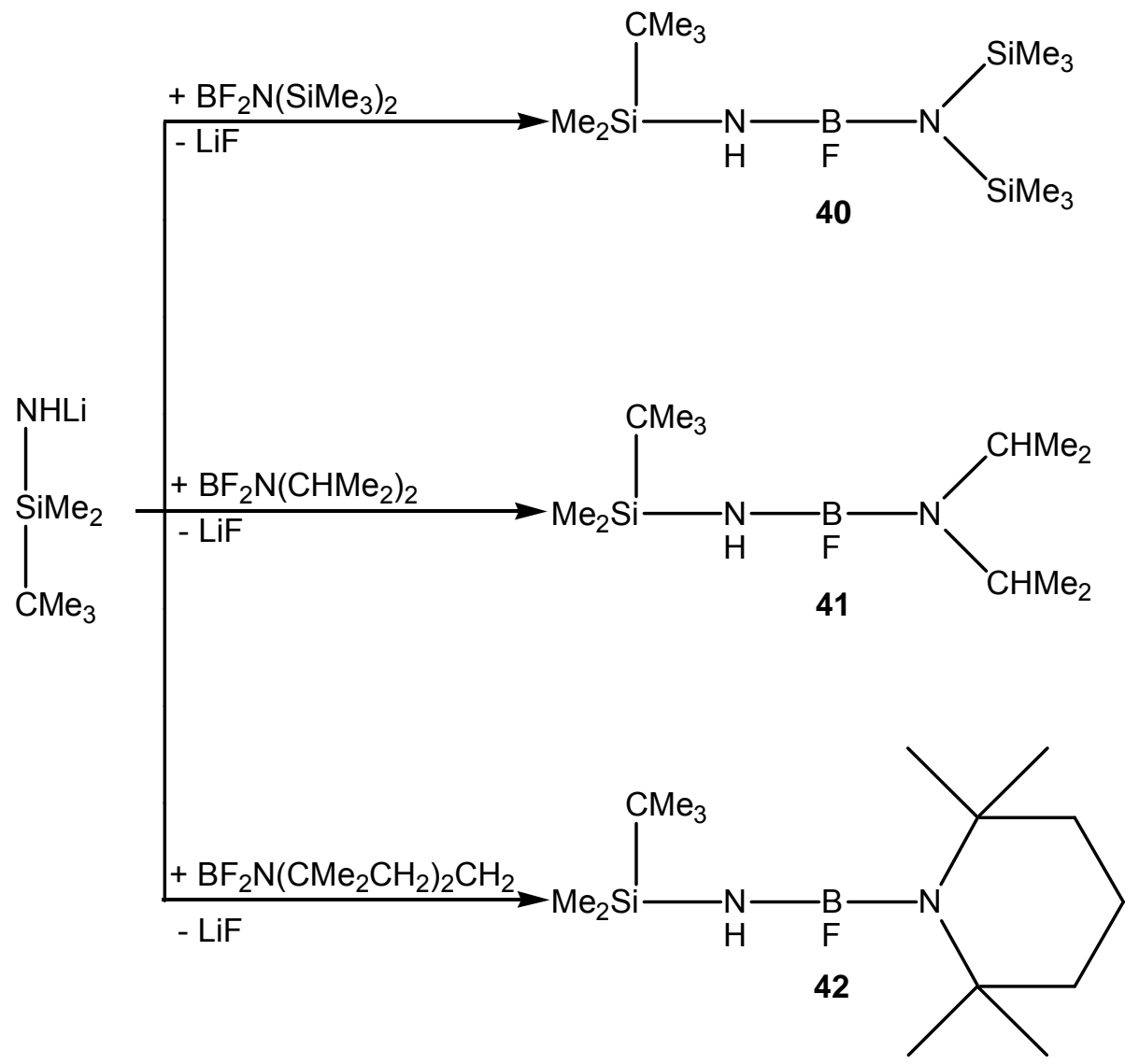

Gleichung 42: Synthese von 40 - 42

40 zersetzt sich bereits bei Temperaturen von $100{ }^{\circ} \mathrm{C}$ im HV unter Fluorsilanabspaltung. 41 und $\mathbf{4 2}$ sind hingegen thermisch stabile Verbindungen. Aus $n$-Hexan gewonnene Kristalle von $\mathbf{4 1}$ schmelzen bereits bei $5{ }^{\circ} \mathrm{C}$. Auf Grund der erwarteten ähnlichen Struktur zu Verbindung $\mathbf{3 8}$ wurde auf das Anfertigen einer Röntgenstrukturanalyse verzichtet.

Die ${ }^{11} \mathrm{~B}-\mathrm{NMR}$-Verschiebungen sind bei den drei untersuchten Verbindungen ähnlich. Der Silylaminsubstituent in $\mathbf{4 0}$ hat jedoch großen Einfluss auf die ${ }^{19} \mathrm{~F}-\mathrm{NMR}$ Verschiebung. Während die Fluorsignale von 41 und 42 im Bereich von $\delta=64$ ppm liegen, werden die Verschiebung bei $\mathbf{4 0} \mathrm{im}$ Vergleich stark Tieffeld verschoben gefunden $(\delta=100 \mathrm{ppm})$. 


\subsubsection{Synthese der tert-Butyldimethylsilylimino-borene, $\mathbf{4 3}$ und $\mathbf{4 4}$}

Bei der Synthese von $\mathbf{4 2}$ werden unerwartet auch die Edukte zurückerhalten. Neben 42 entsteht als Hauptprodukt der Reaktion das Iminoboren 43 (38\% Ausbeute, gaschromatographisch bestimmt). Bei der Reaktion des tert-Butyldimethylsilylamids mit dem Difluor-2,4,6-tri(tert-Butyl)phenyl-boran kann neben den Ausgangssubstanzen bei gleichen Reaktionsbedingungen ausschließlich das Iminoboren $\mathbf{4 4}$ isoliert werden.

Die intermediär entstandenen Aminofluorborane werden demnach durch, noch in Lösung vorhandenes, Amid lithiiert, wobei sich das tert-Butyldimethylsilylamid zurückbildet. Unter LiF-Abspaltungen, welche bereits bei tiefen Temperaturen (unter $-50^{\circ} \mathrm{C}$ ) beobachtet werden, bilden sich die Iminoborene $\mathbf{4 3}$ und $\mathbf{4 4}$.

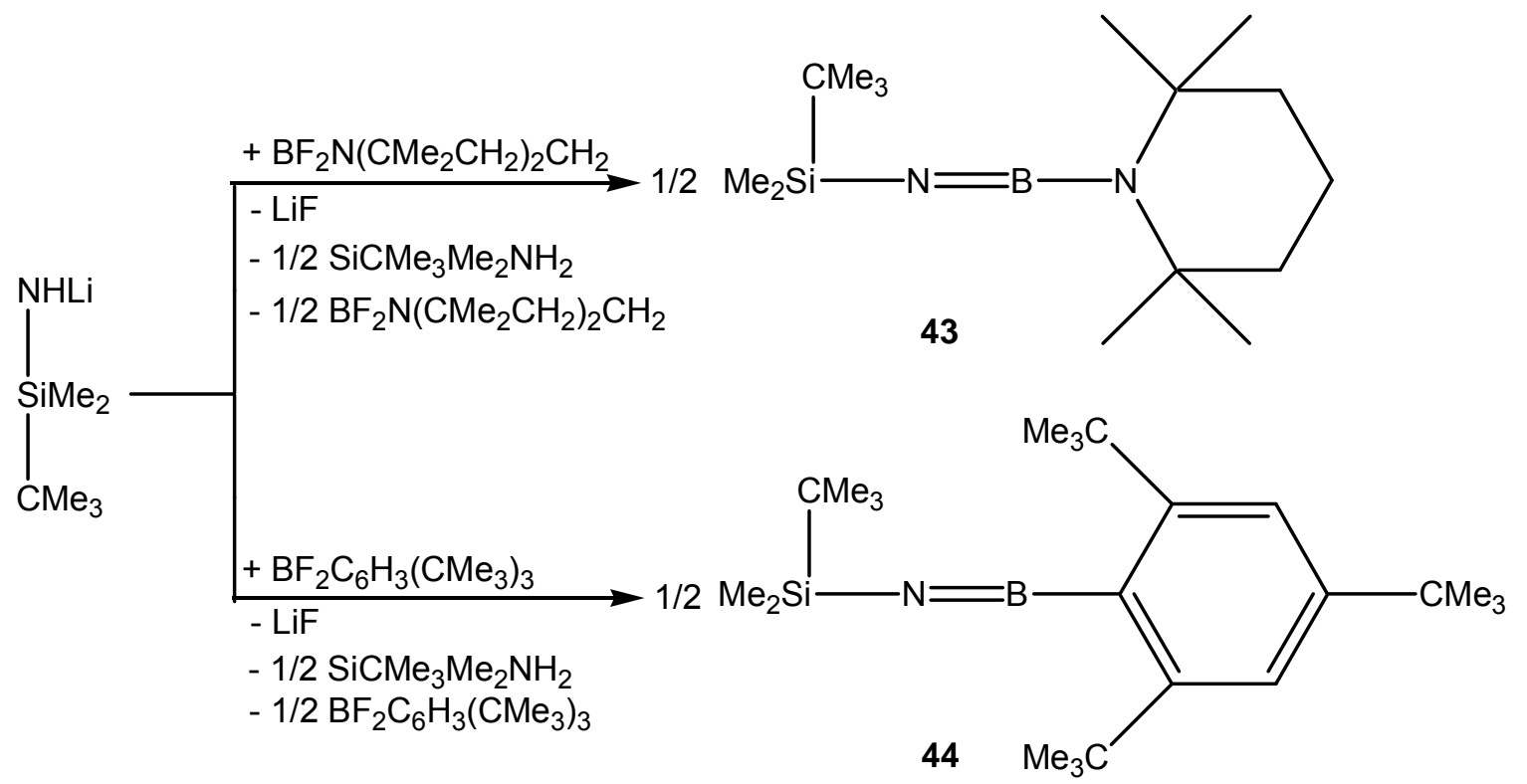

Gleichung 43: Synthese der Iminoborene $\mathbf{4 3}$ und $\mathbf{4 4}$

Es bleibt unklar, warum mit zunehmendem sterischen Anspruch des Borsubstituenten die Bildung des Iminoborens begünstigt wird. Nach Prof. Dr. H. Nöth spielt "gerade bei sperrigen Systemen (Borazine, sperrige Trisaminoborane) [...] die Ummetallierung eine (noch nicht ganz durchsichtige) Rolle. Offenbar liegt ein kinetisches Problem vor" ${ }^{[69]}$. 
44 konnte röntgenstrukturanalytisch untersucht werden. Die gefundene Struktur bestätigt die des kernresonanz- und massenspektroskopisch charakterisierten Iminoborens. Eine Restelektronendichte am Silicium verhindert jedoch das vollständige Lösen der Struktur, so dass auf die Angabe von Bindungslängen und -winkel verzichtet wird.

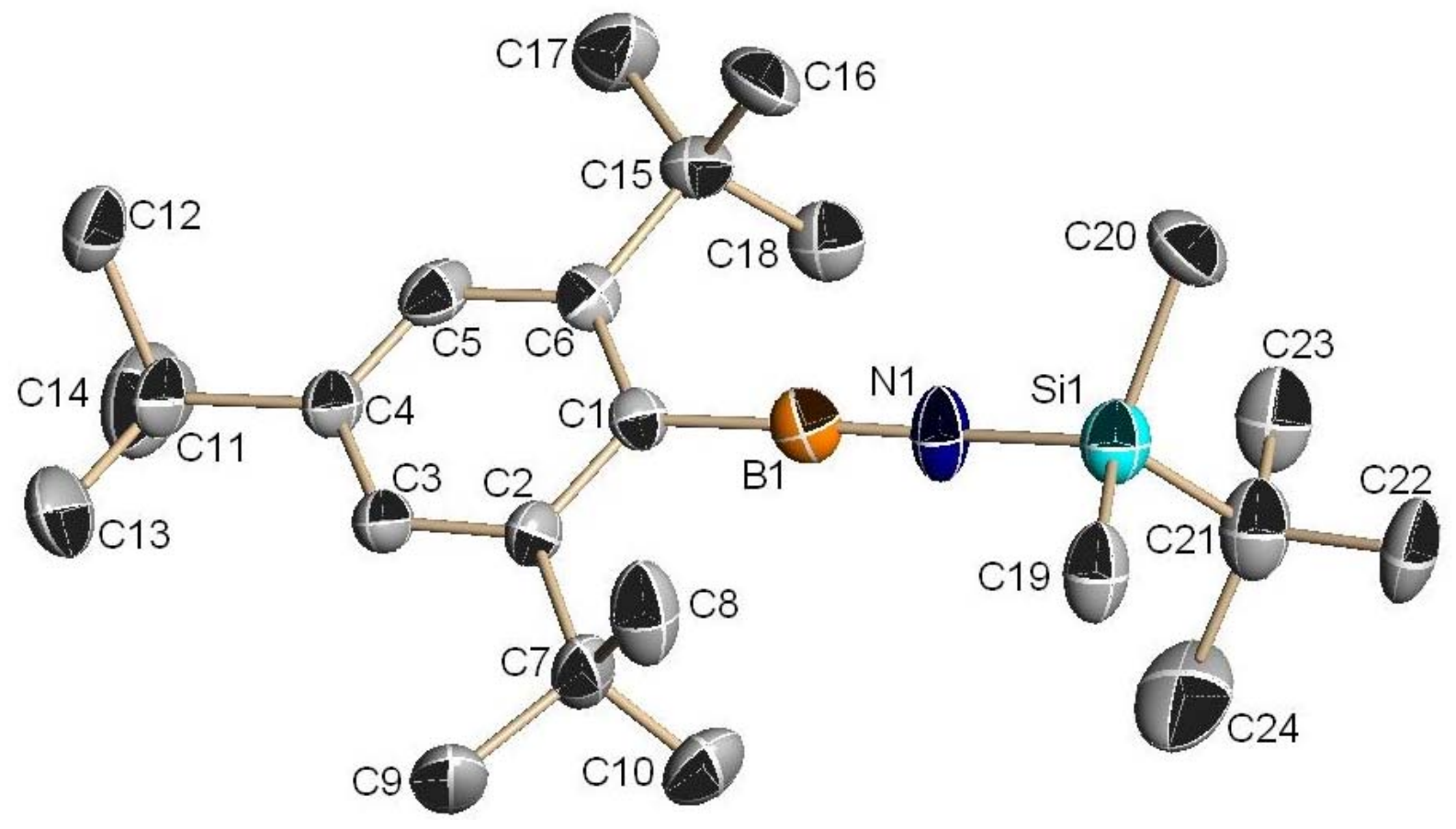

Abbildung 33: Kristallstruktur von $\mathbf{4 4}$ ohne Wasserstoffatome

\subsubsection{Additionsreaktionen an $\mathbf{4 4}$ mit Lewis-Basen}

Während bei Zugabe von Lewis-Säuren wie Trichloralan, aus sterischen Gründen oder auf Grund der geringen Basizität des Stickstoffatoms, keine Addition an 44 beobachtet wird, reagieren Lewis-Basen (z.B. $\mathrm{H}_{2} \mathrm{O}, \mathrm{MeOH}$ und $\mathrm{NH}_{3}$ ) unter Addition an die B-N-Bindung. Bei weiterer Zugabe von Wasser wird nach langer Reaktionszeit die N-Si-Bindung angegriffen. Bordiole können nicht isoliert werden. 


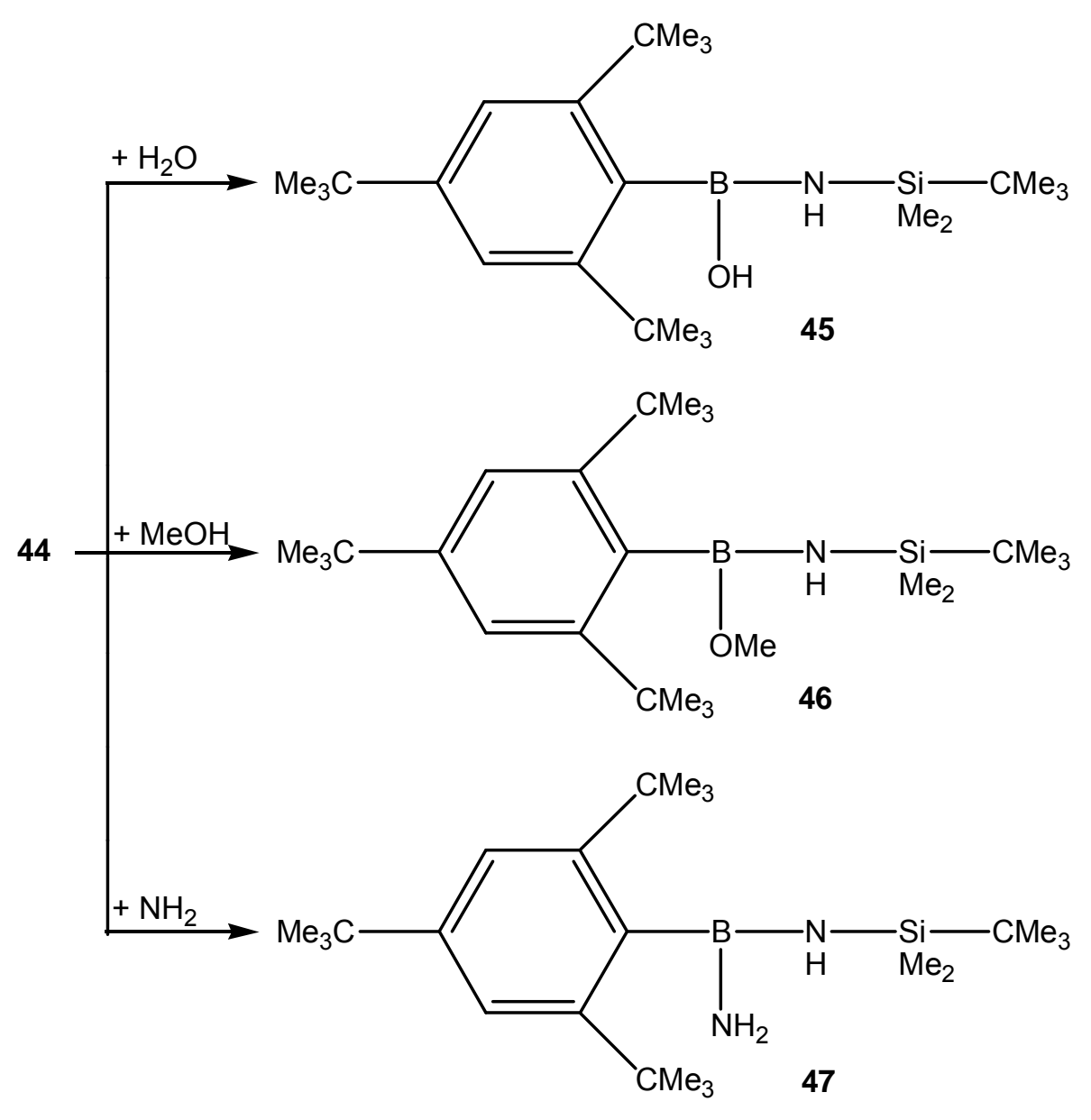

Gleichung 44: Synthese von 45 - 47

Bedingt durch die Drittsubstitution elektronenziehender Sauerstoff- bzw. Stickstoffatome am Bor ist das ${ }^{11} \mathrm{~B}-\mathrm{NMR}$ Signal der Verbindungen 45 - $47 \mathrm{im}$ Vergleich zu 44 um 15 - 16 ppm stärker Tieffeld verschoben, wobei erwartungsgemäß die Methoxigruppierung in Verbindung 46 den stärksten -I-Effekt ausübt. Während die Kohlenstoffatome C2-C6 ähnliche ${ }^{13} \mathrm{C}-\mathrm{NMR}$-Verschiebungen zeigen wie im Iminoboren 44, ist das Signal des C1-Atoms des Phenylrings bei den Verbindungen 45 - 47 jeweils um 17 - 23 ppm Tieffeld verschoben. Die stärkere Entschirmung ist wahrscheinlich auf den zunehmenden -M-Effekt des Boratoms zurückzuführen. Auffällig sind die ${ }^{29}$ Si-NMR-Verschiebungen. Während beim Siliciumatom von 44 eine chemische Verschiebung von $\delta=-4,16$ zu finden ist, führen die Borsubstituenten bei 45 - 47 zu einer Entschirmung des Siliciums. Die OH- substituierte Verbindung 45 zeigt unerwartet mit $\delta=8,46$ die stärkste Tieffeldverschiebung - nicht 46 . 


\subsubsection{Kristallstrukturen der Verbindungen 45 und 47}

Während die Bor- und Stickstoffatome im Iminoboren zweifach koordiniert waren, sind sie nach der Additionsreaktion dreifach substituiert. Dies führt einerseits zur Verlängerung der B-N-Bindung und andererseits zur Verkleinerung des ehemals linearen C-B-N-Winkels. Die Bor- bzw. Stickstoffsubstituenten sind trans-ständig angeordnet.

\subsubsection{Kristallstruktur der Verbindung 45}

Verbindung 45 kristallisiert aus Diethylether orthorhombisch in der Raumgruppe $P c a 2{ }_{1}$. Im Packungsplot sind keine Wasserstoffbrücken zwischen den Hydroxi- bzw. Aminogruppen zweier Moleküle erkennbar, was den relativ niedrigen Schmelzpunkt von $137^{\circ} \mathrm{C}$ erklärt. Die Winkelsummen der Bor- und Stickstoffatome sind exakt $360^{\circ}$, die Hydroxigruppe bzw. das Proton führen nicht zu einem Abweichen des Stickstoffbzw. Boratoms aus der Planarität.

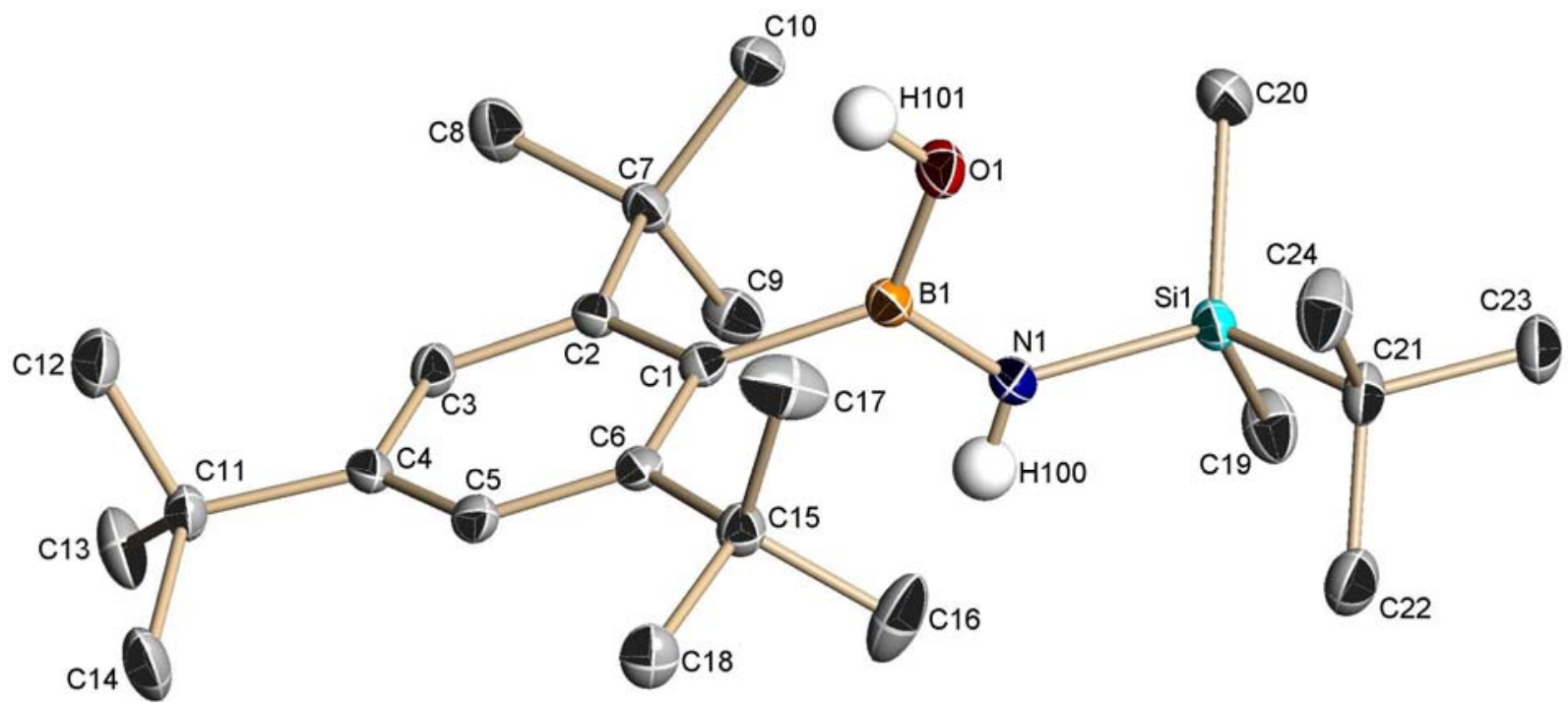

Abbildung 34: Kristallstruktur von $\mathbf{4 5}$ ohne $\mathrm{CH}$-Wasserstoffatome

Bindungslängen/pm mit Standardabweichung

\begin{tabular}{|ll|ll|ll|}
\hline Si1-N1 & $174,51(14)$ & B1-O1 & $137,9(2)$ & B1-N1 & $142,1(2)$ \\
\hline B1-C1 & $160,3(2)$ & B1-H100 & 82,1 & N1-H100 & 84,5 \\
\hline
\end{tabular}

Tabelle 47: Ausgewählte Bindungslängen der Verbindung 45

Bindungswinkel $/{ }^{\circ}$ mit Standardabweichung

\begin{tabular}{|ll|ll|ll|}
\hline O1-B1-N1 & $115,26(14)$ & O1-B1-C1 & $122,32(14)$ & N1-B1-C1 & $122,42(14)$ \\
\hline B1-N1-Si1 & $132,09(12)$ & B1-N1-H100 & 115,69 & Si1-N1-H100 & 112,12 \\
\hline B1-O1-H101 & 113,76 & & & & \\
\hline
\end{tabular}

Tabelle 48: Ausgewählte Bindungswinkel der Verbindung 45 


\subsubsection{Kristallstruktur der Verbindung 47}

Verbindung 47 kristallisiert wie auch 45 aus $n$-Hexan orthorhombisch in der Raumgruppe $P \mathrm{ca} 2{ }_{1}$. Innerhalb der Elementarzelle können keine intermolekularen Wechselwirkungen festgestellt werden. B1 und N1 sind planar umgeben $\left(\Sigma \Varangle \mathrm{B} 1=\Sigma \Varangle \mathrm{N} 1=360^{\circ}\right)$.

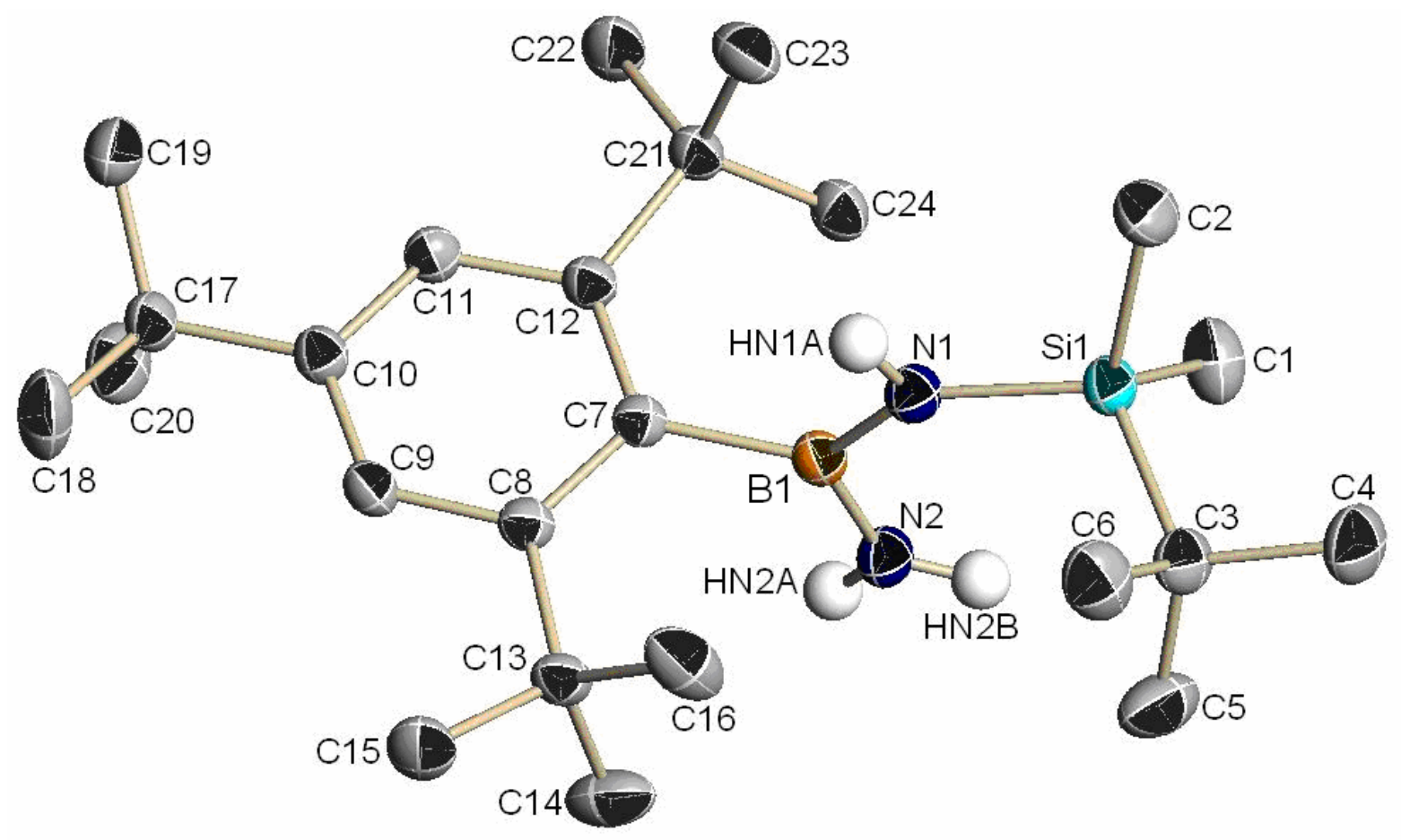

Abbildung 35: Kristallstruktur von $\mathbf{4 7}$ ohne $\mathrm{CH}$-Wasserstoffatome

Bindungslängen/pm mit Standardabweichung

\begin{tabular}{|c|c|c|c|c|}
\hline B1-N1 & $144,0(3)$ & N1-Si1 & $174,3(2)$ & N2-HN2A $\quad 86,2$ \\
\hline B1-N2 & $140,6(3)$ & N1-HN1A & 92,2 & \\
\hline B1-C7 & $160,7(3)$ & N2-HN2A & 92,3 & \\
\hline
\end{tabular}

Tabelle 49: Ausgewählte Bindungslängen der Verbindung 47

Bindungswinkel $/{ }^{\circ}$ mit Standardabweichung

\begin{tabular}{|ll|ll|ll|}
\hline N1-B1-C7 & $121,37(16)$ & N2-B1-N1 & $119,87(17)$ & N2-B1-C7 & $118,76(16)$ \\
\hline B1-N1-Si1 & $134,24(14)$ & B1-N1-HN1A & 114,00 & Si1-N1-HN1A & 111,74 \\
\hline B1-N2-HN2A & 122,81 & B1-N2-HN2A & 127,14 & HN1A-N2-HN2A & 108,84 \\
\hline
\end{tabular}

Tabelle 50: Ausgewählte Bindungswinkel der Verbindung 47 


\subsection{Reaktionen von Fluorboranen mit Lewis-Säuren der Triele}

\subsubsection{Umsetzung des Fluor-bis[bis(trimethylsilyl)amino]-borans mit $\mathrm{AlCl}_{\mathbf{3}}$}

Wird Brom-bis[bis(trimethylsilyl)amino]-boran mit Tribromalan in $\mathrm{CH}_{2} \mathrm{Cl}_{2} / \mathrm{Et}_{2} \mathrm{O}$ zur Reaktion gebracht, erfolgt die Abspaltung des Bromids, so dass ein Boriniumion isoliert werden kann ${ }^{[70]}$.

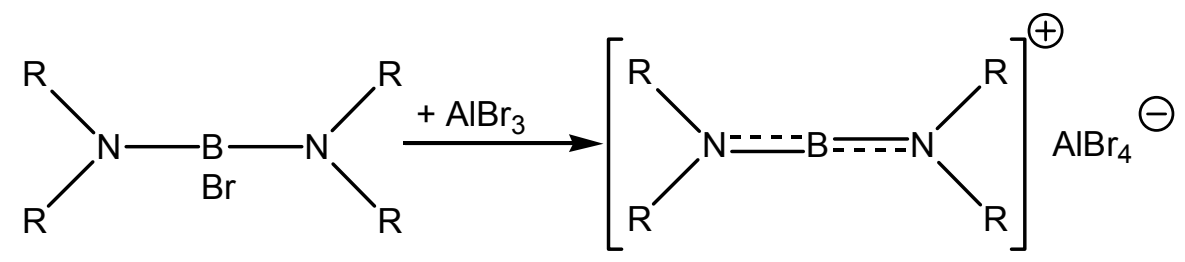

Gleichung 45: Synthese des Bis [bis(trimethylsilyl)amino]-boren-kations ${ }^{\text {[70] }}$

Versuche, beim Chlor-bis[bis(trimethylsilyl)amino]-boran das Halogenidion abzuspalten, gelangen bisher nicht ${ }^{[70]}$.

Im Rahmen dieser Arbeit sollte überprüft werden, ob bei einer Substitution des Chlors durch Fluor eine Borkationenbildung ermöglicht werden kann.

Hierfür wurde das Fluorboran zunächst unter Eiskühlung in Dichlormethan und Diethylether mit $\mathrm{AlCl}_{3}$ (in Diethylether gelöst) versetzt. Spätere Versuche zeigen, dass auf das Lösungsmittel $\mathrm{CH}_{2} \mathrm{Cl}_{2}$ verzichtet werden kann.

Nachdem die Reaktanden für $6 \mathrm{~h}$ unter Rückfluss erhitzt wurden, trat im ${ }^{19} \mathrm{~F}$-NMRSpektrum des Reaktionsgemischs das Signal des Fluortrimethylsilans auf. Die kernresonanz- und massenspektroskopischen Untersuchungen des entstandenen Feststoffs belegen die Bildung des $\mathrm{AlCl}_{3}$-Addukts des Bis(trimethylsilyl)aminotrimethylsilylimino-borens (48).

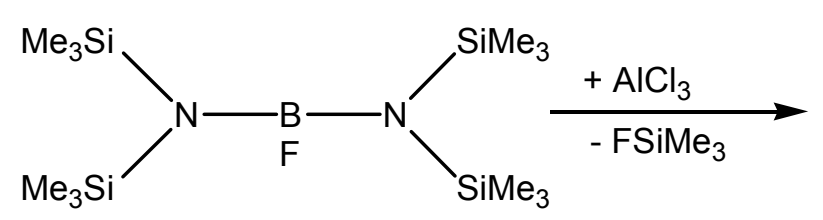

Gleichung 46: Synthese von 48

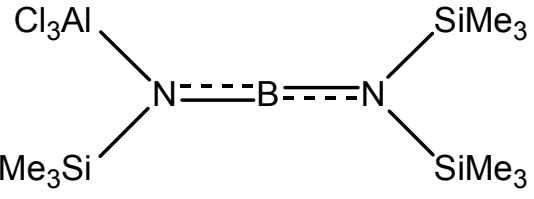

48 
Das Entfernen des Fluorsilans aus dem chemischen Gleichgewicht durch Reaktionstemperaturen über $19^{\circ} \mathrm{C}$ [Sdp.(SiMe $\left.\left.{ }_{3} \mathrm{~F}\right)\right]$ erhöhen die Ausbeute an $\mathbf{4 8}$, so dass eine annähernd quantitative Umsetzung möglich ist.

Eine Fluortrimethylsilanabspaltung vom Fluor-bis[bis(trimethylsilyl)amino]-boran kann auch ausschließlich thermisch erreicht werden, wobei nicht das Iminoboren, sondern dessen Dimer isoliert wird ${ }^{[71,72]}$. Die hier vorgestellte Reaktion zeigt die, durch Trichloralan katalysierte, Fluorsilanabspaltung. Auf Grund der niedrigeren Reaktionstemperaturen ist ein Abfangen des Iminoborens als $\mathrm{AlCl}_{3}$-Addukt möglich.

\subsubsection{Kernresonanzspektroskopische Untersuchungen an $\mathbf{4 8}$}

Die ${ }^{1} \mathrm{H}$ - und ${ }^{13} \mathrm{C}-\mathrm{NMR}$-Verschiebungen der Silylgruppe in Nachbarstellung zum Trichloralan sind Tieffeld verschoben. Das im Vergleich zur Ausgangsverbindung ebenfalls Tieffeld verschobene ${ }^{11} \mathrm{~B}-\mathrm{NMR}$-Signal kann durch die geschwächte ртрпBindung des aluminiumsubstituierten Stickstoffs erklärt werden. Im ${ }^{27} \mathrm{Al}-\mathrm{NMR}$ Spektrum ist ein scharfes Signal erkennbar $(\delta=102,75 \mathrm{ppm})$. Dies weist auf vierfach-koordiniertes Aluminium hin. Die starke Entschirmung des Kerns ist bedingt durch die vier elektronegativen Substituenten $(\mathrm{N}, \mathrm{Cl})$.

Eine Röntgenstrukturanalyse des, in Diethylether umkristallisierten, Feststoffs zeigt, dass bei $170 \mathrm{~K}$ kein Bor-Chlorkontakt auftritt. Somit liegen vierfach koordiniertes Aluminium und zweifach koordiniertes Bor nebeneinander vor, es findet weder, wie bei den vergleichbaren Silylverbindungen, ein Chlortransfer vom Aluminium statt, noch findet sich ein verbrückendes Chloratom.

48 ist die erste röntgenstrukturanalytisch untersuchte Verbindung eines IminoborenAlanaddukts. 


\subsubsection{Kristallstruktur des (Trichloralan-trimethylsilyl-amino)-bis(trimethyl- silyl)amino-borens, 48}

48 kristallisiert aus Diethylether monoklin in der Raumgruppe Pn. Die kurzen BorStickstoffbindungen (132 - $134 \mathrm{pm}$ ) sind vergleichbar mit denen von Aminoboriniumionen ${ }^{[73]}$. Die Stickstoffatome sind jeweils planar umgeben, wobei der $\mathrm{AlCl}_{3}-\mathrm{N}-\mathrm{SiMe}_{3}-$ Winkel mit $126^{\circ}$ aufgeweitet, der N1-B1-N2-Winkel mit 178,7 $7^{\circ}$ annähernd linear ist. Der Aluminium-Stickstoff-Abstand ist auf Grund der Elektronegativitätsunterschiede auf 188 pm verkürzt ( $\Sigma$ Kovalenzradien $\mathrm{Al}, \mathrm{N}=193 \mathrm{pm}^{[56]}$ ). Unerwartet unterscheiden sich die N-Si-Bindungen am N2 um 16 pm.

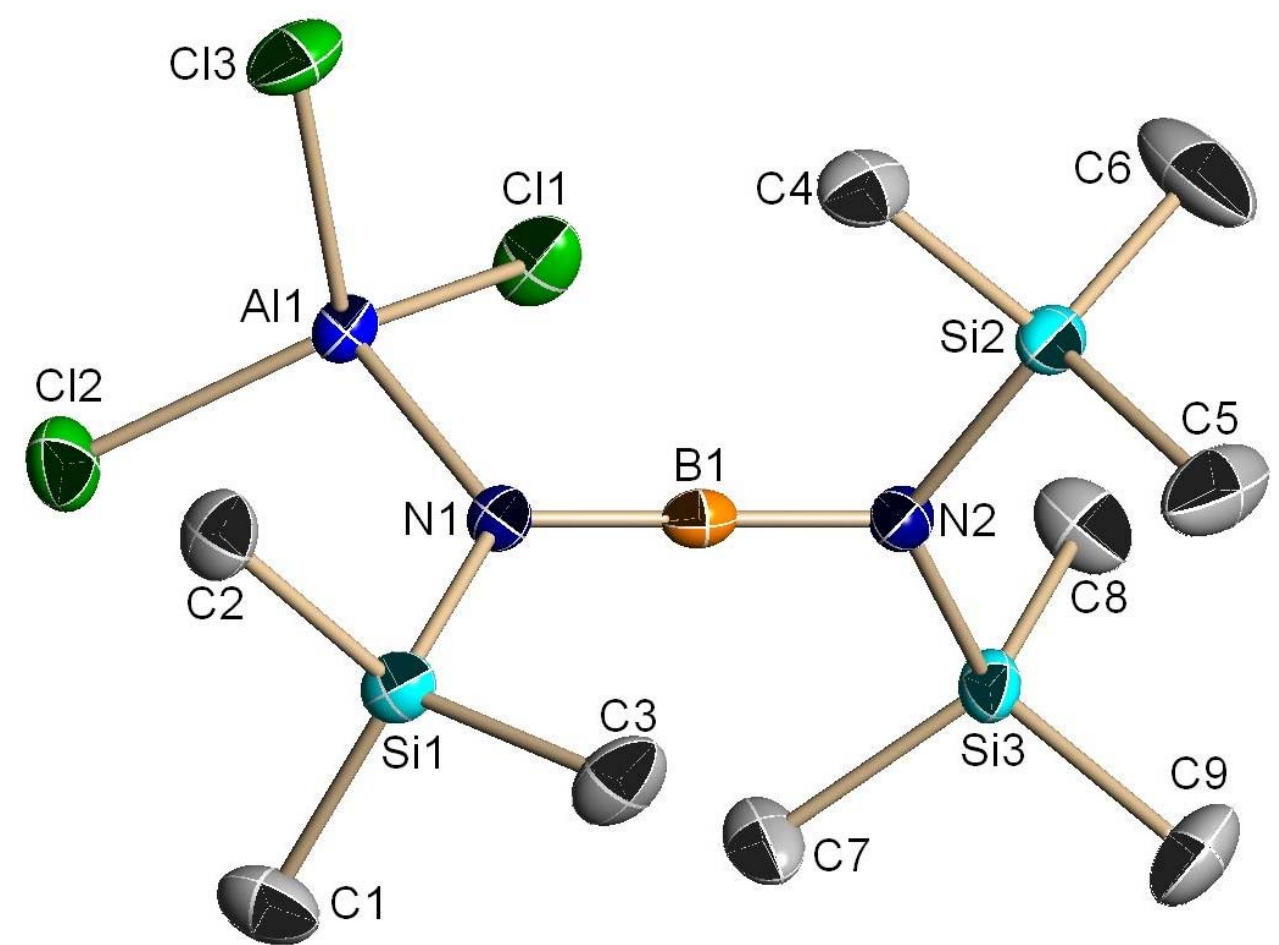

Abbildung 36: Kristallstruktur von $\mathbf{4 8}$ ohne Wasserstoffatome

Bindungslängen (-) bzw. intramolekulare Atomabstände (...)/pm mit Standardabweichung

\begin{tabular}{|ll|ll|ll|}
\hline Al1-N1 & $187,79(15)$ & B1-N1 & $132,3(3)$ & N2-Si2 & $184,7(5)$ \\
\hline Al1-Cl3 & $212,76(8)$ & B1-N2 & $133,7(3)$ & B1 ...Al1 & 272,2 \\
\hline Al1-Cl1 & $213,20(8)$ & N1-Si1 & $179,32(15)$ & B1...Cl1 & 312,3 \\
\hline Al1-Cl2 & $213,38(8)$ & N2-Si3 & $169,0(19)$ & & \\
\hline
\end{tabular}

Tabelle 51: Ausgewählte Bindungslängen bzw. intramolekulare Atomabstände der Verbindung $4 \mathbf{8}$ Bindungswinkel $/{ }^{\circ}$ mit Standardabweichung

\begin{tabular}{|ll|ll|ll|}
\hline N1-Al1-Cl3 & $109,49(6)$ & Cl3-Al1-Cl2 & $109,50(4)$ & B1-N1-Al1 & $115,38(13)$ \\
\hline N1-Al1-Cl1 & $104,40(5)$ & Cl1-Al1-Cl2 & $110,10(4)$ & Si1-N1-Al1 & $125,95(9)$ \\
\hline Cl3-Al1-Cl1 & $112,57(3)$ & N1-B1-N2 & $178,7(2)$ & & \\
\hline N1-Al1-Cl2 & $110,69(5)$ & B1-N1-Si1 & $118,67(13)$ & & \\
\hline
\end{tabular}

Tabelle 52: Ausgewählte Bindungswinkel der Verbindung $\mathbf{4 8}$ 


\subsubsection{Quantenchemische Berechnungen}

Zur Erklärung der experimentellen Ergebnisse wurden von Prof. Dr. S. Schmatz (Abteilung für Theoretische Chemie im Institut für Physikalische Chemie der Universität Göttingen) quantenchemische Berechnungen zu den Reaktionsmechanismen bei der Bildung von $\mathbf{4 8}$ durchgeführt.

Hierbei wurde das Programmpaket Gaussian03 verwendet ${ }^{[74]}$. Die Strukturen und Übergangszustände wurden nach der SCF-Methode unter Verwendung des 6-31G*Basissatzes optimiert und mit Hilfe der Dichtefunktionalmethode (DFT) unter Verwendung der Drei-Parameter-Hybrid-Methode von Becke verfeinert. Dabei wurden das LYP-Funktional (B3LYP) und der 6-31G*-Basissatz verwendet. Frequenzberechnungen wurden durchgeführt, um stationäre Punkte, wie lokale Minima oder Sattelpunkte, zu charakterisieren. Die Übergangsstrukturen wurden durch Verwendung der TS-Routine in Gaussian03 (IRC) ermittelt.

Die Berechnungen an Edukten (Trichloralan, Fluor-bis[bis(trimethylsilyl)amino]boran), und Produkten [Fluortrimethylsilan, (Trichloralan-trimethylsilyl-amino)bis(trimethylsilyl)amino-boren] ergaben, dass die Reaktion exotherm verläuft. Die Differenz der elektronischen Energien beträgt $-23.2 \mathrm{kcal} / \mathrm{mol}$, unter Einbezug der Nullpunktsenergien erhöht sie sich leicht auf $-23.6 \mathrm{kcal} / \mathrm{mol}$. Die freie Reaktionsenthalpie $\Delta G_{0}$ wurde zu $26,6 \mathrm{kcal} / \mathrm{mol}$ berechnet.

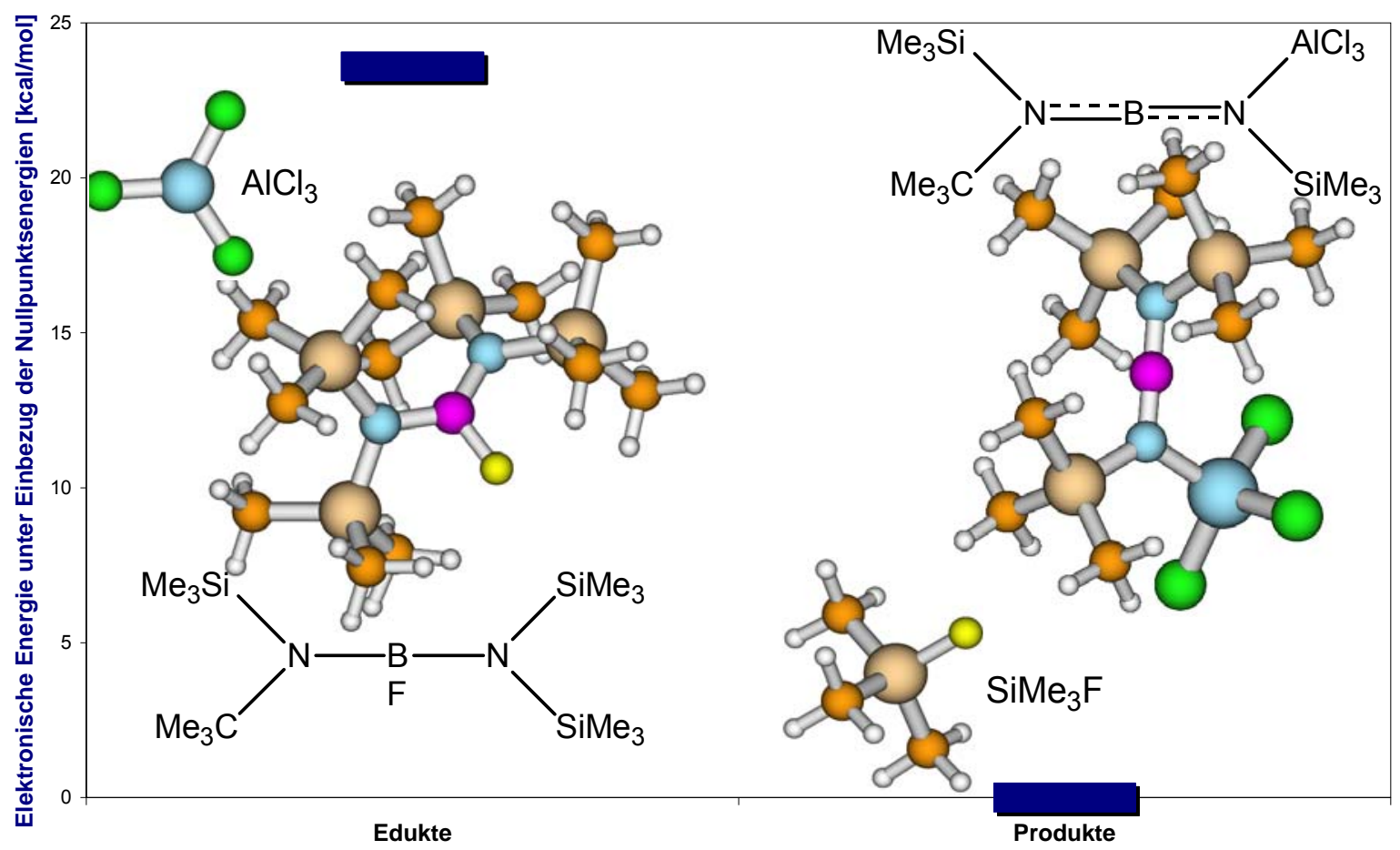

Abbildung 37: Vergleich der Energien der Edukte und Produkte bei der Synthese von 48 
Zur Bestimmung des Übergangszustandes wurden drei mögliche Zwischenprodukte angenommen.

\subsubsection{Berechnungen zu einem $\mathrm{AlCl}_{3} \mathrm{~F}$-Übergangszustand}

Zum einen kann das Aluminium (als Lewis-Säure) am Fluoratom angreifen und dieses abspalten, so dass eine Trennung in Anionen und Kationen erfolgt. In einem nachfolgenden Schritt könnte Fluortrimethylsilan gebildet und Trichloralan an den Iminstickstoff angelagert werden.

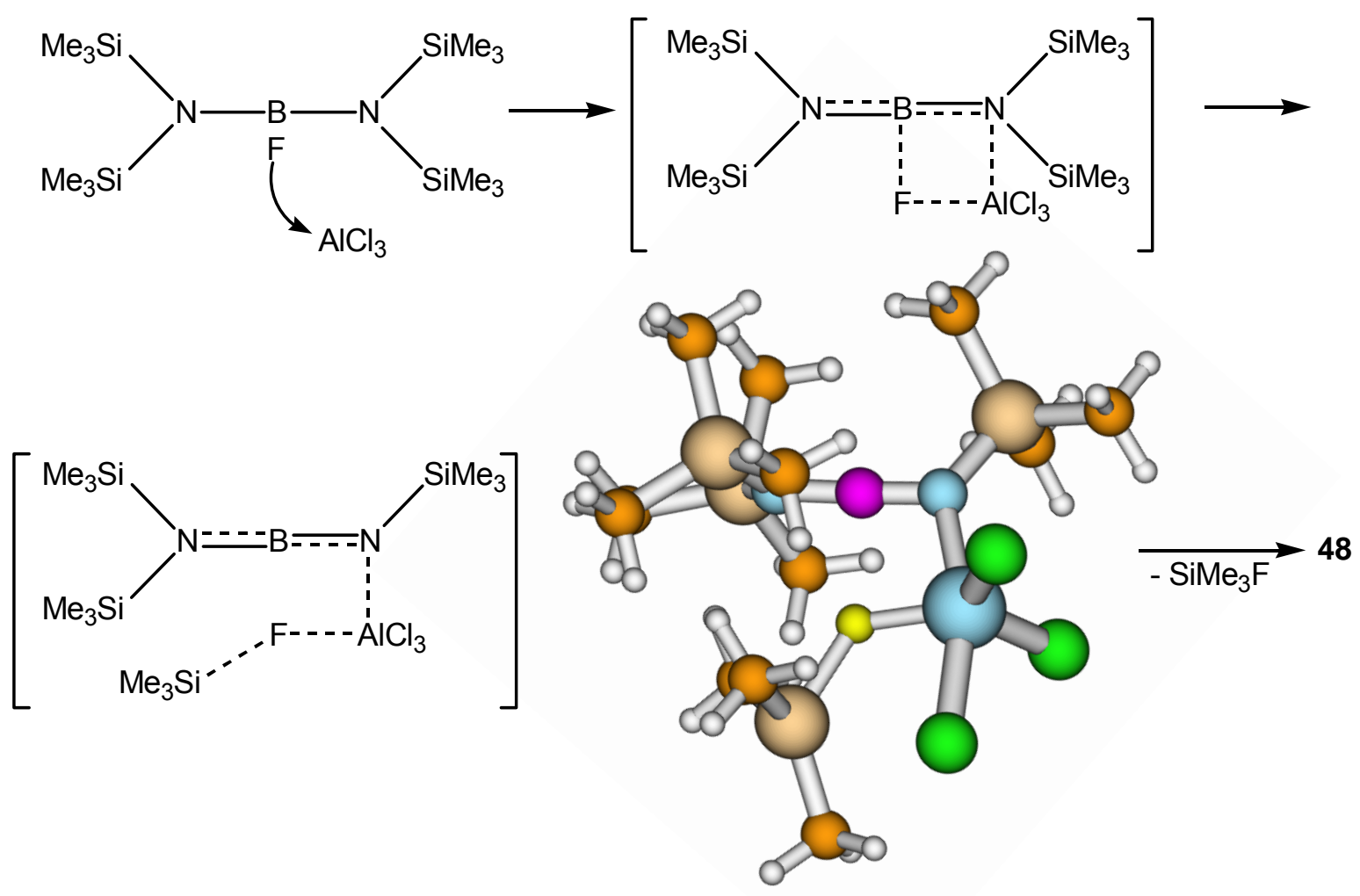

Gleichung 47: Möglicher Reaktionsmechanismus zur Bildung von 48

\subsubsection{Berechnungen zu einem Übergangszustand mit drei- und vierfach ko- ordinierten Stickstoffatomen}

Zum anderen besteht die Möglichkeit, dass sich Aluminium an einem Stickstoffatom anlagert. Die Vierfachkoordination des tetraedrischen Stickstoffs würde die N-SiBindung schwächen, was ein leichteres Abspalten der Trimethylsilylgruppe ermöglichen würde. 


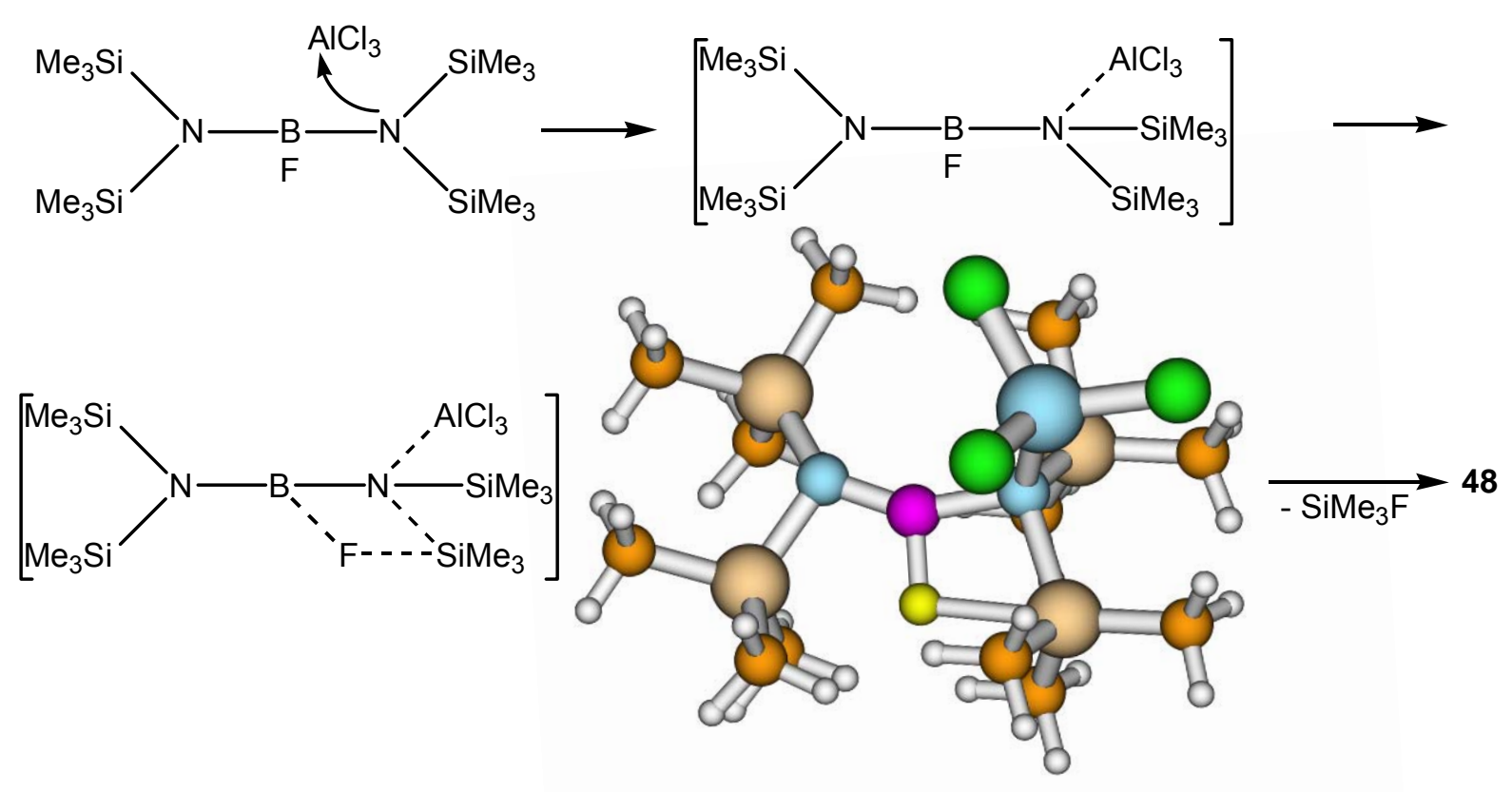

Gleichung 48: Möglicher Reaktionsmechanismus zur Bildung von 48

\subsubsection{Berechnungen zu einem Übergangszustand mit jeweils vierfach koor- dinierten Stickstoffatomen}

Da Aluminium auch pentakoordiniert sein kann ${ }^{[50]}$, ist ein gleichzeitiger Angriff an beiden Stickstoffatomen denkbar.

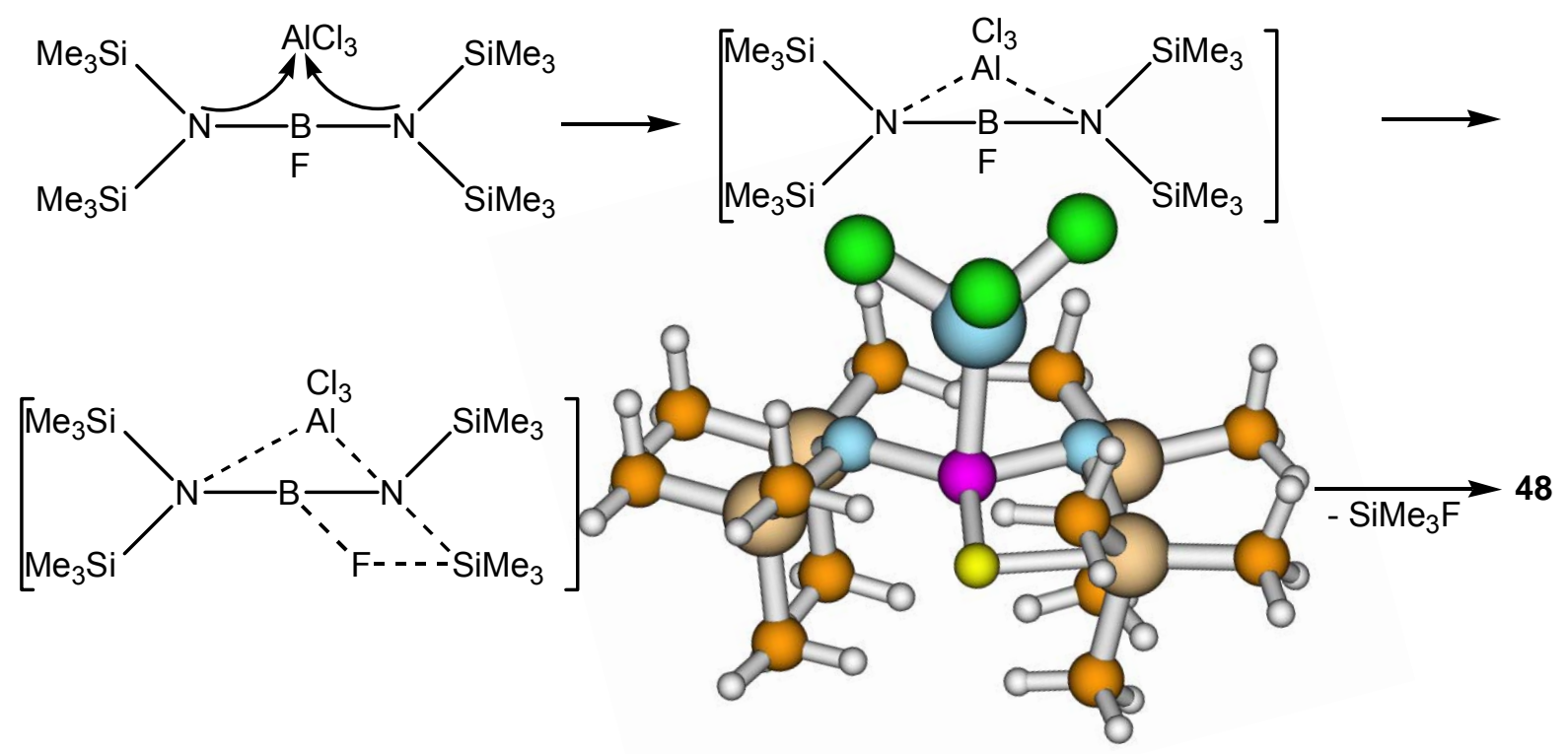

Gleichung 49: Möglicher Reaktionsmechanismus zur Bildung von 48 
Die Berechnungen an den möglichen Übergangszuständen (siehe Gleichungen 47 - 49) gestalteten sich sehr schwierig und aufwendig. Auf Grund der Größe des Systems und der hohen Elektronenzahl waren insbesondere die Berechnungen der Hesse-Matrizen, die für eine gute Konvergenz bei der Sattelpunktssuche notwendig sind, sehr zeitintensiv. Deshalb war es nicht möglich, diese Matrizen in jedem Optimierungsschritt zu erhalten. Trotz intensiver Bemühungen gelang es nicht, eine Struktur zu finden, in welcher die viergliedrigen Übergangszustände B-N-Al-F bzw. B-N-Si-F mit der charakteristischen Struktureinheit als stationärer Punkt auf der Potentialhyperfläche ermittelt werden konnten. Bei den Optimierungen wurde stets eine Bindung aufgebrochen und als stabiles Intermediat ein Addukt von $\mathrm{AlCl}_{3}$ an das Eduktmolekül gefunden. Es ist auf Grund dieser Befunde eher unwahrscheinlich, dass der Mechanismus nach einem derart vorgeschlagenen Reaktionswege verläuft. Mit ziemlicher Sicherheit kann jedoch gesagt werden, dass der komplexe Mechanismus über mehr als einen einzigen Übergangszustand mit mindestens einem stabilen Intermediat verlaufen muss.

Als dritte Möglichkeit blieb die Verbrückung der beiden Stickstoff-Atome über das Trichloralan. Erste Rechnungen ergaben, dass es keine derartige Struktur gibt, in der nicht ein enger Kontakt des Aluminiums zum Bor vorliegt. Die erste Suche nach dem Sattelpunkt zeigt zwar im Energieprofil eine Schulter, die tatsächlich dem postulierten Übergangszustand entspricht, bei einer erneuten Suche mit gerade dieser Startgeometrie divergiert die Struktur jedoch wieder. Es liegt hier offenbar ein Sattelpunkt höherer Ordnung vor, der auf Grund seiner hohen energetischen Lage aus der Diskussion über den Reaktionsmechanismus ausgeschlossen werden kann.

\subsubsection{Umsetzung des Fluor-bis[bis(trimethylsilyl)amino]-borans mit $\mathrm{AlClMe}_{2}$, $\mathrm{AlBr}_{3}$ und $\mathrm{GaCl}_{3}$}

Während Lewis-saure Salze wie $\mathrm{SnCl}_{2}$ oder $\mathrm{FeCl}_{3}$ unter vergleichbaren Reaktionsbedingungen nicht mit Fluor-bis[bis(trimethylsilyl)amino]-boran unter $\mathrm{SiMe}_{3} \mathrm{~F}-$ Eliminierung reagieren und auch bei einer Erhöhung der Reaktionstemperatur (durch Austausch von THF oder Dibutylether anstelle des Diethylethers) keine Fluorsilanabspaltung erkennbar ist, kann in der Reaktion von Chlordimethylalan mit dem Fluorbis[bis(trimethylsilyl)amino]-boran in ähnlich guten Ausbeuten das $\mathrm{AlCIMe}_{2}$-Addukt als wachsartiger Feststoff (49) isoliert werden. $\mathrm{AlBr}_{3}$ und $\mathrm{GaCl}_{3}$ reagieren ebenfalls zu den jeweiligen Iminoborenaddukten $\mathbf{5 0}$ und $\mathbf{5 1}$. 
Die Reaktion des Fluor-bis[bis(trimethylsilyl)amino]-borans mit Trimethylalan ist weitaus langsamer als mit Trichloralan (siehe Gleichung 46). Nachdem das Reaktionsgemisch fünf Tage unter Rückfluss erhitzt wurde, kann auch hier eine Fluortrimethylsilanabspaltung nachgewiesen werden, jedoch kein einheitliches Produkt isoliert werden.
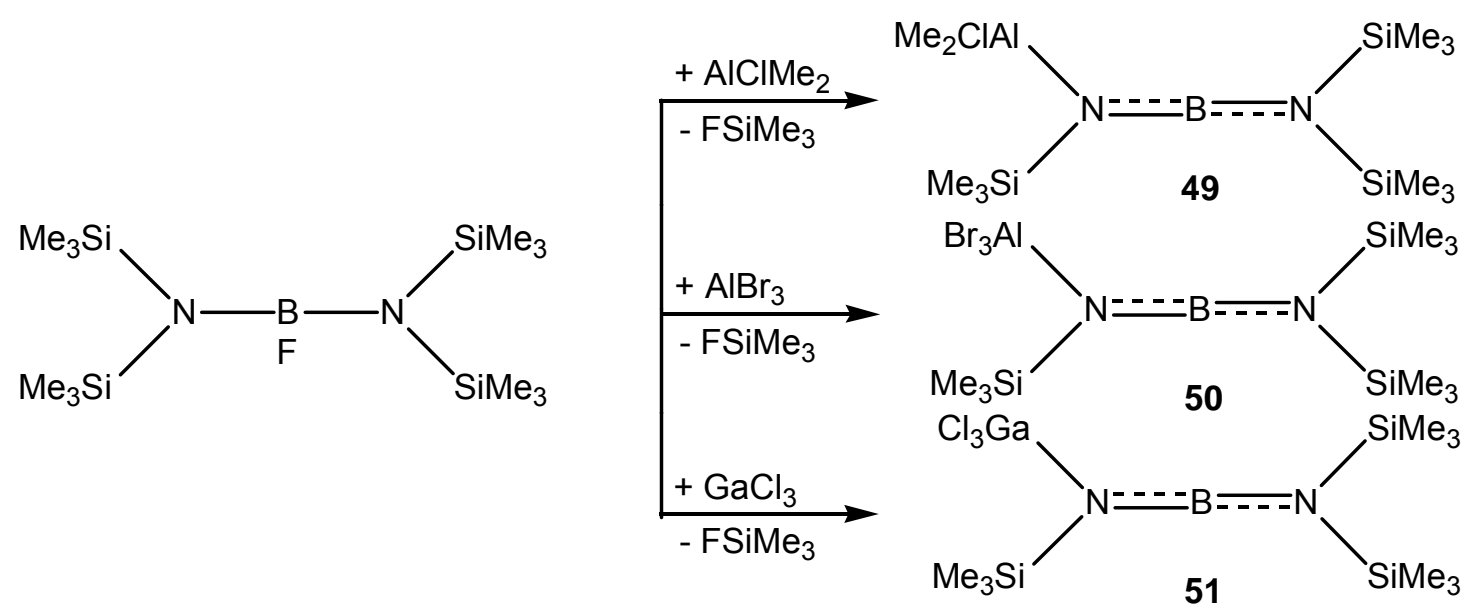

Gleichung 50: Synthese von 49 - 51

Da im ${ }^{1} \mathrm{H}$ - bzw. ${ }^{13} \mathrm{C}-\mathrm{NMR}$-Spektrum beide aluminiumgebundenen Methylgruppen von 49 die gleiche chemische Verschiebung haben, ist eine Methanidwanderung vom Aluminium zum Bor auszuschließen. Alle drei Verbindungen zeigen die, bereits bei 48 beobachtete, Tieffeldverschiebung der ${ }^{11} B-N M R$-Signale im Vergleich zum Fluorbis[bis(trimethylsilyl)amino]-boran. Die Silylgruppen in Nachbarstellung zum Trichloralan sind durch den -l-Effekt der Brom- bzw. Chloratome im Vergleich zum Edukt entschirmt.

Im Rahmen dieser Arbeit sollte untersucht werden, ob bei Substitution einer oder mehrerer Trimethylsilylgruppen des Fluor-bis[bis(trimethylsilyl)amino]-borans durch andere Silyl- (3.5.6), Alkyl- (3.5.7, 3.5.8) oder Arylsubstituenten (3.5.10) ebenfalls eine $\mathrm{AlCl}_{3}$-katalysierte Fluorsilanabspaltung unter Bildung der entsprechenden Iminoborenaddukte beobachtet werden kann. 


\subsubsection{Synthese der Verbindungen 52 - 58}

Wird Lithium-bis(trimethylsilyl)amid mit $\mathrm{BF}_{3} \mathrm{OEt}_{2}$ umgesetzt, bildet sich nicht das Difluor- sondern das Monofluorboran ${ }^{[72]}$. Das gleiche Reaktionsverhalten zeigt Lithium-(tert-butyldimethylsilyl-trimethylsilyl)amid mit Trifluorborandiethyletherat.

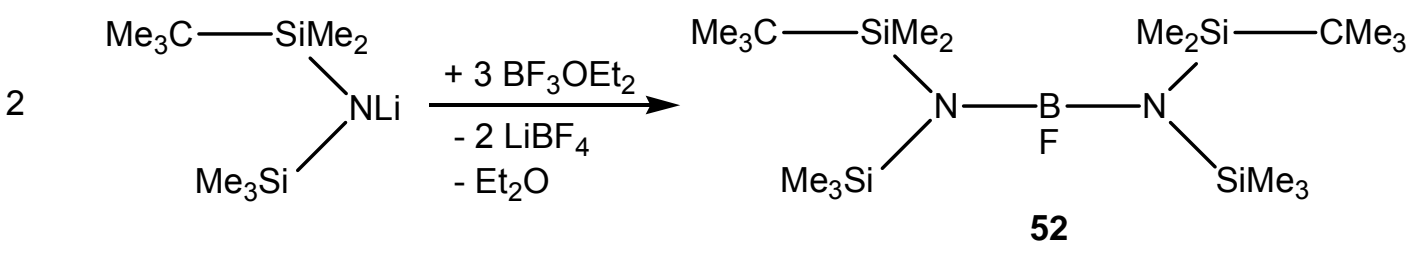

Gleichung 51: Synthese von 52

Bei Verbindung $\mathbf{5 2}$ wird das Auftreten von Rotationsisomeren, auf Grund der sperrigen Silylgruppen bei Raumtemperatur nachgewiesen (siehe ${ }^{1} \mathrm{H}$ - und ${ }^{13} \mathrm{C}-\mathrm{NMR}$ Spektren). Mit Ausnahme der Trimethylsilylgruppen konnten im ${ }^{1} \mathrm{H}-\mathrm{NMR}$-Spektrum Wasserstoff-Fluor-Kopplungen aller Protonen beobachtet werden $(1,1-1,7 \mathrm{~Hz})$. Während bei dem ${ }^{13} \mathrm{C}$-NMR-Signal der Methylgruppen vom tert-Butylsubstituenten eine ${ }^{5} \mathrm{~J}_{\mathrm{CF}}$-Kopplung von $2,4 \mathrm{~Hz}$ zu finden ist, spalten die tertiären Kohlenstoffatome nicht zu einem ${ }^{4} \mathrm{~J}_{\mathrm{CF}}$-Dublett auf.

Bei der Umsetzung von $\mathbf{5 2}$ mit Trichloralan, wird sowohl eine tert-Butylfluordimethylsilan-, als auch eine Fluortrimethylsilanspaltung beobachtet.

Des Weiteren wurden (tert-Butyldimethylsilyl-phenyldimethylsilyl)amin (53) und (tertButylmethylphenylsilyl-trimethylsilyl)amin (55) synthetisiert. Verbindung 53 wird in quantitativen Ausbeuten durch Reaktion von lithiiertem tert-Butyldimethylsilylamid mit Chlordimethylphenylsilan erhalten.
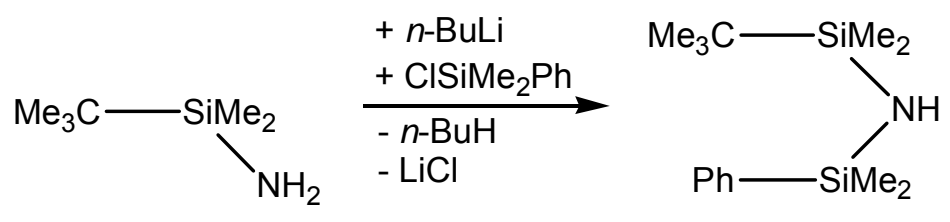
Wird tert-Butylmethylphenylsilylamin (54) mit $n$-Butyllithium metalliert und mit Chlortrimethylsilan umgesetzt, kann in quantitativen Ausbeuten die zu $\mathbf{5 3}$ isomere Verbindung 55 erhalten werden.

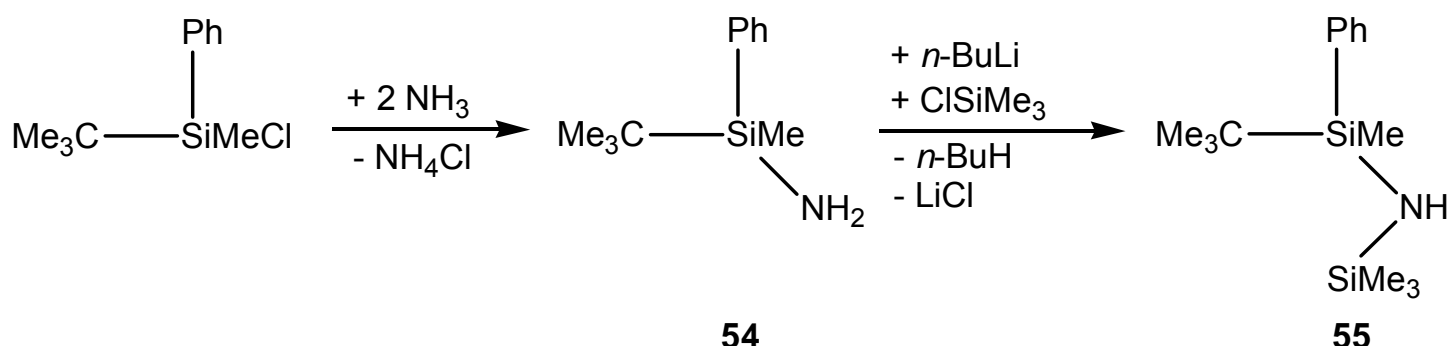

Gleichung 53: Darstellung der Verbindungen 54 und 55

Bei der folgenden Umsetzung des lithiierten Amids (56) mit Trifluorborandiethyletherat wird keine Fluorsilanabspaltung (bis $180^{\circ} \mathrm{C}$ ) beobachtet. Auf Grund des tertButylmethylphenylsilylrests wird das Difluorboran stabilisiert und kann unzersetzt destilliert werden.

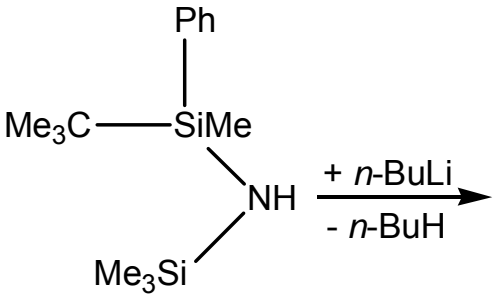

55

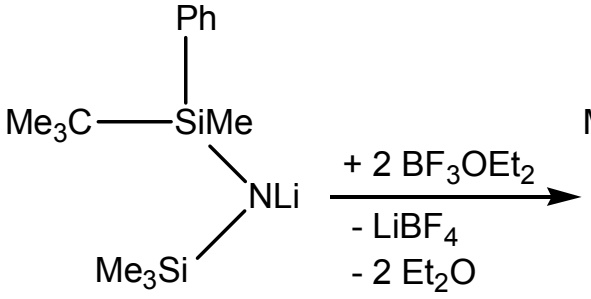

56

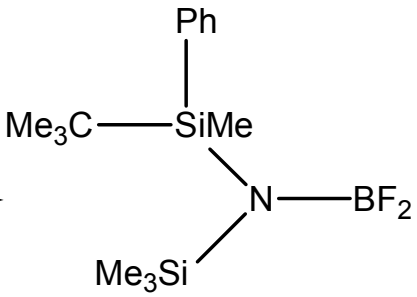

57

Gleichung 54: Synthese von 56 und 57

Eine Umsetzung von $\mathbf{5 7}$ mit Lithium-bis(trimethylsilyl)amid liefert Verbindung $\mathbf{5 8 .}$

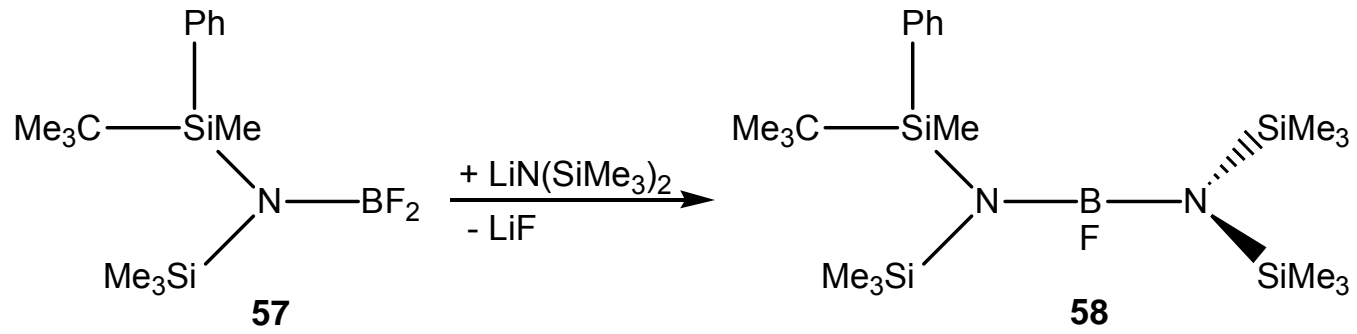


Die Silylgruppen spalten in den ${ }^{1} \mathrm{H}-,{ }^{13} \mathrm{C}$ - und ${ }^{29} \mathrm{Si}-\mathrm{NMR}$-Signale jeweils zu einem Dublett auf. Auf Grund der Chiralität der tert-Butylmethylphenylsilylgruppe treten die äquivalenten Silylgruppen der Bis(trimethylsilyl)-Einheit mit verschiedenen chemischen Verschiebungen auf.

\subsubsection{Kristallstruktur von 58}

Verbindung 58 kristallisiert aus $n$-Hexan monoklin in der Raumgruppe $P 2_{1} / n$.

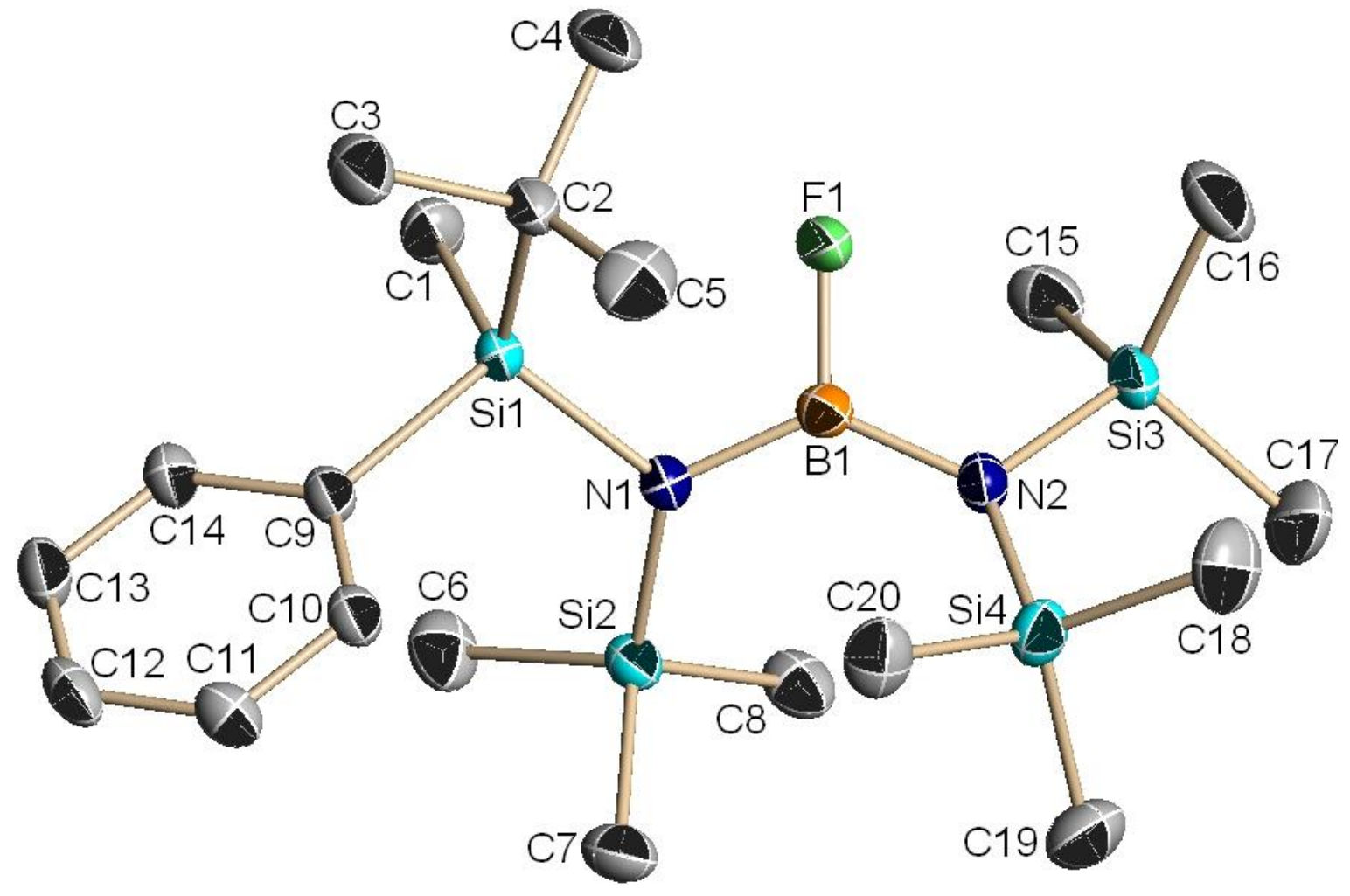

Abbildung 38: Kristallstruktur von $\mathbf{5 8}$ ohne Wasserstoffatome

Bindungslängen/pm mit Standardabweichung

\begin{tabular}{|ll|ll|ll|}
\hline F1-B1 & $137,2(2)$ & B1-N2 & $143,1(2)$ & B1-N1 & $143,4(2)$ \\
\hline Si1-N1 & $177,5(2)$ & Si1-C1 & $187,2(2)$ & Si2-N1 & $177,5(2)$ \\
\hline Si3-N2 & $177,4(2)$ & Si4-N2 & $175,8(2)$ & & \\
\hline
\end{tabular}

Tabelle 53: Ausgewählte Bindungslängen der Verbindung $\mathbf{5 8}$

Bindungswinkel ${ }^{\circ}$ mit Standardabweichung

\begin{tabular}{|ll|ll|ll|}
\hline F1-B1-N2 & $114,63(15)$ & F1-B1-N1 & $114,00(15)$ & N2-B1-N1 & $131,31(17)$ \\
\hline N1-Si1-C9 & $106,02(8)$ & C1-Si1-C9 & $110,01(8)$ & N1-Si1-C2 & $116,65(7)$ \\
\hline N1-Si1-C1 & $110,95(8)$ & C1-Si1-C2 & $107,41(9)$ & C9-Si1-C2 & $105,60(8)$ \\
\hline B1-N1-Si1 & $116,80(12)$ & B1-N1-Si2 & $119,23(12)$ & Si1-N1-Si2 & $120,35(8)$ \\
\hline B1-N2-Si4 & $124,82(12)$ & B1-N2-Si3 & $113,57(12)$ & Si4-N2-Si3 & $120,38(8)$ \\
\hline
\end{tabular}

Tabelle 54: Ausgewählte Bindungslängen der Verbindung $\mathbf{5 8}$ 


\subsubsection{Umsetzung des tert-Butyltrimethylsilylamino-bis(trimethylsilyl)amino- fluor-borans mit $\mathrm{AICl}_{3}$ und $\mathrm{GaCl}_{3}$}

Werden $\mathrm{AlCl}_{3}$ bzw. $\mathrm{GaCl}_{3}$ mit tert-Butyltrimethylsilylamino-bis(trimethylsilyl)aminofluor-boran ${ }^{[70]}$ umgesetzt, wird in beiden Fällen eine Fluortrimethylsilanabspaltung unter Bildung der Addukte 59 und 60 beobachtet. Auf Grund der größeren LewisBasizität des tert-Butyl-Stickstoffs wurde vermutet, dass der Aluminium- bzw. Galliumsubstituent dort bevorzugt koordiniert und zur Abspaltung der benachbarten Trimethylsilylgruppe führt. Die Röntgenstrukturanalysen belegen jedoch, dass eine der Silylgruppen der HMDS-Einheit abgespalten wurde.

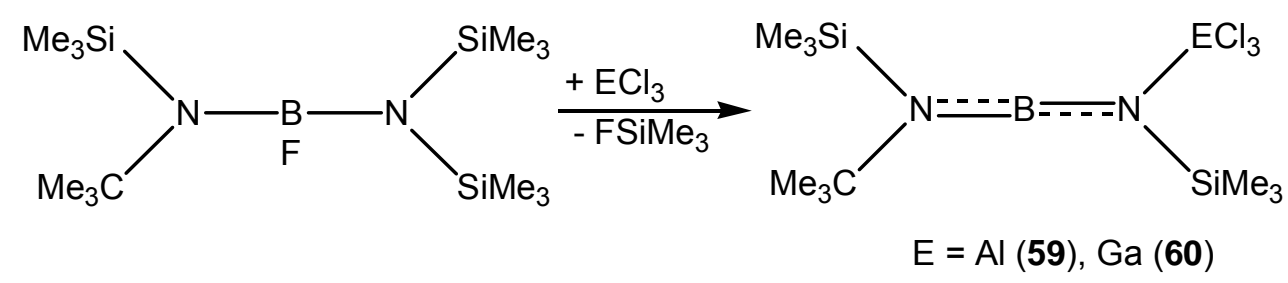

Gleichung 56: Synthese von 59 und 60

Im ${ }^{29}$ Si-NMR-Spektrum des tert-Butyltrimethylsilylamino-bis(trimethylsilyl)amino-fluorborans finden sich die Bis(trimethylsilyl)-gruppen im Vergleich zur tertButylaminosilylgruppe um ca. 3,2 ppm Tieffeld verschoben, was das Abspalten der stärker Lewis-basischen Silylgruppe in der Folgereaktion erklärt.

In den ${ }^{1} \mathrm{H}$-, ${ }^{11} \mathrm{~B}$ - und ${ }^{29}$ Si-NMR-Spektren von 59 und 60 treten ähnliche Verschiebungen wie bei der vollständig silylierten Verbindung 48 auf.

Durch Umkristallisation aus Diethylether werden Einkristalle der Halogentrieladdukte erhalten, welche röntgenstrukturanalytisch untersucht wurden. Die Verbindungen 59 und 60 sind isostrukturell. 


\subsubsection{Kristallstruktur von 59}

Verbindung 58 kristallisiert aus $n$-Hexan monoklin in der Raumgruppe $P 2_{1} / \mathrm{n}$. Der N-B-N-Winkel ist mit $175,8^{\circ}$ annähernd linear. Der Al-N-Abstand ist wie bei 48 verkürzt. Der N1-B1-Abstand ist im Vergleich zu B1-N2 um lediglich ca. 2 pm verkürzt.

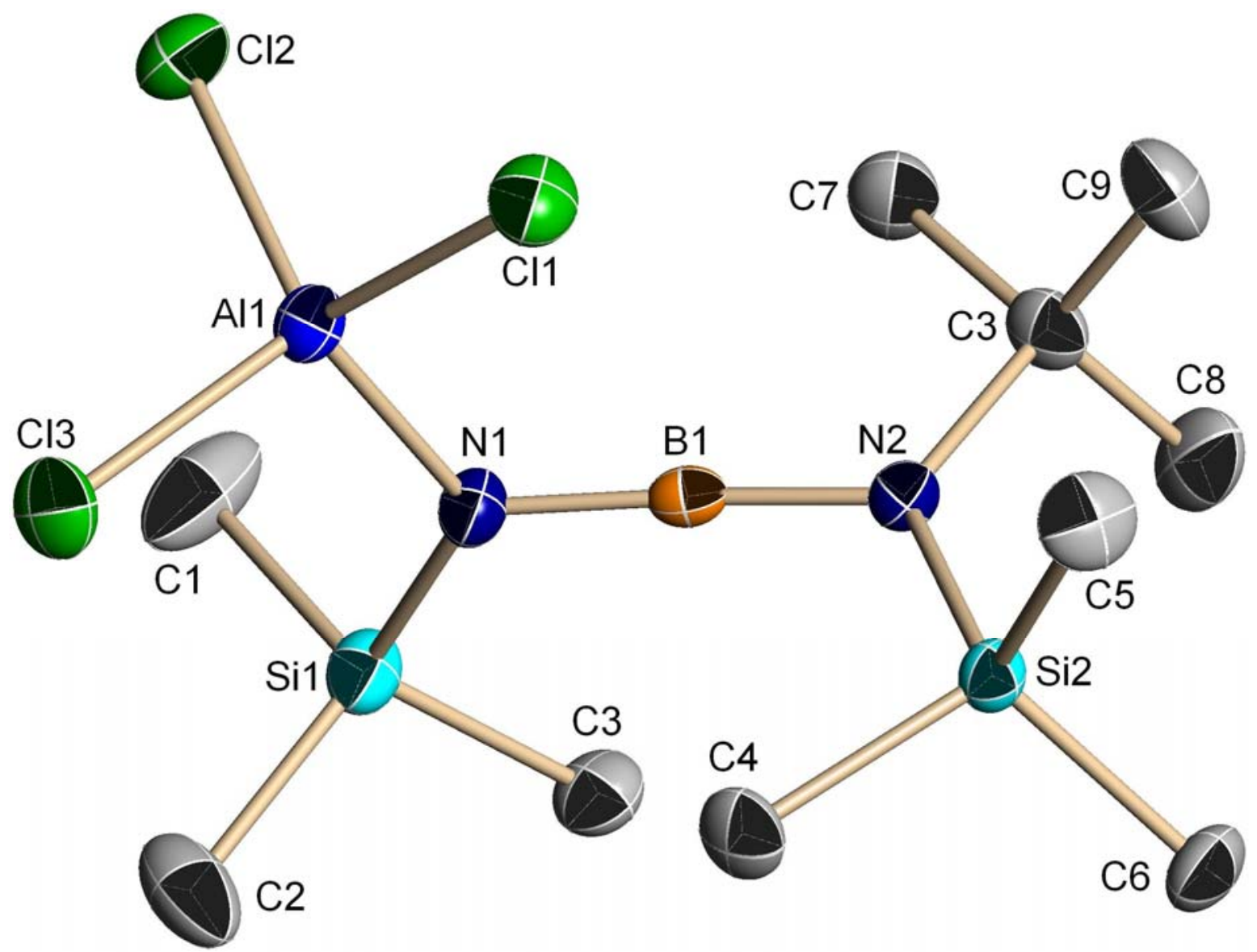

Abbildung 39: Kristallstruktur von 59 ohne Wasserstoffatome

Bindungslängen /pm mit Standardabweichung

\begin{tabular}{|ll|ll|ll|}
\hline N1-B1 & $132,1(5)$ & B1-N2 & $134,2(5)$ & Si1-N1 & $179,73(13)$ \\
\hline N2-C3 & $156,1(13)$ & N2-Si2 & $180,3(4)$ & Al1-N1 & $188,55(13)$ \\
\hline Al1-Cl1 & $213,97(6)$ & Al1-Cl2 & $213,29(6)$ & Al1-Cl3 & $212,43(6)$ \\
\hline
\end{tabular}

Tabelle 55: Ausgewählte Bindungslängen der Verbindung 59

Bindungswinkel $/^{\circ}$ mit Standardabweichung

\begin{tabular}{|ll|ll|ll|}
\hline N1-B1-N2 & $175,8(5)$ & Cl3-Al1-Cl2 & $111,78(3)$ & N1-Al1-Cl1 & $106,52(5)$ \\
\hline B1-N1-Al1 & $115,1(4)$ & Si1-N1-Al1 & $125,83(7)$ & B1-N1-Si1 & $118,7(4)$ \\
\hline B1-N2-C3 & $117,9(4)$ & B1-N2-Si2 & $114,6(3)$ & C3-N2-Si2 & $127,2(4)$ \\
\hline
\end{tabular}

Tabelle 56: Ausgewählte Bindungslängen der Verbindung 59 


\subsubsection{Kristallstruktur von $\mathbf{6 0}$}

Verbindung 60 kristallisiert aus Diethylether monoklin in der Raumgruppe $P 2_{1} / \mathrm{n}$. Der Gallium-Stickstoff-Abstand ist auf Grund der Polaritätssunterschiede auf 193,7 pm verkürzt ( $\Sigma$ Kovalenzradien $B, N=225 \mathrm{pm}^{[56]}$ ), was zu einer, im Vergleich zu 59, kürzeren N1-B1-Bindung führt. Der N-B-N-Winkel ist ca. $176^{\circ}$.

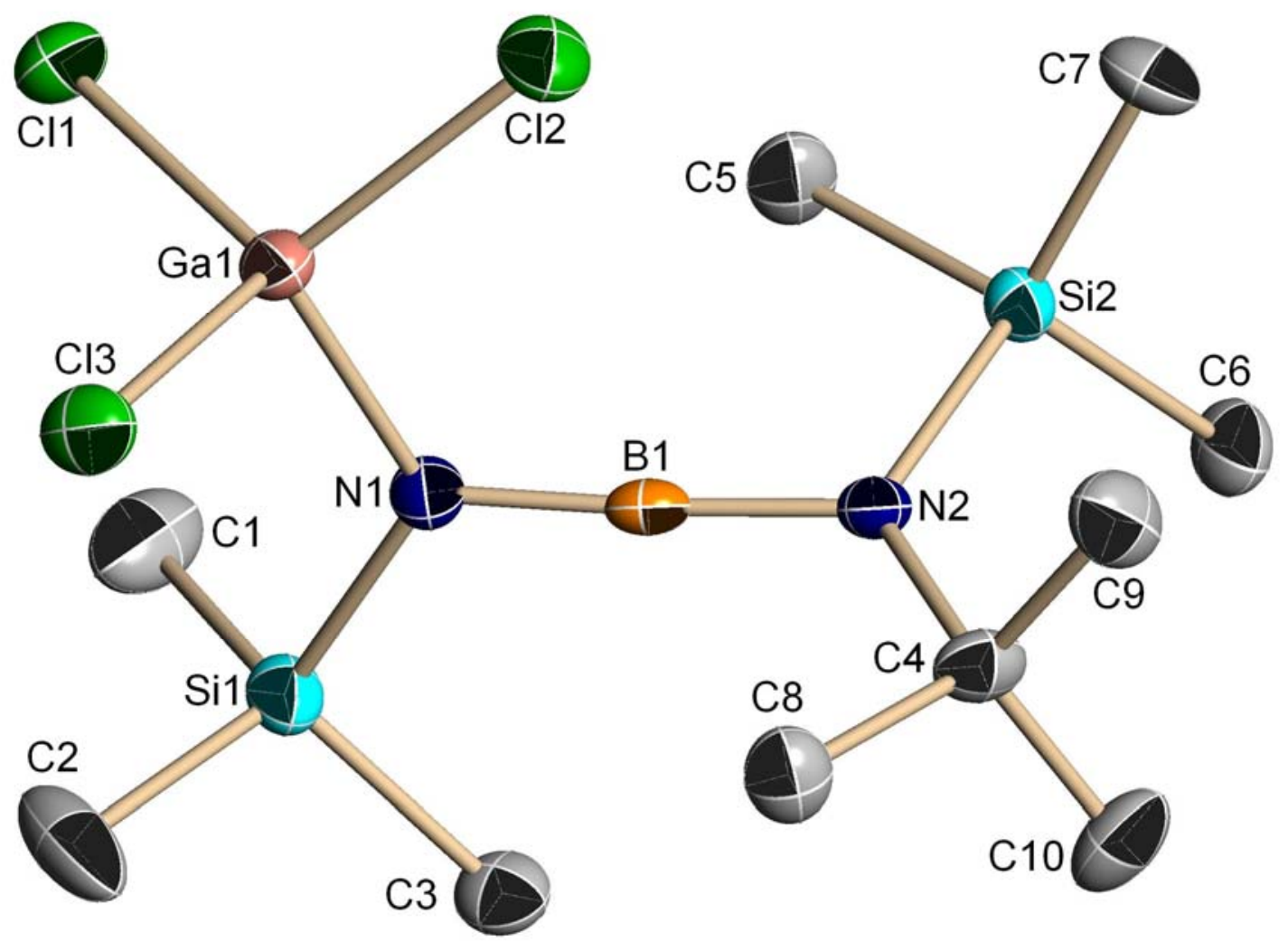

Abbildung 40: Kristallstruktur von 60 ohne Wasserstoffatome

Bindungslängen /pm mit Standardabweichung

\begin{tabular}{|ll|ll|ll|}
\hline Ga1-N1 & $193,67(12)$ & Ga1-Cl1 & $216,16(4)$ & Si1-N1 & $179,38(13)$ \\
\hline N1-B1 & $131,6(5)$ & B1-N2 & $134,4(5)$ & N2-C4 & $155,0(12)$ \\
\hline N2-Si2 & $180,1(3)$ & \multicolumn{3}{|l}{} \\
\hline
\end{tabular}

Tabelle 57: Ausgewählte Bindungslängen der Verbindung 60

Bindungswinkel $/{ }^{\circ}$ mit Standardabweichung

\begin{tabular}{|ll|ll|ll|}
\hline N1-B1-N2 & $176,1(5)$ & N1-Ga1-Cl1 & $109,15(4)$ & Cl1-Ga1-Cl3 & $112,06(18)$ \\
\hline B1-N1-Ga1 & $115,0(4)$ & Si1-N1-Ga1 & $124,79(7)$ & B1-N1-Si1 & $120,0(4)$ \\
\hline B1-N2-C4 & $118,1(4)$ & B1-N2-Si2 & $114,5(3)$ & C4-N2-Si2 & $127,3(4)$ \\
\hline
\end{tabular}

Tabelle 58: Ausgewählte Bindungslängen der Verbindung 60 


\subsubsection{Versuch der Darstellung eines Bis(fluorsilylaminoborans)}

Durch Reaktion der bimolaren Menge von Difluor-diisopropylamino-boran mit Dilithium-1,1,1,3,3,5,5,5-octamethyl-1,3,5-trisila-2,4-diamid sollte ein Bis(fluorsilylaminoboran) dargestellt und im Anschluss mit Trichloralan umgesetzt werden.

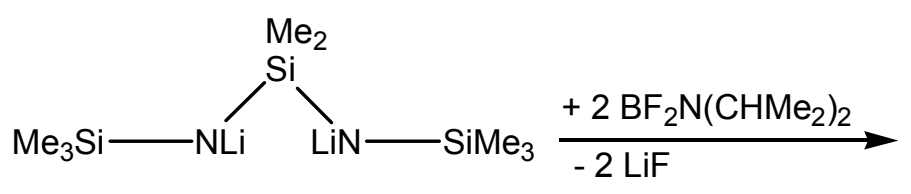

Gleichung 57: Geplante Synthese eines Bis(fluorsilylaminoborans)

Statt des erwarteten Produkts wurde $\mathbf{6 1}$ isoliert. Auch die Reaktion des Monolithiumsalzes in THF ${ }^{[40]}$ mit dem Difluorboran führte, auf Grund einer eintretenden Umlithiierung, zur Bildung der Ringverbindung 61.

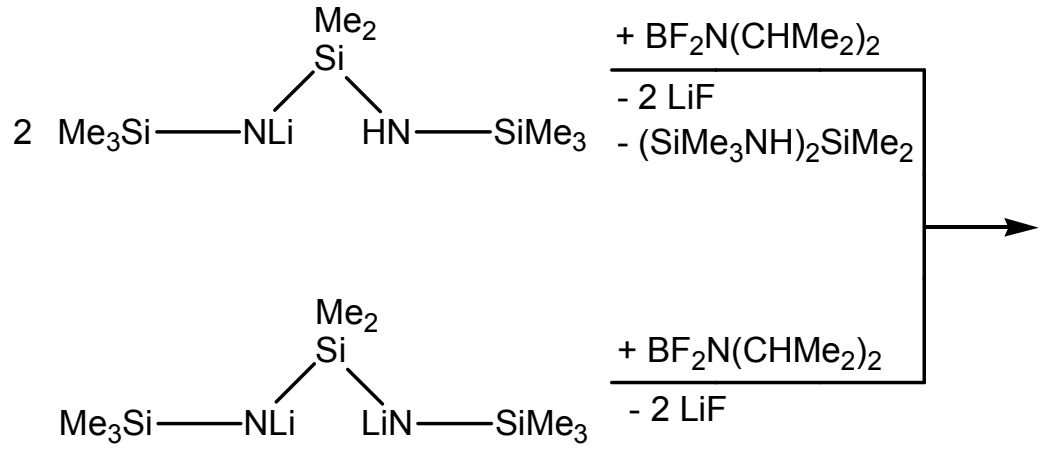

Gleichung 58: Synthese von 61<smiles>CC(C)N(B1[SiH2]N1[SiH3])C(C)C</smiles>

61 


\subsubsection{Kristallstruktur von 61}

Verbindung 61 kristallisiert monoklin in der Raumgruppe $P 2_{1} / \mathrm{c}$. Die endocyclischen Si-N-Abstände sind länger als die exocyclischen.

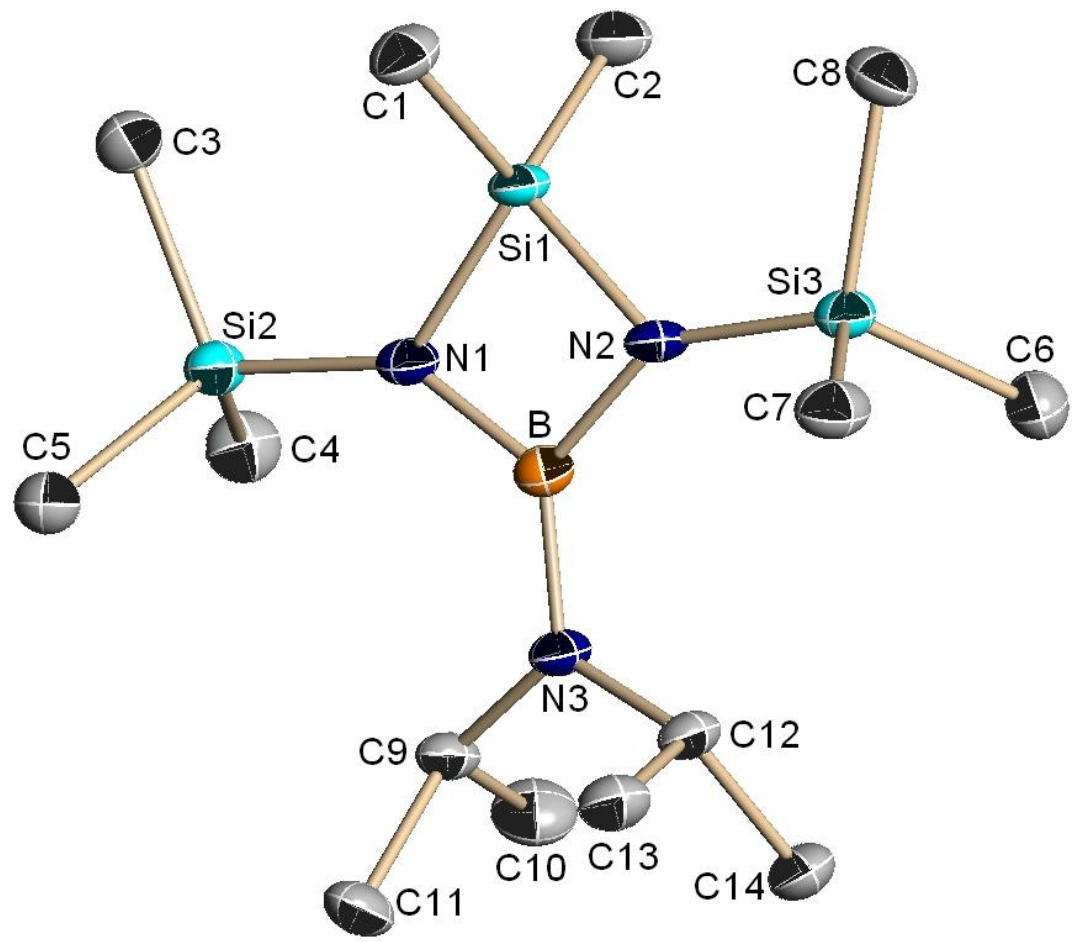

Abbildung 41: Kristallstruktur von 61 ohne Wasserstoffatome

Bindungslängen/pm mit Standardabweichung

\begin{tabular}{|ll|ll|ll|}
\hline B-N1 & $149,3(7)$ & B-N2 & $147,4(13)$ & B-N3 & $141,5(10)$ \\
\hline Si1-N1 & $173,7(17)$ & Si1-N2 & $173,2(4)$ & Si2-N1 & $172,8(4)$ \\
\hline Si3-N2 & $172,7(5)$ & N3-C9 & $147,6(7)$ & N3-C12 & $147,4(7)$ \\
\hline
\end{tabular}

Tabelle 59: Ausgewählte Bindungslängen der Verbindung 61

Bindungswinkel/ ${ }^{\circ}$ mit Standardabweichung

\begin{tabular}{|ll|ll|ll|}
\hline N3-B-N2 & $129(4)$ & N3-B-N1 & 129(2) & N2-B-N1 & $103(2)$ \\
\hline B-N1-Si2 & $144(3)$ & B-N1-Si1 & $86,5(15)$ & Si2-N1-Si1 & $129,5(15)$ \\
\hline B-N2-Si3 & $143,8(10)$ & B-N2-Si1 & $87(3)$ & Si3-N2-Si1 & $129(4)$ \\
\hline B-N3-C12 & $119(4)$ & B-N3-C9 & $119(5)$ & C12-N3-C9 & $121,4(17)$ \\
\hline N2-Si1-N1 & $84(2)$ & \multicolumn{3}{|l|}{} & \\
\hline
\end{tabular}

Tabelle 60: Ausgewählte Bindungslängen der Verbindung 61

\subsubsection{Synthese arylsubstituierter Fluorsilylaminoborane}

Im Folgenden wurde das Reaktionsverhalten Arylsilylamin-substituierter Fluorborane mit $\mathrm{AlCl}_{3}$ untersucht werden. Hierfür wurde zunächst lithiiertes 2,6-Diisopropyltrimethylsilyl-anilin mit Di(iso-butyl)amino-difluor-boran bzw. Difluor-diisopropylaminoboran umgesetzt. 

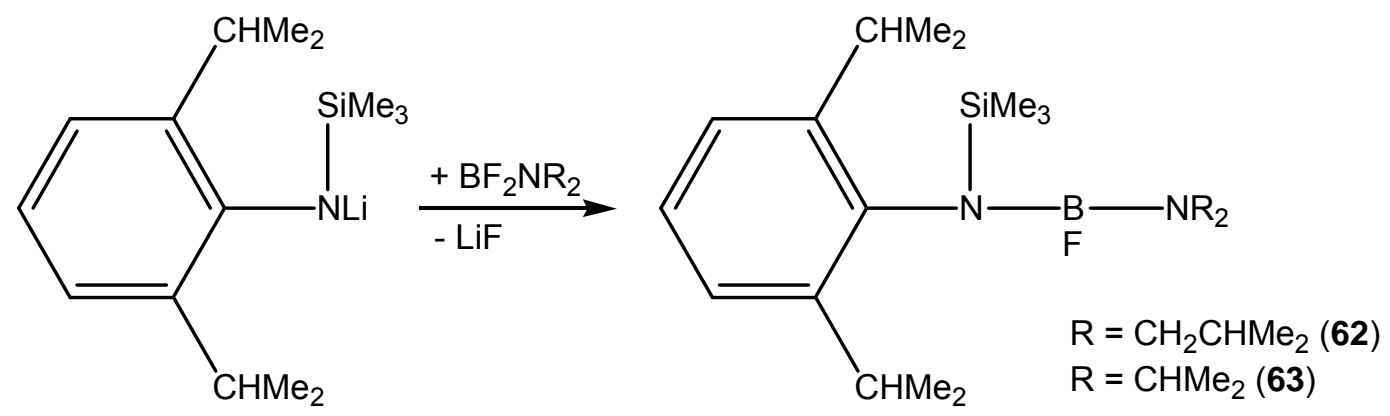

Gleichung 59: Synthese von 62 und 63

Während 62 bei Raumtemperatur flüssig ist, können durch Umkristallisation aus $n$-Hexan Kristalle von 63 erhalten werden, welche röntgenstrukturanalytisch untersucht wurden.

\subsubsection{Kristallstruktur von 63}

Verbindung 63 kristallisiert monoklin in der Raumgruppe $P 2_{1} / \mathrm{c}$. Der N1-Stickstoff ist planar umgeben $\left(\Sigma \Varangle \mathrm{N} 1=359,9^{\circ}\right)$.

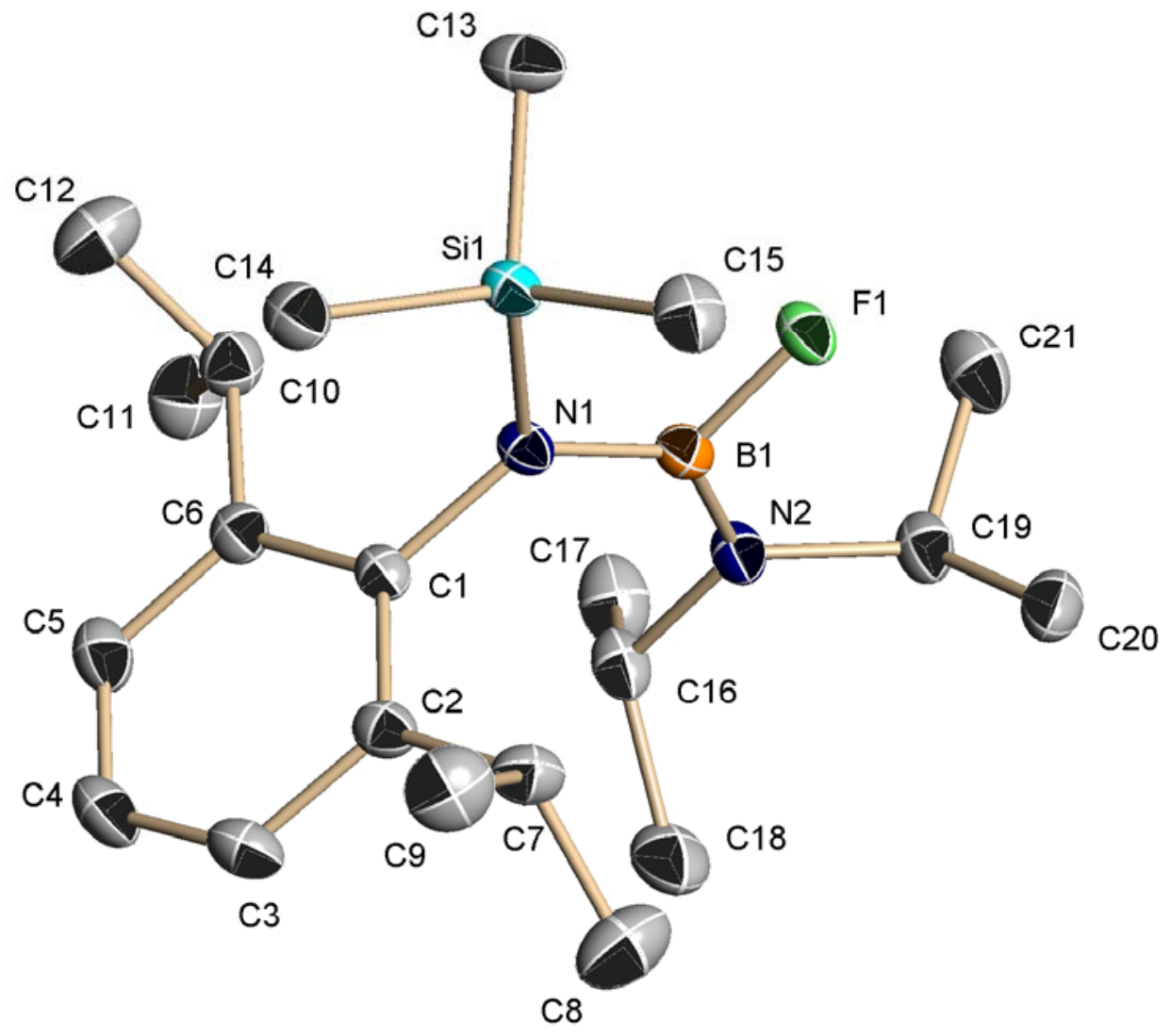

Abbildung 42: Kristallstruktur von 63 ohne Wasserstoffatome 
Bindungslängen/pm mit Standardabweichung

\begin{tabular}{|ll|ll|ll|}
\hline B1-N1 & $143,6(2)$ & B1-F1 & $137,31(19)$ & C1-N1 & $144,64(18)$ \\
\hline Si1-N1 & $177,60(12)$ & & & \\
\hline
\end{tabular}

Tabelle 61: Ausgewählte Bindungslängen der Verbindung 63

Bindungswinkel $/{ }^{\circ}$ mit Standardabweichung

\begin{tabular}{|ll|ll|ll|}
\hline C2-C1-N1 & $119,67(13)$ & N1-B1-F1 & $111,75(13)$ & C1-N1-Si1 & $118,47(9)$ \\
\hline N1-C1-C6 & $119,85(13)$ & F1-B1-N2 & $115,17(13)$ & B1-N2-C19 & $120,43(12)$ \\
\hline C6-C1-C2 & $120,45(13)$ & C1-N1-B1 & $124,14(12)$ & C19-N2-C16 & $114,39(12)$ \\
\hline N1-B1-N2 & $133,08(14)$ & B1-N1-Si1 & $117,28(10)$ & B1-N2-C16 & $125,15(13)$ \\
\hline
\end{tabular}

Tabelle 62: Ausgewählte Bindungswinkel der Verbindung 63

\subsubsection{Umsetzung von 62 und 63 mit $\mathrm{AlCl}_{3}$}

Wird 62 mit $\mathrm{AlCl}_{3}$ in Diethylether/ Dichlormethan versetzt und unter Rückfluss erhitzt, findet eine Spaltung der Bor-Alkylstickstoff-Bindung statt, wobei Di(iso-butyl)amin als $\mathrm{AlCl}_{3}$-Addukt isoliert werden kann (65). Indem die Reaktionstemperatur nicht über $0{ }^{\circ} \mathrm{C}$ erhöht wird, gelingt es die thermodynamisch instabile Zwischenstufe (64) zu isolieren. Wahrscheinlich findet im ersten Schritt eine $\mathrm{AlCl}_{3}$-katalysierte Abspaltung des Trimethylfluorsilans statt (vgl. Gleichung 46), welches an die B-N-Bindung des intermediär entstehenden Iminoborens addiert. Das Friedel-Crafts-Addukt des $\mathrm{AlCl}_{3}$ ist ein möglicher Protonenlieferant, was eine Erklärung für die Protonierung des $\mathrm{Di}($ iso-butyl)stickstoffs sein kann. Verbindung 64 ist das erste bisher bekannte AminAddukt eines Boriniumions.
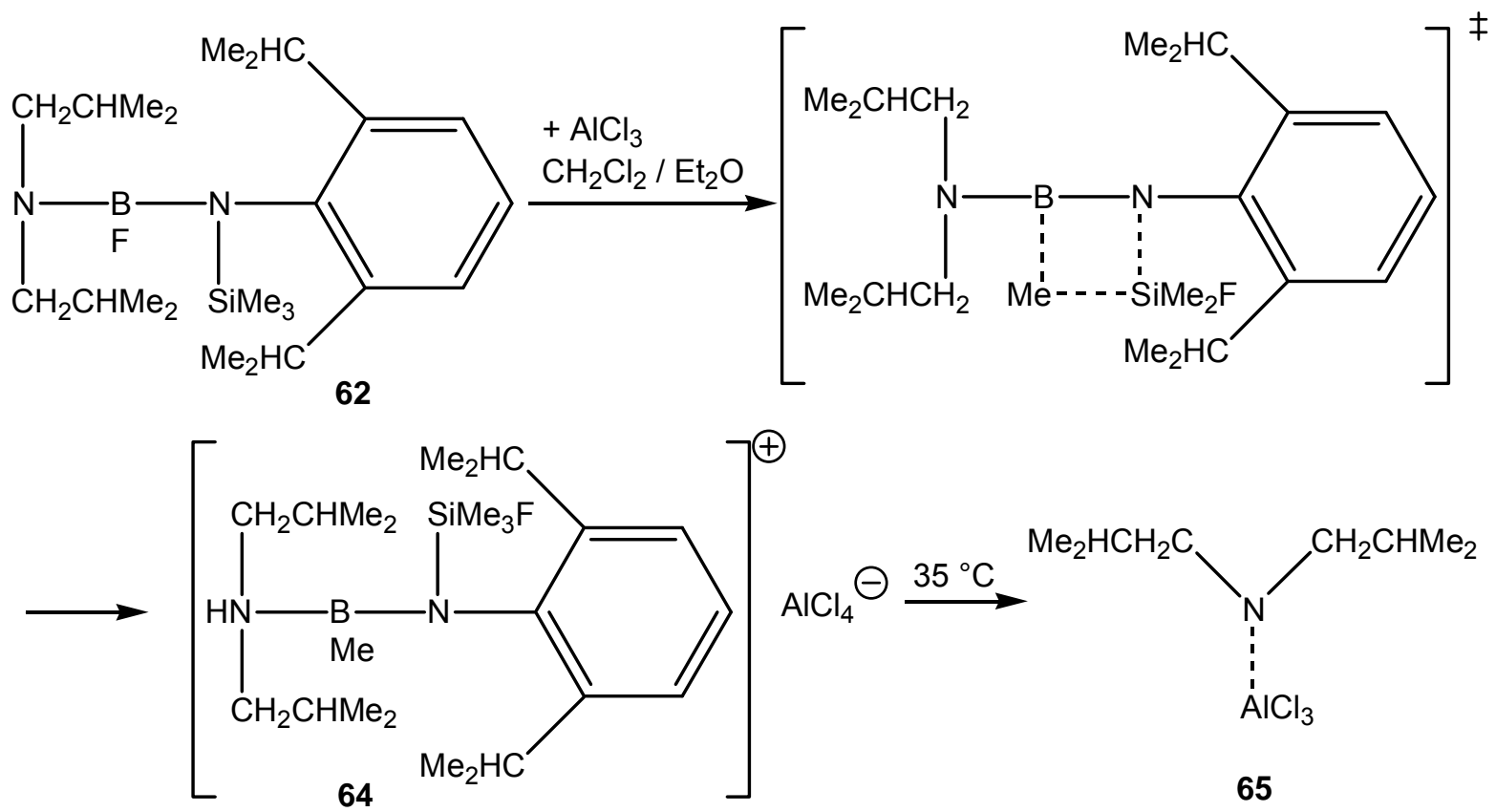
Wird Verbindung 63 mit Trichloralan (nur in Diethylether) versetzt, zeigt die Kristallstruktur des, bei $0{ }^{\circ} \mathrm{C}$ isolierten, Feststoffs ebenfalls die Existenz eines (thermodynamisch instabilen) Diisopropylaminaddukts an einem Boriniumion mit $\mathrm{AlCl}_{4}^{-}$als Gegenion (66).

Die "sehr ungewöhnlichen“ " ${ }^{[69]}$ Reaktionsmechanismen konnten noch nicht vollständig geklärt werden. DFT-Rechnungen können hierbei ein besseres Verständnis der Prozesse liefern. Auf Grund des entstandenen Trimethylfluorsilans bei der Synthese von Verbindung 66 wird jedoch als erste Stufe eine $\mathrm{AlCl}_{3}$-katalysierte Fluorsilanabspaltung angenommen ( $\mathrm{vgl}$. 3.6.1). Es kommt wahrscheinlich aus elektronischen oder sterischen Gründen nicht zu einer Anlagerung des Trichloralans an den Anilinstickstoff. Stattdessen ist die Addition von, aus einer Etherspaltung ${ }^{[65]}$ entstehenden, Ethyl- bzw. Ethenresten am Boratom denkbar. Die Protonierung der beiden Stickstoffatome sowie die Ausbildung des Tetrachloraluminatanions benötigen ebenfalls weitere Studien.

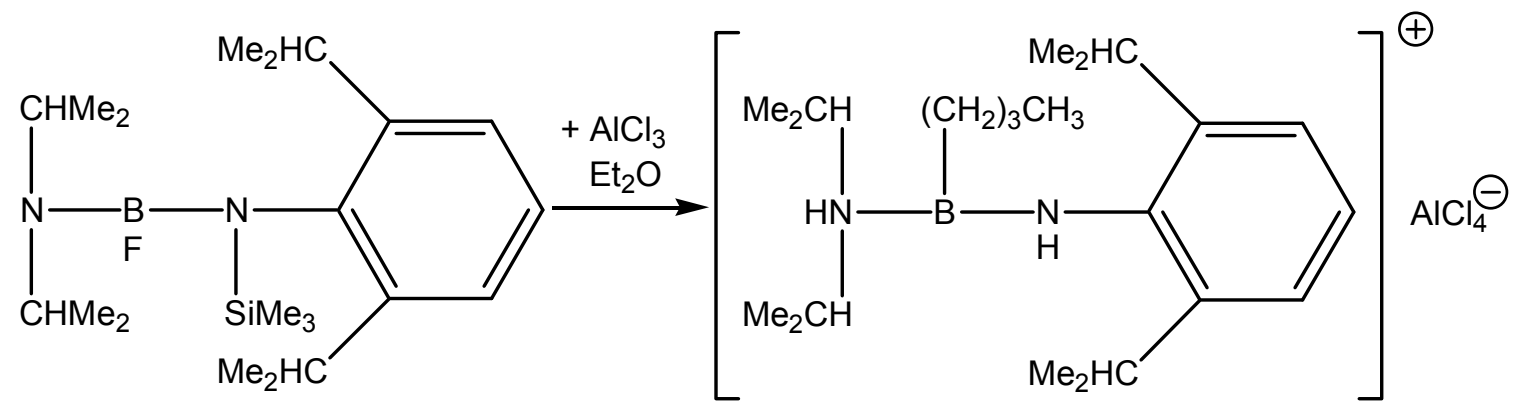

63

66 


\subsubsection{Kristallstruktur von 64}

Auf Grund einer nicht zuzuordnenden Restelektronendichte am Arylring können keine verlässlichen Aussagen über Bindungslängen und -winkel getroffen werden. Weiterhin wird die Struktur auf Grund von Fehlordnungsproblemen am Silicium als ball-and-stick Modell abgebildet.
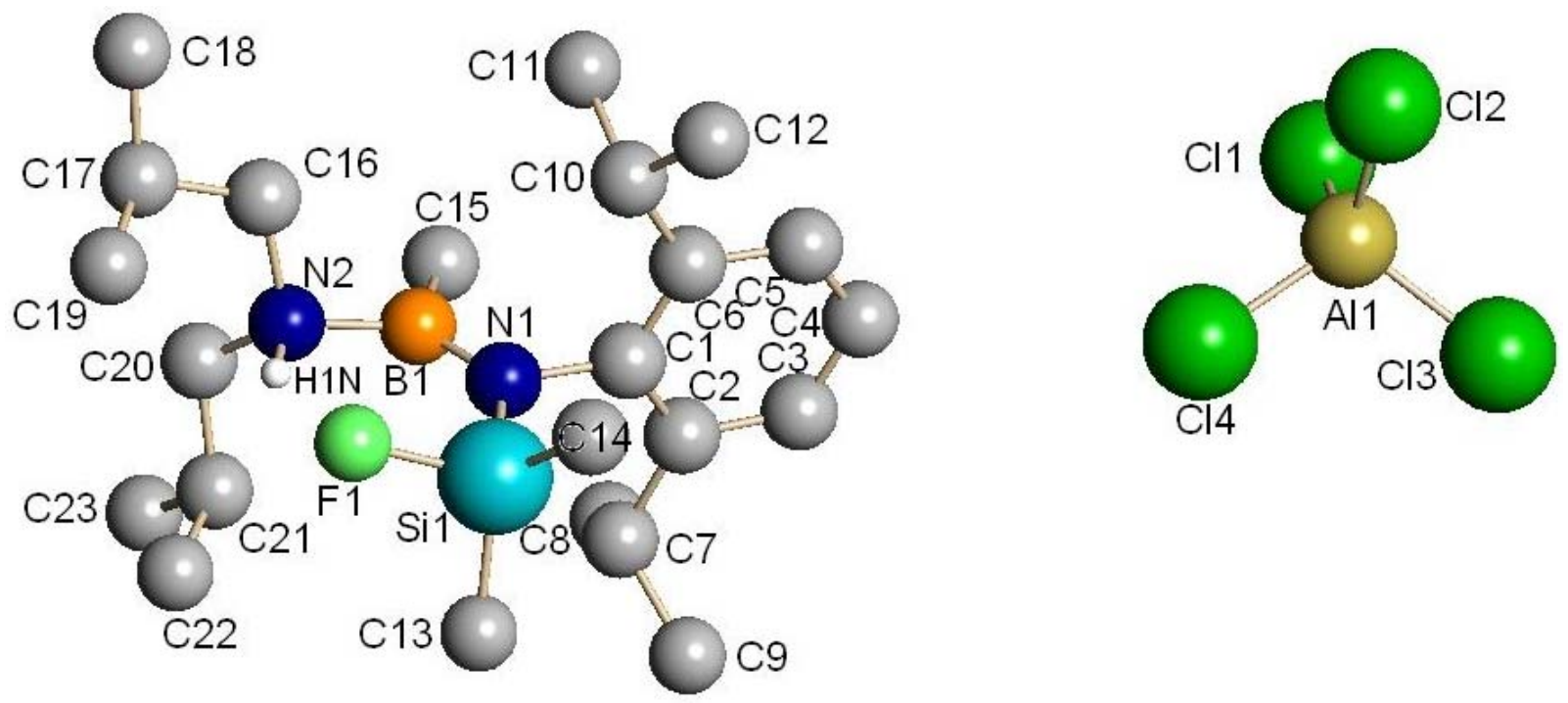

Abbildung 43: Ball-and-stick Modell der Kristallstruktur von 64 ohne CH-Wasserstoffatome

\subsubsection{Kristallstruktur von 65}

Verbindung 65 kristallisiert monoklin in der Raumgruppe $P 2_{1} /$ c. Die StickstoffAluminiumadduktbindung ist mit 196 pm weitaus größer als bei 48 (188 pm).

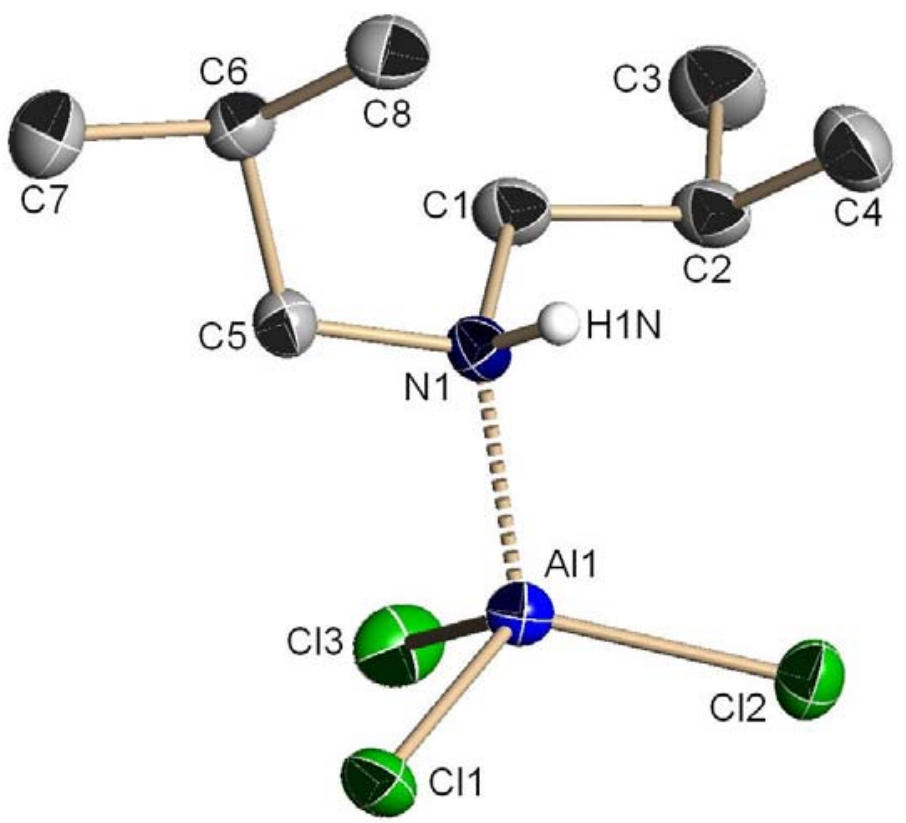


Bindungslängen (-) bzw. intramolekulare Atomabstände (...)/pm mit Standardabweichung

\begin{tabular}{|ll|ll|ll|}
\hline N1-C1 & $150,9(2)$ & N1-C5 & $151,6(2)$ & N1-Al1 & $196,0(1)$ \\
\hline Al1-Cl3 & $211,5(1)$ & Al1-Cl2 & $211,5(1)$ & Al1-Cl1 & $212,8(1)$ \\
\hline
\end{tabular}

Tabelle 63: Ausgewählte Bindungslängen bzw. intramolekulare Atomabstände der Verbindung 65

Bindungswinkel $/{ }^{\circ}$ mit Standardabweichung

\begin{tabular}{|ll|ll|ll|}
\hline C1-N1-C5 & $111,08(10)$ & C1-N1-Al1 & $114,11(8)$ & C5-N1-Al1 & $110,54(8)$ \\
\hline N1-Al1-Cl3 & $105,29(4)$ & N1-Al1-Cl2 & $110,79(4)$ & Cl3-Al1-Cl2 & $112,64(2)$ \\
\hline N1-Al1-Cl1 & $107,44(4)$ & Cl3-Al1-Cl1 & $111,21(2)$ & Cl2-Al1-Cl1 & $109,30(2)$ \\
\hline
\end{tabular}

Tabelle 64: Ausgewählte Bindungswinkel der Verbindung 65

\subsubsection{Kristallstruktur von 66}

Verbindung 66 kristallisiert monoklin in der Raumgruppe $P 2_{1} / \mathrm{n}$. Die B1-N1-Bindung ist mit 137,8 pm im Vergleich zu einer Bor-Stickstoffeinfachbindung verkürzt. Die B1-N2-Bindung ist im Vergleich dazu weitaus länger (157,1 pm). Der Diisopropylaminsubstituent bedingt eine Verkürzung des, beim Boriniuminon linear erwarteten, N1-B1-C13-Winkels $\left(124,4^{\circ}\right)$.

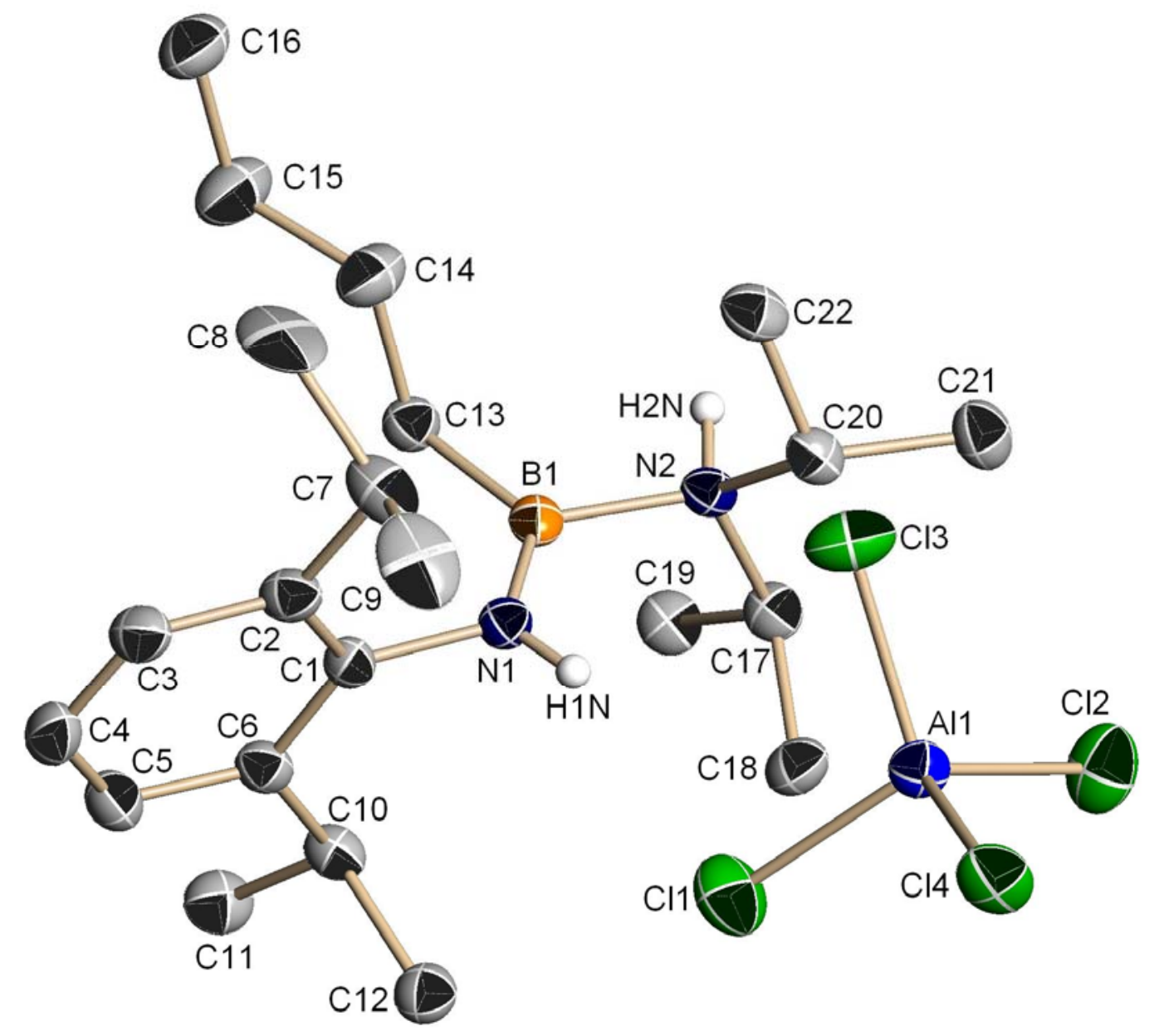

Abbildung 45: Kristallstruktur von 66 ohne $\mathrm{CH}$-Wasserstoffatome 
Bindungslängen/pm mit Standardabweichung

\begin{tabular}{|ll|ll|ll|}
\hline B1-N1 & $137,8(3)$ & B1-N2 & $157,1(3)$ & B1-C13 & $156,4(3)$ \\
\hline N1-C1 & $145,8(3)$ & N1-H1A & 88,0 & N2-C17 & $154,1(3)$ \\
\hline N2-H2N & 86,9 & Al1-Cl3 & $211,9(1)$ & & \\
\hline
\end{tabular}

Tabelle 65: Ausgewählte Bindungslängen der Verbindung 66

Bindungswinkel $/{ }^{\circ}$ mit Standardabweichung

\begin{tabular}{|ll|ll|ll|}
\hline N1-B1-C13 & $124,4(2)$ & N1-B1-N2 & $118,3(2)$ & C13-B1-N2 & $117,3(2)$ \\
\hline B1-N1-C1 & $123,3(2)$ & B1-N1-H1A & 118,34 & H1A-N1-C1 & 118,35 \\
\hline C20-N2-C17 & $114,48(18)$ & C20-N2-B1 & $114,61(17)$ & C17-N2-B1 & $113,56(18)$ \\
\hline H2N-N2-C17 & 102,73 & H2N-N2-B1 & 104,46 & C20-N2-H2N & 105,26 \\
\hline
\end{tabular}

Tabelle 66: Ausgewählte Bindungswinkel der Verbindung 66

Es kann kein intra- oder intermolekularer Kontakt des $\mathrm{AlCl}_{4}$-Anions zum Diisopropylamin oder Boratom festgestellt werden.

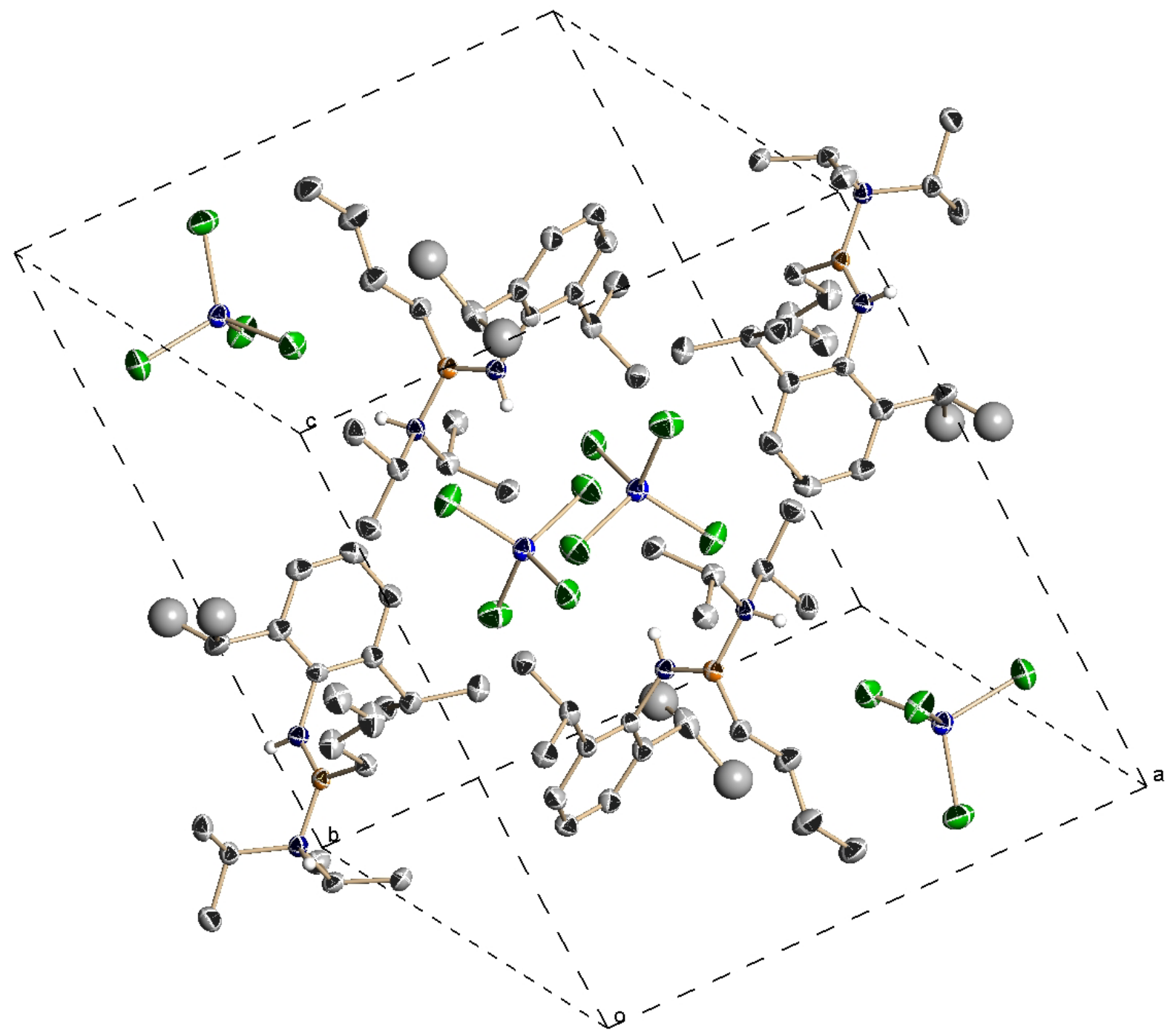

Abbildung 46: Anordnung der Moleküle von 66 in der Elementarzelle ohne $\mathrm{CH}$-Wasserstoffatome 


\subsubsection{Umsetzung von 27 mit $\mathrm{AlCl}_{3}$ in $\mathrm{CH}_{2} \mathrm{Cl}_{2}$}

Verbindung 27 reagiert wie 62 erst nach Zugabe von Methylenchlorid zum $\mathrm{AlCl}_{3}$ Diethylethergemisch. Auch hierbei kommt es zur Protonierung des Stickstoffatoms, wobei unerwartet die Stickstoff-Kohlenstoffbindung gespalten wird. Das IR-Spektrum der Verbindung 67 zeigt drei, jeweils durch $\mathrm{AlCl}_{3}$ verbreiterte, symmetrische und asymmetrische $\mathrm{NH}_{2}$-Valenz- bzw. -Deformationsschwingungen. Weiterhin findet ein Fluor-Chloraustausch am Silicium statt. Auch hierbei konnte der Reaktionsmechanismus noch nicht vollständig aufgeklärt werden.

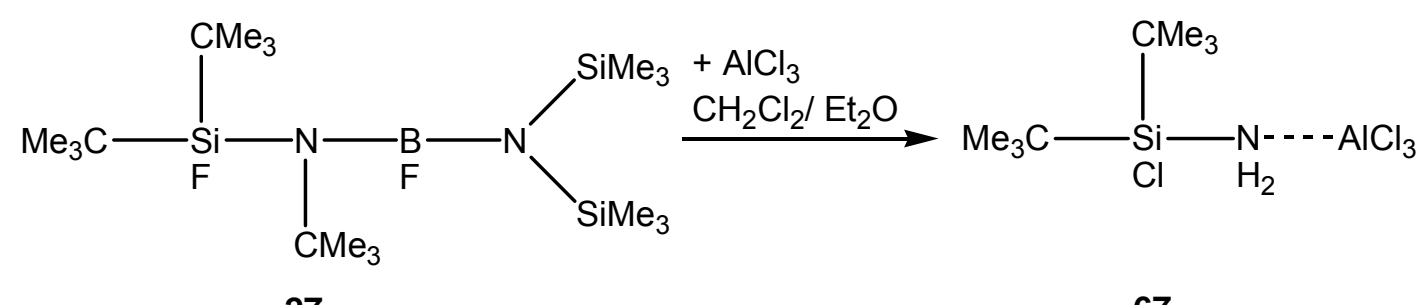

27

67

Schema 5: Synthese von 67

Eine Kristallstrukturanalyse bestätigt die infrarot- und massenspektroskopisch vermutete Verbindung. 


\subsubsection{Kristallstruktur von 67}

Verbindung 67 kristallisiert tetragonal in der Raumgruppe $P 4_{2} / \mathrm{n}$.

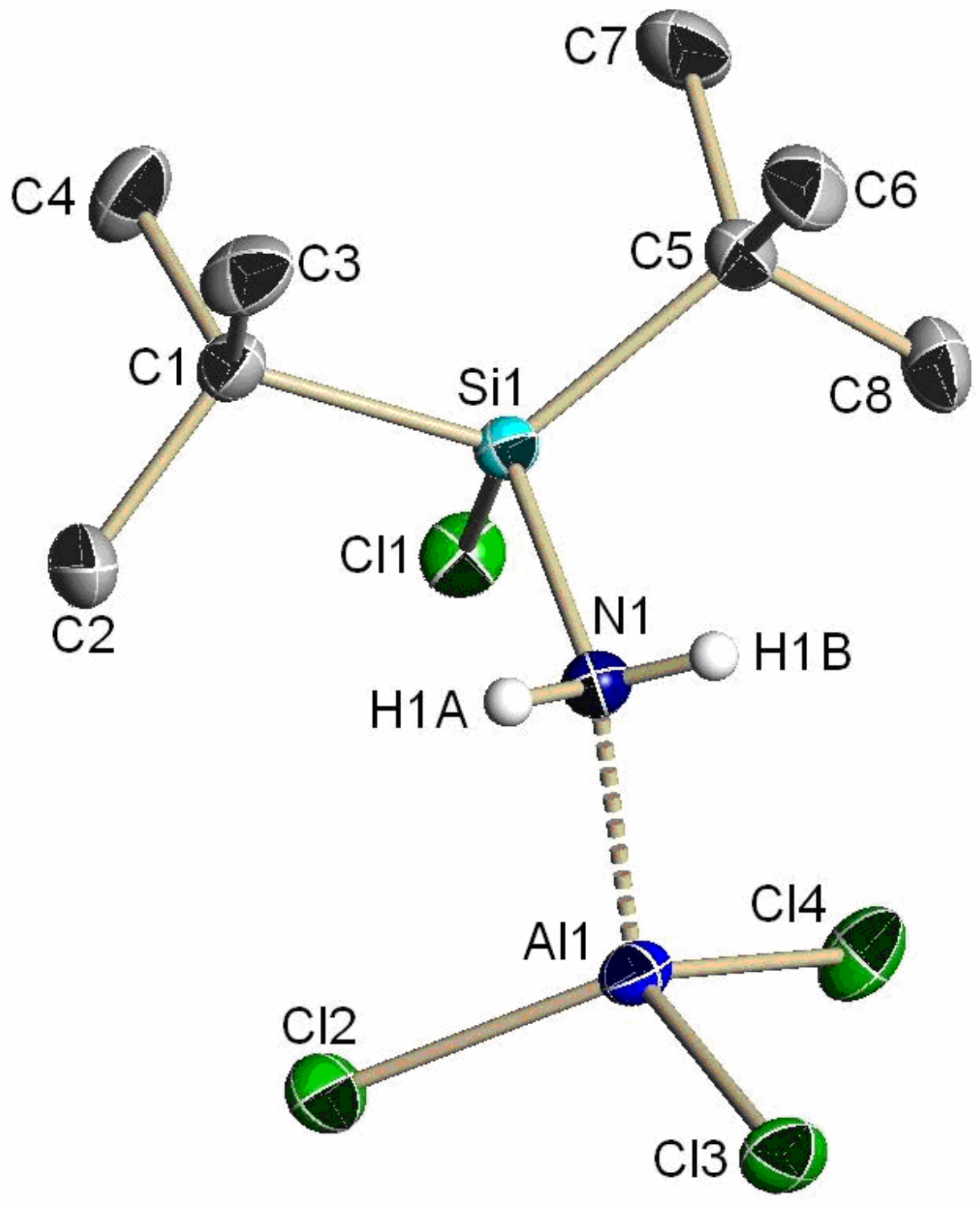

Abbildung 47: Kristallstruktur von 67 ohne Methylwasserstoffatome

Bindungslängen (-) bzw. intramolekulare Atomabstände (...)/pm mit Standardabweichung

\begin{tabular}{|ll|ll|ll|}
\hline Al1...N1 & $195,37(14)$ & Al1-Cl2 & $210,68(6)$ & Cl1-Si1 & $204,78(6)$ \\
\hline N1-Si1 & $183,82(14)$ & N1-H1A & 84,6 & N1-H1B & 82,7 \\
\hline
\end{tabular}

Tabelle 67: Ausgewählte Bindungslängen bzw. intramolekulare Atomabstände der Verbindung 67

Bindungswinkel ${ }^{\circ}$ mit Standardabweichung

\begin{tabular}{|ll|ll|ll|}
\hline Cl4-Al1-Cl3 & $107,10(3)$ & N1-Al1-Cl2 & $107,34(5)$ & N1-Al1-Cl4 & $108,49(5)$ \\
\hline Cl2-Al1-Cl4 & $116,60(3)$ & N1-Al1-Cl3 & $104,98(5)$ & Cl2-Al1-Cl3 & $111,69(3)$ \\
\hline Si1-N1-Al1 & $124,33(7)$ & Si1-N1-H1A & 107,74 & H1A-N1-Al1 & 104,48 \\
\hline
\end{tabular}

Tabelle 68: Ausgewählte Bindungswinkel der Verbindung 67 


\subsubsection{Synthese von Fluor-hydrazon- bzw. Fluor-ketazin-silylamino- boranen ${ }^{[75]}$}

Im Rahmen dieser Arbeit wurden weiterhin bisher unbekannte silylaminsubstituierte Fluorborylhydrazone bzw. -ketazine synthetisiert und mit Trichloralan umgesetzt.

Bis(tert-butylmethyl)ketazin reagiert mit Trifluorborandiethyletherat zum destillierbaren Bis(tert-butylmethyl)ketazin-trifluorboranaddukt (68).

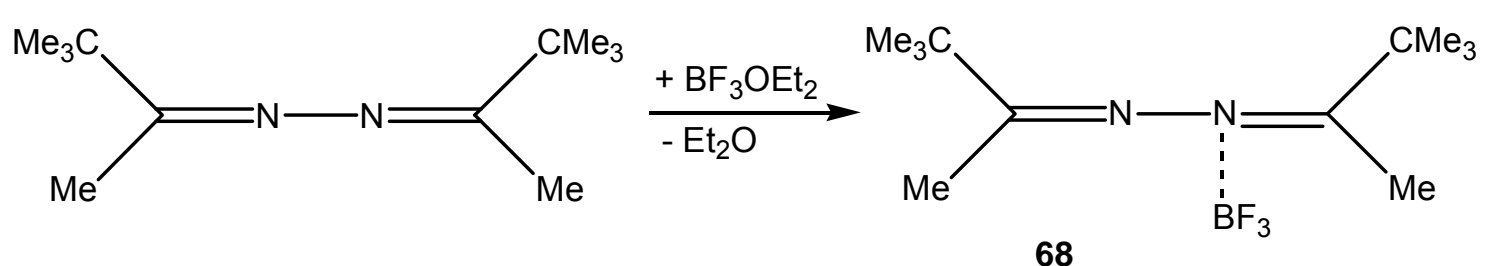

Gleichung 60: Synthese von 68

Der Borkern ist in 68 stark abgeschirmt $\left(\delta^{11} \mathrm{~B}=-1,40 \mathrm{ppm}\right)$, was auf den +M-Effekt der Substituenten Fluor und Stickstoff zurückzuführen ist. Das scharfe ${ }^{11} \mathrm{~B}-\mathrm{NMR}$ Signal zeigt vierfach koordiniertes Bor.

Da die tert-Butyl- sowie Methylgruppen in den ${ }^{1} \mathrm{H}$ - und ${ }^{13} \mathrm{C}-\mathrm{NMR}$-Spektren gleiche Signale zeigen, findet bei Raumtemperatur ein ständiger Austausch der $\mathrm{BF}_{3}$-Einheit statt. Diese Fluktuation wird bei der Kristallisation des Moleküls unterbunden. Die Kristallstruktur von 68 zeigt die Koordination der Fluorborylgruppe zu nur einem Stickstoffatom. 


\subsubsection{Kristallstruktur von 68}

Verbindung 68 kristallisiert aus Diethylether orthorhombisch in der Raumgruppe Pna2 ${ }_{1}$. Auf Grund des B-N-Kontakts ist die N1-C1-Bindung um ca. 2 pm länger als der N2-C7-Abstand.

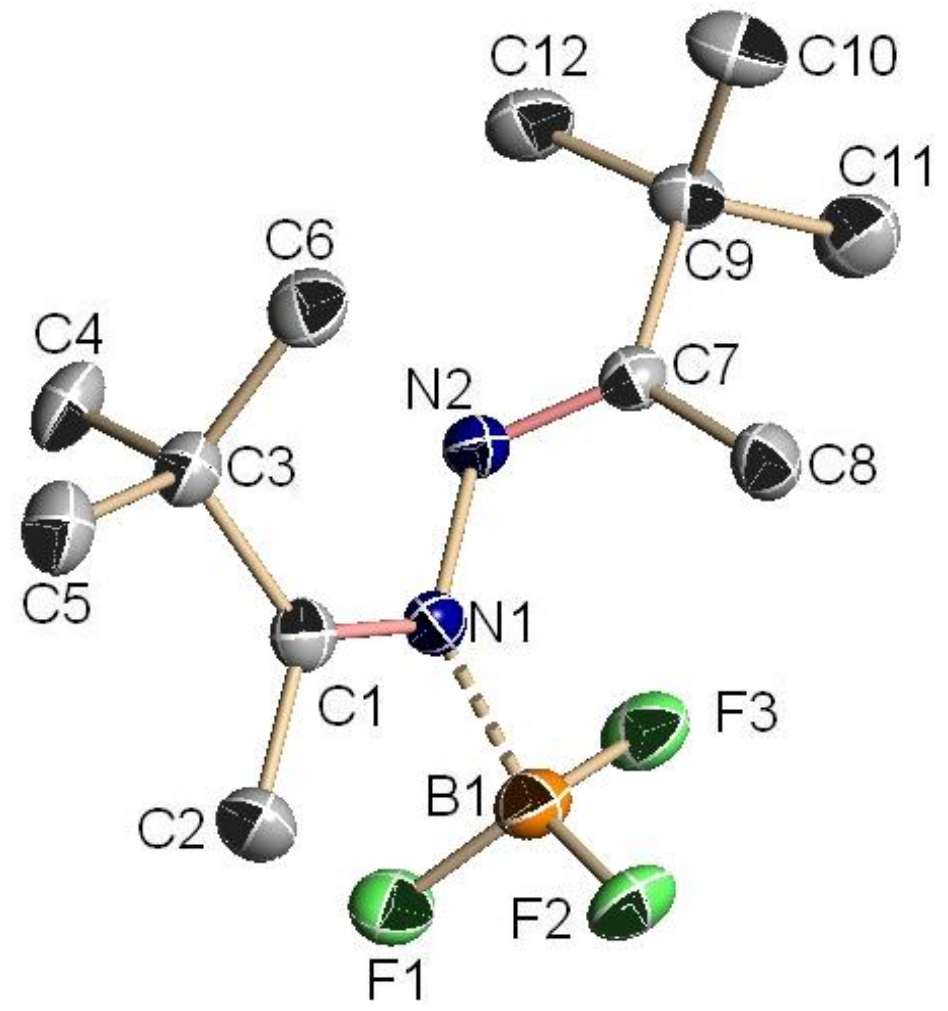

Abbildung 48: Kristallstruktur von 68 ohne Wasserstoffatome

Bindungslängen (-) bzw. intramolekulare Atomabstände (...)/pm mit Standardabweichung

\begin{tabular}{|ll|ll|ll|}
\hline F1-B1 & $137,0(3)$ & N1-C1 & $129,3(3)$ & N1-N2 & $143,3(3)$ \\
\hline N3-N4 & $143,5(3)$ & N2-C7 & $127,2(3)$ & N3-C13 & $128,9(3)$ \\
\hline N3-B2 & $165,0(3)$ & N1..B1 & $166,2(3)$ & & \\
\hline
\end{tabular}

Tabelle 69: Ausgewählte Bindungslängen bzw. intramolekulare Atomabstände der Verbindung 68

Bindungswinkel ${ }^{\circ}$ mit Standardabweichung

\begin{tabular}{|c|c|c|c|c|c|}
\hline C1-N1-N2 & $119,68(19)$ & C1-N1-B1 & $124,99(19)$ & N2-N1-B1 & $113,93(17)$ \\
\hline C7-N2-N1 & $115,53(18)$ & F3-B1-F1 & $111,44(19)$ & F3-B1-F2 & $111,5(2)$ \\
\hline F1-B1-F2 & $112,1(2)$ & F3-B1-N1 & $106,59(18)$ & F1-B1-N1 & $108,39(19)$ \\
\hline F2-B1-N1 & $106,45(18)$ & & & & \\
\hline
\end{tabular}

Tabelle 70: Ausgewählte Bindungswinkel der Verbindung 68 
Wird 68 nochmals mit Trifluorborandiethyletherat umgesetzt, bildet sich nicht das Bis(trifluorboran)addukt, sondern $\mathbf{6 9}^{[76]}$, d.h. $\mathrm{BF}_{3} \mathrm{OEt}_{2}$ fungiert in dieser Reaktion als HF-Fänger.

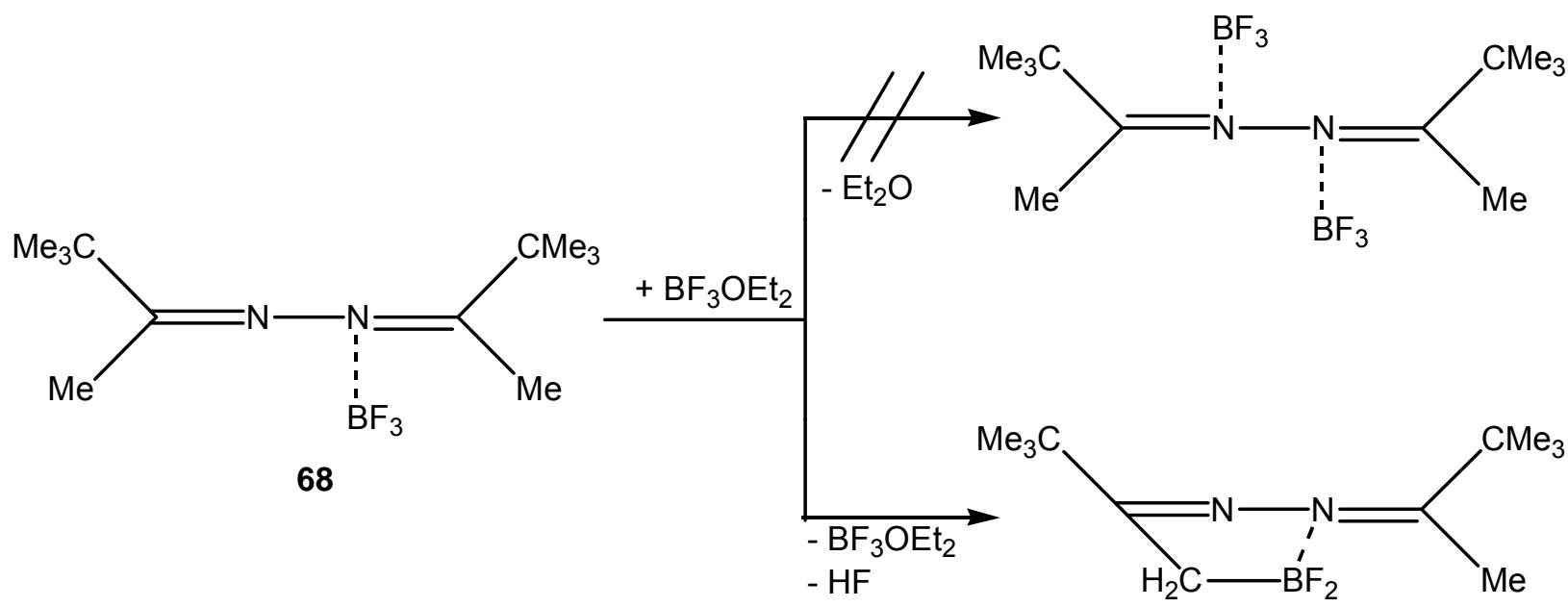

69

Gleichung 61: Synthese von 69

\subsubsection{Kristallstruktur von 69}

Verbindung 69 kristallisiert orthorhombisch in der Raumgruppe Pbca.

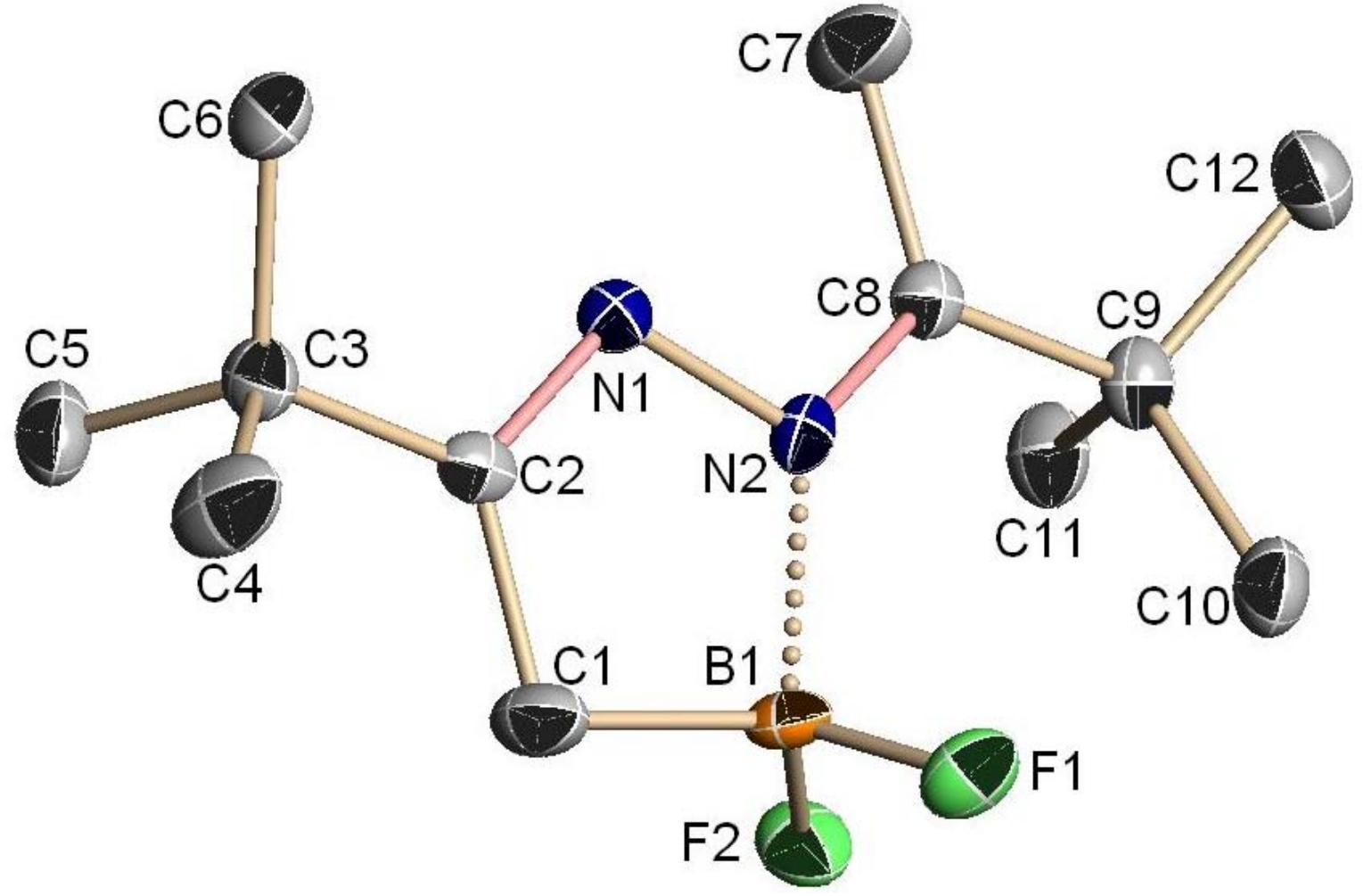

Abbildung 49: Kristallstruktur von 69 ohne Wasserstoffatome 
Bindungslängen (-) bzw. intramolekulare Atomabstände (...)/pm mit Standardabweichung

\begin{tabular}{|ll|ll|ll|}
\hline B1-F1 & $137,8(5)$ & B1-F2 & $139,9(5)$ & B1-C1 & $158,9(6)$ \\
\hline B1...N2 & $168,4(5)$ & N1-C2 & $129,2(4)$ & N1-N2 & $143,8(4)$ \\
\hline N2-C8 & $129,8(4)$ & & & & \\
\hline
\end{tabular}

Tabelle 71: Ausgewählte Bindungslängen bzw. intramolekulare Atomabstände der Verbindung 69

Bindungswinkel $/{ }^{\circ}$ mit Standardabweichung

\begin{tabular}{|ll|ll|ll|}
\hline F1-B1-F2 & $111,1(3)$ & F2-B1-C1 & $115,4(3)$ & F1-B1-N2 & $108,3(3)$ \\
\hline F1-B1-C1 & $115,0(3)$ & F2-B1-N2 & $107,5(3)$ & C1-B1-N2 & $98,2(3)$ \\
\hline C2-N1-N2 & $109,8(3)$ & C8-N2-N1 & $114,1(3)$ & C8-N2-B1 & $137,3(3)$ \\
\hline N1-N2-B1 & $108,4(3)$ & C2-C1-B1 & $104,8(3)$ & & \\
\hline
\end{tabular}

Tabelle 72: Ausgewählte Bindungswinkel der Verbindung 69

Wird Verbindung 69 mit lithiiertem tert-Butylmethyl-trimethylsilyl-hydrazon (70) umgesetzt, bildet sich statt der erwarteten Hydrazon-Ketazinverbindung in einem noch nicht vollständigen Reaktionsverlauf unter Trimethylfluorsilan- und Lithiumfluoridabspaltung das Tris(tert-butylmethyl-hydrazon)boran (71).

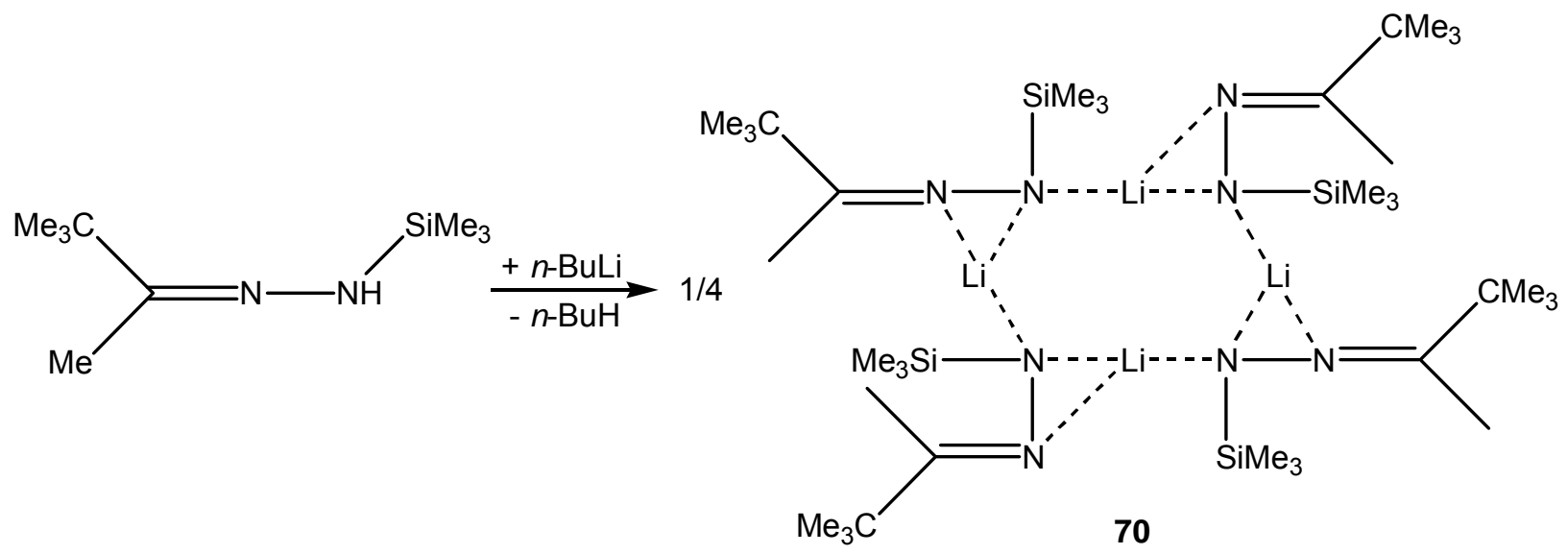

Gleichung 62: Synthese von 70

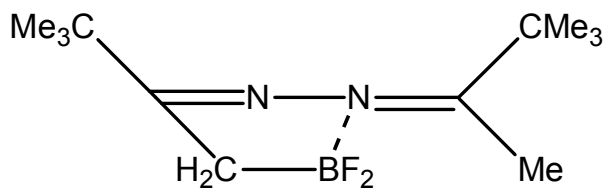

69

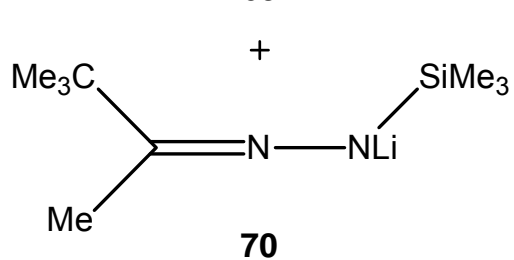

70

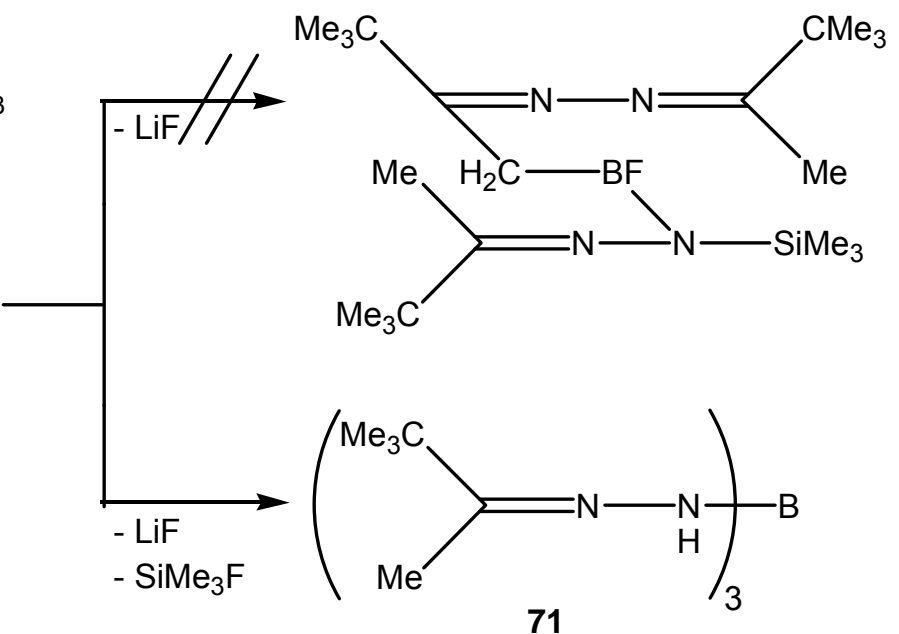

Schema 6: Synthese von $\mathbf{7 1}$ 


\subsubsection{Kristallstruktur von 70}

Verbindung $\mathbf{7 0}$ kristallisiert tetragonal in der Raumgruppe $P 4_{2} / \mathrm{n}$. Die Lithiumionen sind jeweils zu einem Stickstoffatom „end-on“ und zur Hydrazineinheit eines benachbarten Hydrazonmoleküls "side-on" koordiniert, was zur Ausbildung von vier dreigliedrigen Ringen führt. Die Lithiumionen und Stickstoffatome des tetrameren Lithiumsalzes bilden einen zentralen achtgliedrigen Ring.

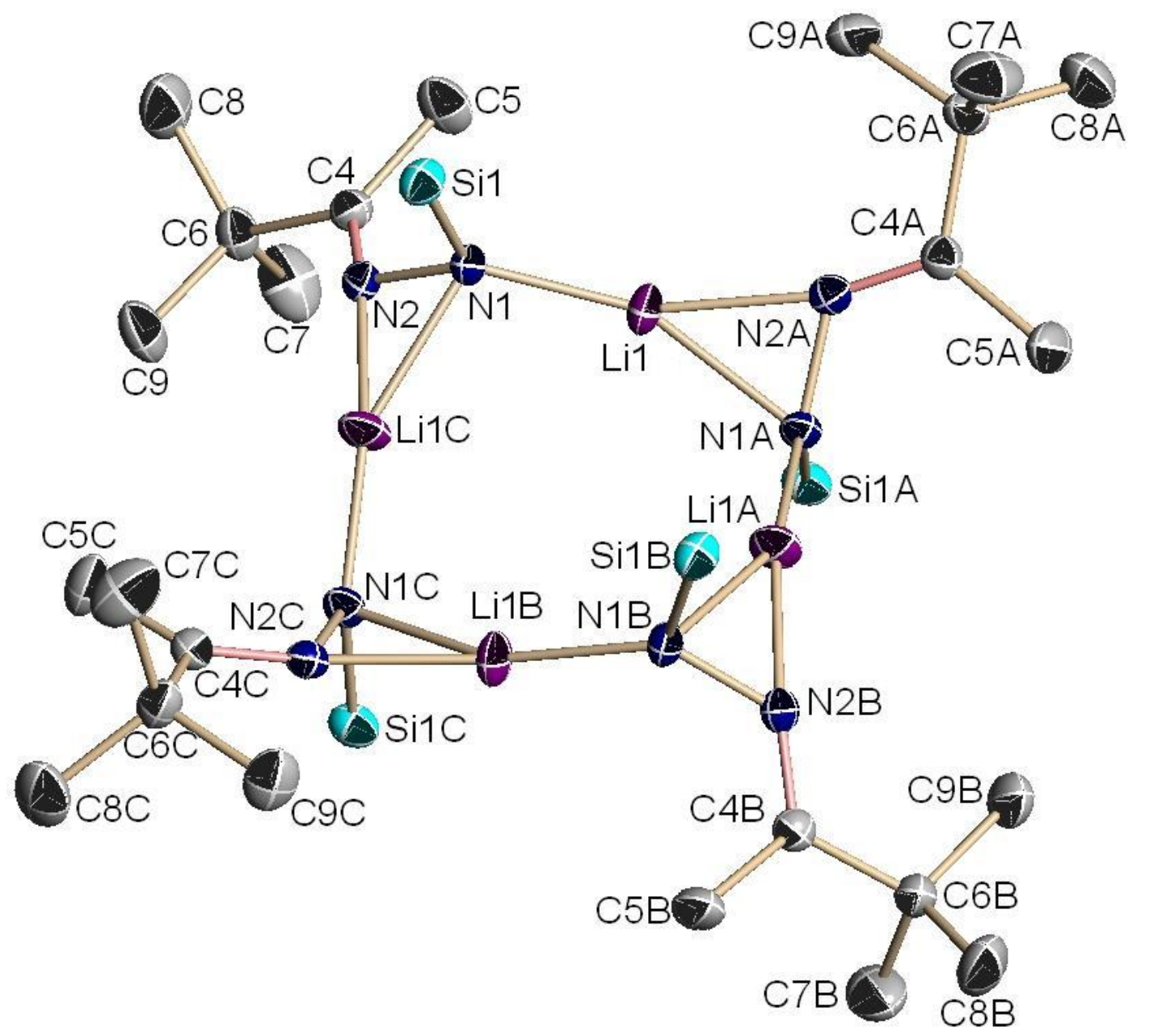

Abbildung 50: Kristallstruktur von $\mathbf{7 0}$ ohne Wasserstoffatome

Bindungslängen/pm mit Standardabweichung

\begin{tabular}{|ll|ll|ll|}
\hline Si1-N1 & $171,8(3)$ & Li1-N1 & $191,0(6)$ & Li1-N2A & $194,0(6)$ \\
\hline Li1-N1A & $205,6(6)$ & N1-N2 & $145,1(3)$ & N2-C4 & $127,6(4)$ \\
\hline
\end{tabular}

Tabelle 73: Ausgewählte Bindungslängen der Verbindung 70

Bindungswinkel ${ }^{\circ}$ mit Standardabweichung

\begin{tabular}{|ll|ll|ll|}
\hline N1-Li1-N2B & $156,8(3)$ & N1-Li1-N1B & $153,8(3)$ & N2B-Li1-N1B & 42,48(14) \\
\hline N2-N1-Si1 & $111,04(18)$ & N2-N1-Li1 & $127,6(2)$ & Si1-N1-Li1 & $121,1(2)$ \\
\hline N2-N1-Li1A & $64,5(2)$ & Si1-N1-Li1A & $100,6(2)$ & Li1-N1-Li1A & $108,5(3)$ \\
\hline C4-N2-N1 & $120,3(2)$ & C4-N2-Li1A & $161,8(3)$ & N1-N2-Li1A & $73,0(2)$ \\
\hline
\end{tabular}

Tabelle 74: Ausgewählte Bindungswinkel der Verbindung 70 


\subsubsection{Kristallstruktur von 71}

Verbindung 14 kristallisiert aus $n$-Hexan orthorhombisch in der Raumgruppe Pbca. Das Boratom ist planar umgeben $\left(\Sigma \nless \mathrm{B} 1=360,0^{\circ}\right)$. Die N-N-Bindungen sind im Vergleich zu 68 oder 70 um 4 - 6 pm verkürzt.

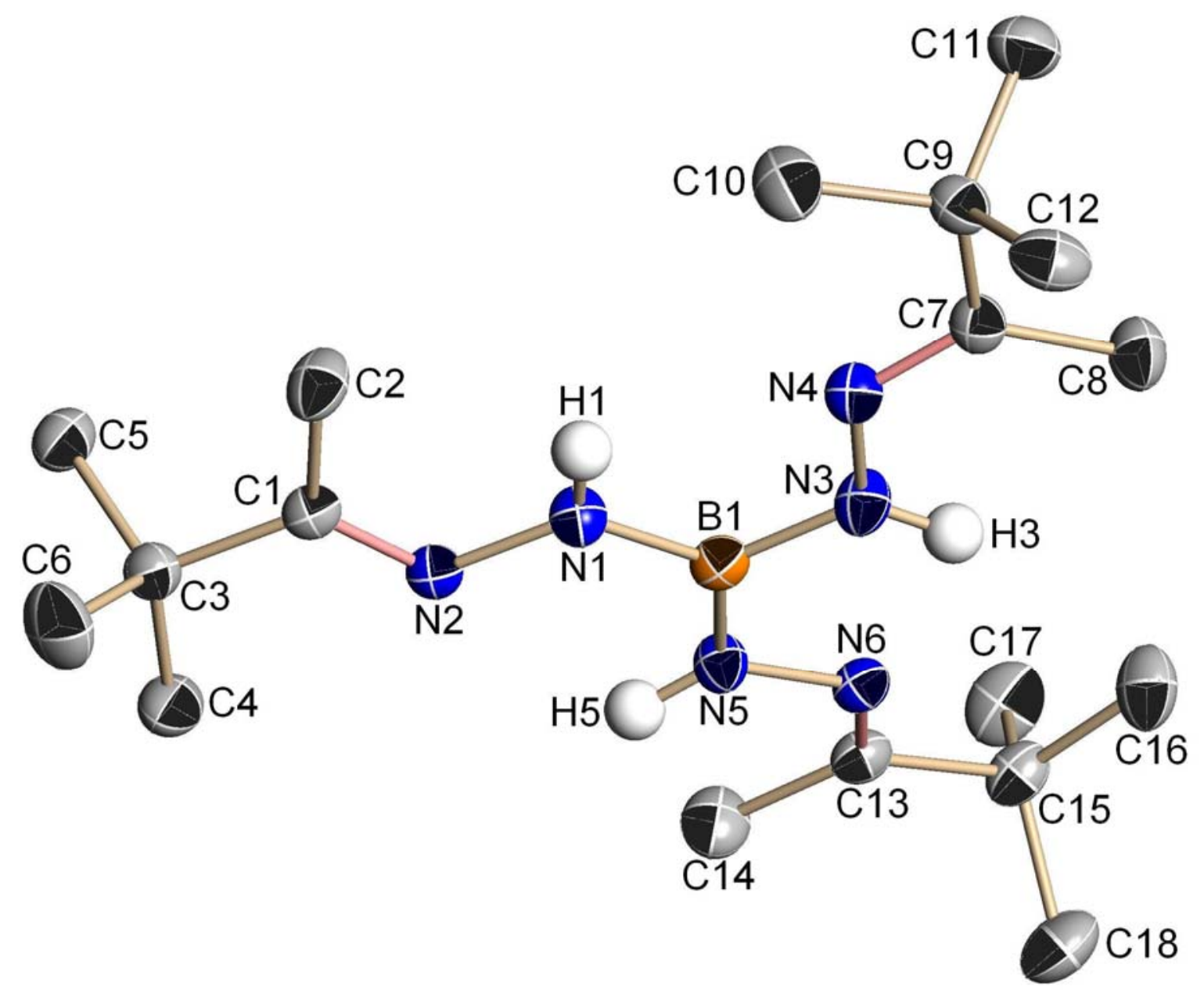

Abbildung 51: Kristallstruktur von 71 ohne Methylwasserstoffatome

Bindungslängen/pm mit Standardabweichung

\begin{tabular}{|ll|ll|ll|}
\hline N1-B1 & $141,86(18)$ & N3-B1 & $142,5(2)$ & N5-B1 & $142,38(19)$ \\
\hline N2-N1 & $138,77(16)$ & N2-C1 & $128,74(17)$ & C1-C2 & $150,4(2)$ \\
\hline C3-C1 & $152,6(2)$ & N1-B1 & 88,0 & & \\
\hline
\end{tabular}

Tabelle 75: Ausgewählte Bindungslängen der Verbindung 71

Bindungswinkel $/^{\circ}$ mit Standardabweichung

\begin{tabular}{|ll|ll|ll|}
\hline N1-B1-N5 & $121,42(13)$ & N1-B1-N3 & $118,58(12)$ & N5-B1-N3 & $119,96(12)$ \\
\hline C1-N2-N1 & $117,59(11)$ & N2-N1-B1 & $121,98(11)$ & N2-N1-H1 & 119,01 \\
\hline H1-N1-B1 & 119,00 & N2-C1-C2 & $123,02(13)$ & N2-C1-C3 & $118,23(12)$ \\
\hline
\end{tabular}

Tabelle 76: Ausgewählte Bindungswinkel der Verbindung 71 


\subsubsection{Synthese von Hydrazonen mit der Struktureinheit N(Si)-BF-N(Si)}

Wird das Lithiumsalz $\mathbf{7 0}$ mit Difluor-bis(trimethylsilyl)amino-boran umgesetzt, so kann das (tert-Butylmethylketon-trimethylsilyl-hydrazono)-fluor-bis(trimethylsilyl)amino-boran (72) isoliert werden.

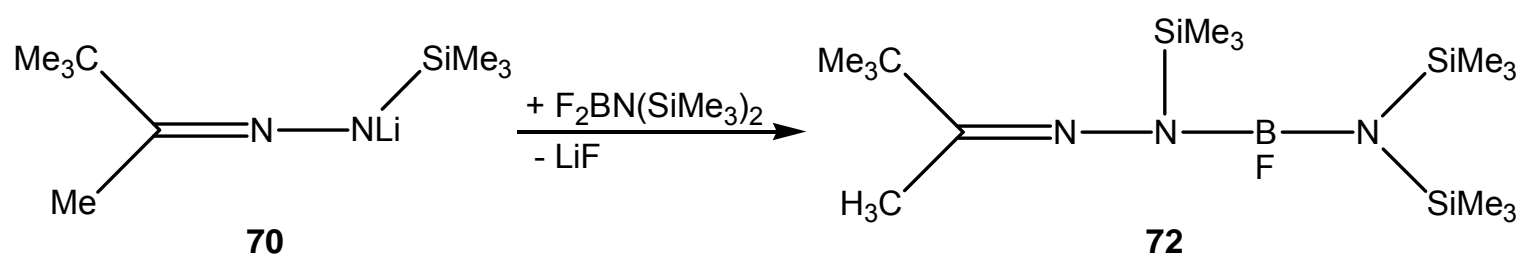

Gleichung 63: Synthese von 72

Das ${ }^{13}$ C-NMR-Signal der Methylkohlenstoffatome der tert-Butylgruppen spaltet zu einem Dublett auf, wobei die ${ }^{6} \mathrm{~J}_{\mathrm{CF}}$-Kopplung mit $3,3 \mathrm{~Hz}$ ungewöhnlich groß ist. Auf Grund des -l-Effekts der Hydrazineinheit ist das Siliciumatom der Hydrazinsilylgruppe mit 8,51 pm stärker Tieffeld verschoben als die Aminosilylgruppen.

Ein weiteres Hydrazon mit der Einheit N(Si)-BF-N(Si) wurde bereits im Arbeitskreis Klingebiel synthetisiert ${ }^{[77]}$ und im Rahmen dieser Arbeit erneut dargestellt.

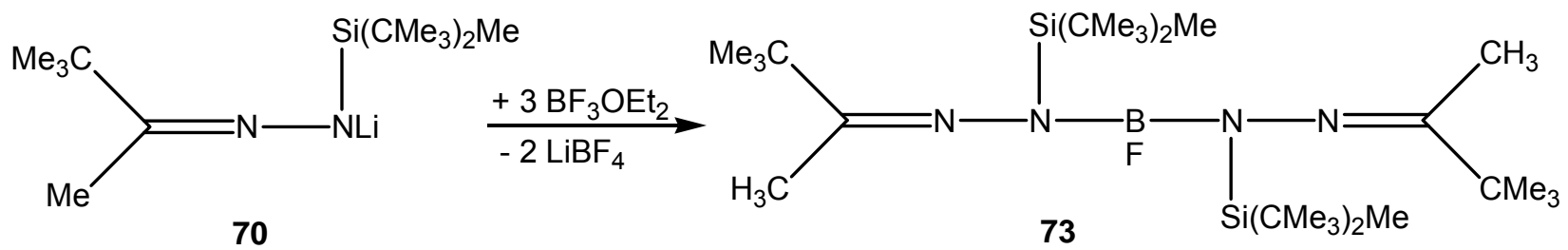

Gleichung 64: Synthese von $73^{[77]}$

Auf Grund fehlender Strukturdaten wurde die Verbindung röntgenographisch untersucht. 


\subsubsection{Kristallstruktur von 73}

73 kristallisiert triklin in der Raumgruppe $P \overline{1}$. Trotz der voluminösen Stickstoffsubstituenten sind $\mathrm{B} 1, \mathrm{~N} 1$ und $\mathrm{N} 2$ planar umgeben $\left(\Sigma \nless \mathrm{B} 1=360,0^{\circ}, \quad \Sigma \Varangle \mathrm{N} 1=359,8^{\circ}\right.$, $\left.\Sigma \nless \mathrm{N} 2=359,8^{\circ}\right)$.

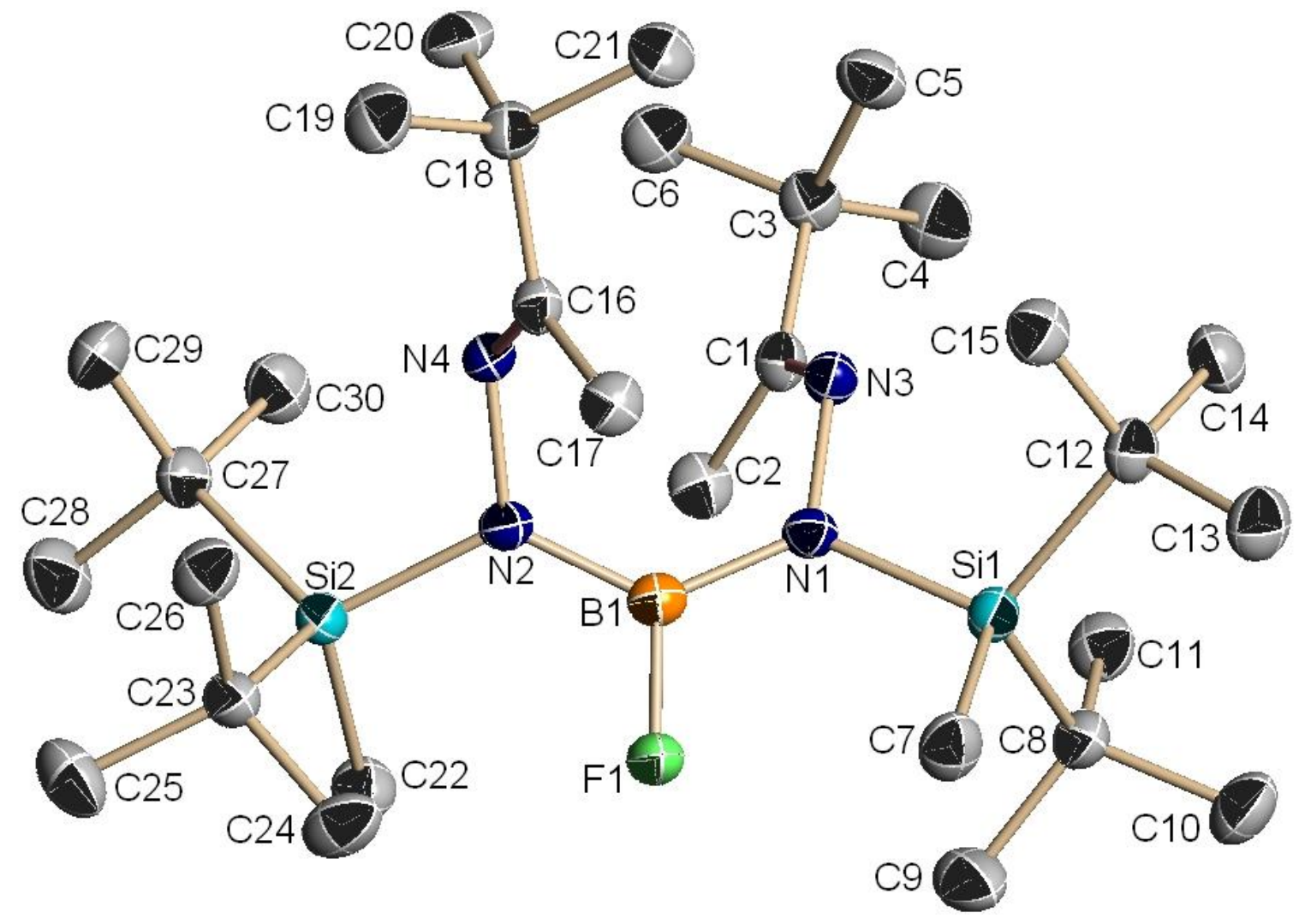

Abbildung 52: Kristallstruktur von 73 ohne Wasserstoffatome

Bindungslängen/pm mit Standardabweichung

\begin{tabular}{|ll|ll|ll|}
\hline B1-F1 & $136,1(2)$ & B1-N1 & $142,6(2)$ & B1-N2 & $142,7(2)$ \\
\hline Si1-N1 & $178,6(1)$ & N1-N3 & $145,0(2)$ & N3-C1 & $128,0(2)$ \\
\hline
\end{tabular}

Tabelle 77: Ausgewählte Bindungslängen der Verbindung 73

Bindungswinkel $/{ }^{\circ}$ mit Standardabweichung

\begin{tabular}{|ll|ll|ll|}
\hline F1-B1-N1 & $114,75(12)$ & F1-B1-N2 & $114,89(12)$ & N1-B1-N2 & $130,36(13)$ \\
\hline B1-N1-N3 & $121,47(10)$ & B1-N1-Si1 & $126,59(9)$ & N3-N1-Si1 & $111,76(8)$ \\
\hline C1-N3-N1 & $116,59(11)$ & & & & \\
\hline
\end{tabular}

Tabelle 78: Ausgewählte Bindungswinkel der Verbindung 73 


\subsubsection{Synthese bisher unbekannter Fluorborane als Vorstufe für die Bildung von Boriniumionen}

Für die Synthese neuartiger Borkationen wurden zwei Fluorborane mit der Struktureinheit $\mathrm{R}_{2} \mathrm{~N}-\mathrm{BF}-\mathrm{NR}_{2}$ und RO-BF-OR synthetisiert, welche röntgenstrukturanalytisch untersucht wurden. Die Difluorboryl-substituierten Verbindungen konnten nicht über die Reaktion der Lithiumsalze mit $\mathrm{BF}_{3} \mathrm{OEt}_{2}$ isoliert werden (vgl. Gleichung $51^{[72]}$ ).

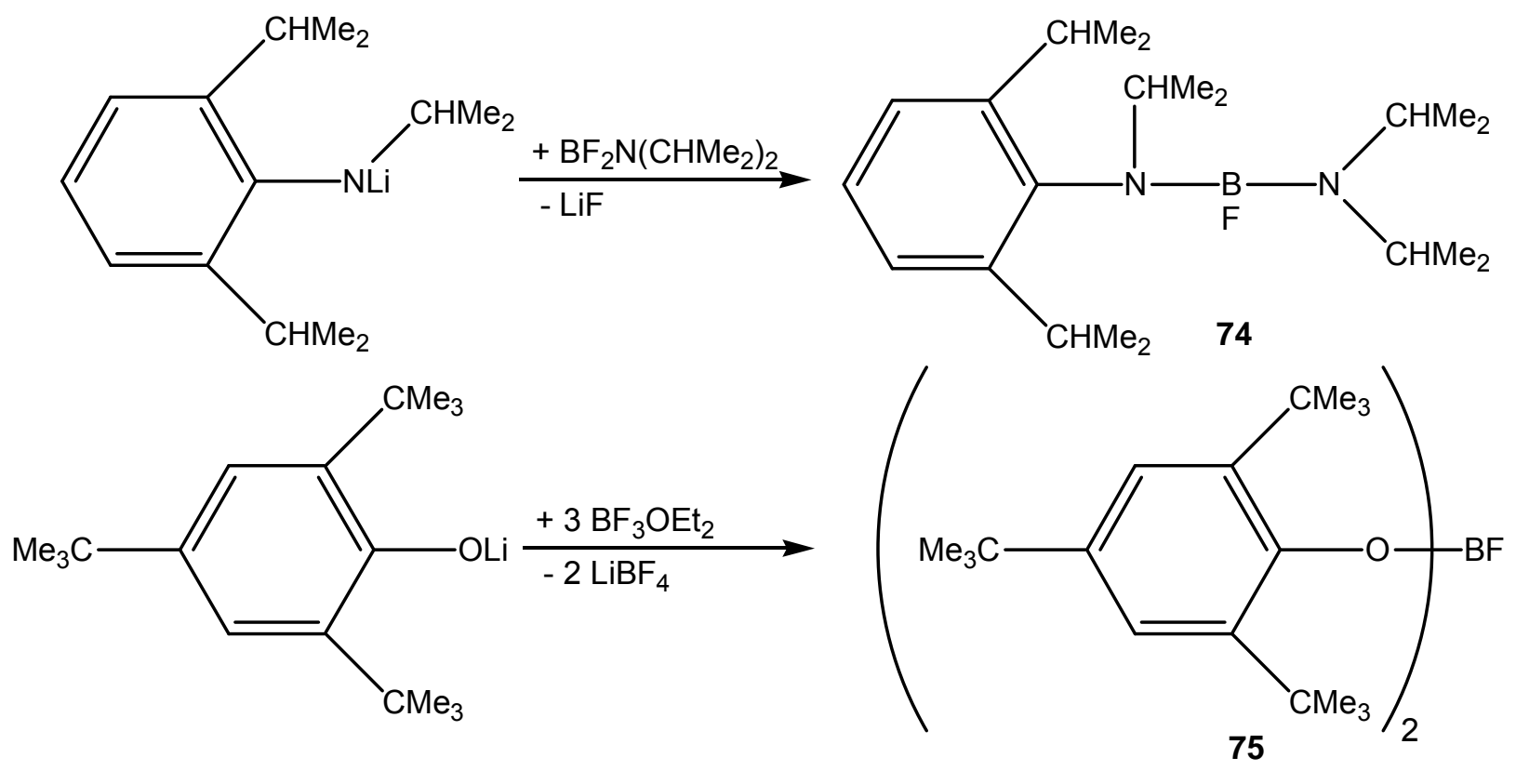

Gleichung 65: Synthese von $\mathbf{7 4}$ und $\mathbf{7 5}$ 


\subsubsection{Kristallstruktur von 74}

Verbindung 74 kristallisiert monoklin in der Raumgruppe $C 2_{1} / \mathrm{C}$. B1, N1 und N2 sind planar umgeben $\left(\Sigma \Varangle \mathrm{B} 1=360,0^{\circ}, \Sigma \Varangle \mathrm{N} 1=359,1^{\circ}, \Sigma \Varangle \mathrm{N} 2=359,6^{\circ}\right)$.

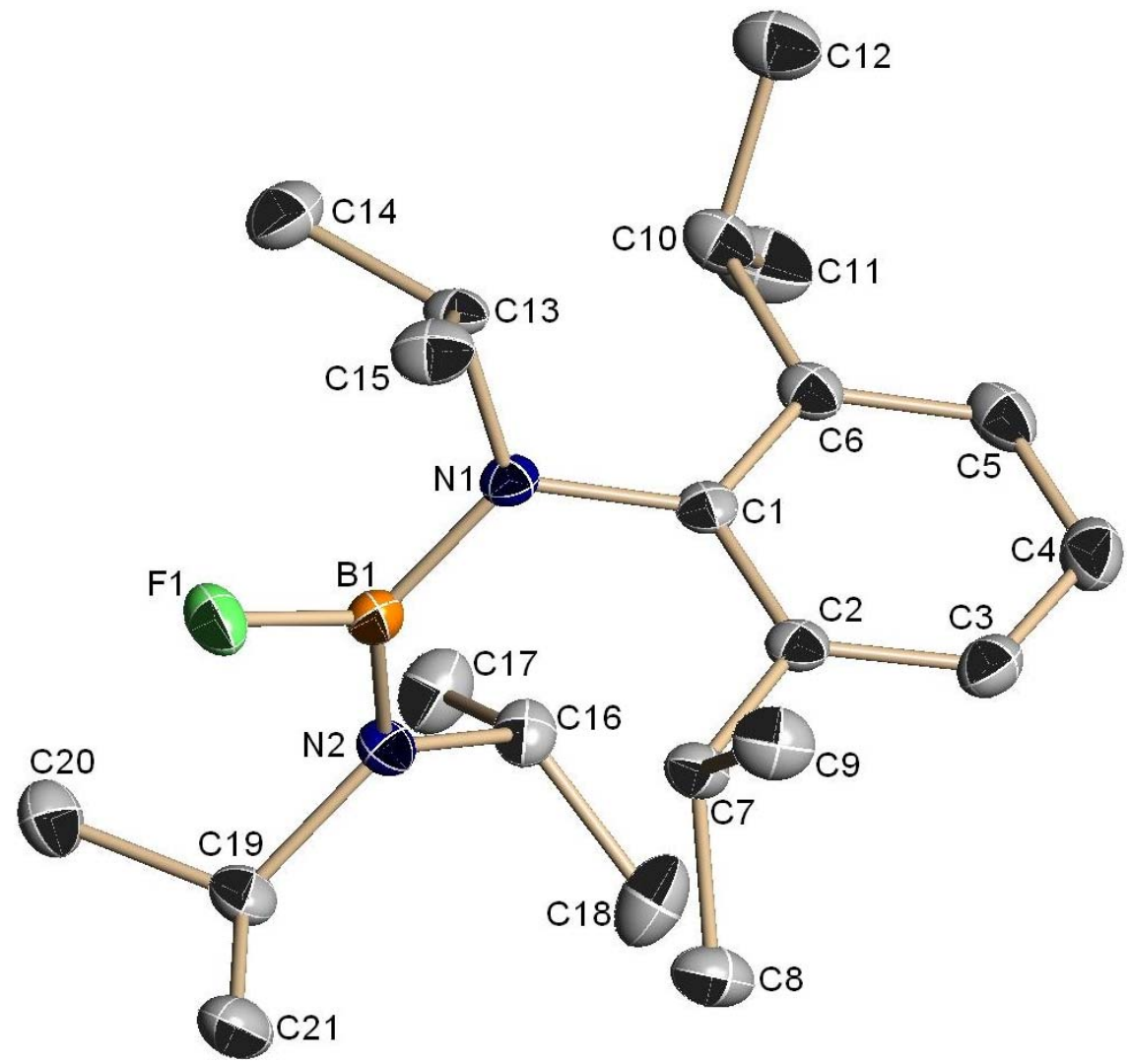

Abbildung 53: Kristallstruktur von 74 ohne Wasserstoffatome

Bindungslängen/pm mit Standardabweichung

\begin{tabular}{|ll|ll|ll|}
\hline N1-B1 & $142,6(3)$ & N2-B1 & $140,8(3)$ & F1-B1 & $136,6(3)$ \\
\hline N1-C1 & $143,4(3)$ & N1-C13 & $149,1(3)$ & N2-C16 & $147,6(3)$ \\
\hline
\end{tabular}

Tabelle 79: Ausgewählte Bindungslängen der Verbindung 74

Bindungswinkel $/^{\circ}$ mit Standardabweichung

\begin{tabular}{|ll|ll|ll|}
\hline F1-B1-N2 & $116,54(19)$ & F1-B1-N1 & $115,6(2)$ & N2-B1-N1 & $127,9(2)$ \\
\hline B1-N1-C1 & $123,47(17)$ & B1-N1-C13 & $121,53(17)$ & C1-N1-C13 & $114,07(16)$ \\
\hline B1-N2-C16 & $122,65(18)$ & B1-N2-C19 & $121,51(18)$ & C16-N2-C19 & $115,41(17)$ \\
\hline
\end{tabular}

Tabelle 80: Ausgewählte Bindungswinkel der Verbindung 74 


\subsubsection{Kristallstruktur von 75}

75 kristallisiert monoklin in der Raumgruppe $P 2_{1} / \mathrm{c}$. Das Boratom ist planar umgeben $\left(\Sigma \nless \mathrm{B} 1=360,0^{\circ}\right)$.

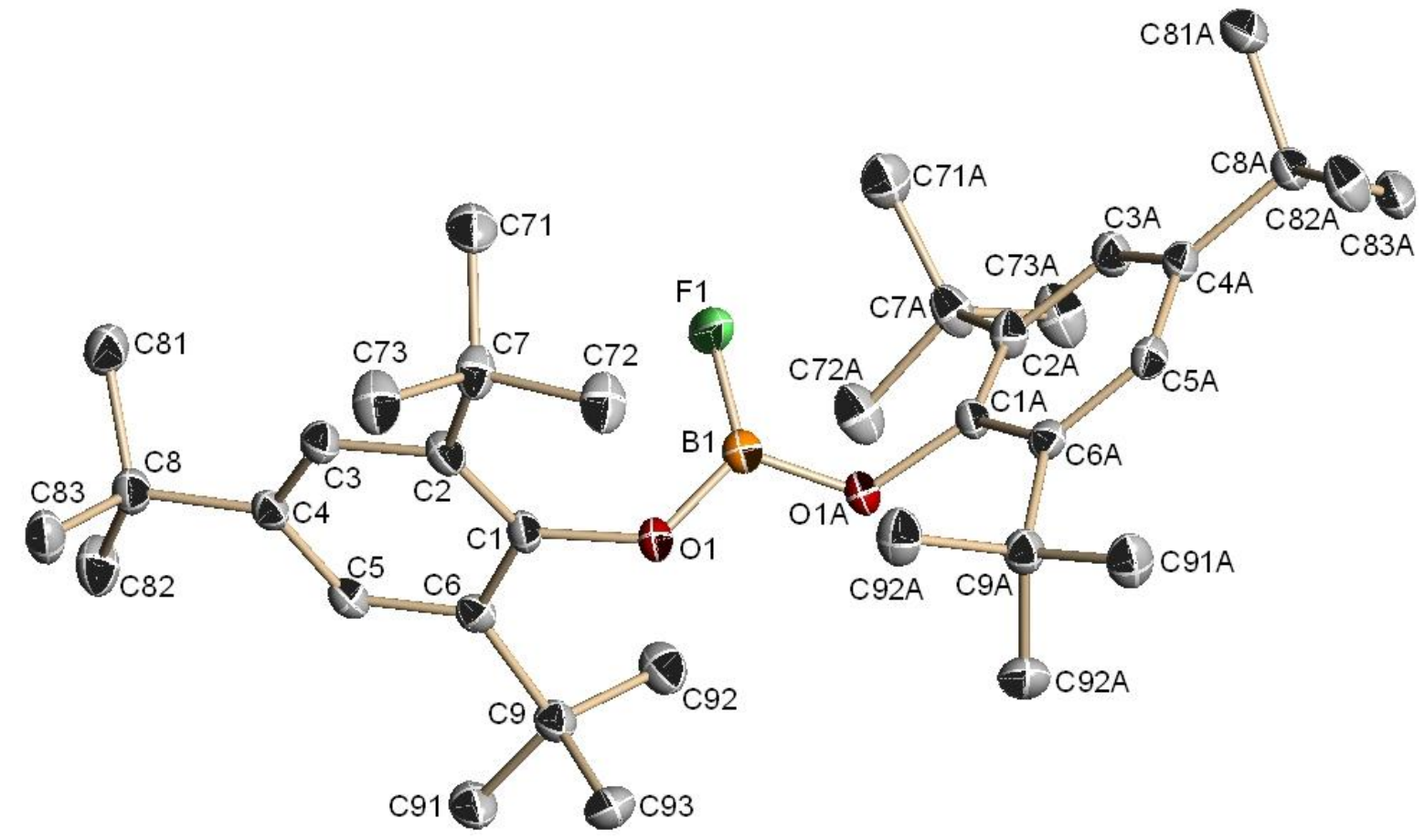

Abbildung 54: Kristallstruktur von 75 ohne Wasserstoffatome

Bindungslängen/pm mit Standardabweichung

\begin{tabular}{|ll|ll|ll|}
\hline O1-B1 & $135,27(13)$ & B1-O1A & $135,26(13)$ & O1-C1 & $140,30(13)$ \\
\hline F1-B1 & $132,5(2)$ & & & \\
\hline
\end{tabular}

Tabelle 81: Ausgewählte Bindungslängen der Verbindung $\mathbf{7 5}$

Bindungswinkel ${ }^{\circ}$ mit Standardabweichung

\begin{tabular}{|ll|ll|ll|}
\hline B1-O1-C1 & $125,61(9)$ & F1-B1-O1A & $121,75(7)$ & F1-B1-O1 & $121,75(7)$ \\
\hline O1A-B1-O1 & $116,50(15)$ & & & \\
\hline
\end{tabular}

Tabelle 82: Ausgewählte Bindungswinkel der Verbindung 75 


\subsection{Keramik 48X}

Das Verhältnis der Silylgruppen zu den Chloratomen in $\mathbf{4 8}$ ist 3:3. Gelänge eine vollständige Abspaltung dieser drei Halogensilaneinheiten, so blieben Aluminium, Bor und Stickstoff im Molverhältnis 1:1:2 übrig. Damit erhielte man eine ternäre kohlenstofffreie AIN / BN - Keramik.

Beim Versuch der destillativen Aufarbeitung von 48 wurde ab $170^{\circ} \mathrm{C}$ eine Chlortrimethylsilanabspaltung beobachtet. Ein anschließendes Erhitzen auf $1200{ }^{\circ} \mathrm{C}$ im Autoklaven führte zur Abspaltung von weiterem Chlorsilan.

Bei REM(SEM)-Aufnahmen von dem zurückbleibenden Feststoff (48X) zeigt sich eine (mit aufsitzenden Staubteilchen) einheitlich glatte Oberfläche.

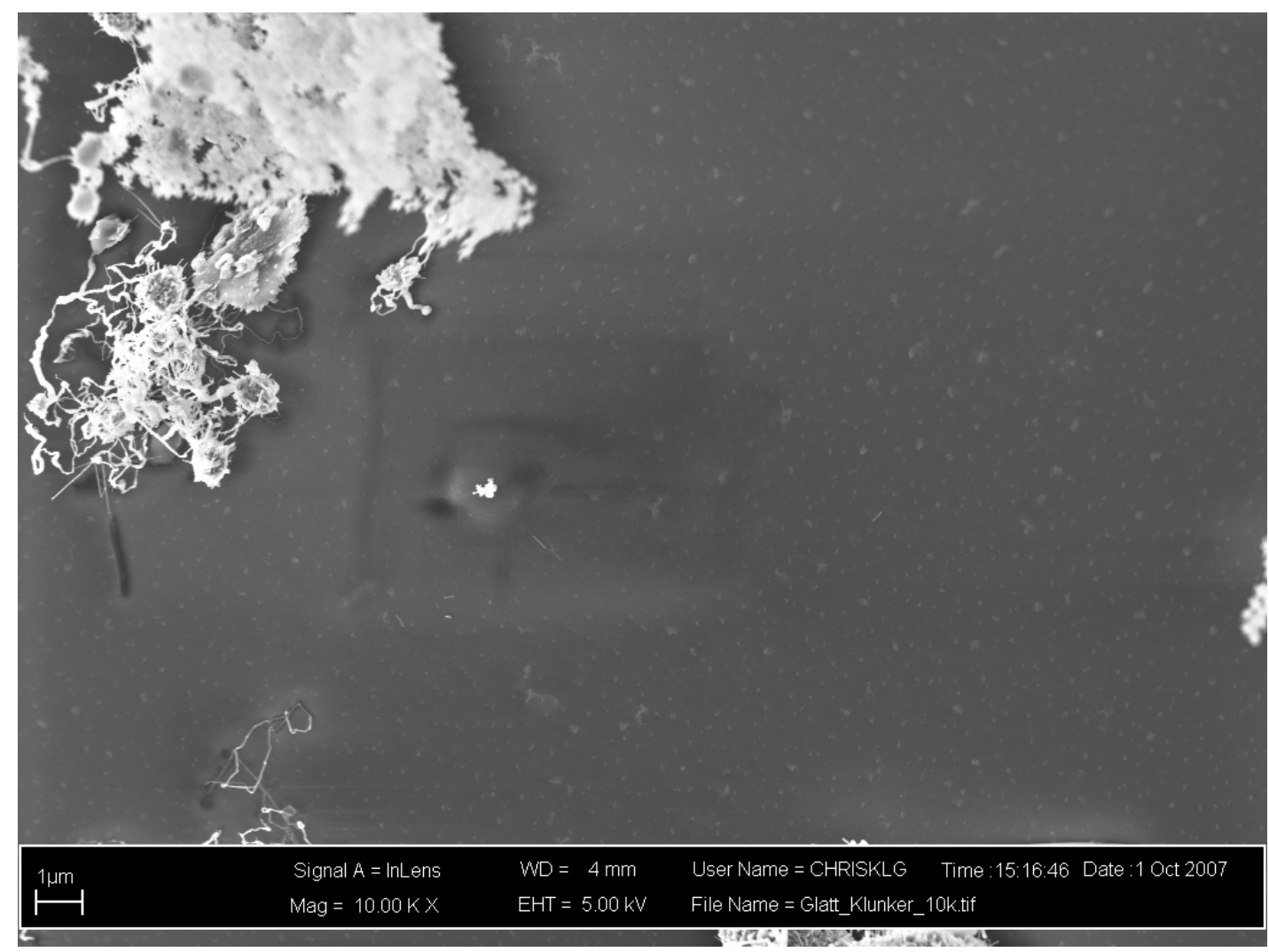

Abbildung 55: 48X, REM(SEM)-Aufnahmen der Oberfläche, 10.000fache Vergrößerung

EDX-Spektren der Oberfläche* belegen, dass diese hauptsächlich aus $\mathrm{SiO}_{2}$ aufgebaut ist. Da bis zum Ende des Thermolyseprozesses unter trockener Argonatmosphäre gearbeitet wurde, wird vermutet, dass während des Abbaus elementares Silicium entstanden ist, welches nach der Reaktion oberflächennah mit Luftsauerstoffbzw. Luftfeuchtigkeit zu Siliciumdioxid reagiert hat.

\footnotetext{
* Auf Grund der Anregungsenergie von $\mathrm{E}_{0}=20 \mathrm{keV}$ findet eine Oberflächenanalyse bis zu einer Tiefe von 7,5 - 3,3 $\mu \mathrm{m}$ statt ${ }^{[a, 56]}$.
} 
Unter der Oberfläche findet man einheitlich eine zweite Spezies vor. Hierzu wurden an Bruchkanten (siehe Abbildung 56) REM(SEM)-Aufnahmen- und EDX-Spektren(siehe Abbildung 57), fokussiert auf die globulären Aggregate (siehe Abbildung 58), aufgenommen.
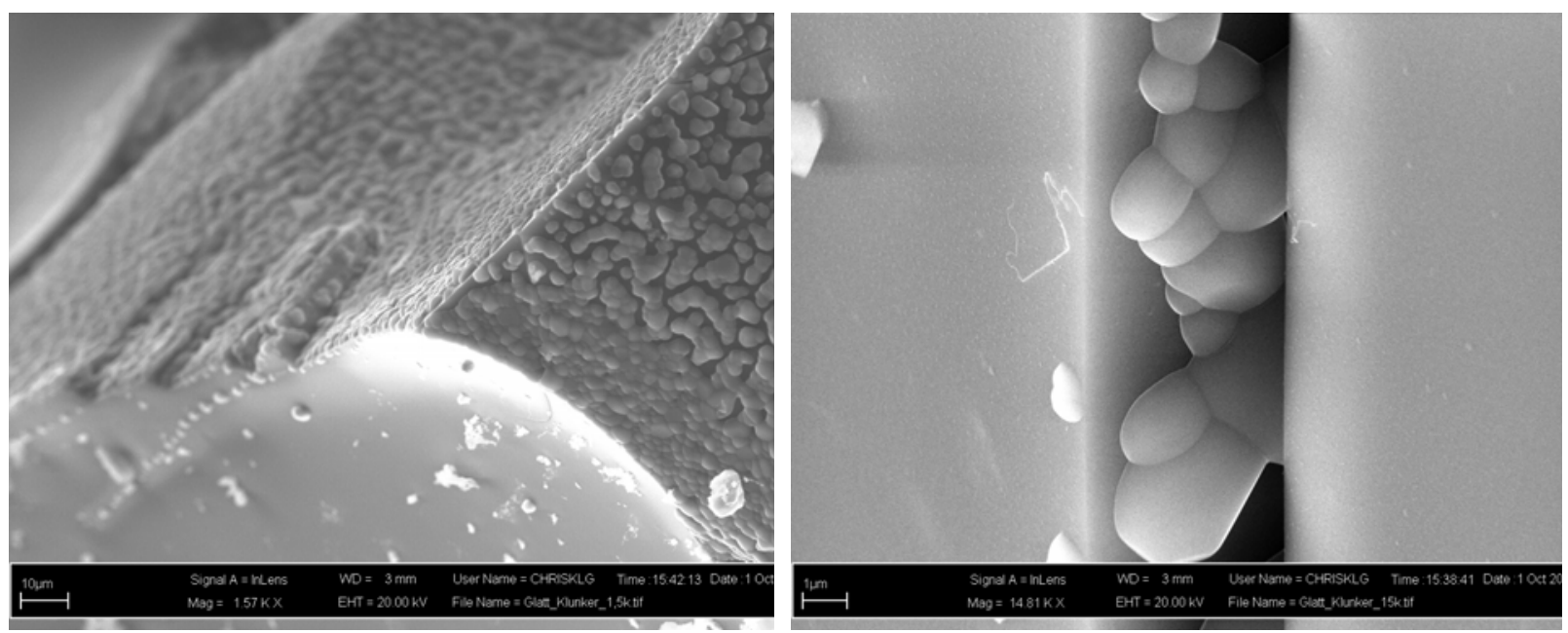

Abbildung 56: 48X, REM(SEM)-Aufnahmen der Bruchkanten, 1.570fache- bzw. 14.810fache Vergrößerung

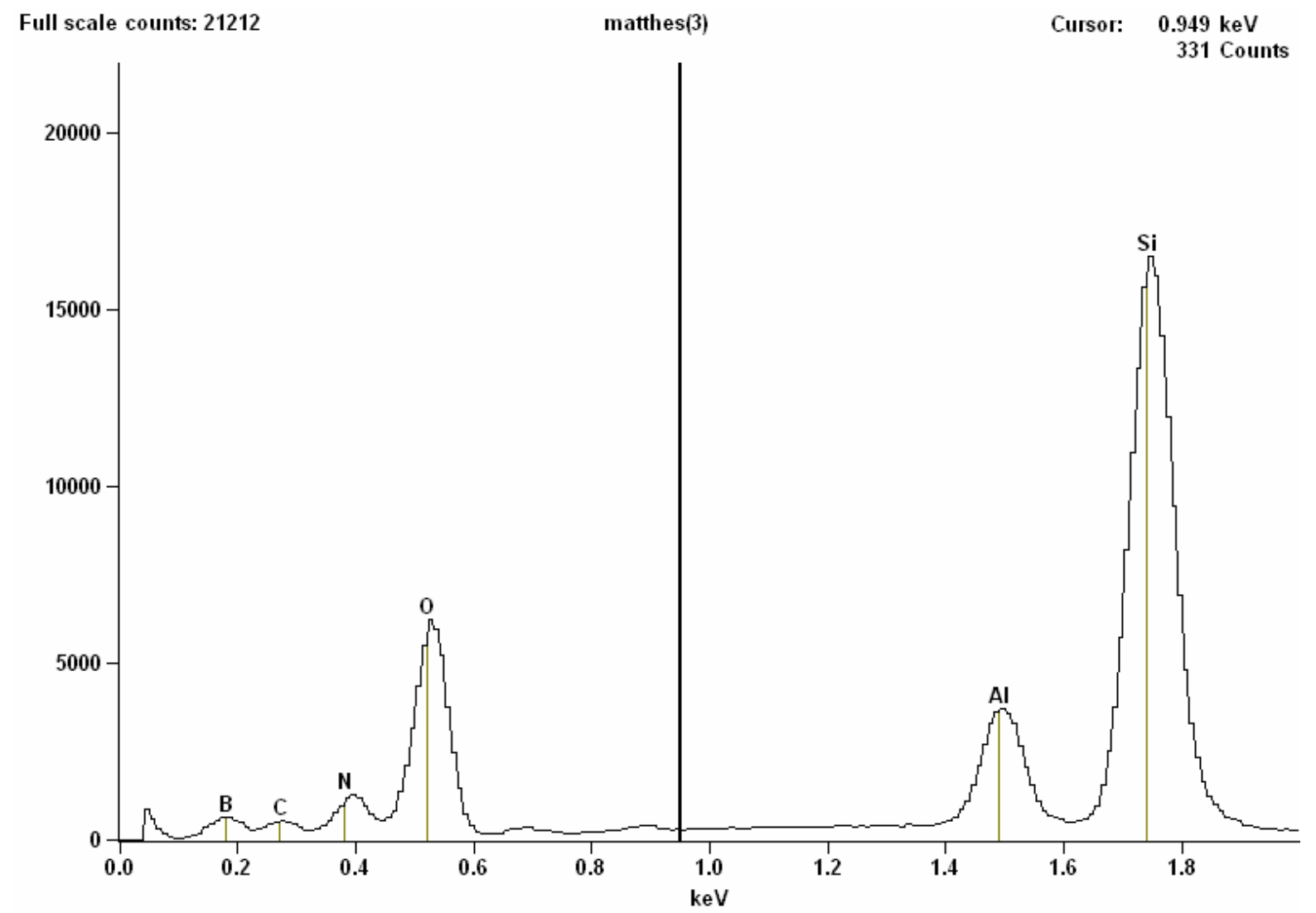

Abbildung 57: EDX-Spektrum, fokusiert auf die globulären Aggregate von $\mathbf{4 8 X}$ 


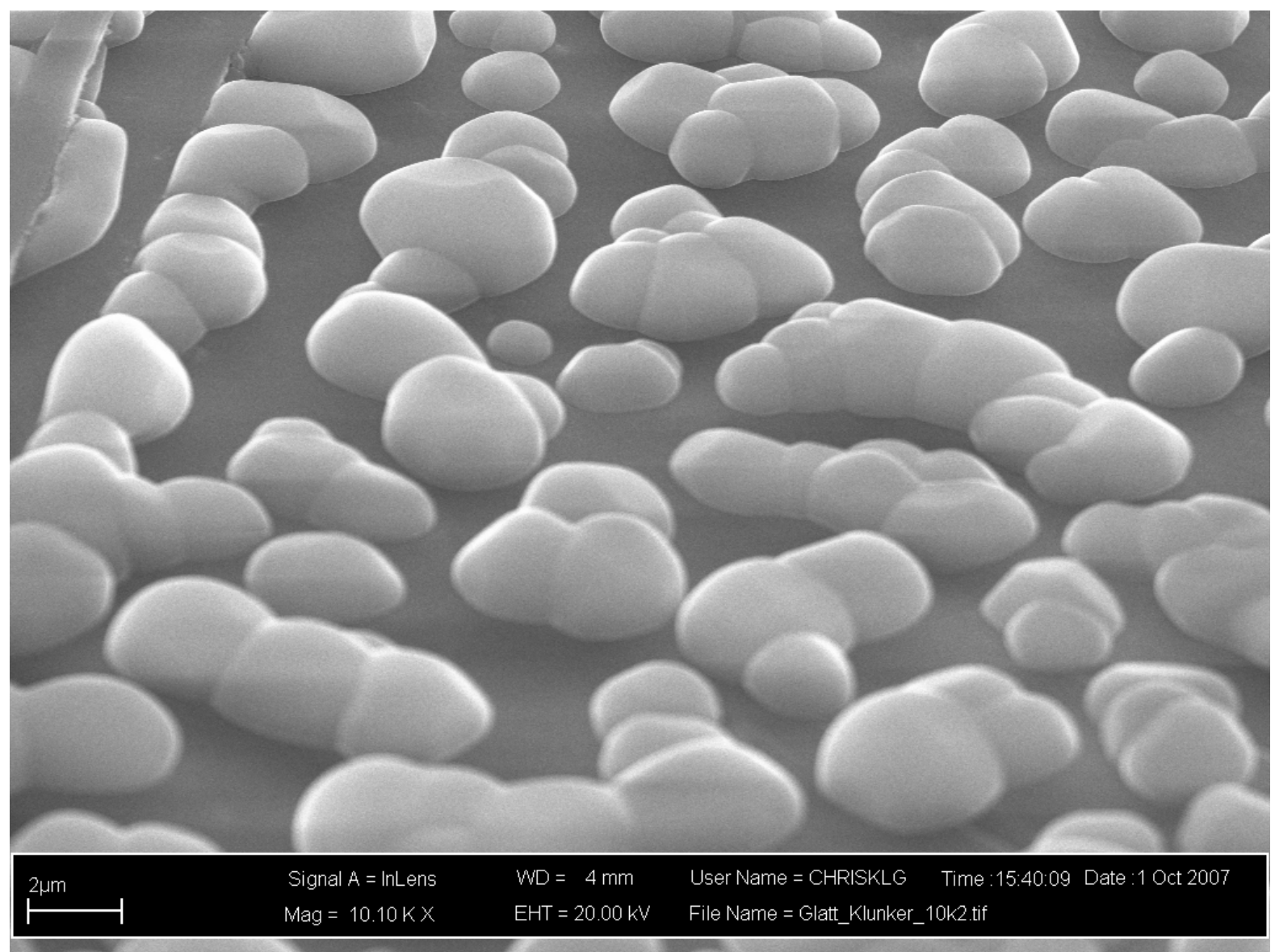

Abbildung 58: 48X, REM(SEM)-Aufnahmen der globulären Aggregate, 10.100fache Vergrößerung

Neben den Oberflächen-SiO ${ }_{2}$-Signalen wird Aluminium, Bor und Stickstoff gefunden. Der Kohlenstoffanteil ist gering*.

Mit Verbindung 48 konnte ein Molekül synthetisiert werden, in welchem das gewünschte Verhältnis B:Al:N von 1:1:2 sowie Silyl:Halogen von 3:3 vorliegt. Leider scheint bei der Thermolyse der Verbindung 48 neben Halogensilan als Abgangsgruppe auch elementares Silicium zu entstehen, welches oberflächennah zu Siliciumdioxid reagiert. Gelingt eine vollständige Halogensilanabspaltung oder eine Trennung der $\mathrm{Al}, \mathrm{B}, \mathrm{N}$ haltigen, globulären Aggregate vom Silicium(dioxid), so sind weitere Untersuchungen wie Leitfähigkeitsmessungen an dieser Substanz von besonderem Interesse. Von mischkeramischen Werkstoffen bestehend aus BN/ AIN werden interessante Halbleitereigenschaften erwartet.

\footnotetext{
* Mit EDX können keine quantitativen Aussagen über den Kohlenstoff- wie auch den Boranteil getroffen werden. Mit Hilfe der Auger-Spektroskopie wären Aussagen über die quantitative Zusammensetzung bezüglich dieser Elemente möglich.
} 


\section{$4 \quad$ Zusammenfassung}

Im Rahmen dieser Arbeit wurden Chlordimethylsilyl-bis(silyl)-amine mit Ethylendiamin bzw. Hydrazin umgesetzt (siehe Gleichungen 22 und 30).
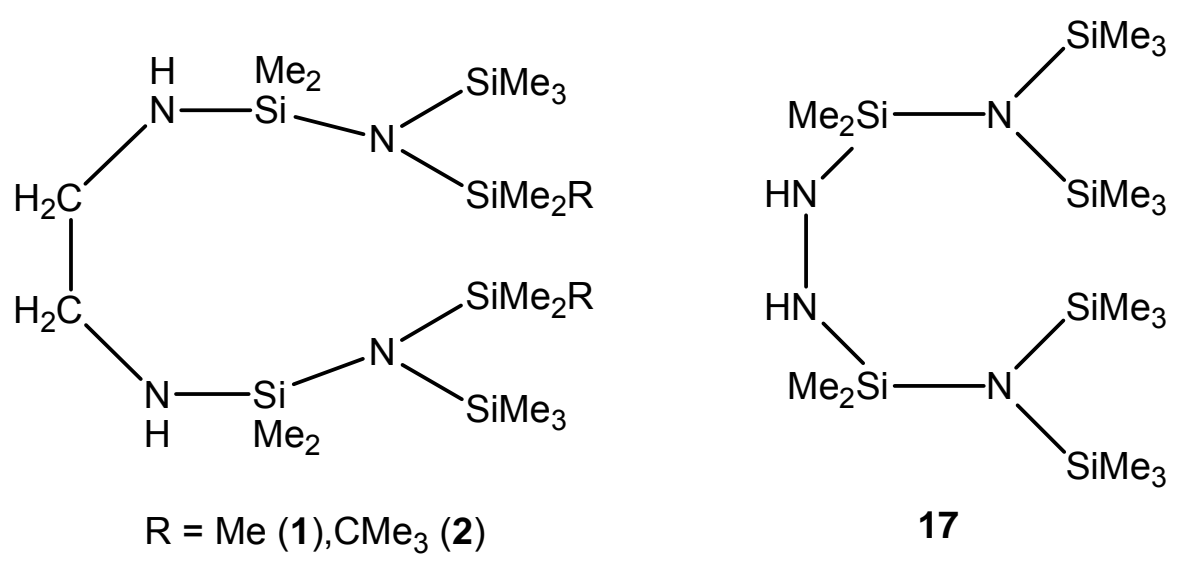

Abbildung 59: Verbindungen 1, 2 und 17

Während Verbindung 1 bis $260{ }^{\circ} \mathrm{C}$ stabil bleibt, isomerisiert 2 unter thermisch induzierter Silatropie bei $200{ }^{\circ} \mathrm{C}$ zu 3.

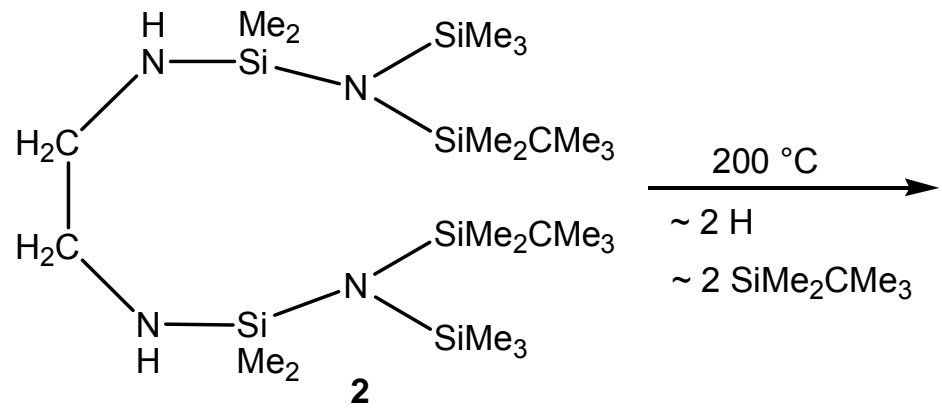

Gleichung 66: Thermisch induzierte Silatropie bei 2

Durch Metallierung mit $n$-Butyllithium können die Dilithiumsalze der Verbindungen 1 und 17 erhalten werden, wobei jeweils zwei Silylgruppen der Verbindung durch anionische Silatropie zu den Ethylendiaminstickstoffatomen gewandert sind (siehe Gleichungen 24 und 31). 


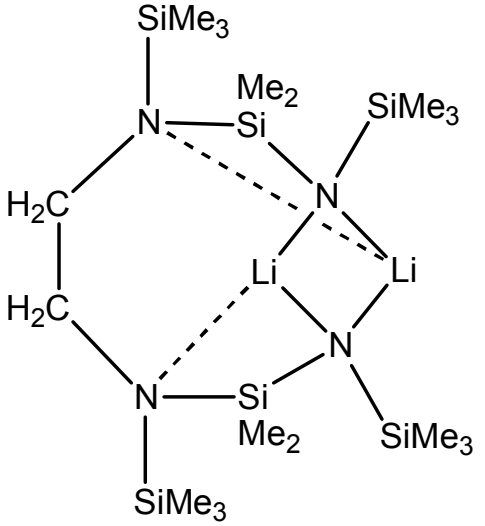

4

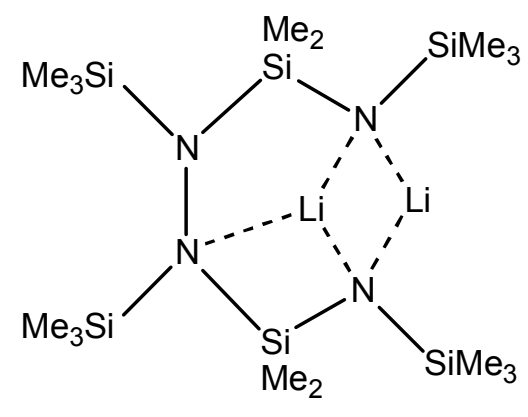

18

Abbildung 60: Dilithiumsalze 4 und 18

Während beide Lithiumionen des monomeren Dilithium-Ethylendiaminsalzes 4 einen intramolekularen Kontakt zum Aminstickstoff haben, ist beim Hydrazinsalz 18 ein Lithiumion zur Methylgruppe eines benachbarten Moleküls koordiniert.
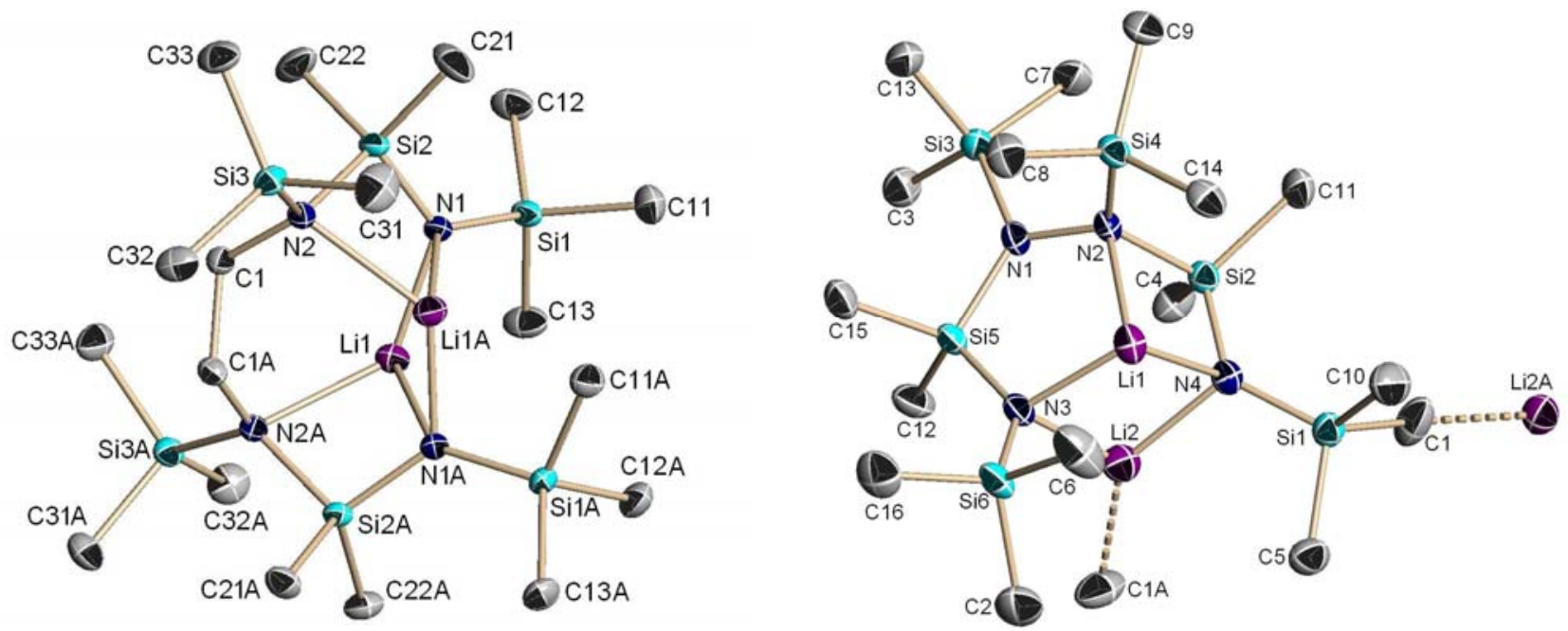

Abbildung 61: Kristallstrukturen der Verbindungen 4 und 18 ohne Wasserstoffatome

Durch Umkristallisation der Dilithiumsalze $\mathbf{4}$ und $\mathbf{1 8}$ aus THF oder TMEDA können Einkristalle gewonnen werden, welche röntgenographisch untersucht wurden. Während die Lithiumionen bei dem asymmetrisch aufgebauten THF-Salze 5 eine Koordination zu beiden Amidstickstoffatomen zeigen (siehe Abbildung 4), wird der neungliedrige Ring durch Zugabe des Komplexbildners TMEDA aufgebrochen, sodass ein symmetrisch aufgebautes Dilthiumsalz isoliert werden kann (6). Die Lithiumionen sind bei Verbindung 6 jeweils zu nur einem Amidstickstoff koordiniert. 

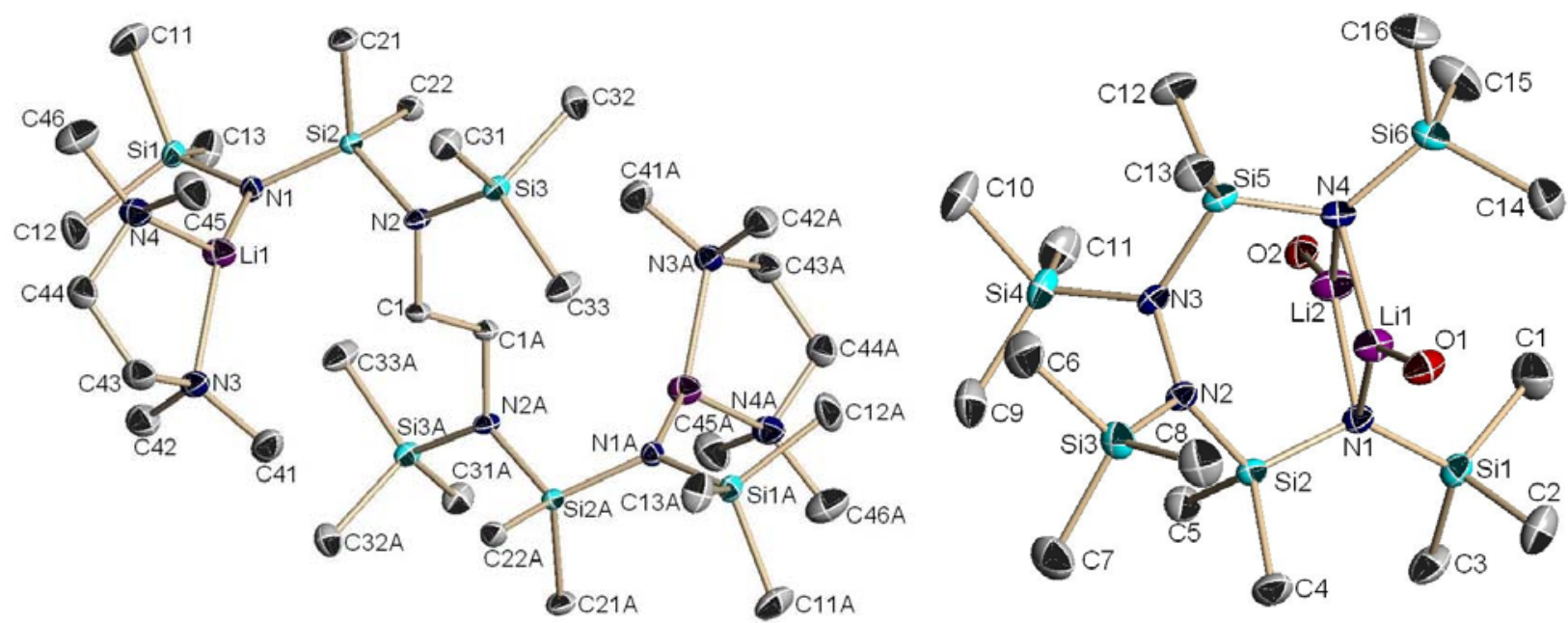

Abbildung 62: Kristallstrukturen der Verbindungen 6 und 19 ohne Wasserstoffatome und Methylgruppen des THF

Durch Reaktion des Dilithiumsalzes 5 mit Halogenboranen und -silanen werden Bis(1,1,3-trisila-2-aza)- (8 - 10) und Bis(1-bora-1,3-disila-2-aza)bis(silyl)ethylendiamine (11 - 13) erhalten (siehe Gleichung 27), welche z.T. röntgenographisch untersucht wurden.

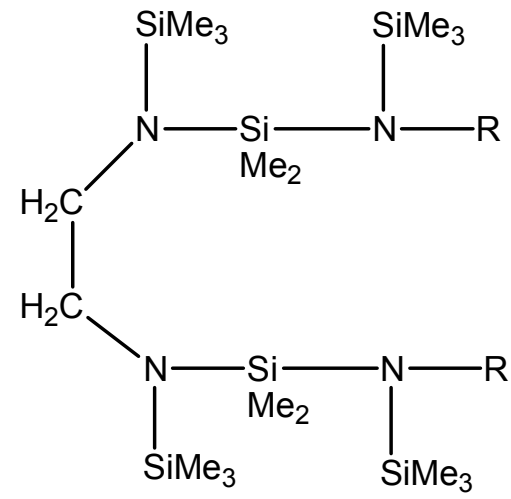

$8-13$

\begin{tabular}{c|c|c|c|c|c|c|}
\multicolumn{2}{c}{8} & $\mathbf{9}$ & $\mathbf{1 0}$ & $\mathbf{1 1}$ & 12 & 13 \\
\hline $\mathrm{R}$ & $\mathrm{F}_{2} \mathrm{SiCMe}_{3}$ & $\mathrm{FSiMe}_{2}$ & $\mathrm{SiMe}_{3}$ & $\mathrm{FBN}\left(\mathrm{CMe}_{2} \mathrm{CH}_{2}\right)_{2} \mathrm{CH}_{2}$ & $\mathrm{FBN}\left(\mathrm{SiMe}_{3}\right)_{2}$ & $\mathrm{FBN}\left(\mathrm{CHMe}_{2}\right)_{2}$ \\
$\mathrm{Hal}$ & $\mathrm{F}$ & $\mathrm{F}$ & $\mathrm{Cl}$ & $\mathrm{F}$ & $\mathrm{F}$ & $\mathrm{F}$
\end{tabular}




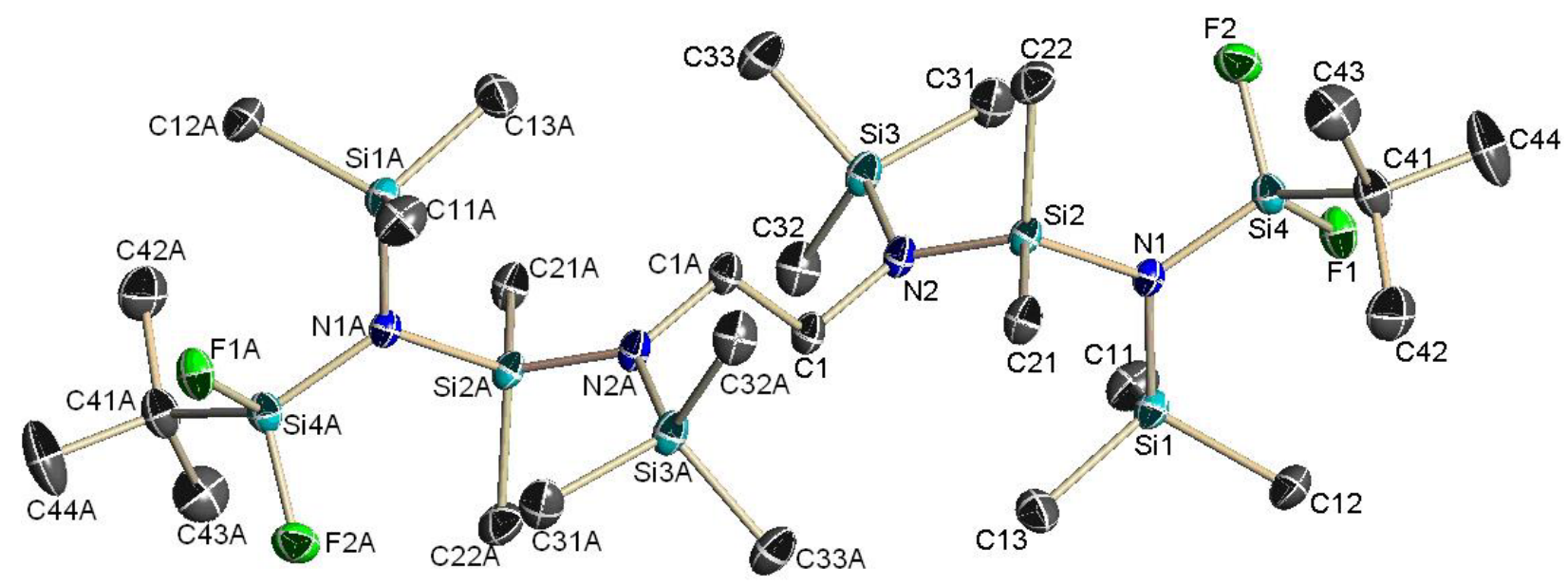

Abbildung 64: Kristallstruktur von 8 ohne Wasserstoffatome

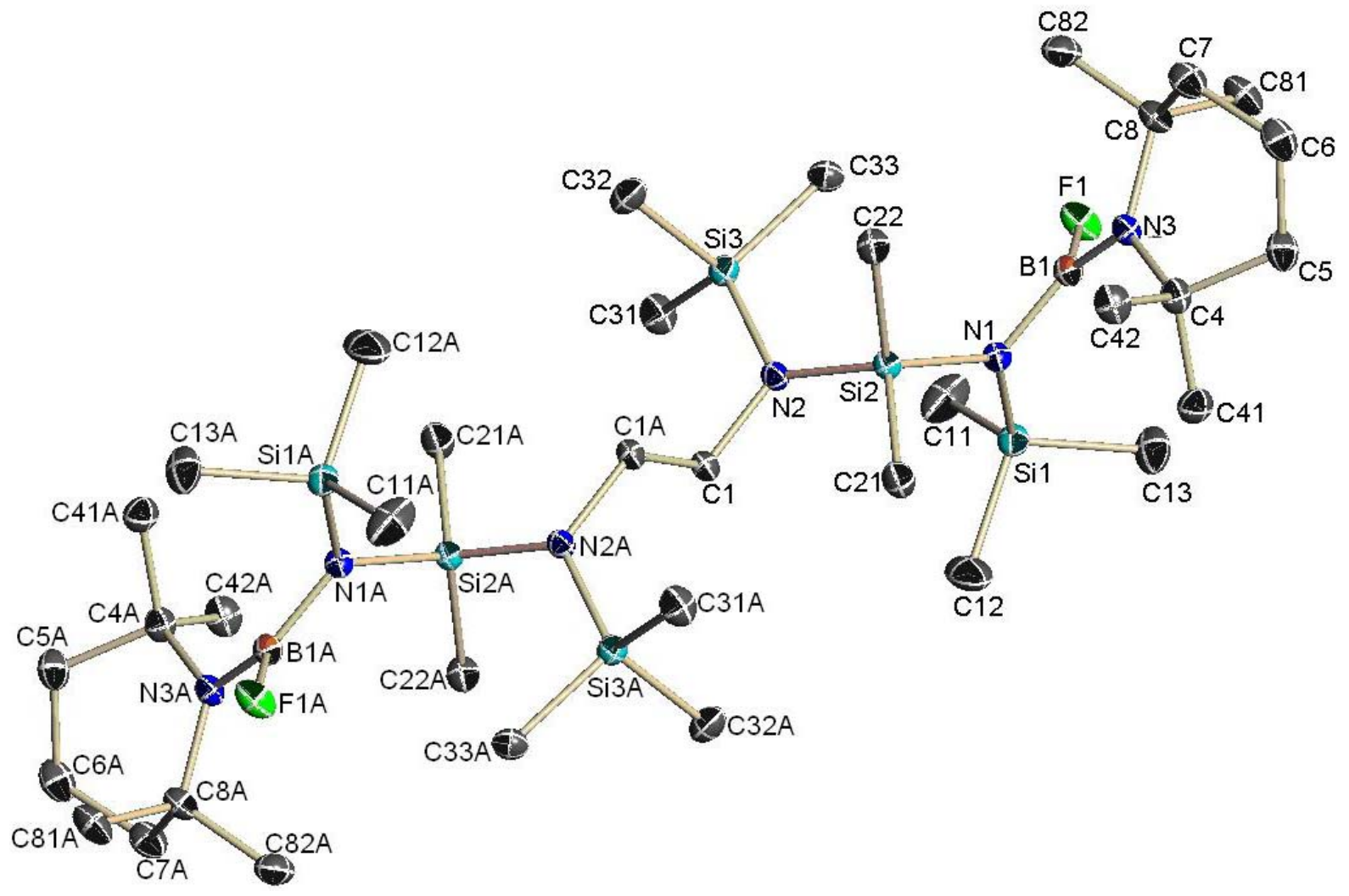

Abbildung 65: Kristallstruktur von 11 ohne Wasserstoffatome

Wird das $N, N^{\prime}$-Bis[(1-fluorboryl-1,1,5,5-tetramethylpiperidino)-1-trimethylsilyl-2-aza-3dimethylsilyl-trimethylsilyl] ethylendiamin (11) auf $150^{\circ} \mathrm{C}$ erhitzt, kommt es zur Fluorierung der Dimethylsilygruppe unter Abspaltung eines Iminoborens, dessen Dimer (14) isoliert und röntgenstrukturanalytisch untersucht werden konnte (siehe Gleichung 28). 


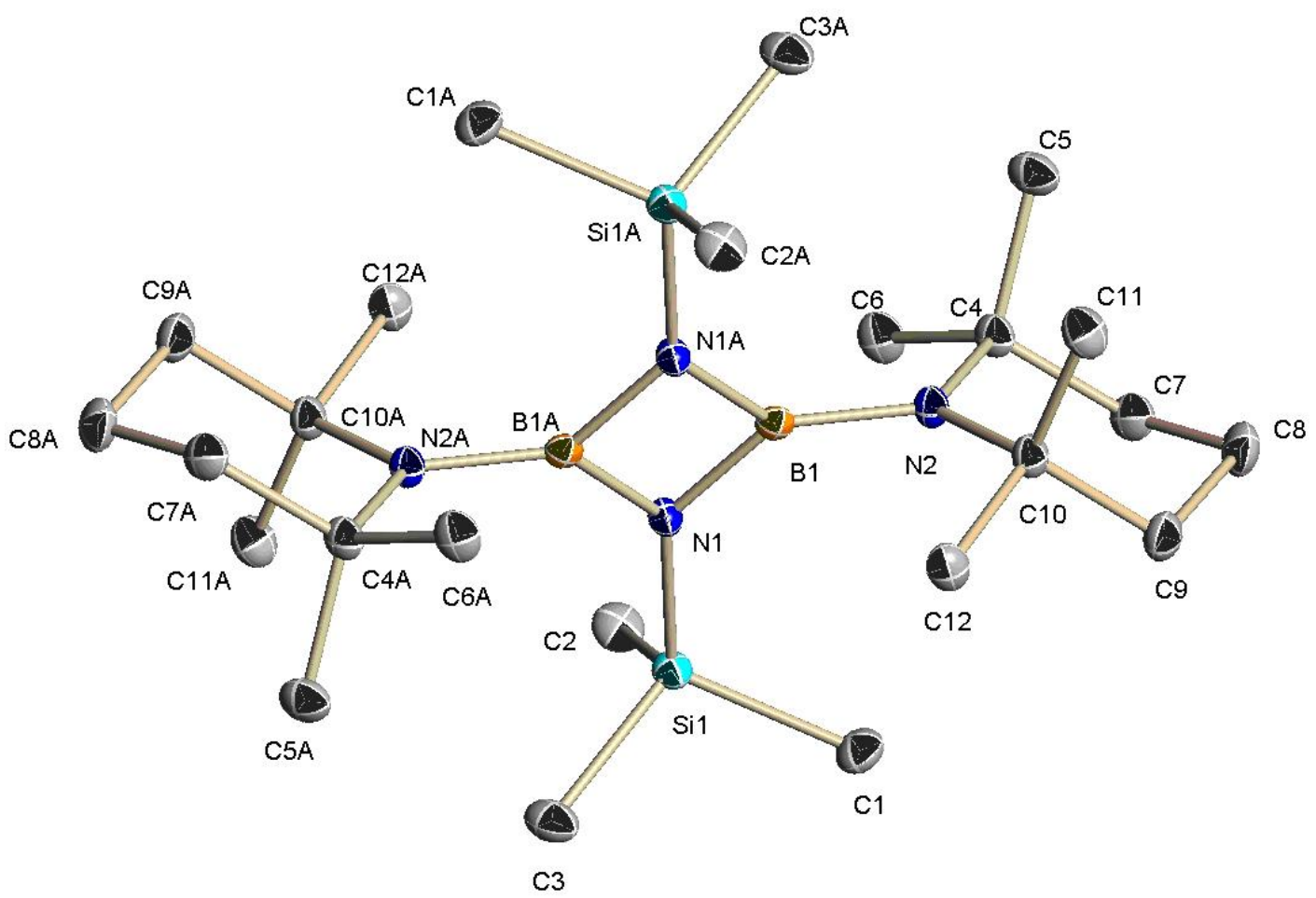

Abbildung 66:Kristallstruktur von 14 ohne Wasserstoffatome

Neben der Synthese offenkettiger Borazine und Silazane können bei Umsetzungen der Dilthiumsalze $\mathbf{4}$ und $\mathbf{1 8}$ mit Tetrafluorsilan bzw. Trifluorboran die cyclischen Verbindungen 15, 16, 21 und 22 erhalten werden (siehe Gleichungen 29 und 34).

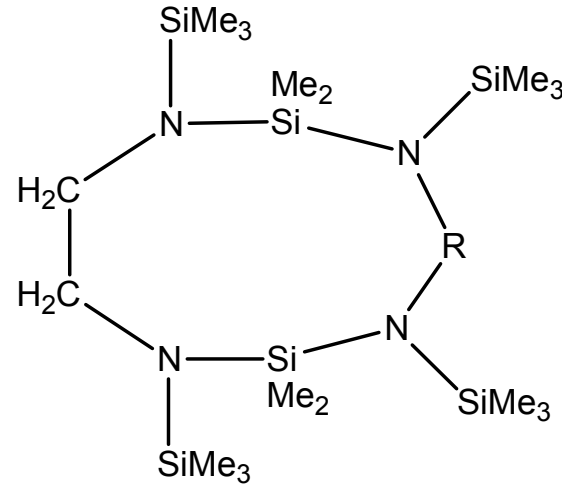

$\mathrm{R}=\mathrm{BF}(\mathbf{1 5}), \mathrm{SiF}_{2}(\mathbf{1 6})$

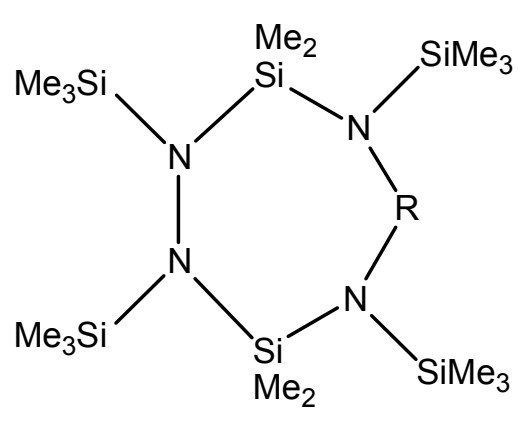

$\mathrm{R}=\mathrm{BF}(\mathbf{2 1}), \mathrm{SiF}_{2}(\mathbf{2 2})$

Abbildung 67: Cyclononane $(15,16)$ und Cycloheptane (21 und 22) 


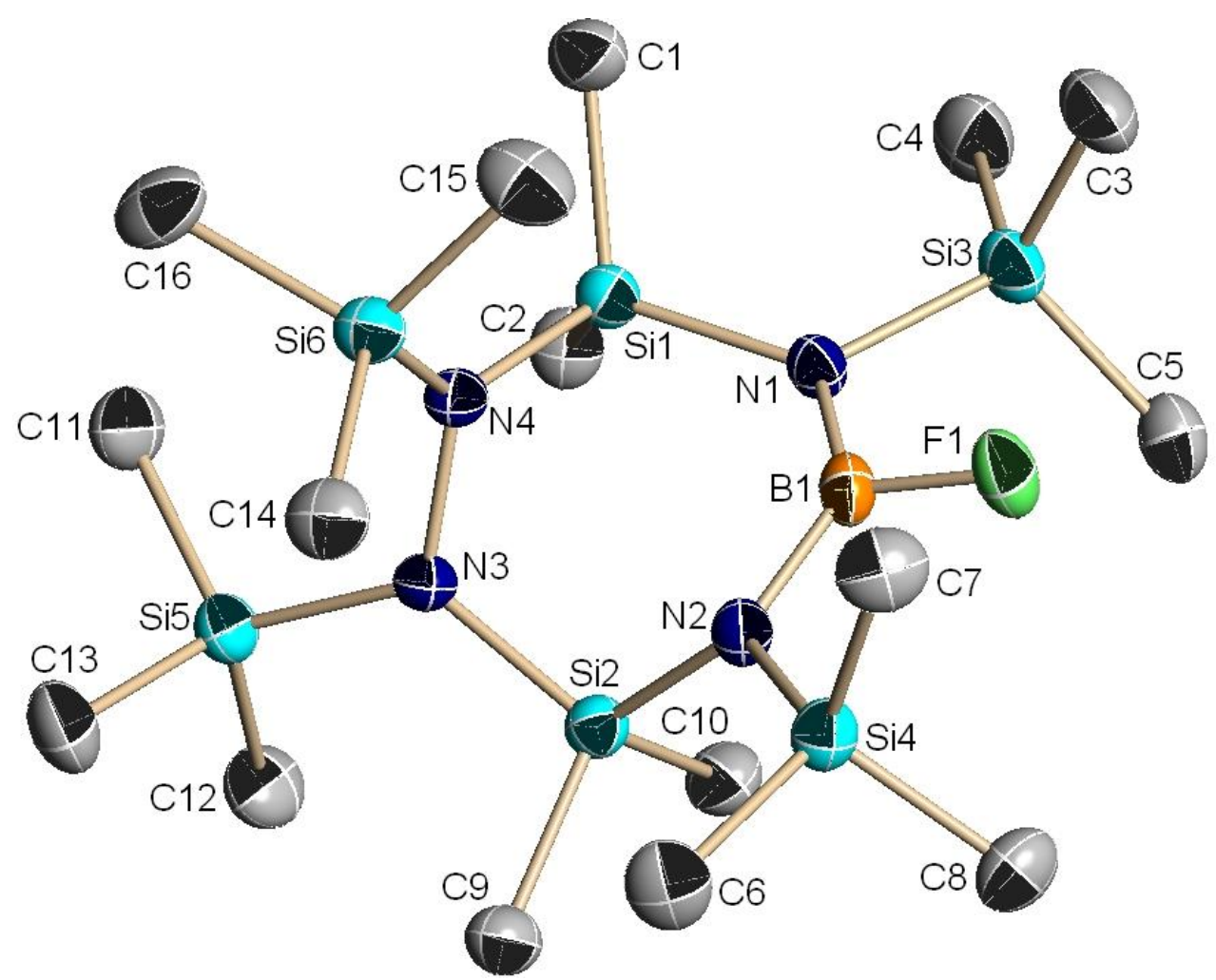

Abbildung 68: Kristallstruktur von 21 ohne Wasserstoffatome

Verbindung 21 zeigt in Diethylether eine reversible Farbänderung. Während sich bei ESR-spektroskopischen Messungen der farblosen Probe kein Signal findet, wird bei der blaugrünen Lösung $\left(\mathbf{2 1}_{\mathrm{E} 2}\right)$ das Auftreten eines Signals in einem komplizierten ESR-Spektrum beobachtet.

Trimethylalan und Fluorborane reagieren jeweils quantitativ mit lithiiertem Di(tertbutyl)fluorsilylamid.

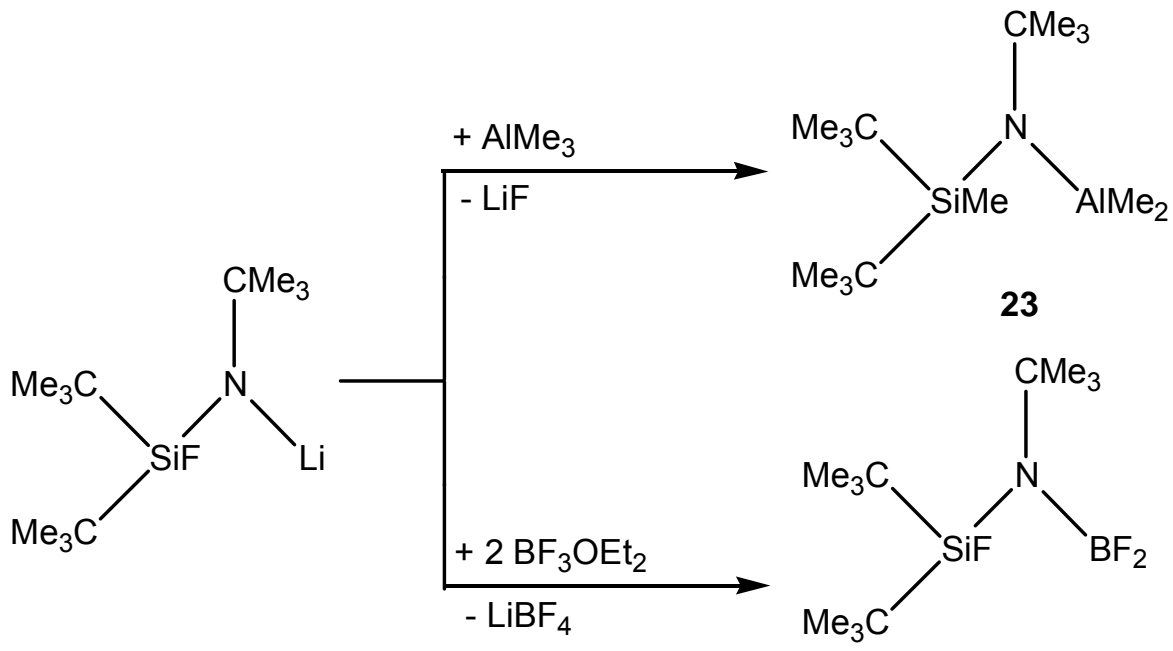


Gleichung 67: Synthese der Verbindungen 23 und 26

Wird das Hydrolyseprodukt von 23 mit $n$-Butyllithium metalliert, kann durch Umkristallisation aus $n$-Hexan das dimere Lithiumsalz isoliert werden konnte, welches Lithium in einer Zweifachkoordination zeigt.

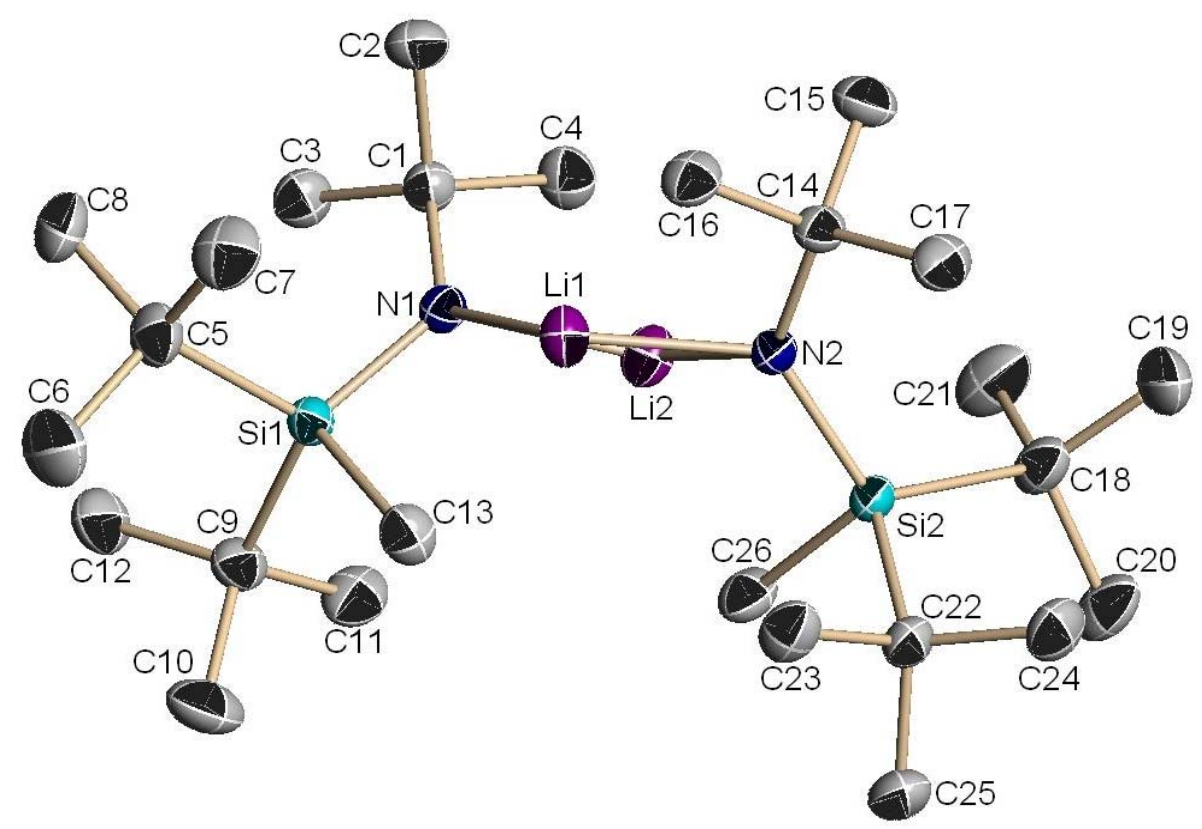

Abbildung 69: Kristallstruktur von 25 ohne Wasserstoffatome

Wird Lithium-bis[di(tert-butyl)fluorsilyl]]amid mit Trimethylalan, Trichloralan bzw. Chlortrimethylsilan in THF umgesetzt, können statt der erwarteten Iminosilen(addukt)e die, unter Methanidionwanderung (28) bzw. Umchlorierung $(29,30)$ entstandenen, Bis[di(tert-butyl)silyl]amine isoliert werden (siehe Schema 2).
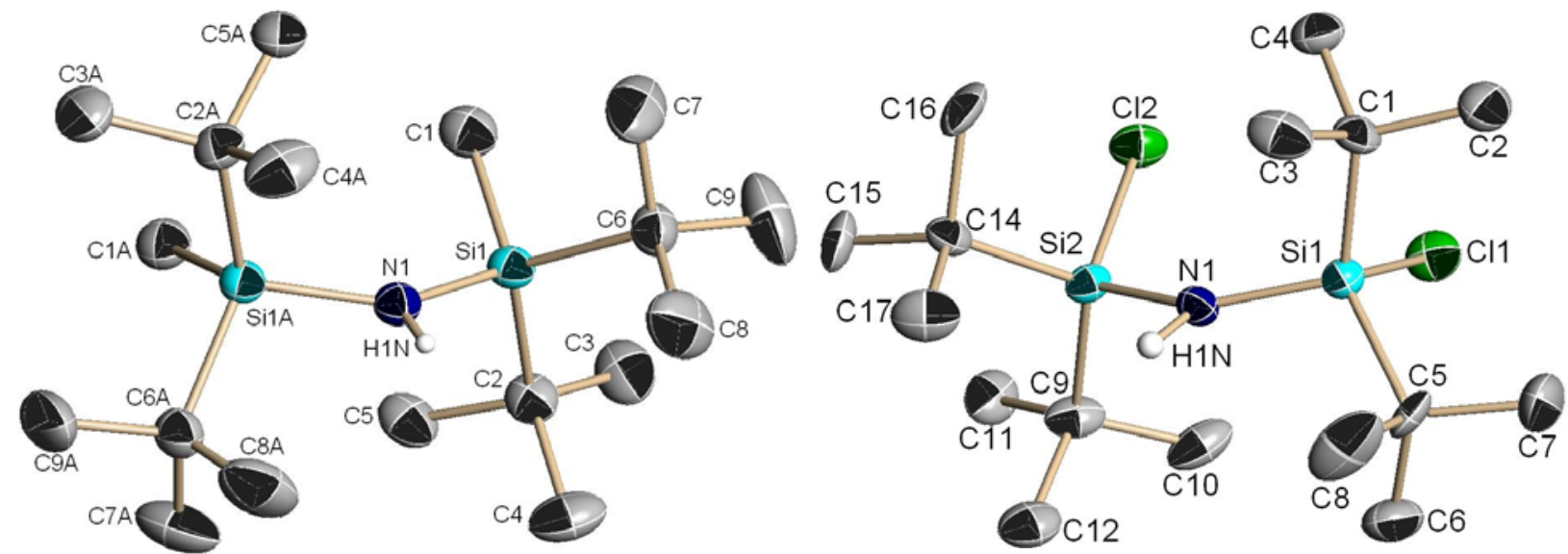

Abbildung 70: Kristallstrukturen der Verbindungen 28 und 29 ohne Methylwasserstoffatome 
Als Vorstufe zur Synthese möglicher Lithium-fluorborylamide wurden Moleküle mit der Struktureinheit RNH-BFR' synthetisiert und z. T. kristallographisch untersucht (siehe Gleichungen 40 - 42).
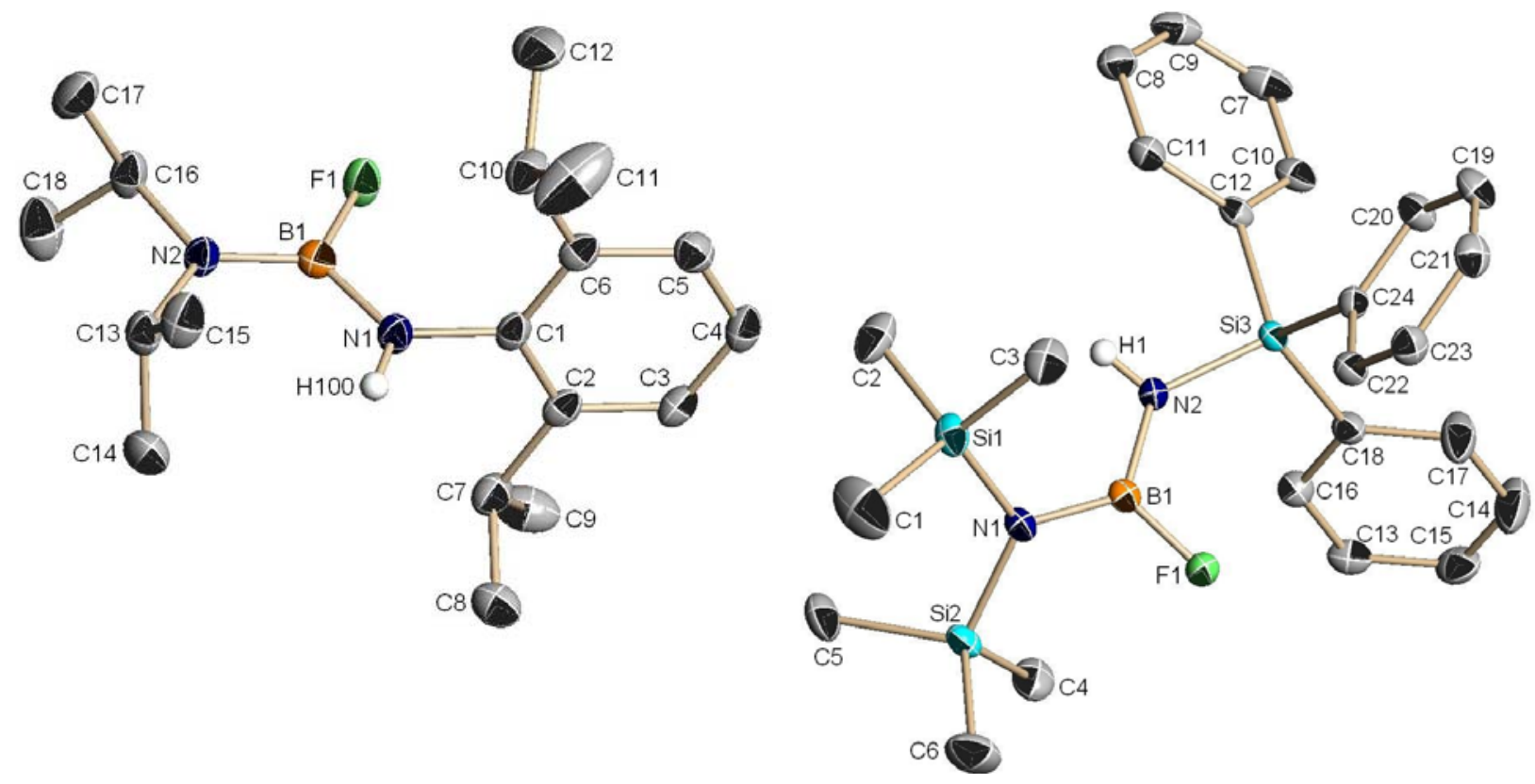

Abbildung 71: Kristallstrukturen der Verbindungen 33 und $\mathbf{3 7}$ ohne $\mathrm{CH}$-Wasserstoffatome

Die Metallierung mit Lithiumorganylen liefert ausschließlich die Organylsubstitutionsprodukte, Iminoborene oder deren Oligomere. Die Lithiumfluoridabspaltungen werden bereits bei $-50{ }^{\circ} \mathrm{C}$ beobachtet.

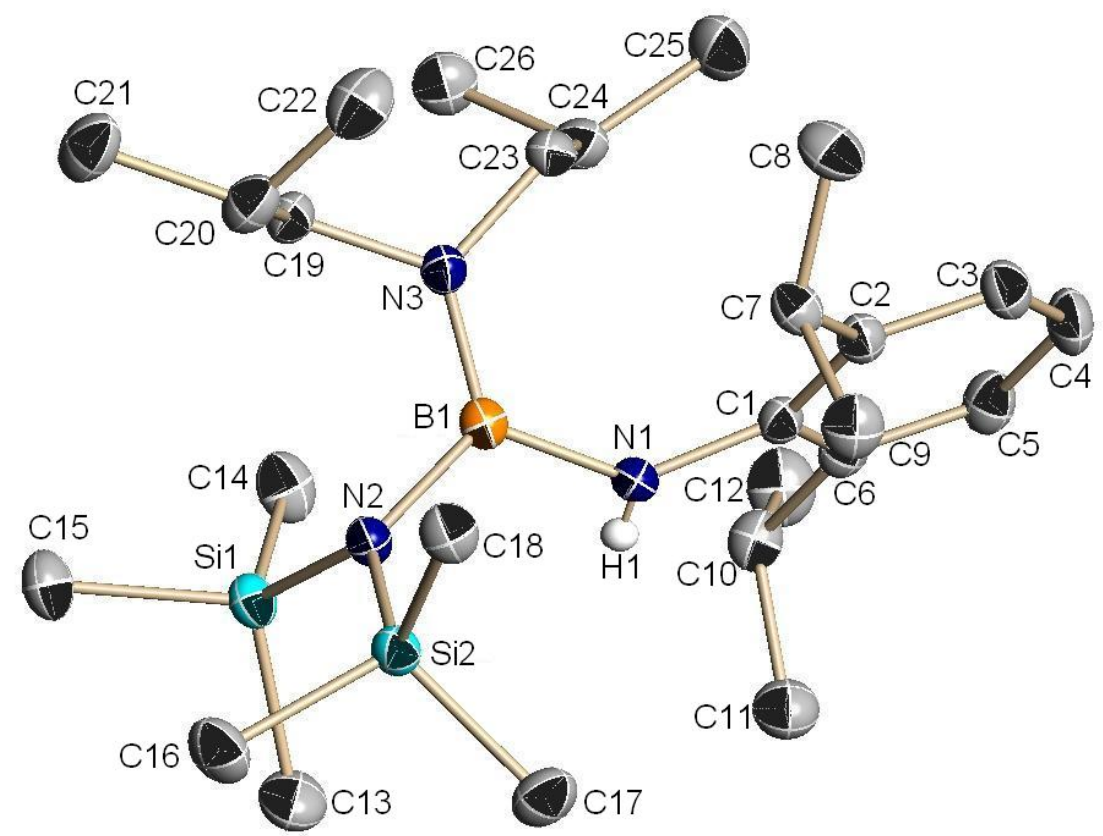

Abbildung 72: Kristallstruktur von 35 ohne $\mathrm{CH}$-Wasserstoffatome 
Wird lithiiertes tert-Butyldimethylsilylamid mit Difluor-1,1,5,5-tetramethylpiperidinoboran bzw. 2,4,6-Tri(tert-butyl)phenyl-difluor-boran umgesetzt, können, auf Grund einer auftretenden Umlithiierung, neben den Fluorboranen die jeweiligen Iminoborene $\mathbf{4 3}$ und $\mathbf{4 4}$ als Reaktionsprodukte isoliert werden (siehe Gleichung 43).
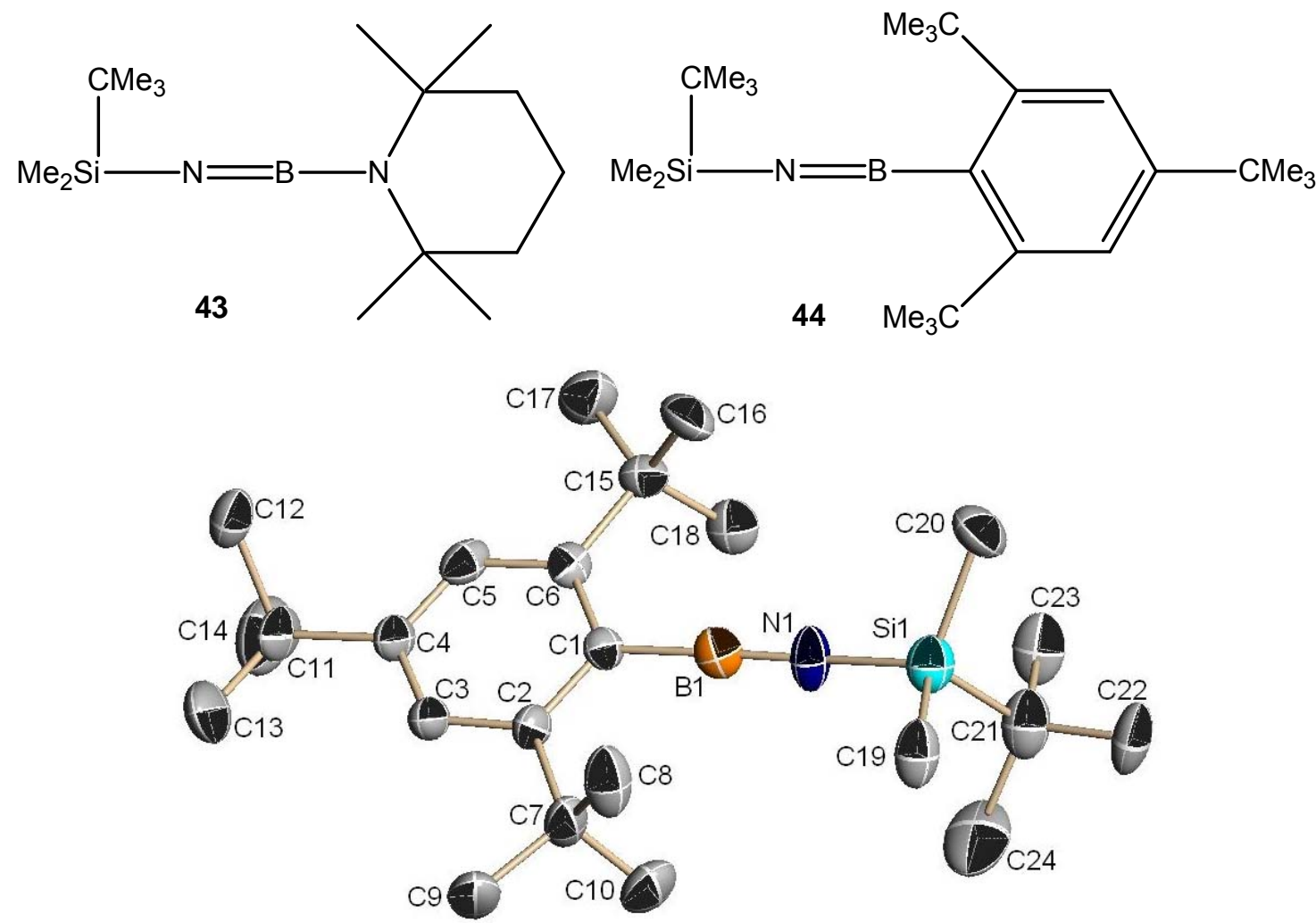

Abbildung 73: Iminoborene (43 und 44) sowie Kristallstruktur von $\mathbf{4 4}$ ohne Wasserstoffatome

Lewis-Säuren wie $\mathrm{H}_{2} \mathrm{O}, \mathrm{MeOH}$ oder $\mathrm{NH}_{3}$ addieren an die $\mathrm{B}-\mathrm{N}-\mathrm{Bindung}$.

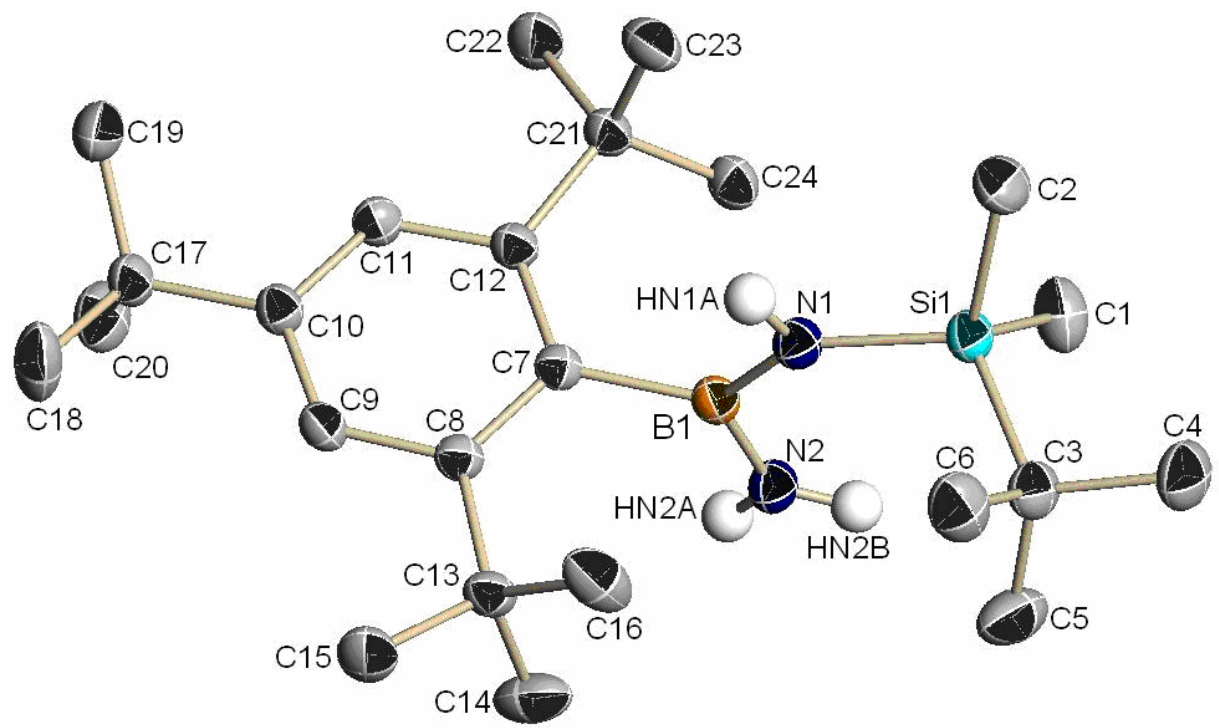


Anstelle einer Kationenbildung wird bei der Reaktion von Fluor-bis[bis(trimethylsilyl)amino]-boran mit Trichloralan eine Fluortrimethylsilanabspaltung bei $35^{\circ} \mathrm{C}$ beobachtet. Es konnte das Bis(trimethylsilyl)amino-trimethylsilylimino-boren als $\mathrm{AlCl}_{3}$ Addukt isoliert und röntgenstrukturanalytisch untersucht werden.

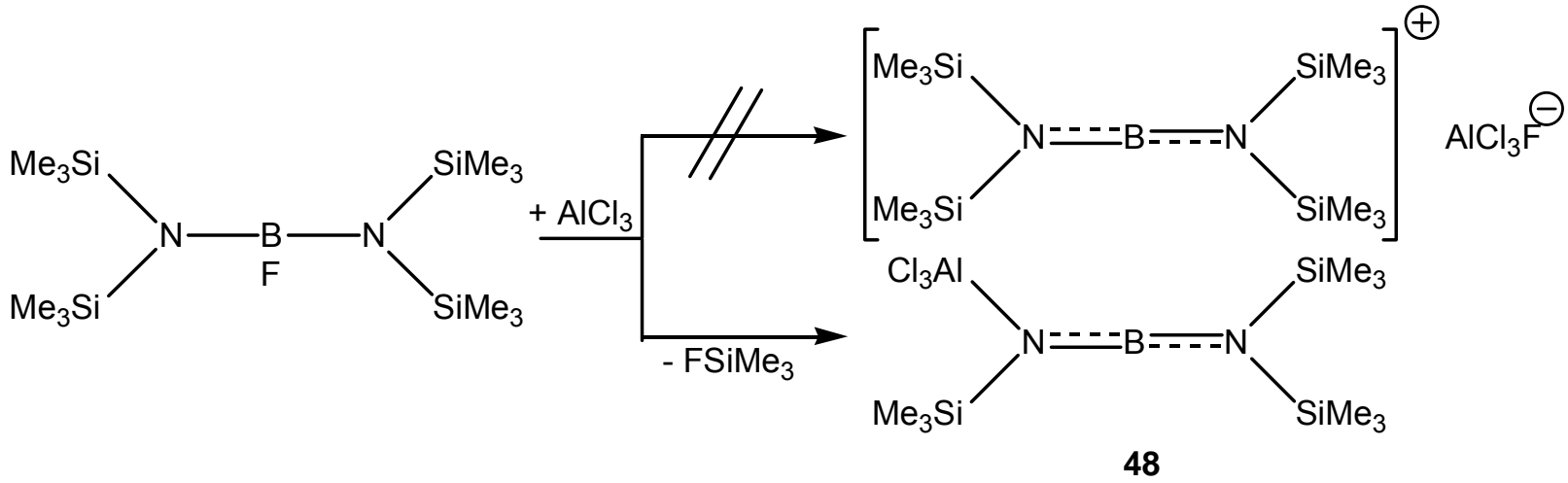

Gleichung 68: Synthese von 48

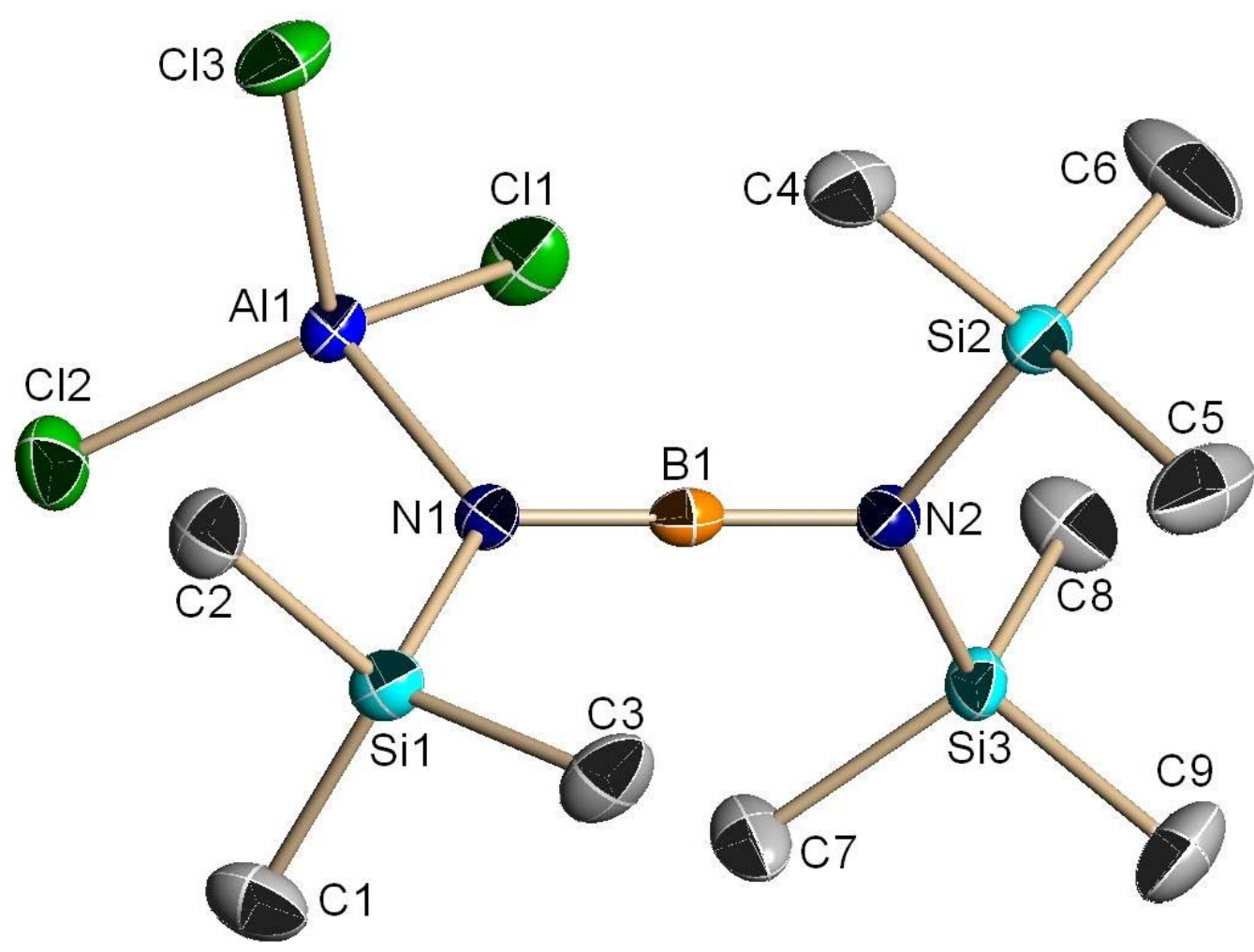

Abbildung 75: Kristallstruktur von 48 ohne Wasserstoffatome

DFT-Rechnungen zeigen einen exothermen Reaktionsverlauf. Der Verlauf der Reaktion über lediglich einen Übergangszustand konnte nicht bestätigt werden. Wahrscheinlich findet der Umlagerungsmechanismus über mehrere Stufen statt. 


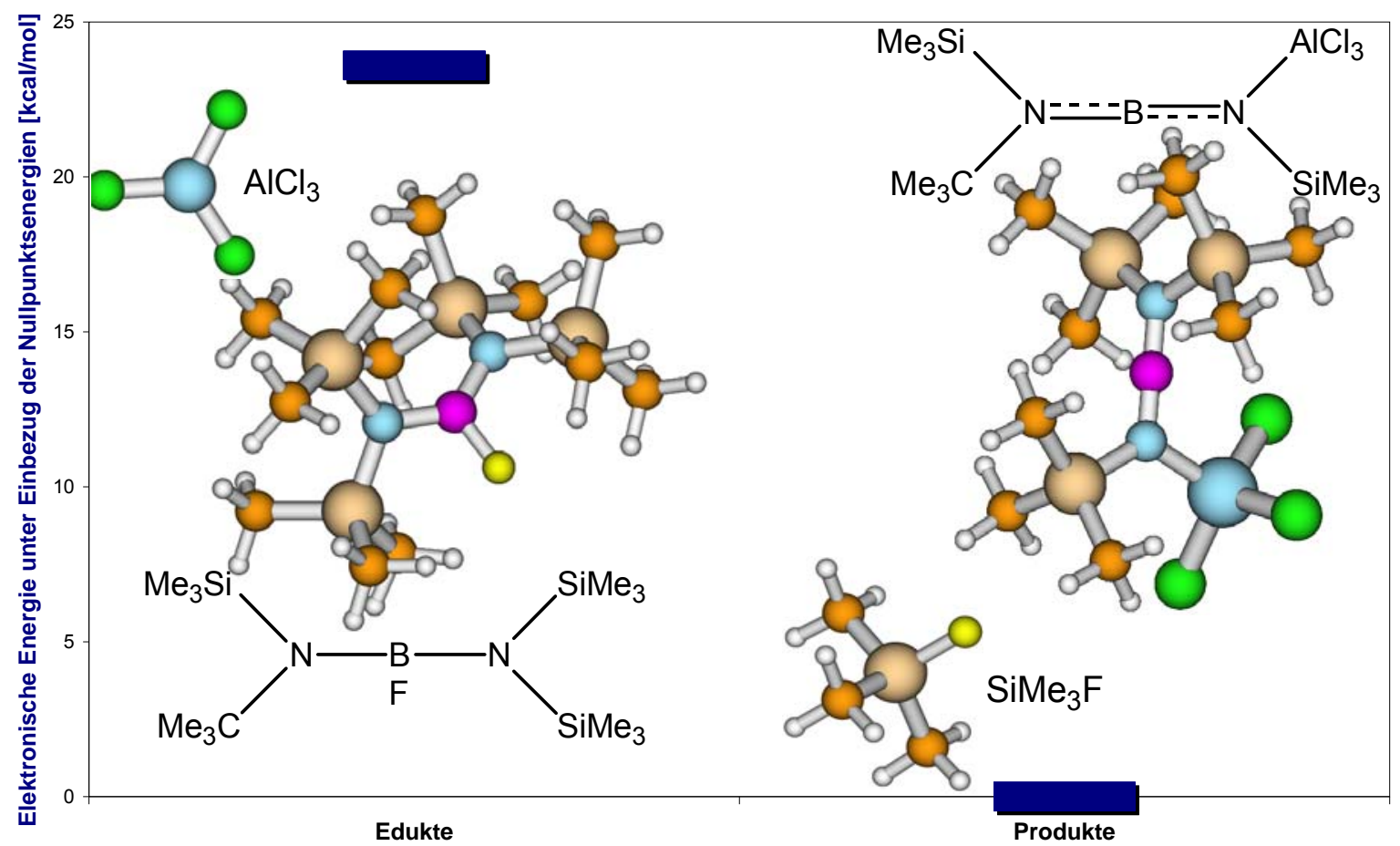

Abbildung 76: Vergleich der Energien der Edukte und Produkte der Synthese von 48

Die katalysierte Fluorsilanabspaltung bei $35^{\circ} \mathrm{C}$ am Fluor-bis[bis(trimethylsilyl)amino]boran gelingt ebenfalls durch eine Reaktion mit $\mathrm{AICIMe}_{2}, \mathrm{AlBr}_{3}$ und $\mathrm{GaCl}_{3}$ (siehe Gleichung 50).

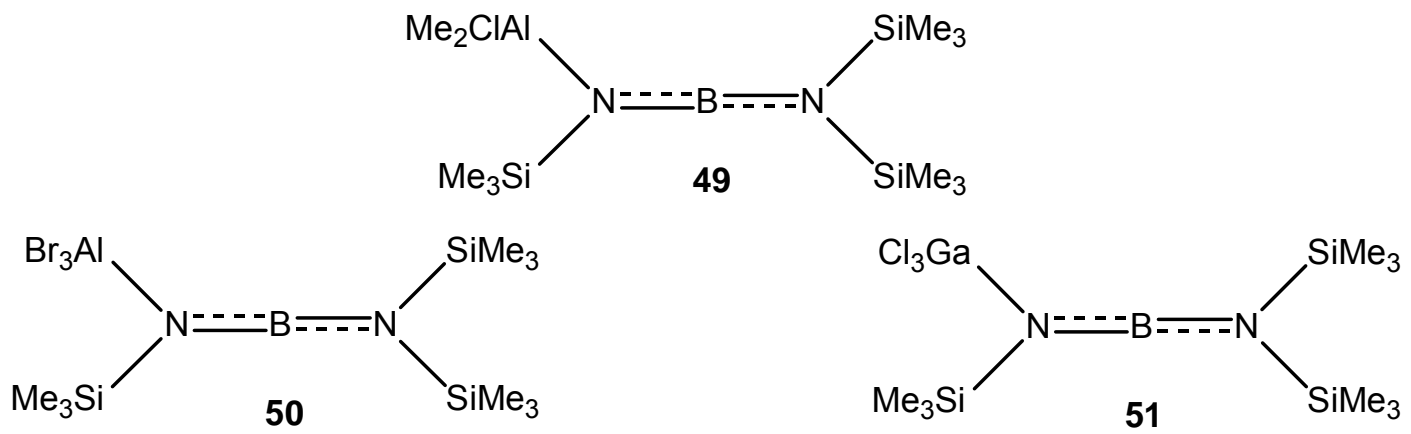

Abbildung 77: Verbindungen 49 - 51

Bei Substitution einer oder mehrerer Trimethylsilylgruppen durch zwei tertButyldimethylsilyleinheiten (52) (siehe Gleichung 51) bzw. einer tert-Butylmethylphenylsilylgruppe (58) am Fluor-bis[bis(trimethylsilyl)amino]-boran, konnten neuartige Fluor-tetrakis(silyl)-borane erhalten werden. 


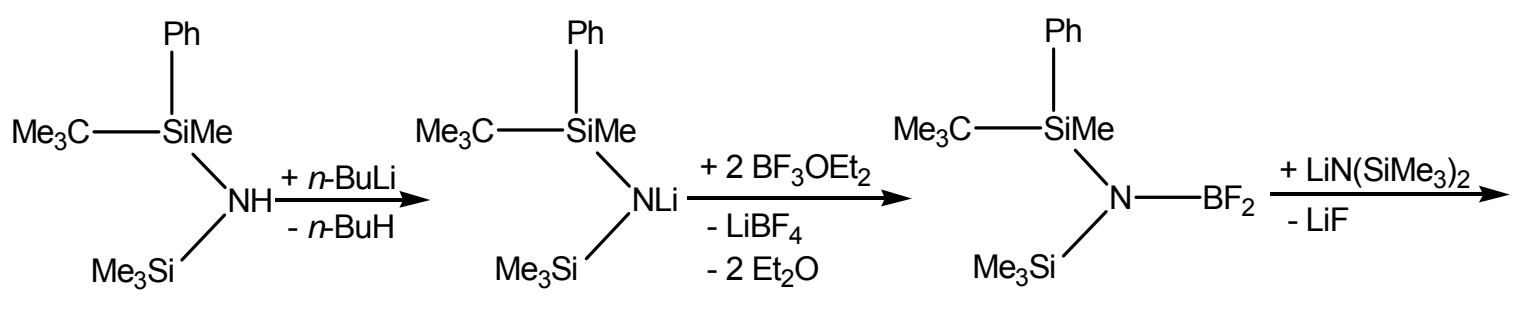

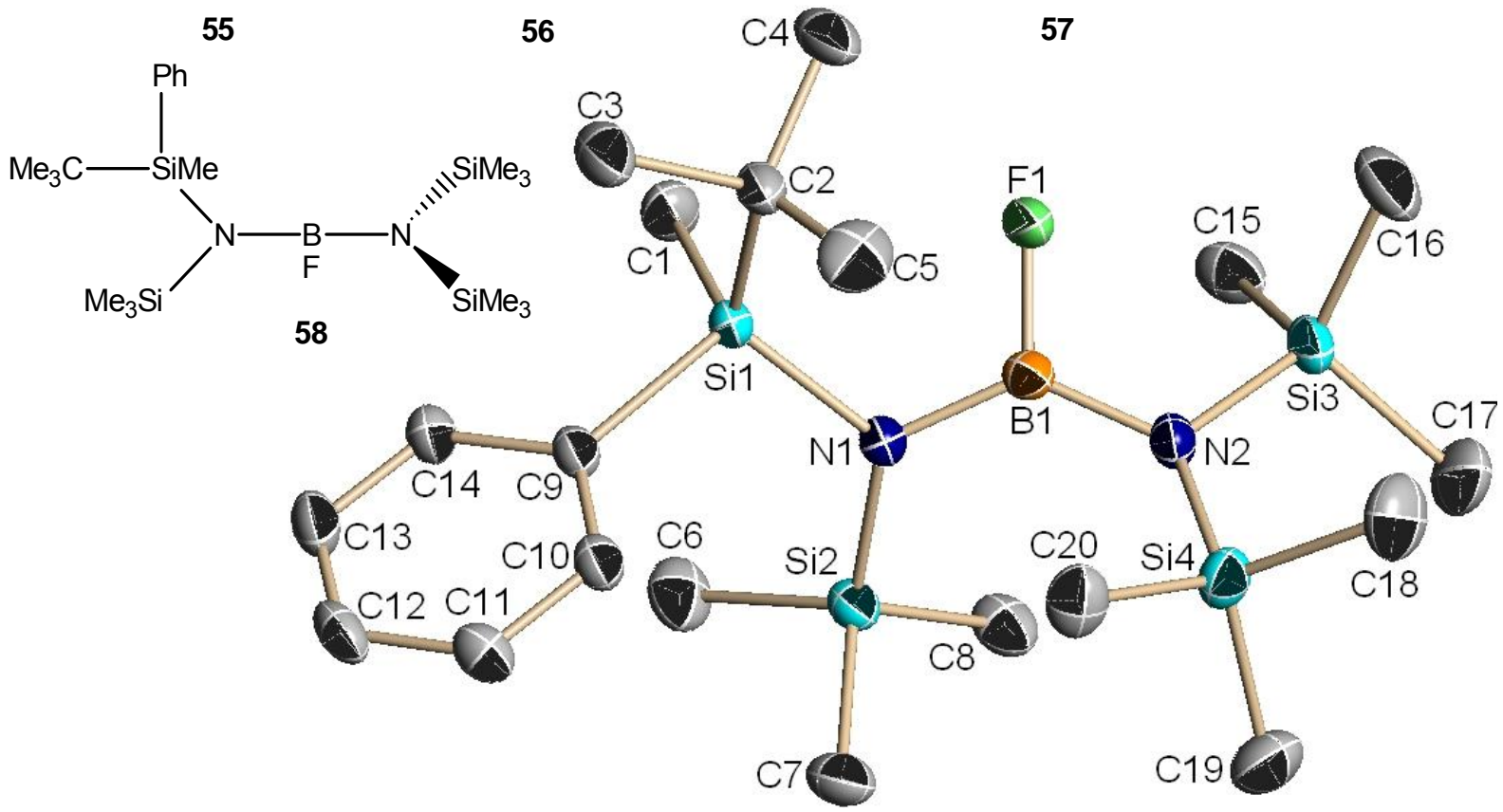

Gleichung 69: Synthese und Kristallstruktur (ohne Wasserstoffatome) von 58

Wird eine Silylgruppe am Fluor-bis[bis(trimethylsilyl)amino]-boran durch eine tertButyleinheit substituiert, kann mit Hilfe von $\mathrm{AlCl}_{3}$ oder $\mathrm{GaCl}_{3}$ ebenfalls eine Fluortrimethylsilaneliminierung bei $35^{\circ} \mathrm{C}$ erreicht werden. Unerwartet wird eine, am Bis(trimethylsilyl)-substituierten Stickstoff sitzende, Trimethylsilylgruppe abgespalten (siehe Gleichung 56).

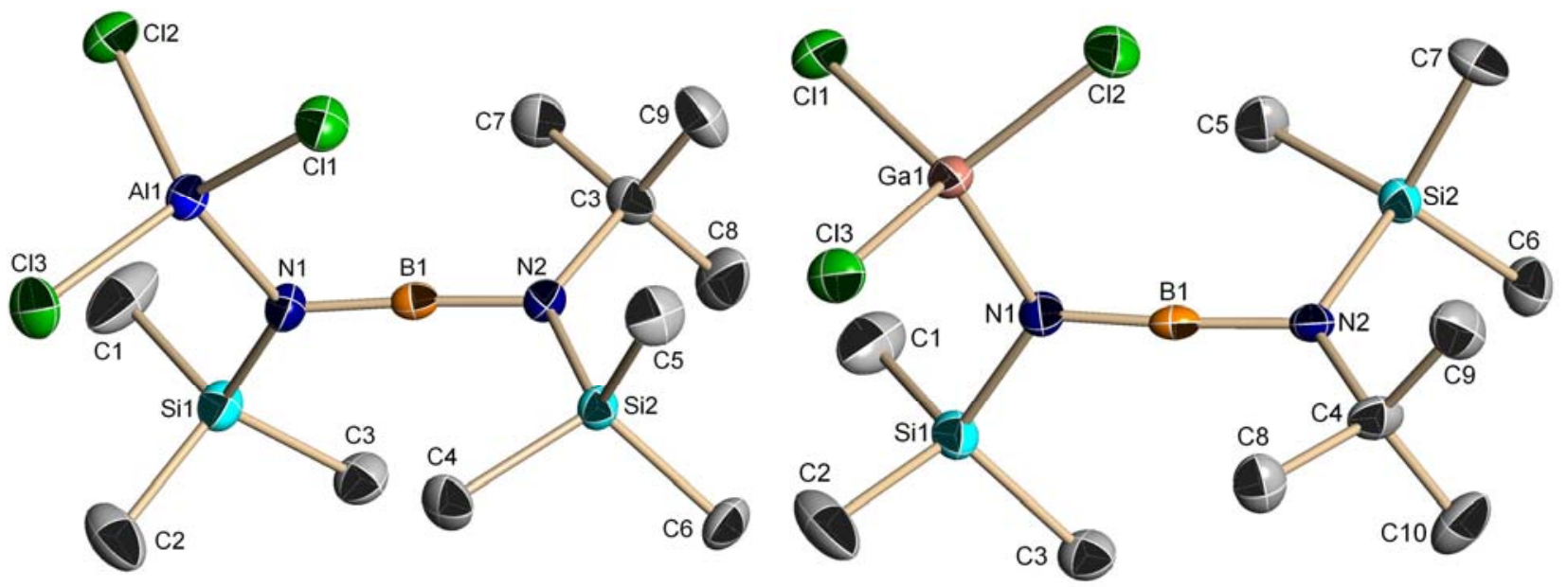

Abbildung 78: Kristallstrukturen der Verbindungen 59 und 60 ohne Wasserstoffatome 
Arylsubstituierte Fluorsilylaminoborane können aus der Reaktion von lithiiertem $N$-Trimethylsilyl-2,6-diisopropylanilin mit Difluorboranen erhalten werden (siehe Gleichung 55).
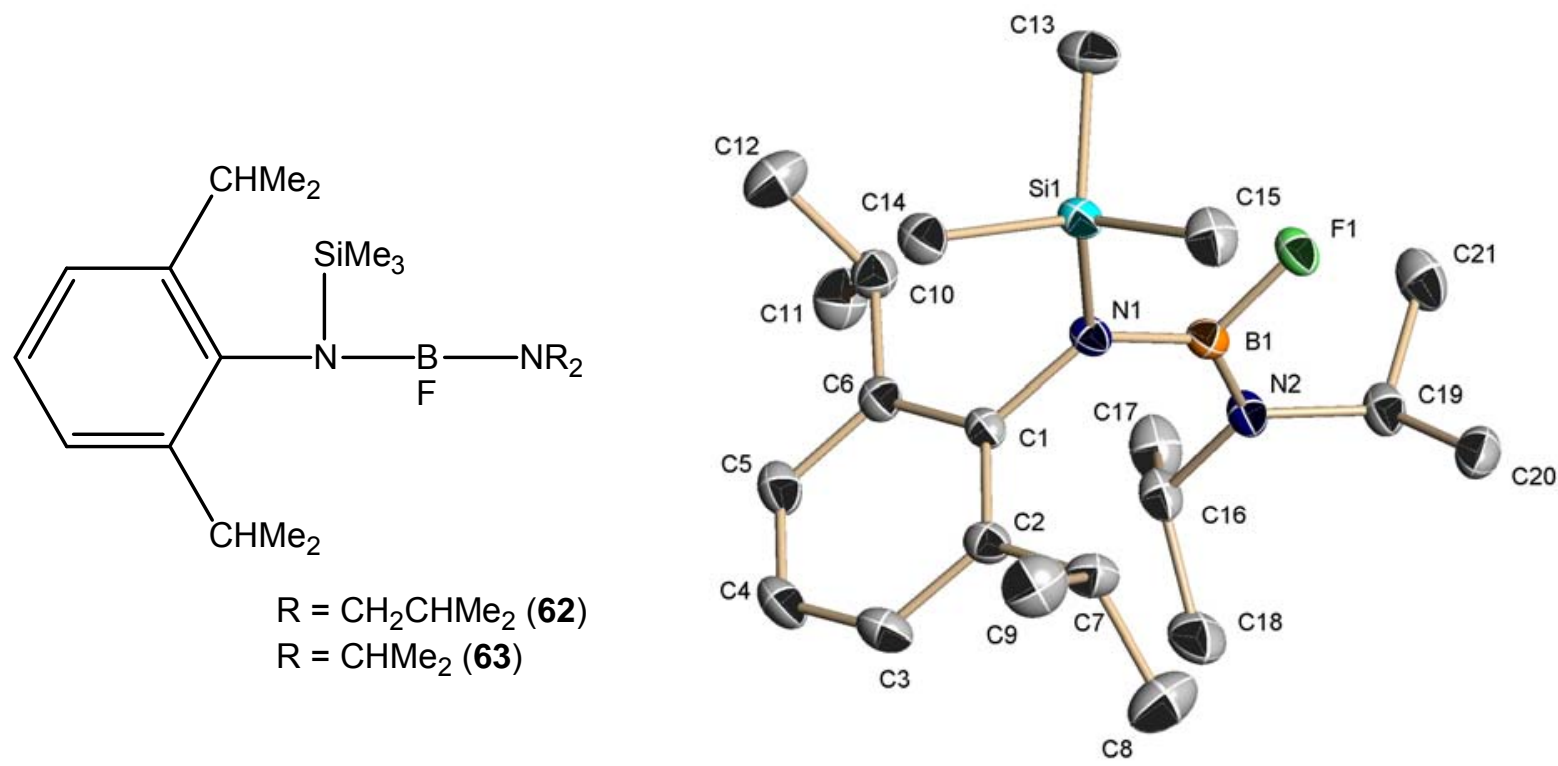

Abbildung 79: Verbindungen 62 und 63 sowie Kristallstruktur von 63 ohne Wasserstoffatome

Bei der Umsetzung von Verbindung 62 mit $\mathrm{AlCl}_{3}$ in Methylenchlorid/ Diethylether konnte das erste strukturell untersuchte, acyclische, unter Umhalogenierung am Bor bzw. Silicium und Protonierung des Aminstickstoffatoms entstandene, BoriniumionAminaddukt isoliert werden (64), welches thermodynamisch instabil ist und zum Di(iso-butyl)amin · trichloralan (65) zerfällt.

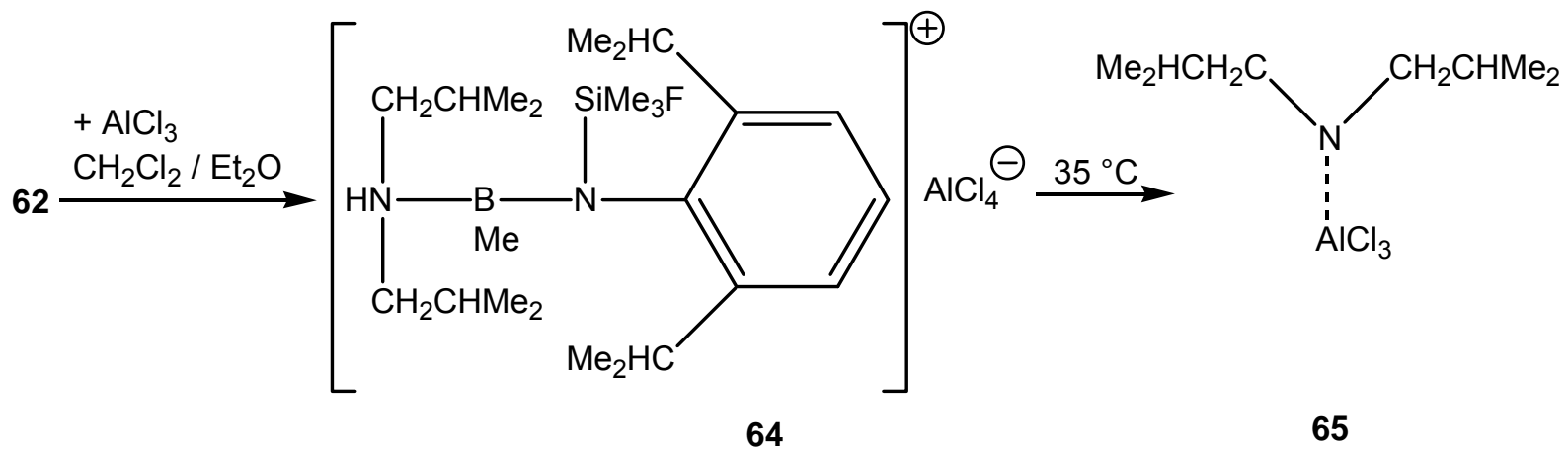



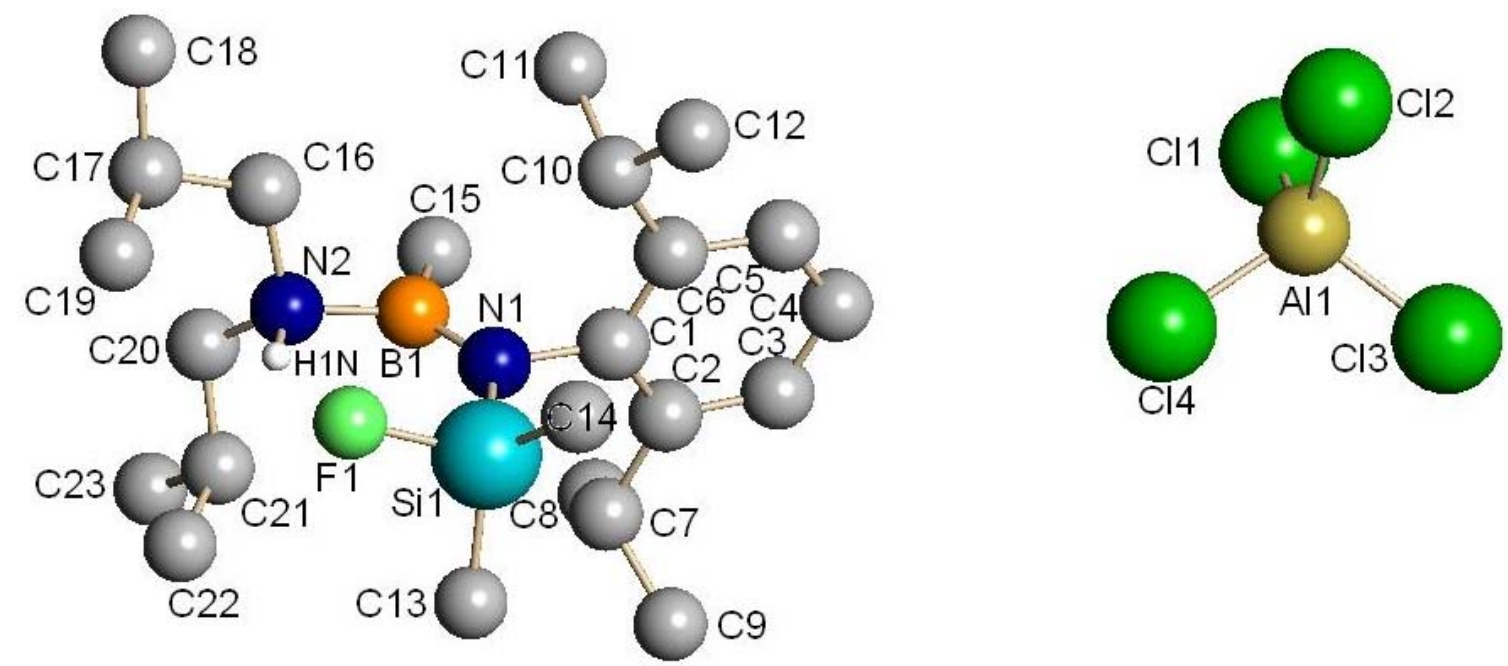

Abbildung 80: Ball-and-stick Modell der Kristallstruktur von 64 ohne CH-Wasserstoffatome

Wird 63 mit Trichloralandiethyletherat unter Ausschluss von Dichlormethan umgesetzt, bildet sich ebenfalls ein, durch Fluortrimethylsilaneliminierung und Diethyletherspaltung entstandenes, Boriniumion-Aminaddukt (66) (siehe Schema 4).

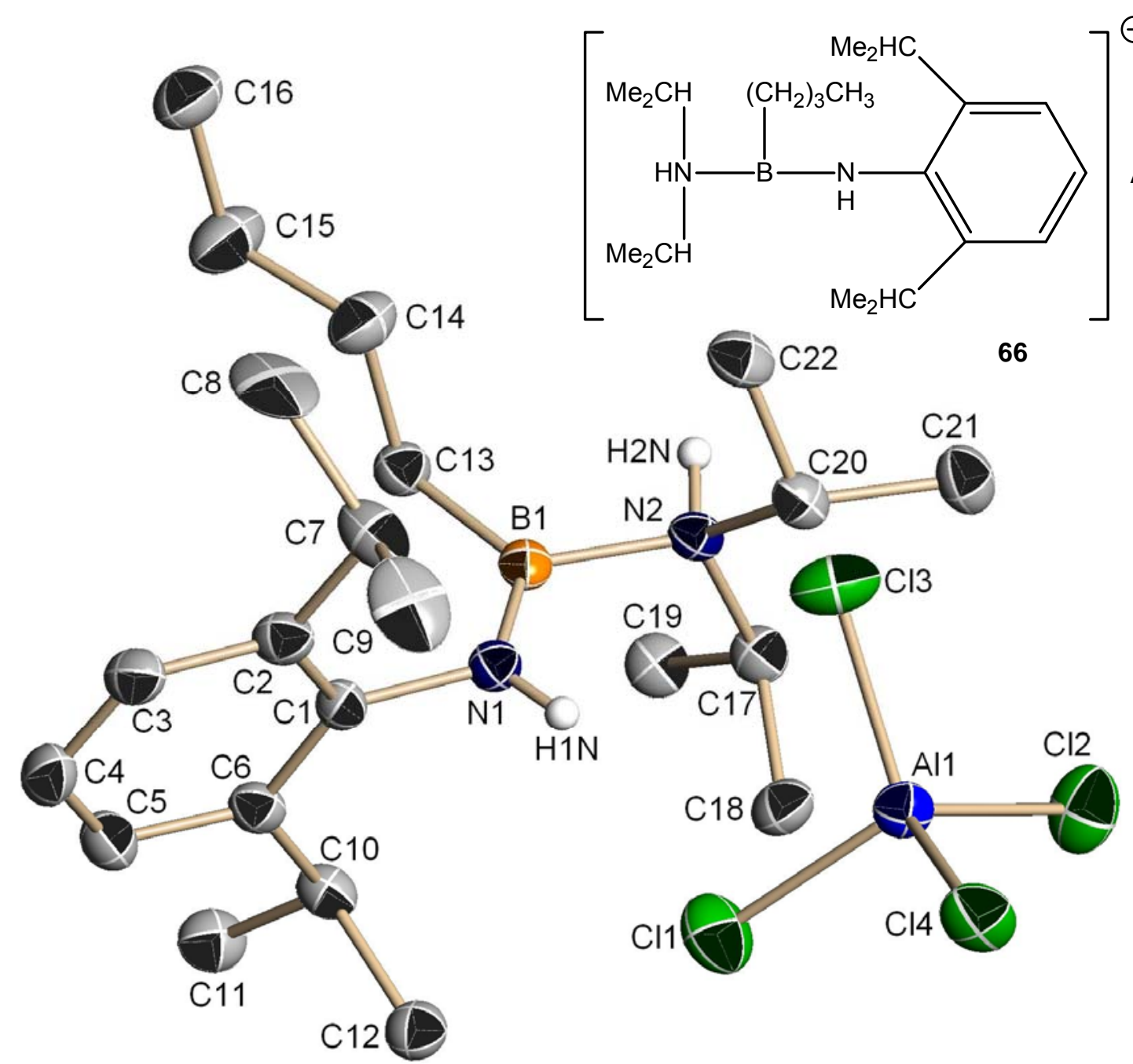

Abbildung 81: Kristallstruktur des Boriniumion-Aminaddukts 66 
Ein ähnlich unerwartetes Reaktionsverhalten zeigt Verbindung $\mathbf{2 7}$ bei der Umsetzung mit $\mathrm{AlCl}_{3}$ in $\mathrm{CH}_{2} \mathrm{Cl}_{2} / \mathrm{Et}_{2} \mathrm{O}$. Auch hier finden eine Umhalogenierung am Silicium und eine Protonierung am Aminstickstoff statt (siehe Schema 5).

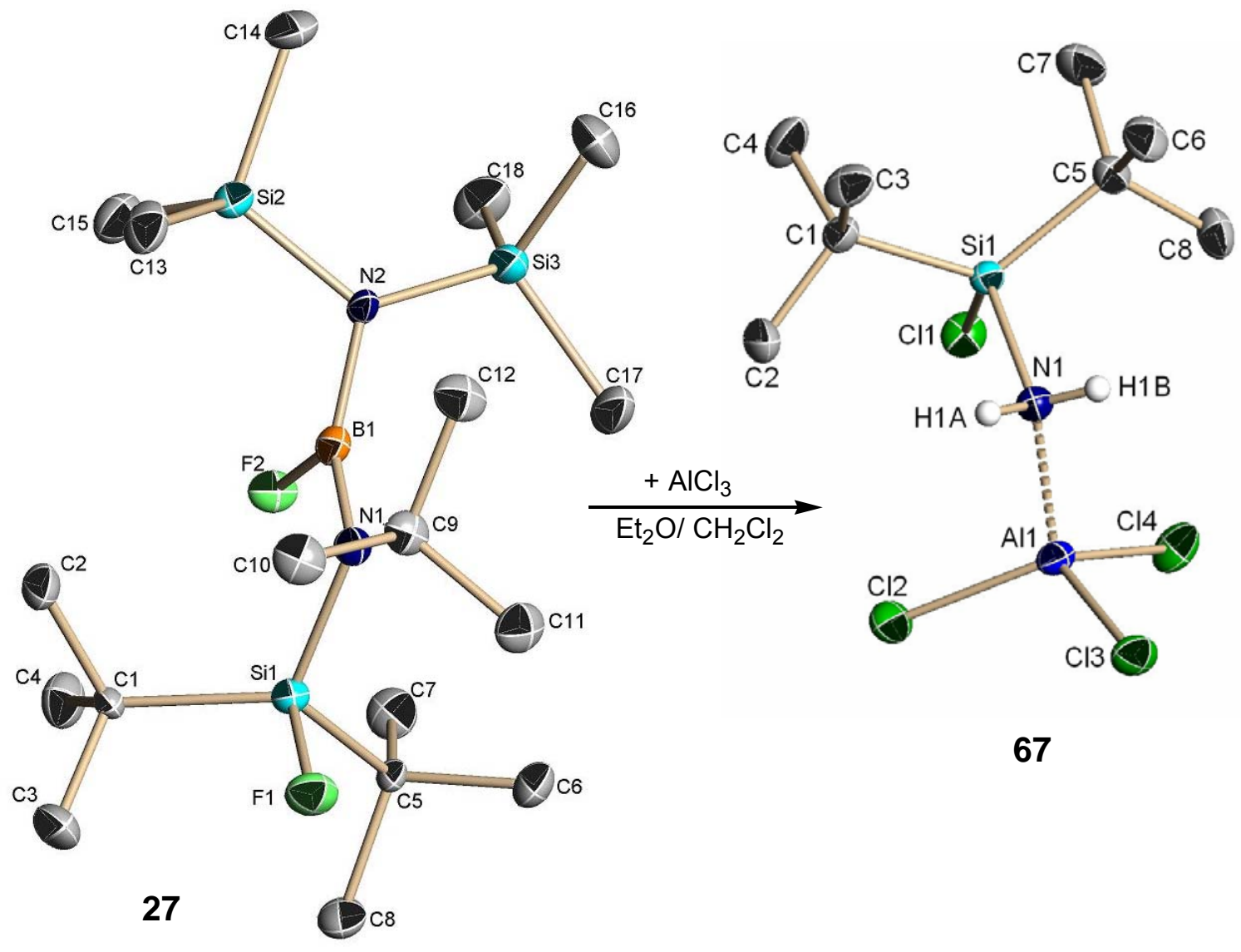

Schema 8: Kristallstruktur von 27 (ohne Wasserstoffatome) sowie

Synthese und Kristallstruktur (ohne Methylwasserstoffatome) von 67

Bis(tert-Butylmethyl)ketazin bildet mit Trifluorboran ein unzersetzt destillierbares Addukt (68). Wird diese Verbindung in Trifluorborandiethyletherat unter Rückfluss erhitzt, kommt es zur Abspaltung von Fluorwasserstoff.

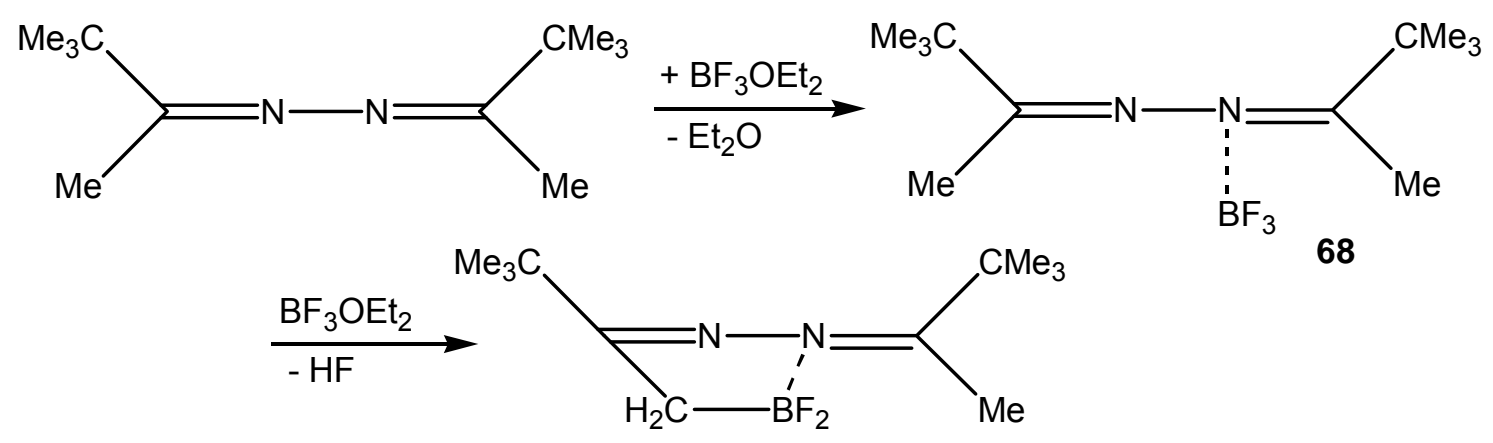

69

Gleichung 66: Synthese von 68 und $69^{[76]}$ 


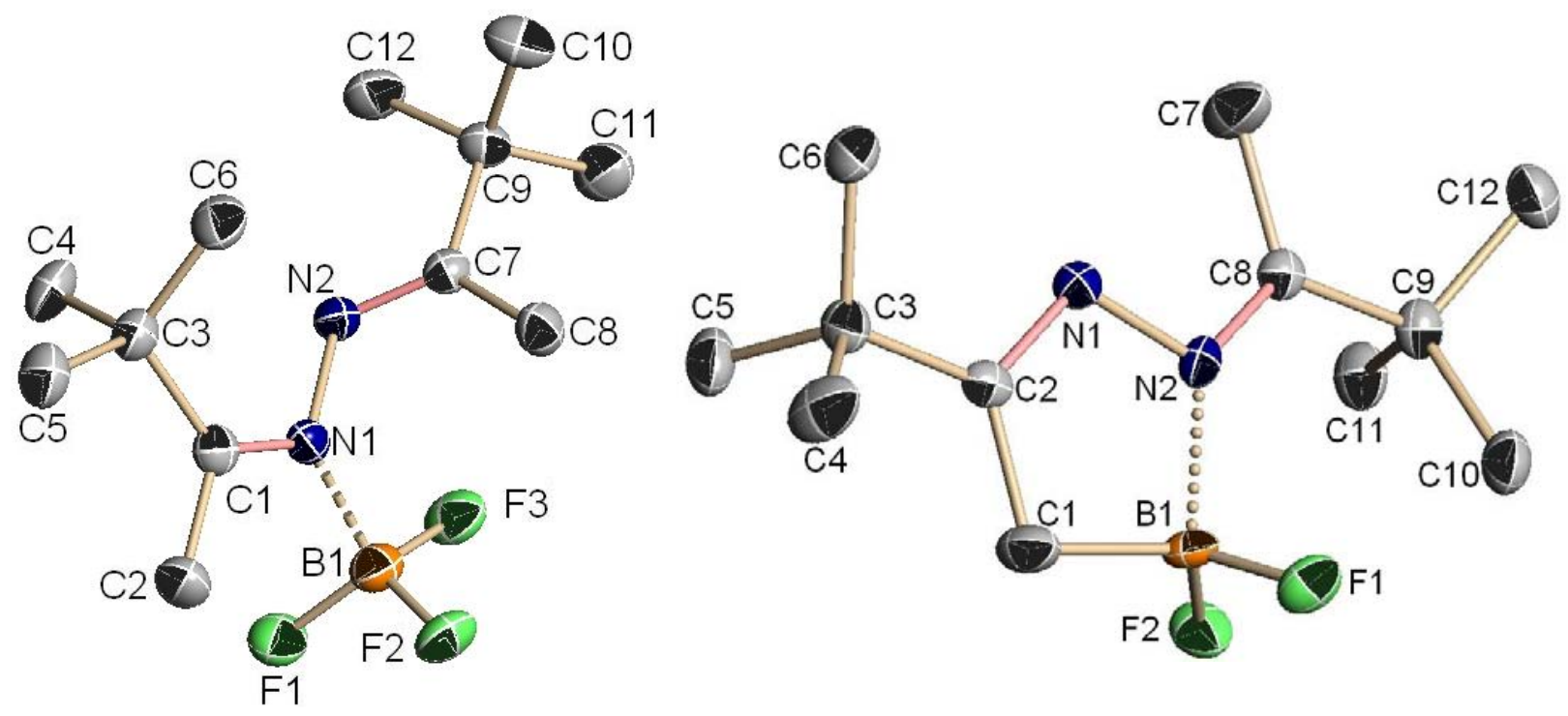

Abbildung 82: Kristallstrukturen der Verbindungen 68 und 69 ohne Wasserstoffatome

Wird Verbindung 69 mit Lithium-tert-butylmethyl-trimethylsilyl-hydrazon (70) umgesetzt, bildet sich das Tris(hyrazon)boran (71).

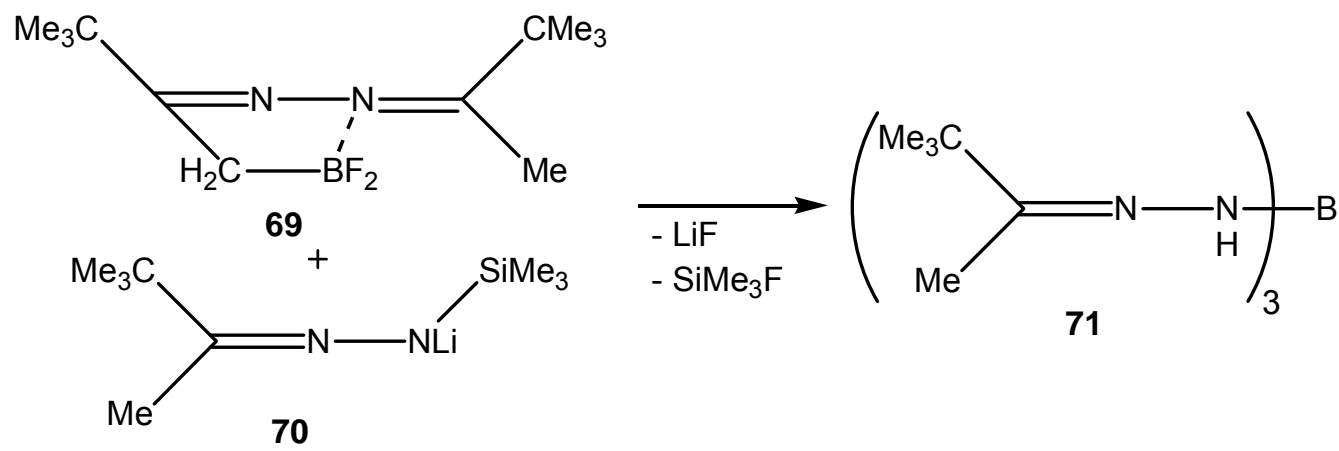

Schema 9: Synthese von $\mathbf{7 1}$

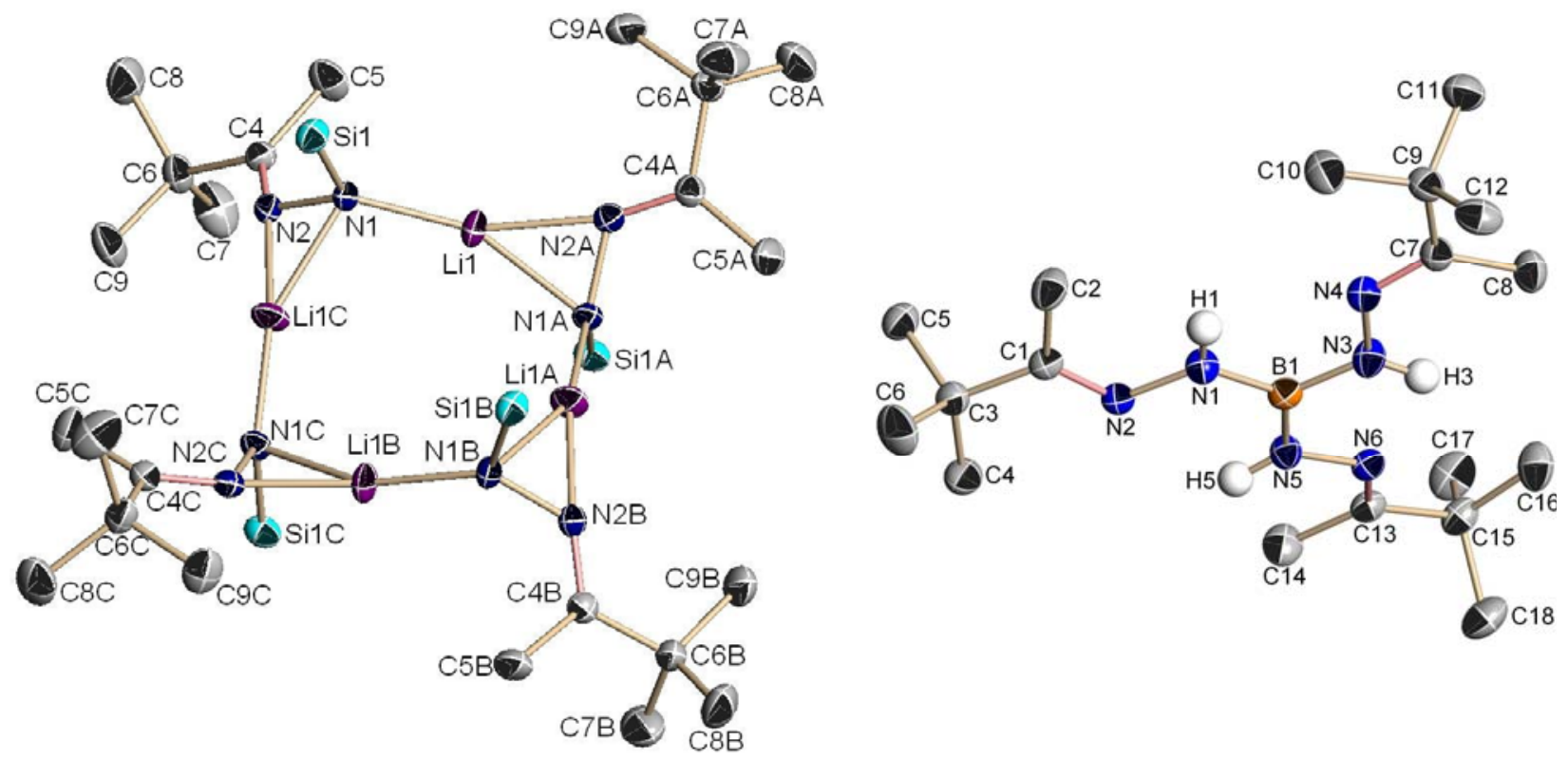

Abbildung 83: Kristallstruktur von 70 und 71 ohne Methylwasserstoffatome 
70 reagiert mit Difluor-bis(trimethylsilyl)-amino-boran zum unzersetzt destillierbaren (tert-Butylmethyl-trimethylsilyl-hydrazon)-fluor-bis(tri-methylsilyl)amino-boran (72) (siehe Gleichung 59).<smiles>BN(N=C(C)C)B(C)N(C)C</smiles>

72

Abbildung 84: Verbindung 72

Als Edukte zur Boreniumionenbildung wurden weitere Fluorborane mit voluminösen Bor-Stickstoffsubstituenten synthetisiert, um eine kinetische Stabilisierung der B-NNiederkoordination zu gewährleisten.

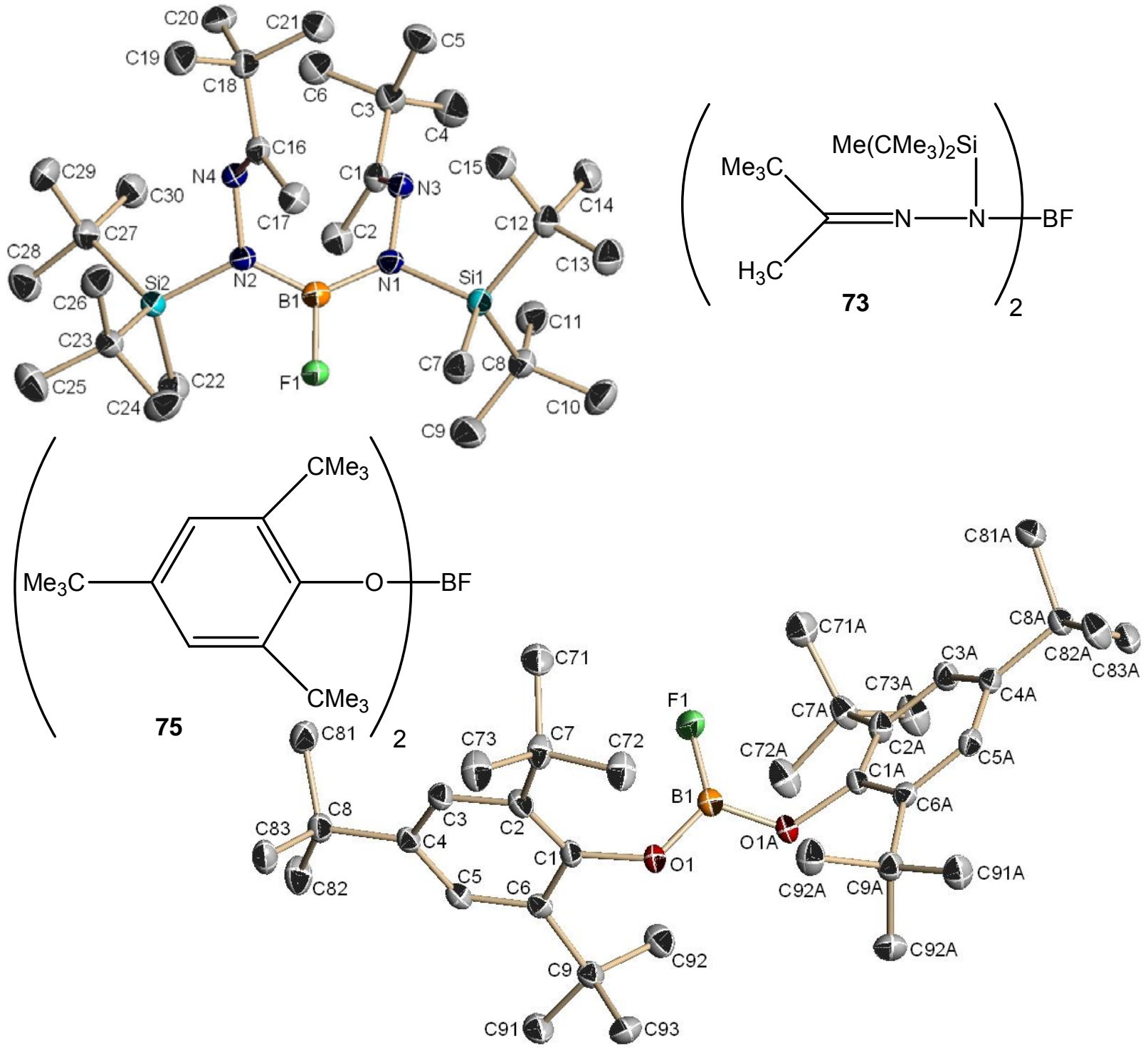


Das Trichloralanaddukt 48 wurde im Autoklaven auf $1200{ }^{\circ} \mathrm{C}$ erhitzt. Die entstandene Substanz wurde via EDX-Spektren und REM-Aufnahmen untersucht. Diese zeigen eine, unter der Oberfläche liegende, Al/B/N-haltige Spezies in Form von globulär verschmolzenden Aggregaten.

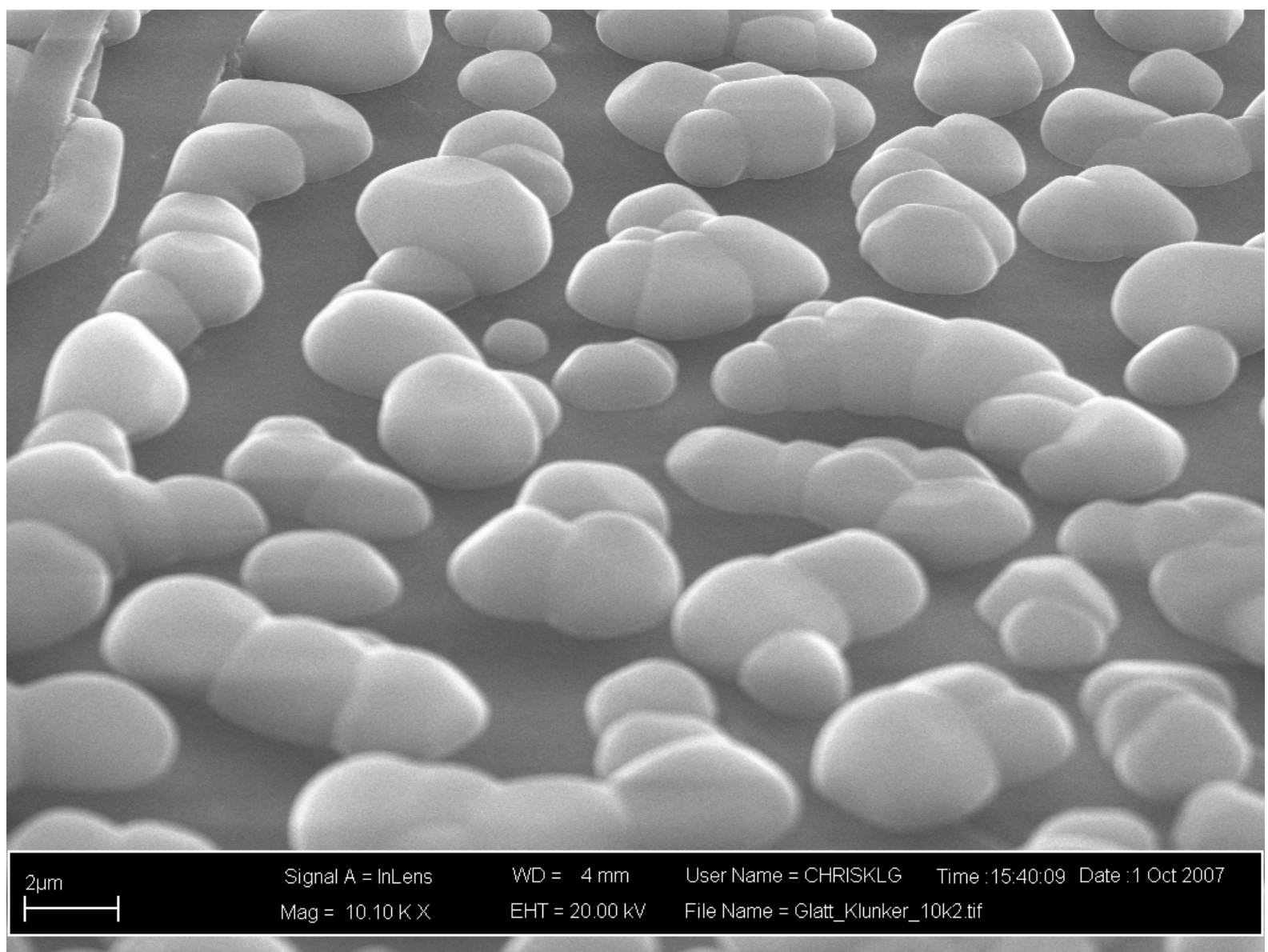

Abbildung 86: 48X, REM(SEM-)Aufnahme der globulär verschmolzenen Aggregate, 10.100fache Vergrößerung 


\section{$5 \quad$ Ausblick}

In der Reaktion der Alkyldiamine mit Chlordimethylsilyl-bis(trimethylsilyl)-amin kann durch Variation des Alkylrests die Kettenlänge der Silazane variiert werden.
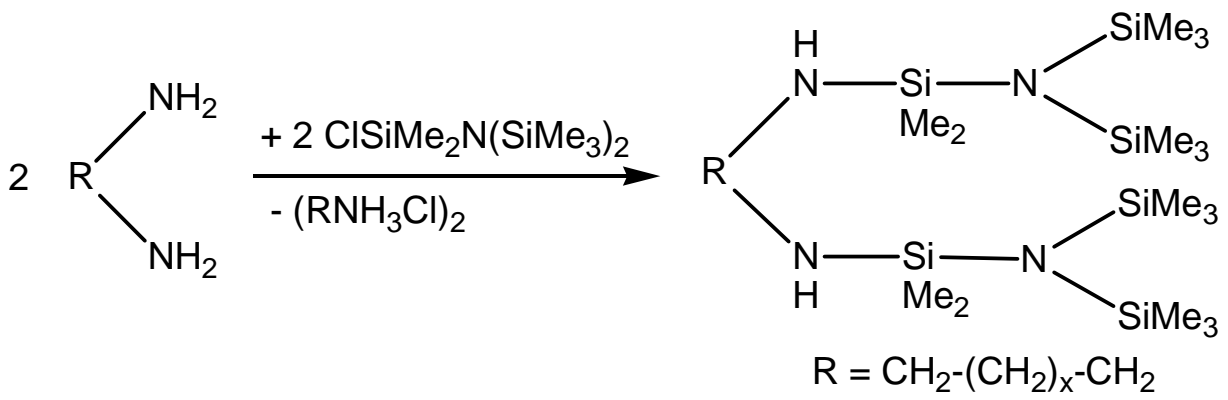

Gleichung 71: Synthese neuartiger Bis(1,1,3-trisila-2-aza)alkyldiamine

Werden diese mit Fluorboranen, -silanen oder auch -phospanen zur Reaktion gebracht, können neuartige offenkettige- oder ringförmige Silazane synthetisiert werden.

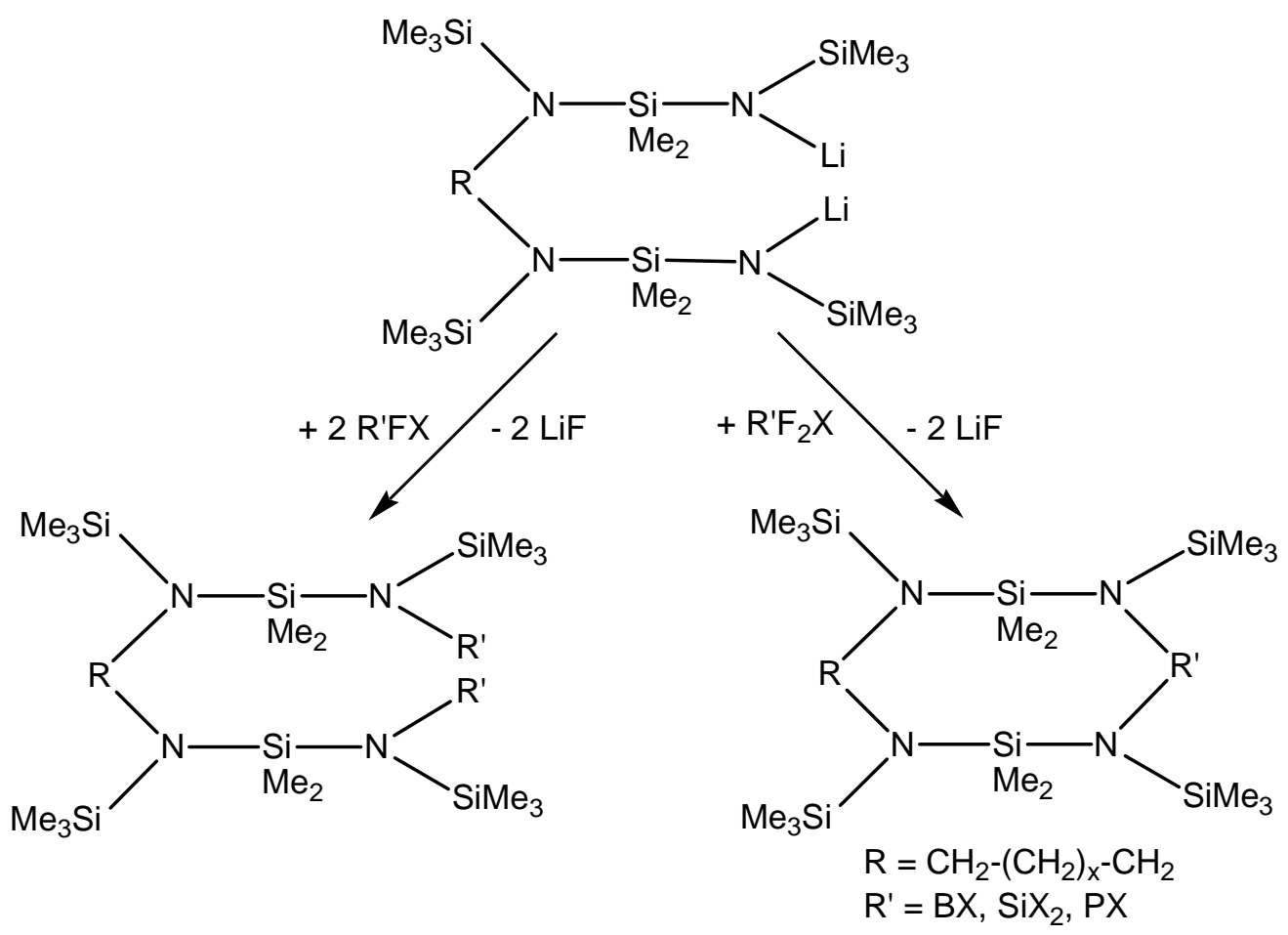

Gleichung 72: Synthese neuartiger Bis(1,3-disila-2-aza-silyl)alkyldiamine bzw. Cyclosilazane

Gelingt eine Fluorsilanabspaltung an diesen ringförmigen Verbindungen und reicht die kinetische Stabilisierung, so könnten Silazane mit einer endocyclischen Iminoboren-, -silen- oder Phosphazaneinheit dargestellt werden. 
Denkbar wäre ebenfalls eine Substitution der Difluorcyclosilazane mit der äquimolaren Menge an LiNHR und anschließender Lithiierung. Möglich wäre dabei die Bildung von exocyclischen Iminosilengruppen an Cyclosilazanen.
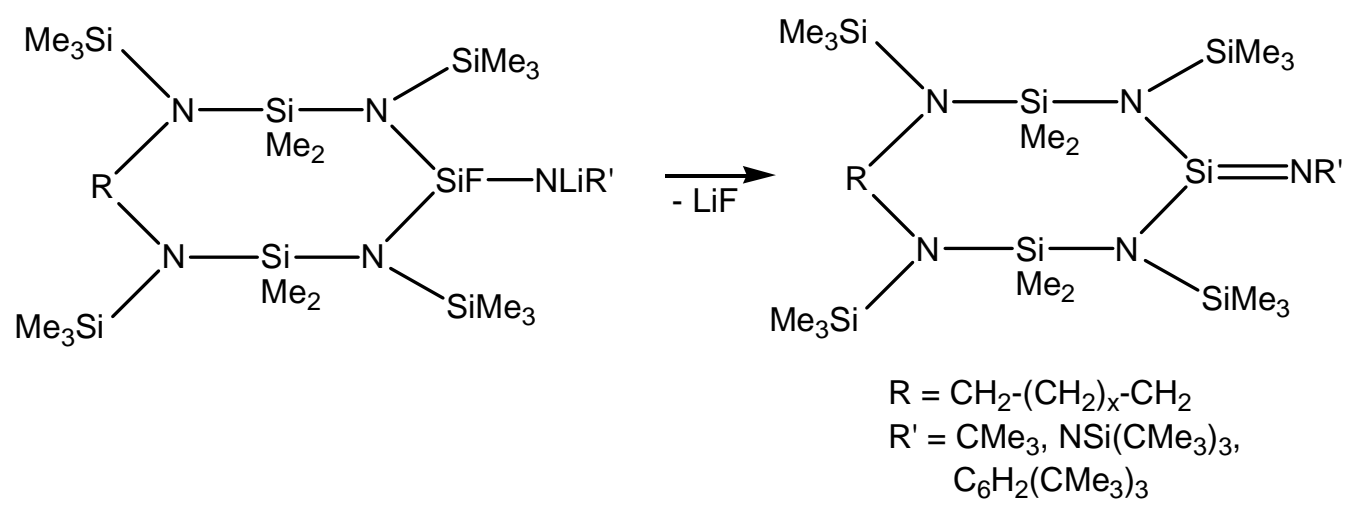

Gleichung 73: Möglicher Syntheseweg von Iminosilenen

Interessant wäre hierbei zu untersuchen, inwieweit die sterische Abschirmung des Rings bzw. der Substituenten am Cyclosilazan ausreicht, um das Iminosilen zu stabilisieren.

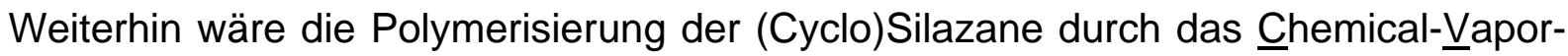
Deposition- oder Pyrolyseverfahren von Interesse. Jüngere Ergebnisse zeigen unerwartete optische Eigenschaften solcher ternären Keramiken ${ }^{[58,78, b]}$.

Durch Variation der Si-C-N-Verhältnisse in den Precursormolekülen werden unterschiedliche keramische Strukturen mit dementsprechend variierenden physikalischen Eigenschaften erwartet.

Durch die Polymerisation der Verbindungen mit Heteroatomen wie Bor oder Phosphor könnten quarternäre Keramiken erhalten werden.

Weiterhin interessant erscheint die Polymerisation der Trichlorgallan-substituierten Verbindung 51. Wie auch einer Aluminiumnitrid/ Bornitrid-Mischkeramik werden einem ternären GaN/BN-Werkstoff interessante elektrische Eigenschaften zugesprochen, welche durch Leitfähigkeitsmessungen untersucht werden können.

Die Möglichkeit der katalytischen Fluorsilanabspaltung bietet neue Möglichkeiten in der Synthese neuer Verbindungen mit Bor in niederer Koordination. So könnten Doppelbindungssysteme z. B. an cyclischen Verbindungen synthetisiert werden. 


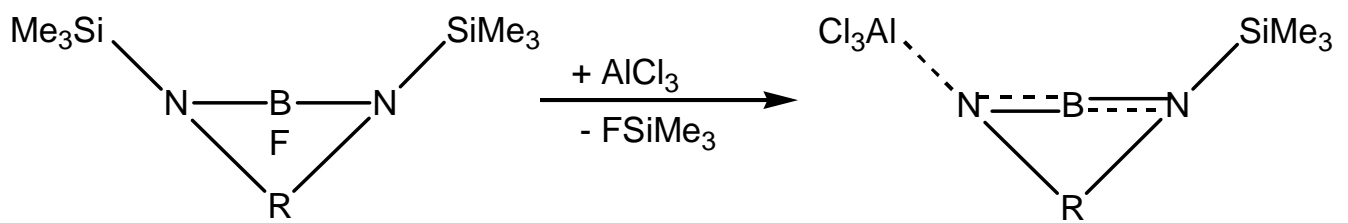

Gleichung 74: Möglichkeit der Synthese ringförmiger Iminoboren-AlCl${ }_{3}-A d d u k t e$

Des Weiteren ist die Aufklärung der Reaktionsmechanismen zur Bildung der Verbindungen 64 - 66 von besonderem Interesse. Durch Variation des Lösungsmittels und der Stickstoffsubstituenten können durch diese Synthesemethode zahlreiche Aminaddukte von Borkationen dargestellt werden.

Gelingt in einer Umsetzung der Verbindungen 73 - 75 mit $\mathrm{AlCl}_{3}$ die Abspaltung des Fluorids, so können hierbei ebenfalls neuartige Borkationen hergestellt werden. Die Bildung eines Dioxoboriniumions wäre von Interesse.

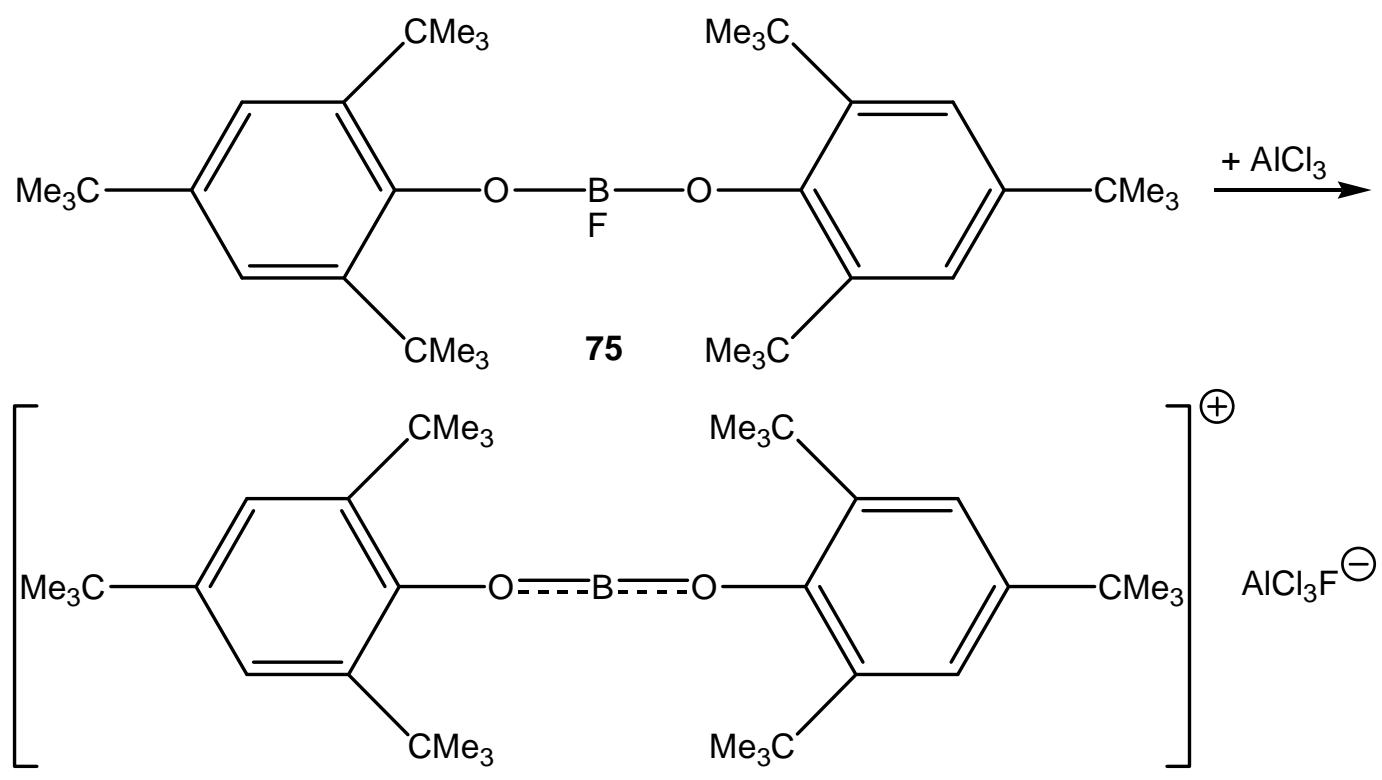

Gleichung 75: Umsetzung von $75 \mathrm{mit} \mathrm{AlCl}_{3}$ 
Weiterhin könnten Untersuchungen bezüglich des Phänomens der Umlithiierung bei der Synthese von Iminoborenen (siehe 3.4.8, Gleichung 43) helfen, einfachere Reaktionswege zu beschreiten. Die, bei der Darstellung entstehenden Edukte, können hierbei (nach destillativer Trennung und erneuter Lithiierung des Amins) wiederholt miteinander zum gewünschten Produkt umgesetzt werden.

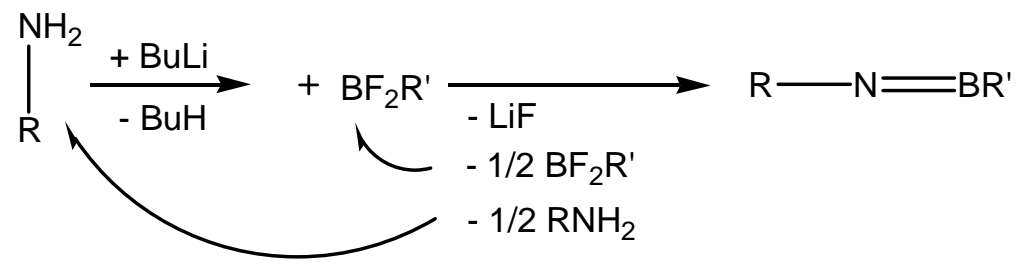

Gleichung 76: Synthesemöglichkeit von Iminoborenen

Durch die Reaktion der Lithium-fluorsilylamide mit Trichloralan in THF konnte eine Umchlorierung beobachtet werden (siehe 3.3.4, Schema 2). Problemlos müsste hierbei die Metallierung der Chlorsilylamine mit tert-Butyllithium sein, wobei die röntgenstrukturanalytische Untersuchung der LiCl-Salze sowie der Iminosilene von besonderem Interesse sind.

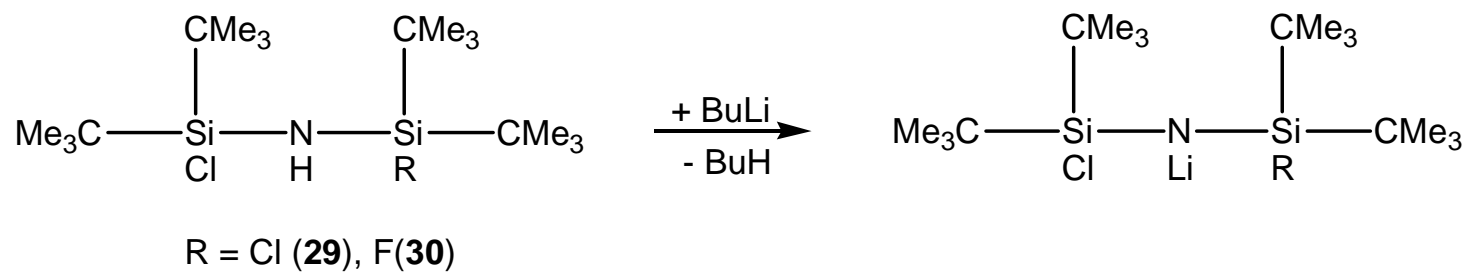<smiles>[R][Si](C)(C)N=[Si](C)(C)C</smiles> 


\section{$6 \quad$ Präparativer Teil}

\subsection{Allgemeine Arbeitsbedingungen}

Alle Reaktionen wurden in einer trockenen Inertgasatmosphäre (Argon oder Stickstoff) durchgeführt.

Soweit die benötigten Ausgangsverbindungen nicht über den Handel erhältlich waren, wurden sie nach den bekannten Literaturvorschriften dargestellt.

Die verwendeten Lösungsmittel ( $n$-Pentan, $n$-Hexan, Tetrahydrofuran, Diethylether, Di( $n$-butyl)ether, Toluol) wurden nach den im Labor üblichen Methoden getrocknet und über Natriumdraht aufbewahrt.

Das bei Umsetzungen verwendete $n$-Butyllithium $\left(\mathrm{C}_{4} \mathrm{H}_{9} \mathrm{Li}\right)$ lag als 23-\%ige Lösung in $n$-Hexan vor. Tert-Butyllithium lag als 15-\%ige Lösung in $n$-Pentan vor.

Bei der Lithiierung frei werdendes Butan wurde in einer Kühlfalle einkondensiert und ausgewogen, wodurch kontrolliert wurde, ob die Lithiierung vollständig abgeschlossen war.

Soweit möglich, wurde der Reaktionsverlauf ${ }^{1} \mathrm{H}$ - bzw. ${ }^{19} \mathrm{~F}-\mathrm{NMR}$-spektroskopisch verfolgt.

Die dargestellten Verbindungen wurden, wenn möglich, massen- und kernresonanzspektroskopisch auf ihre Reinheit überprüft.

Das Kapitel 6.2 enthält die Arbeitsvorschriften für die Herstellung der Verbindungen 1 bis 75 .

Der im folgenden Text als „Hochvakuum“ (HV) bezeichnete Unterdruck beträgt ca. 0,01 mbar. 


\subsection{Arbeitsvorschriften für die dargestellten Verbindungen 1 - 75}

\section{N,N'-Bis[1,1-bis(trimethylsilyl)-2-aza-3-dimethylsilyl]ethylendiamin 1;}

N,N'-Bis(1-tert-butyldimethylsilyl-1-trimethylsilyl-2-aza-3-dimethylsilyl)ethylendiamin 2

Zu 0,1 mol Ethylendiamin in $50 \mathrm{~mL}$ Diethylether werden unter Kühlung $\left(0^{\circ} \mathrm{C}\right) 1 \mathrm{~mol}$ Chlordimethylsilyl-bis(trimethylsilyl)-amin ${ }^{[40]}$ (1) bzw. tert-Butyldimethylsilyl-chlordimethylsilyl-trimethylsilyl-amin (2) gegeben. Nach $4 \mathrm{~h}$ Erhitzen unter Rückfluss wird das Reaktionsgemisch durch eine Fritte vom Hydrochlorid getrennt und fraktioniert destilliert (1) bzw. in Diethylether umkristallisiert (2).

\section{N,N'-Bis(tert-butyldimethylsilyl-1-trimethylsilyl-2-aza-3-dimethylsilyl)ethylen- diamin 3}

$0,03 \mathrm{~mol} 2$ werden für $2 \mathrm{~h}$ bei $200^{\circ} \mathrm{C}$ getempert. Die anschließende fraktionierte Destillation liefert 3.

\section{Dilithium-N,N'-bis(1-trimethylsilyl-2-amido-3-dimethylsilyl-trimethylsilyl)- ethylendiamin 4 - 6}

Zu 0,2 mol 1 werden 0,4 mol $n$-Butyllithium gegeben. Nach 1 h Erhitzen unter Rückfluss wird das Dilithiumsalz im Hochvakuum vom Lösungsmittel getrennt und fraktioniert destilliert. Man erhält die Verbindung 4 rein. Durch Umkristallisation in $n$-Hexan (4), THF (5) bzw. TMEDA (6) werden farblose Kristalle erhalten.

\section{N,N'-Bis(1-trimethylsilyl-2-dimethylsilyl-2-aza-trimethylsilyl)ethylendiamin 7}

0,05 mol 5 werden langsam mit 0,1 mol Wasser (in $50 \mathrm{~mL} n$-Pentan) zur Reaktion gebracht. Das Reaktionsgemisch wird durch Umkondensieren in eine Kühlfalle im HV vom Lithiumhydroxid getrennt. 7 wird durch fraktionierte Destillation rein erhalten.

\section{N,N'-Bis(1-tert-butyldifluorsilyl-1-trimethylsilyl-2-aza-3-dimethylsilyl-trimethyl- silyl)ethylendiamin 8}

$0,1 \mathrm{~mol} 4 \mathrm{in} 100 \mathrm{~mL} n$-Hexan und $100 \mathrm{~mL}$ THF werden unter Kühlung $\left(0^{\circ} \mathrm{C}\right) \mathrm{zu}$ 0,2 mol tert-Butyltrifluorsilan (in $50 \mathrm{~mL}$ THF) hinzugegeben. Nach 5 h Erhitzen unter Rückfluss wird das Gemisch im Hochvakuum vom entstandenen Lithiumfluorid getrennt. Durch fraktionierte Destillation wird die Verbindung 8 rein erhalten. Eine Umkristallisation in $n$-Hexan liefert farblose Kristalle. 
N,N'-Bis(1-fluordimethylsilyl-1-trimethylsilyl-2-aza-3-dimethylsilyl-trimethylsilyl)ethylendiamin 9

0,1 mol 4 in $100 \mathrm{~mL} n$-Hexan und $100 \mathrm{~mL}$ THF werden zu 0,2 mol Difluordimethylsilan (in $50 \mathrm{~mL}$ THF bei $-30^{\circ} \mathrm{C}$ ) langsam hinzugegeben. Nach $5 \mathrm{~h}$ Rühren bei RT unter Rückfluss wird das Gemisch im HV vom entstandenen Lithiumfluorid getrennt. Durch fraktionierte Destillation und Umkristallisation in $n$-Hexan wird die Verbindung 9 rein erhalten.

\section{N,N'-Bis[1,1-bis(trimethylsilyl)-2-aza-3-dimethylsilyl-trimethylsilyl]ethylendi- $\operatorname{amin} 10$}

Zu je 0,1 mol 4 in $100 \mathrm{~mL} n$-Hexan und $100 \mathrm{~mL}$ THF werden 0,2 mol Chlortrimethylsilan langsam hinzugegeben. Nach 2 h Erhitzen unter Rückfluss wird das LiCl durch eine Fritte abgetrennt, das Rohprodukt fraktioniert destilliert und in $n$-Hexan umkristalisiert, sodass farblose Kristalle erhalten werden.

N,N'-Bis[(1-fluorboryl-1,1,5,5-tetramethylpiperidino)-1-trimethylsilyl-2-aza-3-dimethylsilyl-trimethylsilyl]ethylendiamin 11;

N,N'-Bis[1-fluorboryl-bis(trimethylsilyl)amino-1-trimethylsilyl-2-aza-3-dimethylsilyl-trimethylsilyl]ethylendiamin 12;

N,N'-Bis[1-fluorboryl-diisopropylamino-1-trimethylsilyl-2-aza-3-dimethyl-silyltrimethylsilyl]ethylendiamin 13;

1,3-Bis(trimethylsilyl)-2,4-bis(1,1,5,5-tetramethylpiperidino)-1,3-diaza-2,4-dibora-cyclobutan 14

Je 0,1 mol 4 in $100 \mathrm{~mL} n$-Hexan und $100 \mathrm{~mL}$ THF werden zu jeweils 0,2 mol Difluor1,1,5,5-tetramethylpiperidino-boran (11), Difluor-bis(trimethylsilyl)amino-boran (12) bzw. Difluor-diisopropylamino-boran (13), langsam hinzugegeben. Nach Zugabe von $50 \mathrm{~mL}$ THF und 5 h Erhitzen unter Rückfluss wird das jeweilige Gemisch im Hochvakuum vom entstandenen Lithiumfluorid getrennt. Verbindung $\mathbf{1 1}$ wird durch Umkristallisation in $n$-Hexan rein erhalten. 12 - 14 werden fraktioniert destilliert, wobei 14 beim Erhitzen von 11 über $150^{\circ} \mathrm{C}$ entsteht. 12 und 14 werden anschließend in $n$-Hexan umkristallisiert. 
1-Fluor-2,4,7,9-tetrakis(trimethylsilyl)-3,3,7,7-tetramethyl-1-bora-2,4,7,9-tetraaza-3,7-disila-cyclononan 15

Zu 0,1 mol 4 in $100 \mathrm{~mL} n$-Hexan werden 0,3 mol Trifluorboran-Diethylether gegeben. Nach 4 h Erhitzen unter Rückfluss wird das Reaktionsgemisch durch eine Fritte vom $\mathrm{LiBF}_{4}$ getrennt und anschließend fraktioniert destilliert. Durch Umkristallisation in $n$-Hexan werden farblose Kristalle von 15 erhalten.

\section{1,1-Difluor-2,4,7,9-tetrakis(trimethylsilyl)-3,3,8,8-tetramethyl-2,4,7,9-tetraaza-}

\section{1,3,8-trisila-cyclononan 16}

Zu 0,1 mol 4 in $200 \mathrm{~mL} n$-Hexan werden bei $-70{ }^{\circ} \mathrm{C} 0,1 \mathrm{~mol}$ Tetrafluorsilan eingeleitet (10\% Überschuss). Das Gemisch lässt man über einen Zeitraum von 6 h Raumtemperatur annehmen und treibt $1 \mathrm{~h}$ unter Sieden des $n$-Hexans überschüssiges $\mathrm{SiF}_{4}$ aus. Anschließend wird das Rohprodukt im Vakuum vom Lithiumfluorid durch Kondensation in eine Kühlfalle getrennt. Durch fraktionierte Destillation wird die Verbindung 16 rein erhalten.

\section{N,N'-Bis[1,1-bis(trimethylsilyl)-2-aza-3-dimethylsilyl]hydrazin 17}

Zu 1 mol Hydrazin in $250 \mathrm{~mL}$ Diethylether werden unter Kühlung $\left(0^{\circ} \mathrm{C}\right) 1 \mathrm{~mol}$ Chlordimethylsilyl-bis(trimethylsilyl)-amin gegeben. Nach 5 h Erhitzen unter Rückfluss wird das Reaktionsgemisch durch eine Fritte vom Hydrochlorid getrennt und fraktioniert destilliert.

\section{Dilithium-N,N'-bis[1-trimethylsilyl-2-amido-3-dimethylsilyl-trimethylsilyl]hydra-} $\operatorname{zin} 18,19$

Zu 0,2 mol 17 werden 0,4 mol $n$-Butyllithium gegeben. Nach 1 h Erhitzen unter Rückfluss wird das Dilithiumsalz im Hochvakuum vom Lösungsmittel getrennt. Durch Umkristallisation in $n$-Hexan (18) bzw. THF (19) werden farblose Kristalle erhalten.

\section{N,N'-Bis(1-trimethylsilyl-2-aza-3-dimethylsilyl-trimethylsilyl)hydrazin 20}

0,05 mol 19 werden langsam mit 0,1 mol Wasser (in $50 \mathrm{~mL} n$-Pentan) zur Reaktion gebracht. Das Reaktionsgemisch wird durch Umkondensieren in eine Kühlfalle im HV vom Lithiumhydroxid getrennt. 20 wird durch fraktionierte Destillation rein erhalten. 
1-Fluor-2,4,5,7-tetrakis(trimethylsilyl)-3,3,6,6-tetramethyl-1-bora-2,4,5,7tetraaza-3,6-disila-cycloheptan 21

Zu 0,1 mol 18 in $100 \mathrm{~mL} n$-Hexan werden 0,3 mol Trifluorboran-Diethylether gegeben. Nach $4 \mathrm{~h}$ Erhitzen unter Rückfluss wird das Reaktionsgemisch durch eine Fritte vom $\mathrm{LiBF}_{4}$ getrennt und anschließend fraktioniert destilliert. Durch Umkristallisation in n-Hexan werden farblose Kristalle von 21 erhalten.

\section{1,1-Difluor-2,4,5,7-tetrakis(trimethylsilyl)-3,3,6,6-tetramethyl-2,4,5,7-tetraaza-}

\section{1,3,6-trisila-cycloheptan 22}

Zu 0,1 mol 18 in $200 \mathrm{~mL} n$-Hexan werden bei $-70^{\circ} \mathrm{C}$ langsam 0,1 mol Tetrafluorsilan eingeleitet (10\% Überschuss). Das Gemisch lässt man über einen Zeitraum von $6 \mathrm{~h}$ Raumtemperatur annehmen. Anschließend wird das Rohprodukt im Vakuum vom Lithiumfluorid durch Kondensation in eine Kühlfalle getrennt. Durch fraktionierte Destillation wird die Verbindung 22 rein erhalten.

\section{tert-Butyl-di(tert-butyl)methylsilyl-dimethylalan-amin 23}

Zu 0,1 mol tert-Butyl-di(tert-butyl)methylsilyl-amin werden 0,1 mol $n$-Butyllithium gegeben. Nach $1 \mathrm{~h}$ Erhitzen unter Rückfluss wird das Lithiumsalz mit 0,1 mol Trimethylalan (2 molare Lsg. in $n$-Heptan) versetzt. Nach $4 \mathrm{~h}$ Erhitzen unter Rückfluss wird das Reaktionsgemisch durch Kondensation in eine Kühlfalle im HV vom entstandenen Lithiumfluorid getrennt. Eine fraktionierte Destillation bei 10 mbar liefert das wachsartige Produkt 23.

\section{tert-Butyl-di(tert-butyl)methylsilyl-amin 24}

0,05 mol 23 werden langsam mit 0,05 mol Wasser (in $25 \mathrm{~mL} n$-Pentan) zur Reaktion gebracht. Das Reaktionsgemisch wird durch Umkondensieren in eine Kühlfalle im HV vom Alaun getrennt. 24 wird durch fraktionierte Destillation bei 0,1 mbar rein erhalten.

\section{Lithium-tert-butyl-di(tert-butyl)methylsilyl-amid 25}

Zu 0,05 mol 24 in $50 \mathrm{~mL} n$-Hexan werden 0,05 mol $n$-Butyllithium gegeben. Nach $2 \mathrm{~h}$ Erhitzen unter Rückfluss wird das Lithiumsalz durch Umkristallisation in $n$-Hexan kristallin erhalten. 
tert-Butyl-di(tert-butyl)fluorsilyl-difluorboryl-amin 26

\section{1,1,2-Tri(tert-butyl)-2,4-diaza-1,3-difluor-4,4-bis(trimethylsilyl)-1-sila-3-boran 27}

Zu je 0,1 mol tert-Butyl-di(tert-butyl)methylsilyl-amin werden 0,1 mol $n$-Butyllithium gegeben. Nach 1 h Erhitzen unter Rückfluss wird das Lithiumsalz mit 0,2 mol Trifluorboran-Diethylether (26) bzw. 0,1 mol Difluor-bis(trimethylsilyl)-amin-boran (27 - Syntheseweg a) versetzt. Nach $4 \mathrm{~h}$ Erhitzen unter Rückfluss wird das Reaktionsgemisch durch Kondensation in eine Kühlfalle im HV vom entstandenen $\mathrm{LiBF}_{4}$ (26) bzw. LiF (27) getrennt. Eine fraktionierte Destillation liefert die Produkte 26 und 27. Durch Umkristallisation von 27 in $n$-Hexan werden farblose Kristalle erhalten.

27 - Syntheseweg b: Zu 0,05 mol 26 werden die äquimolare Menge LithiumBis(trimethylsilyl)amid gegeben, 2 h unter Rückfluss erhitzt, anschließend in eine Kühlfalle im HV einkondensiert und fraktioniert destilliert.

\section{Bis[di(tert-butyl)methylsilyl]amin 28}

Bis[di(tert-butyl)chlorsilyl]amin ${ }^{[66]} 29$

Di(tert-butyl)chlorsilyl-di(tert-butyl)fluorsilyl-amin ${ }^{[66]} \mathbf{3 0}$

Zu jeweils $3 \mathrm{mmol}$ Bis[di(tert-butyl)methylsilyl]amin werden $3 \mathrm{mmol} n$-Butyllithium gegeben. Nach $1 \mathrm{~h}$ Erhitzen unter Rückfluss wird das Lithiumsalz in $25 \mathrm{~mL}$ THF gelöst und mit jeweils 3 mmol Trimethylalan (28; 2 molare Lsg. in $n$-Heptan), Trichloralan (29, 30 - Syntheseweg a) bzw. frisch destilliertem Chlortrimethylsilan (30 - Syntheseweg b) versetzt. Nach dem Erhitzen unter Rückfluss (28, 168 h; 29 und 30, 48 h), werden die Rohprodukte durch Kondensation in eine Kühlfalle im HV vom LiF getrennt und destillativ gereinigt, wobei Verbindung $\mathbf{3 0}$ sowohl als Produkt der Reaktion des Amids mit Trichloralan (30 - Syntheseweg a), als auch mit Chlortrimethylsilan (30 - Syntheseweg b) charakterisiert werden konnte. Die Verbindungen 28 und 29 werden durch Umkristallisation in $n$-Hexan kristallin erhalten.

Fluor-2,6-diisopropylanilino-(methyl-trimethylsilyl)amino-boran 31

Di(iso-butyl)amino-fluor-2,6-diisopropylanilino-boran 32

\section{Fluor-diisopropylamino-2,6-diisopropylanilino-boran 33}

Jeweils 0,2 mol 2,6-Diisopropylanilin werden unter Eiskühlung mit 0,2 mol n-Butyllithium versetzt, $1 \mathrm{~h}$ unter Rückfluss erhitzt und in je $100 \mathrm{~mL}$ THF gelöst. Die Salzlösungen werden anschließend zu je 0,2 mol Difluor-(methyl-trimethylsilyl)amino-boran (31), Di(iso-butyl)amino-difluor-boran (32) bzw. Difluor-diisopropylamino-boran (33) 
gegeben. Nach dem Erhitzen der Gemische unter Rückfluss für $3 \mathrm{~h}$ werden die Produkte durch Kondensation in eine Kühlfalle im HV vom entstandenen LiF getrennt und durch eine fraktionierte Destillation gereinigt. Anschließend werden die Verbindungen aus $n$-Hexan umkristallisiert.

\section{Diisopropylamino-bis(2,6-diisopropylanilino)-boran 34}

Zu 0,05 mol 32 in $100 \mathrm{~mL} n$-Hexan werden bei $-78^{\circ} \mathrm{C} 0,05 \mathrm{~mol}$ des Lithiumsalzes von 2,6-Diisopropylanilin (in $100 \mathrm{~mL}$ THF, ebenfalls auf $-78{ }^{\circ} \mathrm{C}$ gekühlt) gegeben. Nach dem Rühren des Gemisches bei $-78^{\circ} \mathrm{C}$ für $6 \mathrm{~h}$ wird die Lösung weitere $2 \mathrm{~h}$ unter Rückfluss erhitzt, anschließend durch Umkondensation in eine Kühlfalle im HV vom LiF getrennt, destillativ gereinigt und durch Umkristallisation in $n$-Hexan rein erhalten.

\section{Bis(trimethylsilyl)amino-diisopropylamino-2,6-diisopropylanilino-boran 35}

Di(iso-butyl)amino-(tert-butyl-trimethylsilyl)amino-2,6-diisopropylanilino-boran 36

$\mathrm{Zu}$ je $0,1 \mathrm{~mol} 32$ in $200 \mathrm{~mL} n$-Hexan werden bei $-78^{\circ} \mathrm{C} \quad 0,1 \mathrm{~mol}$ Lithiumbis(trimethylsilyl)amid (35) bzw. 0,1 mol Lithium-tert-butyl-trimethylsilyl-amid (36) (jeweils in $100 \mathrm{~mL} n$-Hexan, auf $-78^{\circ} \mathrm{C}$ gekühlt) gegeben. Nach dem Rühren der Gemische bei $-78{ }^{\circ} \mathrm{C}$ für $6 \mathrm{~h}$ werden die Lösungen weitere $3 \mathrm{~h}$ unter Rückfluss erhitzt, anschließend durch Umkondensation in eine Kühlfalle im HV vom LiF getrennt, destillativ gereinigt und durch Umkristallisation in $n$-Hexan rein erhalten.

\section{Fluor-bis(trimethylsilyl)amino-triphenylsilylamino-boran 37}

\section{Fluor-triphenylsilylamino-diisopropylamino-boran 38}

\section{Fluor-1,1,5,5-tetramethylpiperidino-triphenylsilylamino-boran 39}

Je 0,05 mol Triphenylsilylamin in $50 \mathrm{~mL} n$-Hexan werden unter Eiskühlung mit 0,05 mol $n$-Butyllithium versetzt und $2 \mathrm{~h}$ unter Rückfluss erhitzt. Die Aufschlämmung wird anschließend zu je 0,05 mol Difluor-bis(trimethylsilyl)amino-boran (37), Difluordiisopropylamino-boran (36) bzw. Difluor-1,1,5,5-tetramethyl-piperidino-boran (38) gegeben und für $12 \mathrm{~h}$ unter Rückfluss erhitzt. Anschließend wird das Rohprodukt im HV vom Lithiumfluorid durch Kondensation in eine Kühlfalle getrennt. Eine fraktionierte Destillation liefert die Verbindungen 37 - 39 rein. Röntgentaugliche Einkristalle von $\mathbf{3 7}$ und $\mathbf{3 8}$ werden durch Umkristallisation in Toluol erhalten. 
(tert-Butyldimethylsilyl)amino-fluor-bis(trimethylsilyl)amino-boran 40 (tert-Butyldimethylsilyl)amino-fluor-diisopropylamino-boran 41 (tert-Butyldimethylsilyl)amino-fluor-1,1,5,5-tetramethylpiperidino-boran 42 (tert-Butyldimethylsilyl)imino-1,1,5,5-tetramethylpiperidino-boren 43 (tert-Butyldimethylsilyl)imino-2,4,6-tri(tert-butyl)phenyl-boren 44

Je 0,1 mol (tert-Butyldimethylsilyl)amin (in $100 \mathrm{~mL} n$-Hexan) werden unter Eiskühlung mit 0,1 mol $n$-Butyllithium metalliert und $1 \mathrm{~h}$ unter Rückfluss erhitzt. Die Lösungen werden anschließend bei RT zu je 0,1 mol Difluor-bis(trimethylsilyl)amino-boran (40), Difluor-diisopropylamino-boran (41), Difluor-1,1,5,5-tetramethylpiperidino-boran (42, 43) bzw. 2,4,6-Tri(tert-butyl)phenyl-difluor-boran (44) gegeben und $5 \mathrm{~h}$ unter Rückfluss erhitzt. Anschließend werden die Produktgemische in eine Kühlfalle einkondensiert und durch eine fraktionierte Destillation (40 - 43) bzw. fraktionierte Sublimation (44) gereingt, wobei 40 nicht über $100^{\circ} \mathrm{C}$ erhitzt werden darf. Einkristalle des Iminoborens $\mathbf{4 4}$ werden durch Umkristallisation in Diethylether bei $-20^{\circ} \mathrm{C}$ erhalten.

(tert-Butyldimethylsilyl)amino-2,4,6-tri(tert-butyl)phenyl-hydroxi-boran 45 (tert-Butyldimethylsilyl)amino-2,4,6-tri(tert-butyl)phenyl-methoxi-boran 46 (tert-Butyldimethylsilyl)amino-2,4,6-tri(tert-butyl)phenyl-amino-boran 47 Jeweils $5 \mathrm{mmol}$ von 44 werden mit $5 \mathrm{mmol}$ Wasser (45; in $10 \mathrm{~mL} n$-Pentan), $5 \mathrm{mmol}$ Methanol (46; in $10 \mathrm{~mL} n$-Hexan) bzw. $5 \mathrm{mmol}$ Ammoniak (47; bei $-40^{\circ} \mathrm{C}$ in $25 \mathrm{~mL}$ Diethylether, 10\% Überschuss) versetzt, im Anschluss $1 \mathrm{~h}$ unter Rückfluss erhitzt, im HV vom Lösungsmittel befreit und fraktioniert sublimiert. Einkristalle von 45 und 47 werden durch Umkristallisation in Diethylether erhalten.

(Trichloralan-trimethylsilyl)amino-bis(trimethylsilyl)amino-boran 48 (Chlordimethylalan-trimethylsilyl)amino-bis(trimethylsilyl)amino-boran 49 (Tribromalan-trimethylsilyl)amino-bis(trimethylsilyl)amino-boran 50 (Trichlorgallan-trimethylsilyl)amino-bis(trimethylsilyl)amino-boran 51 Jeweils 0,1 mol Fluor-bis[bis(trimethylsilyl)amino]-boran werden mit je 0,1 mol Trichloralan in $50 \mathrm{~mL}$ Diethylether (48), Chlordimethylalan (2 molare Lösung in $n$-Hexan) (49), Tribromalan in $50 \mathrm{~mL}$ Diethylether (50) bzw. Galliumtrichlorid in $50 \mathrm{~mL}$ Diethylether (51) versetzt. Die Reaktionsgemische werden 48 h unter Rückfluss erhitzt und anschließend bei vermindertem Druck vom Fluortrimethylsilan und Diethyl- 
ether befreit. Die zurückbleibenden Feststoffe werden aus Diethylether umkristallisiert.

\section{Bis[(tert-butyldimethylsilyl-trimethylsilyl)amino]-fluor-boran 52}

0,2 mol tert-Butyldimethylsilyl-trimethylsilyl-amin (in $150 \mathrm{~mL} n$-Hexan) werden mit 0,2 mol $n$-Butyllithium metalliert und $2 \mathrm{~h}$ unter Rückfluss erhitzt. Die Lösung wird anschließend unter Eiskühlung zu 0,3 mol Trifluorborandiethyletherat gegeben und $3 \mathrm{~h}$ unter Rückfluss erhitzt. Das Produktgemisch wird im Anschluss in eine Kühlfalle einkondensiert und durch fraktionierte Destillation gereinigt.

\section{(tert-Butyldimethylsilyl-phenyldimethylsilyl)amin 53}

$0,1 \mathrm{~mol}$ tert-Butyldimethylsilylamin (in $50 \mathrm{~mL} n$-Hexan) werden mit 0,1 mol $n$-Butyllithium metalliert und $1 \mathrm{~h}$ unter Rückfluss erhitzt. Die Lösung wird anschließend mit 0,1 mol Chlordimethylphenylsilan (in $50 \mathrm{~mL}$ THF) versetzt und $7 \mathrm{~h}$ unter Rückfluss erhitzt. Durch Kondensation in eine Kühlfalle im HV wird das Reaktionsgemisch vom Lithiumchlorid getrennt und fraktioniert destilliert, wodurch 53 rein erhalten wird.

\section{tert-Butylmethylphenylsilylamin 54}

In 0,4 mol tert-Butylchlormethylphenylsilan in $200 \mathrm{~mL}$ Diethylether werden bei $0{ }^{\circ} \mathrm{C}$ 0,8 mol Ammoniak eingeleitet. Das Produktgemisch wird durch eine Fritte vom entstandenen Ammoniumchlorid getrennt und fraktioniert destilliert.

\section{(tert-Butylmethylphenylsilyl-trimethylsilyl)amin 55}

0,3 mol tert-Butylmethylphenylsilylamin (in $50 \mathrm{~mL} n$-Hexan) werden mit 0,3 mol $n$-Butyllithium metalliert und $3 \mathrm{~h}$ unter Rückfluss erhitzt. Die Lösung wird anschließend mit 0,1 mol Chlortrimethylsilan (in $50 \mathrm{~mL}$ THF) versetzt und $5 \mathrm{~h}$ unter Rückfluss erhitzt. Durch Kondensation in eine Kühlfalle im HV wird das Reaktionsgemisch vom Lithiumchlorid getrennt. Eine fraktionierte Destillation liefert $\mathbf{5 5 .}$

\section{Lithium-(tert-butylmethylphenylsilyl-trimethylsilyl)amid 56}

Zu 0,2 mol 55 werden 0,2 mol $n$-Butyllithium gegeben. Nach 2 h Erhitzen unter Rückfluss wird das Lithiumsalz durch Umkristallisation in $n$-Hexan rein erhalten. 
(tert-Butylmethylphenylsilyl-trimethylsilyl)amino-difluor-boran 57

0,2 mol 56 werden unter Eiskühlung zu 0,4 mol Trifluorborandiethyletherat gegeben und 4 h unter Rückfluss erhitzt. Das Produktgemisch wird im Anschluss in eine Kühlfalle einkondensiert und durch eine fraktionierte Destillation gereingt.

(tert-Butylmethylphenylsilyl-trimethylsilyl)amino-fluor-bis(trimethylsilyl)-aminoboran 58

Zu 0,1 mol 57 werden 0,1 mol lithiiertes HMDS (in $50 \mathrm{~mL}$ Hexan) gegeben, $3 \mathrm{~h}$ unter Rückfluss erhitzt, durch Kondensation in eine Kühlfalle im HV vom Lithiumfluorid getrennt und anschließend fraktioniert destilliert. Durch Umkristallisation in $n$-Hexan werden farblose Kristalle erhalten.

(tert-Butyl-trimethylsilyl)amino-(trichloralan-trimethylsilyl)amino-boran 59 (tert-Butyl-trimethylsilyl)amino-(trichlorgallan-trimethylsilyl)amino-boran 60

Jeweils $0,1 \mathrm{~mol}$ (tert-Butyl-trimethylsilyl)amino-fluor-bis(trimethylsilyl)amino-boran werden mit je 0,1 mol Trichloralan in $50 \mathrm{~mL}$ Diethylether (59) bzw. Galliumtrichlorid in $50 \mathrm{~mL}$ Diethylether (60) versetzt. Die Reaktionsgemische werden 24 h unter Rückfluss erhitzt und anschließend bei vermindertem Druck vom Fluortrimethylsilan und Diethylether befreit. Die zurückbleibenden Feststoffe werden in Diethylether umkristallisiert.

\section{1-Diisopropylamino-3-dimethyl-2,4-bis(trimethylsilyl)-2,4-diaza-1-bora-3sila- cyclobutan 61}

Zu 0,1 mol Aminodimethylsilyl-bis(trimethylsilyl)-amin werden 0,2 mol $n$-Butyllithium gegeben. Nach 2 h Erhitzen unter Rückfluss wird das Dilithiumsalz mit 0,1 mol Difluor-diisopropylamino-boran und $50 \mathrm{~mL}$ THF versetzt. Nachdem das Reaktionsgemisch für $3 \mathrm{~h}$ unter Rückfluss erhitzt wurde, werden das Produkt und die Lösungsmittel durch Kondensation in eine Kühlfalle im HV vom Lithiumfluorid getrennt. Eine fraktionierte Destillation liefert $\mathbf{6 1}$ rein. Durch Umkristallisation in $n$-Hexan können wachsartige, farblose Kristalle erhalten werden. 


\section{Di(iso-butyl)amino-fluor-(N-trimethylsilyl-2,6-diisopropylanilino)-boran 62} Fluor-diisopropylamino-(N-trimethylsilyl-2,6-diisopropylanilino)-boran 63

Jeweils $0,2 \mathrm{~mol}$ Trimethylsilyl-2,6-diisopropylanilin werden unter Eiskühlung mit 0,2 mol $n$-Butyllithium versetzt, $1 \mathrm{~h}$ unter Rückfluss erhitzt und in je $100 \mathrm{~mL}$ THF gelöst. Die Salzlösungen werden anschließend zu jeweils 0,2 mol Di(iso-butyl)aminodifluor-boran (62) bzw. Difluor-diisopropylamino-boran (63) gegeben. Nach dem Erhitzen der Gemische unter Rückfluss für $4 \mathrm{~h}$, werden die Produkte durch Kondensation in eine Kühlfalle im HV vom entstandenen Lithiumfluorid getrennt und durch eine fraktionierte Destillation gereinigt. Aus $n$-Hexan werden röntgentaugliche Einkristalle von 63 erhalten.

\section{Di(iso-butyl)ammonium-( $N$-fluordimethylsilyl-2,6-diisopropylanilino)-methyl- boran-tetrachloraluminat 64 \\ Di(iso-butyl)amin-trichloralan 65}

Diisopropylammonium-2,6-diisopropylanilino-n-butyl-boran-tetrachloroaluminat 66

\section{Di(tert-butyl)chlorsilylamin-trichloralan 67}

Jeweils 0,1 mol 62 (64 - 66) bzw. 27 (67) in $100 \mathrm{~mL}$ Diethylether werden bei $-20{ }^{\circ} \mathrm{C}$ mit 0,1 mol Trichloralan, in $50 \mathrm{~mL}$ Diethylether (64, 67 - und $50 \mathrm{~mL}$ Dichlormethan) versetzt und $4 \mathrm{~h}$ unter Eiskühlung gerührt. Eine Umkristallisation in Diethylether bei $0{ }^{\circ} \mathrm{C}$ liefert Kristalle von 64 und 67. Wird $641 \mathrm{~h}$ in der Siedehitze des Diethylethers erhitzt, zersetzt sich die Verbindung. Durch Umkristallisation in Diethylether können Kristalle von 65 isoliert werden.

\section{Bis(tert-butylmethyl)ketazin-trifluorboran 68}

0,3 mol Bis(tert-butyl-methyl)ketazin werden unter Eiskühlung mit der äquimolaren Menge an Trifluorborandiethyletherat versetzt und $2 \mathrm{~h}$ unter Rückfluss erhitzt. Eine fraktionierte Destillation liefert 68. Durch Umkristallisation in Diethylether werden farblose Kristalle erhalten. 


\section{5-tert-Butyl-3,3-difluor-2-(1,1-dimethylbutyliden-2)-1,2,3-azaazoniaborata-5-} cyclopenten $^{[76]} 69$

0,2 mol 68 werden mit der äquimolaren Menge an Trifluorborandiethyletherat versetzt und $4 \mathrm{~h}$ unter Rückfluss erhitzt. Eine fraktionierte Destillation liefert 69. Durch Umkristallisation in Diethylether werden farblose Kristalle erhalten.

\section{Lithium-tert-butylmethylketon-trimethylsilyl-hydrazonid 70}

Zu 0,2 mol tert-Butylmethyl-trimethylsilyl-hydrazon werden 0,2 mol $n$-Butyllithium gegeben. Nach 2 h Erhitzen unter Rückfluss wird das Lithiumsalz durch Umkristallisation in $n$-Hexan rein erhalten.

\section{Tris(tert-butylmethylketon-hydrazono)boran 71}

(tert-Butylmethylketon-trimethylsilyl-hydrazono)-fluor-bis(trimethylsilyl)-boran 72

Je 0,1 mol 69 (in 50 mL THF, 71) bzw. Difluor-bis(trimethylsilyl)-amin-boran (in $50 \mathrm{~mL}$ Diethylether, 72) werden mit je 0,1 mol 70 (in $50 \mathrm{~mL} n$-Hexan) versetzt, $4 \mathrm{~h}$ unter Rückfluss erhitzt und durch Umkondensation in eine Kühlfalle im HV vom Lithiumfluorid getrennt. Eine fraktionierte Destillation liefert 71 und 72 rein. Röntgentaugliche Einkristalle von $\mathbf{7 1}$ werden durch Umkristallisation in $n$-Hexan erhalten.

Bis[tert-butylmethylketon-di(tert-butyl)methylsilyl-hydrazono]-fluor-boran ${ }^{[77]} 73$ Verbindung 73 wird gemäß der Literaturvorschrift ${ }^{[77]}$ synthetisiert. Röntgenstrukturtaugliche Einkristalle werden durch Umkristallisation von 73 aus Toluol erhalten.

\section{Fluor-diisopropylamino-N,2,6-triisopropylanilino-boran 74}

0,1 mol N,2,6-Triisopropylanilin werden mit 0,1 mol $n$-Butyllithium versetzt, $2 \mathrm{~h}$ unter Rückfluss erhitzt und in $50 \mathrm{~mL}$ THF gelöst. Die Salzlösung wird anschließend zu 0,1 mol Difluor-diisopropylamino-boran gegeben. Nach dem Erhitzen des Gemisches unter Rückfluss für $4 \mathrm{~h}$, wird das Produkt durch Kondensation in eine Kühlfalle im HV vom entstandenen LiF getrennt und durch eine fraktionierte Destillation gereinigt. Aus $n$-Hexan wird die Verbindungen anschließend umkristallisiert. 


\section{Bis[2,4,6-tri(tert-butyl)-1-oxi-phenyl]-fluor-1-boran 75}

0,1 mol 2,4,6-Tri(tert-butyl)phenol in $100 \mathrm{~mL} n$-Hexan werden bei $0{ }^{\circ} \mathrm{C}$ mit $0,1 \mathrm{~mol}$ n-Butyllithium versetzt und $1 \mathrm{~h}$ unter Rückfluss erhitzt. Die Salzlösung wird anschließend zu 0,15 mol Trifluorborandiethyletherat gegeben. Nach dem Erhitzen des Gemisches unter Rückfluss für $3 \mathrm{~h}$, wird das Produkt durch Kondensation in eine Kühlfalle im HV vom entstandenen $\mathrm{LiBF}_{4}$ getrennt und durch eine fraktionierte Sublimation gereinigt. Aus $n$-Hexan wird die Verbindungen anschließend umkristallisiert.

\subsection{Behandlung und Entsorgung der Abfälle}

Die verwendeten Lösungsmittel wurden abdestilliert oder in Kühlfallen einkondensiert. Falls sie nicht wieder verwendet werden konnten, wurden sie nach halogenierten und nicht halogenierten Lösungsmittelabfällen getrennt und in den dafür bereitgestellten Behältern gesammelt.

Zum Trocknen von Lösungsmitteln verwendetes Natrium wurde mit Ethanol zersetzt und dem alkalischen Ethanolreinigungsbad zugeführt. Andere Trockenmittelreste wie $\mathrm{KOH}$ und $\mathrm{Ca}(\mathrm{OH})_{2}$ wurden nach vollständiger Hydrolyse in Säure- und Basenabfälle überführt.

Zur Kühlung verwendetes Ethanol wurde ebenfalls dem Reinigungsbad zugesetzt.

Die zum Reinigen von Glasgeräten verwendete Kaliumethanolat-Lösung wurde durch Eindampfen eingeengt und in den dafür vorgesehenen Kanistern an die zentrale Sammelstelle gegeben.

Säurebäder wurden mit $\mathrm{Na}_{2} \mathrm{CO}_{3}$ neutralisiert und der zentralen Entsorgung zugeführt.

Die bereitgestellten Behälter, in denen die Abfälle ordnungsgemäß gelagert werden, wurden deklariert und in die ausliegenden Listen eingetragen. 


\section{$7 \quad$ Charakteristische Daten der dargestellten Verbindungen}

\subsection{Verwendete Messgeräte und -methoden}

Die Molmassenbestimmungen wurden massenspektroskopisch an einem Finnigan MAT 8200 oder einem Finnigan MAT 9500 mit einer Elektronenanregungsenergie von $70 \mathrm{eV}$ vorgenommen. Die Charakterisierung aller beschriebenen Verbindungen erfolgte mit Hilfe der Elektronenstoßionisation (E.I.). Hinter der Massenzahl m/z, die in der relativen Atommasseneinheit [u] gemessen wird, ist die relative Intensität, bezogen auf den Basispeak (100\%) angegeben. Die charakteristischen Fragmentpeaks werden mit abnehmender relativer Atommasse aufgeführt.

Bei der Anfertigung der ${ }^{1} \mathrm{H}-,{ }^{7} \mathrm{Li}-,{ }^{13} \mathrm{C}-,{ }^{11} \mathrm{~B}-,{ }^{15} \mathrm{~N}-,{ }^{29} \mathrm{Si}-$ und ${ }^{27} \mathrm{Al}-\mathrm{NMR}-S p e k t r e n$ wurde ein Bruker Avance 300-MHz-Kernresonanzspektrometer bzw. ein Bruker Avance 500-MHz-Kernresonanzspektrometer verwendet. Die ${ }^{19} \mathrm{~F}-\mathrm{NMR}-M e s s u n g e n$ erfolgten an einem Bruker Avance 200-MHz-Kernresonanzspektrometer. Als Standard für die ${ }^{1} \mathrm{H}-,{ }^{13} \mathrm{C}$ - diente TMS als interner-, für die ${ }^{29} \mathrm{Si}-N M R-M e s s u n g e n$ als externer Standard. Bei den ${ }^{7} \mathrm{Li}-\mathrm{NMR}-M e s s u n g e n$ wurde $\mathrm{LiCl}$, , bei ${ }^{11} \mathrm{~B}-\mathrm{BF}_{3} \mathrm{OEt}_{2^{-}}$, bei ${ }^{15} \mathrm{~N}-\mathrm{MeNO}_{2^{-}}$, bei ${ }^{19} \mathrm{~F}-\mathrm{C}_{6} \mathrm{~F}_{6^{-}}$und bei ${ }^{27} \mathrm{Al}-\mathrm{NMR}$-Aufnahmen $\mathrm{AlCl}_{3} \cdot 6 \mathrm{H}_{2} \mathrm{O}$ als externer Standard verwendet. Die Konzentration der zu vermessenden Proben betrug $5 \%$ (bei ${ }^{1} \mathrm{H}-,{ }^{7} \mathrm{Li}-$ ${ }^{11} \mathrm{~B}-{ }^{19} \mathrm{~F}$ - und ${ }^{27} \mathrm{Al}-\mathrm{NMR}-M e s s u n g e n$ ) bzw. $30-40 \%$ (bei ${ }^{13} \mathrm{C}-,{ }^{15} \mathrm{~N}$ - und ${ }^{29} \mathrm{Si}-\mathrm{NMR}$ Messungen) in $\mathrm{CDCl}_{3}$ bzw. $\mathrm{C}_{6} \mathrm{D}_{6}$. Die ${ }^{7} \mathrm{Li}-,{ }^{11} \mathrm{~B}-,{ }^{13} \mathrm{C}-{ }^{19} \mathrm{~F}-$ und ${ }^{27} \mathrm{Al}-\mathrm{NMR}-S p e k t r e n$ wurden protonenbreitbandentkoppelt-, die ${ }^{15} \mathrm{~N}$ - und ${ }^{29} \mathrm{Si}-\mathrm{NMR}$-Spektren teilweise über INEPT oder inverse-gated protonenentkoppelt aufgenommen.

Im Abschnitt 7.3 findet eine Auflistung der neu synthetisierten Verbindungen statt. Hier sind auch die jeweiligen Registernummern zu finden unter denen die Kristalldaten in den Arbeitskreisen verwahrt werden.

Alle Kristalle wurden aus den Schlenkkolben unter Argon entnommen. Proben passender Größe und genügender Qualität wurden in perfluoriniertem Polyether- bzw. Siliconöl, teilweise unter dem kalten Stickstoffstrom eines X-TEMP2 Geräts ${ }^{[79]}$, ausgewählt. Die Kristalle wurden auf der Spitze eines Glasfadens (befestigt am Goniometerkopf) montiert und schockgefroren ${ }^{[80]}$. 
Die Daten von 4 - 6, 8, 11, 12, 14, 27, 31, 37, 38, 44, 45, 48, 63, 67 und 71 wurden mittels graphitmonochromatisierter Mo-K $\mathrm{K}_{\alpha}$-Strahlung $(\lambda=71.073 \mathrm{pm}) \mathrm{im}$ AK Stalke auf einem Bruker D8 Dreikreis-Diffraktometer mit einem APEXII CCD-Detektor aufgenommen $\left(\omega\right.$-Scans, $\left.\Delta \omega=0.3^{\circ}\right)$, wobei die Probe mit einem kalten Stickstoffstrom auf 100(2) K temperiert wurde. Die Messung und eine erste Zellbestimmung wurden mit dem APEX2-Programmpaket ${ }^{[81]}$ vorgenommen. Die Daten wurden mit dem Programm SAINT ${ }^{\left[{ }^{[2]}\right.}$ integriert, wobei genaue Zellparameter erhalten wurden, und empirisch absorptionskorrigiert mit dem Programm SADABS ${ }^{[83]}$.

Die Daten von 9, 15, 18, 19, 25, 28, 29, 32 - 34, 36, 47, 58, 64 - 66, 68 - 70 und 73 wurden im AK Magull mit einem Stoe IPDSII Zweikreis-Diffraktometer bei 133(2) K mittels graphitmonochromatisierter Mo-K $\mathrm{K}_{\alpha}$ Strahlung $(\lambda=71,073 \mathrm{pm})$ und einem Imageplate-Detektor aufgezeichnet ( $\omega$-Scans, $\Delta \omega=1,0^{\circ}$ ). Die Messung, die Zellbestimmung und die Datenreduktion erfolgten mit dem X-AREA Softwarepaket von Stoe \& Cie ${ }^{[84]}$. Eine numerische Korrektur wurde mit dem Programm X-Area ${ }^{[85]}$ durchgeführt.

Die Datensammlung für 10, 35, 61, 74 und 75 erfolgte im AK Sheldrick auf einer Bruker D8 Drehanode mit Cu-K $\mathrm{K}_{\alpha}$-Strahlung $(\lambda=154,184 \mathrm{pm})$ und einem SMART6000 Detektor $\left(\omega\right.$-Scans, $\left.\Delta \omega=0,3^{\circ}\right)$. Messung und Zellbestimmung wurden mit dem PROTEUM-Softwarepaket ${ }^{[86]}$ durchgeführt. Alle weiteren Schritte entsprachen dem Vorgehen bei dem Bruker D8 Diffraktometer mit Mo-Röntgenröhre.

Die jeweiligen Daten wurden in XPREP ${ }^{[87]}$ vereinigt und für die folgenden Schritte aufbereitet. Die Strukturlösung erfolgte mit direkten Methoden in SHELXS ${ }^{[88]}$, die anschließende Verfeinerung nach "full-matrix least-squares procedures" gegen $F^{2}$ mit SHELXL ${ }^{[89]}$. Alle Grafiken und Tabellen wurden mit XSHELL und XCIF im SHELXTLProgrammpaket ${ }^{[90]}$ erstellt.

Der Schmelzpunkt der röntgenstrukturanalytisch untersuchten Verbindungen wurde in einer Apparatur nach Dr. Totolli in abgeschmolzenen Glaskapillaren ermittelt.

Die in der Literatur bereits beschriebenen Verbindungen 29 - 31, 69, und 73 wurden im Rahmen dieser Arbeit erneut bzw. auf neuen Reaktionswegen synthetisiert und charakterisiert, um fehlende NMR-spektroskopische- bzw. röntgenstrukturanalytische Daten zu ergänzen. 
Im folgenden Abschnitt sind die dargestellten Verbindungen unter Angabe folgender Daten aufgeführt:

Systematischer Name, Summenformel, molare Masse, Fest- bzw. Siedepunkt, Ausbeute, massenspektroskopische Ergebnisse, chemische Verschiebungen $\delta[p p m]$, Signalform und Kopplungskonstanten $\mathrm{J}[\mathrm{Hz}]$. Für die ${ }^{1} \mathrm{H}-\mathrm{NMR}$-Signale wird zudem die Anzahl der Protonen aus den Integrationen angegeben.

Zur Beschreibung der Signalformen werden folgende Abkürzungen angegeben: $s$ (Singulett), d (Dublett), dd (Dublett von Dublett), dt (Dublett von Triplett), m (Multiplett), sept (Septett) und $t$ (Triplett). 


\subsection{Physikalische Daten der dargestellten Verbindungen 1 - 75}

$N, N^{\prime}$-Bis[1,1-bis(trimethylsilyl)-2-aza-3-dimethylsilyl]ethylendiamin

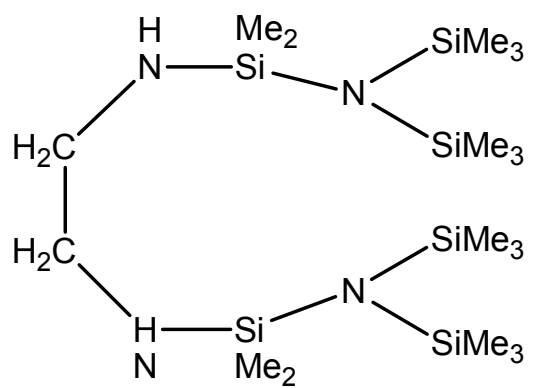

Summenformel:

Molare Masse:

Siedepunkt:

Ausbeute:

Massenspektrum:
(E.I.) $\mathrm{m} / \mathrm{z}$
$\mathrm{C}_{18} \mathrm{H}_{54} \mathrm{~N}_{4} \mathrm{Si}_{6}$

$494,16 \mathrm{~g} / \mathrm{mol}$

$104{ }^{\circ} \mathrm{C} / 0,01 \mathrm{mbar}$

$84 \%$

479

$[\mathrm{M}-\mathrm{Me}]^{+}$

$20 \%$

Kernmagnetische Resonanz (NMR) $\left(\mathrm{CDCl}_{3}\right)$

\begin{tabular}{|c|c|c|c|c|}
\hline Verschiebung & Zuordnung & Signalform & Kopplungskonst. [Hz] & Integration \\
\hline \multicolumn{5}{|l|}{$\delta^{1} \mathrm{H}$ [ppm] } \\
\hline 0,16 & $\mathrm{Si}\left(\mathrm{CH}_{3}\right)_{2}$ & s & & $12 \mathrm{H}$ \\
\hline 0,17 & $\mathrm{Si}\left(\mathrm{CH}_{3}\right)_{3}$ & s & & $36 \mathrm{H}$ \\
\hline 2,66 & $\mathrm{C}_{\mathrm{H}_{2}}$ & $\mathrm{~m}$ & & $4 \mathrm{H}$ \\
\hline \multicolumn{5}{|l|}{$\delta{ }^{13} \mathrm{C}$ [ppm] } \\
\hline 4,07 & $\mathrm{Si}\left(\underline{\mathrm{C}}_{3}\right)_{2}$ & s & & \\
\hline 5,42 & $\mathrm{Si}\left(\underline{\mathrm{CH}}_{3}\right)_{3}$ & $s$ & & \\
\hline 45,32 & $\underline{\mathrm{CH}}_{2}$ & $\mathrm{~s}$ & & \\
\hline \multicolumn{5}{|l|}{$\delta{ }^{15} \mathrm{~N}$ [ppm] } \\
\hline$-348,99$ & $\underline{N} \mathrm{H}$ & $d$ & ${ }^{1} \mathrm{~J}_{\mathrm{NH}}=74,6$ & \\
\hline \multicolumn{5}{|l|}{$\delta^{29} \mathrm{Si}$ [ppm] } \\
\hline$-6,32$ & $\underline{\mathrm{SiMe}}_{2}$ & s & & \\
\hline 1,45 & $\underline{\mathrm{SiMe}}_{3}$ & s & & \\
\hline
\end{tabular}


$N, N^{\prime}$-Bis(1-tert-butyldimethylsilyl-1-trimethylsilyl-2-aza-3-dimethylsilyl)ethylendiamin

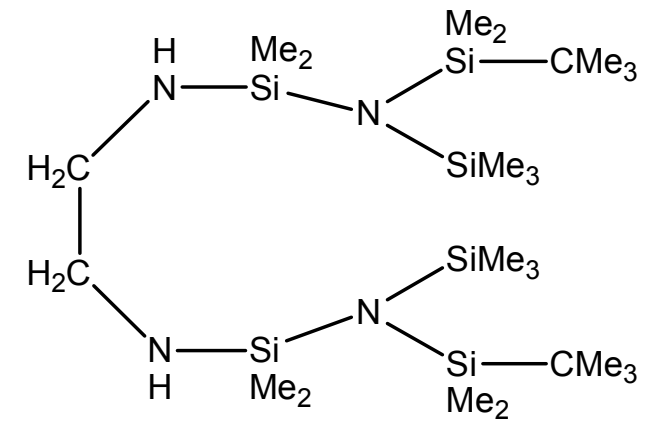

Summenformel:

$$
\mathrm{C}_{24} \mathrm{H}_{66} \mathrm{~N}_{4} \mathrm{Si}_{6}
$$

Molare Masse: $579,32 \mathrm{~g} / \mathrm{mol}$

Festpunkt:

$49^{\circ} \mathrm{C}$

Ausbeute:

$93 \%$

Massenspektrum:

(E.I.) $\mathrm{m} / \mathrm{z}$

563

[M-Me $]^{+}$

$35 \%$

521

[M-Bu] $]^{+}$

$100 \%$

Kernmagnetische Resonanz (NMR) $\left(\mathrm{CDCl}_{3}\right)$

\begin{tabular}{|c|c|c|c|c|}
\hline Verschiebung & Zuordnung & Signalform & Kopplungskonst. [Hz] & Integration \\
\hline \multicolumn{5}{|l|}{$\delta^{1} \mathrm{H}[\mathrm{ppm}]$} \\
\hline 0,17 & $\mathrm{SiCMe}_{3}\left(\mathrm{C}_{3}\right)_{2}$ & s & & $12 \mathrm{H}$ \\
\hline 0,20 & $\mathrm{NH}-\mathrm{Si}\left(\mathrm{C}_{3}\right)_{2}$ & $\mathrm{~s}$ & & $12 \mathrm{H}$ \\
\hline 0,21 & $\mathrm{Si}\left(\mathrm{CH}_{3}\right)_{3}$ & s & & $18 \mathrm{H}$ \\
\hline 0,90 & $\mathrm{SiC}\left(\mathrm{CH}_{3}\right)_{3} \mathrm{Me}_{2}$ & s & & $18 \mathrm{H}$ \\
\hline 2,66 & $\mathrm{CH}_{2}$ & $\mathrm{~m}$ & & $4 \mathrm{H}$ \\
\hline \multicolumn{5}{|l|}{$\delta{ }^{13} \mathrm{C}$ [ppm] } \\
\hline 2,63 & $\mathrm{SiCMe}_{3}\left(\underline{\mathrm{CH}_{3}}\right)_{2}$ & s & & \\
\hline 5,05 & $\mathrm{NH}-\mathrm{Si}\left(\underline{\mathrm{C}}_{3}\right)_{2}$ & $\mathrm{~s}$ & & \\
\hline 6,54 & $\mathrm{Si}\left(\underline{\mathrm{C}}_{3}\right)_{3}$ & $s$ & & \\
\hline 20,05 & $\mathrm{SiCMe}_{3} \mathrm{Me}_{2}$ & $\mathrm{~s}$ & & \\
\hline 28,40 & $\mathrm{SiC}\left(\underline{\mathrm{CH}}_{3}\right)_{3} \mathrm{Me}_{2}$ & s & & \\
\hline 45,74 & $\underline{\mathrm{C}}_{2}$ & $\mathrm{~s}$ & & \\
\hline \multicolumn{5}{|l|}{$\delta{ }^{15} \mathrm{~N}$ [ppm] } \\
\hline$-348,51$ & $\underline{\mathrm{NH}}$ & $s$ & & \\
\hline \multicolumn{5}{|l|}{$\delta^{29} \mathrm{Si}$ [ppm] } \\
\hline$-5,08$ & $\mathrm{NH}-\underline{\mathrm{SiMe}}_{2}$ & $\mathrm{~s}$ & & \\
\hline 1,94 & $\underline{\mathrm{SiMe}}_{3}$ & $\mathrm{~s}$ & & \\
\hline 7,10 & $\underline{\mathrm{SiCMe}_{3} \mathrm{Me}_{2}}$ & $\mathrm{~s}$ & & \\
\hline
\end{tabular}


$N, N^{\prime}$-Bis(tert-butyldimethylsilyl-1-trimethylsilyl-2-aza-3-dimethylsilyl)ethylendiamin

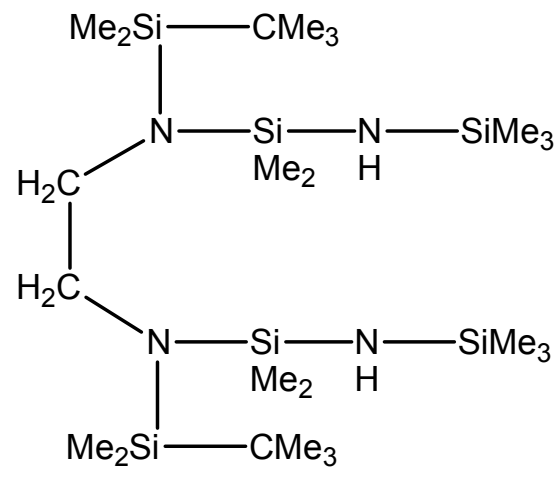

Summenformel:

Molare Masse:

$\mathrm{C}_{24} \mathrm{H}_{66} \mathrm{~N}_{4} \mathrm{Si}_{6}$

$579,32 \mathrm{~g} / \mathrm{mol}$

Siedepunkt:

$142{ }^{\circ} \mathrm{C}, 0,01 \mathrm{mbar}$

Ausbeute:

$90 \%$

Massenspektrum:

(E.I.) $\mathrm{m} / \mathrm{z}$

563

$[\mathrm{M}-\mathrm{Me}]^{+}$

$35 \%$

521

$[\mathrm{M}-\mathrm{Bu}]^{+}$

$100 \%$

Kernmagnetische Resonanz (NMR) $\left(\mathrm{CDCl}_{3}\right)$

\begin{tabular}{|c|c|c|c|c|}
\hline Verschiebung & Zuordnung & Signalform & Kopplungskonst. [Hz] & Integration \\
\hline \multicolumn{5}{|l|}{$\delta^{1} \mathrm{H}$ [ppm] } \\
\hline 0,02 & $\mathrm{SiCMe}_{3}\left(\mathrm{CH}_{3}\right)_{2}$ & s & & $12 \mathrm{H}$ \\
\hline 0,14 & $\mathrm{Si}\left(\mathrm{CH}_{3}\right)_{3}$ & $s$ & & $18 \mathrm{H}$ \\
\hline 0,16 & $\mathrm{NH}-\mathrm{Si}\left(\mathrm{C}_{3}\right)_{2}$ & s & & $12 \mathrm{H}$ \\
\hline 0,85 & $\mathrm{SiC}\left(\mathrm{CH}_{3}\right)_{3} \mathrm{Me}_{2}$ & s & & $18 \mathrm{H}$ \\
\hline 2,79 & $\mathrm{C}_{\mathrm{H}_{2}}$ & $\mathrm{~s}$ & & $4 \mathrm{H}$ \\
\hline \multicolumn{5}{|l|}{$\delta^{13 \mathrm{C}}[\mathrm{ppm}]$} \\
\hline$-2,58$ & $\mathrm{SiCMe}_{3}\left(\underline{\mathrm{CH}}_{3}\right)_{2}$ & s & & \\
\hline 3,15 & $\mathrm{Si}\left(\underline{\mathrm{C}}_{3}\right)_{3}$ & s & & \\
\hline 4,52 & $\mathrm{NH}-\mathrm{Si}\left(\underline{\mathrm{CH}}_{3}\right)_{2}$ & $\mathrm{~s}$ & & \\
\hline 17,90 & $\mathrm{SiC} \mathrm{Me}_{3} \mathrm{Me}_{2}$ & s & & \\
\hline 26,21 & $\mathrm{SiC}\left(\underline{\mathrm{C}} \mathrm{H}_{3}\right)_{3} \mathrm{Me}_{2}$ & $\mathrm{~s}$ & & \\
\hline 48,61 & $\underline{\mathrm{C}} \mathrm{H}_{2}$ & s & & \\
\hline \multicolumn{5}{|l|}{$\delta{ }^{15} \mathrm{~N}$ [ppm] } \\
\hline$-353,27$ & $\underline{\mathrm{NH}}$ & $s$ & & \\
\hline \multicolumn{5}{|l|}{$\delta^{29} \mathrm{Si}$ [ppm] } \\
\hline$-7,52$ & $\mathrm{NH}-\underline{S i M e}_{2}$ & s & & \\
\hline 3,07 & $\underline{\mathrm{SiMe}}_{3}$ & s & & \\
\hline 7,98 & $\underline{\mathrm{SiCMe}} \mathrm{Me}_{2}$ & $s$ & & \\
\hline
\end{tabular}




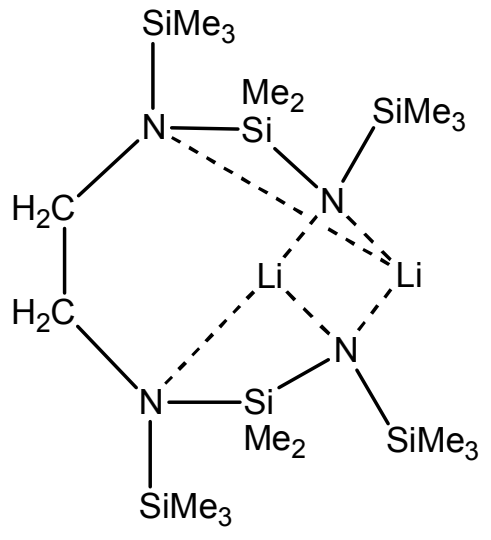

Summenformel:

Molare Masse:

Festpunkt:

Siedepunkt:

Ausbeute:
$\mathrm{C}_{18} \mathrm{H}_{52} \mathrm{Li}_{2} \mathrm{~N}_{4} \mathrm{Si}_{6}$

$507,03 \mathrm{~g} / \mathrm{mol}$

$127^{\circ} \mathrm{C}$

$89^{\circ} \mathrm{C}$

$87^{\circ} \mathrm{C}$

$190^{\circ} \mathrm{C} / 0,01 \mathrm{mbar}$

(4)

$97 \%$

Kernmagnetische Resonanz (NMR) von $4\left(C_{6} D_{6}\right)$

\begin{tabular}{|c|c|c|c|c|}
\hline Verschiebung & Zuordnung & Signalform & Kopplungskonst. [Hz] & Integration \\
\hline \multicolumn{5}{|l|}{$\delta^{1} \mathrm{H}$ [ppm] } \\
\hline 0,09 & $\mathrm{Li}-\mathrm{N}-\mathrm{Si}\left(\mathrm{C}_{\mathrm{H}_{3}}\right)_{3}$ & s & & $18 \mathrm{H}$ \\
\hline 0,19 & $\mathrm{CH}_{2}-\mathrm{N}-\mathrm{Si}\left(\mathrm{CH}_{3}\right)_{3}$ & s & & $18 \mathrm{H}$ \\
\hline 0,24 & $\mathrm{Si}\left(\mathrm{C}_{3}\right)_{2}$ & s & & $12 \mathrm{H}$ \\
\hline 2,91 & $\mathrm{CH}_{2}$ & $\mathrm{~s}$ & & $4 \mathrm{H}$ \\
\hline \multicolumn{5}{|l|}{$\delta{ }^{7} \mathrm{Li}[\mathrm{ppm}]$} \\
\hline 1,64 & $\underline{\mathrm{Li}}$ & $\mathrm{s}$ & & \\
\hline \multicolumn{5}{|l|}{$\delta{ }^{13} \mathrm{C}$ [ppm] } \\
\hline 1,91 & $\mathrm{Li}-\mathrm{N}-\mathrm{Si}\left(\underline{\mathrm{CH}}_{3}\right)_{3}$ & $\mathrm{~s}$ & & \\
\hline 4,75 & $\mathrm{CH}_{2}-\mathrm{N}-\mathrm{Si}\left(\mathrm{CH}_{3}\right)_{3}$ & $\mathrm{~s}$ & & \\
\hline 6,17 & $\mathrm{Si}\left(\underline{\mathrm{CH}}_{3}\right)_{2}$ & $s$ & & \\
\hline 51,27 & $\underline{\mathrm{CH}}_{2}$ & $\mathrm{~s}$ & & \\
\hline \multicolumn{5}{|l|}{$\delta^{29} \mathrm{Si}$ [ppm] } \\
\hline$-12,93$ & $\mathrm{Li}-\mathrm{N}-\mathrm{SiMe}_{3}$ & s & & \\
\hline$-8,11$ & $\underline{\mathrm{SiMe}}_{2}$ & $\mathrm{~s}$ & & \\
\hline 5,73 & $\mathrm{CH}_{2}-\mathrm{N}-\mathrm{SiMe}_{3}$ & $\mathrm{~s}$ & & \\
\hline
\end{tabular}


N,N'-Bis(1-trimethylsilyl-2-dimethylsilyl-2-aza-trimethylsilyl)ethylendiamin<smiles>C[SiH]1NCCN(S(C)(C)C)[SiH](C)NCCS(C)(C)N1</smiles>

Summenformel:

$\mathrm{C}_{18} \mathrm{H}_{54} \mathrm{~N}_{4} \mathrm{Si}_{6}$

Molare Masse: $495,16 \mathrm{~g} / \mathrm{mol}$

Siedepunkt: $104^{\circ} \mathrm{C} / 0,01 \mathrm{mbar}$

Ausbeute: $94 \%$

$\begin{array}{lllll}\text { Massenspektrum: } & \text { (E.I.) } \mathrm{m} / \mathrm{z} & 479 & \text { [M-Me }^{+} & 20 \%\end{array}$

Kernmagnetische Resonanz (NMR) $\left(\mathrm{CDCl}_{3}\right)$

\begin{tabular}{|c|c|c|c|c|}
\hline Verschiebung & Zuordnung & Signalform & Kopplungskonst. [Hz] & Integration \\
\hline \multicolumn{5}{|l|}{$\delta^{1} \mathrm{H}[\mathrm{ppm}]$} \\
\hline 0,06 & $\mathrm{NH}-\mathrm{Si}\left(\mathrm{C}_{3}\right)_{3}$ & s & & $18 \mathrm{H}$ \\
\hline 0,13 & $\mathrm{CH}_{2}-\mathrm{N}-\mathrm{Si}\left(\mathrm{CH}_{3}\right)_{3}$ & s & & $18 \mathrm{H}$ \\
\hline 0,15 & $\mathrm{Si}\left(\mathrm{CH}_{3}\right)_{2}$ & s & & $12 \mathrm{H}$ \\
\hline 2,78 & $\mathrm{CH}_{2}$ & s & & $4 \mathrm{H}$ \\
\hline \multicolumn{5}{|l|}{$\delta{ }^{13} \mathrm{C}[\mathrm{ppm}]$} \\
\hline 2,49 & $\mathrm{NH}-\mathrm{Si}\left(\mathrm{CH}_{3}\right)_{3}$ & $\mathrm{~s}$ & & \\
\hline 3,24 & $\mathrm{CH}_{2}-\mathrm{N}-\mathrm{Si}\left(\mathrm{CH}_{3}\right)_{3}$ & $\mathrm{~s}$ & & \\
\hline 4,42 & $\mathrm{Si}\left(\underline{\mathrm{C}} \mathrm{H}_{3}\right)_{2}$ & $\mathrm{~s}$ & & \\
\hline 47,14 & $\underline{\mathrm{CH}}_{2}$ & $\mathrm{~s}$ & & \\
\hline \multicolumn{5}{|l|}{$\delta{ }^{15} \mathrm{~N}$ [ppm] } \\
\hline$-346,13$ & $\underline{\mathrm{NH}}$ & $d$ & ${ }^{1} \mathrm{~J}_{\mathrm{NH}}=66,2$ & \\
\hline \multicolumn{5}{|l|}{$\delta^{29} \mathrm{Si}[\mathrm{ppm}]$} \\
\hline$-3,95$ & $\mathrm{~N}-\mathrm{SiMe}_{2}$ & s & & \\
\hline 1,42 & $\mathrm{NH}-\mathrm{SiMe}_{3}$ & s & & \\
\hline 5,15 & $\mathrm{CH}_{2}-\mathrm{N}-\mathrm{SiMe}_{3}$ & $\mathrm{~s}$ & & \\
\hline
\end{tabular}


N,N'-Bis(1-tert-butyldifluorsilyl-1-trimethylsilyl-2-aza-3-dimethylsilyl-trimethylsilyl)ethylendiamin

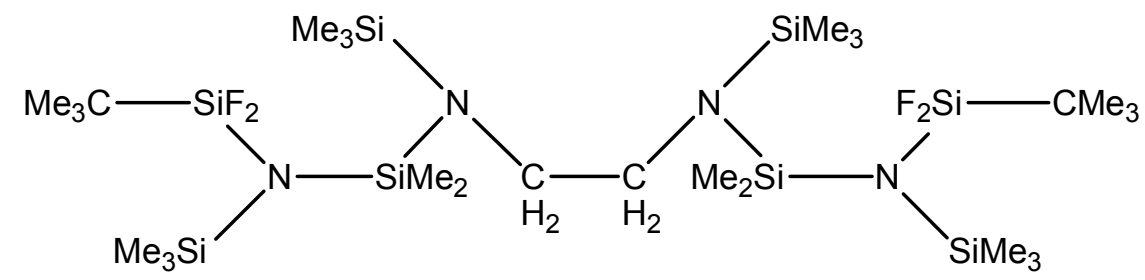

Summenformel:

$\mathrm{C}_{26} \mathrm{H}_{70} \mathrm{~N}_{4} \mathrm{~F}_{4} \mathrm{Si}_{8}$

Molare Masse:

$739,54 \mathrm{~g} / \mathrm{mol}$

Festpunkt:

$112^{\circ} \mathrm{C}$

Siedepunkt:

$170^{\circ} \mathrm{C} / 0,01 \mathrm{mbar}$

Ausbeute:

$85 \%$

Massenspektrum:

(E.I.) $\mathrm{m} / \mathrm{z}$

738

$[\mathrm{M}]^{+}$

$8 \%$

723

[M-Me $^{+}$

$36 \%$

Kernmagnetische Resonanz (NMR) $\left(\mathrm{CDCl}_{3}\right)$

\begin{tabular}{|c|c|c|c|c|}
\hline Verschiebung & Zuordnung & Signalform & Kopplungskonst. [Hz] & Integration \\
\hline \multicolumn{5}{|l|}{$\delta^{1} \mathrm{H}$ [ppm] } \\
\hline 0,21 & $\mathrm{CH}_{2}-\mathrm{N}-\mathrm{Si}\left(\mathrm{C}_{3}\right)_{3}$ & s & & $18 \mathrm{H}$ \\
\hline 0,24 & $\mathrm{SiCMe}_{3} \mathrm{~F}_{2}-\mathrm{N}-\mathrm{Si}\left(\mathrm{CH}_{3}\right)_{3}$ & $\mathrm{t}$ & ${ }^{5} \mathrm{~J}_{\mathrm{HF}}=0,9$ & $18 \mathrm{H}$ \\
\hline 0,37 & $\mathrm{Si}\left(\mathrm{CH}_{3}\right)_{2}$ & $\mathrm{t}$ & ${ }^{5} J_{H F}=1,2$ & $12 \mathrm{H}$ \\
\hline 1,04 & $\mathrm{SiC}\left(\mathrm{CH}_{3}\right)_{3} \mathrm{~F}_{2}$ & $\mathrm{t}$ & ${ }^{4} \mathrm{~J}_{\mathrm{HF}}=1,2$ & $18 \mathrm{H}$ \\
\hline 2,87 & $\mathrm{CH}_{2}$ & $\mathrm{~s}$ & & $4 \mathrm{H}$ \\
\hline \multicolumn{5}{|l|}{$\delta{ }^{13} \mathrm{C}[\mathrm{ppm}]$} \\
\hline 3,19 & $\mathrm{CH}_{2}-\mathrm{N}-\mathrm{Si}\left(\mathrm{CH}_{3}\right)_{3}$ & s & & \\
\hline 4,35 & $\mathrm{SiCMe}_{3} \mathrm{~F}_{2}-\mathrm{N}-\mathrm{Si}\left(\mathrm{C}_{3}\right)_{3}$ & $\mathrm{t}$ & ${ }^{4} J_{C F}=2,0$ & \\
\hline 6,24 & $\mathrm{Si}\left(\underline{\mathrm{CH}}_{3}\right)_{2}$ & $\mathrm{t}$ & ${ }^{4} J_{C F}=2,3$ & \\
\hline 18,89 & $\mathrm{Si} \underline{\mathrm{C}}\left(\mathrm{CH}_{3}\right)_{3} \mathrm{~F}_{2}$ & $\mathrm{t}$ & ${ }^{2} J_{C F}=22,2$ & \\
\hline 27,52 & $\mathrm{SiC}\left(\mathrm{CH}_{3}\right)_{3} \mathrm{~F}_{2}$ & s & & \\
\hline 49,20 & $\underline{\mathrm{C}} \mathrm{H}_{2}$ & s & & \\
\hline \multicolumn{5}{|l|}{$\delta^{19} \mathrm{~F}[\mathrm{ppm}]$} \\
\hline 34,13 & $\underline{F}$ & s & & \\
\hline \multicolumn{5}{|l|}{$\delta^{29} \mathrm{Si}$ [ppm] } \\
\hline$-34,68$ & $\underline{\mathrm{SiCMe}} \mathrm{F}_{2}$ & $\mathrm{t}$ & ${ }^{1} J_{\mathrm{SiF}}=297,1$ & \\
\hline$-2,33$ & $\underline{\mathrm{SiMe}}_{2}$ & $\mathrm{t}$ & ${ }^{3} \mathrm{~J}_{\mathrm{SiF}}=1,7$ & \\
\hline 5,24 & $\mathrm{SiCMe}_{3} \mathrm{~F}_{2}-\mathrm{N}-\mathrm{SiMe}_{3}$ & $t$ & ${ }^{3} \mathrm{~J}_{\mathrm{SiF}}=2,6$ & \\
\hline 6,11 & $\mathrm{CH}_{2}-\mathrm{N}-\mathrm{SiMe}_{3}$ & $\mathrm{~s}$ & & \\
\hline
\end{tabular}


N,N'-Bis(1-fluordimethylsilyl-1-trimethylsilyl-2-aza-3-dimethylsilyl-trimethylsilyl)ethylendiamin 9

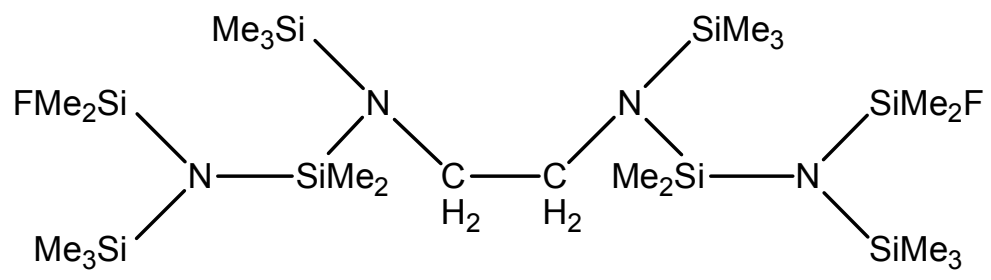

Summenformel:

Molare Masse:

Festpunkt:

Siedepunkt:

Ausbeute:

Massenspektrum:
$\mathrm{C}_{22} \mathrm{H}_{64} \mathrm{~N}_{4} \mathrm{~F}_{2} \mathrm{Si}_{8}$

$647,45 \mathrm{~g} / \mathrm{mol}$

$107^{\circ} \mathrm{C}$

$134{ }^{\circ} \mathrm{C} / 0,01 \mathrm{mbar}$

$90 \%$

$\begin{array}{lll}646 & {[\mathrm{M}]^{+}} & 8 \% \\ 631 & {[\mathrm{M}-\mathrm{Me}]^{+}} & 100 \%\end{array}$

Kernmagnetische Resonanz (NMR) $\left(\mathrm{CDCl}_{3}\right)$

\begin{tabular}{|c|c|c|c|c|}
\hline Verschiebung & Zuordnung & Signalform & Kopplungskonst. [Hz] & Integration \\
\hline \multicolumn{5}{|l|}{$\delta{ }^{1} \mathrm{H}$ [ppm] } \\
\hline 0,07 & $\mathrm{CH}_{2}-\mathrm{N}-\mathrm{Si}\left(\mathrm{C}_{3}\right)_{3}$ & s & & $18 \mathrm{H}$ \\
\hline 0,10 & $\mathrm{SiMe}_{2} \mathrm{~F}-\mathrm{N}-\mathrm{Si}\left(\mathrm{CH}_{3}\right)_{3}$ & $s$ & & $18 \mathrm{H}$ \\
\hline 0,20 & $\mathrm{Si}\left(\mathrm{CH}_{3}\right)_{2} \mathrm{~F}$ & $d$ & ${ }^{3} J_{H F}=7,5$ & $12 \mathrm{H}$ \\
\hline 0,29 & $\mathrm{Si}\left(\mathrm{CH}_{3}\right)_{2}$ & $d$ & ${ }^{5} J_{H F}=0,8$ & $12 \mathrm{H}$ \\
\hline 2,87 & $\mathrm{CH}_{2}$ & $\mathrm{~s}$ & & $4 \mathrm{H}$ \\
\hline \multicolumn{5}{|l|}{$\delta^{13 C}$ [ppm] } \\
\hline 0,17 & $\mathrm{CH}_{2}-\mathrm{N}-\mathrm{Si}\left(\underline{\mathrm{CH}}_{3}\right)_{3}$ & $\mathrm{~s}$ & & \\
\hline 2,31 & $\mathrm{Si}\left(\mathrm{CH}_{3}\right)_{2} \mathrm{~F}$ & $d$ & ${ }^{2} J_{C F}=20,2$ & \\
\hline 5,50 & $\mathrm{Si}\left(\underline{\mathrm{C}}_{3}\right)_{2}$ & $d$ & ${ }^{4} J_{C F}=1,8$ & \\
\hline 6,39 & $\mathrm{SiMe}_{2} \mathrm{~F}-\mathrm{N}-\mathrm{Si}\left(\mathrm{CH}_{3}\right)_{2}$ & d & ${ }^{4} J_{C F}=1,6$ & \\
\hline 46,68 & $\underline{\mathrm{C}}_{2}$ & $\mathrm{~s}$ & & \\
\hline \multicolumn{5}{|l|}{$\delta{ }^{19} \mathrm{~F}$ [ppm] } \\
\hline 30,78 & $\underline{F}$ & sept & ${ }^{3} J_{F H}=7,5$ & \\
\hline \multicolumn{5}{|l|}{$\delta^{29} \mathrm{Si}[\mathrm{ppm}]$} \\
\hline$-6,12$ & $\underline{\mathrm{SiMe}_{2} \mathrm{~F}}$ & $d$ & ${ }^{1} J_{\text {Sif }}=201,3$ & \\
\hline$-3,72$ & $\underline{\mathrm{SiMe}}_{2}$ & $d$ & ${ }^{3} \mathrm{~J}_{\mathrm{SiF}}=3,6$ & \\
\hline 2,29 & $\mathrm{SiMe}_{2} \mathrm{~F}-\mathrm{N}-\underline{\mathrm{SiMe}}_{3}$ & $d$ & ${ }^{3} \mathrm{~J}_{\mathrm{SiF}}=4,5$ & \\
\hline 7,62 & $\mathrm{CH}_{2}-\mathrm{N}-\mathrm{SiMe}_{3}$ & $\mathrm{~s}$ & & \\
\hline
\end{tabular}




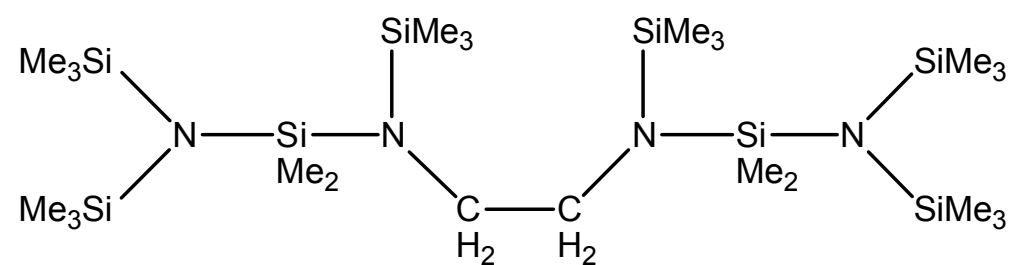

Summenformel:

Molare Masse:

Festpunkt:

Siedepunkt:

Ausbeute:

Massenspektrum:

(E.I.) $\mathrm{m} / \mathrm{z}$

$\mathrm{C}_{24} \mathrm{H}_{70} \mathrm{~N}_{4} \mathrm{Si}_{8}$

$639,52 \mathrm{~g} / \mathrm{mol}$

$106^{\circ} \mathrm{C}$

$158^{\circ} \mathrm{C} / 0,01 \mathrm{mbar}$

$90 \%$

$\begin{array}{lll}638 & {[\mathrm{M}]^{+}} & 5 \% \\ 623 & {[\mathrm{M}-\mathrm{Me}]^{+}} & 100 \%\end{array}$

Kernmagnetische Resonanz (NMR) $\left(\mathrm{CDCl}_{3}\right)$

\begin{tabular}{|c|c|c|c|c|}
\hline Verschiebung & Zuordnung & Signalform & Kopplungskonst. [Hz] & Integration \\
\hline \multicolumn{5}{|l|}{$\delta^{1} \mathrm{H}$ [ppm] } \\
\hline 0,16 & $\mathrm{CH}_{2}-\mathrm{N}-\mathrm{Si}\left(\mathrm{C}_{3}\right)_{3}$ & $s$ & & $18 \mathrm{H}$ \\
\hline 0,17 & $\mathrm{~N}-\left[\mathrm{Si}\left(\mathrm{C}_{3}\right)_{3}\right]_{2}$ & $s$ & & $36 \mathrm{H}$ \\
\hline 0,27 & $\mathrm{Si}\left(\mathrm{CH}_{3}\right)_{2}$ & $\mathrm{~s}$ & & $12 \mathrm{H}$ \\
\hline 2,77 & $\mathrm{C}_{2}$ & $\mathrm{~s}$ & & $4 \mathrm{H}$ \\
\hline \multicolumn{5}{|l|}{$\delta{ }^{13} \mathrm{C}[\mathrm{ppm}]$} \\
\hline 3,21 & $\mathrm{CH}_{2}-\mathrm{N}-\mathrm{Si}\left(\mathrm{CH}_{3}\right)_{3}$ & $\mathrm{~s}$ & & \\
\hline 5,43 & $\mathrm{~N}-\left[\mathrm{Si}\left(\underline{\mathrm{CH}}_{3}\right)_{3}\right]_{2}$ & $\mathrm{~s}$ & & \\
\hline 7,16 & $\mathrm{Si}\left(\underline{\mathrm{CH}}_{3}\right)_{2}$ & $s$ & & \\
\hline 48,88 & $\underline{\mathrm{CH}}_{2}$ & $\mathrm{~s}$ & & \\
\hline \multicolumn{5}{|l|}{$\delta^{29} \mathrm{Si}[\mathrm{ppm}]$} \\
\hline$-2,21$ & $\underline{\mathrm{SiMe}}_{2}$ & $s$ & & \\
\hline 1,67 & $\mathrm{~N}-\left(\underline{\mathrm{SiMe}}_{3}\right)_{2}$ & $s$ & & \\
\hline 4,63 & $\mathrm{CH}_{2}-\mathrm{N}-\underline{\mathrm{SiMe}_{3}}$ & s & & \\
\hline
\end{tabular}


$N, N$ '-Bis[(1-fluorboryl-1,1,5,5-tetramethylpiperidino)-1-trimethylsilyl-2-aza-3-dimethylsilyltrimethylsilyl]ethylendiamin

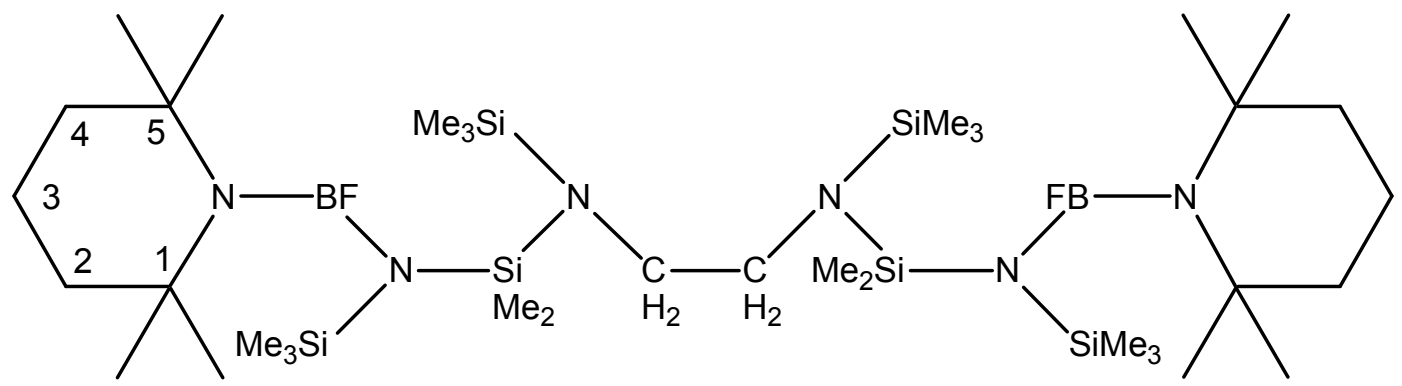

Summenformel:

Molare Masse:

Festpunkt:

Ausbeute:
$\mathrm{C}_{36} \mathrm{H}_{88} \mathrm{~B}_{2} \mathrm{~N}_{6} \mathrm{~F}_{2} \mathrm{Si}_{6}$

$833,26 \mathrm{~g} / \mathrm{mol}$

$158^{\circ} \mathrm{C}$

$86 \%$

Kernmagnetische Resonanz (NMR) $\left(C_{6} D_{6}\right)$

\begin{tabular}{|c|c|c|c|c|}
\hline Verschiebung & Zuordnung & Signalform & Kopplungskonst. [Hz] & Integration \\
\hline \multicolumn{5}{|l|}{$\delta^{1} \mathrm{H}$ [ppm] } \\
\hline 0,36 & $\mathrm{BF}-\mathrm{N}-\mathrm{Si}\left(\mathrm{CH}_{3}\right)_{3}$ & s & & $18 \mathrm{H}$ \\
\hline 0,42 & $\mathrm{CH}_{2}-\mathrm{N}-\mathrm{Si}\left(\mathrm{C}_{3}\right)_{3}$ & $s$ & & $18 \mathrm{H}$ \\
\hline 0,58 & $\mathrm{Si}\left(\mathrm{C}_{3}\right)_{2}$ & s & & $12 \mathrm{H}$ \\
\hline 1,44 & $1 / 5 \mathrm{C}\left(\mathrm{C}_{3}\right)_{2}$ & $\mathrm{~m}$ & & $24 \mathrm{H}$ \\
\hline 1,78 & $2 / 3 / 4 \mathrm{C}_{2}$ & $\mathrm{~m}$ & & $12 \mathrm{H}$ \\
\hline 3,25 & $\mathrm{~N}-\underline{\mathrm{H}}_{2}$ & s & & $4 \mathrm{H}$ \\
\hline \multicolumn{5}{|l|}{$\delta{ }^{11} \mathrm{~B}$ [ppm] } \\
\hline 25,28 & $\underline{B}$ & $s$ & & \\
\hline \multicolumn{5}{|l|}{$\delta^{13} \mathrm{C}$ [ppm] } \\
\hline 3,76 & $\mathrm{BF}-\mathrm{N}-\mathrm{Si}\left(\mathrm{CH}_{3}\right)_{3}$ & s & & \\
\hline 4,26 & $\mathrm{CH}_{2}-\mathrm{N}-\mathrm{Si}\left(\underline{\mathrm{CH}}_{3}\right)_{3}$ & s & & \\
\hline 5,63 & $\mathrm{Si}\left(\underline{\mathrm{CH}}_{3}\right)_{2}$ & $\mathrm{~s}$ & & \\
\hline 15,84 & $3 \underline{\mathrm{CH}}_{2}$ & $\mathrm{~s}$ & & \\
\hline 32,36 & $1 \overline{\mathrm{C}}\left(\mathrm{C}_{3}\right)_{2}$ & $d$ & ${ }^{4} J_{C F}=3,1$ & \\
\hline 32,62 & $5 \mathrm{C}\left(\underline{\mathrm{CH}}_{3}\right)_{2}$ & $d$ & ${ }^{4} J_{C F}=5,3$ & \\
\hline 37,78 & $2 / 4 \underline{\mathrm{CH}}_{2}$ & $\mathrm{~s}$ & & \\
\hline 49,77 & $\mathrm{~N}-\underline{\mathrm{CH}}_{2}$ & $\mathrm{~s}$ & & \\
\hline 53,91 & $1 / 5 \underline{\mathrm{CMe}}_{2}$ & $\mathrm{~s}$ & & \\
\hline \multicolumn{5}{|l|}{$\delta{ }^{19} \mathrm{~F}[\mathrm{ppm}]$} \\
\hline 103,29 & $\underline{F}$ & s & & \\
\hline \multicolumn{5}{|l|}{$\delta^{29} \mathrm{Si}$ [ppm] } \\
\hline$-6,59$ & $\underline{\mathrm{SiMe}}_{2}$ & $d$ & ${ }^{3} J_{S_{\mathrm{iF}}}=0,9$ & \\
\hline 1,34 & BF-N-SiMe ${ }_{3}$ & $d$ & ${ }^{3} J_{S_{i F}}=3,1$ & \\
\hline 5,89 & $\mathrm{CH}_{2}-\mathrm{N}-\mathrm{SiMe}_{3}$ & $\mathrm{~s}$ & & \\
\hline
\end{tabular}


$N, N$ '-Bis[1-fluorboryl-bis(trimethylsilyl)amino-1-trimethylsilyl-2-aza-3-dimethylsilyl-trimethylsilyl]ethylendiamin

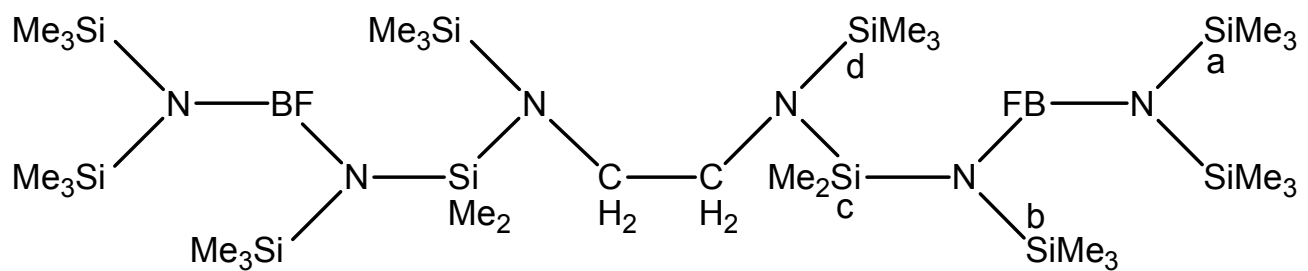

Summenformel:

Molare Masse:

Siedepunkt:

Ausbeute:

Massenspektrum:

(E.I.) $\mathrm{m} / \mathrm{z}$
$\mathrm{C}_{30} \mathrm{H}_{88} \mathrm{~B}_{2} \mathrm{~N}_{6} \mathrm{~F}_{2} \mathrm{Si}_{10}$

$873,53 \mathrm{~g} / \mathrm{mol}$

$170^{\circ} \mathrm{C} / 0,01 \mathrm{mbar}$

$85 \%$

857

$[\mathrm{M}-\mathrm{Me}]^{+}$

$100 \%$

Kernmagnetische Resonanz (NMR) $\left(\mathrm{CDCl}_{3}\right)$

\begin{tabular}{|c|c|c|c|c|}
\hline Verschiebung & Zuordnung & Signalform & Kopplungskonst. [Hz] & Integration \\
\hline \multicolumn{5}{|l|}{$\delta^{1} \mathrm{H}[\mathrm{ppm}]$} \\
\hline 0,18 & b Si $\left(\mathrm{C}_{3}\right)_{3}$ & $d$ & ${ }^{5} \mathrm{H}_{11}=07$ & $18 \mathrm{H}$ \\
\hline 0,19 & $\mathrm{~d} \mathrm{Si}\left(\mathrm{CH}_{3}\right)_{3}$ & s & & $18 \mathrm{H}$ \\
\hline 0,20 & a Si $\left(\underline{C H}_{3}\right)_{3}$ & s & & $36 \mathrm{H}$ \\
\hline 0,34 & c Si $\left(\mathrm{CH}_{3}\right)_{2}$ & $s$ & & $12 \mathrm{H}$ \\
\hline 2,87 & $\mathrm{CH}_{2}$ & $s$ & & $4 \mathrm{H}$ \\
\hline \multicolumn{5}{|l|}{$\delta^{11} \mathrm{~B}$ [ppm] } \\
\hline 27,20 & $\underline{B}$ & $\mathrm{~s}$ & & \\
\hline \multicolumn{5}{|l|}{$\delta{ }^{13} \mathrm{C}[\mathrm{ppm}]$} \\
\hline 3,28 & b Si $\left(\underline{C}_{3}\right)_{3}$ & $d$ & ${ }^{4} J_{C F}=1,5$ & \\
\hline 3,72 & a $\mathrm{Si}\left(\underline{\mathrm{CH}}_{3}\right)_{3}$ & $d$ & ${ }^{4} J_{C F}=1,6$ & \\
\hline 3,75 & $\mathrm{~d} \mathrm{Si}\left(\underline{\mathrm{CH}}_{3}\right)_{3}$ & s & & \\
\hline 5,15 & c Si $\left(\underline{C H}_{3}\right)_{2}$ & $d$ & ${ }^{4} J_{C F}=1,9$ & \\
\hline 49,43 & $\underline{\mathrm{CH}}_{2}$ & s & & \\
\hline \multicolumn{5}{|l|}{$\delta^{19} \mathrm{~F}[\mathrm{ppm}]$} \\
\hline 106,77 & $\underline{F}$ & s & & \\
\hline \multicolumn{5}{|l|}{$\delta^{29} \mathrm{Si}$ [ppm] } \\
\hline$-4,86$ & c $\underline{\mathrm{SiMe}}{ }_{2}$ & $d$ & ${ }^{3} \mathrm{~J}_{\mathrm{SiF}}=3,1$ & \\
\hline 2,80 & b $\underline{\mathrm{SiMe}}_{3}$ & $d$ & ${ }^{3} \mathrm{~J}_{\mathrm{SiF}}=5,4$ & \\
\hline 4,09 & a $\underline{\mathrm{SiMe}}_{3}$ & $d$ & ${ }^{3} \mathrm{~J}_{\mathrm{SiF}}=6,8$ & \\
\hline 5,58 & d $\underline{\mathrm{SiMe}}_{3}$ & $\mathrm{~s}$ & & \\
\hline
\end{tabular}


N,N'-Bis[1-fluorboryl-diisopropylamino-1-trimethylsilyl-2-aza-3-dimethylsilyl-trimethylsilyl]ethylendiamin

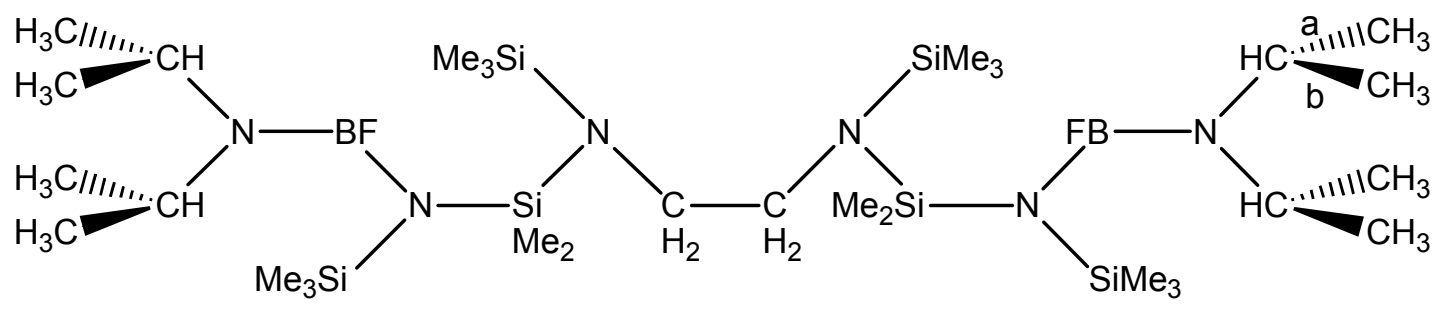

Summenformel:

$\mathrm{C}_{30} \mathrm{H}_{80} \mathrm{~B}_{2} \mathrm{~N}_{6} \mathrm{~F}_{2} \mathrm{Si}_{6}$

Molare Masse:

$753,13 \mathrm{~g} / \mathrm{mol}$

Siedepunkt:

$159{ }^{\circ} \mathrm{C} / 0,01 \mathrm{mbar}$

Ausbeute: $60 \%$

Massenspektrum:

(E.I.) $\mathrm{m} / \mathrm{z}$

737

$[\mathrm{M}-\mathrm{Me}]^{+}$

$20 \%$

Kernmagnetische Resonanz (NMR) $\left(\mathrm{CDCl}_{3}\right)$

\begin{tabular}{|c|c|c|c|c|}
\hline Verschiebung & Zuordnung & Signalform & Kopplungskonst. [Hz] & Integration \\
\hline \multicolumn{5}{|l|}{$\delta{ }^{1} \mathrm{H}[\mathrm{ppm}]$} \\
\hline $\begin{array}{l}0,05 \\
0,12 \\
0,24 \\
1,07 \\
1,18 \\
2,83 \\
3,04 \\
3,91\end{array}$ & $\begin{array}{l}\mathrm{BF}-\mathrm{N}-\mathrm{Si}\left(\mathrm{CH}_{3}\right)_{3} \\
\mathrm{CH} \mathrm{H}_{2}-\mathrm{N}-\mathrm{Si}\left(\underline{\mathrm{CH}}_{3}\right)_{3} \\
\mathrm{Si}\left(\mathrm{C} \mathrm{H}_{3}\right)_{2} \\
\text { a CH}\left(\mathrm{CH}_{3}\right)_{2} \\
\text { b } \mathrm{CH}\left(\mathrm{CH}_{3}\right)_{2} \\
\mathrm{CH}_{2} \\
\text { a } \underline{\mathrm{C}}_{\mathrm{HMe}} \\
\text { b } \underline{\mathrm{C}} \mathrm{HMe}_{2}\end{array}$ & $\begin{array}{c}\text { s } \\
\text { s } \\
\text { s } \\
d \\
d \\
\text { s } \\
\text { sept } \\
\text { sept }\end{array}$ & $\begin{aligned}{ }^{3} \mathrm{~J}_{\mathrm{HH}} & =6,6 \\
{ }^{3} \mathrm{~J}_{\mathrm{HH}} & =6,6 \\
{ }^{3} \mathrm{~J}_{\mathrm{HH}} & =6,6 \\
{ }^{3} \mathrm{~J}_{\mathrm{HH}} & =6,6\end{aligned}$ & $\begin{array}{l}18 \mathrm{H} \\
18 \mathrm{H} \\
12 \mathrm{H} \\
12 \mathrm{H} \\
12 \mathrm{H} \\
4 \mathrm{H} \\
2 \mathrm{H} \\
2 \mathrm{H}\end{array}$ \\
\hline \multicolumn{5}{|l|}{$\delta^{11} \mathrm{~B}$ [ppm] } \\
\hline 24,62 & $\underline{B}$ & $\mathrm{~s}$ & & \\
\hline \multicolumn{5}{|l|}{$\delta{ }^{13} \mathrm{C}[\mathrm{ppm}]$} \\
\hline $\begin{array}{l}2,42 \\
2,82 \\
4,40 \\
21,72 \\
23,70 \\
42,97 \\
47,20 \\
49,35\end{array}$ & $\begin{array}{l}\mathrm{BF}-\mathrm{N}-\mathrm{Si}\left(\underline{\mathrm{CH}}_{3}\right)_{3} \\
\mathrm{CH}{ }_{2}-\mathrm{N}-\mathrm{Si}\left(\underline{\mathrm{C}} \mathrm{H}_{3}\right)_{3} \\
\mathrm{Si}\left(\underline{\mathrm{C}} \mathrm{H}_{3}\right)_{2} \\
\text { a CH}\left({ }_{2} \mathrm{H}_{3}\right)_{2} \\
\mathrm{~b} \mathrm{CH}\left(\underline{\mathrm{C}} \mathrm{H}_{3}\right)_{2} \\
\text { a } \underline{\mathrm{C}} \mathrm{HMe}_{2} \\
\mathrm{~b} \underline{\mathrm{C}} \mathrm{HMe}_{2} \\
\underline{\mathrm{C}} \mathrm{H}_{2}\end{array}$ & $\begin{array}{l}\mathrm{s} \\
\mathrm{s} \\
\mathrm{s} \\
\mathrm{s} \\
\mathrm{s} \\
\mathrm{s} \\
\mathrm{s} \\
\mathrm{s}\end{array}$ & & \\
\hline \multicolumn{5}{|l|}{$\delta^{19} \mathrm{~F}[\mathrm{ppm}]$} \\
\hline 74,54 & $\underline{F}$ & $s$ & & \\
\hline \multicolumn{5}{|l|}{$\delta^{29} \mathrm{Si}$ [ppm] } \\
\hline $\begin{array}{l}-3,81 \\
1,46 \\
5,87\end{array}$ & $\begin{array}{l}\mathrm{SiMe}_{2} \\
\text { BF-N-SiMe } \\
\mathrm{CH}_{2}-\mathrm{N}-\mathrm{SiMe}_{3}\end{array}$ & $\begin{array}{l}\mathrm{s} \\
\mathrm{s} \\
\mathrm{s}\end{array}$ & & \\
\hline
\end{tabular}


1,3-Bis(trimethylsilyl)-2,4-bis(1,1,5,5-tetra-methylpiperidino)-1,3-diaza-2,4-dibora-cyclobutan 14

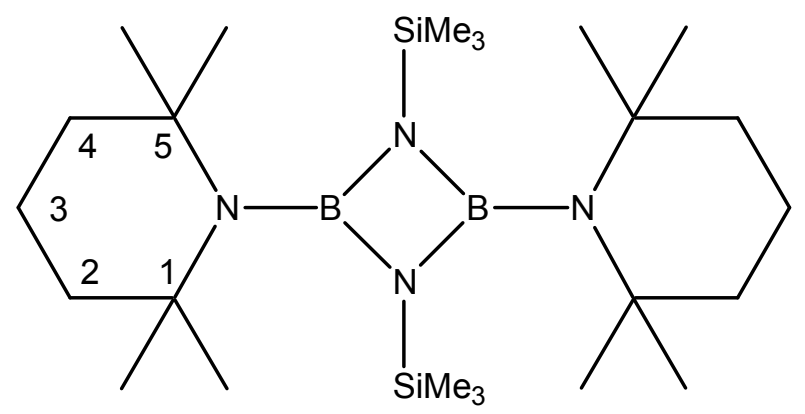

Summenformel:

Molare Masse:

Festpunkt:

Siedepunkt:

Ausbeute:

Massenspektrum:

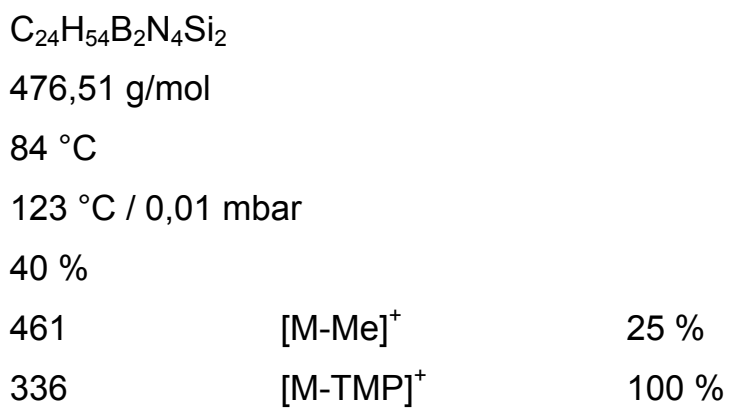

Kernmagnetische Resonanz (NMR) $\left(\mathrm{CDCl}_{3}\right)$

\begin{tabular}{|c|c|c|c|c|}
\hline Verschiebung & Zuordnung & Signalform & Kopplungskonst. [Hz] & Integration \\
\hline \multicolumn{5}{|l|}{$\delta^{1} \mathrm{H}[\mathrm{ppm}]$} \\
\hline 0,24 & $\mathrm{Si}\left(\mathrm{C}_{3}\right)_{3}$ & s & & $18 \mathrm{H}$ \\
\hline 1,29 & $1 / 5 \mathrm{C}\left(\mathrm{C}_{3}\right)_{2}$ & s & & $24 \mathrm{H}$ \\
\hline 1,40 & $2 / 4 \mathrm{C}_{2}$ & $\mathrm{t}$ & ${ }^{3} J_{H H}=6,2$ & $8 \mathrm{H}$ \\
\hline 1,70 & $3 \mathrm{CH}_{2}$ & $\mathrm{~m}$ & & $4 \mathrm{H}$ \\
\hline \multicolumn{5}{|l|}{$\delta^{11} \mathrm{~B}[\mathrm{ppm}]$} \\
\hline 35,16 & $\underline{B}$ & s & & \\
\hline \multicolumn{5}{|l|}{$\delta{ }^{13} \mathrm{C}$ [ppm] } \\
\hline 4,48 & $\mathrm{Si}\left(\underline{\mathrm{CH}}_{3}\right)_{3}$ & s & & \\
\hline 18,39 & $3 \underline{\mathrm{CH}}_{2}$ & s & & \\
\hline 31,63 & $1 / 5 \mathrm{C}\left(\mathrm{CH}_{3}\right)_{2}$ & s & & \\
\hline 39,09 & $2 / 4 \underline{\mathrm{CH}}_{2}$ & s & & \\
\hline 51,19 & $1 / 5 \underline{\mathrm{CMe}}_{2}$ & s & & \\
\hline \multicolumn{5}{|l|}{$\delta^{29} \mathrm{Si}$ [ppm] } \\
\hline$-8,24$ & $\underline{\mathrm{Si}}$ & s & & \\
\hline
\end{tabular}


1-Fluor-2,4,7,9-tetrakis(trimethylsilyl)-3,3,8,8-tetramethyl-1-bora-2,4,7,9-tetraaza-3,8-disilacyclononan<smiles>C[SiH3]N1[BH2-]N([SiH3])[SiH2]N([SiH3])[SiH2]N([SiH3])CCN1[SiH3]</smiles>

Summenformel:

$\mathrm{C}_{18} \mathrm{H}_{52} \mathrm{BN}_{4} \mathrm{FSi}_{6}$

Molare Masse:

$522,95 \mathrm{~g} / \mathrm{mol}$

Festpunkt:

$93^{\circ} \mathrm{C}$

Siedepunkt:

$125^{\circ} \mathrm{C} / 0,01 \mathrm{mbar}$

Ausbeute:

$40 \%$

$\begin{array}{ll}\text { Massenspektrum: } & \text { (E.I.) } \mathrm{m} / \mathrm{z}\end{array}$

507

$[\mathrm{M}-\mathrm{Me}]^{+}$

$5 \%$

503

$[\mathrm{M}-\mathrm{F}]^{+}$

$100 \%$

Kernmagnetische Resonanz (NMR) $\left(\mathrm{CDCl}_{3}\right)$

\begin{tabular}{|c|c|c|c|c|}
\hline Verschiebung & Zuordnung & Signalform & Kopplungskonst. [Hz] & Integration \\
\hline \multicolumn{5}{|l|}{$\delta^{1} \mathrm{H}$ [ppm] } \\
\hline 0,09 & $\mathrm{CH}_{2}-\mathrm{N}-\mathrm{Si}\left(\mathrm{CH}_{3}\right)_{3}$ & s & \multirow{4}{*}{${ }^{5} \mathrm{~J}_{\mathrm{HF}}=1,9$} & $18 \mathrm{H}$ \\
\hline 0,17 & $\mathrm{BF}-\mathrm{N}-\mathrm{Si}\left(\mathrm{CH}_{3}\right)_{3}$ & $d$ & & $18 \mathrm{H}$ \\
\hline 0,27 & $\mathrm{Si}\left(\mathrm{CH}_{3}\right)_{2}$ & s & & $12 \mathrm{H}$ \\
\hline 2,77 & $\mathrm{C}_{\mathrm{H}_{2}}$ & s & & $4 \mathrm{H}$ \\
\hline \multicolumn{5}{|l|}{$\delta{ }^{11} \mathrm{~B}$ [ppm] } \\
\hline 26,32 & $\underline{B}$ & $\mathrm{~s}$ & & \\
\hline \multicolumn{5}{|l|}{$\delta{ }^{13} \mathrm{C}$ [ppm] } \\
\hline 1,61 & $\mathrm{CH}_{2}-\mathrm{N}-\mathrm{Si}\left(\mathrm{CH}_{3}\right)_{3}$ & $s$ & & \\
\hline 3,55 & $\mathrm{BF}-\mathrm{N}-\mathrm{Si}\left(\underline{\mathrm{CH}}_{3}\right)_{3}$ & d & ${ }^{4} J_{C F}=3,5$ & \\
\hline 5,85 & $\mathrm{Si}\left(\underline{\mathrm{CH}}_{3}\right)_{2}$ & d & ${ }^{4} J_{C F}=1,6$ & \\
\hline 45,41 & $\underline{\mathrm{C}} \mathrm{H}_{2}$ & $\mathrm{~s}$ & & \\
\hline \multicolumn{5}{|l|}{$\delta{ }^{19} \mathrm{~F}[\mathrm{ppm}]$} \\
\hline 100,35 & $\underline{F}$ & $s$ & & \\
\hline \multicolumn{5}{|l|}{$\delta^{29} \mathrm{Si}$ [ppm] } \\
\hline$-3,17$ & $\underline{\mathrm{SiMe}}_{2}$ & $d$ & ${ }^{3} \mathrm{~J}_{\mathrm{SiF}}=6,6$ & \\
\hline 4,47 & $\mathrm{CH}_{2}-\mathrm{N}-\mathrm{SiMe}_{3}$ & s & & \\
\hline 4,73 & BF-N-SiMe ${ }_{3}$ & $d$ & ${ }^{3} J_{\mathrm{SiF}_{\mathrm{F}}}=9,1$ & \\
\hline
\end{tabular}


1,1-Difluor-2,4,7,9-tetrakis(trimethylsilyl)-3,3,8,8-tetramethyl-1,3,8-trisila-2,4,7,9-tetraaza-cyclononan<smiles>C[SiH3]N1CCN([SiH3])[SiH](C)N([AsH3])[SiH2]N([AsH3])[SiH2]N([SiH3])[SiH2]1</smiles>

Summenformel:

Molare Masse:

Siedepunkt:

Ausbeute:

Massenspektrum:
$\mathrm{C}_{18} \mathrm{H}_{52} \mathrm{~N}_{4} \mathrm{~F}_{2} \mathrm{Si}_{7}$

$559,23 \mathrm{~g} / \mathrm{mol}$

$125^{\circ} \mathrm{C} / 0,01 \mathrm{mbar}$

$20 \%$

Kernmagnetische Resonanz (NMR) $\left(\mathrm{CDCl}_{3}\right)$

\begin{tabular}{|c|c|c|c|c|}
\hline \multicolumn{4}{|l|}{$\delta^{1} \mathrm{H}[\mathrm{ppm}]$} & Integration \\
\hline 0,10 & $\mathrm{CH}_{2}-\mathrm{N}-\mathrm{Si}\left(\mathrm{C}_{3}\right)_{3}$ & s & & $18 \mathrm{H}$ \\
\hline 0,24 & $\mathrm{SiF}_{2}-\mathrm{N}-\mathrm{Si}\left(\mathrm{CH}_{3}\right)_{3}$ & $\mathrm{t}$ & ${ }^{5} \mathrm{~J}_{\mathrm{HF}}=1,2$ & $18 \mathrm{H}$ \\
\hline 0,35 & $\mathrm{Si}\left(\mathrm{C}_{3}\right)_{2}$ & $\mathrm{~s}$ & & $12 \mathrm{H}$ \\
\hline 2,91 & $\mathrm{CH}_{2}$ & $\mathrm{~s}$ & & $4 \mathrm{H}$ \\
\hline \multicolumn{5}{|l|}{$\delta^{13} \mathrm{C}$ [ppm] } \\
\hline 2,08 & $\mathrm{CH}_{2}-\mathrm{N}-\mathrm{Si}\left(\underline{\mathrm{CH}_{3}}\right)_{3}$ & $\mathrm{~s}$ & & \\
\hline 4,48 & $\mathrm{SiF}_{2}-\mathrm{N}-\mathrm{Si}\left(\underline{\mathrm{CH}}_{3}\right)_{3}$ & $\mathrm{t}$ & ${ }^{4} J_{C F}=2,3$ & \\
\hline 5,37 & $\mathrm{Si}\left(\underline{\mathrm{CH}}_{3}\right)_{2}$ & $\mathrm{~s}$ & & \\
\hline 48,60 & $\underline{\mathrm{C}} \mathrm{H}_{2}$ & $\mathrm{~s}$ & & \\
\hline \multicolumn{5}{|l|}{$\delta{ }^{19} \mathrm{~F}$ [ppm] } \\
\hline 51,07 & $\underline{F}$ & $\mathrm{~s}$ & & \\
\hline \multicolumn{5}{|l|}{$\delta^{29} \mathrm{Si}[\mathrm{ppm}]$} \\
\hline$-61,08$ & $\underline{\mathrm{SiF}}_{2}$ & $\mathrm{t}$ & ${ }^{1} \mathrm{~J}_{\mathrm{SiF}}=232,1$ & \\
\hline$-2,08$ & $\underline{\mathrm{SiMe}_{2}}$ & $\mathrm{t}$ & ${ }^{3} \mathrm{~J}_{\mathrm{SiF}}=1,3$ & \\
\hline 5,34 & $\mathrm{CH}_{2}-\mathrm{N}-\underline{\mathrm{SiMe}}{ }_{3}$ & $\mathrm{~s}$ & & \\
\hline 6,55 & $\mathrm{SiF}_{2}-\mathrm{N}-\underline{\mathrm{SiMe}_{3}}$ & $\mathrm{t}$ & ${ }^{3} \mathrm{~J}_{\mathrm{SiF}}=1,2$ & \\
\hline
\end{tabular}




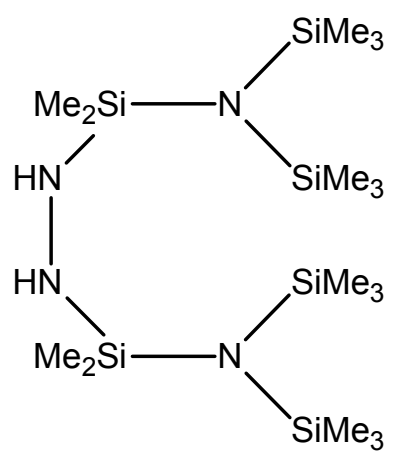

Summenformel:

Molare Masse:

Siedepunkt:

Ausbeute:

Massenspektrum:
(E.I.) $\mathrm{m} / \mathrm{z}$
$\mathrm{C}_{16} \mathrm{H}_{50} \mathrm{~N}_{4} \mathrm{Si}_{6}$

$467,11 \mathrm{~g} / \mathrm{mol}$

$113^{\circ} \mathrm{C} / 0,01 \mathrm{mbar}$

$84 \%$

$\begin{array}{lll}468 & {[\mathrm{M}]^{+}} & 30 \% \\ 467 & {[\mathrm{M}-\mathrm{H}]^{+}} & 40 \%\end{array}$

Kernmagnetische Resonanz (NMR) $\left(\mathrm{CDCl}_{3}\right)$

\begin{tabular}{|c|c|c|c|c|}
\hline Verschiebung & Zuordnung & Signalform & Kopplungskonst. [Hz] & Integration \\
\hline \multicolumn{5}{|l|}{$\delta^{1} \mathrm{H}$ [ppm] } \\
\hline 0,18 & $\mathrm{Si}\left(\mathrm{CH}_{3}\right)_{2}$ & $s$ & & $12 \mathrm{H}$ \\
\hline 0,19 & $\mathrm{Si}\left(\mathrm{CH}_{3}\right)_{3}$ & $s$ & & $36 \mathrm{H}$ \\
\hline 2,28 & $\mathrm{NH}$ & $s$ & & $2 \mathrm{H}$ \\
\hline \multicolumn{5}{|l|}{$\delta^{13} \mathrm{C}$ [ppm] } \\
\hline 2,82 & $\mathrm{Si}\left(\underline{\mathrm{C}}_{3}\right)_{2}$ & s & & \\
\hline 5,47 & $\mathrm{Si}\left(\underline{\mathrm{CH}}_{3}\right)_{3}$ & s & & \\
\hline \multicolumn{5}{|l|}{$\delta^{15} \mathrm{~N}$ [ppm] } \\
\hline$-323,96$ & $\underline{\mathrm{NH}}$ & $d$ & ${ }^{1} \mathrm{~J}_{\mathrm{NH}}=76,6$ & \\
\hline \multicolumn{5}{|l|}{$\delta^{29} \mathrm{Si}$ [ppm] } \\
\hline$-4,51$ & $\underline{\mathrm{SiMe}}_{2}$ & s & & \\
\hline 1,98 & $\underline{\mathrm{SiMe}}_{3}$ & $s$ & & \\
\hline
\end{tabular}




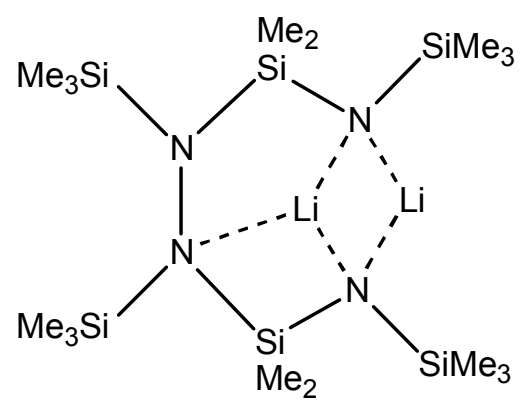

Summenformel:

$$
\mathrm{C}_{16} \mathrm{H}_{48} \mathrm{Li}_{2} \mathrm{~N}_{4} \mathrm{Si}_{6}
$$

Molare Masse: $478,97 \mathrm{~g} / \mathrm{mol}$

Festpunkt: $95{ }^{\circ} \mathrm{C}$

Siedepunkt: $124{ }^{\circ} \mathrm{C} / 0,01 \mathrm{mbar}$

Ausbeute: $93 \%$

Kernmagnetische Resonanz (NMR) $\left(C_{6} D_{6}\right)$

\begin{tabular}{|c|c|c|c|c|}
\hline Verschiebung & Zuordnung & Signalform & Kopplungskonst. [Hz] & Integration \\
\hline \multicolumn{5}{|l|}{$\delta^{1} \mathrm{H}[\mathrm{ppm}]$} \\
\hline 0,12 & $\mathrm{Li}-\mathrm{N}-\mathrm{Si}\left(\mathrm{C}_{3}\right)_{3}$ & s & & $18 \mathrm{H}$ \\
\hline 0,15 & $\mathrm{~N}-\mathrm{N}-\mathrm{Si}\left(\mathrm{C}_{3}\right)_{3}$ & s & & $18 \mathrm{H}$ \\
\hline 0,31 & $\mathrm{Si}\left(\mathrm{CH}_{3}\right)_{2}$ & s & & $12 \mathrm{H}$ \\
\hline \multicolumn{5}{|l|}{$\delta^{7} \mathrm{Li}[\mathrm{ppm}]$} \\
\hline 1,46 & $\underline{\mathrm{Li}}$ & s & & \\
\hline \multicolumn{5}{|l|}{$\delta{ }^{13} \mathrm{C}[\mathrm{ppm}]$} \\
\hline 3,39 & $\mathrm{Li}-\mathrm{N}-\mathrm{Si}\left(\underline{\mathrm{CH}}_{3}\right)_{3}$ & s & & \\
\hline 5,69 & $\mathrm{~N}-\mathrm{N}-\mathrm{Si}\left(\underline{\mathrm{CH}}_{3}\right)_{3}$ & s & & \\
\hline 7,66 & $\mathrm{Li}-\mathrm{N}-\mathrm{Si}\left(\underline{\mathrm{C}} \mathrm{H}_{3}\right)_{2}$ & s & & \\
\hline \multicolumn{5}{|l|}{$\delta^{29} \mathrm{Si}$ [ppm] } \\
\hline$-12,86$ & $\mathrm{Li}-\mathrm{N}-\mathrm{SiMe}_{3}$ & $\mathrm{~s}$ & & \\
\hline$-5,87$ & $\underline{\mathrm{SiMe}}_{2}$ & s & & \\
\hline 6,74 & $\mathrm{~N}-\mathrm{N}-\mathrm{SiMe}_{3}$ & s & & \\
\hline
\end{tabular}




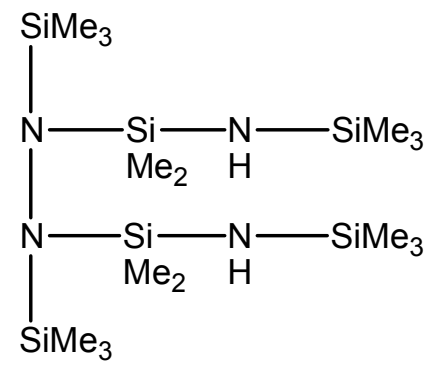

Summenformel:

Molare Masse:

Siedepunkt:

Ausbeute:

Massenspektrum:
$\mathrm{C}_{16} \mathrm{H}_{50} \mathrm{~N}_{4} \mathrm{Si}_{6}$

$467,11 \mathrm{~g} / \mathrm{mol}$

$104{ }^{\circ} \mathrm{C} / 0,01 \mathrm{mbar}$

$94 \%$

$451 \quad[\mathrm{M}-\mathrm{Me}]^{+} \quad 30 \%$

Kernmagnetische Resonanz (NMR) $\left(C_{6} D_{6}\right)$

\begin{tabular}{|c|c|c|c|c|}
\hline Verschiebung & Zuordnung & Signalform & Kopplungskonst. [Hz] & Integration \\
\hline \multicolumn{5}{|l|}{$\delta^{1} \mathrm{H}$ [ppm] } \\
\hline 0,15 & $\mathrm{NH}-\mathrm{Si}\left(\mathrm{C}_{3}\right)_{3}$ & $s$ & & $18 \mathrm{H}$ \\
\hline 0,28 & $\mathrm{~N}-\mathrm{N}-\mathrm{Si}\left(\mathrm{CH}_{3}\right)_{3}$ & $\mathrm{~s}$ & & $18 \mathrm{H}$ \\
\hline 0,33 & $\mathrm{Si}\left(\mathrm{C}_{3}\right)_{2}$ & s & & $12 \mathrm{H}$ \\
\hline \multicolumn{5}{|l|}{$\delta^{13} \mathrm{C}$ [ppm] } \\
\hline 3,20 & $\mathrm{NH}-\mathrm{Si}\left(\underline{\mathrm{C}}_{3}\right)_{3}$ & s & & \\
\hline 3,81 & $\mathrm{~N}-\mathrm{N}-\mathrm{Si}\left(\mathrm{CH}_{3}\right)_{3}$ & $\mathrm{~s}$ & & \\
\hline 7,49 & $\mathrm{Si}\left(\underline{\mathrm{C}}_{3}\right)_{2}$ & $s$ & & \\
\hline \multicolumn{5}{|l|}{$\delta{ }^{29} \mathrm{Si}[\mathrm{ppm}]$} \\
\hline$-0,08$ & $\mathrm{~N}-\underline{\mathrm{SiMe}}_{2}$ & $\mathrm{~s}$ & & \\
\hline 8,80 & $\mathrm{NH}-\mathrm{SiMe}_{3}$ & s & & \\
\hline 9,21 & $\mathrm{~N}-\mathrm{N}-\mathrm{SiMe}_{3}$ & s & & \\
\hline
\end{tabular}


1-Fluor-2,4,5,7-tetrakis(trimethylsilyl)-3,3,6,6-tetramethyl-1-bora-2,4,5,7-tetraaza-3,6-disilacycloheptan

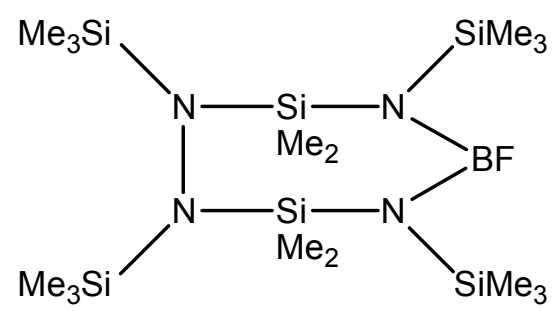

Summenformel:

Molare Masse:

Festpunkt:

Siedepunkt:

Ausbeute:

Massenspektrum:

(E.I.) $\mathrm{m} / \mathrm{z}$

$\mathrm{C}_{16} \mathrm{H}_{48} \mathrm{BN}_{4} \mathrm{FSi}_{6}$

$494,90 \mathrm{~g} / \mathrm{mol}$

$93^{\circ} \mathrm{C}$

$133^{\circ} \mathrm{C} / 0,01 \mathrm{mbar}$

$40 \%$

494

$[\mathrm{M}]^{+}$

$100 \%$

479

[M-Me]

$80 \%$

Kernmagnetische Resonanz (NMR) $\left(\mathrm{CDCl}_{3}\right)$

\begin{tabular}{|c|c|c|c|c|}
\hline Verschiebung & Zuordnung & Signalform & Kopplungskonst. [Hz] & Integration \\
\hline \multicolumn{5}{|l|}{$\delta^{1} \mathrm{H}$ [ppm] } \\
\hline 0,23 & $\mathrm{~N}-\mathrm{N}-\mathrm{Si}\left(\mathrm{CH}_{3}\right)_{3}$ & $s$ & \multirow{3}{*}{${ }^{5} \mathrm{~J}_{\mathrm{HF}}=2,3$} & $18 \mathrm{H}$ \\
\hline 0,29 & $\mathrm{BF}-\mathrm{N}-\mathrm{Si}\left(\mathrm{C}_{3}\right)_{3}$ & d & & $18 \mathrm{H}$ \\
\hline 0,33 & $\mathrm{Si}\left(\mathrm{CH}_{3}\right)_{2}$ & s & & $12 \mathrm{H}$ \\
\hline \multicolumn{5}{|l|}{$\delta{ }^{11} \mathrm{~B}$ [ppm] } \\
\hline 26,58 & $\underline{B}$ & s & & \\
\hline \multicolumn{5}{|l|}{$\delta{ }^{13} \mathrm{C}$ [ppm] } \\
\hline 3,20 & $\mathrm{~N}-\mathrm{N}-\mathrm{Si}\left(\underline{\mathrm{CH}}_{3}\right)_{3}$ & s & & \\
\hline 3,85 & BF-N-Si( $\left(\mathrm{CH}_{3}\right)_{3}$ & $d$ & ${ }^{4} J_{C F}=4,3$ & \\
\hline 5,17 & $\mathrm{Si}\left(\underline{\mathrm{C}} \mathrm{H}_{3}\right)_{2}$ & $d$ & ${ }^{4} J_{C F}=1,9$ & \\
\hline \multicolumn{5}{|l|}{$\delta^{19} \mathrm{~F}[\mathrm{ppm}]$} \\
\hline 94,52 & $\underline{F}$ & s & & \\
\hline \multicolumn{5}{|l|}{$\delta^{29} \mathrm{Si}$ [ppm] } \\
\hline$-1,37$ & $\underline{\mathrm{SiMe}}_{2}$ & $d$ & ${ }^{3} \mathrm{~J}_{\mathrm{SiF}}=7,4$ & \\
\hline 5,40 & BF-N-SiMe 3 & d & ${ }^{3} \mathrm{~J}_{\mathrm{SiF}}=10,9$ & \\
\hline 9,34 & $\mathrm{~N}-\mathrm{N}-\mathrm{SiMe}_{3}$ & s & & \\
\hline
\end{tabular}


1,1-Difluor-2,4,5,7-tetra(trimethylsilyl)-3,3,6,6-tetramethyl-2,4,5,7-tetraaza-1,3,6-trisila-cycloheptan

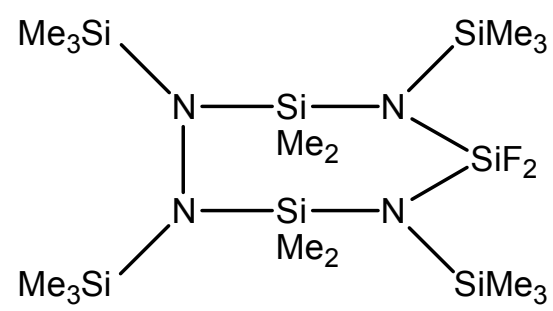

Summenformel:

Molare Masse:

Siedepunkt:

Ausbeute:

Massenspektrum:
(E.I.) $\mathrm{m} / \mathrm{z}$

$$
\mathrm{C}_{16} \mathrm{H}_{48} \mathrm{~N}_{4} \mathrm{~F}_{2} \mathrm{Si}_{7}
$$

$531,17 \mathrm{~g} / \mathrm{mol}$

$136{ }^{\circ} \mathrm{C} / 0,01 \mathrm{mbar}$ $42 \%$

$\begin{array}{lll}530 & {[\mathrm{M}]^{+}} & 100 \% \\ 515 & {[\mathrm{M}-\mathrm{Me}]^{+}} & 80 \%\end{array}$

Kernmagnetische Resonanz (NMR) $\left(\mathrm{CDCl}_{3}\right)$

\begin{tabular}{|c|c|c|c|c|}
\hline Verschiebung & Zuordnung & Signalform & Kopplungskonst. [Hz] & Integration \\
\hline \multicolumn{5}{|l|}{$\delta^{1} \mathrm{H}$ [ppm] } \\
\hline 0,13 & $\mathrm{~N}-\mathrm{N}-\mathrm{Si}\left(\mathrm{C}_{\mathrm{H}_{3}}\right)_{3}$ & $\mathrm{~s}$ & & $18 \mathrm{H}$ \\
\hline 0,25 & $\mathrm{SiF}_{2}-\mathrm{N}-\mathrm{Si}\left(\mathrm{CH}_{3}\right)_{3}$ & $\mathrm{t}$ & ${ }^{5} J_{H E}=1.1$ & $18 \mathrm{H}$ \\
\hline 0,32 & $\mathrm{Si}\left(\mathrm{CH}_{3}\right)_{2}$ & $\mathrm{t}$ & ${ }^{5} \mathrm{~J}_{\mathrm{HF}}=1,3$ & $12 \mathrm{H}$ \\
\hline \multicolumn{5}{|l|}{$\delta{ }^{13} \mathrm{C}$ [ppm] } \\
\hline 2,60 & $\mathrm{~N}-\mathrm{N}-\mathrm{Si}\left(\mathrm{CH}_{3}\right)_{3}$ & $\mathrm{t}$ & ${ }^{4} J_{C F}=4,3$ & \\
\hline 4,06 & $\mathrm{SiF}_{2}-\mathrm{N}-\mathrm{Si}\left(\underline{\mathrm{C}}_{3}\right)_{3}$ & $\mathrm{t}$ & ${ }^{4} J_{C F}=4,3$ & \\
\hline 5,34 & $\mathrm{Si}\left(\underline{\mathrm{C}} \mathrm{H}_{3}\right)_{2}$ & $\mathrm{t}$ & ${ }^{4} J_{C F}=1,9$ & \\
\hline \multicolumn{5}{|l|}{$\delta{ }^{19} \mathrm{~F}[\mathrm{ppm}]$} \\
\hline 48,32 & $\underline{F}$ & s & & \\
\hline \multicolumn{5}{|l|}{$\delta^{29} \mathrm{Si}[\mathrm{ppm}]$} \\
\hline$-61,08$ & $\underline{\mathrm{SiF}}_{2}$ & $\mathrm{t}$ & ${ }^{1} \mathrm{~J}_{\mathrm{SiF}}=232,1$ & \\
\hline 0,48 & $\underline{\mathrm{SiMe}}_{2}$ & $\mathrm{t}$ & ${ }^{3} \mathrm{~J}_{\mathrm{SiF}}=1,3$ & \\
\hline 5,71 & $\mathrm{SiF}_{2}-\mathrm{N}-\underline{\mathrm{SiMe}}_{3}$ & $\mathrm{t}$ & ${ }^{3} \mathrm{~J}_{\mathrm{SiF}}=1,4$ & \\
\hline 9,24 & $\mathrm{~N}-\mathrm{N}-\underline{\mathrm{SiMe}}_{3}$ & $\mathrm{~s}$ & & \\
\hline
\end{tabular}


<smiles>CN(C)[Si](C)(C)C</smiles>

Summenformel:

Molare Masse:

Siedepunkt:

Ausbeute:
$\mathrm{C}_{15} \mathrm{H}_{36} \mathrm{NAISi}$

$285,52 \mathrm{~g} / \mathrm{mol}$

$90^{\circ} \mathrm{C} / 10 \mathrm{mbar}$

$87 \%$

Kernmagnetische Resonanz (NMR) $\left(C_{6} D_{6}\right)$

\begin{tabular}{|c|c|c|c|c|}
\hline Verschiebung & Zuordnung & Signalform & Kopplungskonst. [Hz] & Integration \\
\hline \multicolumn{5}{|l|}{$\delta^{1} \mathrm{H}[\mathrm{ppm}]$} \\
\hline$-0,34$ & $\mathrm{Al}\left(\mathrm{C}_{3}\right)_{2}$ & s & & $6 \mathrm{H}$ \\
\hline 0,21 & $\mathrm{Si}\left(\mathrm{CMe}_{3}\right)_{2} \mathrm{C}_{3}$ & $s$ & & $3 \mathrm{H}$ \\
\hline 1,04 & $\mathrm{Si}\left[\mathrm{C}\left(\mathrm{CH}_{3}\right)_{3}\right]_{2} \mathrm{Me}$ & $\mathrm{s}$ & & $18 \mathrm{H}$ \\
\hline 1,26 & $\mathrm{~N}-\mathrm{C}\left(\mathrm{CH}_{3}\right)_{3}$ & $\mathrm{~s}$ & & $9 \mathrm{H}$ \\
\hline \multicolumn{5}{|l|}{$\delta{ }^{13} \mathrm{C}[\mathrm{ppm}]$} \\
\hline$-3,31$ & $\mathrm{Al}\left(\underline{\mathrm{C}}_{3}\right)_{2}$ & $\mathrm{~s}$ & & \\
\hline$-0,70$ & $\mathrm{Si}\left(\mathrm{CMe}_{3}\right)_{2} \underline{\mathrm{CH}}_{3}$ & s & & \\
\hline 21,94 & $\left.\mathrm{Si}(\underline{\mathrm{CMe}})_{3}\right)_{2} \mathrm{CH}_{3}$ & s & & \\
\hline 29,82 & $\mathrm{Si}\left[\mathrm{C}\left(\underline{\mathrm{C}} \mathrm{H}_{3}\right)_{3}\right]_{2} \mathrm{Me}$ & s & & \\
\hline 36,11 & $\mathrm{~N}-\mathrm{C}\left(\mathrm{CH}_{3}\right)_{3}$ & s & & \\
\hline 52,82 & $\mathrm{~N}-\underline{-}-\mathrm{Me}_{3}$ & s & & \\
\hline \multicolumn{5}{|l|}{$\delta^{27} \mathrm{Al}$ [ppm] } \\
\hline 68,16 & $\underline{\mathrm{Al}}$ & $\mathrm{s}$ & & \\
\hline \multicolumn{5}{|l|}{$\delta^{29} \mathrm{Si}$ [ppm] } \\
\hline$-0,84$ & $\underline{\mathrm{Si}}$ & $s$ & & \\
\hline
\end{tabular}


<smiles>CN[Si](C)(C)C</smiles>

Summenformel:

Molare Masse:

Siedepunkt:

Ausbeute:

Massenspektrum:

(E.I.) $\mathrm{m} / \mathrm{z}$

$\mathrm{C}_{13} \mathrm{H}_{31} \mathrm{NSi}$

$229,48 \mathrm{~g} / \mathrm{mol}$

$28{ }^{\circ} \mathrm{C} / 0,1 \mathrm{mbar}$

$80 \%$

$229 \quad[\mathrm{M}-\mathrm{Me}]^{+} \quad 10 \%$

$172 \quad[\mathrm{M}-\mathrm{Bu}]^{+} \quad 100 \%$

Kernmagnetische Resonanz (NMR) $\left(\mathrm{CDCl}_{3}\right)$

\begin{tabular}{|c|c|c|c|c|}
\hline Verschiebung & Zuordnung & Signalform & Kopplungskonst. [Hz] & Integration \\
\hline \multicolumn{5}{|l|}{$\delta^{1} \mathrm{H}$ [ppm] } \\
\hline 0,10 & $\mathrm{Si}\left(\mathrm{CMe}_{3}\right)_{2} \mathrm{CH}_{3}$ & s & & $3 \mathrm{H}$ \\
\hline 0,92 & $\mathrm{Si}\left[\mathrm{C}\left(\mathrm{CH}_{3}\right)_{3}\right]_{2} \mathrm{Me}$ & s & & $18 \mathrm{H}$ \\
\hline 1,14 & $\mathrm{~N}-\mathrm{C}\left(\mathrm{CH}_{3}\right)_{3}$ & s & & $9 \mathrm{H}$ \\
\hline \multicolumn{5}{|l|}{$\delta{ }^{13} \mathrm{C}$ [ppm] } \\
\hline$-6,43$ & $\mathrm{Si}\left(\mathrm{CMe}_{3}\right)_{2} \underline{\mathrm{CH}_{3}}$ & $\mathrm{~s}$ & & \\
\hline 20,37 & $\left.\mathrm{Si}(\underline{\mathrm{CMe}})_{3}\right)_{2} \mathrm{Me}$ & s & & \\
\hline 28,47 & $\mathrm{Si}\left[\mathrm{C}\left(\mathrm{CH}_{3}\right)_{3}\right]_{2} \mathrm{Me}$ & s & & \\
\hline 34,36 & $\mathrm{~N}-\mathrm{C}\left(\mathrm{CH}_{3}\right)_{3}$ & s & & \\
\hline 49,37 & $\mathrm{~N}-\underline{\mathrm{C}} \mathrm{Me}_{3}$ & $\mathrm{~s}$ & & \\
\hline \multicolumn{5}{|l|}{${ }^{29} \mathrm{Si}[\mathrm{ppm}]$} \\
\hline 4,47 & $\underline{\mathrm{Si}}$ & s & & \\
\hline
\end{tabular}




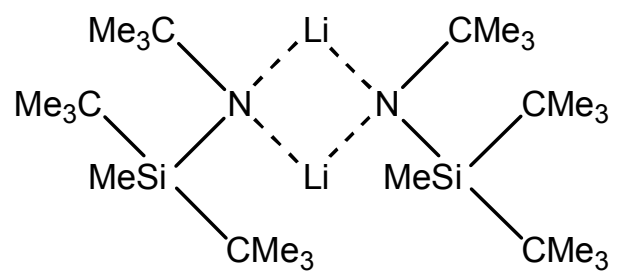

Summenformel:

$\mathrm{C}_{26} \mathrm{H}_{60} \mathrm{Li}_{2} \mathrm{~N}_{2} \mathrm{Si}_{2}$

Molare Masse:

$470,82 \mathrm{~g} / \mathrm{mol}$

Festpunkt:

$68^{\circ} \mathrm{C}$

Ausbeute:

$95 \%$

Kernmagnetische Resonanz (NMR) $\left(C_{6} D_{6}\right)$

\begin{tabular}{|c|c|c|c|c|}
\hline Verschiebung & Zuordnung & Signalform & Kopplungskonst. [Hz] & Integration \\
\hline \multicolumn{5}{|l|}{$\delta^{1} \mathrm{H}$ [ppm] } \\
\hline 0,01 & $\mathrm{Si}\left(\mathrm{CMe}_{3}\right)_{2} \underline{\mathrm{CH}}_{3}$ & s & & $6 \mathrm{H}$ \\
\hline 1,16 & $\mathrm{Si}\left[\mathrm{C}\left(\mathrm{CH}_{3}\right)_{3}\right]_{2} \mathrm{Me}$ & s & & $36 \mathrm{H}$ \\
\hline 1,33 & $\mathrm{~N}-\mathrm{C}\left(\mathrm{CH}_{3}\right)_{3}$ & s & & $18 \mathrm{H}$ \\
\hline \multicolumn{5}{|l|}{$\delta^{7} \mathrm{Li}[\mathrm{ppm}]$} \\
\hline 1,45 & $\underline{\mathrm{Li}}$ & $s$ & & \\
\hline \multicolumn{5}{|l|}{$\delta{ }^{13} \mathrm{C}$ [ppm] } \\
\hline$-2,30$ & $\mathrm{Si}\left(\mathrm{CMe}_{3}\right)_{2} \underline{\mathrm{C}} \mathrm{H}_{3}$ & s & & \\
\hline 13,94 & $\mathrm{Si}\left(\underline{\mathrm{CMe}_{3}}\right)_{2} \mathrm{Me}$ & s & & \\
\hline 31,01 & $\mathrm{Si}\left[\mathrm{C}\left(\mathrm{CH}_{3}\right)_{3}\right]_{2} \mathrm{Me}$ & s & & \\
\hline 36,81 & $\mathrm{~N}-\mathrm{C}\left(\mathrm{CH}_{3}\right)_{3}$ & s & & \\
\hline 51,22 & $\mathrm{~N}-\underline{\mathrm{C}} \mathrm{Me}_{3}$ & s & & \\
\hline \multicolumn{5}{|l|}{$\delta^{29} \mathrm{Si}$ [ppm] } \\
\hline$-5,32$ & $\underline{\mathrm{Si}}$ & s & & \\
\hline
\end{tabular}


<smiles>CN(C)[Si](C)(C)C</smiles>

Summenformel:

Molare Masse:

Siedepunkt:

Ausbeute:

Massenspektrum:
$\mathrm{C}_{12} \mathrm{H}_{27} \mathrm{BNF}_{3} \mathrm{Si}$

$281,24 \mathrm{~g} / \mathrm{mol}$

$80^{\circ} \mathrm{C} / 10 \mathrm{mbar}$

$60 \%$

$266 \quad[\mathrm{M}-\mathrm{Me}]^{+} \quad 18 \%$

Kernmagnetische Resonanz (NMR) $\left(\mathrm{CDCl}_{3}\right)$

\begin{tabular}{|c|c|c|c|c|c|}
\hline Verschiebung & Zuordnung & Signalform & \multicolumn{2}{|c|}{ Kopplungskonst. [Hz] } & Integration \\
\hline \multicolumn{6}{|l|}{$\delta^{1} \mathrm{H}$ [ppm] } \\
\hline 1,06 & $\operatorname{SiF}\left[\mathrm{C}\left(\mathrm{CH}_{3}\right)_{3}\right]_{2}$ & $d$ & & ${ }^{4} J_{H F}=0,8$ & $18 \mathrm{H}$ \\
\hline \multirow[t]{2}{*}{1,37} & $\mathrm{~N}-\mathrm{C}\left(\mathrm{CH}_{3}\right)_{3}$ & $\mathrm{dt}$ & d & ${ }^{5} J_{H F}=3,1$ & $9 \mathrm{H}$ \\
\hline & & & $\mathrm{t}$ & ${ }^{5} J_{H F}=1,3$ & \\
\hline \multicolumn{6}{|l|}{$\delta^{11} B$ [ppm] } \\
\hline 25,38 & $\underline{B}$ & $\mathrm{~s}$ & & & \\
\hline \multicolumn{6}{|l|}{$\delta{ }^{13} \mathrm{C}$ [ppm] } \\
\hline 22,13 & $\mathrm{SiF}\left(\underline{\mathrm{C} M \mathrm{Me}_{3}}\right)_{2}$ & d & & ${ }^{2} J_{C F}=13,5$ & \\
\hline \multirow[t]{2}{*}{28,36} & $\operatorname{SiF}\left[\mathrm{C}\left(\underline{\mathrm{C}} \mathrm{H}_{3}\right)_{3}\right]_{2}$ & $\mathrm{dt}$ & $\mathrm{t}$ & ${ }^{3} J_{C F}=3,6$ & \\
\hline & & & $d$ & ${ }^{5} J_{C F}=1,8$ & \\
\hline 32,92 & $\mathrm{~N}-\mathrm{C}\left(\mathrm{CH}_{3}\right)_{3}$ & $\mathrm{dt}$ & $\begin{array}{l}\mathrm{t} \\
\mathrm{d}\end{array}$ & $\begin{array}{l}{ }^{4} J_{C F}=2,6 \\
{ }^{4} J_{C F}=6,1\end{array}$ & \\
\hline 55,16 & $\mathrm{~N}-\underline{\mathrm{C}} \mathrm{Me}_{3}$ & $\mathrm{t}$ & & ${ }^{3} J_{C F}=0,6$ & \\
\hline \multicolumn{6}{|l|}{$\delta{ }^{19} \mathrm{~F}$ [ppm] } \\
\hline 6,35 & SiF & s & & & \\
\hline 54,26 & $\mathrm{BE}$ & $s$ & & & \\
\hline \multicolumn{6}{|l|}{$\delta^{29} \mathrm{Si}[\mathrm{ppm}]$} \\
\hline \multirow[t]{2}{*}{10,68} & $\underline{\operatorname{SiF}}\left(\mathrm{CMe}_{3}\right)_{2}$ & $\mathrm{dt}$ & $d$ & ${ }^{1} J_{\mathrm{SiF}}=301,2$ & \\
\hline & & & $\mathrm{t}$ & ${ }^{3} \mathrm{~J}_{\mathrm{SiF}}=4,9$ & \\
\hline
\end{tabular}


1,1,2-Tri(tert-butyl)-2,4-diaza-1,3-difluor-4,4-bis(trimethylsilyl)-1-sila-3-boran<smiles>CN(C)[Si](C)(C)C</smiles>

Summenformel:

Molare Masse:

Siedepunkt:

Ausbeute:

Massenspektrum:
$\mathrm{C}_{18} \mathrm{H}_{45} \mathrm{BN}_{2} \mathrm{~F}_{2} \mathrm{Si}_{3}$

$422,63 \mathrm{~g} / \mathrm{mol}$

$93{ }^{\circ} \mathrm{C} / 0,01 \mathrm{mbar}$

$92 \%$ (Syntheseweg a), $87 \%$ (Syntheseweg b)

$407 \quad[\mathrm{M}-\mathrm{Me}]^{+} \quad 5 \%$

$365 \quad[\mathrm{M}-\mathrm{Bu}]^{+} \quad 100 \%$

Kernmagnetische Resonanz (NMR) $\left(\mathrm{CDCl}_{3}\right)$

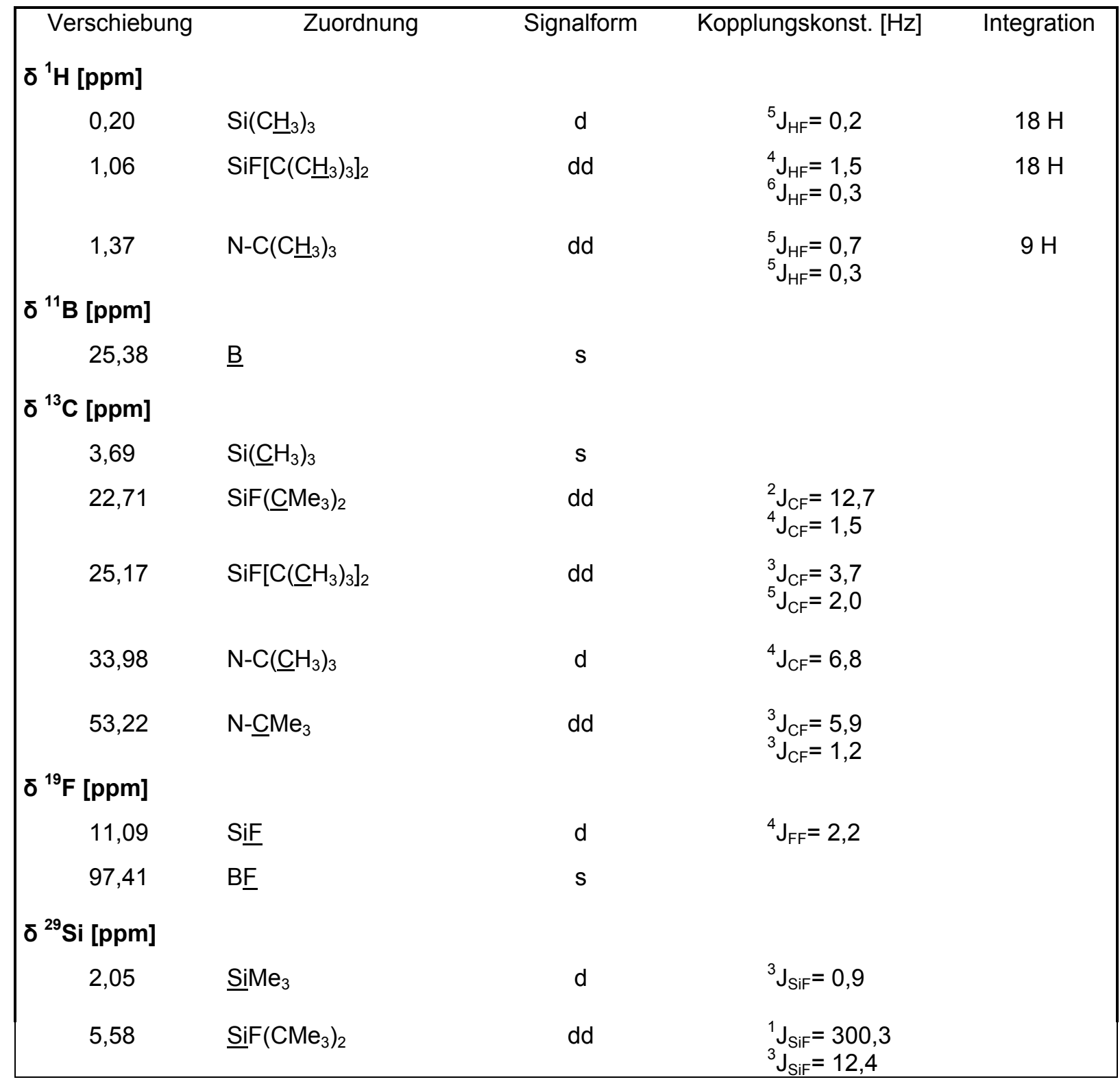


<smiles>C[Si](C)(C)N[Si](C)(C)C</smiles>

Summenformel:

Molare Masse:

Festpunkt:

Siedepunkt:

Ausbeute:

Massenspektrum:
$\mathrm{C}_{18} \mathrm{H}_{43} \mathrm{NSi}_{2}$

$329,71 \mathrm{~g} / \mathrm{mol}$

$57^{\circ} \mathrm{C}$

$75{ }^{\circ} \mathrm{C} / 0,01 \mathrm{mbar}$

$30 \%$

$\begin{array}{lll}329 & {[\mathrm{M}]^{+}} & 20 \% \\ 272 & {[\mathrm{M}-\mathrm{Bu}]^{+}} & 70 \%\end{array}$

Kernmagnetische Resonanz (NMR) $\left(\mathrm{CDCl}_{3}\right)$

\begin{tabular}{|c|c|c|c|c|}
\hline Verschiebung & Zuordnung & Signalform & Kopplungskonst. [Hz] & Integration \\
\hline \multicolumn{5}{|l|}{$\delta^{1} \mathrm{H}$ [ppm] } \\
\hline 0,07 & $\mathrm{Si}\left(\mathrm{CMe}_{3}\right)_{2} \mathrm{C}_{3}$ & $\mathrm{~s}$ & & $6 \mathrm{H}$ \\
\hline 0,99 & $\mathrm{~S}\left[\mathrm{C}\left(\mathrm{CH}_{3}\right)_{3}\right]_{2} \mathrm{Me}$ & $\mathrm{s}$ & & $36 \mathrm{H}$ \\
\hline \multicolumn{5}{|l|}{$\delta{ }^{13} \mathrm{C}[\mathrm{ppm}]$} \\
\hline 1,09 & $\mathrm{Si}\left(\mathrm{CMe}_{3}\right)_{2} \underline{\mathrm{C}} \mathrm{H}_{3}$ & s & & \\
\hline 21,04 & $\mathrm{Si}\left(\underline{\mathrm{C}} \mathrm{Me}_{3}\right)_{2} \mathrm{Me}$ & $\mathrm{s}$ & & \\
\hline 28,71 & $\mathrm{Si}\left[\mathrm{C}\left(\mathrm{CH}_{3}\right)_{3}\right]_{2} \mathrm{Me}$ & $\mathrm{s}$ & & \\
\hline \multicolumn{5}{|l|}{$\delta{ }^{15} \mathrm{~N}$ [ppm] } \\
\hline$-378,81$ & $\underline{N}$ & $\mathrm{~s}$ & & \\
\hline \multicolumn{5}{|l|}{$\delta^{29} \mathrm{Si}$ [ppm] } \\
\hline 8,62 & $\underline{\mathrm{Si}}$ & s & & \\
\hline
\end{tabular}


Bis[di(tert-butyl)chlorsilyl]amin ${ }^{[66]}$<smiles>C[Si](C)(C)N[Si](C)(C)Cl</smiles>

Summenformel:

$$
\mathrm{C}_{16} \mathrm{H}_{37} \mathrm{NSi}_{2} \mathrm{Cl}_{2}
$$

Molare Masse:

$370,55 \mathrm{~g} / \mathrm{mol}$

Ausbeute:

$30 \%$

Kernmagnetische Resonanz (NMR) $\left(\mathrm{CDCl}_{3}\right)$

\begin{tabular}{|ccc|}
\hline Verschiebung & Zuordnung & Signalform \\
$\delta{ }^{15} \mathrm{~N}$ [ppm] & & \\
$-364,84$ & $\underline{\mathrm{N}}$ & $\mathrm{S}$ \\
& & \\
\hline
\end{tabular}


Di(tert-butyl)chlorsilyl-di(tert-butyl)fluorsilyl-amin ${ }^{[66]}$<smiles>C[Si](C)(C)N[Si](C)(C)C</smiles>

Summenformel:

$\mathrm{C}_{16} \mathrm{H}_{37} \mathrm{NFSi}_{2} \mathrm{Cl}$

Molare Masse:

$354,09 \mathrm{~g} / \mathrm{mol}$

Ausbeute:

30 \% (Syntheseweg a); 40 \% (Syntheseweg b)

Kernmagnetische Resonanz (NMR) $\left(\mathrm{CDCl}_{3}\right)$

\begin{tabular}{|cccc|}
\hline $\begin{array}{c}\text { Verschiebung } \\
\delta{ }^{15} \mathrm{~N} \text { [ppm] }\end{array}$ & Zuordnung & Signalform & Kopplungskonst. [Hz] \\
$-364,11$ & $\underline{N}$ & $d$ & \\
$\delta{ }^{19} \mathrm{~F}[\mathrm{ppm}]$ & & & \\
2,72 & $\underline{\mathrm{F}}$ & $\mathrm{d}$ & \\
& & & ${ }^{3} \mathrm{~J}_{\mathrm{FF}}=6,2$ \\
\hline
\end{tabular}


Fluor-2,6-diisopropylanilino-(methyl-trimethylsilyl)amino-boran ${ }^{\text {[68] }}$

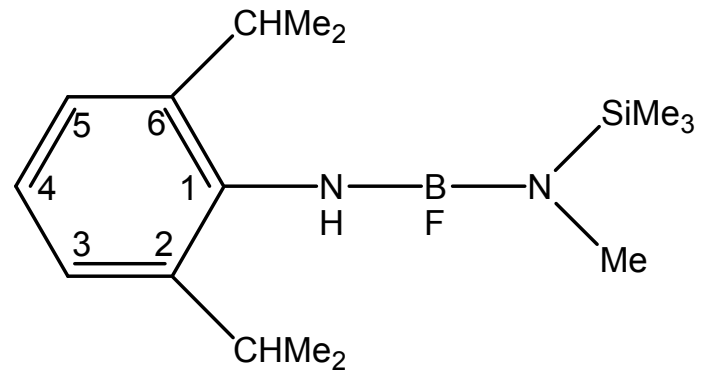

Summenformel:

$\mathrm{C}_{20} \mathrm{H}_{37} \mathrm{BN}_{2} \mathrm{~F}$

Molare Masse:

$334,32 \mathrm{~g} / \mathrm{mol}$

Festpunkt:

$62{ }^{\circ} \mathrm{C}$

Verbindung 31 wird hier, auf Grund bekannter kernresonanz- und massenspektroskopischer Daten ${ }^{[68]}$, ausschließlich röntgenstrukturanalytisch beschrieben. 


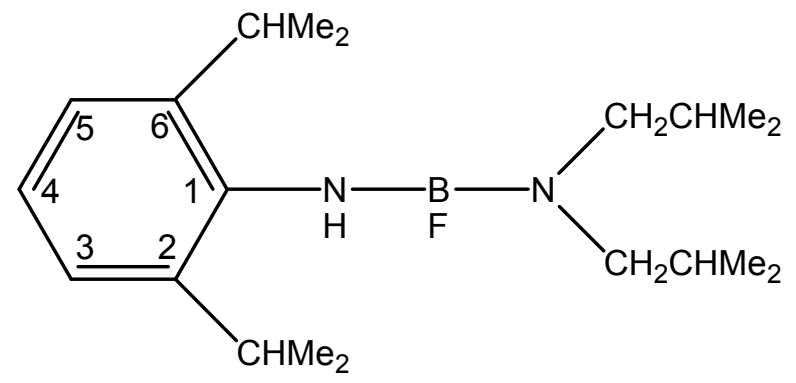

Summenformel:

Molare Masse:

Festpunkt:

Siedepunkt:

Ausbeute:

Massenspektrum:
$\mathrm{C}_{20} \mathrm{H}_{37} \mathrm{BN}_{2} \mathrm{~F}$

$334,32 \mathrm{~g} / \mathrm{mol}$

$81{ }^{\circ} \mathrm{C}$

$80^{\circ} \mathrm{C} / 0,01 \mathrm{mbar}$

$95 \%$

$334 \quad[\mathrm{M}]^{+} \quad 18 \%$

$291 \quad\left[\mathrm{M}-\mathrm{CHMe}_{2}\right]^{+} \quad 18 \%$

Kernmagnetische Resonanz (NMR) $\left(\mathrm{CDCl}_{3}\right)$

\begin{tabular}{|c|c|c|c|c|}
\hline Verschiebung & Zuordnung & Signalform & Kopplungskonst. [Hz] & Integration \\
\hline \multicolumn{5}{|l|}{$\delta^{1} \mathrm{H}$ [ppm] } \\
\hline 0,91 & $\mathrm{CH}_{2}-\mathrm{CH}\left(\mathrm{CH}_{3}\right)_{2}$ & $d$ & ${ }^{3} \mathrm{~J}_{\mathrm{HH}}=6,6$ & $12 \mathrm{H}$ \\
\hline 1,19 & $2 / 6 \mathrm{C}-\mathrm{CH}\left(\mathrm{CH}_{3}\right)_{2}$ & $d$ & ${ }^{3} \mathrm{~J}_{\mathrm{HH}}=6,9$ & $12 \mathrm{H}$ \\
\hline 1,88 & $\mathrm{CH}_{2}-\mathrm{CHMe}_{2}$ & $\mathrm{~m}$ & & $2 \mathrm{H}$ \\
\hline 2,72 & $\mathrm{C}_{2}-\mathrm{CHMe}_{2}$ & $d$ & ${ }^{3} \mathrm{~J}_{\mathrm{HH}}=7,4$ & $4 \mathrm{H}$ \\
\hline 3,29 & 2/6 C-C $\underline{\mathrm{CHM}}{ }_{2}$ & sept & ${ }^{3} \mathrm{~J}_{\mathrm{HH}}=6,9$ & $2 \mathrm{H}$ \\
\hline 3,49 & $\mathrm{NH}$ & $d$ & ${ }^{3} \mathrm{~J}_{\mathrm{HF}}=17,2$ & $1 \mathrm{H}$ \\
\hline $7,13-7,36$ & $3 / 4 / 5 \mathrm{C} \underline{\mathrm{H}}$ & $\mathrm{m}$ & & $3 \mathrm{H}$ \\
\hline \multicolumn{5}{|l|}{$\delta^{11} \mathrm{~B}$ [ppm] } \\
\hline 21,99 & $\underline{B}$ & $\mathrm{~s}$ & & \\
\hline \multicolumn{5}{|l|}{$\delta{ }^{13} \mathrm{C}$ [ppm] } \\
\hline 20,13 & $\mathrm{CH}_{2}-\mathrm{CH}\left(\mathrm{CH}_{3}\right)_{2}$ & $s$ & & \\
\hline 23,46 & $2 / 6 \mathrm{C}-\mathrm{CH}\left(\underline{\mathrm{CH}}_{3}\right)_{2}$ & s & & \\
\hline 26,93 & $\mathrm{CH}_{2}-\underline{\mathrm{C}} \mathrm{HMe}_{2}$ & s & & \\
\hline 28,43 & 2/6 C- $\underline{\mathrm{C}} \mathrm{HMe}_{2}$ & $s$ & & \\
\hline 53,56 & $\underline{\mathrm{C}} \mathrm{H}_{2}-\mathrm{CHMe}_{2}$ & $s$ & & \\
\hline 122,85 & $3 / 5 \underline{C}$ & s & & \\
\hline 125,17 & $4 \underline{C}$ & s & & \\
\hline 136,04 & $2 / 6 \underline{C}$ & $\mathrm{~s}$ & & \\
\hline 145,13 & $1 \underline{\mathrm{C}}$ & $\mathrm{s}$ & & \\
\hline \multicolumn{5}{|l|}{$\delta{ }^{19} \mathrm{~F}$ [ppm] } \\
\hline 22,75 & $\underline{F}$ & $\mathrm{~s}$ & & \\
\hline
\end{tabular}


Fluor-diisopropylamino-2,6-diisopropylanilino-boran

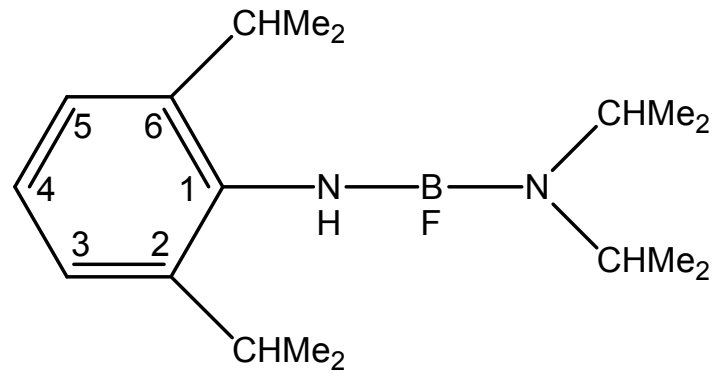

Summenformel:

$\mathrm{C}_{18} \mathrm{H}_{32} \mathrm{BN}_{2} \mathrm{~F}$

Molare Masse:

$306,27 \mathrm{~g} / \mathrm{mol}$

Festpunkt:

$83^{\circ} \mathrm{C}$

Siedepunkt:

$70^{\circ} \mathrm{C} / 0,01 \mathrm{mbar}$

Ausbeute:

$97 \%$

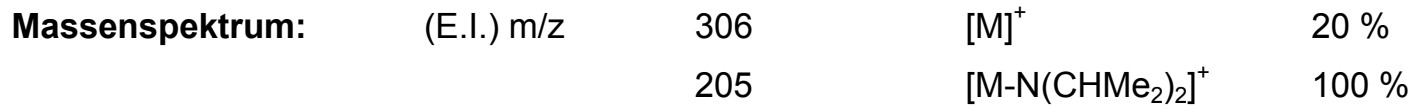

Kernmagnetische Resonanz (NMR) $\left(\mathrm{CDCl}_{3}\right)$

\begin{tabular}{|c|c|c|c|c|}
\hline Verschiebung & Zuordnung & Signalform & Kopplungskonst. [Hz] & Integration \\
\hline \multicolumn{5}{|l|}{$\delta^{1} \mathrm{H}$ [ppm] } \\
\hline 1,19 & $2 / 6 \mathrm{C}-\mathrm{CH}\left(\mathrm{CH}_{3}\right)_{2}$ & d & ${ }^{3} \mathrm{~J}_{\mathrm{HH}}=6,8$ & $12 \mathrm{H}$ \\
\hline 1,19 & $\mathrm{~N}-\mathrm{CH}\left(\mathrm{CH}_{3}\right)_{2}$ & $d$ & ${ }^{3} \mathrm{~J}_{\mathrm{HH}}=6,8$ & $12 \mathrm{H}$ \\
\hline 3,27 & 2/6 C-C $\underline{\mathrm{HMe}} 2$ & sept & ${ }^{3} \mathrm{~J}_{\mathrm{HH}}=6,8$ & $2 \mathrm{H}$ \\
\hline 3,37 & $\mathrm{~N}-\mathrm{C} \underline{\mathrm{HMe}} \mathrm{M}_{2}$ & sept & ${ }^{3} \mathrm{~J}_{\mathrm{HH}}=6,8$ & $2 \mathrm{H}$ \\
\hline 3,39 & $\mathrm{~N} \underline{\mathrm{H}}$ & $d$ & ${ }^{3} J_{H F}=0,6$ & $1 \mathrm{H}$ \\
\hline $7,02-7,11$ & $3 / 4 / 5 \mathrm{C} \underline{\mathrm{H}}$ & $\mathrm{m}$ & & $3 \mathrm{H}$ \\
\hline \multicolumn{5}{|l|}{$\delta{ }^{11} B$ [ppm] } \\
\hline 21,78 & $\underline{B}$ & $\mathrm{~s}$ & & \\
\hline \multicolumn{5}{|l|}{$\delta{ }^{13} \mathrm{C}$ [ppm] } \\
\hline 22,43 & $\mathrm{~N}-\mathrm{CH}\left(\underline{\mathrm{C}} \mathrm{H}_{3}\right)_{2}$ & $s$ & & \\
\hline 23,40 & $2 / 6 \mathrm{C}-\mathrm{CH}\left(\mathrm{CH}_{3}\right)_{2}$ & $\mathrm{~s}$ & & \\
\hline 28,51 & 2/6 C- $\underline{\mathrm{C}} \mathrm{HMe}_{2}$ & $\mathrm{~s}$ & & \\
\hline 44,58 & $\mathrm{~N}-\underline{\mathrm{C}} \mathrm{HMe}_{2}$ & $\mathrm{~s}$ & & \\
\hline 122,88 & $3 / 5 \underline{C}$ & $s$ & & \\
\hline 125,10 & $4 \underline{C}$ & $s$ & & \\
\hline 132,43 & $2 / 6 \underline{C}$ & $\mathrm{~s}$ & & \\
\hline 145,11 & $1 \underline{\mathrm{C}}$ & $\mathrm{s}$ & & \\
\hline \multicolumn{5}{|l|}{$\delta{ }^{19} \mathrm{~F}$ [ppm] } \\
\hline 28,98 & $\underline{F}$ & $\mathrm{~s}$ & & \\
\hline
\end{tabular}


Diisopropylamino-bis(2,6-diisopropylanilino)-boran

34

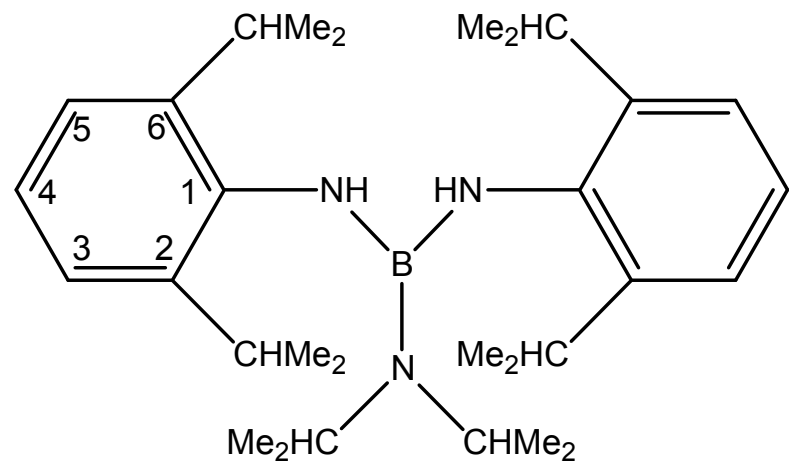

Summenformel:

$\mathrm{C}_{30} \mathrm{H}_{50} \mathrm{BN}_{3}$

Molare Masse:

$463,55 \mathrm{~g} / \mathrm{mol}$

Siedepunkt:

$90^{\circ} \mathrm{C} / 0,01 \mathrm{mbar}$

Ausbeute:

$77 \%$

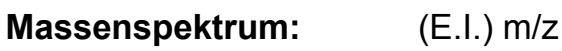

463

[M] ${ }^{+}$

$10 \%$

420

$\left[\mathrm{M}-\mathrm{CHMe}_{2}\right]^{+}$

$100 \%$

Kernmagnetische Resonanz (NMR) $\left(\mathrm{CDCl}_{3}\right)$

\begin{tabular}{|c|c|c|c|c|}
\hline Verschiebung & Zuordnung & Signalform & Kopplungskonst. [Hz] & Integration \\
\hline \multicolumn{5}{|l|}{$\delta^{1} \mathrm{H}$ [ppm] } \\
\hline 1,19 & $\mathrm{~N}-\mathrm{CH}\left(\mathrm{CH}_{3}\right)_{2}$ & $d$ & ${ }^{3} \mathrm{~J}_{\mathrm{HH}}=6,4$ & $12 \mathrm{H}$ \\
\hline 1,26 & $2 / 6 \mathrm{C}-\mathrm{CH}\left(\mathrm{CH}_{3}\right)_{2}$ & d & ${ }^{3} \mathrm{~J}_{\mathrm{HH}}=6,7$ & $24 \mathrm{H}$ \\
\hline 2,92 & 2/6 C-C $\underline{H M e}_{2}$ & sept & ${ }^{3} \mathrm{~J}_{\mathrm{HH}}=6,7$ & $4 \mathrm{H}$ \\
\hline 3,13 & $\mathrm{~N}-\mathrm{C} \underline{\mathrm{HMe}} \mathrm{H}_{2}$ & sept & ${ }^{3} J_{H H}=6,4$ & $2 \mathrm{H}$ \\
\hline 3,71 & $\mathrm{~N} \underline{\mathrm{H}}$ & $s$ & & $2 \mathrm{H}$ \\
\hline 6,79 & $4 \mathrm{CH}$ & $\mathrm{t}$ & ${ }^{3} \mathrm{~J}_{\mathrm{HH}}=7,6$ & $2 \mathrm{H}$ \\
\hline 7,03 & $3 / 5 \mathrm{C} \underline{\mathrm{H}}$ & $d$ & ${ }^{3} \mathrm{~J}_{\mathrm{HH}}=7,6$ & $4 \mathrm{H}$ \\
\hline \multicolumn{5}{|l|}{$\delta^{11} B$ [ppm] } \\
\hline 23,49 & $\underline{B}$ & $s$ & & \\
\hline \multicolumn{5}{|l|}{$\delta{ }^{13} \mathrm{C}[\mathrm{ppm}]$} \\
\hline 21,14 & $\mathrm{~N}-\mathrm{CH}\left(\mathrm{CH}_{3}\right)_{2}$ & $\mathrm{~s}$ & & \\
\hline 22,43 & $2 / 6 \mathrm{C}-\mathrm{CH}\left(\mathrm{CH}_{3}\right)_{2}$ & $s$ & & \\
\hline 27,82 & 2/6 C- $\underline{\mathrm{C}} \mathrm{HMe}_{2}$ & $s$ & & \\
\hline 46,47 & $\mathrm{~N}-\underline{\mathrm{C}} \mathrm{HMe}_{2}$ & $\mathrm{~s}$ & & \\
\hline 118,51 & $3 / 5 \underline{C}$ & s & & \\
\hline 122,75 & $4 \underline{C}$ & $s$ & & \\
\hline 132,43 & $2 / 6 \underline{C}$ & $s$ & & \\
\hline 140,23 & $1 \underline{C}$ & s & & \\
\hline
\end{tabular}


Bis(trimethylsilyl)amino-diisopropylamino-2,6-diisopropylanilino-boran

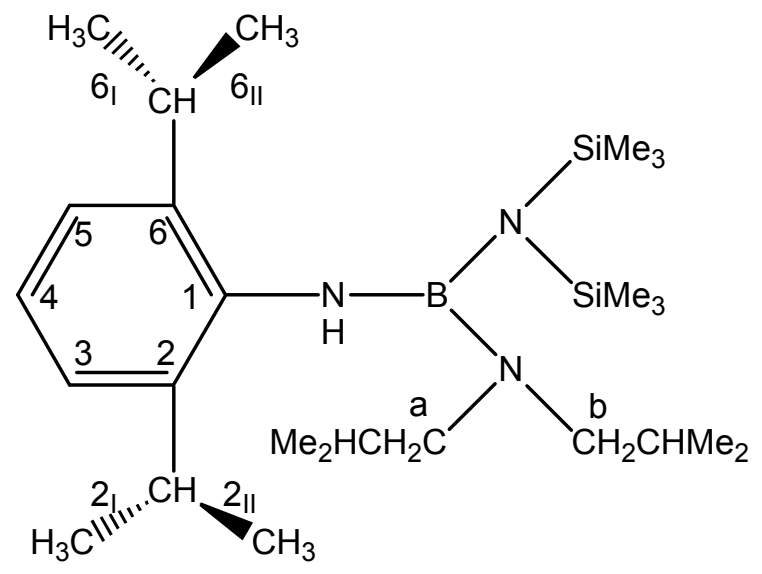

Summenformel:

$\mathrm{C}_{26} \mathrm{H}_{54} \mathrm{BN}_{3} \mathrm{Si}_{2}$

Molare Masse:

$475,71 \mathrm{~g} / \mathrm{mol}$

Siedepunkt:

$100^{\circ} \mathrm{C} / 0,01 \mathrm{mbar}$

Ausbeute: $95 \%$

\begin{tabular}{|c|c|c|}
\hline Massenspektrum: & (E.I.) $\mathrm{m} / \mathrm{z}$ & \\
\hline
\end{tabular}

Kernmagnetische Resonanz (NMR) $\left(\mathrm{CDCl}_{3}\right)$

\begin{tabular}{|c|c|c|c|c|}
\hline Verschiebung & Zuordnung & Signalform & Kopplungskonst. [Hz] & Integration \\
\hline \multicolumn{5}{|l|}{$\delta^{1} \mathrm{H}[\mathrm{ppm}]$} \\
\hline 0,21 & $\mathrm{Si}\left(\mathrm{C}_{3}\right)_{3}$ & $\mathrm{~s}$ & & $18 \mathrm{H}$ \\
\hline 0,35 & $\mathrm{a} \mathrm{CH}_{2}-\mathrm{CH}\left(\mathrm{CH}_{3}\right)_{2}$ & $d$ & ${ }^{3} \mathrm{~J}_{\mathrm{HH}}=6,6$ & $6 \mathrm{H}$ \\
\hline 0,86 & b $\mathrm{CH}_{2}-\mathrm{CH}\left(\mathrm{CH}_{3}\right)_{2}$ & $d$ & ${ }^{3} J_{H H}=6,6$ & $6 \mathrm{H}$ \\
\hline 1,09 & $2, / 6, \mathrm{C}-\mathrm{CH}\left(\mathrm{C}_{\mathrm{H}_{3}}\right)_{2}$ & $d$ & ${ }^{3} \mathrm{~J}_{\mathrm{HH}}=6,9$ & $6 \mathrm{H}$ \\
\hline 1,25 & ${ }_{2 \|} / 6_{\|} \mathrm{C}-\mathrm{CH}\left(\mathrm{CH}_{3}\right)_{2}$ & $d$ & ${ }^{3} \mathrm{~J}_{\mathrm{HH}}=6,9$ & $6 \mathrm{H}$ \\
\hline 1,87 & $\mathrm{a} / \mathrm{b} \mathrm{CH}{ }_{2}-\underline{C H M e}_{2}$ & $\mathrm{~m}$ & & $2 \mathrm{H}$ \\
\hline 2,36 & $\mathrm{a} \mathrm{C} \underline{H}_{2}-\mathrm{CHMe}_{2}$ & $d$ & ${ }^{3} \mathrm{~J}_{\mathrm{HH}}=6,4$ & $2 \mathrm{H}$ \\
\hline 2,89 & $\mathrm{~b} \mathrm{C} \underline{\mathrm{H}}_{2}-\mathrm{CHMe}_{2}$ & $d$ & ${ }^{3} \mathrm{~J}_{\mathrm{HH}}=7,6$ & $2 \mathrm{H}$ \\
\hline 3,47 & 2/6 C-C $\underline{\mathrm{CHMe}} 2$ & sept & ${ }^{3} J_{H H}=6,9$ & $2 \mathrm{H}$ \\
\hline 3,63 & $\mathrm{~N} \underline{\mathrm{H}}$ & $s$ & & $1 \mathrm{H}$ \\
\hline 7,04 & $3 / 4 / 5 \mathrm{C} \underline{\mathrm{H}}$ & $s$ & & $3 \mathrm{H}$ \\
\hline \multicolumn{5}{|l|}{$\delta{ }^{11} \mathrm{~B}[\mathrm{ppm}]$} \\
\hline 25,04 & $\underline{B}$ & $s$ & & \\
\hline \multicolumn{5}{|l|}{$\delta{ }^{13} \mathrm{C}$ [ppm] } \\
\hline 3,76 & $\mathrm{Si}\left(\underline{\mathrm{C}} \mathrm{H}_{3}\right)_{3}$ & s & & \\
\hline 20,45 & a $\mathrm{CH}_{2}-\mathrm{CH}\left(\mathrm{CH}_{3}\right)_{2}$ & $\mathrm{~s}$ & & \\
\hline
\end{tabular}




\begin{tabular}{|c|c|c|}
\hline 21,03 & b $\mathrm{CH}_{2}-\mathrm{CH}\left(\mathrm{CH}_{3}\right)_{2}$ & $\mathrm{~s}$ \\
\hline 21,76 & $2, / 6, \mathrm{C}-\mathrm{CH}\left(\mathrm{CH}_{3}\right)_{2}$ & $\mathrm{~s}$ \\
\hline 25,37 & ${ }_{2 \|} / 6_{\|} \mathrm{C}-\mathrm{CH}\left(\mathrm{CH}_{3}\right)_{2}$ & $\mathrm{~s}$ \\
\hline 27,31 & a $\mathrm{CH}_{2}-\underline{\mathrm{C}} \mathrm{HMe}_{2}$ & $\mathrm{~s}$ \\
\hline 28,09 & 2/6 C- $\underline{\mathrm{C}} \mathrm{HMe}_{2}$ & $\mathrm{~s}$ \\
\hline 29,51 & b CH${ }_{2}-\underline{C} \mathrm{HMe}_{2}$ & $s$ \\
\hline 53,93 & a $\underline{\mathrm{CH}}_{2}-\mathrm{CHMe}_{2}$ & $s$ \\
\hline 57,79 & $\mathrm{~b} \underline{\mathrm{C}} \mathrm{H}_{2}-\mathrm{CHMe}_{2}$ & $\mathrm{~s}$ \\
\hline 122,43 & $3 / 5 \underline{C}$ & $s$ \\
\hline 124,38 & $4 \underline{\mathrm{C}}$ & $s$ \\
\hline 140,03 & $2 / 6 \underline{C}$ & $s$ \\
\hline 144,25 & $1 \underline{\mathrm{C}}$ & $\mathrm{s}$ \\
\hline$\delta{ }^{29} \mathrm{Si}$ [ppm] & & \\
\hline$-0,01$ & $\underline{\mathrm{Si}}$ & $\mathrm{s}$ \\
\hline
\end{tabular}




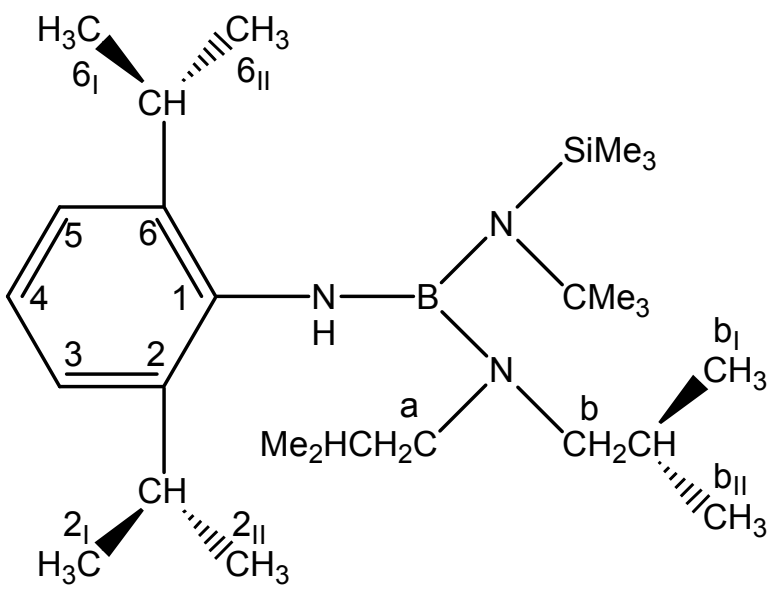

Summenformel:

Molare Masse:

Festpunkt:

Siedepunkt:

Ausbeute:

Massenspektrum:

(E.I.) $\mathrm{m} / \mathrm{z}$

$\mathrm{C}_{27} \mathrm{H}_{54} \mathrm{BN}_{3} \mathrm{Si}$

$459,63 \mathrm{~g} / \mathrm{mol}$

$116^{\circ} \mathrm{C}$

$104{ }^{\circ} \mathrm{C} / 0,01 \mathrm{mbar}$

$95 \%$

$459 \quad[\mathrm{M}]^{+} \quad 8 \%$

$416 \quad\left[\mathrm{M}-\mathrm{CHMe}_{2}\right]^{+} \quad 35 \%$

Kernmagnetische Resonanz (NMR) $\left(\mathrm{CDCl}_{3}\right)$

\begin{tabular}{|c|c|c|c|c|}
\hline Verschiebung & Zuordnung & Signalform & Kopplungskonst. [Hz] & Integration \\
\hline \multicolumn{5}{|l|}{$\delta^{1} \mathrm{H}[\mathrm{ppm}]$} \\
\hline 0,24 & $\mathrm{Si}\left(\mathrm{CH}_{3}\right)_{3}$ & s & & $9 \mathrm{H}$ \\
\hline 0,53 & a $\mathrm{CH}_{2}-\mathrm{CH}\left(\mathrm{CH}_{3}\right)_{2}$ & $d$ & ${ }^{3} \mathrm{~J}_{\mathrm{HH}}=6,6$ & $6 \mathrm{H}$ \\
\hline 0,89 & ${ }_{2} \mathrm{C}-\mathrm{CH}\left(\mathrm{CH}_{3}\right)_{2}$ & d & ${ }^{3} \mathrm{~J}_{\mathrm{HH}}=7,0$ & $3 \mathrm{H}$ \\
\hline 0,90 & ${ }_{2 \|} \mathrm{C}-\mathrm{CH}\left(\mathrm{C}_{3}\right)_{2}$ & $d$ & ${ }^{3} \mathrm{~J}_{\mathrm{HH}}=7,0$ & $3 \mathrm{H}$ \\
\hline 1,11 & $\mathrm{~b}_{1} \mathrm{CH}_{2}-\mathrm{CH}\left(\mathrm{CH}_{3}\right)_{2}$ & $d$ & ${ }^{3} \mathrm{~J}_{\mathrm{HH}}=6,8$ & $3 \mathrm{H}$ \\
\hline 1,12 & $\mathrm{~b}_{\mathrm{II}} \mathrm{CH}_{2}-\mathrm{CH}\left(\mathrm{C}_{3}\right)_{2}$ & d & ${ }^{3} \mathrm{~J}_{\mathrm{HH}}=6,8$ & $3 \mathrm{H}$ \\
\hline 1,27 & $6, \mathrm{C}-\mathrm{CH}\left(\mathrm{CH}_{3}\right)_{2}$ & $d$ & ${ }^{3} \mathrm{~J}_{\mathrm{HH}}=7,2$ & $3 \mathrm{H}$ \\
\hline 1,28 & $6_{\|} \mathrm{C}-\mathrm{CH}\left(\mathrm{CH}_{3}\right)_{2}$ & $d$ & ${ }^{3} \mathrm{~J}_{\mathrm{HH}}=7,2$ & $3 \mathrm{H}$ \\
\hline 1,39 & $\mathrm{~N}-\mathrm{C}\left(\mathrm{CH}_{3}\right)_{3}$ & $s$ & & $9 \mathrm{H}$ \\
\hline 1,93 & a $\mathrm{CH}_{2}-\mathrm{CHMe}_{2}$ & $\mathrm{~m}$ & & $1 \mathrm{H}$ \\
\hline 1,93 & $\mathrm{~b} \mathrm{CH}_{2}-\mathrm{CHMe}_{2}$ & $\mathrm{~m}$ & & $1 \mathrm{H}$ \\
\hline 2,27 & a $\underline{\mathrm{C}}_{2}-\mathrm{CHMe}_{2}$ & $d$ & ${ }^{3} \mathrm{~J}_{\mathrm{HH}}=6,8$ & $2 \mathrm{H}$ \\
\hline 2,51 & $\mathrm{~b} \underline{\mathrm{C}}_{2}-\mathrm{CHMe}_{2}$ & $d$ & ${ }^{3} J_{H H}=5,6$ & $2 \mathrm{H}$ \\
\hline
\end{tabular}




\begin{tabular}{|c|c|c|c|c|}
\hline 3,46 & $2 \mathrm{C}-\mathrm{C} \underline{\mathrm{H}} \mathrm{Me}_{2}$ & sept & ${ }^{3} \mathrm{~J}_{\mathrm{HH}}=7,0$ & $1 \mathrm{H}$ \\
\hline 3,52 & $6 \mathrm{C}-\mathrm{C}-\mathrm{HMe}_{2}$ & sept & ${ }^{3} \mathrm{~J}_{\mathrm{HH}}=7,2$ & $1 \mathrm{H}$ \\
\hline 3,83 & $\mathrm{~N} \underline{\mathrm{H}}$ & $s$ & & $1 \mathrm{H}$ \\
\hline $7,11-7,35$ & $3 / 4 / 5 \mathrm{C} \underline{\mathrm{H}}$ & $\mathrm{m}$ & & $3 \mathrm{H}$ \\
\hline \multicolumn{5}{|l|}{$\delta^{11} \mathrm{~B}[\mathrm{ppm}]$} \\
\hline 25,70 & $\underline{\mathrm{B}}$ & $s$ & & \\
\hline \multicolumn{5}{|l|}{$\delta{ }^{13} \mathrm{C}[\mathrm{ppm}]$} \\
\hline 4,93 & $\mathrm{Si}\left(\underline{\mathrm{C}}_{3}\right)_{3}$ & $\mathrm{~s}$ & & \\
\hline 20,24 & ${ }_{2} \mathrm{CH}_{2}-\mathrm{CH}\left(\mathrm{CH}_{3}\right)_{2}$ & s & & \\
\hline 20,83 & ${ }_{2 \|} \mathrm{CH}_{2}-\mathrm{CH}\left(\mathrm{CH}_{3}\right)_{2}$ & $\mathrm{~s}$ & & \\
\hline 21,00 & a $\mathrm{CH}_{2}-\mathrm{CH}\left(\underline{\mathrm{CH}}_{3}\right)_{2}$ & $\mathrm{~s}$ & & \\
\hline 21,77 & $6, \mathrm{CH}_{2}-\mathrm{CH}\left(\mathrm{CH}_{3}\right)_{2}$ & $\mathrm{~s}$ & & \\
\hline 21,99 & $6_{\|} \mathrm{C}-\mathrm{CH}\left(\mathrm{CH}_{3}\right)_{2}$ & s & & \\
\hline 24,92 & $b_{1} \mathrm{CH}_{2}-\mathrm{CH}\left(\mathrm{CH}_{3}\right)_{2}$ & $\mathrm{~s}$ & & \\
\hline 25,81 & $\mathrm{~b}_{\mathrm{II}} \mathrm{CH}_{2}-\mathrm{CH}\left(\underline{\mathrm{CH}}_{3}\right)_{2}$ & s & & \\
\hline 26,37 & a $\mathrm{CH}_{2}-\underline{\mathrm{C}} \mathrm{HMe}_{2}$ & $\mathrm{~s}$ & & \\
\hline 28,03 & $2 \mathrm{C}-\underline{\mathrm{C}} \mathrm{HMe}_{2}$ & s & & \\
\hline 28,10 & $6 \mathrm{C}-\underline{\mathrm{C}} \mathrm{HMe}_{2}$ & s & & \\
\hline 28,72 & $\mathrm{~b} \mathrm{CH}{ }_{2}-\underline{C} \mathrm{HMe}_{2}$ & s & & \\
\hline 33,64 & $\mathrm{~N}-\mathrm{C}\left(\mathrm{CH}_{3}\right)_{3}$ & $\mathrm{~s}$ & & \\
\hline 52,39 & $\mathrm{~N}-\underline{\mathrm{C}} \mathrm{Me}_{3}$ & $\mathrm{~s}$ & & \\
\hline 53,04 & $\mathrm{a} \underline{\mathrm{C}} \mathrm{H}_{2}-\mathrm{CHMe}_{2}$ & $\mathrm{~s}$ & & \\
\hline 56,81 & b $\underline{\mathrm{C}} \mathrm{H}_{2}-\mathrm{CHMe}_{2}$ & $\mathrm{~s}$ & & \\
\hline 122,47 & $3 / 5 \underline{C}$ & $\mathrm{~s}$ & & \\
\hline 123,90 & $4 \underline{C}$ & $\mathrm{~s}$ & & \\
\hline 140,49 & $2 / 6 \underline{C}$ & $\mathrm{~s}$ & & \\
\hline 144,03 & $1 \underline{C}$ & $\mathrm{~s}$ & & \\
\hline \multicolumn{5}{|l|}{$\delta^{29} \mathrm{Si}$ [ppm] } \\
\hline$-4,80$ & $\underline{\mathrm{Si}}$ & $\mathrm{s}$ & & \\
\hline
\end{tabular}




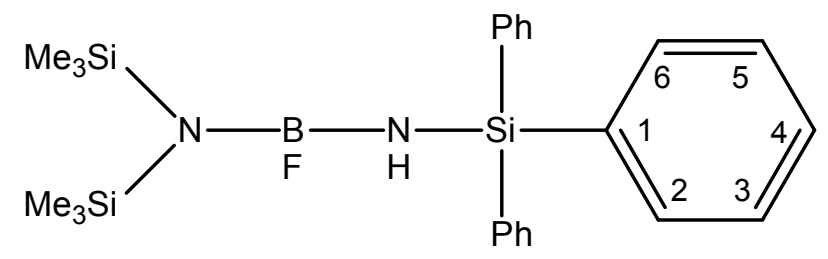

Summenformel:

Molare Masse:

Festpunkt:

Siedepunkt:

Ausbeute:

Massenspektrum:
$\mathrm{C}_{24} \mathrm{H}_{34} \mathrm{BN}_{2} \mathrm{FSi}_{3}$

$464,61 \mathrm{~g} / \mathrm{mol}$

$105^{\circ} \mathrm{C}$

$132{ }^{\circ} \mathrm{C} / 0,01 \mathrm{mbar}$

$70 \%$

$\begin{array}{lll}305 & {[\mathrm{M}-\mathrm{Me}]^{+}} & 20 \% \\ 263 & {[\mathrm{M}-\mathrm{Bu}]^{+}} & 100 \%\end{array}$

Kernmagnetische Resonanz (NMR) $\left(\mathrm{CDCl}_{3}\right)$

\begin{tabular}{|c|c|c|c|c|}
\hline Verschiebung & Zuordnung & Signalform & Kopplungskonst. [Hz] & Integration \\
\hline \multicolumn{5}{|l|}{$\delta^{1} \mathrm{H}$ [ppm] } \\
\hline 0,09 & $\mathrm{Si}\left(\mathrm{CH}_{3}\right)_{3}$ & s & & $18 \mathrm{H}$ \\
\hline $7,19-7,79$ & 2/3/4/5/6 C므 & $\mathrm{m}$ & & $15 \mathrm{H}$ \\
\hline \multicolumn{5}{|l|}{$\delta^{11} \mathrm{~B}$ [ppm] } \\
\hline 25,62 & $\underline{B}$ & $\mathrm{~s}$ & & \\
\hline \multicolumn{5}{|l|}{$\delta{ }^{13} \mathrm{C}[\mathrm{ppm}]$} \\
\hline 3,25 & $\mathrm{Si}\left(\underline{\mathrm{CH}}_{3}\right)_{2}$ & s & & \\
\hline 127,79 & $3 / 5 \underline{C}$ & s & & \\
\hline 129,68 & $4 \underline{\mathrm{C}}$ & s & & \\
\hline 135,31 & $2 / 6 \underline{\mathrm{C}}$ & s & & \\
\hline 135,28 & $1 \underline{\mathrm{C}}$ & s & & \\
\hline \multicolumn{5}{|l|}{$\delta{ }^{19} \mathrm{~F}[\mathrm{ppm}]$} \\
\hline 72,98 & $\underline{F}$ & $\mathrm{~s}$ & & \\
\hline \multicolumn{5}{|l|}{$\delta^{29} \mathrm{Si}$ [ppm] } \\
\hline$-15,93$ & $\underline{\mathrm{Si}}\left(\mathrm{C}_{6} \mathrm{H}_{5}\right)_{3}$ & s & & \\
\hline 4,45 & $\underline{\mathrm{SiMe}}_{3}$ & $d$ & ${ }^{3} \mathrm{~J}_{\mathrm{SiF}}=6,3$ & \\
\hline
\end{tabular}


Fluor-triphenylsilylamino-diisopropylamino-boran

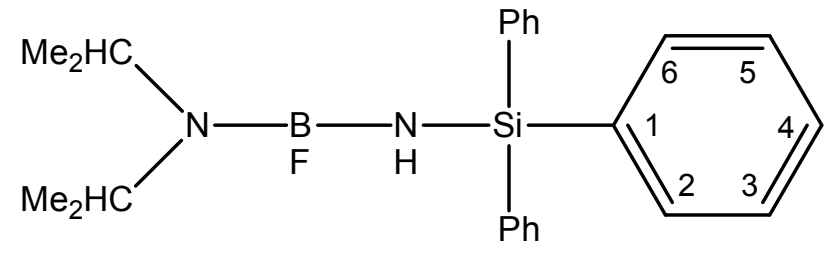

Summenformel:

Molare Masse:

Siedepunkt:

Ausbeute:

Massenspektrum:

$\mathrm{C}_{24} \mathrm{H}_{30} \mathrm{BN}_{2} \mathrm{FSi}$

$404,40 \mathrm{~g} / \mathrm{mol}$

$88^{\circ} \mathrm{C} / 0,01 \mathrm{mbar}$

$85 \%$

$\begin{array}{lll}404 & {[\mathrm{M}]^{+}} & 5 \% \\ 393 & {[\mathrm{M}-\mathrm{Me}]^{+}} & 100 \%\end{array}$

Kernmagnetische Resonanz (NMR) $\left(\mathrm{CDCl}_{3}\right)$

\begin{tabular}{|c|c|c|c|c|}
\hline Verschiebung & Zuordnung & Signalform & Kopplungskonst. [Hz] & Integration \\
\hline \multicolumn{5}{|l|}{$\delta^{1} \mathrm{H}$ [ppm] } \\
\hline 1,08 & $\mathrm{CH}\left(\mathrm{C}_{\mathrm{H}_{3}}\right)_{2}$ & $d$ & ${ }^{3} \mathrm{~J}_{\mathrm{HH}}=6,2$ & $12 \mathrm{H}$ \\
\hline 2,96 & $\mathrm{CHMe}_{2}$ & sept & ${ }^{3} \mathrm{~J}_{\mathrm{HH}}=6,2$ & $2 \mathrm{H}$ \\
\hline $7,11-7,73$ & 2/3/4/5/6 C & $\mathrm{m}$ & & $15 \mathrm{H}$ \\
\hline \multicolumn{5}{|l|}{$\delta^{11} \mathrm{~B}$ [ppm] } \\
\hline 15,72 & $\underline{B}$ & s & & \\
\hline \multicolumn{5}{|l|}{$\delta{ }^{13} \mathrm{C}$ [ppm] } \\
\hline 22,37 & $\mathrm{CH}\left(\underline{\mathrm{CH}}_{3}\right)_{2}$ & s & & \\
\hline 45,83 & $\underline{\mathrm{C}} \mathrm{HMe}_{2}$ & $s$ & & \\
\hline 127,59 & $3 / 5 \underline{C}$ & $s$ & & \\
\hline 129,98 & $4 \underline{\mathrm{C}}$ & $\mathrm{s}$ & & \\
\hline 134,57 & $2 / 6 \underline{C}$ & s & & \\
\hline 135,35 & $1 \underline{C}$ & $s$ & & \\
\hline \multicolumn{5}{|l|}{$\delta{ }^{19} \mathrm{~F}[\mathrm{ppm}]$} \\
\hline 41,19 & $\underline{F}$ & s & & \\
\hline \multicolumn{5}{|l|}{$\delta^{29} \mathrm{Si}$ [ppm] } \\
\hline$-16,79$ & $\underline{\mathrm{Si}}$ & s & & \\
\hline
\end{tabular}




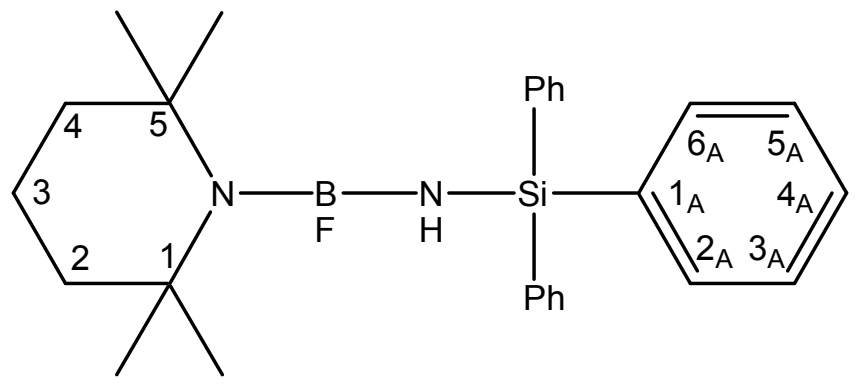

Summenformel:

Molare Masse:

Siedepunkt:

Ausbeute:

Massenspektrum:

\begin{abstract}
$\mathrm{C}_{27} \mathrm{H}_{34} \mathrm{BN}_{2} \mathrm{FSi}$
\end{abstract}
$444,26 \mathrm{~g} / \mathrm{mol}$

$121^{\circ} \mathrm{C} / 0,01 \mathrm{mbar}$

$64 \%$

$429 \quad[\mathrm{M}-\mathrm{Me}]^{+} \quad 100 \%$

Kernmagnetische Resonanz (NMR) $\left(\mathrm{CDCl}_{3}\right)$

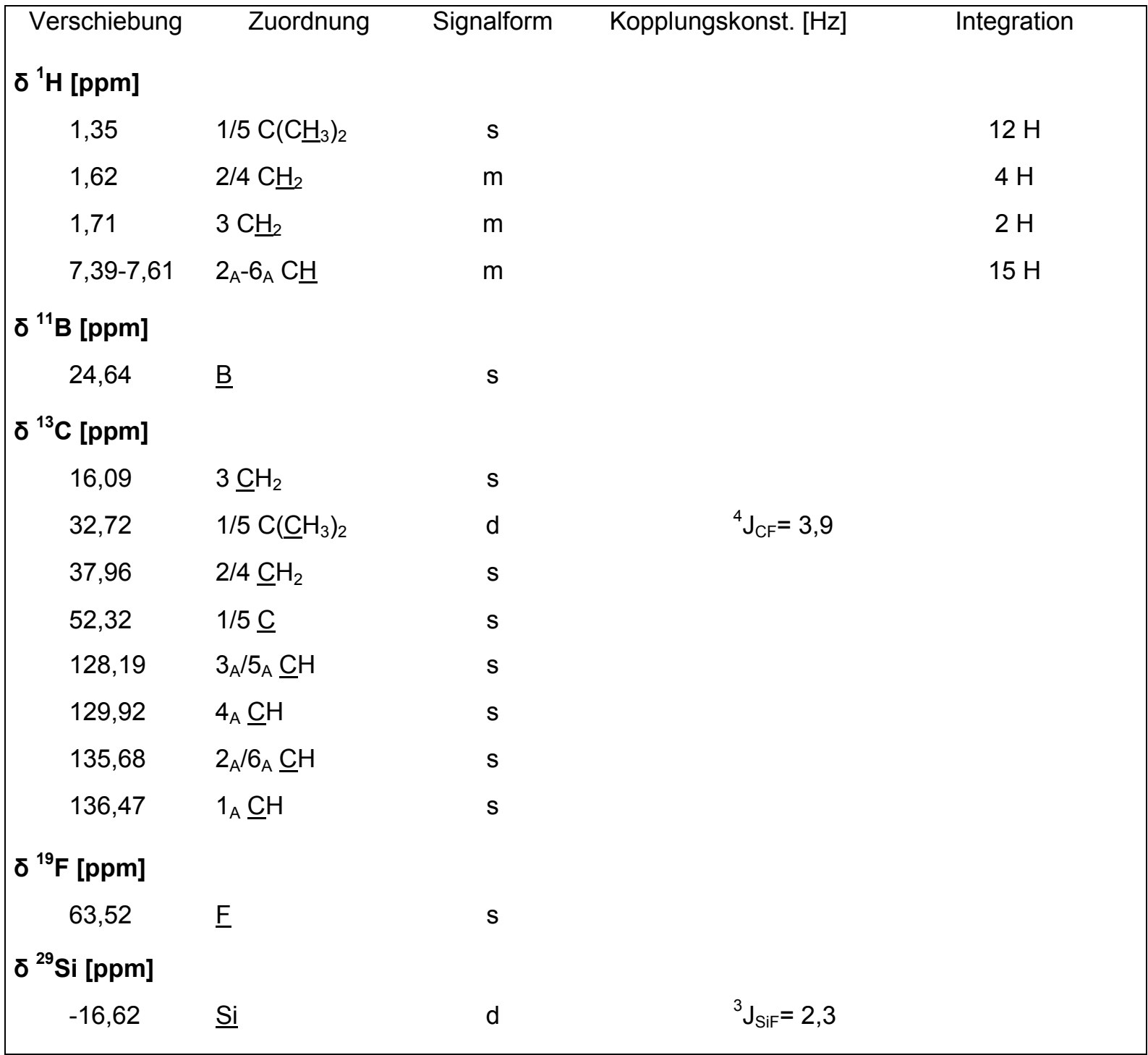


(tert-Butyldimethylsilyl)amino-fluor-bis(trimethylsilyl)amino-boran

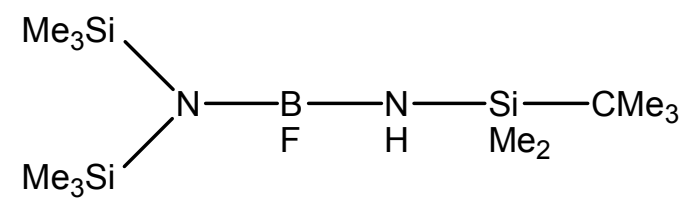

Summenformel:

Molare Masse:

Siedepunkt:

Zersetzungspunkt:

Ausbeute:

Massenspektrum:
$\mathrm{C}_{12} \mathrm{H}_{34} \mathrm{BN}_{2} \mathrm{FSi}_{3}$

$320,48 \mathrm{~g} / \mathrm{mol}$

$52{ }^{\circ} \mathrm{C} / 0,01 \mathrm{mbar}$

$100^{\circ} \mathrm{C}$

$70 \%$

305

263
[M-Me $^{+}$

$[\mathrm{M}-\mathrm{Bu}]^{+}$
$20 \%$

$100 \%$

Kernmagnetische Resonanz (NMR) $\left(C D C l_{3}\right)$

\begin{tabular}{|c|c|c|c|c|}
\hline Verschiebung & Zuordnung & Signalform & Kopplungskonst. [Hz] & Integration \\
\hline \multicolumn{5}{|l|}{$\delta^{1} \mathrm{H}$ [ppm] } \\
\hline 0,09 & $\mathrm{SiCMe}_{3}\left(\mathrm{C}_{3}\right)_{2}$ & s & & $6 \mathrm{H}$ \\
\hline 0,32 & $\mathrm{Si}\left(\mathrm{CH}_{3}\right)_{3}$ & s & & $18 \mathrm{H}$ \\
\hline 1,07 & $\operatorname{SiC}\left(\mathrm{CH}_{3}\right)_{3} \mathrm{Me}_{2}$ & $\mathrm{~s}$ & & $9 \mathrm{H}$ \\
\hline \multicolumn{5}{|l|}{$\delta^{11} \mathrm{~B}[\mathrm{ppm}]$} \\
\hline 25,08 & $\underline{B}$ & s & & \\
\hline \multicolumn{5}{|l|}{$\delta{ }^{13} \mathrm{C}[\mathrm{ppm}]$} \\
\hline 2,59 & $\mathrm{SiCMe}_{3}\left(\underline{\mathrm{C}} \mathrm{H}_{3}\right)_{2}$ & s & & \\
\hline 4,31 & $\mathrm{Si}\left(\underline{\mathrm{C}}_{3}\right)_{3}$ & s & & \\
\hline 19,86 & $\mathrm{Si}^{\mathrm{C}} \mathrm{Me}_{3} \mathrm{Me}_{2}$ & $\mathrm{~s}$ & & \\
\hline 27,13 & $\mathrm{SiC}\left(\underline{\mathrm{CH}}_{3}\right)_{3} \mathrm{Me}_{2}$ & $\mathrm{~s}$ & & \\
\hline \multicolumn{5}{|l|}{$\delta{ }^{19} \mathrm{~F}[\mathrm{ppm}]$} \\
\hline 100,45 & $\underline{F}$ & s & & \\
\hline \multicolumn{5}{|l|}{$\delta^{29} \mathrm{Si}$ [ppm] } \\
\hline 2,31 & $\underline{\mathrm{SiMe}}_{3}$ & $s$ & & \\
\hline 5,62 & $\underline{\mathrm{SiCMe}} \mathrm{Me}_{2}$ & $d$ & ${ }^{3} J_{S_{i F}}=7,0$ & \\
\hline
\end{tabular}


(tert-Butyldimethylsilyl)amino-fluor-diisopropylamino-boran<smiles>C[SiH2]NPBN(C(C)C)C(C)C</smiles>

Summenformel:

Molare Masse:

Festpunkt:

Siedepunkt:

Ausbeute:
$\mathrm{C}_{12} \mathrm{H}_{30} \mathrm{BN}_{2} \mathrm{FSi}$

$260,27 \mathrm{~g} / \mathrm{mol}$

$5{ }^{\circ} \mathrm{C}$

$25^{\circ} \mathrm{C} / 0,01 \mathrm{mbar}$

$85 \%$

Kernmagnetische Resonanz (NMR) $\left(\mathrm{CDCl}_{3}\right)$

\begin{tabular}{|c|c|c|c|c|}
\hline Verschiebung & Zuordnung & Signalform & Kopplungskonst. [Hz] & Integration \\
\hline \multicolumn{5}{|l|}{$\delta^{1} \mathrm{H}$ [ppm] } \\
\hline 0,05 & $\mathrm{SiCMe}_{3}\left(\mathrm{C}_{3}\right)_{2}$ & $d$ & ${ }^{5} \mathrm{~J}_{\mathrm{HF}}=1,4$ & $6 \mathrm{H}$ \\
\hline 0,84 & $\mathrm{SiC}\left(\mathrm{C}_{3}\right)_{3} \mathrm{Me}_{2}$ & s & & $9 \mathrm{H}$ \\
\hline 1,08 & a $\mathrm{CH}\left(\mathrm{C}_{\mathrm{H}_{3}}\right)_{2}$ & d & ${ }^{3} J_{H H}=6,3$ & $6 \mathrm{H}$ \\
\hline 1,10 & b $\mathrm{CH}\left(\mathrm{C}_{\mathrm{H}_{3}}\right)_{2}$ & dd & ${ }^{3} J_{H H}=6,8$ & $6 \mathrm{H}$ \\
\hline & & & ${ }^{5} \mathrm{~J}_{\mathrm{HF}}=3,3$ & \\
\hline 2,96 & $\mathrm{CHMe}_{2}$ & sept & ${ }^{3} \mathrm{~J}_{\mathrm{HH}}=6,3$ & $1 \mathrm{H}$ \\
\hline 3,23 & $\mathrm{CHMe}_{2}$ & sept & ${ }^{3} \mathrm{~J}_{\mathrm{HH}}=6,8$ & $1 \mathrm{H}$ \\
\hline \multicolumn{5}{|l|}{$\delta{ }^{11} B$ [ppm] } \\
\hline 23,59 & $\underline{B}$ & $\mathrm{~s}$ & & \\
\hline \multicolumn{5}{|l|}{$\delta{ }^{13} \mathrm{C}$ [ppm] } \\
\hline$-3,69$ & $\mathrm{SiCMe}_{3}\left(\underline{\mathrm{CH}}_{3}\right)_{2}$ & $d$ & ${ }^{4} J_{C F}=2,6$ & \\
\hline 17,77 & $\mathrm{Si}^{\mathrm{C}} \mathrm{Me}_{3} \mathrm{Me}_{2}$ & s & & \\
\hline 22,74 & a CH$\left(\underline{\mathrm{C}} \mathrm{H}_{3}\right)_{2}$ & $s$ & & \\
\hline 23,11 & b $\mathrm{CH}\left(\underline{\mathrm{CH}}_{3}\right)_{2}$ & d & ${ }^{4} \mathrm{~J}_{\mathrm{CF}}=1,4$ & \\
\hline 25,94 & $\mathrm{SiC}\left(\underline{\mathrm{C}} \mathrm{H}_{3}\right) \mathrm{Me}_{2}$ & s & & \\
\hline 44,47 & $\mathrm{~b} \underline{\mathrm{C}} \mathrm{HMe}_{2}$ & d & ${ }^{3} \mathrm{~J}_{\mathrm{CF}}=2,9$ & \\
\hline 45,68 & a $\underline{\mathrm{C}} \mathrm{HMe}_{2}$ & $s$ & & \\
\hline \multicolumn{5}{|l|}{$\delta^{19} \mathrm{~F}[\mathrm{ppm}]$} \\
\hline 63,88 & $\underline{F}$ & s & & \\
\hline \multicolumn{5}{|l|}{$\delta^{29} \mathrm{Si}[\mathrm{ppm}]$} \\
\hline 9,19 & $\underline{\mathrm{Si}}$ & $d$ & ${ }^{3} \mathrm{~J}_{\mathrm{SiF}}=2,7$ & \\
\hline
\end{tabular}


(tert-Butyldimethylsilyl)amino-fluor-1,1,5,5-tetramethylpiperidino-boran

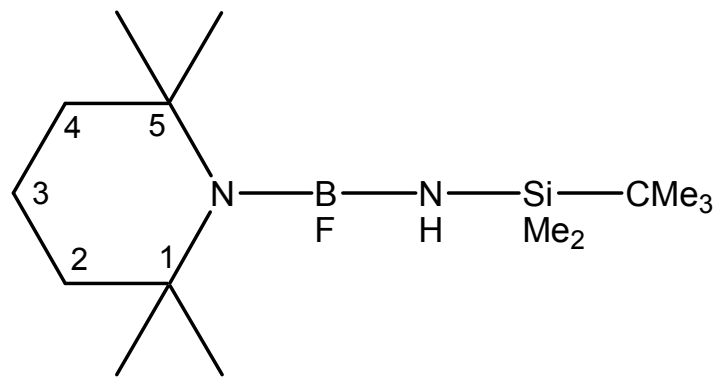

Summenformel:

$\mathrm{C}_{15} \mathrm{H}_{34} \mathrm{BN}_{2} \mathrm{FSi}$

Molare Masse:

$300,34 \mathrm{~g} / \mathrm{mol}$

Siedepunkt:

$64{ }^{\circ} \mathrm{C} / 0,01 \mathrm{mbar}$

Ausbeute:

$15 \%$

$\begin{array}{lllll}\text { Massenspektrum: } & \text { (E.I.) } \mathrm{m} / \mathrm{z} & 285 & {[\mathrm{M}-\mathrm{Me}]^{+}} & 100 \%\end{array}$

Kernmagnetische Resonanz (NMR) $\left(\mathrm{CDCl}_{3}\right)$

\begin{tabular}{|c|c|c|c|c|}
\hline \multicolumn{5}{|l|}{$\delta^{1} \mathrm{H}[\mathrm{ppm}]$} \\
\hline 0,06 & $\mathrm{SiCMe}_{3}\left(\mathrm{CH}_{3}\right)_{2}$ & $d$ & ${ }^{5} \mathrm{JHF}_{\mathrm{HF}}=1,5$ & $6 \mathrm{H}$ \\
\hline 0,86 & $\mathrm{SiC}\left(\mathrm{CH}_{3}\right)_{3} \mathrm{Me}_{2}$ & s & & $9 \mathrm{H}$ \\
\hline 1,24 & $1 / 5 \mathrm{C}\left(\mathrm{CH}_{3}\right)_{2}$ & $\mathrm{~s}$ & ${ }^{5} \mathrm{~J}_{\mathrm{HE}}=1,4$ & $12 \mathrm{H}$ \\
\hline 1,48 & $2 / 4 \mathrm{CH}_{2}$ & $\mathrm{~m}$ & & $4 \mathrm{H}$ \\
\hline 1,60 & $3 \mathrm{CH}_{2}$ & $\mathrm{~m}$ & & $2 \mathrm{H}$ \\
\hline \multicolumn{5}{|l|}{$\delta^{11} B$ [ppm] } \\
\hline 29,50 & $\underline{B}$ & s & & \\
\hline \multicolumn{5}{|l|}{$\delta{ }^{13} \mathrm{C}$ [ppm] } \\
\hline$-3,50$ & $\mathrm{SiCMe}_{3}\left(\underline{\mathrm{CH}}_{3}\right)_{2}$ & $d$ & ${ }^{4} J_{C F}=2,8$ & \\
\hline 17,40 & $\mathrm{SiC} \mathrm{Me}_{3} \mathrm{Me}_{2}$ & s & & \\
\hline 18,12 & $3 \underline{\mathrm{CH}}_{2}$ & $\mathrm{~s}$ & & \\
\hline 26,19 & $\mathrm{SiC}\left(\underline{\mathrm{CH}}_{3}\right)_{3} \mathrm{Me}_{2}$ & s & & \\
\hline 32,14 & $1 / 5 \mathrm{C}\left(\underline{\mathrm{C}}_{3}\right)_{2}$ & $d$ & ${ }^{4} J_{C F}=4,1$ & \\
\hline 37,61 & $2 / 4 \underline{\mathrm{CH}}_{2}$ & d & ${ }^{4} J_{C F}=0,7$ & \\
\hline 51,28 & $1 / 5 \underline{C}$ & $\mathrm{~s}$ & & \\
\hline \multicolumn{5}{|l|}{$\delta{ }^{19} \mathrm{~F}$ [ppm] } \\
\hline 64,9 & $\underline{F}$ & $s$ & & \\
\hline \multicolumn{5}{|l|}{$\delta{ }^{29} \mathrm{Si}$ [ppm] } \\
\hline 9,89 & $\underline{\mathrm{Si}}$ & $d$ & ${ }^{3} J_{\mathrm{SiF}_{\mathrm{F}}}=7,9$ & \\
\hline
\end{tabular}


(tert-Butyldimethylsilyl)imino-1,1,5,5-tetramethylpiperidino-boren

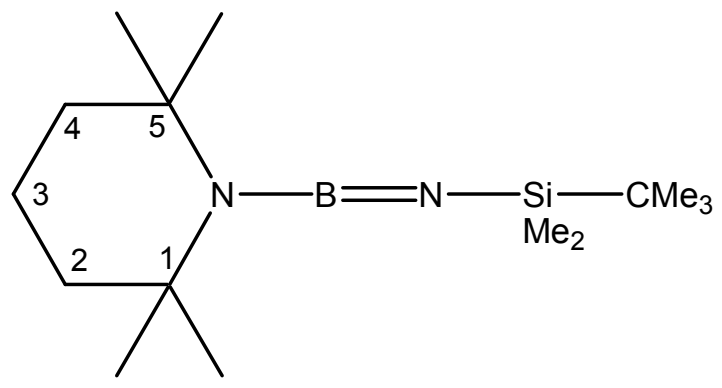

Summenformel:

$\mathrm{C}_{15} \mathrm{H}_{33} \mathrm{BN} \mathrm{N}_{2} \mathrm{Si}$

Molare Masse:

$280,33 \mathrm{~g} / \mathrm{mol}$

Siedepunkt:

$64{ }^{\circ} \mathrm{C} / 0,01 \mathrm{mbar}$

Ausbeute:

$40 \%$

$\begin{array}{lllll}\text { Massenspektrum: } & \text { (E.I.) } \mathrm{m} / \mathrm{z} & 223 & {[\mathrm{M}-\mathrm{Bu}]^{+}} & 100 \%\end{array}$

Kernmagnetische Resonanz (NMR) $\left(\mathrm{CDCl}_{3}\right)$

\begin{tabular}{|c|c|c|c|c|}
\hline Verschiebung & Zuordnung & Signalform & Kopplungskonst. [Hz] & Integration \\
\hline \multicolumn{5}{|l|}{$\delta^{1} \mathrm{H}$ [ppm] } \\
\hline$-0,03$ & $\mathrm{SiCMe}_{3}\left(\mathrm{C}_{3}\right)_{2}$ & s & & $6 \mathrm{H}$ \\
\hline 0,86 & $\mathrm{SiC}\left(\mathrm{CH}_{3}\right)_{3} \mathrm{Me}_{2}$ & s & & $9 \mathrm{H}$ \\
\hline 1,29 & $1 / 5 \mathrm{C}\left(\mathrm{CH}_{3}\right)_{2}$ & s & & $12 \mathrm{H}$ \\
\hline 1,38 & $2 / 4 \mathrm{CH}_{2}$ & $\mathrm{~m}$ & & $4 \mathrm{H}$ \\
\hline 1,53 & $3 \mathrm{CH}_{2}$ & $\mathrm{~m}$ & & $2 \mathrm{H}$ \\
\hline \multicolumn{5}{|l|}{$\delta^{11} \mathrm{~B}$ [ppm] } \\
\hline 17,97 & $\underline{B}$ & $\mathrm{~s}$ & & \\
\hline \multicolumn{5}{|l|}{$\delta{ }^{13} \mathrm{C}$ [ppm] } \\
\hline$-2,28$ & $\mathrm{SiCMe}_{3}\left(\underline{\mathrm{CH}}_{3}\right)_{2}$ & s & & \\
\hline 17,38 & $\mathrm{SiC} \mathrm{Me}_{3} \mathrm{Me}_{2}$ & $s$ & & \\
\hline 18,12 & $3 \underline{\mathrm{CH}}_{2}$ & s & & \\
\hline 26,24 & $\mathrm{SiC}\left(\underline{\mathrm{CH}}_{3}\right)_{3} \mathrm{Me}_{2}$ & $s$ & & \\
\hline 32,12 & $1 / 5 \mathrm{C}\left(\underline{\mathrm{C}} \mathrm{H}_{3}\right)_{2}$ & $s$ & & \\
\hline 38,10 & $2 / 4 \underline{\mathrm{CH}}_{2}$ & $\mathrm{~s}$ & & \\
\hline 51,28 & $1 / 5 \underline{C}$ & s & & \\
\hline \multicolumn{5}{|l|}{$\delta^{29} \mathrm{Si}$ [ppm] } \\
\hline$-4,20$ & $\underline{\mathrm{Si}}$ & $s$ & & \\
\hline
\end{tabular}


(tert-Butyldimethylsilyl)imino-2,4,6-tri(tert-butyl)phenyl-boren

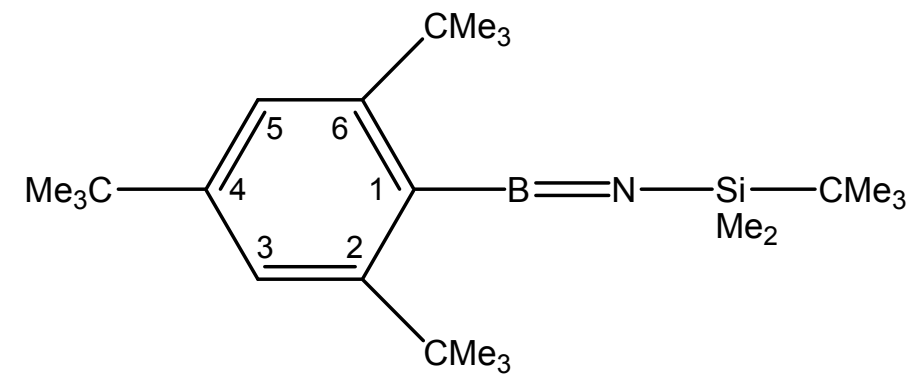

Summenformel:

Molare Masse:

Siedepunkt:

Ausbeute:

Massenspektrum:

$$
\mathrm{C}_{24} \mathrm{H}_{44} \mathrm{BNSi}
$$

$385,51 \mathrm{~g} / \mathrm{mol}$

$95^{\circ} \mathrm{C} / 0,01 \mathrm{mbar}$

$40 \%$

$\begin{array}{lll}370 & {[\mathrm{M}-\mathrm{Me}]^{+}} & 5 \% \\ 328 & {[\mathrm{M}-\mathrm{Bu}]^{+}} & 100 \%\end{array}$

Kernmagnetische Resonanz (NMR) $\left(\mathrm{CDCl}_{3}\right)$

\begin{tabular}{|c|c|c|c|c|}
\hline Verschiebung & Zuordnung & Signalform & Kopplungskonst. [Hz] & Integration \\
\hline \multicolumn{5}{|l|}{$\delta^{1} \mathrm{H}$ [ppm] } \\
\hline 0,12 & $\mathrm{SiCMe}_{3}\left(\mathrm{CH}_{3}\right)_{2}$ & $s$ & & $6 \mathrm{H}$ \\
\hline 0,97 & $\mathrm{SiC}\left(\mathrm{CH}_{3}\right)_{3} \mathrm{Me}_{2}$ & $s$ & & $9 \mathrm{H}$ \\
\hline 1,34 & $4 \mathrm{C}-\mathrm{C}\left(\mathrm{C}_{3}\right)_{3}$ & $s$ & & $9 \mathrm{H}$ \\
\hline 1,59 & $2 / 6 \mathrm{C}-\mathrm{C}\left(\mathrm{CH}_{3}\right)_{3}$ & $\mathrm{~s}$ & & $18 \mathrm{H}$ \\
\hline 7,34 & $3 / 5 \mathrm{CH}$ & $s$ & & $2 \mathrm{H}$ \\
\hline \multicolumn{5}{|l|}{$\delta{ }^{11} \mathrm{~B}$ [ppm] } \\
\hline 20,18 & $\underline{B}$ & $s$ & & \\
\hline \multicolumn{5}{|l|}{$\delta{ }^{13} \mathrm{C}$ [ppm] } \\
\hline$-3,23$ & $\mathrm{SiCMe}_{3}\left(\underline{\mathrm{CH}}_{3}\right)_{2}$ & $s$ & & \\
\hline 17,91 & $\mathrm{SiCMe}_{3} \mathrm{Me}_{2}$ & $\mathrm{~s}$ & & \\
\hline 26,14 & $\mathrm{SiC}\left(\underline{\mathrm{CH}}_{3}\right)_{3} \mathrm{Me}_{2}$ & s & & \\
\hline 31,23 & $4 \mathrm{C}-\mathrm{C}\left(\underline{\mathrm{C}}_{3}\right)_{3}$ & $s$ & & \\
\hline 32,03 & $2 / 6 \mathrm{C}-\mathrm{C}\left(\underline{\mathrm{CH}}_{3}\right)_{3}$ & $\mathrm{~s}$ & & \\
\hline 35,45 & $4 \mathrm{C}-\underline{\mathrm{C}}\left(\mathrm{CH}_{3}\right)_{3}$ & $\mathrm{~s}$ & & \\
\hline 36,79 & $2 / 6 \mathrm{C}-\underline{\mathrm{CMe}}{ }_{3}$ & $\mathrm{~s}$ & & \\
\hline 113,10 & $1 \underline{C}$ & $\mathrm{~s}$ & & \\
\hline 119,72 & $3 / 5 \underline{C}$ & $s$ & & \\
\hline 153,46 & $4 \underline{C}$ & $\mathrm{~s}$ & & \\
\hline 159,98 & $2 / 6 \underline{\mathrm{C}}$ & $\mathrm{s}$ & & \\
\hline \multicolumn{5}{|l|}{$\delta^{29} \mathrm{Si}$ [ppm] } \\
\hline$-4,16$ & $\underline{\mathrm{Si}}$ & $s$ & & \\
\hline
\end{tabular}


(tert-Butyldimethylsilyl)amino-2,4,6-tri(tert-butyl)phenyl-hydroxi-boran

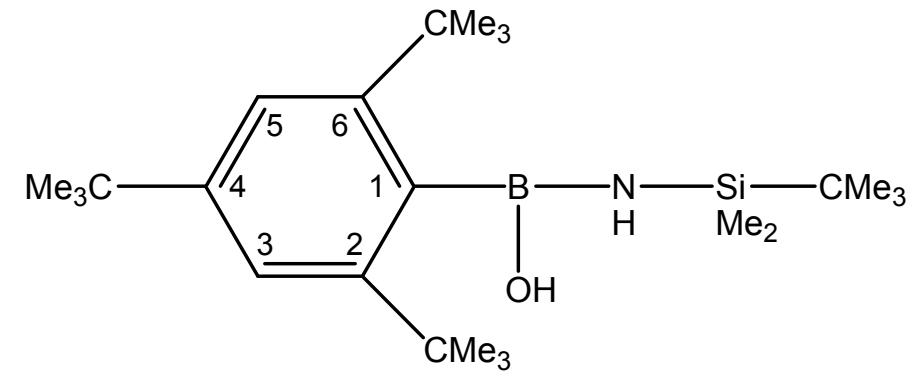

Summenformel:

$\mathrm{C}_{24} \mathrm{H}_{46} \mathrm{BNOSi}$

Molare Masse:

$403,52 \mathrm{~g} / \mathrm{mol}$

Siedepunkt:

$97^{\circ} \mathrm{C} / 0,01 \mathrm{mbar}$

Ausbeute:

$97 \%$

Massenspektrum:

(E.I.) $\mathrm{m} / \mathrm{z}$

403

$[\mathrm{M}]^{+}$

$45 \%$

346

$[\mathrm{M}-\mathrm{Bu}]^{+}$

$60 \%$

Kernmagnetische Resonanz (NMR) $\left(\mathrm{CDCl}_{3}\right)$

\begin{tabular}{|c|c|c|c|c|}
\hline Verschiebung & Zuordnung & Signalform & Kopplungskonst. [Hz] & Integration \\
\hline \multicolumn{5}{|l|}{$\delta^{1} \mathrm{H}$ [ppm] } \\
\hline 0,21 & $\mathrm{SiCMe}_{3}\left(\mathrm{CH}_{3}\right)_{2}$ & s & & $6 \mathrm{H}$ \\
\hline 0,89 & $\mathrm{SiC}\left(\mathrm{CH}_{3}\right)_{3} \mathrm{Me}_{2}$ & $s$ & & $9 \mathrm{H}$ \\
\hline 1,32 & $4 \mathrm{C}-\mathrm{C}\left(\mathrm{CH}_{3}\right)_{3}$ & $\mathrm{~s}$ & & $9 \mathrm{H}$ \\
\hline 1,47 & $2 / 6 \mathrm{C}-\mathrm{C}\left(\mathrm{CH}_{3}\right)_{3}$ & s & & $18 \mathrm{H}$ \\
\hline 2,61 & $\mathrm{~N} \underline{\mathrm{H}}$ & $d$ & ${ }^{4} \mathrm{~J}_{\mathrm{HH}}=2,3$ & $1 \mathrm{H}$ \\
\hline 3,94 & $\mathrm{BO} \underline{\mathrm{H}}$ & $d$ & ${ }^{4} J_{H H}=2,3$ & $1 \mathrm{H}$ \\
\hline 7,43 & $3 / 5 \mathrm{C} \underline{\mathrm{H}}$ & $s$ & & $2 \mathrm{H}$ \\
\hline \multicolumn{5}{|l|}{$\delta^{11} \mathrm{~B}$ [ppm] } \\
\hline 36,04 & $\underline{B}$ & s & & \\
\hline \multicolumn{5}{|l|}{$\delta^{13} \mathrm{C}[\mathrm{ppm}]$} \\
\hline$-3,91$ & $\mathrm{SiCMe}_{3}\left(\underline{\mathrm{CH}}_{3}\right)_{2}$ & $\mathrm{~s}$ & & \\
\hline 17,63 & $\mathrm{SiCMe}_{3} \mathrm{Me}_{2}$ & $\mathrm{~s}$ & & \\
\hline 26,47 & $\mathrm{SiC}\left(\underline{\mathrm{CH}}_{3}\right)_{3} \mathrm{Me}_{2}$ & $s$ & & \\
\hline 31,72 & $4 \mathrm{C}-\mathrm{C}\left(\mathrm{CH}_{3}\right)_{3}$ & $\mathrm{~s}$ & & \\
\hline 32,92 & $2 / 6 \mathrm{C}-\mathrm{C}\left(\underline{\mathrm{C}} \mathrm{H}_{3}\right)_{3}$ & $s$ & & \\
\hline 37,52 & $4 \mathrm{C}-\underline{\mathrm{C}} \mathrm{Me}_{3}$ & s & & \\
\hline 38,29 & $2 / 6 \mathrm{C}-\underline{\mathrm{CMe}}{ }_{3}$ & s & & \\
\hline 121,91 & $3 / 5 \underline{C}$ & s & & \\
\hline 133,61 & $1 \underline{\mathrm{C}}$ & s & & \\
\hline 149,47 & $4 \underline{C}$ & $\mathrm{~s}$ & & \\
\hline 153,14 & $2 / 6 \underline{C}$ & s & & \\
\hline \multicolumn{5}{|l|}{$\delta^{29} \mathrm{Si}$ [ppm] } \\
\hline 8,46 & $\underline{\mathrm{Si}}$ & s & & \\
\hline
\end{tabular}


(tert-Butyldimethylsilyl)amino-2,4,6-tri(tert-butyl)phenyl-methoxi-boran

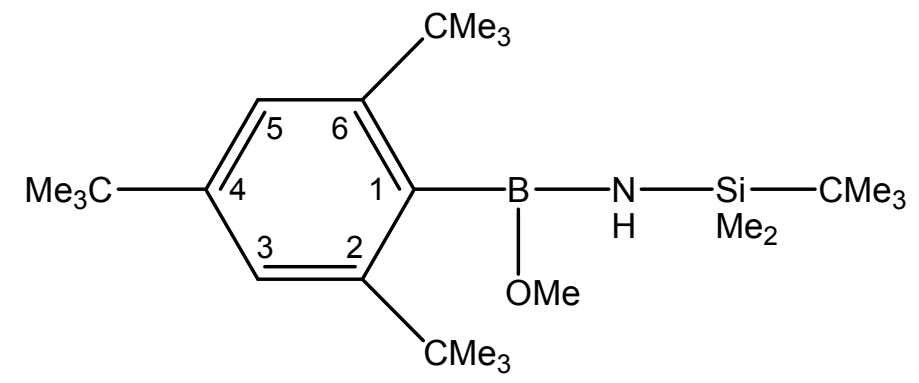

Summenformel:

Molare Masse:

Siedepunkt:

Ausbeute:

Massenspektrum:
$\mathrm{C}_{25} \mathrm{H}_{48} \mathrm{BNOSi}$

$417 \mathrm{~g} / \mathrm{mol}$

$99{ }^{\circ} \mathrm{C} / 0,01 \mathrm{mbar}$

$90 \%$

$\begin{array}{lll}370 & {[\mathrm{M}-\mathrm{Me}]^{+}} & 5 \% \\ 328 & {[\mathrm{M}-\mathrm{Bu}]^{+}} & 100 \%\end{array}$

Kernmagnetische Resonanz (NMR) $\left(\mathrm{CDCl}_{3}\right)$

\begin{tabular}{|c|c|c|c|c|}
\hline Verschiebung & Zuordnung & Signalform & Kopplungskonst. [Hz] & Integration \\
\hline \multicolumn{5}{|l|}{$\delta^{1} \mathrm{H}[\mathrm{ppm}]$} \\
\hline 0,06 & $\mathrm{SiCMe}_{3}\left(\mathrm{C}_{3}\right)_{2}$ & s & & $6 \mathrm{H}$ \\
\hline 0,87 & $\mathrm{SiC}\left(\mathrm{CH}_{3}\right)_{3} \mathrm{Me}_{2}$ & s & & $9 \mathrm{H}$ \\
\hline 1,31 & $4 \mathrm{C}-\mathrm{C}\left(\mathrm{C}_{3}\right)_{3}$ & $\mathrm{~s}$ & & $9 \mathrm{H}$ \\
\hline 1,43 & $2 / 6 \mathrm{C}-\mathrm{C}\left(\mathrm{C}_{3}\right)_{3}$ & $s$ & & $18 \mathrm{H}$ \\
\hline 2,47 & $\mathrm{NH}$ & s & & $1 \mathrm{H}$ \\
\hline 3,31 & $\mathrm{OC}_{3}$ & $s$ & & $3 \mathrm{H}$ \\
\hline 7,37 & $3 / 5 \mathrm{C} \underline{\mathrm{H}}$ & s & & $2 \mathrm{H}$ \\
\hline \multicolumn{5}{|l|}{$\delta^{11} \mathrm{~B}$ [ppm] } \\
\hline 36,26 & $\underline{B}$ & s & & \\
\hline \multicolumn{5}{|l|}{$\delta^{13} \mathrm{C}$ [ppm] } \\
\hline$-4,23$ & $\mathrm{SiCMe}_{3}\left(\underline{\mathrm{CH}}_{3}\right)_{2}$ & s & & \\
\hline 17,18 & $\mathrm{SiCMe}_{3} \mathrm{Me}_{2}$ & $\mathrm{~s}$ & & \\
\hline 26,17 & $\mathrm{SiC}\left(\underline{\mathrm{CH}}_{3}\right)_{3} \mathrm{Me}_{2}$ & s & & \\
\hline 32,51 & $4 \mathrm{C}-\mathrm{C}\left(\underline{\mathrm{CH}}_{3}\right)_{3}$ & s & & \\
\hline 33,10 & $2 / 6 \mathrm{C}-\mathrm{C}\left(\underline{\mathrm{CH}}_{3}\right)_{3}$ & s & & \\
\hline 34,79 & $4 \underline{\mathrm{CMe}}_{3}$ & $s$ & & \\
\hline 37,99 & $2 / 6 \mathrm{C}-\underline{\mathrm{C}} \mathrm{Me}_{3}$ & $\mathrm{~s}$ & & \\
\hline 53,92 & $\mathrm{O}_{\mathbf{C H}} \mathrm{H}_{3}$ & s & & \\
\hline 121,78 & $3 / 5 \underline{C}$ & $\mathrm{~s}$ & & \\
\hline 130,82 & $1 \underline{\mathrm{C}}$ & s & & \\
\hline 148,61 & $4 \underline{C}$ & s & & \\
\hline 152,13 & $2 / 6 \underline{\mathrm{C}}$ & $\mathrm{s}$ & & \\
\hline \multicolumn{5}{|l|}{$\delta^{29} \mathrm{Si}$ [ppm] } \\
\hline 7,65 & $\underline{\mathrm{Si}}$ & s & & \\
\hline
\end{tabular}


(tert-Butyldimethylsilyl)amino-2,4,6-tri(tert-butyl)phenyl-amino-boran

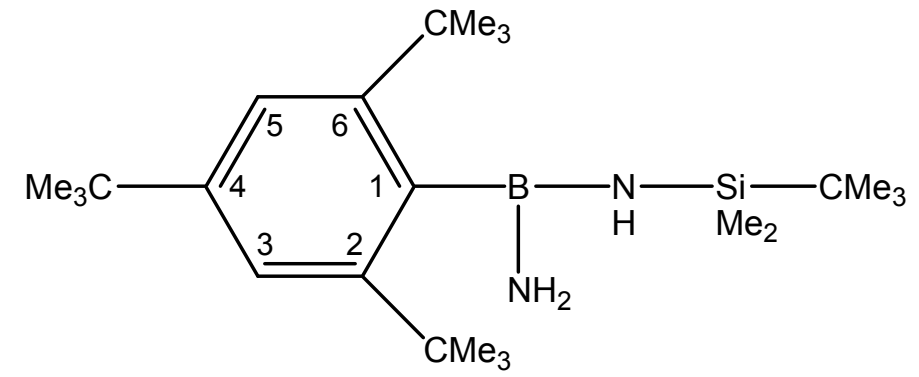

Summenformel:

$\mathrm{C}_{24} \mathrm{H}_{47} \mathrm{BN}_{2} \mathrm{Si}$

Molare Masse:

$402,54 \mathrm{~g} / \mathrm{mol}$

Siedepunkt:

$96{ }^{\circ} \mathrm{C} / 0,01 \mathrm{mbar}$

Ausbeute:

$90 \%$

Massenspektrum:

(E.I.) $\mathrm{m} / \mathrm{z}$

370

$[\mathrm{M}-\mathrm{Me}]^{+}$

$5 \%$

328

$[\mathrm{M}-\mathrm{Bu}]^{+}$

$100 \%$

Kernmagnetische Resonanz (NMR) $\left(\mathrm{CDCl}_{3}\right)$

\begin{tabular}{|c|c|c|c|c|}
\hline Verschiebung & Zuordnung & Signalform & Kopplungskonst. [Hz] & Integration \\
\hline \multicolumn{5}{|l|}{$\delta^{1} \mathrm{H}$ [ppm] } \\
\hline 0,17 & $\mathrm{SiCMe}_{3}\left(\mathrm{C}_{3}\right)_{2}$ & s & & $6 \mathrm{H}$ \\
\hline 0,89 & $\mathrm{SiC}\left(\mathrm{CH}_{3}\right)_{3} \mathrm{Me}_{2}$ & s & & $9 \mathrm{H}$ \\
\hline 1,32 & $4 \mathrm{C}-\mathrm{C}\left(\mathrm{C}_{3}\right)_{3}$ & $s$ & & $9 \mathrm{H}$ \\
\hline 1,50 & $2 / 6 \mathrm{C}-\mathrm{C}\left(\mathrm{CH}_{3}\right)_{3}$ & s & & $18 \mathrm{H}$ \\
\hline 2,24 & $\mathrm{SiCMe}_{3} \mathrm{Me}_{2}-\mathrm{N} \underline{\mathrm{H}}$ & s & & $1 \mathrm{H}$ \\
\hline 2,82 & $\mathrm{NH}_{2}$ & $\mathrm{~s}$ & & $2 \mathrm{H}$ \\
\hline 7,42 & $3 / 5 \mathrm{CH}$ & $\mathrm{s}$ & & $2 \mathrm{H}$ \\
\hline \multicolumn{5}{|l|}{$\delta^{11} \mathrm{~B}$ [ppm] } \\
\hline 35,29 & $\underline{B}$ & $\mathrm{~s}$ & & \\
\hline \multicolumn{5}{|l|}{$\delta{ }^{13} \mathrm{C}$ [ppm] } \\
\hline$-3,89$ & $\mathrm{SiCMe}_{3}\left(\underline{\mathrm{CH}}_{3}\right)_{2}$ & s & & \\
\hline 17,68 & $\mathrm{SiCMe}_{3} \mathrm{Me}_{2}$ & $\mathrm{~s}$ & & \\
\hline 26,04 & $\mathrm{SiC}\left(\underline{\mathrm{C}} \mathrm{H}_{3}\right)_{3} \mathrm{Me}_{2}$ & s & & \\
\hline 31,41 & $4 \mathrm{C}-\mathrm{C}\left(\underline{\mathrm{C}} \mathrm{H}_{3}\right)_{3}$ & $s$ & & \\
\hline 33,85 & $2 / 6 \mathrm{C}-\mathrm{C}\left(\mathrm{CH}_{3}\right)_{3}$ & $\mathrm{~s}$ & & \\
\hline 34,84 & $4 \mathrm{C}-\underline{\mathrm{CM}} \mathrm{M}_{3}$ & $\mathrm{~s}$ & & \\
\hline 38,35 & $2 / 6 \mathrm{C}-\underline{\mathrm{CMe}} \mathrm{M}_{3}$ & $s$ & & \\
\hline 121,47 & $3 / 5 \underline{\mathrm{C}}$ & $\mathrm{s}$ & & \\
\hline 136,22 & $1 \underline{\mathrm{C}}$ & s & & \\
\hline 148,06 & $4 \underline{\mathrm{C}}$ & $\mathrm{s}$ & & \\
\hline 152,67 & $2 / 6 \underline{C}$ & $s$ & & \\
\hline \multicolumn{5}{|l|}{$\delta^{29} \mathrm{Si}$ [ppm] } \\
\hline 6,64 & $\underline{\mathrm{Si}}$ & s & & \\
\hline
\end{tabular}




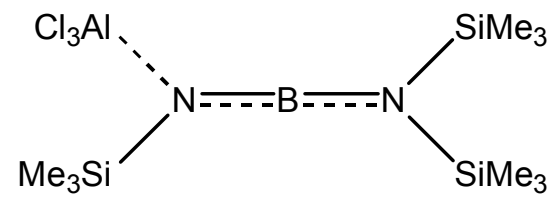

Summenformel:

Molare Masse:

Festpunkt:

Ausbeute:

Massenspektrum:

(E.I.) $\mathrm{m} / \mathrm{z}$

$\mathrm{C}_{9} \mathrm{H}_{27} \mathrm{BN}_{2} \mathrm{AISi}_{3} \mathrm{Cl}_{3}$ $391,73 \mathrm{~g} / \mathrm{mol}$

$56{ }^{\circ} \mathrm{C}$

$94 \%$
$[\mathrm{M}-\mathrm{Me}]^{+}$

$[\mathrm{M}-\mathrm{Cl}]^{+}$
$95 \%$

$25 \%$

Kernmagnetische Resonanz (NMR) $\left(C_{6} D_{6}\right)$

\begin{tabular}{|c|c|c|c|c|}
\hline Verschiebung & Zuordnung & Signalform & Kopplungskonst. [Hz] & Integration \\
\hline \multicolumn{5}{|l|}{$\delta^{1} \mathrm{H}$ [ppm] } \\
\hline 0,10 & $\mathrm{~N}-\left[\mathrm{Si}\left(\mathrm{C}_{3}\right)_{3}\right]_{2}$ & s & & $18 \mathrm{H}$ \\
\hline 0,30 & $\mathrm{AlCl}_{3}-\mathrm{N}-\mathrm{Si}\left(\mathrm{CH}_{3}\right)_{3}$ & s & & $9 \mathrm{H}$ \\
\hline \multicolumn{5}{|l|}{$\delta^{11} \mathrm{~B}[\mathrm{ppm}]$} \\
\hline 38,63 & $\underline{B}$ & s & & \\
\hline \multicolumn{5}{|l|}{$\delta{ }^{13} \mathrm{C}[\mathrm{ppm}]$} \\
\hline 1,57 & $\mathrm{~N}-\left[\mathrm{Si}\left(\underline{\mathrm{CH}}_{3}\right)_{3}\right]_{2}$ & $s$ & & \\
\hline 2,01 & $\mathrm{AlCl}_{3}-\mathrm{N}-\mathrm{Si}\left(\underline{\mathrm{CH}}_{3}\right)_{3}$ & s & & \\
\hline \multicolumn{5}{|l|}{$\delta{ }^{27} \mathrm{Al}$ [ppm] } \\
\hline 102,75 & $\underline{\mathrm{Al}}$ & $s$ & & \\
\hline \multicolumn{5}{|l|}{$\delta^{29} \mathrm{Si}$ [ppm] } \\
\hline 12,81 & $\mathrm{AlCl}_{3}-\mathrm{N}-\mathrm{SiMe}_{3}$ & $\mathrm{~s}$ & & \\
\hline 15,35 & $\mathrm{~N}-\left(\underline{\mathrm{SiMe}_{3}}\right)_{2}$ & $s$ & & \\
\hline
\end{tabular}




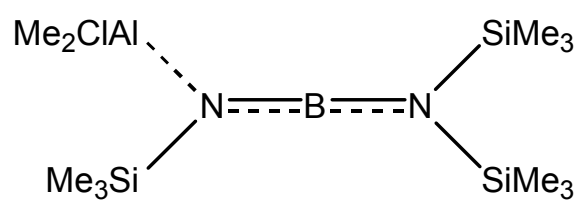

Summenformel:

Molare Masse:

Festpunkt:

Ausbeute:

Massenspektrum:
(E.I.) $\mathrm{m} / \mathrm{z}$
$\mathrm{C}_{11} \mathrm{H}_{33} \mathrm{BN}_{2} \mathrm{AlSi}_{3} \mathrm{Cl}$

$350,89 \mathrm{~g} / \mathrm{mol}$

$42{ }^{\circ} \mathrm{C}$

$91 \%$

$\begin{array}{lll}350 & {[\mathrm{M}]^{+}} & 8 \% \\ 335 & {[\mathrm{M}-\mathrm{Me}]^{+}} & 45 \% \\ 315 & {[\mathrm{M}-\mathrm{Cl}]^{+}} & 5 \% \\ 259 & {\left[\mathrm{M}-\mathrm{AlMe}_{2} \mathrm{Cl}\right]^{+}} & 20 \%\end{array}$

Kernmagnetische Resonanz (NMR) $\left(\mathrm{CDCl}_{3}\right)$

\begin{tabular}{|c|c|c|c|c|}
\hline Verschiebung & Zuordnung & Signalform & Kopplungskonst. [Hz] & Integration \\
\hline \multicolumn{5}{|l|}{$\delta^{1} \mathrm{H}$ [ppm] } \\
\hline$-0,32$ & $\mathrm{AICl}\left(\mathrm{C}_{3}\right)_{2}$ & $\mathrm{~s}$ & & $6 \mathrm{H}$ \\
\hline 0,10 & $\mathrm{~N}-\left[\mathrm{Si}\left(\mathrm{CH}_{3}\right)_{3}\right]_{2}$ & $\mathrm{~s}$ & & $18 \mathrm{H}$ \\
\hline 0,42 & $\mathrm{AlCl}_{3}-\mathrm{N}-\mathrm{Si}\left(\mathrm{CH}_{3}\right)_{3}$ & $\mathrm{~s}$ & & $9 \mathrm{H}$ \\
\hline \multicolumn{5}{|l|}{$\delta{ }^{11} \mathrm{~B}$ [ppm] } \\
\hline 33,63 & $\underline{B}$ & $s$ & & \\
\hline \multicolumn{5}{|l|}{$\delta^{13} \mathrm{C}$ [ppm] } \\
\hline 1,03 & $\mathrm{AICl}\left(\underline{\mathrm{C}}_{3}\right)_{2}$ & s & & \\
\hline 1,94 & $\mathrm{~N}-\left[\mathrm{Si}\left(\mathrm{CH}_{3}\right)_{3}\right]_{2}$ & $s$ & & \\
\hline 2,48 & $\mathrm{AlCl}_{3}-\mathrm{N}-\mathrm{Si}\left(\mathrm{CH}_{3}\right)_{3}$ & $\mathrm{~s}$ & & \\
\hline \multicolumn{5}{|l|}{$\delta{ }^{27} \mathrm{Al}$ [ppm] } \\
\hline 63,24 & $\underline{\mathrm{Al}}$ & $\mathrm{s}$ & & \\
\hline \multicolumn{5}{|l|}{$\delta^{29} \mathrm{Si}[\mathrm{ppm}]$} \\
\hline 11,15 & $\mathrm{AlCl}_{3}-\mathrm{N}-\mathrm{SiMe}_{3}$ & $s$ & & \\
\hline 12,85 & $\mathrm{~N}-\left(\underline{\mathrm{SiMe}}_{3}\right)_{2}$ & $s$ & & \\
\hline
\end{tabular}




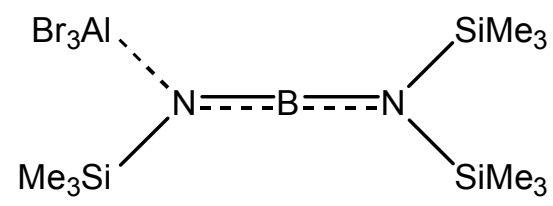

Summenformel:

Molare Masse:

Festpunkt:

Ausbeute:
$\mathrm{C}_{9} \mathrm{H}_{27} \mathrm{BN}_{2} \mathrm{AlSi}_{3} \mathrm{Br}_{3}$

$525,09 \mathrm{~g} / \mathrm{mol}$

$122{ }^{\circ} \mathrm{C}$

$95 \%$

Kernmagnetische Resonanz (NMR) $\left(\mathrm{CDCl}_{3}\right)$

\begin{tabular}{|c|c|c|c|c|}
\hline Verschiebung & Zuordnung & Signalform & Kopplungskonst. [Hz] & Integration \\
\hline \multicolumn{5}{|l|}{$\delta^{1} \mathrm{H}$ [ppm] } \\
\hline 0,39 & $\mathrm{~N}-\left[\mathrm{Si}\left(\mathrm{CH}_{3}\right)_{3}\right]_{2}$ & $\mathrm{~s}$ & & $18 \mathrm{H}$ \\
\hline 0,51 & $\mathrm{AlBr}_{3}-\mathrm{N}-\mathrm{Si}\left(\mathrm{CH}_{3}\right)_{3}$ & s & & $9 \mathrm{H}$ \\
\hline \multicolumn{5}{|l|}{$\delta{ }^{11} \mathrm{~B}$ [ppm] } \\
\hline 26,19 & $\underline{B}$ & $\mathrm{~s}$ & & \\
\hline \multicolumn{5}{|l|}{$\delta{ }^{13} \mathrm{C}[\mathrm{ppm}]$} \\
\hline 1,94 & $\mathrm{~N}-\left[\mathrm{Si}\left(\mathrm{CH}_{3}\right)_{3}\right]_{2}$ & s & & \\
\hline 2,30 & $\mathrm{AlBr}_{3}-\mathrm{N}-\mathrm{Si}\left(\underline{\mathrm{CH}}_{3}\right)_{3}$ & s & & \\
\hline \multicolumn{5}{|l|}{$\delta^{27} \mathrm{Al}[\mathrm{ppm}]$} \\
\hline 91,47 & $\underline{\mathrm{Al}}$ & s & & \\
\hline \multicolumn{5}{|l|}{$\delta^{29} \mathrm{Si}$ [ppm] } \\
\hline 13,70 & $\mathrm{AlBr}_{3}-\mathrm{N}-\underline{\mathrm{SiMe}_{3}}$ & s & & \\
\hline 15,74 & $\mathrm{~N}-\left(\underline{\mathrm{SiMe}_{3}}\right)_{2}$ & s & & \\
\hline
\end{tabular}


<smiles>C[AsH]N([13CH3])[AsH3]</smiles>

Summenformel:

Molare Masse:

Festpunkt:

Ausbeute:
$\mathrm{C}_{9} \mathrm{H}_{27} \mathrm{BN}_{2} \mathrm{Si}_{3} \mathrm{Cl}_{3} \mathrm{Ga}$

$434,47 \mathrm{~g} / \mathrm{mol}$

$157^{\circ} \mathrm{C}$

$82 \%$

Kernmagnetische Resonanz (NMR) $\left(\mathrm{CDCl}_{3}\right)$

\begin{tabular}{|c|c|c|c|c|}
\hline Verschiebung & Zuordnung & Signalform & Kopplungskonst. [Hz] & Integration \\
\hline \multicolumn{5}{|l|}{$\delta{ }^{1} \mathrm{H}$ [ppm] } \\
\hline 0,33 & $\mathrm{GaCl}_{3}-\mathrm{N}-\mathrm{Si}\left(\mathrm{CH}_{3}\right)_{3}$ & s & & $9 \mathrm{H}$ \\
\hline 0,36 & $\mathrm{~N}-\left[\mathrm{Si}\left(\mathrm{CH}_{3}\right)_{3}\right]_{2}$ & $\mathrm{~s}$ & & $18 \mathrm{H}$ \\
\hline \multicolumn{5}{|l|}{$\delta{ }^{11} \mathrm{~B}[\mathrm{ppm}]$} \\
\hline 30,10 & $\underline{B}$ & $\mathrm{~s}$ & & \\
\hline \multicolumn{5}{|l|}{$\delta{ }^{13} \mathrm{C}[\mathrm{ppm}]$} \\
\hline 1,74 & $\mathrm{~N}-\left[\mathrm{Si}\left(\mathrm{CH}_{3}\right)_{3}\right]_{2}$ & $\mathrm{~s}$ & & \\
\hline 1,83 & $\mathrm{GaCl}_{3}-\mathrm{N}-\mathrm{Si}\left(\underline{\mathrm{CH}}_{3}\right)_{3}$ & $\mathrm{~s}$ & & \\
\hline \multicolumn{5}{|l|}{$\delta^{29} \mathrm{Si}$ [ppm] } \\
\hline 13,68 & $\mathrm{GaCl}_{3}-\mathrm{N}-\mathrm{SiMe}_{3}$ & $s$ & & \\
\hline 15,67 & $\left.\mathrm{~N}-(\underline{\mathrm{SiMe}})_{3}\right)_{2}$ & $\mathrm{~s}$ & & \\
\hline
\end{tabular}


Bis[(tert-butyldimethylsilyl-trimethylsilyl)amino]-fluor-boran<smiles></smiles>

Summenformel:

$\mathrm{C}_{18} \mathrm{H}_{48} \mathrm{BN}_{2} \mathrm{FSi}_{4}$

Molare Masse:

$434,74 \mathrm{~g} / \mathrm{mol}$

Siedepunkt:

$128^{\circ} \mathrm{C} / 0,01 \mathrm{mbar}$

Ausbeute:

$97 \%$

Massenspektrum:

(E.I.) $\mathrm{m} / \mathrm{z}$

$\begin{array}{ll}419 & {[\mathrm{M}-\mathrm{Me}]^{+}} \\ 377 & {\left[\mathrm{M}-\mathrm{CMe}_{3}\right]^{+}}\end{array}$

$18 \%$

$100 \%$

Kernmagnetische Resonanz (NMR) $\left(\mathrm{CDCl}_{3}\right)$

\begin{tabular}{|c|c|c|c|c|}
\hline Verschiebung & Zuordnung & Signalform & Kopplungskonst. [Hz] & Integration \\
\hline \multicolumn{5}{|l|}{$\delta^{1} \mathrm{H}$ [ppm] } \\
\hline 0,18 & a SiCMe $3\left(\underline{C H}_{3}\right)_{2}$ & $d$ & ${ }^{5} \mathrm{~J}_{\mathrm{HF}}=1,7$ & $6 \mathrm{H}$ \\
\hline 0,20 & b SiCMe ${ }_{3}\left(\underline{C H}_{3}\right)_{2}$ & $d$ & ${ }^{5} \mathrm{~J}_{\mathrm{HF}}=1,5$ & $6 \mathrm{H}$ \\
\hline 0,25 & $\mathrm{Si}\left(\mathrm{CH}_{3}\right)_{3}$ & s & & $18 \mathrm{H}$ \\
\hline 0,93 & $\mathrm{SiC}\left(\mathrm{CH}_{3}\right)_{3} \mathrm{Me}_{2}$ & $d$ & ${ }^{5} \mathrm{~J}_{\mathrm{HF}}=1,1$ & $18 \mathrm{H}$ \\
\hline \multicolumn{5}{|l|}{$\delta^{11} \mathrm{~B}$ [ppm] } \\
\hline 26,73 & $\underline{B}$ & s & & \\
\hline \multicolumn{5}{|l|}{$\delta{ }^{13} \mathrm{C}[\mathrm{ppm}]$} \\
\hline$-0,16$ & a $\mathrm{SiCMe}_{3}\left(\underline{\mathrm{CH}}_{3}\right)_{2}$ & $d$ & ${ }^{4} J_{C F}=3,7$ & \\
\hline$-0,04$ & b SiCMe ${ }_{3}\left(\underline{\mathrm{CH}}_{3}\right)_{2}$ & $d$ & ${ }^{4} \mathrm{~J}_{\mathrm{CF}}=3,5$ & \\
\hline 4,98 & $\mathrm{Si}\left(\underline{\mathrm{C}} \mathrm{H}_{3}\right)_{3}$ & $d$ & ${ }^{4} J_{C F}=0,7$ & \\
\hline 19,87 & $\mathrm{SiC} \mathrm{Me}_{3} \mathrm{Me}_{2}$ & $s$ & & \\
\hline 28,15 & $\mathrm{SiC}\left(\underline{\mathrm{C}} \mathrm{H}_{3}\right)_{3} \mathrm{Me}_{2}$ & $d$ & ${ }^{5} J_{C F}=2,4$ & \\
\hline \multicolumn{5}{|l|}{$\delta^{19} \mathrm{~F}$ [ppm] } \\
\hline 116,22 & $\underline{F}$ & s & & \\
\hline \multicolumn{5}{|l|}{$\delta^{29} \mathrm{Si}$ [ppm] } \\
\hline 2,88 & $\underline{\mathrm{SiMe}}_{3}$ & $d$ & ${ }^{3} \mathrm{~J}_{\mathrm{SiF}}=5,5$ & \\
\hline 9,51 & $\underline{\mathrm{SiCMe}} \mathrm{Me}_{2}$ & $d$ & ${ }^{3} \mathrm{~J}_{\mathrm{SiF}}=4,9$ & \\
\hline
\end{tabular}




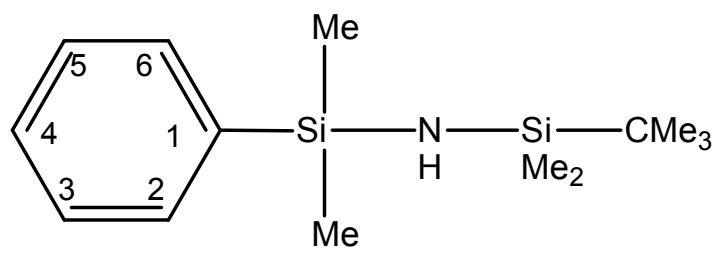

Summenformel:

Molare Masse:

Siedepunkt:

Ausbeute:

Massenspektrum:

\section{$\mathrm{C}_{14} \mathrm{H}_{27} \mathrm{NSi}_{2}$}

$265,54 \mathrm{~g} / \mathrm{mol}$

$65{ }^{\circ} \mathrm{C} / 0,01 \mathrm{mbar}$

$95 \%$

$208 \quad[\mathrm{M}-\mathrm{Bu}]^{+} \quad 100 \%$

Kernmagnetische Resonanz (NMR) $\left(\mathrm{CDCl}_{3}\right)$

\begin{tabular}{|c|c|c|c|c|}
\hline Verschiebung & Zuordnung & Signalform & Kopplungskonst. [Hz] & Integration \\
\hline \multicolumn{5}{|l|}{$\delta^{1} \mathrm{H}$ [ppm] } \\
\hline 0,08 & $\mathrm{SiCMe}_{3}\left(\mathrm{C}_{3}\right)_{2}$ & s & & $6 \mathrm{H}$ \\
\hline 0,38 & $\mathrm{Si}\left(\mathrm{C}_{6} \mathrm{H}_{5}\right)\left(\mathrm{C}_{3}\right)_{2}$ & s & & $6 \mathrm{H}$ \\
\hline 0,93 & $\mathrm{SiC}\left(\mathrm{CH}_{3}\right)_{3} \mathrm{Me}_{2}$ & s & & $9 \mathrm{H}$ \\
\hline $7,36-7,71$ & 2/3/4/5/6 C & $\mathrm{m}$ & & $5 \mathrm{H}$ \\
\hline \multicolumn{5}{|l|}{$\delta{ }^{13} \mathrm{C}[\mathrm{ppm}]$} \\
\hline$-2,88$ & $\mathrm{SiCMe}_{3}\left(\underline{\mathrm{CH}}_{3}\right)_{2}$ & $\mathrm{~s}$ & & \\
\hline 0,92 & $\mathrm{Si}\left(\mathrm{C}_{6} \mathrm{H}_{5}\right)\left(\underline{\mathrm{C}} \mathrm{H}_{3}\right)_{2}$ & s & & \\
\hline 18,09 & $\mathrm{Si}^{\mathrm{C}} \mathrm{Me}_{3} \mathrm{Me}_{2}$ & $\mathrm{~s}$ & & \\
\hline 25,65 & $\mathrm{SiC}\left(\underline{\mathrm{C}} \mathrm{H}_{3}\right)_{3} \mathrm{Me}_{2}$ & s & & \\
\hline 127,67 & $3 / 5 \underline{C}$ & $s$ & & \\
\hline 129,13 & $4 \underline{C}$ & s & & \\
\hline 132,95 & $2 / 6 \underline{\mathrm{C}}$ & s & & \\
\hline 140,20 & $1 \underline{\mathrm{C}}$ & $s$ & & \\
\hline \multicolumn{5}{|l|}{$\delta^{29} \mathrm{Si}$ [ppm] } \\
\hline$-2,73$ & $\underline{\mathrm{Si}}\left(\mathrm{C}_{6} \mathrm{H}_{5}\right) \mathrm{Me}_{2}$ & $s$ & & \\
\hline 11,54 & $\underline{\mathrm{SiCMe}} \mathrm{Me}_{2}$ & s & & \\
\hline
\end{tabular}




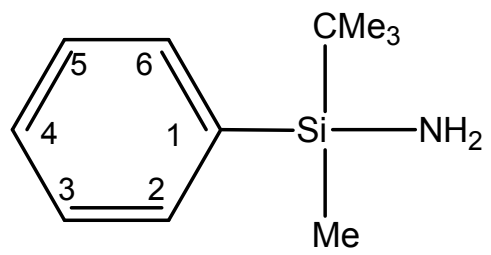

Summenformel:

Molare Masse:

Siedepunkt:

Ausbeute:

Massenspektrum:

(E.I.) $\mathrm{m} / \mathrm{z}$

$\mathrm{C}_{11} \mathrm{H}_{19} \mathrm{NSi}$

$193,36 \mathrm{~g} / \mathrm{mol}$

$35^{\circ} \mathrm{C} / 0,1 \mathrm{mbar}$

$98 \%$

$\begin{array}{lll}193 & {[\mathrm{M}]^{+}} & 55 \% \\ 178 & {[\mathrm{M}-\mathrm{Me}]^{+}} & 100 \%\end{array}$

Kernmagnetische Resonanz (NMR) $\left(\mathrm{CDCl}_{3}\right)$

\begin{tabular}{|c|c|c|c|c|}
\hline Verschiebung & Zuordnung & Signalform & Kopplungskonst. [Hz] & Integration \\
\hline \multicolumn{5}{|l|}{$\delta^{1} \mathrm{H}$ [ppm] } \\
\hline 0,37 & $\mathrm{SiC}_{\mathrm{H}_{3}}$ & s & & $3 \mathrm{H}$ \\
\hline 0,95 & $\mathrm{SiC}\left(\mathrm{CH}_{3}\right)_{3}$ & s & & $9 \mathrm{H}$ \\
\hline $7,38-7,63$ & 2/3/4/5/6 Cㅡㅡ & $\mathrm{m}$ & & $5 \mathrm{H}$ \\
\hline \multicolumn{5}{|l|}{$\delta{ }^{13} \mathrm{C}$ [ppm] } \\
\hline 4,60 & $\mathrm{SiCH}_{3}$ & $\mathrm{~s}$ & & \\
\hline 17,62 & $\mathrm{SiC}\left(\mathrm{CH}_{3}\right)_{3}$ & $\mathrm{~s}$ & & \\
\hline 26,19 & $\mathrm{SiC}\left(\underline{\mathrm{CH}}_{3}\right)_{3}$ & s & & \\
\hline 127,37 & $3 / 5 \underline{C}$ & $\mathrm{~s}$ & & \\
\hline 128,86 & $4 \underline{C}$ & $\mathrm{~s}$ & & \\
\hline 134,24 & $2 / 6 \underline{\mathrm{C}}$ & s & & \\
\hline 138,20 & $1 \underline{C}$ & s & & \\
\hline \multicolumn{5}{|l|}{$\delta{ }^{15} \mathrm{~N}$ [ppm] } \\
\hline$-380,41$ & $\underline{N}$ & $\mathrm{t}$ & ${ }^{1} \mathrm{~J}_{\mathrm{NH}}=71,7$ & \\
\hline \multicolumn{5}{|l|}{${ }^{29} \mathrm{Si}$ [ppm] } \\
\hline 1,02 & $\underline{\mathrm{Si}}$ & s & & \\
\hline
\end{tabular}




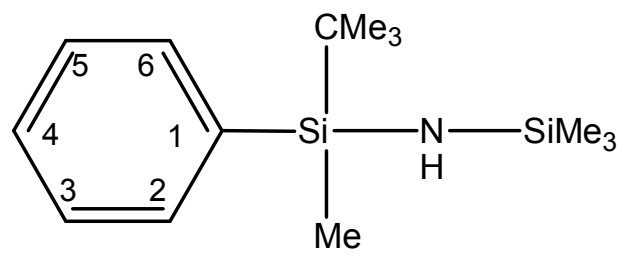

Summenformel:

Molare Masse:

Siedepunkt:

Ausbeute:

Massenspektrum:
(E.I.) $\mathrm{m} / \mathrm{z}$
$\mathrm{C}_{14} \mathrm{H}_{27} \mathrm{NSi}_{2}$

$265,54 \mathrm{~g} / \mathrm{mol}$

$70^{\circ} \mathrm{C} / 0,01 \mathrm{mbar}$

$95 \%$

$\begin{array}{lll}250 & {[\mathrm{M}-\mathrm{Me}]^{+}} & 10 \% \\ 208 & {[\mathrm{M}-\mathrm{Bu}]^{+}} & 100 \%\end{array}$

Kernmagnetische Resonanz (NMR) $\left(\mathrm{CDCl}_{3}\right)$

\begin{tabular}{|c|c|c|c|c|}
\hline Verschiebung & Zuordnung & Signalform & Kopplungskonst. [Hz] & Integration \\
\hline \multicolumn{5}{|l|}{$\delta^{1} \mathrm{H}$ [ppm] } \\
\hline 0,04 & $\mathrm{Si}\left(\mathrm{CH}_{3}\right)_{3}$ & s & & $9 \mathrm{H}$ \\
\hline 0,38 & $\mathrm{Si}\left(\mathrm{C}_{6} \mathrm{H}_{5}\right) \mathrm{CMe}_{3} \mathrm{C}_{3}$ & s & & $3 \mathrm{H}$ \\
\hline 0,89 & $\mathrm{Si}\left(\mathrm{C}_{6} \mathrm{H}_{5}\right) \mathrm{C}\left(\mathrm{C}_{3}\right)_{3} \mathrm{Me}$ & s & & $9 \mathrm{H}$ \\
\hline $7,16-7,31$ & 2/3/4/5/6 C므 & $\mathrm{m}$ & & $5 \mathrm{H}$ \\
\hline \multicolumn{5}{|l|}{$\delta{ }^{13} \mathrm{C}[\mathrm{ppm}]$} \\
\hline$-4,31$ & $\mathrm{Si}\left(\mathrm{C}_{6} \mathrm{H}_{5}\right) \mathrm{CMe}_{3} \underline{\mathrm{CH}}_{3}$ & $\mathrm{~s}$ & & \\
\hline 2,56 & $\mathrm{Si}\left(\mathrm{CH}_{3}\right)_{3}$ & $\mathrm{~s}$ & & \\
\hline 17,82 & $\mathrm{Si}\left(\mathrm{C}_{6} \mathrm{H}_{5}\right) \underline{\mathrm{C}} \mathrm{Me}_{3} \mathrm{Me}$ & s & & \\
\hline 26,32 & $\mathrm{Si}\left(\mathrm{C}_{6} \mathrm{H}_{5}\right) \mathrm{C}\left(\underline{\mathrm{C}}_{3}\right)_{3} \mathrm{Me}$ & s & & \\
\hline 127,18 & $3 / 5 \underline{C}$ & $\mathrm{~s}$ & & \\
\hline 128,67 & $4 \underline{C}$ & s & & \\
\hline 134,54 & $2 / 6 \underline{C}$ & $s$ & & \\
\hline 139,04 & $1 \underline{C}$ & s & & \\
\hline \multicolumn{5}{|l|}{$\delta^{15} \mathrm{~N}$ [ppm] } \\
\hline$-366,42$ & $\underline{N}$ & $d$ & ${ }^{1} \mathrm{~J}_{\mathrm{NH}}=66,4$ & \\
\hline \multicolumn{5}{|l|}{$\delta^{29} \mathrm{Si}$ [ppm] } \\
\hline 0,53 & $\underline{\mathrm{Si}}\left(\mathrm{C}_{6} \mathrm{H}_{5}\right) \mathrm{CMe}_{3} \mathrm{Me}$ & $s$ & & \\
\hline 4,07 & $\underline{\mathrm{SiMe}}_{3}$ & s & & \\
\hline
\end{tabular}




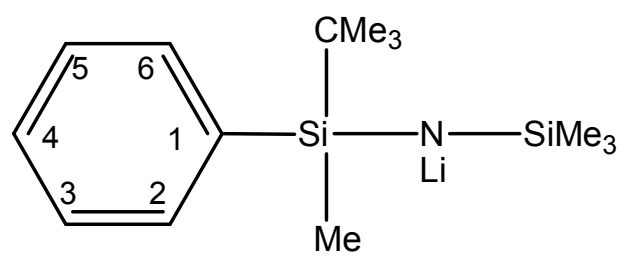

Summenformel:

$\mathrm{C}_{14} \mathrm{H}_{26} \mathrm{LiNSi}_{2}$

Molare Masse:

$271,47 \mathrm{~g} / \mathrm{mol}$

Festpunkt:

$117^{\circ} \mathrm{C}$

Ausbeute:

$95 \%$

Kernmagnetische Resonanz (NMR) $\left(\mathrm{CDCl}_{3}\right)$

\begin{tabular}{|c|c|c|c|c|}
\hline Verschiebung & Zuordnung & Signalform & Kopplungskonst. [Hz] & Integration \\
\hline \multicolumn{5}{|l|}{$\delta^{1} H$ [ppm] } \\
\hline 0,05 & $\mathrm{Si}\left(\mathrm{C}_{3}\right)_{3}$ & s & & $9 \mathrm{H}$ \\
\hline 0,38 & $\mathrm{Si}\left(\mathrm{C}_{6} \mathrm{H}_{5}\right) \mathrm{CMe}_{3} \mathrm{CH}_{3}$ & s & & $3 \mathrm{H}$ \\
\hline 0,88 & $\mathrm{Si}\left(\mathrm{C}_{6} \mathrm{H}_{5}\right) \mathrm{C}\left(\mathrm{C}_{3}\right)_{3} \mathrm{Me}$ & s & & $9 \mathrm{H}$ \\
\hline $7,11-7,31$ & $2 / 3 / 4 / 5 / 6 \mathrm{CH}$ & $\mathrm{m}$ & & $5 \mathrm{H}$ \\
\hline \multicolumn{5}{|l|}{$\delta^{7} \mathrm{Li}$ [ppm] } \\
\hline 0,88 & $\underline{\mathrm{Li}}$ & s & & \\
\hline \multicolumn{5}{|l|}{$\delta{ }^{13} \mathrm{C}[\mathrm{ppm}]$} \\
\hline$-4,01$ & $\mathrm{Si}\left(\mathrm{C}_{6} \mathrm{H}_{5}\right) \mathrm{CMe}_{3} \underline{\mathrm{CH}_{3}}$ & $s$ & & \\
\hline 2,63 & $\mathrm{Si}\left(\underline{\mathrm{CH}}_{3}\right)_{3}$ & s & & \\
\hline 17,94 & $\mathrm{Si}\left(\mathrm{C}_{6} \mathrm{H}_{5}\right) \underline{\mathrm{C}} \mathrm{Me}_{3} \mathrm{Me}$ & $\mathrm{s}$ & & \\
\hline 26,46 & $\mathrm{Si}\left(\mathrm{C}_{6} \mathrm{H}_{5}\right) \mathrm{C}\left(\underline{\mathrm{C}}_{3}\right)_{3} \mathrm{Me}$ & s & & \\
\hline 127,65 & $3 / 5 \underline{C}$ & s & & \\
\hline 129,16 & $4 \underline{\mathrm{C}}$ & $\mathrm{s}$ & & \\
\hline 134,85 & $2 / 6 \underline{C}$ & $s$ & & \\
\hline 139,05 & $1 \underline{\mathrm{C}}$ & $s$ & & \\
\hline \multicolumn{5}{|l|}{$\delta^{29} \mathrm{Si}$ [ppm] } \\
\hline$-0,17$ & $\underline{\mathrm{Si}}\left(\mathrm{C}_{6} \mathrm{H}_{5}\right) \mathrm{CMe}_{3} \mathrm{Me}$ & $s$ & & \\
\hline 4,13 & $\underline{\mathrm{SiMe}}_{3}$ & $s$ & & \\
\hline
\end{tabular}


(tert-Butylmethylphenylsilyl-trimethylsilyl)amino-difluor-boran

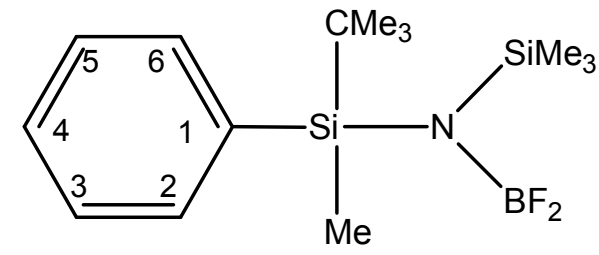

Summenformel:

Molare Masse:

Siedepunkt:

Ausbeute:

Massenspektrum:
$\mathrm{C}_{14} \mathrm{H}_{26} \mathrm{BNF}_{2} \mathrm{Si}_{2}$

$313,34 \mathrm{~g} / \mathrm{mol}$

$72{ }^{\circ} \mathrm{C} / 0,01 \mathrm{mbar}$

$95 \%$

298

256
[M-Me] $^{+}$

[M-Bu $]^{+}$
$10 \%$

$100 \%$

Kernmagnetische Resonanz (NMR) $\left(\mathrm{CDCl}_{3}\right)$

\begin{tabular}{|c|c|c|c|c|}
\hline Verschiebung & Zuordnung & Signalform & Kopplungskonst. [Hz] & Integration \\
\hline \multicolumn{5}{|l|}{$\delta^{1} \mathrm{H}$ [ppm] } \\
\hline 0,07 & $\mathrm{Si}\left(\mathrm{CH}_{3}\right)_{3}$ & $\mathrm{t}$ & ${ }^{5} \mathrm{~J}_{\mathrm{HF}}=1,2$ & $9 \mathrm{H}$ \\
\hline 0,46 & $\mathrm{Si}\left(\mathrm{C}_{6} \mathrm{H}_{5}\right) \mathrm{CMe}_{3} \mathrm{C}_{3}$ & $\mathrm{t}$ & ${ }^{5} \mathrm{~J}_{\mathrm{HF}}=1,8$ & $3 \mathrm{H}$ \\
\hline 1,14 & $\mathrm{SiC}\left(\mathrm{CH}_{3}\right)_{3}$ & $\mathrm{t}$ & ${ }^{6} \mathrm{~J}_{\mathrm{HF}}=0,5$ & $9 \mathrm{H}$ \\
\hline $7,31-7,72$ & $2 / 3 / 4 / 5 / 6 \mathrm{CH}$ & $\mathrm{m}$ & & $5 \mathrm{H}$ \\
\hline \multicolumn{5}{|l|}{$\delta^{11} \mathrm{~B}$ [ppm] } \\
\hline 18,44 & $\underline{B}$ & $\mathrm{~s}$ & & \\
\hline \multicolumn{5}{|l|}{$\delta^{13} \mathrm{C}$ [ppm] } \\
\hline 0,64 & $\mathrm{Si}\left(\mathrm{C}_{6} \mathrm{H}_{5}\right) \mathrm{CMe}_{3} \underline{\mathrm{CH}_{3}}$ & $\mathrm{t}$ & ${ }^{4} J_{C F}=3,2$ & \\
\hline 2,82 & $\mathrm{Si}\left(\underline{\mathrm{C}}_{3}\right)_{3}$ & $\mathrm{t}$ & ${ }^{4} \mathrm{~J}_{\mathrm{CF}}=2,0$ & \\
\hline 19,52 & $\mathrm{Si}\left(\mathrm{C}_{6} \mathrm{H}_{5}\right) \underline{\mathrm{C}} \mathrm{Me}_{3} \mathrm{Me}$ & $s$ & & \\
\hline 27,66 & $\mathrm{Si}\left(\mathrm{C}_{6} \mathrm{H}_{5}\right) \mathrm{C}\left(\underline{\mathrm{CH}}_{3}\right)_{3} \mathrm{Me}$ & $\mathrm{t}$ & ${ }^{5} J_{C F}=1,3$ & \\
\hline 127,75 & $3 / 5 \underline{C}$ & $\mathrm{~s}$ & & \\
\hline 129,17 & $4 \underline{C}$ & s & & \\
\hline 134,13 & $2 / 6 \underline{C}$ & $s$ & & \\
\hline 138,59 & $1 \underline{\mathrm{C}}$ & $\mathrm{t}$ & ${ }^{4} J_{C F}=0,8$ & \\
\hline \multicolumn{5}{|l|}{$\delta{ }^{19} \mathrm{~F}[\mathrm{ppm}]$} \\
\hline \multicolumn{5}{|l|}{$\delta^{29} \mathrm{Si}[\mathrm{ppm}]$} \\
\hline 4,16 & $\underline{\mathrm{Si}}\left(\mathrm{C}_{6} \mathrm{H}_{5}\right) \mathrm{CMe}_{3} \mathrm{Me}$ & $\mathrm{t}$ & ${ }^{3} J_{\mathrm{SiF}}=3,3$ & \\
\hline 11,52 & $\underline{\mathrm{SiMe}}_{3}$ & $\mathrm{t}$ & ${ }^{3} \mathrm{~J}_{\mathrm{SiF}}=5,4$ & \\
\hline
\end{tabular}


(tert-Butylmethylphenylsilyl-trimethylsilyl)amino-fluor-bis(trimethylsilyl)amino-boran

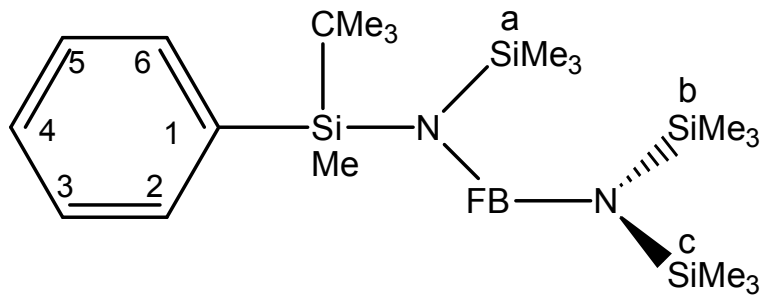

Summenformel:

$\mathrm{C}_{20} \mathrm{H}_{44} \mathrm{BN}_{2} \mathrm{FSi}_{4}$

Molare Masse:

Festpunkt: $454,73 \mathrm{~g} / \mathrm{mol}$ $95{ }^{\circ} \mathrm{C}$

Siedepunkt: $128^{\circ} \mathrm{C} / 0,01 \mathrm{mbar}$

Ausbeute: $95 \%$

Massenspektrum:

(E.I.) $\mathrm{m} / \mathrm{z}$

439

$[\mathrm{M}-\mathrm{Me}]^{+}$

$15 \%$

397

$[\mathrm{M}-\mathrm{Bu}]^{+}$

$100 \%$

Kernmagnetische Resonanz (NMR) $\left(\mathrm{CDCl}_{3}\right)$

\begin{tabular}{|c|c|c|c|c|}
\hline Verschiebung & Zuordnung & Signalform & Kopplungskonst. [Hz] & Integration \\
\hline \multicolumn{5}{|l|}{$\delta^{1} \mathrm{H}$ [ppm] } \\
\hline 0,06 & a Si $\left(\mathrm{CH}_{3}\right)_{3}$ & $s$ & & $9 \mathrm{H}$ \\
\hline 0,18 & b Si $\left(\mathrm{C}_{3}\right)_{3}$ & $d$ & ${ }^{5} J_{H F}=1,0$ & $9 \mathrm{H}$ \\
\hline 0,27 & c Si $\left(\mathrm{CH}_{3}\right)_{3}$ & $d$ & ${ }^{5} \mathrm{~J}_{\mathrm{HF}}=0,7$ & $9 \mathrm{H}$ \\
\hline 0,52 & $\mathrm{Si}\left(\mathrm{C}_{6} \mathrm{H}_{5}\right) \mathrm{CMe}_{3} \mathrm{C}_{3}$ & $\mathrm{~s}$ & & $3 \mathrm{H}$ \\
\hline 0,93 & $\operatorname{SiC}\left(\mathrm{CH}_{3}\right)_{3}$ & s & & $9 \mathrm{H}$ \\
\hline $7,30-7,32$ & 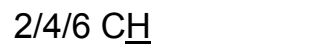 & $\mathrm{m}$ & & $3 \mathrm{H}$ \\
\hline $7,62-7,64$ & $3 / 5 \mathrm{C} \underline{\mathrm{H}}$ & $\mathrm{m}$ & & $2 \mathrm{H}$ \\
\hline \multicolumn{5}{|l|}{$\delta^{11} \mathrm{~B}[\mathrm{ppm}]$} \\
\hline 26,47 & $\underline{B}$ & $\mathrm{~s}$ & & \\
\hline \multicolumn{5}{|l|}{$\delta^{13} \mathrm{C}[\mathrm{ppm}]$} \\
\hline$-2,05$ & $\mathrm{Si}\left(\mathrm{C}_{6} \mathrm{H}_{5}\right) \mathrm{CMe}_{3} \underline{\mathrm{CH}}_{3}$ & $d$ & ${ }^{4} J_{C F}=0,9$ & \\
\hline 3,75 & b Si $\left(\underline{\mathrm{C}} \mathrm{H}_{3}\right)_{3}$ & $d$ & ${ }^{4} J_{C F}=2,5$ & \\
\hline 4,03 & c Si $\left(\underline{C} \mathrm{H}_{3}\right)_{3}$ & $d$ & ${ }^{4} J_{C F}=2,2$ & \\
\hline 4,34 & a Si $\left(\underline{\mathrm{C}} \mathrm{H}_{3}\right)_{3}$ & s & & \\
\hline 20,75 & $\mathrm{Si}\left(\mathrm{C}_{6} \mathrm{H}_{5}\right) \underline{\mathrm{CMe}_{3} \mathrm{Me}}$ & s & & \\
\hline 28,75 & $\mathrm{Si}\left(\mathrm{C}_{6} \mathrm{H}_{5}\right) \mathrm{C}\left(\underline{\mathrm{C}}_{3}\right)_{3} \mathrm{Me}$ & $d$ & ${ }^{5} \mathrm{~J}_{\mathrm{CF}}=3,3$ & \\
\hline 127,24 & $3 / 5 \underline{C}$ & $s$ & & \\
\hline 128,77 & $4 \underline{C}$ & $\mathrm{~s}$ & & \\
\hline 135,02 & $2 / 6 \underline{C}$ & $d$ & ${ }^{4} J_{C F}=1,5$ & \\
\hline 138,86 & $1 \underline{\mathrm{C}}$ & $\mathrm{s}$ & & \\
\hline \multicolumn{5}{|l|}{$\delta{ }^{19} \mathrm{~F}[\mathrm{ppm}]$} \\
\hline 109.94 & $\underline{\mathrm{F}}$ & $\mathrm{s}$ & & \\
\hline \multicolumn{5}{|l|}{${ }^{29} \mathrm{Si}$ [ppm] } \\
\hline 1,52 & $\underline{\mathrm{Si}}\left(\mathrm{C}_{6} \mathrm{H}_{5}\right) \mathrm{CMe}_{3} \mathrm{Me}$ & s & & \\
\hline 2,93 & a $\underline{\mathrm{SiMe}}_{3}$ & $d$ & ${ }^{3} J_{S_{i F}}=3,3$ & \\
\hline 4,54 & b $\underline{S i M e}_{3}$ & $d$ & ${ }^{3} J_{\mathrm{SiF}}=9,4$ & \\
\hline 5,05 & c $\underline{\mathrm{SiMe}}_{3}$ & $d$ & ${ }^{3} \mathrm{~J}_{\mathrm{SiF}}=8,8$ & \\
\hline
\end{tabular}


<smiles></smiles>

Summenformel:

Molare Masse:

Festpunkt:

Ausbeute:

Massenspektrum:
(E.I.) $\mathrm{m} / \mathrm{z}$
$\mathrm{C}_{10} \mathrm{H}_{27} \mathrm{BN}_{2} \mathrm{AlSi}_{2} \mathrm{Cl}_{3}$

$375,66 \mathrm{~g} / \mathrm{mol}$

$72{ }^{\circ} \mathrm{C}$

$91 \%$

355

$[\mathrm{M}-\mathrm{Me}]^{+}$
$\left[\mathrm{M}-\mathrm{AlCl}_{3}\right]^{+}$
$5 \%$

$40 \%$

Kernmagnetische Resonanz (NMR) $\left(C_{6} D_{6}\right)$

\begin{tabular}{|c|c|c|c|c|}
\hline Verschiebung & Zuordnung & Signalform & Kopplungskonst. [Hz] & Integration \\
\hline \multicolumn{5}{|l|}{$\delta^{1} \mathrm{H}$ [ppm] } \\
\hline 0,12 & $\mathrm{CMe}_{3}-\mathrm{N}-\mathrm{Si}\left(\mathrm{CH}_{3}\right)_{3}$ & s & & $9 \mathrm{H}$ \\
\hline 0,32 & $\mathrm{AlCl}_{3}-\mathrm{N}-\mathrm{Si}\left(\mathrm{CH}_{3}\right)_{3}$ & s & & $9 \mathrm{H}$ \\
\hline 1,07 & $\mathrm{~N}-\mathrm{C}\left(\mathrm{CH}_{3}\right)_{3}$ & $\mathrm{~s}$ & & $9 \mathrm{H}$ \\
\hline \multicolumn{5}{|l|}{$\delta^{11} B$ [ppm] } \\
\hline 31,88 & $\underline{B}$ & $\mathrm{~s}$ & & \\
\hline \multicolumn{5}{|l|}{$\delta{ }^{13} \mathrm{C}$ [ppm] } \\
\hline 1,97 & $\mathrm{CMe}_{3}-\mathrm{N}-\mathrm{Si}\left(\underline{\mathrm{CH}}_{3}\right)_{3}$ & s & & \\
\hline 1,99 & $\mathrm{AlCl}_{3}-\mathrm{N}-\mathrm{Si}\left(\mathrm{CH}_{3}\right)_{3}$ & s & & \\
\hline 32,28 & $\mathrm{~N}-\mathrm{C}\left(\underline{\mathrm{C}} \mathrm{H}_{3}\right)_{3}$ & $\mathrm{~s}$ & & \\
\hline 58,35 & $\mathrm{~N}-\underline{C} \mathrm{Me}_{3}$ & $\mathrm{~s}$ & & \\
\hline \multicolumn{5}{|l|}{$\delta{ }^{27} \mathrm{Al}$ [ppm] } \\
\hline 103,07 & $\underline{\mathrm{Al}}$ & s & & \\
\hline \multicolumn{5}{|l|}{${ }^{29} \mathrm{Si}$ [ppm] } \\
\hline 11,33 & $\mathrm{AlCl}_{3}-\mathrm{N}-\underline{\mathrm{SiMe}}_{3}$ & $\mathrm{~s}$ & & \\
\hline 13,25 & $\mathrm{CMe}_{3}-\mathrm{N}-\mathrm{SiMe}_{3}$ & $s$ & & \\
\hline
\end{tabular}




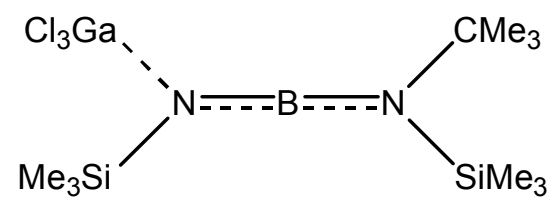

Summenformel:

Molare Masse:

Festpunkt:

Ausbeute:

Massenspektrum:
(E.I.) $\mathrm{m} / \mathrm{z}$
$\mathrm{C}_{10} \mathrm{H}_{27} \mathrm{BN}_{2} \mathrm{Si}_{2} \mathrm{Cl}_{3} \mathrm{Ga}$

$418,40 \mathrm{~g} / \mathrm{mol}$

$72{ }^{\circ} \mathrm{C}$

$98 \%$

$[\mathrm{M}-\mathrm{Me}]^{+}$

$5 \%$

Kernmagnetische Resonanz (NMR) $\left(\mathrm{CDCl}_{3}\right)$

\begin{tabular}{|c|c|c|c|c|}
\hline Verschiebung & Zuordnung & Signalform & Kopplungskonst. [Hz] & Integration \\
\hline \multicolumn{5}{|l|}{$\delta^{1} \mathrm{H}[\mathrm{ppm}]$} \\
\hline 0,40 & $\mathrm{CMe}_{3}-\mathrm{N}-\mathrm{Si}\left(\mathrm{CH}_{3}\right)_{3}$ & $\mathrm{~s}$ & & $9 \mathrm{H}$ \\
\hline 0,44 & $\mathrm{GaCl}_{3}-\mathrm{N}-\mathrm{Si}\left(\mathrm{CH}_{3}\right)_{3}$ & s & & $9 \mathrm{H}$ \\
\hline 1,47 & $\mathrm{~N}-\mathrm{C}\left(\mathrm{CH}_{3}\right)_{3}$ & s & & $9 \mathrm{H}$ \\
\hline \multicolumn{5}{|l|}{$\delta^{11} \mathrm{~B}$ [ppm] } \\
\hline 31,92 & $\underline{B}$ & s & & \\
\hline \multicolumn{5}{|l|}{$\delta{ }^{13} \mathrm{C}[\mathrm{ppm}]$} \\
\hline 1,93 & $\mathrm{CMe}_{3}-\mathrm{N}-\mathrm{Si}\left(\underline{\mathrm{C}}_{3}\right)_{3}$ & $\mathrm{~s}$ & & \\
\hline 2,30 & $\mathrm{GaCl}_{3}-\mathrm{N}-\mathrm{Si}\left(\underline{\mathrm{CH}}_{3}\right)_{3}$ & s & & \\
\hline 32,64 & $\mathrm{~N}-\mathrm{C}\left(\mathrm{C}_{3}\right)_{3}$ & s & & \\
\hline 58,54 & $\mathrm{~N}-\underline{\mathrm{C}} \mathrm{Me}_{3}$ & s & & \\
\hline \multicolumn{5}{|l|}{$\delta^{29} \mathrm{Si}$ [ppm] } \\
\hline 11,77 & $\mathrm{GaCl}_{3}-\mathrm{N}-\mathrm{SiMe}_{3}$ & $\mathrm{~s}$ & & \\
\hline 14,26 & $\mathrm{CMe}_{3}-\mathrm{N}-\mathrm{SiMe}_{3}$ & $\mathrm{~s}$ & & \\
\hline
\end{tabular}




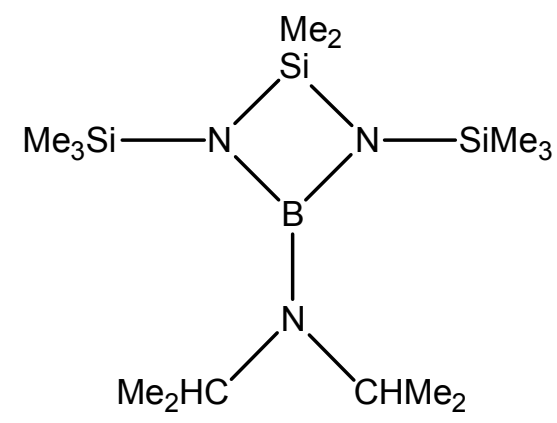

Summenformel:

$\mathrm{C}_{14} \mathrm{H}_{38} \mathrm{BN}_{3} \mathrm{Si}_{3}$

Molare Masse:

$343,54 \mathrm{~g} / \mathrm{mol}$

Festpunkt:

$81^{\circ} \mathrm{C}$

Siedepunkt:

$72{ }^{\circ} \mathrm{C} / 0,01 \mathrm{mbar}$

Ausbeute:

$80 \%$

Massenspektrum:

(E.I.) $\mathrm{m} / \mathrm{z}$

343

$[\mathrm{M}]^{+}$

$5 \%$

328

$[\mathrm{M}-\mathrm{Me}]^{+}$

$100 \%$

Kernmagnetische Resonanz (NMR) $\left(\mathrm{CDCl}_{3}\right)$

\begin{tabular}{|c|c|c|c|c|}
\hline Verschiebung & Zuordnung & Signalform & Kopplungskonst. [Hz] & Integration \\
\hline \multicolumn{5}{|l|}{$\delta^{1} \mathrm{H}$ [ppm] } \\
\hline 0,06 & $\mathrm{Si}\left(\mathrm{CH}_{3}\right)_{3}$ & $s$ & & $18 \mathrm{H}$ \\
\hline 0,29 & $\mathrm{Si}\left(\mathrm{CH}_{3}\right)_{2}$ & $\mathrm{~s}$ & & $6 \mathrm{H}$ \\
\hline 1,23 & $\mathrm{CH}\left(\mathrm{CH}_{3}\right)_{2}$ & $d$ & ${ }^{3} \mathrm{~J}_{\mathrm{HH}}=7,1$ & $12 \mathrm{H}$ \\
\hline 3,84 & $\mathrm{CHMe}_{2}$ & sept & ${ }^{3} \mathrm{~J}_{\mathrm{HH}}=7,1$ & $2 \mathrm{H}$ \\
\hline \multicolumn{5}{|l|}{$\delta^{11} B$ [ppm] } \\
\hline 24,57 & & $\mathrm{~s}$ & & \\
\hline \multicolumn{5}{|l|}{$\delta^{13} \mathrm{C}$ [ppm] } \\
\hline 2,82 & $\mathrm{Si}\left(\underline{\mathrm{C}} \mathrm{H}_{3}\right)_{3}$ & $\mathrm{~s}$ & & \\
\hline 3,87 & $\mathrm{Si}\left(\underline{\mathrm{C}}_{3}\right)_{2}$ & s & & \\
\hline 25,51 & $\mathrm{CH}\left(\underline{\mathrm{C}} \mathrm{H}_{3}\right)_{2}$ & $\mathrm{~s}$ & & \\
\hline 46,95 & $\underline{\mathrm{C}} \mathrm{HMe}_{2}$ & $\mathrm{~s}$ & & \\
\hline \multicolumn{5}{|l|}{${ }^{29} \mathrm{Si}$ [ppm] } \\
\hline$-6,13$ & $\mathrm{SiMe}_{3}$ & $s$ & & \\
\hline 4,72 & $\mathrm{SiMe}_{2}$ & $\mathrm{~s}$ & & \\
\hline
\end{tabular}




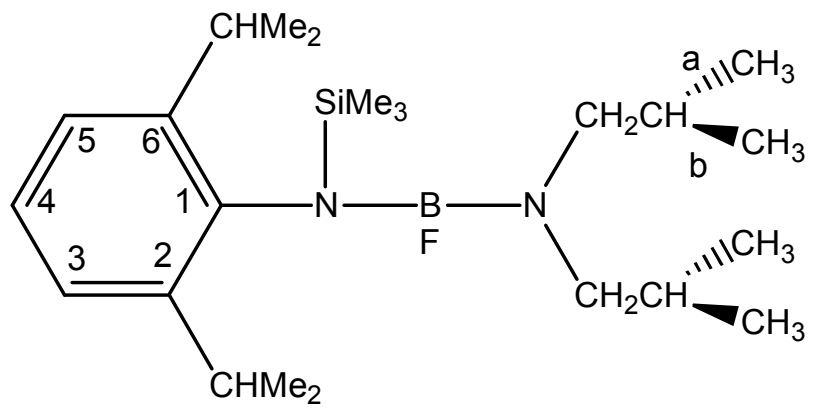

Summenformel:

Molare Masse:

Siedepunkt:

Ausbeute:

Massenspektrum:
(E.I.) $\mathrm{m} / \mathrm{z}$
$\mathrm{C}_{23} \mathrm{H}_{44} \mathrm{BFN}_{2} \mathrm{Si}$

$406,50 \mathrm{~g} / \mathrm{mol}$

$89^{\circ} \mathrm{C} / 0,01 \mathrm{mbar}$

$88 \%$

$\begin{array}{lll}406 & {[\mathrm{M}]^{+}} & 6 \% \\ 391 & {[\mathrm{M}-\mathrm{Me}]^{+}} & 18 \% \\ 363 & {[\mathrm{M}-\mathrm{CHMe}]^{+}} & 100 \%\end{array}$

Kernmagnetische Resonanz (NMR) $\left(\mathrm{CDCl}_{3}\right)$

\begin{tabular}{|c|c|c|c|c|}
\hline Verschiebung & Zuordnung & Signalform & Kopplungskonst. [Hz] & Integration \\
\hline \multicolumn{5}{|l|}{$\delta^{1} \mathrm{H}$ [ppm] } \\
\hline 0,15 & $\mathrm{Si}\left(\mathrm{CH}_{3}\right)_{3}$ & $d$ & ${ }^{5} \mathrm{~J}_{\mathrm{HF}}=2,2$ & $9 \mathrm{H}$ \\
\hline 0,71 & $2 / 6 \mathrm{C}-\mathrm{CH}\left(\mathrm{CH}_{3}\right)_{2}$ & $d$ & ${ }^{3} \mathrm{~J}_{\mathrm{HH}}=6,7$ & $12 \mathrm{H}$ \\
\hline 1,22 & $\mathrm{a} \mathrm{CH}_{2}-\mathrm{CH}\left(\mathrm{C}_{3}\right)_{2}$ & $d$ & ${ }^{3} \mathrm{~J}_{\mathrm{HH}}=6,9$ & $6 \mathrm{H}$ \\
\hline 1,25 & b CH${ }_{2}-\mathrm{CH}\left(\mathrm{CH}_{3}\right)_{2}$ & $d$ & ${ }^{3} J_{H H}=6,9$ & $6 \mathrm{H}$ \\
\hline 2,43 & $\mathrm{CH}_{2} \mathrm{CHMe}_{2}$ & $d$ & ${ }^{3} J_{H H}=6,1$ & $4 \mathrm{H}$ \\
\hline 2,92 & 2/6 C-CHMe ${ }_{2}$ & sept & ${ }^{3} \mathrm{~J}_{\mathrm{HH}}=6,7$ & $2 \mathrm{H}$ \\
\hline 3,39 & $\mathrm{CH}_{2} \mathrm{CHMe}_{2}$ & $\mathrm{~m}$ & & $2 \mathrm{H}$ \\
\hline $7,06-7,13$ & $3 / 4 / 5 \mathrm{C} \underline{\mathrm{H}}$ & $\mathrm{m}$ & & $3 \mathrm{H}$ \\
\hline \multicolumn{5}{|l|}{$\delta{ }^{13} \mathrm{C}$ [ppm] } \\
\hline 1,75 & $\mathrm{Si}\left(\underline{\mathrm{CH}}_{3}\right)_{3}$ & $d$ & ${ }^{4} \mathrm{~J}_{\mathrm{CF}}=4,5$ & \\
\hline 20,18 & $2 / 6 \mathrm{C}-\mathrm{CH}\left(\mathrm{CH}_{3}\right)_{2}$ & $\mathrm{~s}$ & & \\
\hline 24,28 & a $\mathrm{CH}_{2} \mathrm{CH}\left(\underline{\mathrm{CH}}_{3}\right)_{2}$ & $\mathrm{~s}$ & & \\
\hline 25,09 & b CH${ }_{2} \mathrm{CH}\left(\underline{\mathrm{CH}}_{3}\right)_{2}$ & s & & \\
\hline 27,29 & 2/6 C-ㅡㅡㅂㄹ ${ }_{2}$ & $\mathrm{~s}$ & & \\
\hline 27,81 & $\mathrm{CH}_{2} \underline{\mathrm{C}} \mathrm{HMe}_{2}$ & s & & \\
\hline 52,56 & $\mathrm{CH}_{2} \mathrm{CHMe}_{2}$ & $d$ & ${ }^{3} J_{C F}=4,7$ & \\
\hline 123,16 & $4 \underline{C}$ & $\mathrm{~s}$ & & \\
\hline 125,25 & $3 / 5 \underline{C}$ & $\mathrm{~s}$ & & \\
\hline
\end{tabular}




$\mid \begin{array}{clll}140.75 & 2 / 6 \underline{\mathrm{C}} & \mathrm{d} & \\ 145,87 & 1 \underline{\mathrm{C}} & \mathrm{s} & \\ & & & \\ \delta \mathrm{J}_{\mathrm{CF}}=8,4 \\ { }^{11} \mathrm{~B}[\mathrm{ppm}] & & \mathrm{s} & \\ 21,73 & \underline{\mathrm{B}} & & \\ \delta{ }^{19} \mathrm{~F}[\mathrm{ppm}] & & \mathrm{s} & \\ 46,06 & \underline{\mathrm{F}} & & \\ \delta^{29} \mathrm{Si}[\mathrm{ppm}] & & \mathrm{d} & { }^{3} \mathrm{~J}_{\mathrm{SiF}}=9,5 \\ 8,00 & \underline{S i} & & \end{array}$




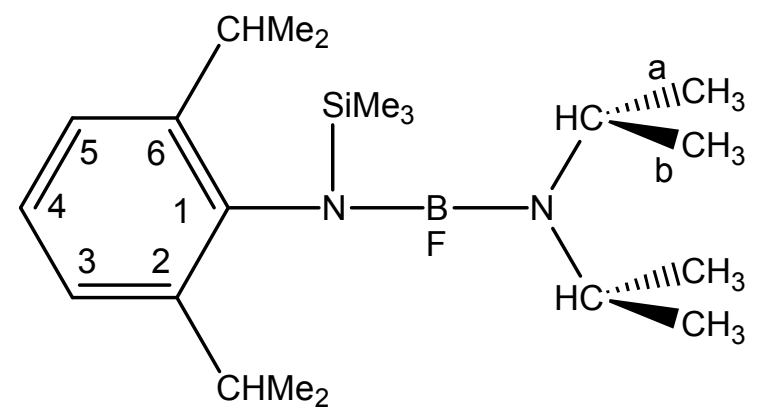

Summenformel:

Molare Masse:

Festpunkt:

Siedepunkt:

Ausbeute:

Massenspektrum:
(E.I.) $\mathrm{m} / \mathrm{z}$
$\mathrm{C}_{21} \mathrm{H}_{40} \mathrm{BFN} \mathrm{Si}$

$378,46 \mathrm{~g} / \mathrm{mol}$

$83^{\circ} \mathrm{C}$

$80^{\circ} \mathrm{C} / 0,01 \mathrm{mbar}$

$95 \%$

$\begin{array}{lll}378 & {[\mathrm{M}]^{+}} & 3 \% \\ 363 & {[\mathrm{M}-\mathrm{Me}]^{+}} & 100 \% \\ 335 & {\left[\mathrm{M}-\mathrm{CHMe}_{2}\right]^{+}} & 90 \%\end{array}$

Kernmagnetische Resonanz (NMR) $\left(C D C l_{3}\right)$

\begin{tabular}{|c|c|c|c|c|}
\hline Verschiebung & Zuordnung & Signalform & Kopplungskonst. [Hz] & Integration \\
\hline \multicolumn{5}{|l|}{$\delta^{1} \mathrm{H}[\mathrm{ppm}]$} \\
\hline 0,10 & $\mathrm{Si}\left(\mathrm{CH}_{3}\right)_{3}$ & $d$ & ${ }^{5} J_{H F}=2,2$ & $9 \mathrm{H}$ \\
\hline 0,97 & $2 / 6 \mathrm{C}-\mathrm{CH}\left(\mathrm{CH}_{3}\right)_{2}$ & $d$ & ${ }^{3} \mathrm{~J}_{\mathrm{HH}}=6,5$ & $12 \mathrm{H}$ \\
\hline 1,18 & a N-CH$\left(\mathrm{CH}_{3}\right)_{2}$ & d & ${ }^{3} \mathrm{~J}_{\mathrm{HH}}=6,8$ & $6 \mathrm{H}$ \\
\hline 1,25 & $\mathrm{~b} \mathrm{~N}-\mathrm{CH}\left(\mathrm{CH}_{3}\right)_{2}$ & d & ${ }^{3} \mathrm{~J}_{\mathrm{HH}}=6,8$ & $6 \mathrm{H}$ \\
\hline 2,94 & 2/6 C-C $\underline{\mathrm{CHMe}} 2$ & sept & ${ }^{3} \mathrm{~J}_{\mathrm{HH}}=6,5$ & $2 \mathrm{H}$ \\
\hline 3,41 & $\mathrm{~N}-\mathrm{C} \underline{\mathrm{HMe}} \mathrm{H}_{2}$ & sept & ${ }^{3} \mathrm{~J}_{\mathrm{HH}}=6,8$ & $2 \mathrm{H}$ \\
\hline $6,8-7,07$ & $3 / 4 / 5 \mathrm{C} \underline{\mathrm{H}}$ & $\mathrm{m}$ & & $3 \mathrm{H}$ \\
\hline \multicolumn{5}{|l|}{$\delta{ }^{13} \mathrm{C}[\mathrm{ppm}]$} \\
\hline 1,75 & $\mathrm{Si}\left(\mathrm{CH}_{3}\right)_{3}$ & s & & \\
\hline 22,44 & $2 / 6 \mathrm{C}-\mathrm{CH}\left(\mathrm{CH}_{3}\right)_{2}$ & s & & \\
\hline 24,19 & a N-CH$\left(\mathrm{CH}_{3}\right)_{2}$ & $\mathrm{~s}$ & & \\
\hline 25,20 & b N-CH$\left(\underline{C H}_{3}\right)_{2}$ & $s$ & & \\
\hline 27,77 & 2/6 C- $\underline{C} \mathrm{HMe}_{2}$ & $\mathrm{~s}$ & & \\
\hline 44,07 & $\mathrm{~N}-\underline{\mathrm{C}} \mathrm{HMe}_{2}$ & d & ${ }^{3} J_{C F}=2,4$ & \\
\hline 123,59 & $4 \underline{C}$ & $s$ & & \\
\hline 124,99 & $3 / 5 \underline{C}$ & $\mathrm{~s}$ & & \\
\hline
\end{tabular}




\begin{tabular}{|c|c|c|c|}
\hline 140,81 & $2 / 6 \underline{\mathrm{C}}$ & $\mathrm{s}$ & \\
\hline 145,74 & $1 \underline{C}$ & $\mathrm{~s}$ & \\
\hline \multicolumn{4}{|c|}{$\delta^{11} \mathrm{~B}$ [ppm] } \\
\hline 22,61 & $\underline{B}$ & $\mathrm{~s}$ & \\
\hline \multicolumn{4}{|c|}{$\delta{ }^{19} \mathrm{~F}[\mathrm{ppm}]$} \\
\hline 53,00 & $\underline{F}$ & $s$ & \\
\hline \multicolumn{4}{|c|}{$\delta^{29} \mathrm{Si}$ [ppm] } \\
\hline 8,30 & $\underline{\mathrm{Si}}$ & $d$ & ${ }^{3} J_{S_{i F}}=9,6$ \\
\hline
\end{tabular}


Di(iso-butyl)ammonium-(N-fluordimethylsilyl-2,6-diisopropylanilino)-methyl-boran-tetrachloroaluminat

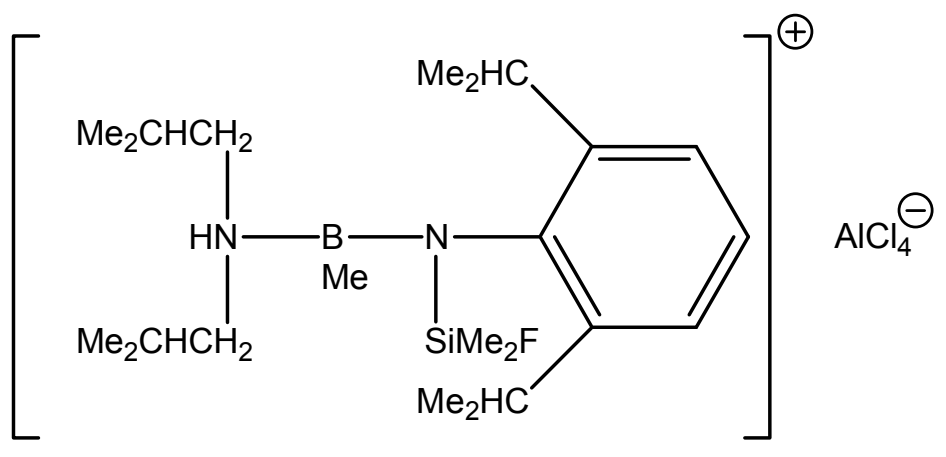

Summenformel:

Molare Masse:

Zersetzungspunkt:

Ausbeute:

Massenspektrum:

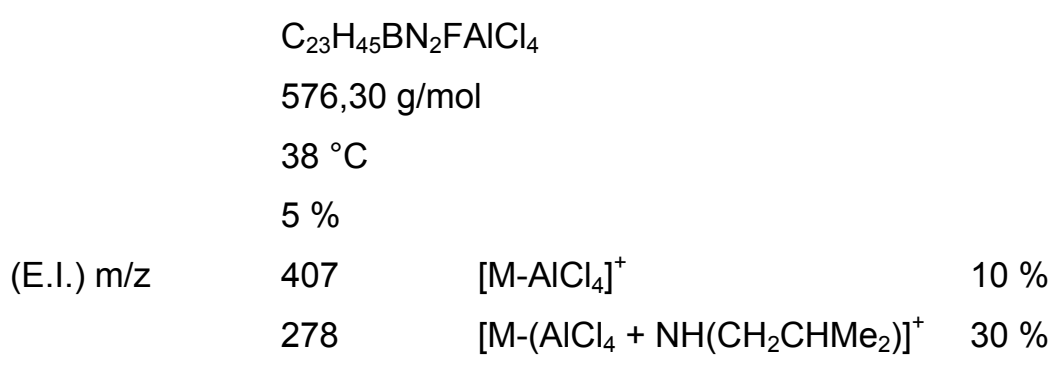

Verbindung 64 wird hier ausschließlich röntgenstrukturanalytisch und massenspektroskopisch beschrieben. 


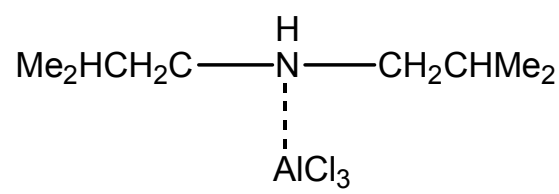

Summenformel:

Molare Masse:

Festpunkt:
$\mathrm{C}_{8} \mathrm{H}_{19} \mathrm{NAICl}_{3}$ $262,58 \mathrm{~g} / \mathrm{mol}$

$77^{\circ} \mathrm{C}$

Kernmagnetische Resonanz (NMR) $\left(\mathrm{CDCl}_{3}\right)$

\begin{tabular}{|c|c|c|c|c|}
\hline Verschiebung & Zuordnung & Signalform & Kopplungskonst. [Hz] & Integration \\
\hline \multicolumn{5}{|l|}{$\delta^{1} \mathrm{H}$ [ppm] } \\
\hline 1,06 & $\mathrm{CH}\left(\mathrm{C}_{3}\right)_{2}$ & $d$ & ${ }^{3} \mathrm{~J}_{\mathrm{HH}}=6,7$ & $12 \mathrm{H}$ \\
\hline 2,19 & $\mathrm{CHMe}_{2}$ & $\mathrm{~m}$ & & $2 \mathrm{H}$ \\
\hline 2,94 & $\mathrm{C}_{2} \mathrm{CHMe}_{2}$ & $\mathrm{~m}$ & & $4 \mathrm{H}$ \\
\hline \multicolumn{5}{|l|}{$\delta{ }^{13} \mathrm{C}[\mathrm{ppm}]$} \\
\hline 20,24 & $\mathrm{CH}\left(\underline{\mathrm{CH}}_{3}\right)_{2}$ & $\mathrm{~s}$ & & \\
\hline 25,63 & $\underline{\mathrm{C}} \mathrm{HMe}_{2}$ & $\mathrm{~s}$ & & \\
\hline 56,04 & $\underline{\mathrm{C}} \mathrm{H}_{2} \mathrm{CHMe}_{2}$ & s & & \\
\hline \multicolumn{5}{|l|}{$\delta{ }^{27} \mathrm{Al}$ [ppm] } \\
\hline 103,41 & $\underline{\mathrm{Al}}$ & s & & \\
\hline
\end{tabular}




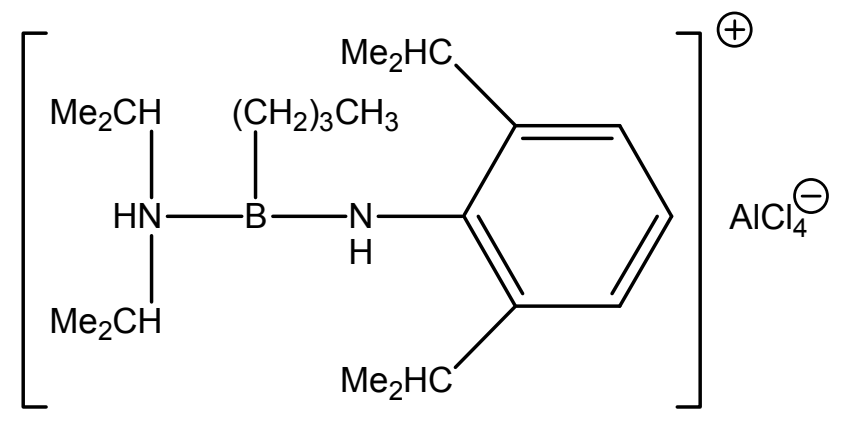

Summenformel:

Molare Masse:

Zersetzungspunkt:

Ausbeute:
$\mathrm{C}_{22} \mathrm{H}_{42} \mathrm{BN}_{2} \mathrm{AlCl}_{4}$

$514,19 \mathrm{~g} / \mathrm{mol}$

$35{ }^{\circ} \mathrm{C}$

$20 \%$

Verbindung 66 wird hier ausschließlich röntgenstrukturanalytisch beschrieben. 
<smiles>CC(C)(C)[Si](Cl)(NC(Cl)(Cl)Cl)C(C)(C)C</smiles>

Summenformel:

Molare Masse:

Festpunkt:

Massenspektrum:

IR-Spektrum:

$$
\begin{aligned}
& \mathrm{C}_{8} \mathrm{H}_{20} \mathrm{NAISiCl}_{4} \\
& 327,13 \mathrm{~g} / \mathrm{mol} \\
& 85^{\circ} \mathrm{C}
\end{aligned}
$$

$\begin{array}{lll}\text { (E.I.) } \mathrm{m} / \mathrm{z} & 325 & {[\mathrm{M}]^{+}} \\ {\left[\mathrm{cm}^{-1}\right]} & \delta_{\mathrm{NH} 2} & 1566,3 \\ & \text { UNH2 S } & 3152,4 \\ & \text { UNH2 AS } & 3219,7\end{array}$

Verbindung 67 wird hier ausschließlich röntgenstrukturanalytisch und massenspektroskopisch beschrieben. 


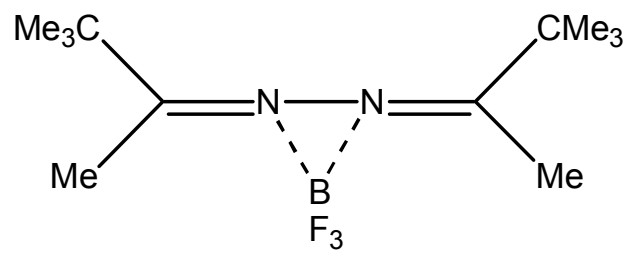

Summenformel:

Molare Masse:

Festpunkt:

Siedepunkt:

Ausbeute:

Massenspektrum:
(E.I.) $\mathrm{m} / \mathrm{z}$
$\mathrm{C}_{12} \mathrm{H}_{24} \mathrm{BN}_{2} \mathrm{~F}_{3}$

$264,14 \mathrm{~g} / \mathrm{mol}$

$70^{\circ} \mathrm{C}$

$61^{\circ} \mathrm{C} / 0,01$ mbar

$97 \%$

$\begin{array}{lll}264 & {[\mathrm{M}]^{+}} & 30 \% \\ 196 & {\left[\mathrm{M}-\mathrm{BF}_{3}\right]^{+}} & 80 \%\end{array}$

Kernmagnetische Resonanz (NMR) $\left(C D C l_{3}\right)$

\begin{tabular}{|c|c|c|c|c|}
\hline Verschiebung & Zuordnung & Signalform & Kopplungskonst. [Hz] & Integration \\
\hline \multicolumn{5}{|l|}{$\delta^{1} H$ [ppm] } \\
\hline 1,32 & $\mathrm{C}-\mathrm{C}\left(\mathrm{CH}_{3}\right)_{3}$ & s & & $18 \mathrm{H}$ \\
\hline 2,26 & $\mathrm{CMe}_{3}-\mathrm{C}-\mathrm{C}_{3}$ & $s$ & & $6 \mathrm{H}$ \\
\hline \multicolumn{5}{|l|}{$\delta^{11} \mathrm{~B}[\mathrm{ppm}]$} \\
\hline$-1,40$ & $\underline{B}$ & s & & \\
\hline \multicolumn{5}{|l|}{$\delta^{13} \mathrm{C}$ [ppm] } \\
\hline 15,87 & $\mathrm{CMe}_{3}-\mathrm{C}-\underline{\mathrm{C}} \mathrm{H}_{3}$ & $s$ & & \\
\hline 27,05 & $\mathrm{C}-\mathrm{C}\left(\underline{\mathrm{C}}_{3}\right)_{3}$ & $s$ & & \\
\hline 40,48 & $\mathrm{C}-\underline{\mathrm{C}} \mathrm{Me}_{3}$ & s & & \\
\hline 189,15 & $\mathrm{~N}=\underline{\mathrm{C}}$ & s & & \\
\hline \multicolumn{5}{|l|}{$\delta^{19} \mathrm{~F}[\mathrm{ppm}]$} \\
\hline 11,82 & ${ }^{10} \mathrm{BF}$ & s & & \\
\hline 11,87 & ${ }^{11} \mathrm{BE}$ & s & & \\
\hline
\end{tabular}


5-tert-Butyl-3,3-difluor-2-(1,1-dimethylbutyliden-2)-1,2,3-azaazoniaborata-5-cyclopenten ${ }^{\text {[76] }}$

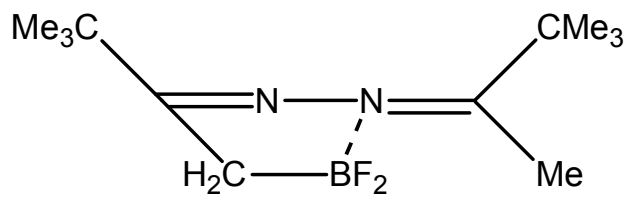

$\begin{array}{ll}\text { Summenformel: } & \mathrm{C}_{12} \mathrm{H}_{23} \mathrm{BN}_{2} \mathrm{~F}_{2} \\ \text { Molare Masse: } & 244,13 \mathrm{~g} / \mathrm{mol} \\ \text { Festpunkt: } & 72{ }^{\circ} \mathrm{C} \\ \text { Ausbeute: } & 94 \%\end{array}$

Auf Grund der bekannten kernresonanz- und massenspektroskopischen Daten wird Verbindung 69 [76] hier ausschließlich röntgenstrukturanalytisch besprochen. 


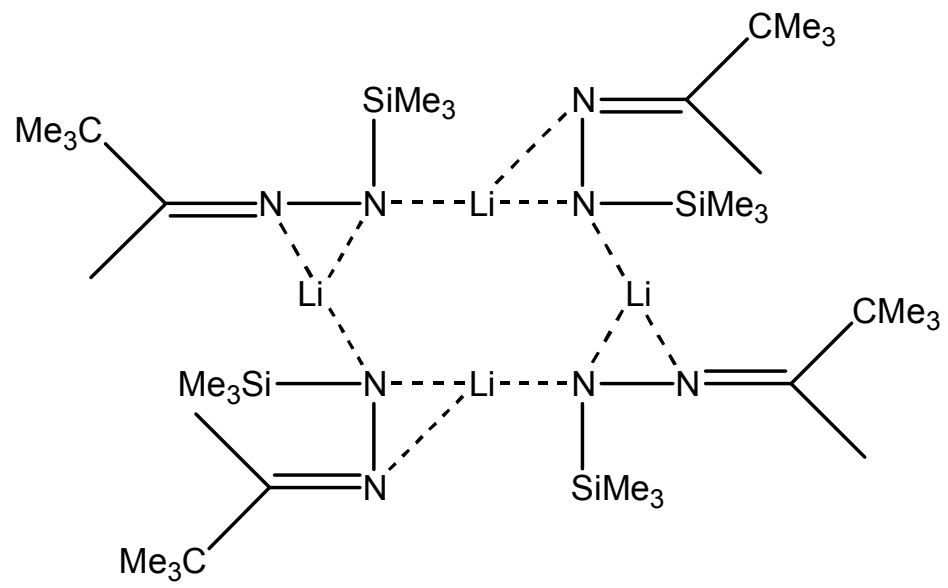

Summenformel:

$\mathrm{C}_{36} \mathrm{H}_{84} \mathrm{Li}_{4} \mathrm{~N}_{8} \mathrm{Si}_{4}$

Molare Masse:

$769,21 \mathrm{~g} / \mathrm{mol}$

Festpunkt:

$132{ }^{\circ} \mathrm{C}$

Ausbeute:

$95 \%$

Kernmagnetische Resonanz (NMR) $\left(C_{6} D_{6}\right)$

\begin{tabular}{|c|c|c|c|c|}
\hline Verschiebung & Zuordnung & Signalform & Kopplungskonst. [Hz] & Integration \\
\hline \multicolumn{5}{|l|}{$\delta^{1} \mathrm{H}[\mathrm{ppm}]$} \\
\hline 0,25 & $\mathrm{~N}-\mathrm{N}-\mathrm{Si}\left(\mathrm{CH}_{3}\right)_{3}$ & s & & $36 \mathrm{H}$ \\
\hline 1,09 & $\mathrm{~N}=\mathrm{C}-\mathrm{C}\left(\mathrm{CH}_{3}\right)_{3}$ & s & & $36 \mathrm{H}$ \\
\hline 1,28 & $\mathrm{~N}=\mathrm{C}-\mathrm{CH}_{3}$ & s & & $12 \mathrm{H}$ \\
\hline \multicolumn{5}{|l|}{$\delta^{7} \mathrm{Li}$ [ppm] } \\
\hline $0,3-0,9$ & $\underline{\mathrm{Li}}$ & $\mathrm{m}$ & & \\
\hline \multicolumn{5}{|l|}{$\delta{ }^{13} \mathrm{C}[\mathrm{ppm}]$} \\
\hline$-0,45$ & $\mathrm{~N}-\mathrm{N}-\mathrm{Si}\left(\underline{\mathrm{CH}}_{3}\right)_{3}$ & $\mathrm{~s}$ & & \\
\hline 8,48 & $\mathrm{~N}=\mathrm{C}-\underline{\mathrm{C}} \mathrm{H}_{3}$ & s & & \\
\hline 28,17 & $\mathrm{~N}=\mathrm{C}-\underline{\mathrm{C}} \mathrm{Me}_{3}$ & $s$ & & \\
\hline 38,09 & $\mathrm{~N}=\mathrm{C}-\mathrm{C}\left(\underline{\mathrm{C}}_{3}\right)_{3}$ & s & & \\
\hline 149,38 & $\mathrm{~N}=\underline{\mathrm{C}}$ & s & & \\
\hline \multicolumn{5}{|l|}{${ }^{29} \mathrm{Si}$ [ppm] } \\
\hline 5,03 & $\underline{\mathrm{Si}}$ & s & & \\
\hline
\end{tabular}




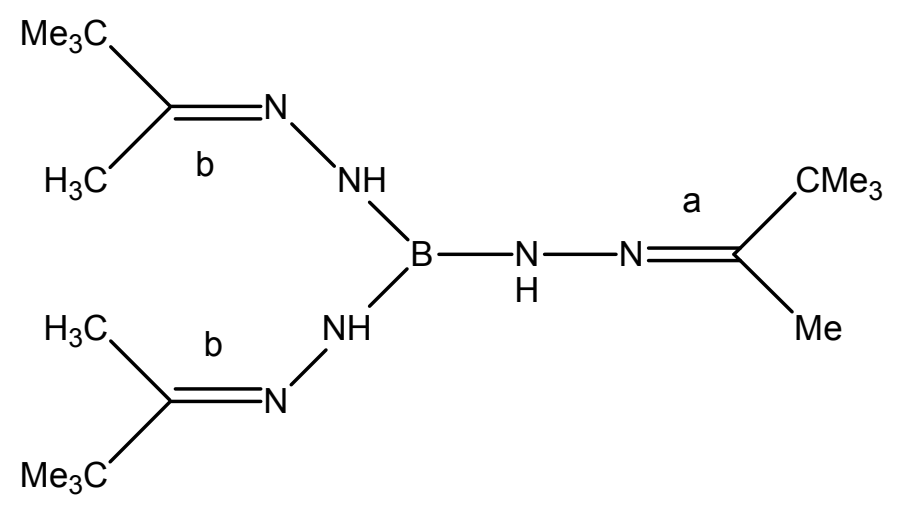

Summenformel:

$\mathrm{C}_{18} \mathrm{H}_{39} \mathrm{BN}_{6}$

Molare Masse:

$350,35 \mathrm{~g} / \mathrm{mol}$

Siedepunkt:

$96{ }^{\circ} \mathrm{C} / 0,01 \mathrm{mbar}$

Ausbeute:

$30 \%$

Massenspektrum:

(E.I.) $\mathrm{m} / \mathrm{z}$

350

$[\mathrm{M}]^{+}$

$15 \%$

293

$[\mathrm{M}-\mathrm{Bu}]^{+}$

$100 \%$

Kernmagnetische Resonanz (NMR) $\left(\mathrm{CDCl}_{3}\right)$

\begin{tabular}{|c|c|c|c|c|}
\hline Verschiebung & Zuordnung & Signalform & Kopplungskonst. [Hz] & Integration \\
\hline \multicolumn{5}{|l|}{$\delta^{1} \mathrm{H}$ [ppm] } \\
\hline 1,07 & a $\mathrm{N}=\mathrm{C}-\mathrm{C}\left(\mathrm{CH}_{3}\right)_{3}$ & s & & $9 \mathrm{H}$ \\
\hline 1,14 & b N=C-C $\left(\mathrm{CH}_{3}\right)_{3}$ & s & & $18 \mathrm{H}$ \\
\hline 1,66 & b N=C-C $\underline{H}_{3}$ & $\mathrm{~s}$ & & $6 \mathrm{H}$ \\
\hline 1,70 & a N=C-C $\underline{H}_{3}$ & $\mathrm{~s}$ & & $3 \mathrm{H}$ \\
\hline 4,82 & $\mathrm{NH}$ & s & & $3 \mathrm{H}$ \\
\hline \multicolumn{5}{|l|}{$\delta^{11} \mathrm{~B}$ [ppm] } \\
\hline 0,37 & $\underline{B}$ & s & & \\
\hline \multicolumn{5}{|l|}{$\delta{ }^{13} \mathrm{C}$ [ppm] } \\
\hline 9,93 & a N=C- $\underline{C} \mathrm{H}_{3}$ & $\mathrm{~s}$ & & \\
\hline 12,16 & b N=C- $\underline{C} \mathrm{H}_{3}$ & s & & \\
\hline 27,70 & a N=C-C( $\left(\mathrm{CH}_{3}\right)_{3}$ & $\mathrm{~s}$ & & \\
\hline 27,80 & b N=C-C( $\left(\mathrm{CH}_{3}\right)_{3}$ & s & & \\
\hline 38,14 & a N=C- $\underline{\mathrm{C}} \mathrm{Me}_{3}$ & $\mathrm{~s}$ & & \\
\hline 38,40 & $\mathrm{~b} N=\mathrm{C}-\underline{\mathrm{C}} \mathrm{Me}_{3}$ & $\mathrm{~s}$ & & \\
\hline 157,82 & a N $=\underline{C}$ & s & & \\
\hline 164,49 & b N $=\underline{C}$ & s & & \\
\hline
\end{tabular}




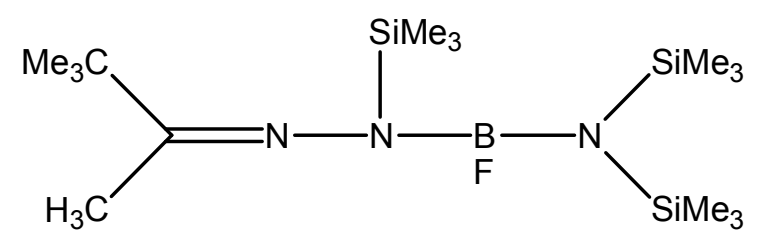

Summenformel:

Molare Masse:

Siedepunkt:

Ausbeute:

Massenspektrum:

(E.I.) $\mathrm{m} / \mathrm{z}$

$\mathrm{C}_{15} \mathrm{H}_{39} \mathrm{BN}_{3} \mathrm{FSi}_{3}$

$375,56 \mathrm{~g} / \mathrm{mol}$

$86^{\circ} \mathrm{C} / 0,01 \mathrm{mbar}$

$95 \%$

$375 \quad[\mathrm{M}]^{+} \quad 65 \%$

$360 \quad[\mathrm{M}-\mathrm{Me}]^{+} \quad 75 \%$

Kernmagnetische Resonanz (NMR) $\left(\mathrm{CDCl}_{3}\right)$

\begin{tabular}{|c|c|c|c|c|}
\hline Verschiebung & Zuordnung & Signalform & Kopplungskonst. [Hz] & Integration \\
\hline \multicolumn{5}{|l|}{$\delta^{1} \mathrm{H}[\mathrm{ppm}]$} \\
\hline 0,11 & $\mathrm{~N}-\mathrm{N}-\mathrm{Si}\left(\mathrm{CH}_{3}\right)_{3}$ & $d$ & ${ }^{5} \mathrm{~J}_{\mathrm{HF}}=1,8$ & $9 \mathrm{H}$ \\
\hline 0,14 & $\mathrm{~N}-\left[\mathrm{Si}\left(\mathrm{C}_{3}\right)_{3}\right]_{2}$ & $d$ & 5 & $18 \mathrm{H}$ \\
\hline 1,15 & $\mathrm{~N}=\mathrm{C}-\mathrm{C}\left(\mathrm{C}_{\mathrm{H}_{3}}\right)_{3}$ & $\mathrm{~s}$ & & $9 \mathrm{H}$ \\
\hline 1,77 & $\mathrm{~N}=\mathrm{C}-\mathrm{CH}_{3}$ & $\mathrm{~s}$ & & $3 \mathrm{H}$ \\
\hline \multicolumn{5}{|l|}{$\delta^{11} \mathrm{~B}$ [ppm] } \\
\hline 24,09 & $\underline{B}$ & $s$ & & \\
\hline \multicolumn{5}{|l|}{$\delta{ }^{13} \mathrm{C}[\mathrm{ppm}]$} \\
\hline 0,34 & $\mathrm{~N}-\mathrm{N}-\mathrm{Si}\left(\mathrm{CH}_{3}\right)_{3}$ & $d$ & ${ }^{4} J_{C F}=3,3$ & \\
\hline 3,12 & $\mathrm{~N}-\left[\mathrm{Si}\left(\underline{\mathrm{C}}_{3}\right)_{3}\right]_{2}$ & $d$ & ${ }^{4} J_{C F}=1,1$ & \\
\hline 13,72 & $\mathrm{~N}=\mathrm{C}-\underline{\mathrm{C}} \mathrm{H}_{3}$ & $d$ & ${ }^{5} \mathrm{~J}_{\mathrm{CF}}=0,8$ & \\
\hline 27,83 & $\mathrm{~N}=\mathrm{C}-\mathrm{C}\left(\underline{\mathrm{CH}}_{3}\right)_{3}$ & $d$ & ${ }^{6} J_{C F}=3,3$ & \\
\hline 39,55 & $\mathrm{~N}=\mathrm{C}-\underline{\mathrm{C}} \mathrm{Me}_{3}$ & $d$ & ${ }^{5} J_{C F}=1,4$ & \\
\hline 177,93 & $\mathrm{~N}=\underline{\mathrm{C}}$ & $\mathrm{s}$ & & \\
\hline \multicolumn{5}{|l|}{$\delta{ }^{19} \mathrm{~F}[\mathrm{ppm}]$} \\
\hline 66,24 & $\underline{F}$ & s & & \\
\hline \multicolumn{5}{|l|}{$\delta^{29} \mathrm{Si}$ [ppm] } \\
\hline 2,62 & $\mathrm{~N}-(\underline{\mathrm{SiMe}})_{3}$ & $d$ & ${ }^{3} J_{\mathrm{SiF}}=3,9$ & \\
\hline 8,51 & $\mathrm{~N}-\mathrm{N}-\mathrm{SiMe}_{3}$ & $d$ & ${ }^{3} \mathrm{~J}_{\mathrm{SiF}}=1,0$ & \\
\hline
\end{tabular}




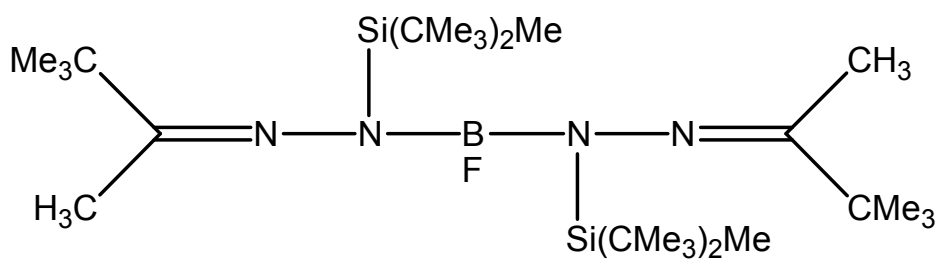

Summenformel:

Molare Masse:

Festpunkt:

Ausbeute:
$\mathrm{C}_{30} \mathrm{H}_{66} \mathrm{BN}_{4} \mathrm{FSi}_{2}$

$568,85 \mathrm{~g} / \mathrm{mol}$

$183^{\circ} \mathrm{C}$

$95 \%$

Auf Grund bekannter Kernresonanz- und Massenspektroskopischer Daten ${ }^{[77]}$ wird Verbindung 73 hier ausschließlich röntgenstrukturanalytisch besprochen. 
Fluor-diisopropylamino-N,2,6-triisopropylanilino-boran

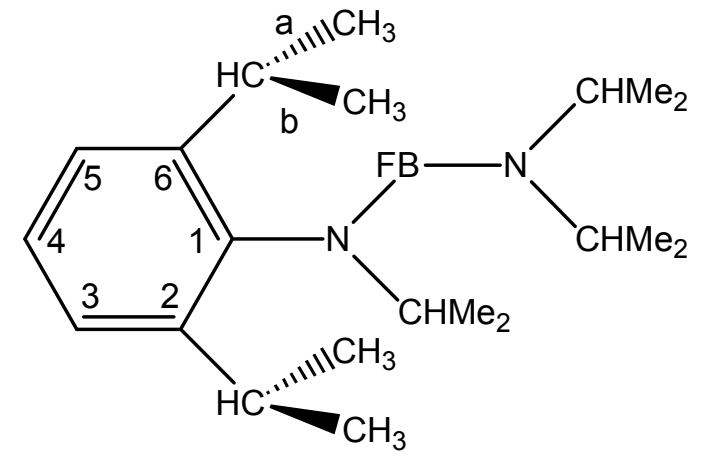

Summenformel:

$\mathrm{C}_{21} \mathrm{H}_{38} \mathrm{BN}_{2} \mathrm{~F}$

Molare Masse:

$348,35 \mathrm{~g} / \mathrm{mol}$

Siedepunkt:

$110^{\circ} \mathrm{C} / 0,01 \mathrm{mbar}$

Ausbeute:

$97 \%$

Massenspektrum:

(E.I.) $\mathrm{m} / \mathrm{z}$

348

$[\mathrm{M}]^{+}$

$15 \%$

333

$[\mathrm{M}-\mathrm{Me}]^{+}$

$100 \%$

Kernmagnetische Resonanz (NMR) $\left(\mathrm{CDCl}_{3}\right)$

\begin{tabular}{|c|c|c|c|c|}
\hline Verschiebung & Zuordnung & Signalform & Kopplungskonst. [Hz] & Integration \\
\hline \multicolumn{5}{|l|}{$\delta^{1} \mathrm{H}$ [ppm] } \\
\hline 0,92 & $\mathrm{~N}-\left[\mathrm{CH}\left(\mathrm{CH}_{3}\right)_{2}\right]_{2}$ & $d$ & ${ }^{3} \mathrm{~J}_{\mathrm{HH}}=6,7$ & $12 \mathrm{H}$ \\
\hline 1,19 & a $\mathrm{CH}\left(\mathrm{CH}_{3}\right)_{2}$ & $d$ & ${ }^{3} \mathrm{~J}_{\mathrm{HH}}=6,8$ & $6 \mathrm{H}$ \\
\hline 1,22 & b $\mathrm{CH}\left(\mathrm{C}_{3}\right)_{2}$ & $d$ & ${ }^{3} J_{H H}=6,8$ & $6 \mathrm{H}$ \\
\hline 1,29 & $\mathrm{C}_{12} \mathrm{H}_{17}-\mathrm{N}-\mathrm{CH}\left(\mathrm{C}_{3}\right)_{2}$ & dd & $\begin{array}{l}{ }^{3} \mathrm{~J}_{\mathrm{HH}}=6,7 \\
{ }^{3} \mathrm{~J}_{\mathrm{HF}}=1,9\end{array}$ & $6 \mathrm{H}$ \\
\hline 3,00 & $\mathrm{~N}-\left(\mathrm{CH}_{\mathrm{H}} \mathrm{Me}_{2}\right)_{2}$ & sept & ${ }^{3} \mathrm{~J}_{\mathrm{HH}}=6,7$ & $2 \mathrm{H}$ \\
\hline 3,02 & $\mathrm{C}_{12} \mathrm{H}_{17}-\mathrm{N}-\mathrm{C} \underline{\mathrm{H} M \mathrm{Me}_{2}}$ & sept & ${ }^{3} \mathrm{~J}_{\mathrm{HH}}=6,7$ & $1 \mathrm{H}$ \\
\hline 3,30 & $2 / 6 \mathrm{C}-\mathrm{CHMe}_{2}$ & sept & ${ }^{3} \mathrm{~J}_{\mathrm{HH}}=6,8$ & $2 \mathrm{H}$ \\
\hline $7,01-7,15$ & $3 / 4 / 5 \mathrm{C} \underline{\mathrm{H}}$ & $\mathrm{m}$ & & $3 \mathrm{H}$ \\
\hline \multicolumn{5}{|l|}{$\delta{ }^{11} B$ [ppm] } \\
\hline 23,39 & $\underline{B}$ & $\mathrm{~s}$ & & \\
\hline \multicolumn{5}{|l|}{$\delta{ }^{13} \mathrm{C}$ [ppm] } \\
\hline 22,56 & $\mathrm{C}_{12} \mathrm{H}_{17}-\mathrm{N}-\mathrm{CH}\left(\underline{\mathrm{CH}}_{3}\right)_{2}$ & d & ${ }^{3} J_{C F}=4,3$ & \\
\hline 22,87 & $\mathrm{~N}-\left[\mathrm{CH}\left(\underline{\mathrm{CH}}_{3}\right)_{2}\right]_{2}$ & $\mathrm{~s}$ & & \\
\hline 23,46 & a C-CH( $\left(\mathrm{CH}_{3}\right)_{2}$ & $\mathrm{~s}$ & & \\
\hline 24,64 & b C-CH( $\left(\mathrm{CH}_{3}\right)_{2}$ & $s$ & & \\
\hline 27,78 & 2/6 C- $\underline{\mathrm{C}} \mathrm{HMe}_{2}$ & $\mathrm{~s}$ & & \\
\hline 44,35 & $\mathrm{~N}-\left(\underline{\mathrm{CHMe}} \mathrm{HM}_{2}\right)_{2}$ & $\mathrm{~s}$ & & \\
\hline 55,29 & $\mathrm{C}_{12} \mathrm{H}_{17}-\mathrm{N}-\underline{\mathrm{C}} \mathrm{HMe}_{2}$ & $\mathrm{~s}$ & & \\
\hline 124,26 & $3 / 5 \underline{C}$ & $s$ & & \\
\hline 125,54 & $4 \underline{C}^{-}$ & $s$ & & \\
\hline 142,14 & $2 / 6 \underline{C}$ & $s$ & & \\
\hline 146,94 & $1 \underline{C}$ & $d$ & ${ }^{3} J_{C F}=1,9$ & \\
\hline \multicolumn{5}{|l|}{$\delta{ }^{19} \mathrm{~F}$ [ppm] } \\
\hline 48,86 & $\underline{F}$ & $s$ & & \\
\hline
\end{tabular}


Bis[2,4,6-tri(tert-butyl)-1-oxi-phenyl]-fluor-boran

75

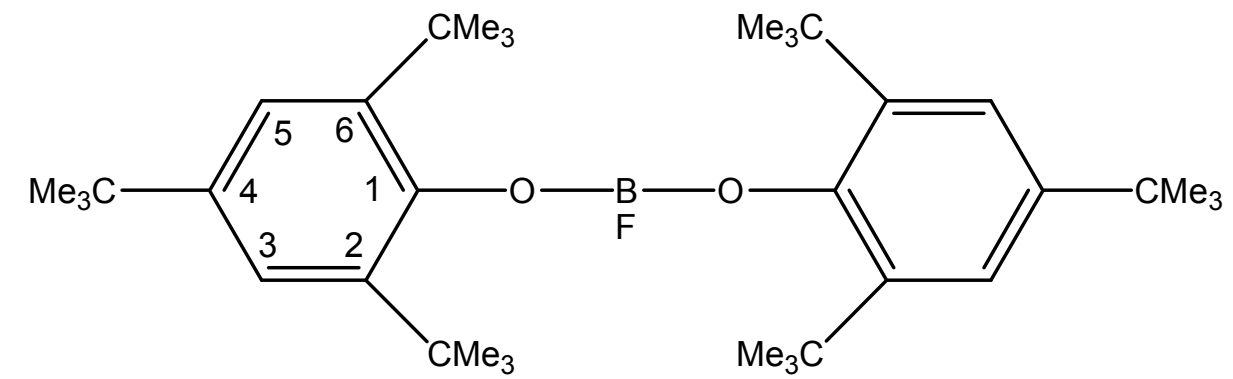

Summenformel:

$\mathrm{C}_{36} \mathrm{H}_{58} \mathrm{BO}_{2} \mathrm{~F}$

Molare Masse:

$552,65 \mathrm{~g} / \mathrm{mol}$

SFestpunkt:

$169{ }^{\circ} \mathrm{C} / 0,01 \mathrm{mbar}$

Ausbeute:

$80 \%$

$\begin{array}{ll}\text { Massenspektrum: } \quad \text { (E.I.) } \mathrm{m} / \mathrm{z} & \end{array}$

552

[M]

$100 \%$

537

$[\mathrm{M}-\mathrm{Me}]^{+}$

$40 \%$

Kernmagnetische Resonanz (NMR) $\left(\mathrm{CDCl}_{3}\right)$

\begin{tabular}{|c|c|c|c|c|}
\hline Verschiebung & Zuordnung & Signalform & Kopplungskonst. [Hz] & Integration \\
\hline \multicolumn{5}{|l|}{$\delta^{1} \mathrm{H}$ [ppm] } \\
\hline 1,30 & $4 \mathrm{C}-\mathrm{C}\left(\mathrm{CH}_{3}\right)_{3}$ & s & & $18 \mathrm{H}$ \\
\hline 1,43 & $2 / 6 \mathrm{C}-\mathrm{C}\left(\mathrm{CH}_{3}\right)_{3}$ & s & & $36 \mathrm{H}$ \\
\hline 7,25 & $3 / 5 \mathrm{C} \underline{\mathrm{H}}$ & s & & $4 \mathrm{H}$ \\
\hline \multicolumn{5}{|l|}{$\delta{ }^{11} B$ [ppm] } \\
\hline 13,63 & $\underline{B}$ & & & \\
\hline \multicolumn{5}{|l|}{$\delta{ }^{13} \mathrm{C}$ [ppm] } \\
\hline 30,43 & $2 / 6 \mathrm{C}-\mathrm{C}\left(\underline{\mathrm{C}}_{3}\right)_{3}$ & $s$ & & \\
\hline 31,53 & $4 \mathrm{C}-\mathrm{C}\left(\underline{\mathrm{C}} \mathrm{H}_{3}\right)_{3}$ & $\mathrm{~s}$ & & \\
\hline 34,52 & $2 / 6 \mathrm{C}-\underline{\mathrm{C}} \mathrm{Me}_{3}$ & s & & \\
\hline 35,94 & $4 \mathrm{C}-\underline{\mathrm{C}} \mathrm{Me}_{3}$ & s & & \\
\hline 123,13 & $3 / 5 \underline{C}$ & $s$ & & \\
\hline 140,10 & $2 / 6 \underline{C}$ & $\mathrm{~s}$ & & \\
\hline 144,66 & $4 \underline{C}$ & s & & \\
\hline 147,78 & $1 \underline{C}$ & $\mathrm{~s}$ & & \\
\hline \multicolumn{5}{|l|}{$\delta{ }^{19} \mathrm{~F}$ [ppm] } \\
\hline 55,17 & $\underline{F}$ & $\mathrm{~s}$ & & \\
\hline
\end{tabular}




\section{3 Übersicht über die neuen Verbindungen}

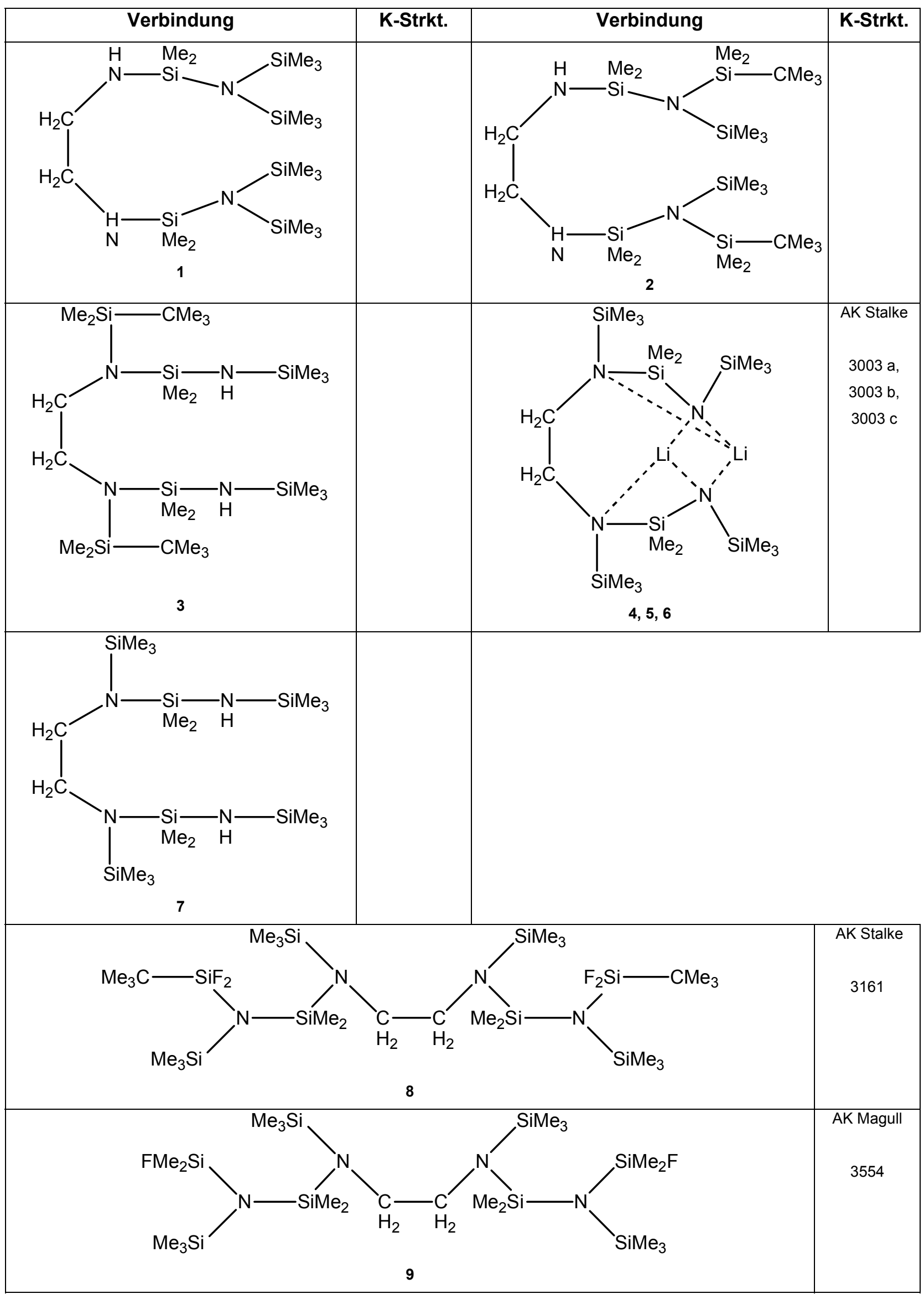




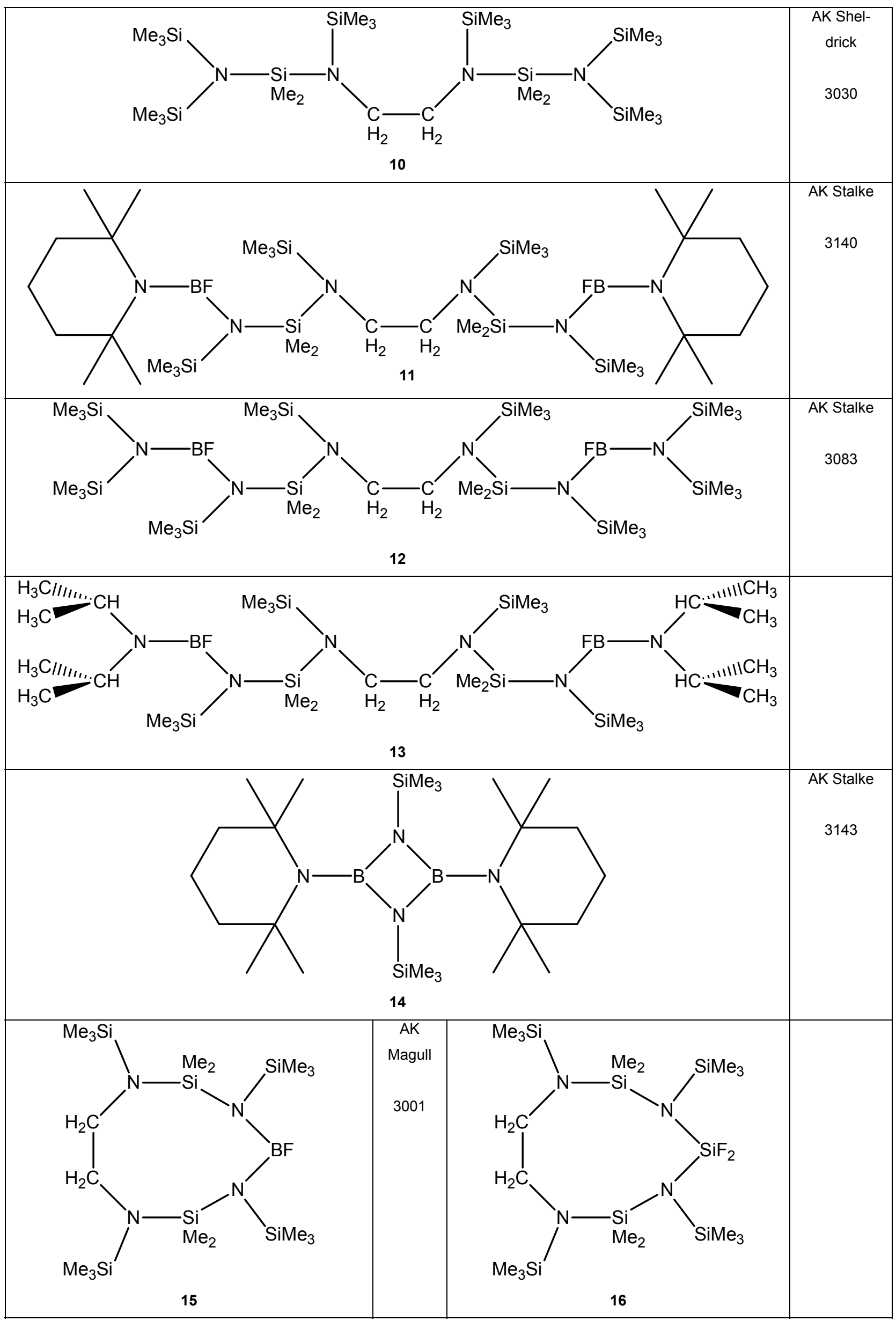




(1)




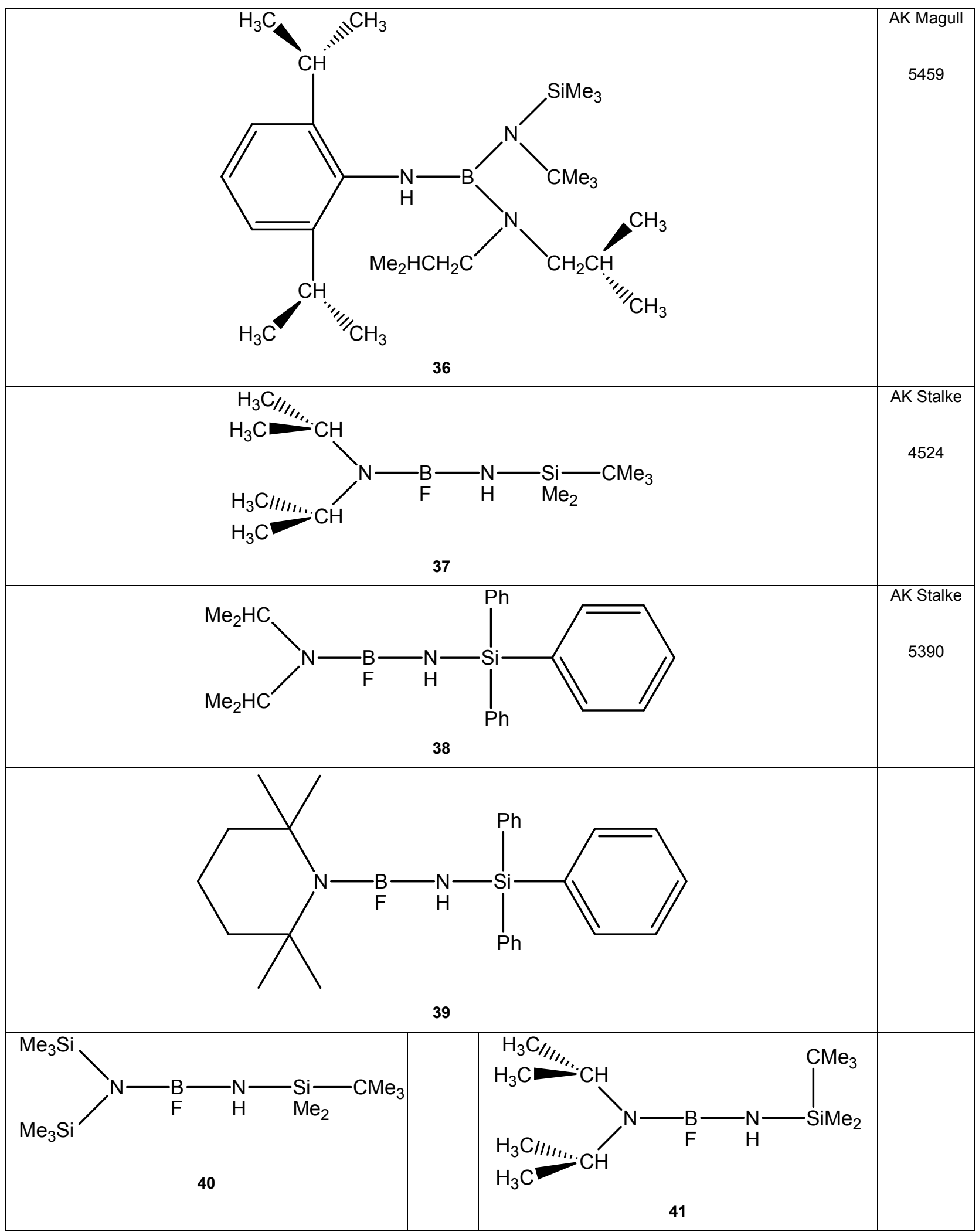




(2)




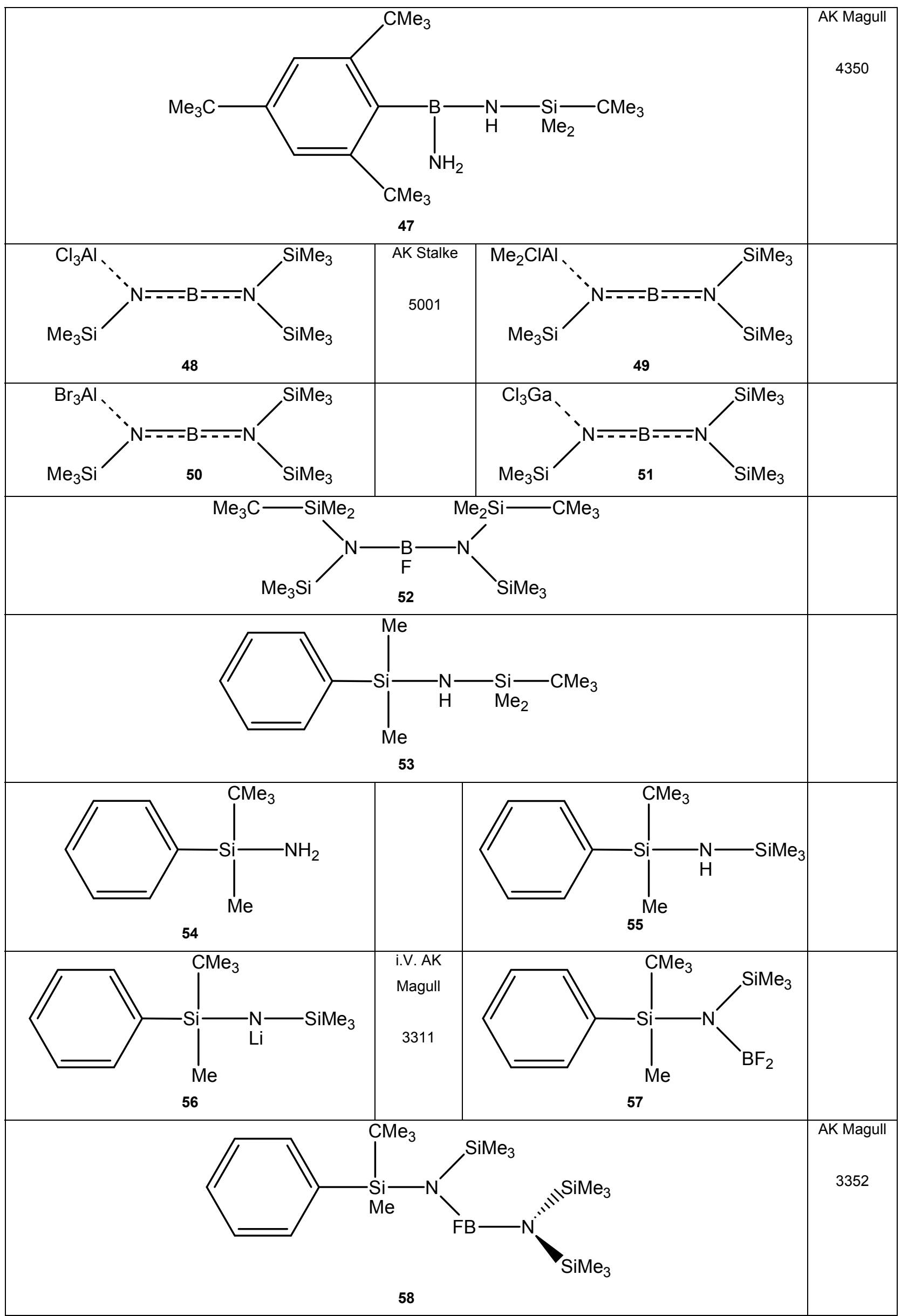




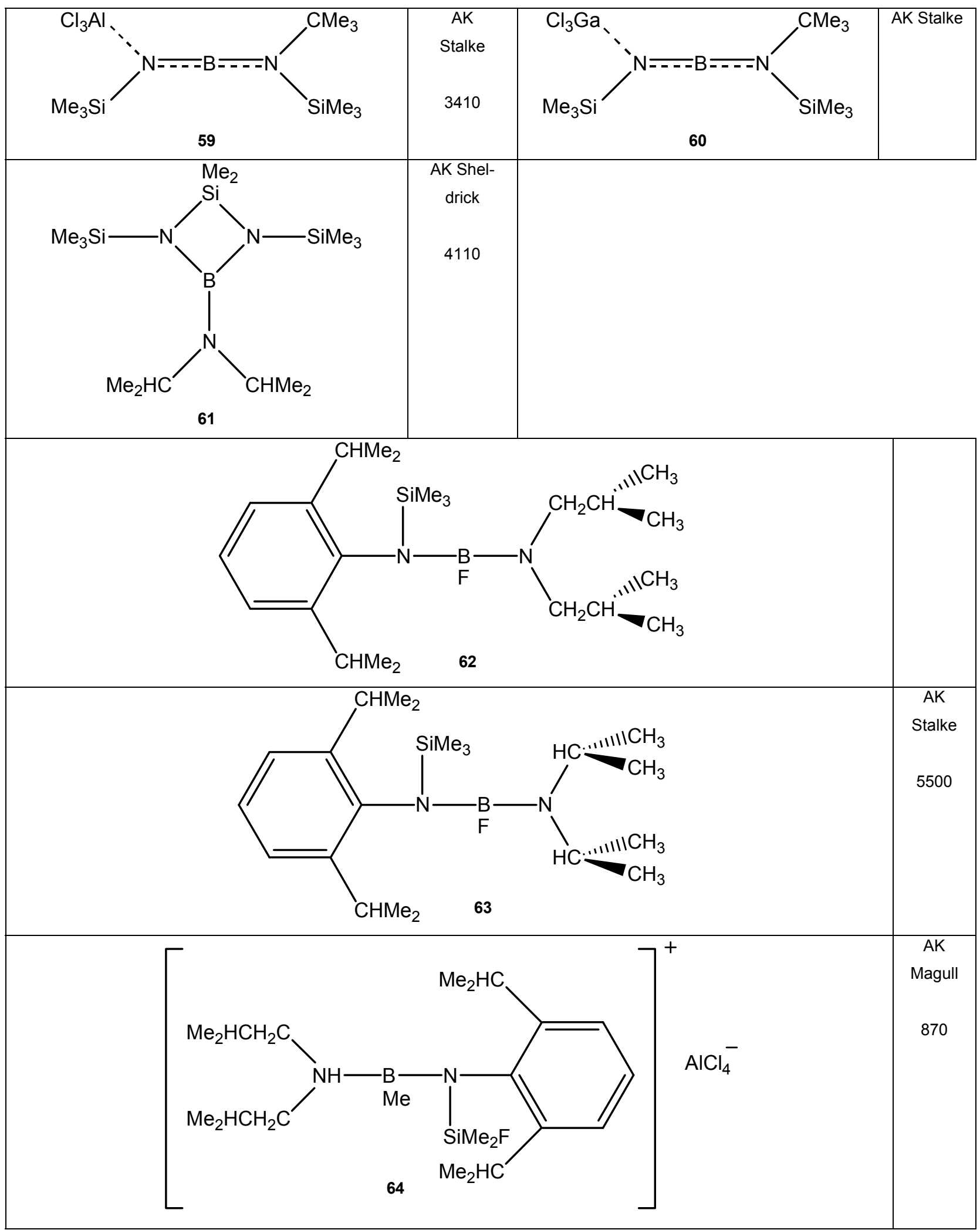




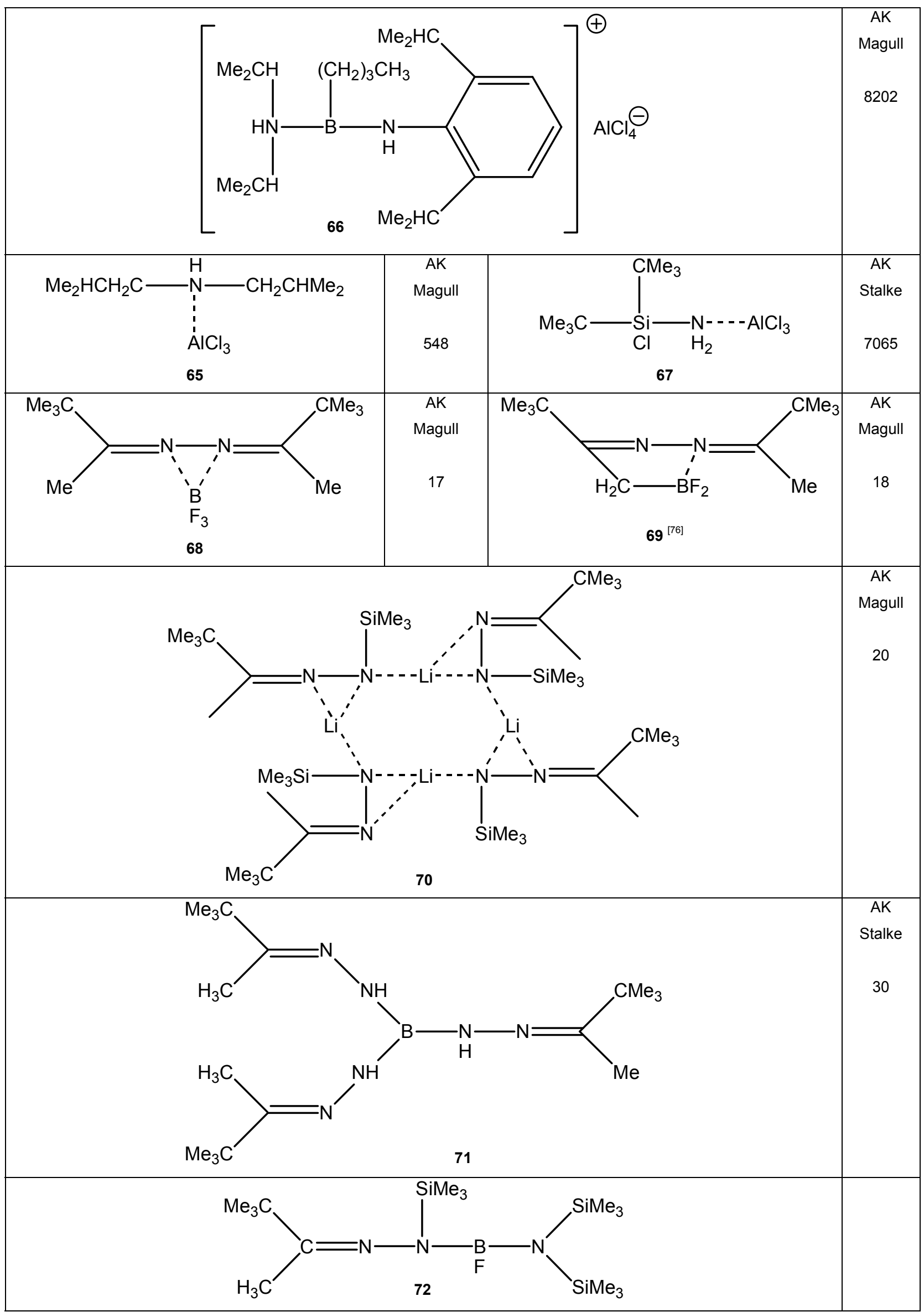




(n)




\section{Literaturverzeichnis}

[1] W.G. Mixter, Am. Chem. J. 2, (1881)153.

[2] E. Frankland, Ann. Chem. 124, (1862) 129.

[3] C.A. Kraus, E.H. Brown, J. Am. Chem. Soc. 51, (1929) 2690.

[4] A. Stock, E. Pohland, Ber. Dt. Chem. Ges. 59, (1926) 2215.

[5] E.L. Mutterties, The Chemistry of Boron and its Compounds, John Wiley \& Sons, New York (1967).

[6] V.I. Matkovich (Hrsg.), Boron and Refractory Borides, Springer-Verlag, Berlin, Heidelberg, New York (1977).

[7] W.N. Lipscomb, Boron Hydrides, Benjamin, New York (1963).

[8] K. Wade, Electron Deficient Compounds, Nelson \& Sons, London (1971).

[9] K. Kawaguchi, Y. Endo, E. Hirota, J. Mol. Spectrosc. 93, (1982) 38.

[10] E.F. Pearson, R.V. McCormick, J. Chem. Phys. 58, (1973) 1619.

[11] C. Kirby, H.W. Kroto, J. Mol. Spectrosc. 83, (1980) 130.

[12] J. Henkelmann, G. Maier, H.P. Reisenauer, Angew. Chem. 97, (1985) 1061.

[13] P. Paetzold, Adv. Inorg. Chem. 31, (1987) 123.

[14] M. Haase, U. Klingebiel, Chem. Ber. 119, (1986) 1117.

[15] H. Nöth, Angew. Chem. 12, (1988) 1664.

[16] H. Nöth, S. Weber, Chem. Ber. 118, (1985) 2554.

[17] H. Nöth, S. Weber, Z. Naturforsch. 38 b, (1983) 1460.

[18] W. Dilthey, F. Eduardoff, F.J. Schumacher, Liebigs Ann. Chem. 344, (1906) 300.

[19] R.W. Parry, S.G. Shore, J. Am. Chem. Soc. 80, (1958) 4.

[20] H. Nöth, Nachr. Chem. Tech. Lab. 32, (1984) 956.

[21] J. Davis, Philos. Trans. 1, (1812) 352.

[22] A. Ladenburg, Ann. Chem. Pharm. 164, (1872) 300.

[23] U. Wannagat, Adv. Inorg. Radiochem. 6, (1964) 225.

[24] M. F. Lappert, P. P. Power, A. R. Sanger, R. C. Srivastava, Metal and Metalloid Amides, John Wiley \& Sons, Chichester (1980).

[25] S. D. Brewer, C. P. Haber, J. Am. Chem. Soc. 70, (1948) 3888.

[26] W. Fink, Angew. Chem. 73, (1961) 736.

[27] W. Fink, Chem. Ber. 96, (1963) 1071. 
[28] J. Haiduc, D. B. Sowerby, The Chemistry of Inorganic Homo- and Heterocycles, Academic Press, London (1987).

[29] U. Klingebiel, S. Schmatz, E. Gellermann, C. Drost, M. Noltemeyer, Monatsh. f. Chem. 132, (2001) 1105.

[30] U. Wannagat, W. Liehr, Angew. Chem. 69, (1957) 783.

[31] U. Wannagat, W. Liehr, Z. Anorg. Allg. Chem. 297, (1958) 129.

[32] U. Wannagat, W. Liehr, Z. Anorg. Allg. Chem. 299, (1959) 341.

[33] U. Wannagat, H. Niederprüm, Angew. Chem. 71, (1959) 574.

[34] U. Wannagat, H. Niederprüm, Z. Anorg. Allg. Chem. 310, (1961) 32.

[35] K. Bode, U. Klingebiel, Adv. Organomet. Chem. 40, (1996) 1.

[36] C. Drost, U. Klingebiel, Chem. Ber. 126, (1993) 1413.

[37] K. Bode, U. Klingebiel, H. Witte-Abel, M.W. Gluth, M. Noltemeyer, R. HerbstIrmer, M. Schäfer, Phosphorus, Sulfur, and Silicon. 108, (1996) 121.

[38] R. West, Adv. Organomet. Chem. 16, (1977) 1.

[39] R. West, Pure Appl. Chem. 19, (1969) 291.

[40] C. Matthes, M. Noltemeyer, U. Klingebiel, S. Schmatz, Organometallics 26, (2007) 838.

[41] D. Grosskopf, L. Markus, U. Klingebiel, M. Noltemeyer, Phosphorus, Sulfur, and Silicon. 97, (1994) 113.

[42] D. Stalke, N. Keweloh, U. Klingebiel, M. Noltemeyer, G.M. Sheldrick, Z. Naturforsch. 42b, (1987) 1237.

[43] D. Stalke, U. Klingebiel, S. Vollbrecht, Z. Naturforsch. 47b, (1992) 27.

[44] D. Stalke, U. Klingebiel, G.M. Sheldrick, J. Organomet. Chem. 344, (1988) 37.

[45] H. Nöth, H. Sachdev, M. Schmidt, H. Schwenk, Chem. Ber. 128, (1995) 105.

[46] M. Metzler, H. Nöth, H. Sachdev, Angew. Chem. 106, (1994) 1837.

[47] I. Hemme, U. Klingebiel, Adv. Organomet. Chem. 39, (1996), 159.

[48] U. Klingebiel, J. Niesmann, Phosphorus, Sulfur, and Silicon 124 \& 125, (1997) 113.

[49] U. Klingebiel, M. Noltemeyer, H-G Schmidt, D. Schmidt-Bäse, Chem. Ber. 130, (1997) 753.

[50] W. Clegg, U. Klingebiel, J. Neemann, G.M. Sheldrick, J. Organomet. Chem. $\underline{249},(1983) 47$.

[51] N. Helmold, U. Klingebiel, S. Schmatz, Adv. Organomet. Chem. 54, (2006) 1. 
[52] S. Schaible, R. Riedel, R. Boese, E. Werner, U. Klingebiel, M. Nieger, Appl. Organomet. Chem. 8, (1994) 1.

[53] U. Klingebiel, L. Skoda, Z. Naturforsch. 40b, (1985) 913.

[54] Ber. Dt. Keram. Ges. 44, (1967) 209.

[55] E. Werner, U. Klingebiel, Z. Anorg. Allg. Chem. 620, (1994) 1093.

[56] A.F. Holleman, N. Wiberg, Lehrbuch der Anorganischen Chemie, de Gruyter, Berlin, (1994).

[57] H. Lange, G. Wötting, G. Winter, Angew. Chem. 103, (1991) 1606.

[58] B. Jaschke, U. Klingebiel, R. Riedel, N. Doslik, R. Gadow, Appl. Organomet. Chem. 14, (2000) 671.

[59] K.J. Wynne, R.W. Rice, Ann. Rev. Mater. Sci. 14, (1984) 297.

[60] T. Ballweg, H. Wolter, W. Storch, Keram. Z. 53, (2001) 678.

[61] S. Schaible, R. Riedel, E. Werner, U. Klingebiel, Appl. Organomet. Chem. 7, (1993) 53.

[62] D.J. Brauer, H. Bürger, W. Geschwandtner, G.R. Liewald, J. Organomet. Chem. 248, (1983) 1.

[63] U. Pieper, S. Walter, U. Klingebiel, D. Stalke, Angew. Chem. 102, (1990) 218.

[64] M. Jendras, U. Klingebiel, M. Noltemeyer, Z. Naturforsch. 58, B: Chem. Sciences, (2003) 246.

[65] B. Jaschke, R. Herbst-Irmer, U. Klingebiel, P. Neugebauer, T. Pape, Z. Anorg. Allg. Chem. 625, (1999) 1035.

[66] J.P. Schneider, E. Popowski, K. Junge, H. Reinke, Z. Anorg. Allg. Chem. 627, (2001) 2680.

[67] H.W. Lerner, N. Wiberg, J.W. Bats, J. Organomet. Chem. 690, (2005) 3898.

[68] W. Luthin, J.G. Stratmann, G. Elter, A. Meller, A. Heine, H. Gornitzka, Z. Anorg. Allg. Chem. 621, (1995) 1995.

[69] H. Nöth, persönliche Mitteilung vom 11.10.2007.

[70] P. Kölle, H. Nöth, Chem. Ber. 119, (1986) 3849.

[71] P. Geymayer, E.G. Rochow, U. Wannagat, Angew. Chem. 11, (1964) 499.

[72] C. R. Russ, A. G. MacDiarmid, Angew. Chem. 11, (1964) 500.

[73] H. Nöth, S. Weber, B. Rasthofer, C.K. Navula, A. Konstantinov, Pure and Appl. Chem. 55, (1983) 1453.

[74] M. J. Frisch et al., Gaussian 03, Revision C.02, Gaussian Inc., Wallingford CT (2004). 
[75] C. Matthes, M. Görth, U. Klingebiel, A. Ringe, J. Magull, Z. Anorg. Allg. Chem., in Vorbereitung.

[76] T. Groh, Dissertation, Göttingen: Georg-August-Universität (2000).

[77] C. Drost, Dissertation, Göttingen: Georg-August-Universität (1992).

[78] M. Mänz, Diplomarbeit, Göttingen: Georg-August-Universität (2005).

[79] D. Stalke, Chem. Soc. Rev. 27, (1998) 171; T. Kottke, R. J. Lagow, D. Stalke, J. Appl. Crystallogr. 29, (1996) 465.

[80] H. Hope, Acta Crystallogr. B44, (1988) 22.

[81] Bruker APEX v2.1-0, Bruker AXS Inst. Inc., Madison (WI, USA), (2007).

[82] SAINT v7.34A in Bruker APEX v2.1-0, Bruker AXS Inst. Inc., Madison (WI, USA), (2005).

[83] G.M. Sheldrick, SADABS 2004/1, Göttingen, (2004).

[84] X-AREA, Stoe \& Cie GmbH, Darmstadt, (2002).

[85] X-RED32, Stoe \& Cie GmbH, Darmstadt, (2002).

[86] Bruker PROTEUM v1.40, Bruker AXS Inst. Inc., Madison (WI, USA), (2002).

[87] G.M. Sheldrick, XPREP in SHELXTL v6.12, Bruker AXS Inst. Inc., Madison (WI, USA), (2000).

[88] G.M. Sheldrick, SHELXS in SHELXTL v6.12, Bruker AXS Inst. Inc., Madison (WI, USA), (2000).

[89] G.M. Sheldrick, SHELXL in SHELXTL v6.12, Bruker AXS Inst. Inc., Madison (WI, USA), (2000).

[90] XSHELL v4.01 in SHELXTL v6.12, B. A. I. Inc., Madison (WI, USA), (2000).

Quellen aus dem world wide web:

[a] http://www.diss.fu-berlin.de/2001/27/kap2.pdf, S. 19, Stand 24.11.2007.

[b] http://www.dfg.de, Stand 24.11.2007.

\section{Publikationen}

C. Matthes, M. Noltemeyer, U. Klingebiel, S. Schmatz, Intramolecular Rearrangement of Organosilyl Groups in Silylamines: A Combined ExperimentalTheoretical Study, Organometallics 26, (2007) 838.

U. Klingebiel, C. Matthes, From Lithiumhalosilylamides to small and large Ring compounds, Iminosilenes and Iminosilanes, J. Organomet. Chem. 692, (2007) 2633. 
C. Matthes, U. Klingebiel, S. Deuerlein, B. Heisen, H. Ott, D. Stalke, A. Ringe, J. Magull, $\quad N, N^{\prime}$-Bis(1,3-disila-2-amino-) und $\quad N, N^{\prime}-B i s(1,3-d i s i l a-2-a m i n o(-2-$ amido)ethylendiamine, Sila- und Boracyclononane, Z. Anorg. Allg. Chem., in Vorbereitung.

C.Matthes, U. Klingebiel, H. Ott, D. Stalke, Trichloroaluminium Adducts of Iminoborens: Synthesis and Crystal Structures, Chem. Com., in Vorbereitung.

C. Matthes, M. Görth, U. Klingebiel, A. Ringe, J. Magull, Lithium- und Borsubstituierte Hydrazone und Ketazine, Z. Anorg. Allg. Chem., in Vorbereitung.

C.Matthes, U. Klingebiel, A. Ringe, J. Magull, Aminoadducts of Boriniumkations, Chem. Com., in Vorbereitung.

\section{Vorträge (*) und Poster $\left(^{* *}\right)$}

C. Matthes, U. Klingebiel

From Lithiumhalosilylamides to small and large Ring compounds, Iminosilenes and Iminosilanes $\left(^{\star}\right)$, 12. Deutscher Fluortag, Schmitten i. Taunus, September 2006.

C. Matthes, U. Klingebiel, S. Schmatz,

Intramolecular Rearrangement of Organosilyl Groups in Silylamines - A Joint Experimental-Theoretical Study- (**),

11th International Symposium on Inorganic Ring Systems i. Oulu, Juli/August 2006.

C. Matthes, U. Klingebiel, S. Schmatz, Intramolecular Rearrangement of Organosilyl Groups in Silylamines - A Joint Experimental-Theoretical Study- $\left.{ }^{* \star}\right)$,

1. Fakultätstag der Göttinger Chemie, i. Göttingen, 29. Juni 2007.

C. Matthes, U. Klingebiel, S. Schmatz,

Thermal and Anionic Rearrangements of Silylamines and -amides- $\left.{ }^{(\star *}\right)$, 4th European Silicon Days, i. Bath, September 2007.

C. Matthes, U. Klingebiel, S. Schmatz,

Thermal and Anionic Rearrangements of Silylamines and -amides- $\left.{ }^{\star \star}\right)$, Chemisches Kolloquium der GDCh, i. Ulm, September 2007. 


\section{Lebenslauf}

Am 08.05.1980 wurde ich als Sohn von Peter Matthes und seiner Ehefrau Sibylle Matthes, geb. Delitzsch, in Eisenach geboren.

Von 1986 - 1991 besuchte ich die 8. POS in Eisenach bzw. 114. POS in Dresden und wechselte anschließend auf das Ernst-Abbe-Gymnasium in Eisenach, wo ich am 09.07.1998 meine Reifeprüfung ablegte.

Vom November 1998 bis August 1999 leistete ich meinen Wehrdienst bei der 1. Gebirgsdivision ab.

Im Wintersemester 1999/2000 immatrikulierte ich mich an der Georg-AugustUniversität zu Göttingen in den Studienfächern Biologie und Chemie für das Lehramt an Gymnasien. Im Wintersemester 2000/2001 immatrikulierte ich mich im Erweiterungsfach Sport für das Lehramt an Gymnasien. Im Wintersemester 2003/2004 nahm ich am ERASMUS-Austauschprogramm teil und studierte Sciences et Techniques des Activités Physiques et Sportives in Nizza.

Nach der Anfertigung einer experimentellen Staatsexamensarbeit in der Anorganischen Chemie bei Prof. Dr. U. Klingebiel, bei der ich durch ein Stipendium des Verbandes der Chemischen Industrie gefördert wurde, schloss ich am 22.11.2005 das wissenschaftliche Studium in den Fächern Biologie und Chemie-, am 11.05.2006 im Erweiterungsfach Sport mit dem Ersten Staatsexamen ab.

Am 01.01.2006 begann ich unter Leitung von Prof. Dr. U. Klingebiel mit den Arbeiten der hier vorliegenden Dissertation.

Im Zeitraum 01.10.2002 - 31.07.2003 sowie 01.07.2004 - 21.11.2005 war ich als studentische Hilfskraft, vom 22.11.2005 - 14.06.2007 als wissenschaftliche Hilfskraft der Georg-August-Universität Göttingen beschäftigt. Seit dem 15.06.2007 bin ich als wissenschaftlicher Mitarbeiter an der Universität Göttingen angestellt. Zum 01.08.2007 war ich für ein Stipendium der Otto-Vahlbruch-Stiftung vorgesehen. Neben der Betreuung von Tutorien, Seminaren und Praktika als Assistent leitete ich im Zeitraum März 2006 bis August 2007 das Schulversuchspraktikum für Lehramtskandidaten. 